\title{
Thermal Plume Transport From Sand and Gravel Pits Potential Thermal Impacts on Cool-Water Streams
}

Jeffrey M. Markle, The University of Western Ontario

Supervisor: Robert Schincariol, The University of Western Ontario

A thesis submitted in partial fulfillment of the requirements for the Doctor of Philosophy degree in Geophysics

(C) Jeffrey M. Markle 2011

Follow this and additional works at: https://ir.lib.uwo.ca/etd

Part of the Hydrology Commons

\section{Recommended Citation}

Markle, Jeffrey M., "Thermal Plume Transport From Sand and Gravel Pits Potential Thermal Impacts on Cool-Water Streams" (2011). Electronic Thesis and Dissertation Repository. 360.

https://ir.lib.uwo.ca/etd/360

This Dissertation/Thesis is brought to you for free and open access by Scholarship@Western. It has been accepted for inclusion in Electronic Thesis and Dissertation Repository by an authorized administrator of Scholarship@Western. For more information, please contact wlswadmin@uwo.ca. 
(Thesis format: Integrated-Article)

Jeffrey M. Markle

Graduate Program

in

Geophysics

\begin{abstract}
A thesis submitted in partial fulfilment of the requirements for the degree of Doctor of Philosophy
\end{abstract}

The School of Graduate and Postdoctoral Studies

The University of Western Ontario London, Ontario, Canada

(c) Jeffrey M. Markle 2011 
THE UNIVERSITY OF WESTERN ONTARIO

School of Graduate and Postdoctoral Studies

\section{CERTIFICATE OF EXAMINATION}

$\underline{\text { Supervisor }}$

Dr. Robert A. Schincariol

Supervisory Committee

Dr. John Sass

Dr. R. Kerry Rowe $\underline{\text { Examiners }}$

Dr. Edward Sudicky

Dr. Kristy Tiampo

Dr. Stephen Hicock

Dr. Clare Robinson

The thesis by

\section{Jeffrey Michael Markle}

entitled:

\section{Thermal Plume Transport From Sand and Gravel Pits Potential Thermal Impacts on Cool-Water Streams}

is accepted in partial fulfillment of the requirements for the degree of

Doctor of Philosophy

\section{Date}

Chair of the Thesis Examination Board 


\section{Abstract}

Potential thermal impacts from below-water-table aggregate extraction on a coolwater stream were investigated by monitoring thermal plumes, moving through an unconfined glacial-outwash aquifer, and assessing their subsurface persistence. The growing demand for aggregate and increased pressure to pursue extraction in ecologically sensitive areas has driven the need for this work. During a 10-year period, ground and surface water temperatures were measured monthly, including two periods of intensive monitoring (22 months and 2.5 years). The aquifer hydraulic conductivity $(K)$ is quantified at the laboratory and field scale. The mean $K$ 's from the multi-scale tests depend on test-support volume and span two-orders of magnitude, $1.8 \times 10^{-4}$ to $1.7 \times 10^{-2}$ $\mathrm{m} \mathrm{s}^{-1}$. The apparent thermal conductivity $\lambda$ is characterized at an unprecedented level of detail by: (i) measuring the thermal conductivity of the soil solids, $\lambda_{\mathrm{s}}$ using the steadystate divided-bar apparatus and estimating conductivity from mineral composition; (ii) measuring the volumetric water content and porosity using cross-hole ground-penetrating radar; (iii) evaluating four models used to predict the apparent thermal conductivity, $\lambda$, of variably-saturated soils (iv) calculating the $\lambda$ field on a $0.25-\mathrm{m}$ square cell grid using measured data and the selected model, and (v) simulating thermal transport within the two-dimensional domain using a finite-element numerical model. The apparent thermal conductivity in the saturated aquifer ranges from 2.14 to $2.69 \mathrm{~W} \mathrm{~m}^{-1} \mathrm{~K}^{-1}$ with a mean of $2.42 \mathrm{~W} \mathrm{~m}^{-1} \mathrm{~K}^{-1}$. These measurement and model methods may be used at other sites to construct thermal conductivity distributions for similar glacial soils. The annual temperature amplitude in the pit is $10^{\circ} \mathrm{C}$ greater than the up gradient groundwater, resulting in alternating warm and cool plumes that persist in the aquifer for 11-months and migrate up to $250 \mathrm{~m}$ down gradient. The observed plume velocity $\left(1.2 \mathrm{~m} \mathrm{~d}^{-1}\right)$ lags the groundwater velocity $\left(2.8 \mathrm{~m} \mathrm{~d}^{-1}\right)$ due to thermal retardation. Using field data a conceptual model is developed, and implemented in a three-dimensional finite-element numerical model. While this work focused on plume migration, these results demonstrate that assessing impacts on the aquatic community requires an integrated, multi-disciplinary study. This work can guide such assessments. 


\section{Keywords}

Groundwater / surface water interaction, Heat transport, Thermal conductivity, Groundpenetrating radar, Brook trout, Hydraulic conductivity, Numerical modelling, Akaike's information criterion. 


\section{Co-Authorship}

I wish to acknowledge the following three co-authors: Dr. Robert Schincariol, Dr. John Sass, and Dr. John Molson.

Dr. John Molson developed the heat transport code, Heatflow, used in this work. He guided me in making a number of significant modifications to the code to adapt Heatflow to this study. He also reviewed chapter 2 and Appendix B, and provided constructive criticism and comments.

Dr. John Sass provided guidance on the thermal conductivity sample collection, preparation, and interpretation. He also arranged for the measurement of the solid fraction thermal conductivities by the USGS in Sacramento, CA. Dr. Sass made available his temperature logging system, and assisted with the collection of temperature profiles in the bedrock aquifer at 2 domestic water wells. Finally, Dr. Sass reviewed Chapter 2 and provided constructive criticism and comments.

Dr. Robert Schincariol provided guidance on the direction of all the research, and on the analysis and interpretation of the results presented in this thesis. He reviewed the entire document and provided numerous helpful suggestions and comments. 


\section{Acknowledgments}

I am grateful to Tom Sinclair, Township of Goderich, for providing access to the site, Dr. Kent Novakowski for providing drilling equipment in the initial phases of the investigation and assistance with pond temperature measurements; John McNeil and Greg Powers for their assistance with the field work; Dr. Ron Griffiths for providing his data on the macroinvertebrates, his insightful comments on stream ecology, and for his guidance on the appropriate use of the ANOVA analysis and multiple comparison tests; Fred Grubb, USGS, Sacramento, CA, for measuring the thermal conductivity of the aquifer solids with the divided-bar apparatus; Dr. David Aldridge for providing a copy of Pronto used to invert the GPR data; Dr. Hansreudi Maurer for providing an independent inversion of the GPR velocity data, and Dr.Yasushi Mori for providing the thermal conductivity value for the dry Tottori dune sand. I also wish to acknowledge the constructive comments provided by the anonymous reviews of the manuscripts that form Chapters 1 and 2. I wish to thank Mike Malhiot, Ontario Ministry of Natural Resources, for bring this interesting topic to my attention.

I would like to thank Dr. John Molson for providing so much of his time and guidance as I made changes to his finite-element numerical model Heatflow. He graciously answered my numerous questions on how parts of the code worked, and on several occasions he tolerated long e-mail exchanges as I tried to understand. Moreover, his experience with groundwater flow and transport modelling was invaluable when it came to deciding what physical processes were important and needed to be considered by the model, and what processes could be ignored or approximated.

Dr. John Sass kindly made available his years of experience with and knowledge in making measurements of thermal conductivity of unconsolidated sediments. He provided financial support and access to the USGS laboratory in Sacramento, CA., so that thermal conductivity measurements of the overburden materials could be made. He also graciously provided his borehole temperature logging system and assisted with measuring bedrock temperature profiles with the logging system.

I would like to thank Dr. Rob Schincariol for making it possible for me to 
undertake this endeavour. Not only did he provided me an unusually high level of financial support so that I could afford to return to school for two years, but also he took the chance that I would never finish this degree. His friendship and moral support throughout my studies are greatly appreciated.

I am indebted to Rob McLaren for providing copies of GridBuilder and Grok, and explaining how to use many of the important features of these programs. Without these programs, constructing the three-dimensional, finite-element grids would have been impossible. He also kindly made a number of modifications to GridBuilder.

Finally, I wish to thank my family. My wife Angela, for graciously taking on more duties while I pursued this work, for her support and her encouragement when I felt like giving up, and for helping me persevere at the end when I needed it. And my son Cole, for giving up so much of his time with me, and for his understanding and patience when I was not able to play.

This work was funded by the Department of Fisheries and Oceans Canada, the Ontario Aggregate Resources Corporation, the Province of Ontario, the Natural Science and Engineering Research Council of Canada through grants awarded to R. A. Schincariol, student grants from the Geological Society of America, the American Association of Petroleum Geologists, and scholarships through the Province of Ontario and Meloche Monnex and the Canadian Council of Professional Engineers. 


\section{Table of Contents}

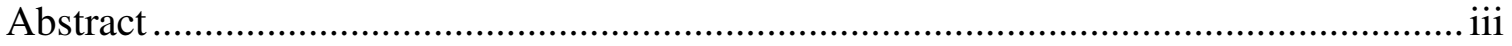

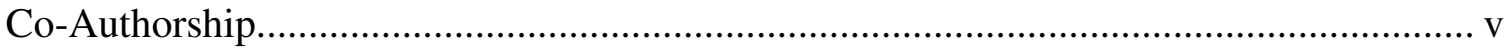

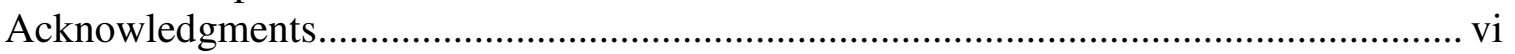

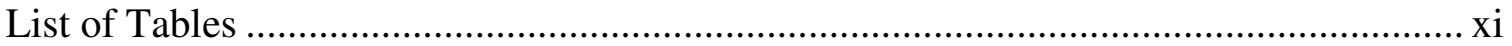

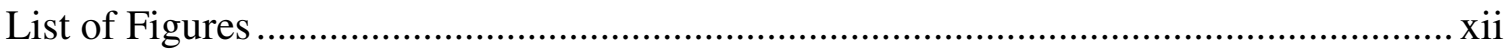

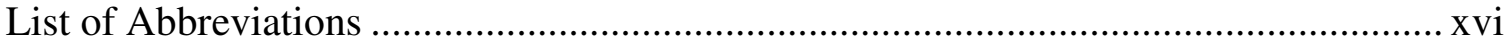

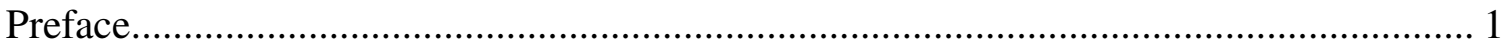

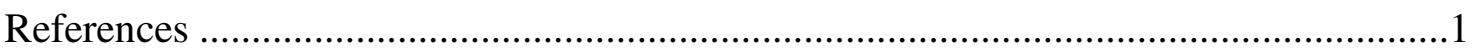

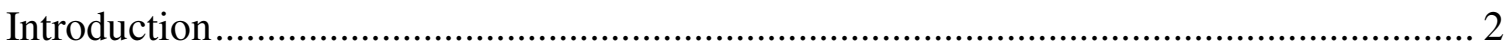

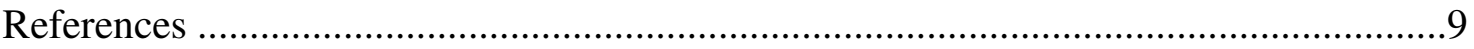

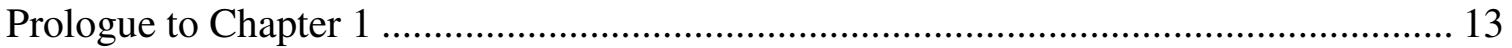

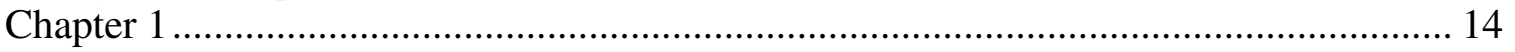

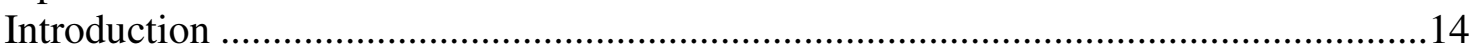

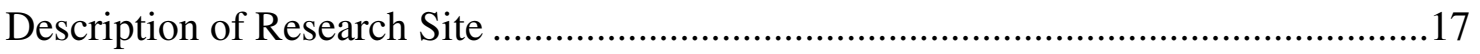

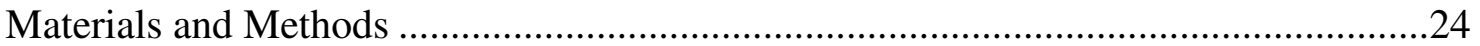

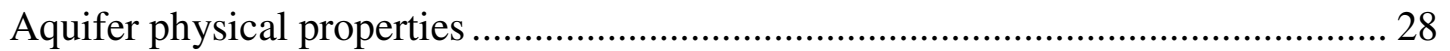

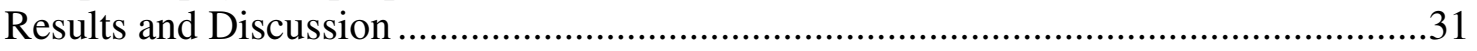

Aquifer hydraulic properties ................................................................................. 34

Aquifer thermal properties ............................................................................ 40

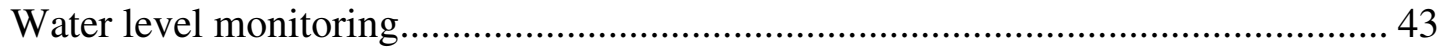

Air - ground surface temperature and heat flux................................................... 45

Pond water temperatures ................................................................................... 48

Thermal plume monitoring..................................................................................... 49

Groundwater - surface water interaction .......................................................... 52

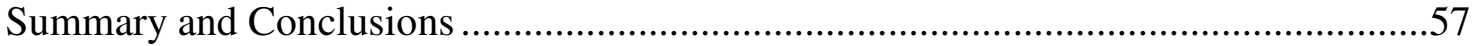

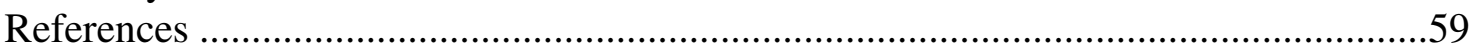

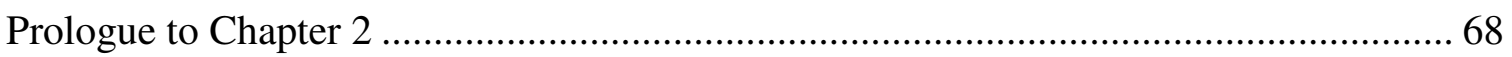

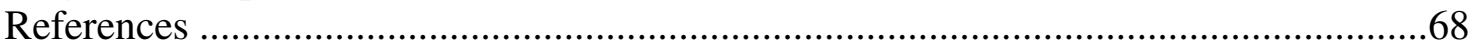

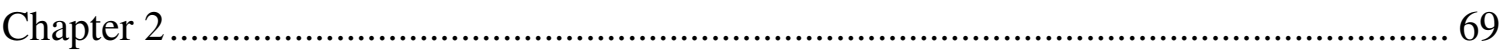

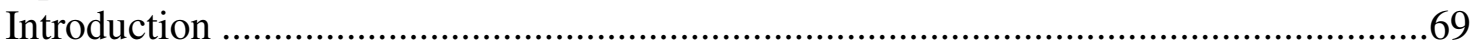

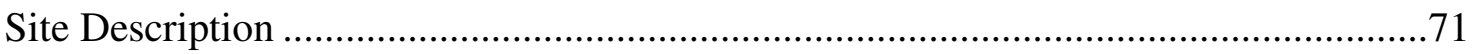

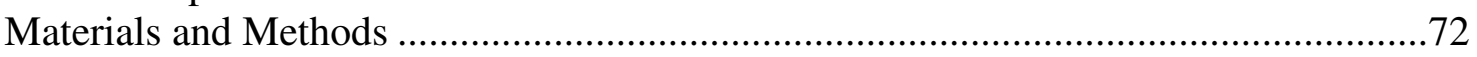

Field and laboratory methods ............................................................................... 72

Thermal conductivity of soil solids ................................................................... 72

Volumetric water content and porosity ……………........................................ 74

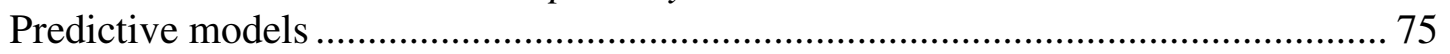

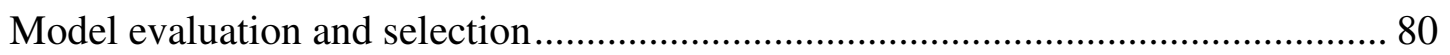

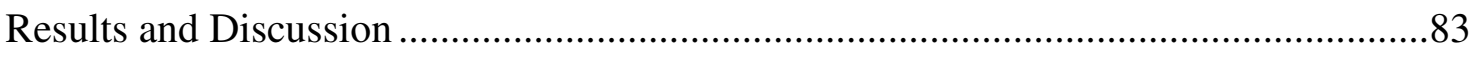

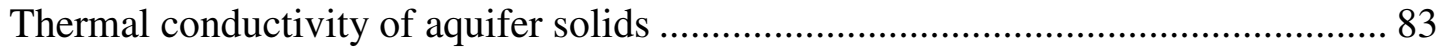

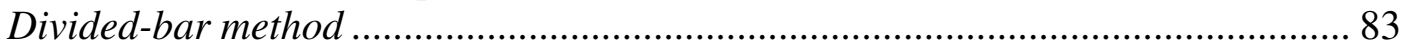

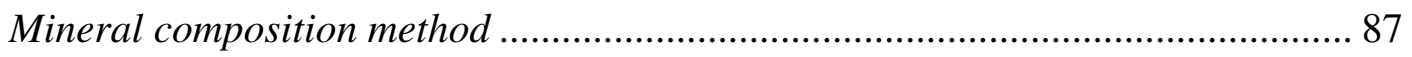

Volumetric water content and porosity …………………….................................. 91

Evaluation of candidate models ................................................................................ 93 
Model predicted apparent thermal conductivities .................................................. 97

Numerical simulations of heat transport ……………….................................... 100

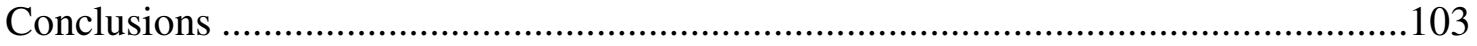

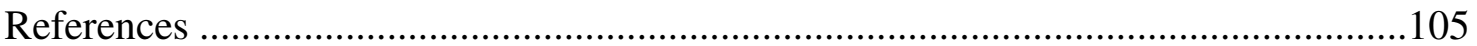

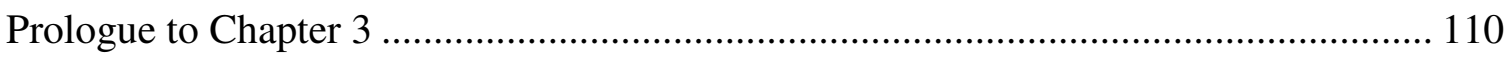

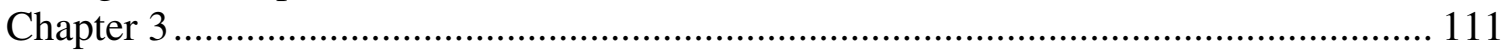

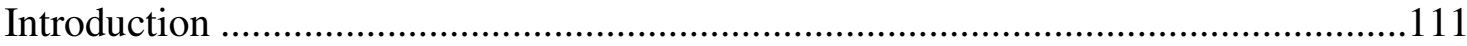

Geological and Hydrogeological Setting of Tricks Creek..........................................112

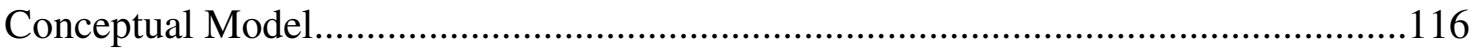

Governing Equations and Numerical Model .....................................................122

Verification of Modified Model ...................................................................... 124

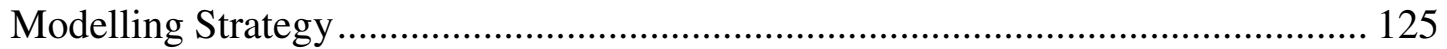

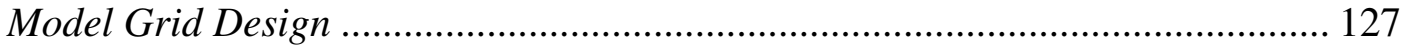

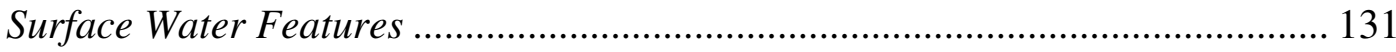

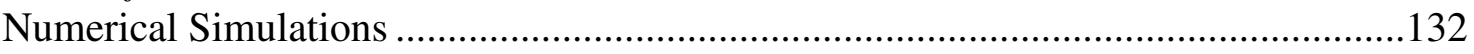

Hydraulic properties of the glacial outwash and till .............................................. 132

Hydraulic properties of the streams and ponds ..................................................... 133

Hydraulic boundary conditions .......................................................................... 134

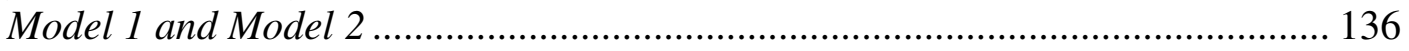

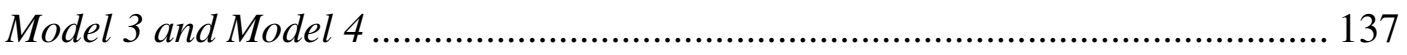

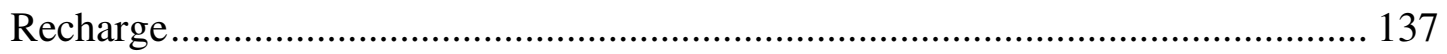

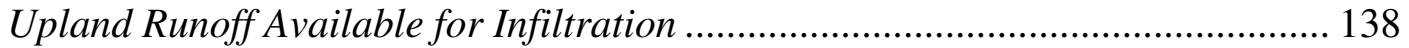

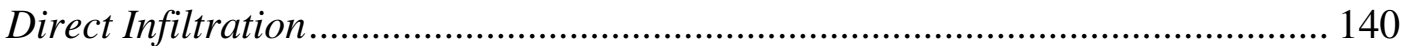

Thermal boundary conditions.............................................................................. 140

Thermal properties of the glacial outwash and till .............................................. 145

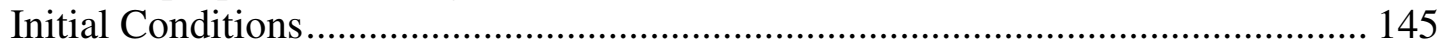

Steady-State and Transient Groundwater Flow Models..................................... 145

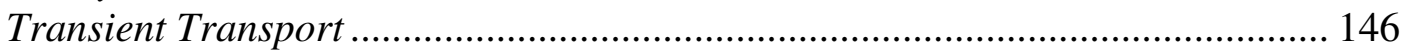

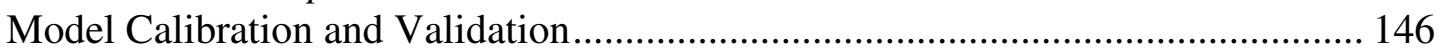

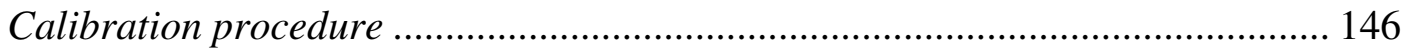

Final calibrated model parameters .............................................................. 149

Transient Groundwater Flow Model - Model 2................................................... 154

Transient Groundwater Flow and Transport - Model 3 and Model 4 ..................... 156

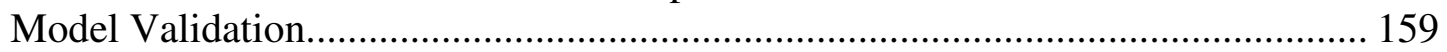

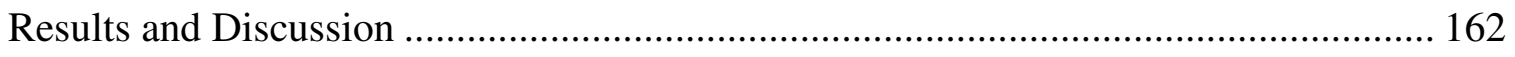

Influence of Hydraulic Conductivity................................................................. 162

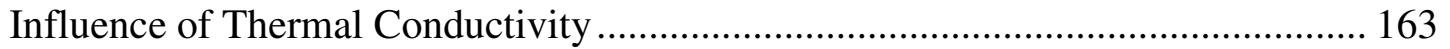

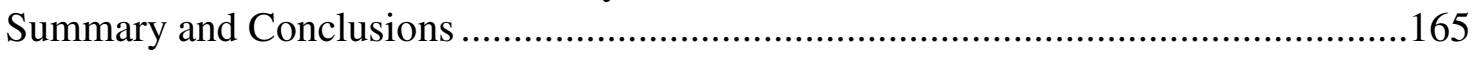

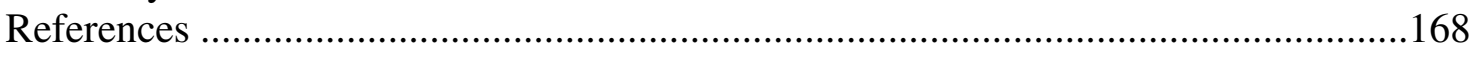

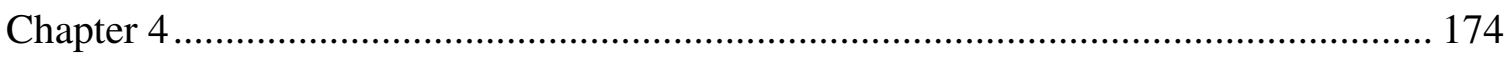

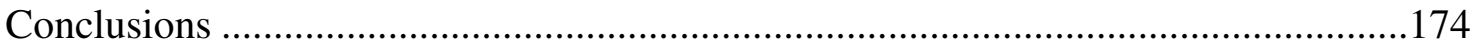

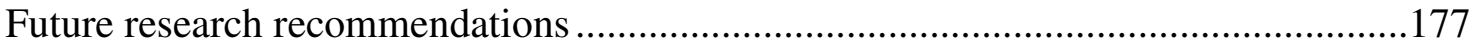

Appendix A: Watershed Hydrology and Climate .......................................................... 180

Appendix A1: Basin characteristics and stream discharge..........................................180

Appendix A2: Climate data (Temperature and Precipitation).......................................183 


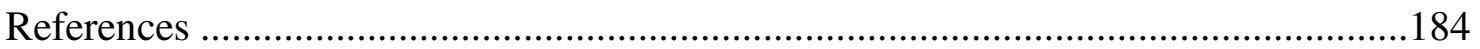

Appendix B: Numerical Method.......................................................................... 185

Appendix B1: Equations for Flow in Saturated Porous Media .....................................185

Appendix B2: Equations for Thermal Transport in Variably-Saturated Porous

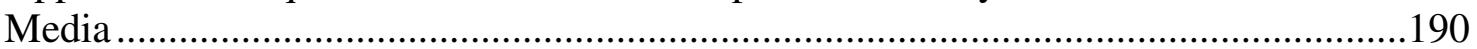

Appendix B3: Basis of the Galerkin Finite Element Method..........................................199

Appendix B4: Derivation of the Galerkin Finite Element Equation for

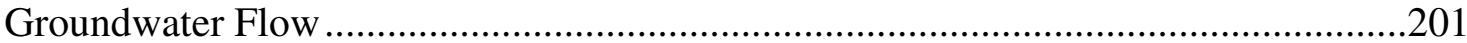

Appendix B5: Derivation of the Finite-Element Equations for Thermal Transport.....203

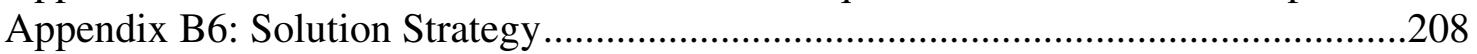

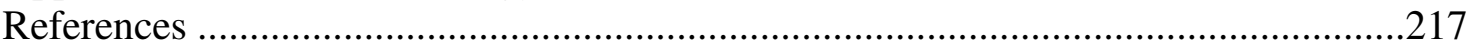

Appendix C: Benchmark Tests .......................................................................... 219

Appendix C1: One-dimensional heat conduction benchmark ………….....................219

Appendix C2: Checks of the consistency of the velocity approximation.....................223

Appendix C3: One-dimensional heat transport benchmark (Stallman, 1965)..............225

Appendix C4: Elder Short Heater Problem (Elder, 1967).........................................228

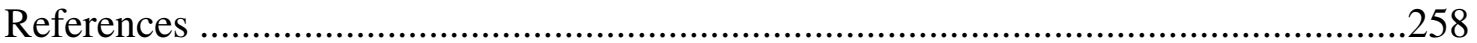

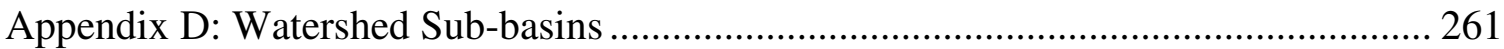

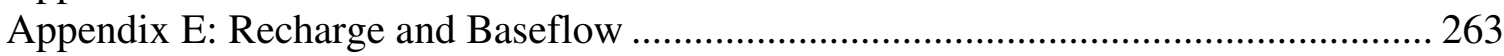

Appendix E1: Groundwater recharge ………………..............................................263

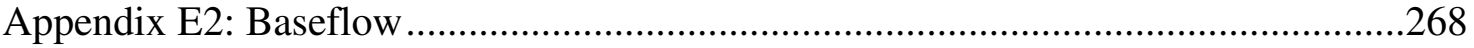

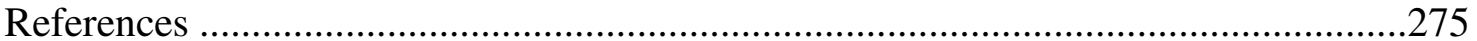

Appendix F: Bedrock Well Temperature Profiles ....................................................... 278

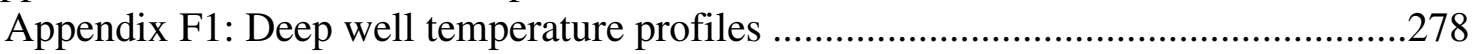

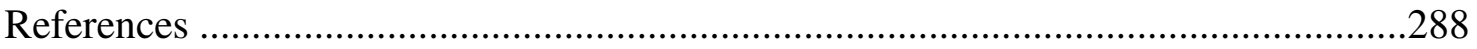

Appendix G: Calibration Targets .................................................................... 289

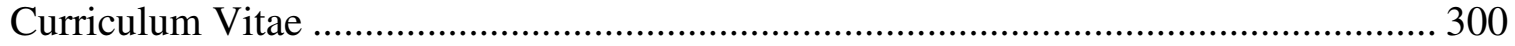




\section{List of Tables}

Table 1-1 Average mineral composition determined by X-ray diffraction and calculated using the integrated peak area method.

Table 1-2 Statistical summary of aquifer porosity, bulk density, and particle density measurements.

Table 1-3 Hydraulic conductivity $K$ of the outwash sand and gravel at $10^{\circ} \mathrm{C}$

Table 1-4 Values of specific heat and density for minerals found in the outwash and till.

Table 1-5 Average thermal properties of the porous medium solids.

Table 1-6 Lag times determined from cross-correlation of the periodic annual temperature variation in the pond with the periodic temperature variation in the aquifer down gradient of the pond.

Table 2-1 Criteria for evaluating the fit of the apparent thermal conductivity, estimated with the candidate models, to the measured thermal conductivity.

Table 2-2 Thermal conductivity of the porous media solids $\left(\lambda_{\mathrm{s}}\right)$ measured by the divided-bar apparatus and estimated from the mineral composition measured by X-ray diffraction.

Table 2-3 The ANOVA table (one-way unbalanced analysis) shows the between-groups and within-groups sources of variance for the thermal conductivity data. The null hypothesis, $H_{0}$, was rejected at $p$ $<0.05$.

Table 2-4 Mean value $\pm 95 \%$ confidence interval, standard deviation, and upper and lower quartiles of the measured thermal conductivity of the porous media solids for individual stratigraphic units and for the grouped sand units.

Table 2-5 Mineral composition of the porous media measured by X-ray diffraction and calculated using the integrated peak area method.

Table 2-6 Thermal conductivity of selected minerals and rocks. The underlined values are considered to be representative of the minerals in the glaciofluvial outwash sands and gravels.

Table 2-7 Parameter values input into the candidate models used to estimate the apparent thermal conductivity of the soil.

Table 2-8 Summary of Akaike Information Criterion $\left(\mathrm{AIC}_{\mathrm{C}}\right)$ measures between the apparent thermal conductivity predicted by the candidate models and the measured values reported in the literature...................................96

Table 3-1 Model domains considered in study.

Table 3-2 Boundary conditions for the groundwater flow and transport models. ...135

Table 3-3 Parameter values for the calibrated models. 150 


\section{List of Figures}

Figure 1-1 Study area location map and three-dimensional view of the Tricks

Creek watershed and wetland.

Figure 1-2 Quaternary geology for the Tricks Creek watershed (Fig. 1-2a, adapted from Cooper and Fitzgerald, 1977) and a geological crosssection (Fig. 1-2b).

Figure 1-3 Monitoring well locations and potentiometric surface in the glacialoutwash sand and gravel aquifer. The location of the detailed study area is shown by the dashed polygon.

Figure 1-4 Detailed study area and monitoring well location map. The initial pit configuration and monitoring well network in the first phase of the study is shown at the top (Fig. 1-4a). The pit configuration and monitoring well network during the second phase of the study is shown at the bottom (Fig. 1-4b) and the inset shows in detail the well locations. The hatched area indicates where the majority of the aggregate in the unsaturated zone has been excavated.

Figure 1-5

Geologic cross-section A-A' through the outwash deposit.

Figure 1-6

Distribution of $\ln (\mathrm{K})$ with the normal distribution curve shown by the thick line: (a) measured with the constant-head permeameter, (b) measured by in situ falling-head tests, and estimated from (c) grainsize analyses and (d) cross-hole ground-penetrating radar using the Kozeny-Carman equation.

Figure 1-7 Vertical profiles of hydraulic conductivity $K$ at 10EC. (a) $K_{\mathrm{v}}$ measured by permeameter. Values of $K$, estimated from the groundpenetrating radar (GPR) using the Kozeny-Carman empirical equation, are from a zero-offset profile completed between M17 and M19. These are representative of an average of the porous medium between the two wells. (b) $K$ estimated from grain size using the Kozeny-Carman equation. These are depth-averaged values from the cores for wells M17 and M19.

Figure 1-8 Relationship of hydraulic conductivity to scale of measurement in glacial outwash sand and gravel. The upper bound, shown by the dashed line, is assumed to be the hydraulic conductivity obtained from the constant-rate pumping test which has a support volume $>$ $10^{4} \mathrm{~m}^{3}$ (Schulz-Makuch et al., 1999).

Figure 1-9 Temperature profile for the up-gradient multilevel well M0. The depths of the thermistors are reported as metres bgs (below ground surface). For clarity, the temperatures for only seven of the 12 thermistors are shown.

Figure 1-10 Water levels in the pond and two down-gradient monitoring wells (M8 and M13). Precipitation is shown at the top.

Figure 1-11 Shown are: (top) Air temperature at $2 \mathrm{~m}$ ags (above ground surface), (middle) near-surface soil temperature $0.02 \mathrm{~m} \mathrm{bgs} \mathrm{(below} \mathrm{ground}$ surface), and (bottom) soil heat flux $0.05 \mathrm{~m}$ bgs at M14. 
Figure 1-12 Pond temperature profile at the surface and 4.5, 2.4, and $0.35 \mathrm{~m}$ apb (above the pond bottom).

Figure 1-13 Thermal plume migration along section A-A' for period January to December 1996.

Figure 1-14 Surface water temperature and groundwater temperature (1 $\mathrm{m}$ below the base of the streambed) at (a) MP1, (b) MP2, (c) MP4, and (d) MP5. Also shown are the spawning and incubation time periods and temperature criteria for brook trout. The top and bottom of the boxes define the optimum temperature range for spawning and incubation (Hokanson et al., 1973; Witzel and MacCrimmon, 1983), and the width defines the time period during which spawning and incubation commonly occur. The open triangles define the upper and lower temperatures at which brook trout commonly spawn in Ontario (Witzel and MacCrimmon, 1983). The open circle shows the ET50 (Hokanson et al., 1973; Scott and Crossman, 1973), which is the upper mean effective temperature giving the median sublethal response, and the $\times$ shows the LT50 (Hokanson et al., 1973) which is the temperature at which 50\% normal hatch occurs. Finally, the horizontal bars indicate the temperatures beyond which spawning and incubation are unsuccessful (Hokanson et al., 1973; Curry et al., 2002

Figure 2-1

Site location map.

Figure 2-2

Box-whisker plot of the measured thermal conductivity for the solid fraction of porous media grouped by stratigraphic unit. The caps at the end of each box indicate the minimum and maximum values, the box is defined by the lower and upper quartiles (25th and 75th percentiles), and the line in the centre of the box is the median. No outliers were present in the data.

Figure 2-3 (a) Two-dimensional volumetric water content tomogram for the saturated zone, calculated from the interwell velocity tomogram and the Burggeman-Hanai-Sen mixing formula (Sen et al., 1981; Feng and Sen, 1985). The white $X$ s indicate the transmitter and receiver station locations. (b) Measured water content (2) variation vs. depth between the two boreholes on the right side of the section. In the saturated zone, the water content is equal to the porosity $(\mathrm{N})$. Above the water table, the porosity was measured in the laboratory on core collected during drilling. The locations and measured values of the porosity are indicated by filled circles.

Figure 2-4

(a) Geological cross-section of the area over which the GPR (ground-penetrating radar) survey was completed. The three major stratigraphic units shown are gravel and sand, sand, and till. The vertical lines are the locations of the boreholes where core samples were collected during drilling and where the cross-hole GPR survey was conducted. The unsaturated zone is $\sim 1.5 \mathrm{~m}$ thick. (b) Envelope of the annual temperature variation (minimum observed temperature 
on the left and maximum on the right) and the temperature profile on 1 July for this section of the aquifer

Figure 2-5 Two-dimensional distribution of the apparent thermal conductivity, $\lambda\left(\mathrm{W} \mathrm{m}^{-1} \mathrm{~K}^{-1}\right)$ for the glaciofluvial outwash sand and gravel aquifer as calculated using the Campbell et al. (1994) model.

Figure 2-6 Simulated thermal plumes (left column) and corresponding temperature differences (right column) at $10 \mathrm{~d}$ using various thermal conductivity distributions. The thermal plumes on the left are shown using (a) a $10 \%$ decrease in the observed field, (b) the observed field, and (c) a $10 \%$ increase in the observed field. The corresponding temperature differences on the right were obtained by subtracting the plume temperatures simulated in the heterogeneous fields $(\mathrm{a}, \mathrm{b}$, and $\mathrm{c})$ from plume temperatures simulated using equivalent mean thermal conductivity fields in the saturated and unsaturated zones of (d) 2.16 and $1.57 \mathrm{~W} \mathrm{~m}^{-1} \mathrm{~K}^{-1}$, (e) 2.40 and 1.73 $\mathrm{W} \mathrm{m}{ }^{-1} \mathrm{~K}^{-1}$, and (f) 2.64 and $1.89 \mathrm{~W} \mathrm{~m}^{-1} \mathrm{~K}^{-1}$, respectively. 102

Figure 3-1 Study area location map and three-dimensional view of the Tricks Creek watershed and wetland.

Figure 3-2 Glacial-outwash aquifer within the Tricks Creek and Bridgewater Creek watersheds.

Figure 3-3 Study site location within the Tricks Creek watershed is shown by the dashed line. Also shown are the potentiometric surface and monitoring locations in the glacial-outwash aquifer.

Figure 3-4

Ground surface elevation overlain by the stream network (light blue), Bridgewater Creek watershed in the north (dark blue), and Tricks Creek watershed in the south (dark blue). The boundary of the glacial-outwash is shown by the dashed line.

Figure 3-5 Till surface elevation overlain by Bridgewater Creek watershed in the north (dark blue), and Tricks Creek watershed in the south (dark blue). The glacial-outwash channel is the distinct north-south trending feature incised into the surface of the till moraine. The white dots indicate the location of water well record and borehole data used to construct the till surface.

Figure 3-6

Bedrock surface elevation overlain by Bridgewater Creek watershed in the north (dark blue), and Tricks Creek watershed in the south (dark blue). The white dots indicate the location of water well record and borehole data used to construct the bedrock surface..... 120

Figure 3-7 Conceptual hydrogeological model of groundwater and surface water flow in a valley-filled glacial-outwash aquifer incised into a till moraine.

Figure 3-8 Three-dimensional model domains showing telescopic mesh refinement: a) model 1 - outwash aquifer spanning Tricks Creek and Bridgewater Creek watersheds; b) model 2 - Tricks Creek groundwater catchment; c) model 3 - sub-domain 1; and d) model 4 - final sub-domain focusing on detailed study area. 
Figure 3-9 Comparison of observed mean daily surface temperature at $0.02 \mathrm{~m}$ bgs with simplified model input sinusoidal temperature function.......... 143

Figure 3-10 Comparison of observed pond temperatures with simplified model input sinusoidal temperature functions (smooth lines).

Figure 3-11 Monitoring locations used for model calibration. Monitoring locations across the watershed are shown at the top and monitoring wells in detailed study area are shown at the bottom.

Figure 3-12 Residual plot of the calibrated steady-state groundwater flow model... 151

Figure 3-13 3-d domain for steady-state groundwater flow model. RMS $=0.18 \mathrm{~m}$ and RMS/total head drop $=0.02$. Black dots denote the location of calibration points

Figure 3-14 Hydraulic conductivity distribution, $-\operatorname{Ln}\left(K_{\mathrm{x}}\right) \mathrm{m} \mathrm{s}^{-1}$, for the final calibrated steady-state groundwater flow model.

Figure 3-15 Model 2 calibrated transient water levels for selected monitoring wells: TR-1 (northern portion of watershed); M0 to M8 (monitoring points in detailed study area); and TR-9 (southern portion of watershed). The black line is the simulation result, the blue is the observed water level recorded with a pressure transducer, and the red symbols are manual measurements. Daily recharge is shown at the top.

Figure 3-16

Model 4 calibrated locations.

Figure 3-17 Model 4 calibrated transient ground water temperatures for selected monitoring wells down gradient of the pond. The black lines show the observed temperatures and the red show the simulated temperatures. The top left shows the calibration plot for the monitoring well M2 located $10 \mathrm{~m}$ down gradient of the pond and the bottom right shows the calibration plot for a well M12 located $190 \mathrm{~m}$ down gradient.

Figure 3-18 Model validation points and the final pond configuration. 159

Figure 3-19 Model 4 transient ground water temperatures for the validation simulation at selected monitoring wells down gradient of the expanded pond. The black lines show the observed temperatures and the red show the simulated temperatures. The top left shows the temperature plot for the monitoring well M10 located $45 \mathrm{~m}$ down gradient of the pond and the bottom right shows the temperature plot for a well M12 located 100 m down gradient.

Figure 3-20 Comparison of simulated temperatures for $K$ decreased by 50\% (red lines) and $K$ increased by $50 \%$ (blue lines) at points in the upper (solid line), middle (long dash) and lower (short dash) portions of the aquifer.

Figure 3-21 Comparison of simulated temperatures for $\lambda=2.18 \mathrm{~W} \mathrm{~m}^{-1} \mathrm{~K}^{-1}$ (red lines) and $\lambda=2.66 \mathrm{~W} \mathrm{~m}^{-1} \mathrm{~K}^{-1}$ (blue lines) at points in the upper (solid line), middle (long dash) and lower (short dash) portions of the aquifer. 


\section{List of Abbreviations}

ags: above ground surface

AIC: Akaike's information criterion

$\mathrm{AIC}_{\mathrm{C}}$ : Akaike's information criterion for small sample sizes

ANOVA: analysis of variance

asl: above sea-level

bgs: below ground surface

BHS: Bruggeman-Hanai-Sen

CS: coarse sand

FS: fine sand

G: gravel

GPR: ground-penetrating radar

K-L: Kullback-Leibler

MCT: multiple comparison tests

MOP: multiple-offset profile

MS: medium sand

PVC: polyvinyl chloride

T: till

XRD: X-ray diffraction

ZOP: zero-offset profile

$n$ : number of data points

$r$ : correlation coefficient

RSS: residual sum of squares

SD: standard deviation

CI: confidence interval 


\section{Preface}

I have written this thesis in the integrated-article format such that each of the three chapters forms the basis of a journal manuscript. Chapter 1 was published in the Journal of Hydrology in 2007 (Markle and Schincariol, 2007) and provides a detailed description of the problem and the study site. This chapter summarizes much of the field data focusing mainly on the physical characteristics of the watershed, aquifer hydraulic properties and temperature measurements collected at the site in support of this study. It includes an analysis of the field information as it relates to groundwater flow and heat transport. Chapter 2 was published in the Soil Science Society of America Journal in 2006 (Markle, Schincariol, Sass, and Molson, 2006) and focuses on characterizing the thermal properties of the glacial outwash aquifer. Methods of measuring the thermal conductivity are compared, predictive models for estimating the thermal conductivity of porous sediments are evaluated, and a preferred model for calculating thermal conductivity is selected using the Akaike's Information Criterion. Chapter 3 has been prepared for submission to the Journal of Hydrology and presents the results from numerical simulations of transient fluid and heat flow through the glacial outwash aquifer.

I have provided several appendices. These include background information and additional analysis not typically included in a manuscript, but nevertheless, providing important details on methods used and analyses completed.

\section{References}

Markle, J.M., R.A. Schincariol, J.H. Sass, J.W. Molson. 2006. Characterizing the twodimensional thermal conductivity distribution in a sand and gravel aquifer. Soil Science Society of America 70:1281-1294.

Markle, J.M., R.A. Schincariol. 2007. Thermal plume transport from sand and gravel pits - Potential thermal impacts on cool water streams. Journal of Hydrology 338:174195. 


\section{Introduction}

In 2010, 205 million tonnes of sand and gravel, worth 1.5 billion dollars, were mined and used in Canada, one-third of which was used in the province of Ontario (NRC, 2010). Over 80 percent of all the aggregate produced in Ontario is used in the construction industry, making sand and gravel indispensable to infrastructure construction and maintenance (e.g., roads, bridges, railways, buildings, building foundations, etc.). In the last 20 years, 1.84 billion tonnes of sand and gravel have been produced in Ontario and production is projected to remain constant during the next 20 years (AltusGroup, 2009). However, within the last 10 years the licensing of replacement reserves has declined resulting in a 2.5 to one consumption to replacement ratio (MNR, 2010). This has led to a gradual depletion of our existing reserve base and, though not an immediate issue, at some point in the future this trend will need to be altered through a combination of a reduction in consumption and an increase in approval of replacement reserves. While sand and gravel is a fundamental element of urban development, increasingly the location of aggregate pits is becoming a concern. Opposition to new aggregate operations is rising resulting in the need to maximize the extraction at new and existing licensed sites. This has increased pressure to reduce or eliminate regulatory setbacks and pursue extraction in sensitive habitats. This pressure is further increased by the fact it may take 5 to 10 years and several million dollars to complete the regulatory approvals process and the associated studies required to evaluate the feasibility of proposed operations. Given the time and cost associated with the approval process, increasing the certainty surrounding the permitting process is important and requires that the influence extraction operations can have on the natural environment is well understood and that the methods for evaluating these potential effects are clear and well-founded.

There are numerous factors that need to be considered when evaluating the feasibility of establishing an aggregate operation. These include environmental factors such as nearby or adjacent natural features and water resources, as well as agriculture, traffic, noise, and social factors. The development of aggregate extraction operations involves extensive construction and results in disruptions to the surrounding natural 
environment. These developments have the potential for environmental degradation such as threats to groundwater and surface water resources; disruption of fish and wildlife habitat; and degradation of environmentally sensitive areas. Evaluation of the potential impacts requires a comprehensive understanding of the pre-development environmental conditions (i.e., flora, fauna, groundwater and surface water resources); the interactions of these various elements; and the effects that pit development will have on these environmental conditions. Without undertaking comprehensive assessments, establishing appropriate setbacks, and implementing pit designs which have consideration of the surrounding environment, unacceptable impacts to the existing natural environment may occur. In most jurisdictions in North America the approval process for new or expanding aggregate pits requires the proponent complete an environmental assessment of the potential impacts of the proposal. Traditionally, these environmental assessments have considered the presence of endangered or threatened flora and fauna and the potential effects on existing domestic groundwater supplies, groundwater and surface water levels, and discharge rates of nearby streams and rivers. More recently, several studies have shown that anthropogenic activities can alter groundwater and surface water temperatures causing measurable changes to the biotic communities. For example, forestry operations have been shown to alter the temperature of discharging groundwater changing the structure and density of both the macroinvertebrate and fish communities (e.g., Lynch and Rishel, 1984; Curry et al., 1995; Curry et al., 2002; Nislow and Lowe, 2006). Increases in discharging groundwater temperature were shown to alter all levels of the aquatic community including both the plant and benthic invertebrate communities, as well as changing the rates of organic matter decomposition and thus energy cycling (e.g., Vannote and Sweeney, 1980; Hogg and Williams, 1996; Lakly and McArthur, 2000; Taylor and Dykstra, 2005; Nislow and Lowe, 2006).

In Ontario, many of the top quality sand and gravel deposits formed in glacial outwash channels during the last glaciation. These outwash channels coincidentally give rise to, and are collocated with, woodlands, wetlands, and cool and cold-water streams which are important habitat for several species of cool-water fish and other flora and fauna. As a result, there has been speculation that aggregate extraction may alter the 
temperature of groundwater and nearby streams. The excavation of aggregate material below the water table involves removal of the forest cover and soil, followed by excavation of the unsaturated and saturated porous media. The result is a pond, often hectares in area, where forest cover existed previously. Removal of the forest cover and the unsaturated porous media exposes the water directly to the solar radiation (e.g., Deardorff, 1978; Kaufmann et al., 2003; Nitoiu and Beltrami, 2005) and eliminates the insulating effect of the unsaturated zone. This results in an energy transfer across the airwater interface of the pond that is many times larger than the energy transfer across the water table under forested conditions. As a result, the temperature of the water in the pond is different from the groundwater under forested conditions. In summer months, the water in the pond is much warmer and in the winter it is colder. Under the influence of the hydraulic gradient in the surrounding aquifer, the water in these ponds moves back into the groundwater system. Then it moves through the aquifer as a series of alternating warm and cool thermal plumes. If this thermally altered groundwater discharges before reaching background temperature, it may adversely affect the stream temperature and the aquatic biota in the discharge area. In response to this recognition, where cool or coldwater streams are present, approval agencies are requesting the proponent provide an evaluation of the potential thermal impacts from the proposed development. As this is a relatively new consideration, no comprehensive field assessments of the thermal impacts have been completed, and the factors that need to be considered and the methods to use in the assessments are not clear. This lack of clarity increases the uncertainty in the approvals process which can result in delays, unnecessary costs and conflict. The completion of scientifically sound and defensible assessments of required separation distances (setbacks) between the pit and nearby surface water features (i.e., creeks, streams, rivers and wetlands) is necessary to ensure that a balance is maintained between protecting the stream aquatic habitat and maximize the development potential of the sand and gravel resource.

The intent of this work is to further our understanding of the potential magnitude of the thermal alteration these operations may have on groundwater and nearby surface 
water; establish a framework that researchers and practitioners may use to design investigations integrating hydrogeology and stream ecology where impacts from thermal disturbances to groundwater and surface water are of concern; and to provide a basis to better inform conservation decisions and management of developments involving new or expanding aggregate extraction operations. To meet these objectives this research comprises two main elements. The first element is a detailed field investigation comprising the measurement of thermal plume movement from an aggregate pit under natural gradient conditions through an unconfined, glaciofluvial outwash sand and gravel aquifer in southwestern Ontario, Canada. The groundwater from this aquifer discharges to a wetland and stream, supporting a cool-water fishery. The second element is the application of a numerical model to simulate subsurface movement of the observed thermal plumes. By completing a detailed field investigation and collecting high quality data I hope to demonstrate appropriate methods for field investigations, and data collection, analysis and interpretation, as well as provide insight to the data density (spatial and temporal) necessary for these types of studies. Through the application of a numerical model the suitability and limitations of these models for their use in predicting subsurface movement of thermal plumes will be demonstrated. By furthering our understanding of the processes involved in subsurface plume movement in these settings and by demonstrating appropriate field and numerical assessment tools, I hope to increase certainty around estimating the potential thermal effects that pit development may have on groundwater and surface water resources, and the associated aquatic habitat.

This thesis is written in manuscript format and is divided into three chapters and seven appendices. The appendices provide detailed data, interpretation and analysis supporting the information presented in the chapters. Chapter 1 presents a description of the physical characteristics of the field site, the investigation methods, the aquifer physical properties, the groundwater and surface water levels, the interaction of groundwater and surface water at this site, and thermal plume migration observed along a two-dimensional section. This data is used to develop a conceptual site model. In Chapter 1 , the link between the groundwater - surface water interaction and the aquatic ecosystem 
is discussed. For example, the distribution of aquatic animals often reflects the timing and availability of groundwater discharge volume, distribution, and temperature in order to fulfill various life cycle requirements. The temperature of discharging groundwater is an important factor in determining if temperature-sensitive aquatic animals can be supported in groundwater discharge areas of river, streams, and wetlands (e.g., Garside, 1966; Hynes, 1983; Pugsley and Hynes, 1986; Elliott, 1994; Acornley, 1999; Power et al., 1999). Many temperature-sensitive species have narrow ranges of thermal tolerance. Areas of cool discharging groundwater moderate stream temperatures by cooling the stream in the summer and warming the stream in the winter. This provides areas of thermal refuge for aquatic animals and creates thermal conditions suitable for cool- and cold-water aquatic fauna. Several studies have shown that the moderating influence of discharging groundwater on surface water temperature enhances the spawning and nursery habitat potential for several species of trout (e.g., Cunjak and Power, 1986; Curry et al., 1995; Acornley, 1999; Baxter and McPhail, 1999). As will be discussed in Chapter 1 , even small changes in the timing and temperature of discharging groundwater can adversely affect temperature-sensitive species present in the discharge areas.

The movement of thermal plumes through the subsurface is controlled by the groundwater velocity and by the aquifer thermal properties. Key thermal properties are volumetric heat capacity and thermal conductivity. Chapter 2 focuses on characterizing and quantifying the thermal characteristics of the hydrostratigraphic units at the site which include a glacial outwash aquifer and an underlying till aquitard. Since an aquifer is a granular medium consisting of solid, liquid, and gaseous phases, the volumetric heat capacity and thermal conductivity will depend on the volumetric proportions of these components. The volumetric heat capacity of an aquifer can be calculated accurately from the heat capacities and volume fractions of these three phases (Smith, 1939, 1942; Woodside and Messmer, 1961; de Vries, 1963). The thermal conductivity, $\lambda$, is more complicated to calculate. It depends mainly on the mineral composition of the aquifer solids, and the porosity and degree of saturation. To a lesser extent, it depends on the bulk density of the aquifer solids, the shapes, sizes, and arrangement of the solid 
particles, the contact area between the particles, the interfacial contact between the solid and liquid phases, the vapor diffusion in the unsaturated pores, and the temperature and pressure conditions (Smith 1939, 1942; de Vries, 1963; Hopmans and Dane, 1986; Ewen and Thomas, 1987). There are several methods available for estimating the apparent thermal conductivity of unconsolidated porous media. The most common methods include the direct measurement of conductivity using probes (e.g., Lubimova et al., 1961; Sass et al., 1981; Bristow et al., 1994; Bristow, 1998) or thermal conductivity measurement cells (e.g., Sass, 1965; Sass et al., 1971), and the estimation of apparent conductivity using either empirical or mixing models (de Vries, 1963; Johansen, 1975; Gori, 1983; Campbell et al., 1994). In Chapter 2 the thermal conductivity of the solid fraction of the porous media is estimated using two approaches: direct measurement using the steady-state divided-bar apparatus (Sass et al., 1971); and estimation based on the mineralogy determined by X-ray diffraction methods. Empirical and mixing models are explored for estimating the apparent thermal conductivity of the variably-saturated porous media at the site (Johansen, 1975; de Vries, 1963; Gori, 1983; Campbell et al., 1994). A preferred mixing model is selected using an information-theoretic procedure (Akaike, 1973). The volumetric water content and porosity of the aquifer were estimated from a series of cross-hole GPR (ground-penetrating-radar) surveys completed across a 12.3-m-wide by 7.6-m-thick portion of the aquifer as well as measurements of porosity on recovered borehole cores. These data are used in the mixing model to calculate the apparent thermal conductivity and the mixing model is implemented in a finite-element numerical model.

Modelling the movement of these plumes is a primary method used for evaluating the potential impacts these operations may have on the stream temperature. In Chapter 3 , the conceptual site model developed in Chapter 1 along with the thermal properties of the porous media presented in Chapter 2 are implemented in a three-dimensional finiteelement numerical model used to simulate the coupled density-dependent groundwater flow and thermal energy transport of the observed thermal plumes through the subsurface. Transient flow and heat transport were simulated and compared to field 
observations. The calibrated model may be used to assess the important physical processes controlling heat transport in this shallow unconfined aquifer. The modeling method and model limitations are discussed. 


\section{References}

Acornley, R.M. 1999. Water temperature within spawning beds in two chalk streams and implications for salmoniod egg development. Hydrological Processes 13:439_ 446.

Akaike, H. 1973. Information theory as an extension of the maximum likelihood principle. p. 267-281. In B.N. Petrov and F. Csaki (ed.) Second Symposium in Information Theory. Akadémiai Kiad $\therefore$, Budapest.

Altus Group Economic Consulting. December 18, 2009. The State of the Aggregate Resource in Ontario Study SAROS), Paper 1 - Aggregate Consumption and Demand. MNR Number 52654. Queen's Printer for Ontario. pp. 26.

Baxter, J.S., J.D. McPhail. 1999. The influence of redd site selection, ground water upwelling, and over-winter incubation temperature on survival of bull trout (Salvelinus confluentus) from egg to alevin. Canadian Journal Zoology 77:12331239.

Bristow, K.L., G.J. Kluitenberg, R. Horton. 1994. Measurement of soil thermal properties with a dual-probe heat-pulse technique. Soil Science Society of America Journal 58:1288-1294.

Bristow, K.L. 1998. Measurement of thermal properties and water content of unsaturated soil using dual-probe heat-pulse probes. Agricultural and Forest Meteorology 89:75-84.

Campbell, G.S., J.D. Jr. Jungbauer, W.R. Bidlake, R.D. Hungerford. 1994. Predicting the effect of temperature on soil thermal conductivity. Soil Science 158:307-313.

Cunjak, R.A., G. Power. 1986. Winter habitat utilization by stream resident brook trout (Salvelinus fontinalis) and brown trout (Salmo trutta). Canadian Journal of Fisheries and Aquatic Science 43:1970-1981.

Curry, R.A., L.G. Noakes, G.E. Morgan. 1995. Ground water and the incubation and emergence of brook trout (Salvelinus fontinalis). Canadian Journal of Fisheries and Aquatic Sciences 52:1741-1749.

Curry R.A., D.A. Scruton, K.D. Clarke. 2002. The thermal regimes of brook trout incubation habitats and evidence of changes during forestry operations. Canadian Journal of Forest Research 32:1200-1207, doi:10.1139/X02-046.

Deardorff, J.W. 1978. Efficient prediction of ground surface temperature and moisture, with inclusion of a layer of vegetation. Journal of Geophysical Research 83:1889- 
1903.

de Vries, D.A. 1963. Thermal properties of soils. p. 210-235. In W.R. Van Wijk (ed.) Physics of Plant Environment. North-Holland, Amsterdam.

Elliott, J.M., 1994. Quantative Ecology and Brown trout. Oxford University Press: Oxford.

Ewen, J., H.R. Thomas. 1987. The thermal probe - a new method and its use on a saturated sand. Géotechnique 37:91-105.

Garside, E.T. 1966. Effects of oxygen in relation to temperature on the development of embryos of brook trout and rainbow trout. Journal of Fisheries Resources Board of Canada 23:1121-1134.

Gori, F. 1983. A theoretical model for predicting the effective thermal conductivity of unsaturated frozen soils. p. 363-368. In Proceedings of the Fourth International Conference on Permafrost. Fairbanks, Alaska, National Academy Press, Washington, DC.

Hogg, I.D., D.D. Williams. 1996. Response of stream invertebrates to a global-warming thermal regime: An ecosystem-level manipulation. Ecology 77:395-407.

Hopmans, J.W., J.H. Dane. 1986. Thermal conductivity of two porous media as a function of water content, temperature, and density. Soil Science 142:187-195.

Hynes, H.B.N. 1983. Groundwater and stream ecology. Hydrobiologia 100:93-99.

Johansen, O. 1975. Thermal conductivity of soils. Ph.D. diss. Trondheim, Norway, (CRREL Draft Translation 637, 1977), ADA 044002.

Kaufmann, R.K., L. Zhou, R.B. Mynemi, C.J. Tucker, D. Slayback, N.V. Shabanov. 2003. The effect of vegetation on surface temperature: A statistical analysis of NDVI and climate data. Journal of Geophysical Research Letters 30, 2147, doi:10.1029/2003GL018251, 2003.

Lakly, M.B., J.V. McArthur. 2000. Macroinvertebrate recovery of a post-thermal stream: habitat structure and biotic function. Ecological Engineering 15:S87-S100.

Lubimova, H.A., L.M. Lusova, F.V. Firsov, G.N. Starkova, A.P. Shushpanov. 1961. Determination of surface heat flow in Mazesta (USSR). Annals of Geophysics XIV:157-167. 
Lynch, J.A., G.B. Rishel. 1984. Thermal alteration of streams draining clearcut watersheds: quantification and biological implications. Hydrobiologia 111:161169.

Natural Resources Canada. Minerals and Metals Sector. accessed 1 July 2011. http://mmsd.mms.nrcan.gc.ca/stat-stat/prod-prod/ann-ann-eng.aspx

Nislow, K.H., W.H. Lowe. 2006. Influences of logging history and riparian forest characteristics on macroinvertebrates and brook trout (Salvelinus fontinalis) in headwater streams (New Hapmshire, U.S.A.), Freshwater Biology 51, 388-397, doi:10.1111/j.1365-2427.2005.01492.x.

Nitoiu, D., H. Beltrami. 2005. Subsurface thermal effects of land use changes. Journal of Geophysical Research 110, FO1005, doi:10.1029/2004JF000151.

Ontario Ministry of Natural Resources. February 2010. The State of the Aggregate Resource in Ontario Study, Consolidated Report. MNR Number 52624. Queen's Printer for Ontario. pp. 26.

Power, G., R.S. Brown, J.G. Imhof. 1999. Groundwater and fish - insights from northern North America. Hydrological Processes 13:401-422.

Pugsley, C.W., H.B.N. Hynes. 1986. Three-dimensional distribution of winter stonefly nymphs, allocapnia pygmaea, within the substrate of a southern Ontario river. Canadian Journal of Fisheries and Aquatic Sciences 43:1812-1817.

Sass, J.H. 1965. The thermal conductivity of fifteen feldspar specimens. Journal of Geophysical Research 70:4064-4065.

Sass, J.H., A.H. Lachenbruch, R.J. Munroe. 1971. Thermal conductivity of rocks from measurements on fragments and its application to heat-flow determinations. Journal of Geophysical Research 76:3391-3401.

Sass, J.H., J.P. Kennelly, Jr., W.E. Wendt, T.H. Moses, Jr., J.P. Ziagos. 1981. In-situ determination of heat flow in unconsolidated sediments. Geophysics 46:76-83.

Smith, W.O. 1939. Thermal conductivity in moist soils. Soil Science Society of America Proceedings 4:32-40.

Smith, W.O. 1942. The thermal conductivity of dry soil. Soil Science 53:435-459.

Taylor, B.R., A.N. Dykstra. 2005. Effects of hot ground water on a small swamp-stream in Nova Scotia, Canada. Hydrobiologia 545:129-144. 
Vannote, R.L., B.W. Sweeney. 1980. Geographic analysis of thermal equilibria: A conceptual model for evaluating the effect of natural and modified thermal regimes on aquatic insect communities, The American Naturalist 115:667-695.

Woodside, W., J.H. Messmer. 1961. Thermal conductivity of porous media. I. Unconsolidated sands. Journal of Applied Physics 32:1688-1706. 


\section{Prologue to Chapter 1}

Chapter 1 was published in the Journal of Hydrology in 2007 (Markle and Schincariol, 2007) and provides a detailed description of the problem considered in this research and describes the linkages between the development of aggregate resources, the thermal characteristics of the groundwater and surface water, and ultimately the influence of thermally altered groundwater on the stream ecosystem. In this chapter, I describe the physical characteristics of the Trick's Creek watershed and show how the hydrological functions are related to the hydrogeological setting of the watershed. Since the core question to be addressed by this research is "Can aggregate pits cause thermal groundwater plumes that may discharge to streams or rivers and adversely affect the instream ecosystem?", an effort is made in Chapter 1 to demonstrate the linkage between groundwater and the stream ecosystem.

A significant portion of the work completed for this research involved collecting field data. These data were collected mainly by drilling boreholes, collecting overburden samples, installing wells, and measuring groundwater and surface water levels and temperatures. Also, the aquifer physical properties were measured in the field and laboratory. These data form the basis for understanding the groundwater flow conditions in the Trick's Creek watershed and the interaction between groundwater and surface water in this watershed. These data are presented and described in this chapter. While the hydraulic properties of the aquifer are discussed in Chapter 1, it is in Chapter 2 where the thermal properties are discussed in detail. 


\section{Chapter 1}

\section{Introduction}

Groundwater discharge to streams and rivers exerts a fundamental influence on stream and river ecology (Elliott, 1994). The distribution of aquatic animals often reflects the timing and availability of groundwater discharge volume, distribution, and temperature in order to fulfill various life cycle requirements. Areas of cool discharging groundwater moderate stream temperatures by cooling the stream in the summer and warming the stream in the winter. Thus the diel and annual temperature fluctuations of the stream are subdued compared to air temperature. This provides areas of thermal refuge for aquatic animals and creates thermal conditions suitable for cool- and cold-water aquatic fauna. For example, many macroinvertebrates use the streambed substrate (the hyporheic zone) in areas of discharging groundwater for critical development stages and as refuge from adverse conditions within the stream (Hynes, 1983; Pugsley and Hynes, 1986). In particular, during the egg and pupal stages, these insects are not mobile and must tolerate the temperature conditions present within the streambed. Furthermore, aquatic insects do not acclimate generally, and some species have critical temperature thresholds above which acute mortality occurs (e.g., deKozlowski and Bunting, 1981; Quinn et al., 1994; Chadwick and Feminella, 2001). For some aquatic insects, there is evidence that increases in ambient winter stream temperatures result in early emergence, and diminished adult size and fecundity (Vannote and Sweeney, 1980; Hogg and Williams, 1996; Taylor and Dykstra, 2005). Reduced adult size affects reproduction potential and the competitive ability of the affected species within the aquatic community. As well, seasonal temperature patterns may be a critical factor in maintaining temporal segregation of competing macroinvertebrates and in determining the stability and number of species in a given community (Vannote and Sweeney, 1980). Moreover, temperature changes affecting one species within a macroinvertebrate community may have a positive or negative effect on other species within the community either directly or indirectly through complex interactions with processes involving energy cycling and organic matter 
dynamics (Lakly and McArthur, 2000; Nislow and Lowe, 2006). Such perturbations can affect the natural stream ecosystem, and these effects may even extend to the higher trophic levels such as fish.

Several studies have shown that the moderating influence of discharging groundwater on the surface water temperature enhances the spawning and nursery habitat potential for several species of trout (e.g., Cunjak and Power, 1986; Curry et al., 1995; Acornley, 1999; Baxter and McPhail, 1999). Thus, in addition to indirectly affecting higher trophic levels, such as fish, changes to groundwater and surface water temperatures may directly affect this group. In southern Ontario, Canada, native brook trout (Salvelinus fontinalis) spawn most commonly at stream temperatures between 6 and $8^{\circ} \mathrm{C}$ between early October and mid- to late November (Witzel and MacCrimmon, 1983). The fertilized eggs are buried in the gravel and sand substrate of the stream where they incubate and hatch as free embryos (alevins) in midwinter. The alevins remain in the streambed substrate and emerge in late March to early May (Power, 1980). Successful incubation relies on stable temperature conditions with the optimum incubation temperature between 6 and $8^{\circ} \mathrm{C}$ (Marten, 1992), and with 50\% mortality above $11.7^{\circ} \mathrm{C}$ (Hokanson et al., 1973). During the winter, the embryos are not mobile and must survive the thermal regime present in the hyporheic zone. During this period, groundwater discharge keeps stream temperatures above $0^{\circ} \mathrm{C}$, preventing anchor ice formation that would freeze the immobile embryos and alevins. Also, the water temperature greatly influences growth rates and development time decreases with increasing incubation temperatures (Garside, 1966). Early emergence, resulting from increased incubation temperatures, may increase exposure of fry to high-flow events, and alter the natural synchrony between emergence and the presence of a food supply (Noakes, 1989; Curry et al., 1995; Power et al., 1999). Thus, even small changes in the timing and temperature of discharging groundwater can adversely affect temperature-sensitive species present in the discharge areas.

The link between the groundwater and surface water interaction and the biotic system has recently become the topic of renewed interest as we move toward integrated watershed management (e.g., Hynes, 1983; Brunke and Gonser, 1997; Holmes, 2000; 
Hunt and Wilcox, 2003; Hunt et al., 2006). Several studies have shown that anthropogenic activities can alter groundwater and surface water temperatures causing measurable changes to the biotic communities. For example, forestry operations have been shown to alter the temperature of discharging groundwater changing the structure and density of both the macroinvertebrate and fish communities (e.g., Lynch and Rishel, 1984; Curry et al., 1995; Curry et al., 2002; Nislow and Lowe, 2006). Increases in discharging groundwater temperature were shown to alter all levels of the aquatic community including both the plant and benthic invertebrate communities, as well as changing the rates of organic matter decomposition and thus energy cycling (Taylor and Dykstra, 2005). Andrews and Anderson (1979) investigated the influence of a 200-ha power plant cooling lake in Wisconsin on the groundwater temperature. Their monitoring showed that the thermal disturbance to the groundwater was limited to $100 \mathrm{~m}$ down gradient from the lake. There has been speculation that aggregate extraction may alter the temperature of groundwater and nearby streams. Aggregate resources are commonly associated with glacial outwash deposits that also often support cool-water streams. The excavation of aggregate material below the water table involves removal of the forest cover and soil, followed by excavation of the unsaturated and saturated porous medium. The result is a pond, often hectares in area, where forest cover existed previously. The energy transfer across the air-water interface of the pond is many times larger than the energy transfer across the water table prior to extraction due to the removal of the vegetation and unsaturated zone. As a result, the temperature of the water in the pond is different from the pre-existing groundwater. In summer months, the water in the pond is much warmer and in the winter it is colder. Since the water in these ponds is simply exposed groundwater, it continues to move under the influence of the hydraulic gradient in the surrounding aquifer. Thus, the water moves back into the groundwater system down gradient of the ponds and through the aquifer as a series of alternating warm and cool thermal plumes. If sufficient subsurface travel time (to enable equilibration to background temperatures) is not provided prior to discharge to the stream, altered stream temperature may result. When temperature changes are sufficient, adverse impacts on thermally sensitive fauna, such as macroinvertebrates and cold-water fish communities, 
will result. The increasing demand for aggregates has created pressure to extract aggregates in areas associated with sensitive cool-water streams and wetlands. Thus the need to better understand the processes involved in the movement of these plumes is increasing.

In this study, we measured the groundwater temperature down gradient of an aggregate pit, during a 22-month period, using a dense network of monitoring wells. We quantified hydraulic properties of the glacial outwash aquifer at the laboratory and field scale, and characterized the aquifer thermal properties at a level of detail not previously reported in the literature. Our objectives were to measure thermal plume movement from an aggregate pit through an unconfined, sand and gravel aquifer, to quantify the distance across which thermal plumes persist in the subsurface under natural gradient conditions, and to establish a framework that researchers and practitioners may use to design investigations integrating hydrogeology and stream ecology where the impacts from thermal disturbances to the groundwater are of concern. Here we present a description of the field site, the investigation methods, the aquifer physical properties, the linkage between the groundwater and surface water, and thermal plume migration observed along a two-dimensional section. In this study, we focused on the migration of the thermal plumes through the aquifer, and we did not attempt to measure specific impacts on the biotic community (macroinvertebrates and fish) in a nearby creek. Such an assessment will require a highly integrated, multi-disciplinary study that is beyond the scope of this study; however, this work can be used to design and plan such an assessment at this or other locations, and to better inform conservation decisions and management of developments involving aggregate extraction.

\section{Description of Research Site}

A multi-year study of thermal plume migration through an unconfined aquifer was completed in the Tricks Creek watershed. The Tricks Creek watershed is a small headwater system $\left(26 \mathrm{~km}^{2}\right)$ located in southwestern Ontario, Canada, $\sim 180 \mathrm{~km}$ west of Toronto (Fig. 1-1). Tricks Creek lies within a wetland complex that encompasses an area 
of $\sim 105$ ha (4\% of watershed). The Tricks Creek wetland complex is a long, narrow, riverine type wetland. The headwaters for Tricks Creek originate in the northern portion of the wetland, flow to the south for approximately $4 \mathrm{~km}$, and discharge into the Bayfield River (43E34N55.5O N, 81E35N14O W). Two small tributaries enter the creek, one from the west and one from the east. We have extended the watershed to include the small creek to the west as it discharges to the Bayfield River at the same point as Tricks Creek. In the upper reach, Tricks Creek is slow moving and meanders through the wetland. The streambed is predominantly silt with thick organic sediments in most locations. In the lower reach, the creek is fast moving with alternating riffles and pools. Here the streambed is dominated by sand and gravel substrate with small cobbles and boulders. The creek has an average streambed gradient of $0.002 \mathrm{~m} \mathrm{~m}^{-1}$, and is generally small, ranging from 1 to $4 \mathrm{~m}$ in width, and from 0.25 to $1.5 \mathrm{~m}$ in depth. The mean annual flow is $0.45 \mathrm{~m}^{3} \mathrm{~s}^{-1}$, with peak flows of $2.3 \mathrm{~m}^{3} \mathrm{~s}^{-1}$. Under baseflow conditions (July to September), the average flow of $0.22 \mathrm{~m}^{3} \mathrm{~s}^{-1}$ is maintained mainly from groundwater discharge from the glacial outwash. Tricks Creek is characterized by cool water with a mean annual temperature of $12.9^{\circ} \mathrm{C}$. The maximum average daily water temperature is $22.2^{\circ} \mathrm{C}$ (July to August), and the minimum average daily water temperature is $3.2^{\circ} \mathrm{C}$ (February). These stream temperatures support resident brook and rainbow trout (Salmo Gairdneri) populations, and a diverse assemblage of macroinvertebrates, typical of a cool, headwater stream. Benthic invertebrate sampling at one location on Tricks Creek found between 23 and 37 different taxa comprising Ephemeroptera (mayflies), Plecoptera (stoneflies), Trichoptera (caddisflies), and Diptera (R. Griffiths, unpublished data). In order of abundance were Ephemeroptera (main taxa included epemerellidae, baetidae and leptophlebiidae mayflies), Diptera (main taxa included chironominae tanypodinae, and tipulidae), Plecoptera (main taxa included chloroperlidae, leuctridae and perlodidae stoneflies), and Trichoptera (main taxa included goeridae, hydropsychidae, lepidostomatidae and philopotamidae caddisflies). 


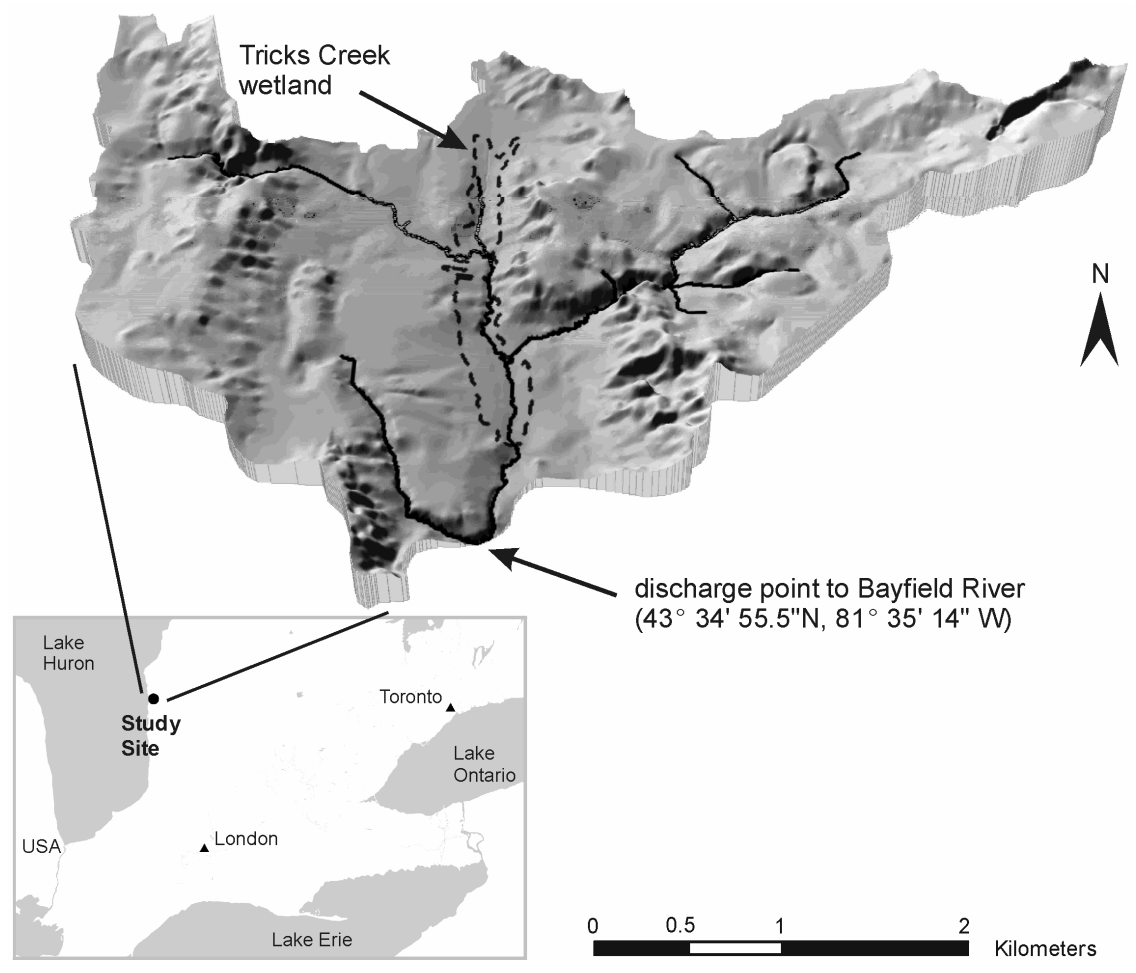

Figure 1-1 Study area location map and three-dimensional view of the Tricks Creek watershed and wetland.

The topography of the watershed is characterized by a broad north-south trending valley that slopes from an elevation of $260 \mathrm{~m}$ asl (above sea-level) at the northern boundary to $245.8 \mathrm{~m}$ asl at the southern point where the creek discharges to the Bayfield River. The valley is bounded on the east and west by gently rising hills which reach maximum elevations of $300 \mathrm{~m}$ asl on the east and $285 \mathrm{~m}$ asl on the west. Climatic records for the Goderich, Ontario weather station (43E46N N, 81E43N W), $19 \mathrm{~km}$ to the northwest, and the Blyth, Ontario weather station (43E43N N, 81E22N W), $15 \mathrm{~km}$ to the northeast, indicate the mean monthly temperature from 1971-2000 varied from a low of $-7.5^{\circ} \mathrm{C}$ in January to a high of $20.2^{\circ} \mathrm{C}$ in July. The average annual temperature is $6.8^{\circ} \mathrm{C}$ and the mean annual precipitation is $1184.3 \mathrm{~mm} \mathrm{yr}^{-1}$, with $350.4 \mathrm{~cm}$ as snow (Environment Canada, 2005).

Tricks Creek and the surrounding wetland complex lie on the eastern edge of a former glacial outwash channel that trends north-south. The outwash deposits are 
laterally confined to the west by the Wyoming Moraine and to the east by the Wawanosh Moraine, and unconformably overlie the contact between these moraines (Fig. 1-2a).

Formed 13,000 years before present during the Port Huron Stadial of the Wisconsinan glaciation, the outwash was deposited in a meltwater channel that was cut into the underlying sediments during the last glacial retreat, (Cooper and Fitzgerald, 1977; Barnett, 1992). The outwash is composed of poorly-sorted to well-sorted sandy gravel to gravely sand with cobbles, boulders, and traces of silt. It is mainly gravel and sand in the northern portion of the valley becoming progressively finer to the south where it is mainly fine to medium sand. The meltwater channel scoured into the tills most deeply along the western edge of the channel, depositing between 20 and $30 \mathrm{~m}$ of outwash material along this edge in the northern portion of the watershed and 10-15 $\mathrm{m}$ in the south. To the east, the channel was much shallower with approximately 5-10 $\mathrm{m}$ of sand and gravel beneath Tricks Creek (Fig. 1-2b). In the northern portion of the watershed a glacial outwash channel flowing from the northeast to the southwest merged with the main channel. The resulting increase in flow may have contributed to the larger amount of scouring of the till surface in this area and along the western edge of the main channel. This glaciofluvial outwash deposit represents a significant aggregate resource, and several sand and gravel extraction operations are active along the western edge of the wetland. 

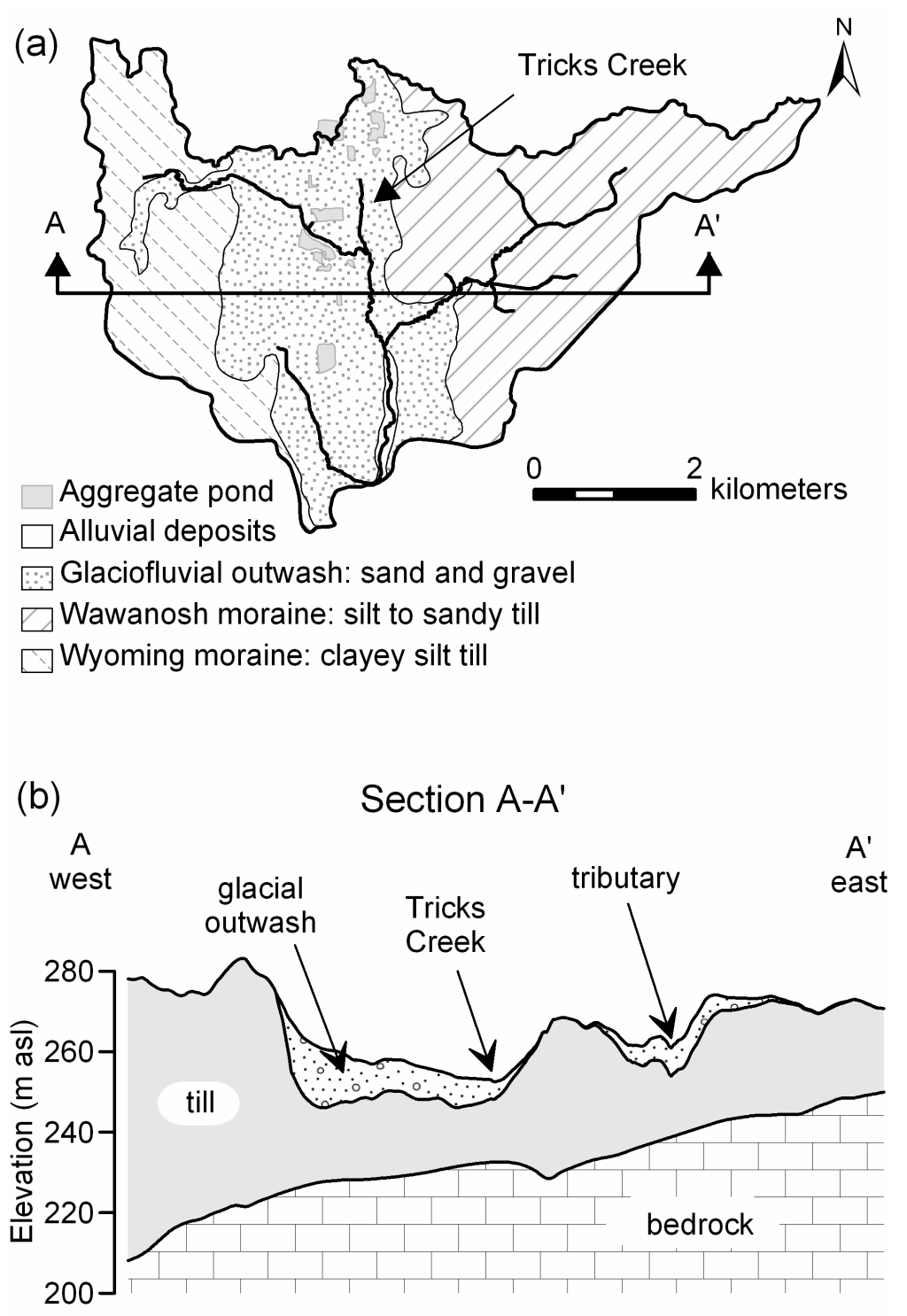

Figure 1-2 Quaternary geology for the Tricks Creek watershed (Fig. 1-2a, adapted from Cooper and Fitzgerald, 1977) and a geological cross-section (Fig. 1-2b).

Glacial moraine deposits are present across the entire study area. The Wyoming Moraine consists of the St. Joseph Till which is a moderately-stony clayey-silt till. The Wawanosh Moraine consists primarily of ice contact stratified drift deposits, and some occurrences of the silt to sandy silt Rannoch Till along the western margin of the moraine (Cooper and Fitzgerald, 1977). Within the watershed, the moraine deposits vary in thickness from approximately 25 to $45 \mathrm{~m}$. The moraine deposits sit unconformably on top 
of the truncated surface of the Middle Devonian Dundee Formation. Generally, the Dundee is a grey brownish grey, medium- to fine-grained, fossiliferous limestone and dolomitic limestone (Liberty and Bolton, 1971). Within the Tricks Creek catchment area, the bedrock surface slopes gently in a westerly direction $\left(0.006 \mathrm{~m} \mathrm{~m}^{-1}\right)$.

The glacial outwash forms an unconfined sand and gravel aquifer. The depth to the water table ranges between 0.2 and $4 \mathrm{~m}$ bgs (below ground surface). The regional groundwater flow direction within the catchment is from north to south where groundwater discharges to the Bayfield River (Fig. 1-3). The water table elevation at the top of the catchment is approximately $255.5 \mathrm{~m}$ asl, dropping to $244 \mathrm{~m}$ asl at the southern end of the catchment. In the north portion of the catchment, the horizontal hydraulic gradients are very low $\left(<0.001 \mathrm{~m} \mathrm{~m}^{-1}\right)$. Near Tricks Creek the gradients increase significantly and groundwater flows toward the creek. At the southern end of the catchment, the influence of the Bayfield River increases the horizontal gradient $(>0.005$ $\mathrm{m} \mathrm{m}^{-1}$ ). The unconfined aquifer is bounded laterally and from below by a till aquitard. To the north, the aquifer is bounded by a groundwater and surface water divide. The water table aquifer is recharged only by precipitation that falls within the catchment, and all the groundwater eventually discharges to Tricks Creek or the Bayfield River. 


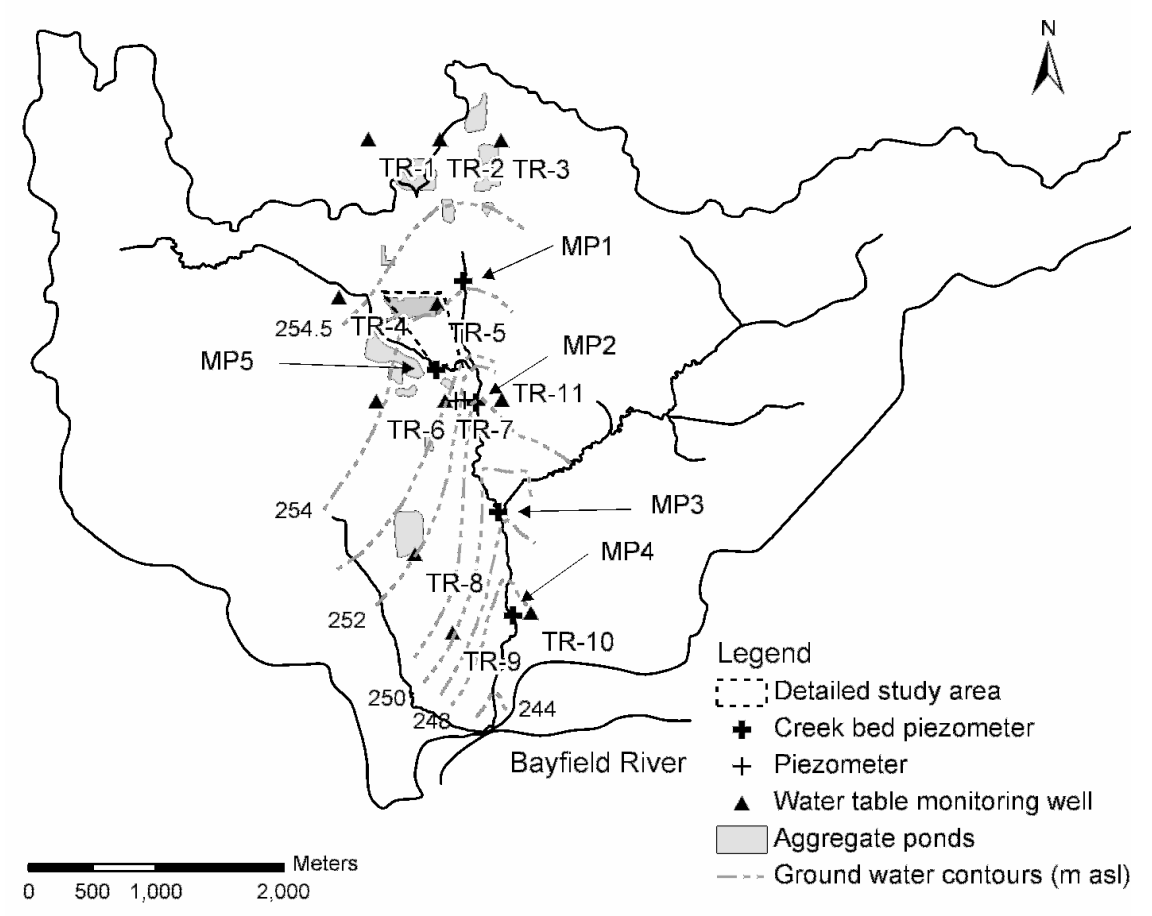

Figure 1-3 Monitoring well locations and potentiometric surface in the glacialoutwash sand and gravel aquifer. The location of the detailed study area is shown by the dashed polygon.

In this study, we observed thermal plume migration down gradient of an existing aggregate operation. The site is located approximately $1 / 3$ of the way down the catchment (Fig. 1-3). The sand and gravel extraction proceeds by first clearing the forest cover and removing the soil and porous medium above the water table, and then excavating the porous medium below the water table. The pond, created by the extraction operation, covers approximately 5 ha and varies between 4 and $6 \mathrm{~m}$ in depth. The pond is about 50 $\mathrm{m}$ west of the wetland and $100 \mathrm{~m}$ west of Tricks Creek. The regional potentiometric surface shows that the groundwater flow path from the pond to Tricks Creek is $\sim 750 \mathrm{~m}$ in length. The western tributary of Tricks Creek crosses this flow path about $400 \mathrm{~m}$ down gradient of the pit. The horizontal hydraulic gradient in the area of the pit is between 0.001 and $0.004 \mathrm{~m} \mathrm{~m}^{-1}$.

Temperature measurements made in the monitoring wells shown in Figure 1-3 demonstrate that the annual range of groundwater temperature in the outwash decreases 
with depth. At a depth of $3 \mathrm{~m}$ bgs, temperatures range from an average maximum of $12.7^{\circ} \mathrm{C}$ (September) to an average minimum of $5.0^{\circ} \mathrm{C}$ (March), with a mean annual temperature of $9.2^{\circ} \mathrm{C}$. This annual variation of approximately $7^{\circ} \mathrm{C}$ is the combined result of heat transport from the ground surface by conduction as well as by convection with water infiltrating through the unsaturated zone and into the saturated porous medium. From measurements at a series of streambed piezometers, we determined the mean annual temperature of groundwater discharging to Tricks Creek to be approximately $10.3^{\circ} \mathrm{C}$.

\section{Materials and Methods}

Twenty-nine monitoring wells were installed in two phases during the course of this study. In phase one, 17 wells (M0-M16) were installed and temperatures were monitored for a 22-month period (Fig. 1-4a). During this period only a limited amount of aggregate extraction occurred. Subsequent aggregate extraction resulted in the removal, or destruction, of the first $35 \mathrm{~m}$ of the monitoring well network (eight wells) as the downgradient pit face advanced south. Extraction ceased again and we installed 12 additional wells (M17-M28) for the purposes of a large-scale aquifer test and a cross-hole GPR (ground-penetrating radar) survey (Fig. 1-4b). 

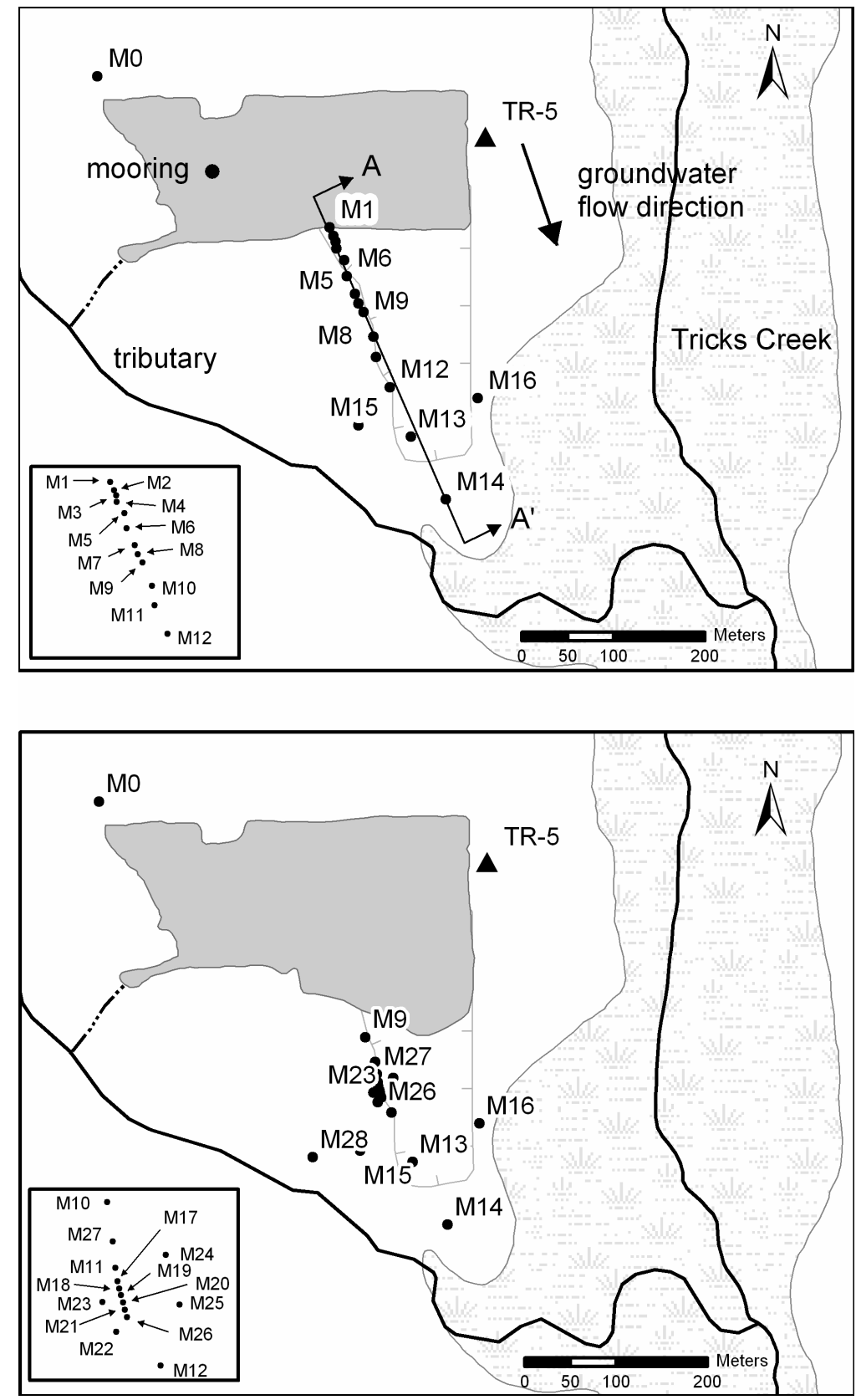

Figure 1-4 Detailed study area and monitoring well location map. The initial pit configuration and monitoring well network in the first phase of the study is shown at the top (Fig. 1-4a). The pit configuration and monitoring well network during the second phase of the study is shown at the bottom (Fig. 1-4b) and the inset shows in detail the well locations. The hatched area indicates where the majority of the aggregate in the unsaturated zone has been excavated. 


\section{Monitoring network and instrumentation}

In phase one, 15 multilevel piezometers and two water table monitoring wells (M8 and M15) were installed in February 1995 (Fig. 1-4a). We installed the majority of the wells within the first $150 \mathrm{~m}$ down gradient of the pond based on preliminary modeling results (Yang, 1995). The most distant well was $325 \mathrm{~m}$ down gradient. Well TR-5, along the east side of the pit, is a water table well installed in a preliminary investigation. Each multilevel piezometer consisted of 0.0095 -m outside diameter polyethylene tubes bundled to a $0.025-\mathrm{m}$-diameter PVC (polyvinyl chloride) pipe. A 0.10-m section of each polyethylene tube was perforated and covered with nylon screen. In most cases, a $0.3-$ 0.6-m section of the PVC pipe was also screened. These wells provide measurements at discrete points vertically within the aquifer. The water table monitoring wells were constructed with $0.05-\mathrm{m}$ diameter, schedule $40 \mathrm{PVC}$ pipe, and each well was screened across the aquifer with a 6.1-m long, 20 slot PVC screen. The abundance of cobbles precluded sample collection and we determined the stratigraphy based on observations during drilling.

We installed 12 additional monitoring wells in a second phase of drilling in January 2001 for use in a large-scale aquifer test and a GPR survey (Fig. 1-4b). These wells included eight multilevel wells, three water table wells (M17, M19, and M21), and one pumping well (M26). The pumping well was completed as a 0.2-m diameter, schedule 40 well with a 3-m long, 50 slot PVC screen. The well was backfilled with No. 3 sand up to $1 \mathrm{~m}$ above the well screen and natural collapse material above. For this phase of the drilling, the auger rig was equipped with a $1.8-\mathrm{m}$ long continuous soil sampler that facilitated the collection of relatively undisturbed soil cores with a $0.127-\mathrm{m}$ diameter. Core recovery rates with this method were approximately $50 \%$.

We monitored groundwater levels periodically at the array of piezometers. During the initial 22-month monitoring period, water levels were monitored continuously in the pond and at wells, M8 and M13, by data loggers with Druck pressure transducers. Groundwater temperature measurements were taken at an array of 128 thermistors installed in the wells (M0-M14) and connected to a series of Campbell Scientific, CR10 data loggers. The accuracy of the thermistors across the observed temperature range was 
$\pm 0.2^{\circ} \mathrm{C}$. Groundwater temperatures were recorded from July 1995 to May 1997 for wells M0-M14. In May 1997, the instrumentation in wells M1-M9 was removed due to the advance of the pit face. Monitoring continued at the remaining locations until December 1997. Monthly groundwater temperatures were collected manually at M15 and M16. Soil heat flux was monitored with two soil heat flux plates (HFT-3 Heat Flow Transducer manufactured by Radiation and Energy Balance Systems) installed 0.05 m BGS. One plate was located at M12 and the other was located at M14. Near surface atmospheric conditions were monitored near M14. Air temperature was monitored at heights of 1 and $2 \mathrm{~m}$ ags (above ground surface) with two Campbell Scientific 207 Temperature probes. Precipitation was recorded with a Geneq P-1000 tipping-bucket rain gauge during the spring, summer, and fall. During the winter, the snow depth was measured with a Campbell Scientific UDG01 Ultrasonic Depth Gauge from which an equivalent rainfall was estimated. Data loggers measured the sensors every $30 \mathrm{~s}$ and stored average values every $30 \mathrm{~min}$.

We established a mooring in the pond at which we measured surface water temperature at four elevations for a 51-month period, from June 1993 to August 1997 (Fig. 1-4). Three submersible Brankner temperature data loggers monitored the temperature at $0.35 \mathrm{~m}, 2.4 \mathrm{~m}$ and $4.5 \mathrm{~m}$ above the pond bottom. The water temperature near the pond surface was monitored by a thermistor floated $0.05 \mathrm{~m}$ below the water surface. The average annual depth of the pond at the mooring was $6 \mathrm{~m}$.

We installed mini piezometers in the creek bed at five locations (MP1-MP5) during the course of the study (Fig. 1-3). At each location, three piezometers were driven to depths ranging between 0.35 and $1.8 \mathrm{~m}$ below the streambed. We manually measured both surface water and groundwater levels and temperatures at these locations with two exceptions. At MP4 and MP5 water levels and temperatures were measured with a combination of electronic and manual methods. Two of these monitoring locations (MP2 and MP4) are brook trout redds (spawning and nursery sites). At the remaining sites the streambed substrates are fine grained making them unsuitable spawning locations. However, we monitored temperatures and water levels at these locations to observe the groundwater - surface water interactions at locations distributed along the creek. 


\section{Aquifer physical properties}

Glacial outwash deposits are heterogeneous deposits, having large variations in hydraulic conductivity (e.g., Hess et al., 1992; Rehfeldt et al., 1992; Anderson et al., 1999;

Oldenborger et al., 2003). Properly characterizing these spatial variations in hydraulic conductivity, at a scale appropriate for the field problem under investigation, is critical for understanding the movement of fluid and heat through the porous medium. To this end, we completed a multi-scale assessment of the hydraulic conductivity using a variety of direct and indirect methods. These included grain-size analysis and constant-head permeameter tests on the soil cores in the laboratory, falling-head tests in the monitoring wells, a cross-hole GPR survey, and a two-day constant discharge pumping test. We completed constant-head permeameter tests on 32 soil cores obtained from nine boreholes. The permeameter design allowed multiple values of hydraulic conductivity to be determined along the length of the core (Boggs et al., 1990; Wolf et al., 1991) and we

obtained estimates of permeability for 160 subsections of the core with a mean length of $0.1 \mathrm{~m}$. For each core, we applied five or more different head drops across the permeameter and measured the flow rate. The estimated permeability was determined from the average of these tests. Viscosity and density corrections were applied, and hydraulic conductivities are reported at $10^{\circ} \mathrm{C}$.

After permeameter testing was completed, we sectioned the cores from M17 and M19 at each pressure measurement point, and completed grain-size analysis and determined the porosity for each subsection. Estimates of the permeability were obtained with the empirical relation of Kozeny-Carman (Kozeny, 1927; Carman, 1937, 1956) given as

$$
K=\left(\frac{\rho_{w} g}{\mu_{w}}\right) \frac{\phi^{3}}{C S_{S}^{2}(1-\phi)^{2}},
$$

where $C$ is a factor accounting for the tortuosity of the pore spaces, $g$ is the gravitational acceleration, $\rho_{\mathrm{w}}$ is the density of water, $\mu_{\mathrm{w}}$ is the dynamic viscosity of water, and $S_{\mathrm{S}}$ is the 
specific surface area of the soil particles per unit volume of particles. Carman (1956) reported the value of $C$ to be $4.8 \pm 0.3$ for uniform spheres, and $C$ is generally taken to be 5. The specific surface area may be estimated from the particle size distribution curves (Carrier, 2003). We selected the Kozeny-Carman formula as it provides direct estimates of $K$ with porosities obtained from both the GPR survey and the soil cores, and it is preferred over other common relations such as Hazen (Carrier, 2003).

We conducted falling-head tests in 75 piezometers in the multilevel wells and eight water table wells, and determined the aquifer hydraulic conductivity by methods for high conductivity formations (Butler, 1997). A minimum of three tests, with different initial heads, were completed in each piezometer and the hydraulic conductivity was estimated from the average of the measured values.

The average hydraulic properties of the aquifer were obtained from an aquifer pump test. We conducted the test at a constant pumping rate of $0.0228 \mathrm{~m}^{3} \mathrm{~s}^{-1}$ for a period of $48 \mathrm{~h}$. Discharge was measured by a flow rate meter and totalizer. During the test, we measured water levels with a water level meter in 35 piezometers (14 multilevel wells), four water table monitoring wells, and six 0.025-m-diameter PVC piezometers. In addition, drawdown data were measured in the pumping well and nine piezometers with pressure transducers connected to data loggers. All the observation wells were within a 155-m radius of the pumping well. We applied Moench's (1997) method of analysis for pumping tests in anisotropic, unconfined aquifers to the data to obtain estimates of aquifer hydraulic properties.

We collected GPR tomographic data across six boreholes (M17-M21, and M26), which span a 12.3-m-wide by 7.6-m-thick portion of the aquifer, with a Sensors and Software (Mississauga, ON) pulseEKKO 100 GPR system equipped with borehole antennas (Fig. 1-4b). We completed surveys using two different antenna configurations. For the first configuration, ZOP (zero-offset profile), we moved the transmitter and receiver antennas down their respective boreholes in unison. For the second configuration, MOP (multiple-offset profile), we held the receiving antenna fixed and moved the transmitting antenna down the borehole until it had occupied all possible positions. Then the receiver was moved and the process repeated until both antennas had 
occupied all possible positions. We completed five ZOP surveys using $100 \mathrm{MHz}$ antennas and a pulser voltage of $400 \mathrm{~V}$. The borehole separation ranged from 4.85 to $13.53 \mathrm{~m}$, and the profiles began at the ground surface and proceeded to the bottom of the borehole with a step size of $0.125 \mathrm{~m}$. Six MOPs were completed with $200 \mathrm{MHz}$ antennas and a pulser voltage of $400 \mathrm{~V}$. The borehole separation ranged from 2.35 to $6.92 \mathrm{~m}$. For the MOPs we collected the first trace just below the water table and the final trace at the bottom of the borehole with a step size of $0.25 \mathrm{~m}$.

We inverted the travel times to reconstruct the horizontal and vertical structure with Pronto (Aldridge and Oldenburg, 1993), a curved-ray tracing tomographic inversion code. The inversions were performed with the domain divided into $0.25-\mathrm{m}$ square cells. The porosity for each cell block was estimated from the inverted slowness field with the BHS (Bruggeman-Hanai-Sen) mixing formula (Sen et al., 1981; Feng and Sen, 1985) given by

$$
\phi=\frac{\left(\varepsilon_{e f f}-\varepsilon_{s}\right)}{\left(\varepsilon_{w a}-\varepsilon_{s}\right)}\left(\frac{\varepsilon_{w a}}{\varepsilon_{e f f}}\right)^{C},
$$

where $\varepsilon_{\mathrm{wa}}$ and $\varepsilon_{\mathrm{s}}$ are the dielectric constants of water and air respectively, $\varepsilon_{\mathrm{eff}}$ is the measured dielectric constant of the saturated soil, and $C$ is a shape factor $(1 / 3$ for spherical grains). Using the GPR-determined porosities and the Kozeny-Carman equation (Eq. (1.1)), we estimated the aquifer hydraulic conductivity.

In addition to GPR-determined porosity, measurements of aquifer porosity, as well as bulk density and soil particle density were made for 31 subsections of soil cores obtained from boreholes M17 and M19. The bulk density was estimated from the measured dimensions and dry mass of the core segments. The sample porosity was taken to be the average of the porosity estimated from the difference in mass between saturated and oven dried samples, and the porosity estimated from the measured sample volume and mean grain density.

The heat capacity of the aquifer solids $C_{\mathrm{s}}$ can be determined from the known 
mineral composition using

$$
C_{s}=\sum_{i=1}^{N} f_{i}\left(c_{s} \rho_{s}\right)_{i}
$$

where $N$ is the number of mineral phases, $f_{i}$ is the volume fraction of the mineral phase $i$, and $c_{\mathrm{s}}$ and $\rho_{\mathrm{s}}$ are the specific heat and density of each mineral phase, respectively. The heat capacity of a variably-saturated porous medium $C_{0}$ can be determined by

$$
C_{0}=(1-S) \phi c_{a} \rho_{a}+S \phi c_{w} \rho_{w}+(1-\phi) C_{s},
$$

where $S$ is the saturation level, $c_{\mathrm{a}}$ and $\rho_{\mathrm{a}}$ are the specific heat and density of air, and $c_{\mathrm{w}}$ and $\rho_{\mathrm{w}}$ are the specific heat and density of water. For temperatures ranging from 0 to $50^{\circ} \mathrm{C}$, the heat capacity of the air phase is negligible relative to the water and solids (Luckner and Schestakow, 1991), and the first term of Eq. (1.4) is generally ignored.

We measured the thermal conductivity of the aquifer solids $\lambda_{\mathrm{s}}$ in the laboratory on 41 samples using the divided-bar apparatus (Sass et al., 1971). On a subset of 27 samples, we determined the mineralogy by XRD (X-ray diffraction) techniques. From the known mineral compositions, we estimated the bulk thermal conductivity for the aquifer solids $\lambda_{\mathrm{s}}$, having $n$ mineral components with a volume fraction $x_{i}$ and conductivity $\lambda_{i}$, using the geometric mean equation given by

$$
\lambda_{s}=\prod_{i=1}^{n}\left(\lambda_{i}^{x_{i}}\right) .
$$

\section{Results and Discussion}

Figure 1-5 is a geological cross-section from the pit south along the monitoring well network. The ground surface elevation varies along the section as the result of the 
extraction activity. In the first $50 \mathrm{~m}$ down gradient of the pond, most of the unsaturated sand and gravel have been excavated, and the top soil has been removed in area up to 290 $\mathrm{m}$ down gradient of the pond. As well, between 200 and $290 \mathrm{~m}$, the unsaturated sand and gravel has been removed (Fig. 1-4a and Fig. 1-5). Surface extraction has not proceeded beyond $300 \mathrm{~m}$ down gradient of the pond edge. Along the section, approximately $6 \mathrm{~m}$ of gravel and sand overlie the till. The majority of the material is undifferentiated gravel and sand with boulders and cobbles. A 1- to 2-m-thick layer of medium to coarse sand overlies the till along a portion of the section with the occasional lense of medium to coarse sand found within the gravel. From the grain-size analyses, the material ranges from poorly-sorted to well-sorted gravel and sand with little or no fines (generally $<5 \%$ silt). Over $65 \%$ of the material recovered was gravel with the remaining being fine to coarse sand. The geometric mean particle diameter (Shiozawa and Campbell, 1991), $d_{\mathrm{g}}$, for the gravel ranges from 4.3 to $19.0 \mathrm{~mm}$ with an average value of $11.3 \mathrm{~mm}$. For the sand, $d_{\mathrm{g}}$ ranges from 0.3 to $8.9 \mathrm{~mm}$ with an average value of $4.2 \mathrm{~mm}$. The mineral composition of the 27 outwash samples (Table 1-1) is primarily calcite, dolomite, quartz, and plagioclase feldspar (Markle et al., 2006). Some samples contain hornblende, illite, montmorillonite, and chlorite or possibly kaolinite in minor quantities ( $<5 \%$ total).

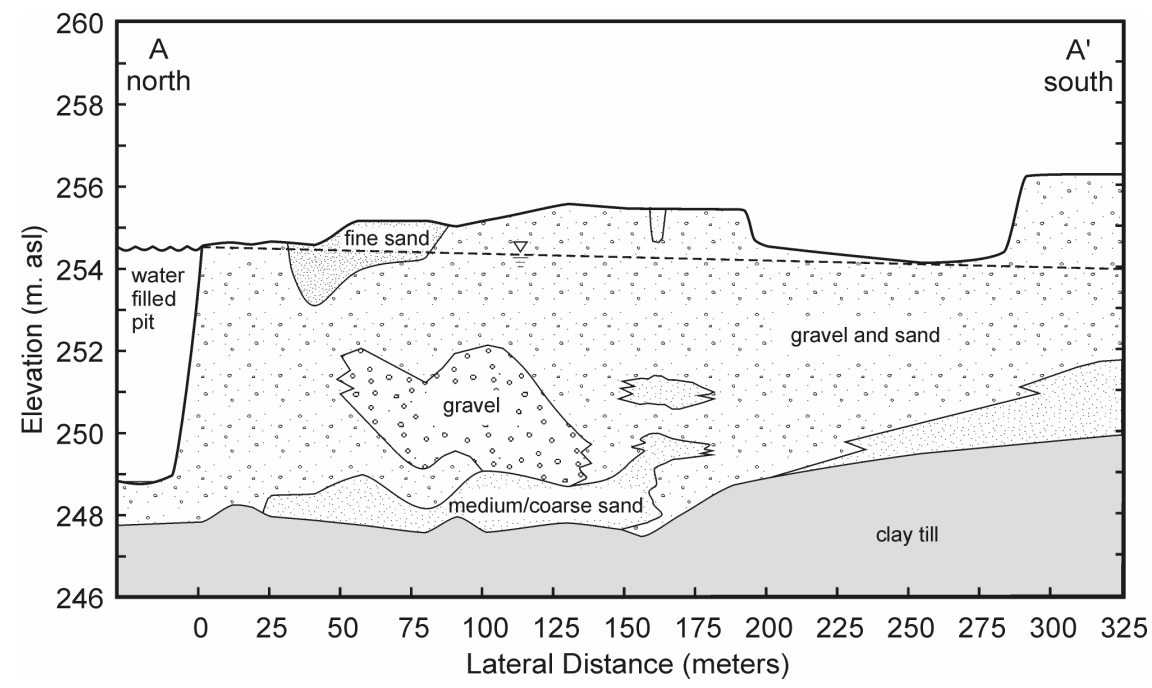

Figure 1-5 Geologic cross-section A-A' through the outwash deposit. 
The porosity of the aquifer ranges from 0.18 to 0.39 (Table 1-2). GPR-determined porosities compare well with porosities measured on the aquifer cores. The mean and standard deviation of the GPR-determined porosities were $0.294 \pm 0.002$ and $0.031 \mathrm{~m}^{3} \mathrm{~m}^{-}$ ${ }^{3}$, respectively, and the mean and standard deviation of the core-determined porosities were $0.274 \pm 0.022$ and $0.057 \mathrm{~m}^{3} \mathrm{~m}^{-3}$, respectively. The mean porosity from the core segments is $7 \%$ lower than that determined from the GPR. This difference may be due to consolidation during core extraction (Wolf et al., 1991) or under representation of higher porosity layers in the recovered core. Therefore, we assume a porosity of 0.29 . We found the porosity was not correlated to stratigraphic units, but rather it varied with depth. We believe the variation may be related to small changes in the depositional environment which occurred as the vertical sequence of aquifer material was deposited. The mean bulk density of the 29 cores was $1.92 \pm 0.058 \mathrm{~g} \mathrm{~cm}^{-3}$, and the standard deviation of the measurements was $0.160 \mathrm{~g} \mathrm{~cm}^{-3}$. The mean and standard deviation of the particle density measurements were $2.64 \pm 0.033 \mathrm{~g} \mathrm{~cm}^{-3}$ and $0.090 \mathrm{~g} \mathrm{~cm}^{-3}$, respectively.

Table 1-1 Average mineral composition determined by X-ray diffraction and calculated using the integrated peak area method.

\begin{tabular}{|c|c|c|c|}
\hline \multirow[b]{2}{*}{ Mineral } & \multicolumn{3}{|c|}{ Mineralogical composition (average volumetric fraction $†$ ) } \\
\hline & Gravel, $n=6$ & Fine to coarse sand, $n=17$ & Till, $n=3$ \\
\hline Calcite & $0.44 \pm 0.07$ & $0.29 \pm 0.04$ & $0.32 \pm 0.04$ \\
\hline Dolomite & $0.36 \pm 0.05$ & $0.36 \pm 0.04$ & $0.40 \pm 0.09$ \\
\hline Anorthite & $0.02 \pm 0.04$ & $0.08 \pm 0.04$ & $0.04 \pm 0.03$ \\
\hline Hornblende & $0.006 \pm 0.005$ & $0.006 \pm 0.002$ & $0.003 \pm 0.003$ \\
\hline Quartz & $0.17 \pm 0.04$ & $0.25 \pm 0.06$ & $0.22 \pm 0.07$ \\
\hline Illite & $0.005 \ddagger$ & 0.003 & $0.009 \pm 0.006$ \\
\hline Montmorillonite & & $0.004 \pm 0.002$ & \\
\hline Chlorite/kaolinite & $0.0014 \pm 0.002$ & $0.001 \pm 0.001$ & $0.0032 \pm 0.003$ \\
\hline
\end{tabular}

Table 1-2 Statistical summary of aquifer porosity, bulk density, and particle density measurements.

\begin{tabular}{|c|c|c|c|c|c|c|c|}
\hline \multirow[t]{2}{*}{ Data source } & \multirow{2}{*}{$\begin{array}{c}\text { Number of } \\
\text { values } n\end{array}$} & \multicolumn{2}{|c|}{ Porosity N } & \multicolumn{2}{|c|}{ Bulk density $\rho_{b}$} & \multicolumn{2}{|c|}{ Specific gravity } \\
\hline & & Mean \pm C.I. $\dagger$ & $S D \ddagger$ & Mean \pm C.I. & $S D$ & Mean \pm C.I. & $S D$ \\
\hline $\begin{array}{l}\text { Core sections } \\
\text { GPR }\end{array}$ & $\begin{array}{c}29 \\
1164\end{array}$ & $\begin{array}{l}\mathrm{m}^{3} \\
0.274 \pm 0.022 \\
0.294 \pm 0.002\end{array}$ & $\begin{array}{l}0.057 \\
0.031\end{array}$ & $\begin{array}{l}\mathrm{g} \mathrm{cn} \\
1.92 \pm 0.058\end{array}$ & 0.160 & $2.64 \pm 0.033$ & 0.090 \\
\hline
\end{tabular}




\section{Aquifer hydraulic properties}

Estimates of hydraulic conductivity for the outwash sand and gravel range from $10^{-5}$ to $2.7 \times 10^{-2} \mathrm{~m} \mathrm{~s}^{-1}$ (Table 1-3). The hydraulic conductivity for the till ranges from $10^{-9}$ to $10^{-}$ ${ }^{11} \mathrm{~m} \mathrm{~s}^{-1}$ based on field-based falling-head tests. For the outwash, the hydraulic conductivity values are lognormally distributed (Fig. 1-6), and the geometric mean hydraulic conductivity, determined by permeameter and grain-size analyses, agrees well with values measured in the field by falling-head tests and cross-hole GPR with the MOP configuration (Table 1-3). We used Student's t-test to compare the mean hydraulic conductivities for the gravel and the sand, obtained by permeameter, grain size, and GPR. No significant difference $(\alpha=0.05)$ between the mean hydraulic conductivities was found. The horizontal hydraulic conductivity determined by GPR with the ZOP configuration was $1.5 \times 10^{-3} \mathrm{~m} \mathrm{~s}^{-1}$, and the horizontal hydraulic conductivity estimated from the constant discharge test was $1.7 \times 10^{-2} \mathrm{~m} \mathrm{~s}^{-1}$. The ratio of horizontal to vertical hydraulic conductivity determined from the pumping test was $5: 1$, which is within the range of anisotropy ratios reported for other sand and gravel aquifers: 7:1 to 17:1 for an alluvial terrace deposit (Boggs et al., 1990); 2:1 to 5:1 for glacial outwash (Hess et al., 1991), and 1.6:1 for glacial outwash (Moench, 2004). The variation in hydraulic conductivity with depth is shown in Figure 1-7. The vertical hydraulic conductivity, measured by the permeameter, varies across two-orders of magnitude (Fig. 1-7a) while estimates from grain-size analysis, which are isotropic values, vary across one-order of magnitude (Fig. 1-7b). Both profiles show that the hydraulic conductivity increases with depth. Also, the GPR-determined hydraulic conductivities show a step increase at a depth of $4.5 \mathrm{~m}$, but have significantly less variation than the permeameter or grain-size values. 
Table 1-3

Hydraulic conductivity $K$ of the outwash sand and gravel at $10^{\circ} \mathrm{C}$.

\begin{tabular}{|c|c|c|c|c|c|c|}
\hline Test Method & $\begin{array}{c}\text { Number of } \\
\text { values } \\
n\end{array}$ & $\begin{array}{c}\text { Geometric } \\
\text { mean hydraulic } \\
\text { conductivity } K\end{array}$ & $\begin{array}{c}\text { Variance } \\
\ln (K)\end{array}$ & Minimum & Maximum & $\begin{array}{c}\text { Estimated } \\
\text { support } \\
\text { volume } V\end{array}$ \\
\hline Grain size $\dagger$ & 60 & $5.2 \times 10^{-4}$ & $\mathrm{~m} \mathrm{~s}^{-1}$ & $4.9 \times 10^{-5}$ & $5.0 \times 10^{-3}$ & $\begin{array}{c}\mathrm{m}^{3} \\
1.24 \times 10^{-3}\end{array}$ \\
\hline Permeameter & 158 & $4.1 \times 10^{-4}$ & 1.927 & $1.0 \times 10^{-5}$ & $2.7 \times 10^{-2}$ & $1.24 \times 10^{-3}$ \\
\hline Falling-head test $§$ & 75 & $1.8 \times 10^{-4}$ & 0.127 & $1.6 \times 10^{-5}$ & $2.9 \times 10^{-4}$ & $9.8 \times 10^{-4}$ \\
\hline Falling-head test $t[$ & 8 & $9.1 \times 10^{-4}$ & 1.551 & $1.9 \times 10^{-4}$ & $9.0 \times 10^{-3}$ & $7.0 \times 10^{-3}$ \\
\hline Ground-penetrating radar, MOP\# & 1249 & $6.0 \times 10^{-4}$ & 0.287 & $1.7 \times 10^{-4}$ & $2.1 \times 10^{-3}$ & 1.2 \\
\hline Ground-penetrating radar, $\mathrm{ZOP} \dagger \dagger$ & 169 & $1.5 \times 10^{-3}$ & 0.202 & $5.7 \times 10^{-4}$ & $4.0 \times 10^{-3}$ & 17.8 \\
\hline Heat plume & 1 & $7.8 \times 10^{-3} \dagger \ddagger$ & & & & $4.0 \times 10^{2} \S \S$ \\
\hline 2-day pumping test & 1 & $1.7 \times 10^{-2}$ & & & & $1.36 \times 10^{4}$ \\
\hline \multicolumn{7}{|c|}{$\dagger K$ was estimated using the Kozeny-Carman empirical equation. } \\
\hline \multicolumn{7}{|c|}{$\$$ The estimated $K$ is representative of the vertical hydraulic conductivity. } \\
\hline \multicolumn{7}{|c|}{$\S$ Tests completed in 0.0095 -m-diameter piezometers. } \\
\hline \multicolumn{7}{|c|}{ II Tests completed in 0.05 -m-diameters wells. } \\
\hline \multirow{2}{*}{\multicolumn{7}{|c|}{$\begin{array}{l}\text { \# } K \text { estimated from the MOP (multiple-offset-profiles) for } 0.25 \text {-m square cells using the Kozeny-Carman empirical equation. } \\
\dagger \dagger K \text { estimated from the ZOP (zero-offset-profiles) using the Kozeny-Carman empirical equation. }\end{array}$}} \\
\hline & & & & & & \\
\hline \multicolumn{7}{|c|}{$\ddagger \ddagger K$ estimated from cross-correlation of the observed thermal plumes, with a porosity of 0.29 and hydraulic gradient of 0.0012 . } \\
\hline \multicolumn{7}{|c|}{$\S \S$ Support volume estimated by the length of aquifer considered in the cross-correlation analysis, the average thickness of the } \\
\hline
\end{tabular}
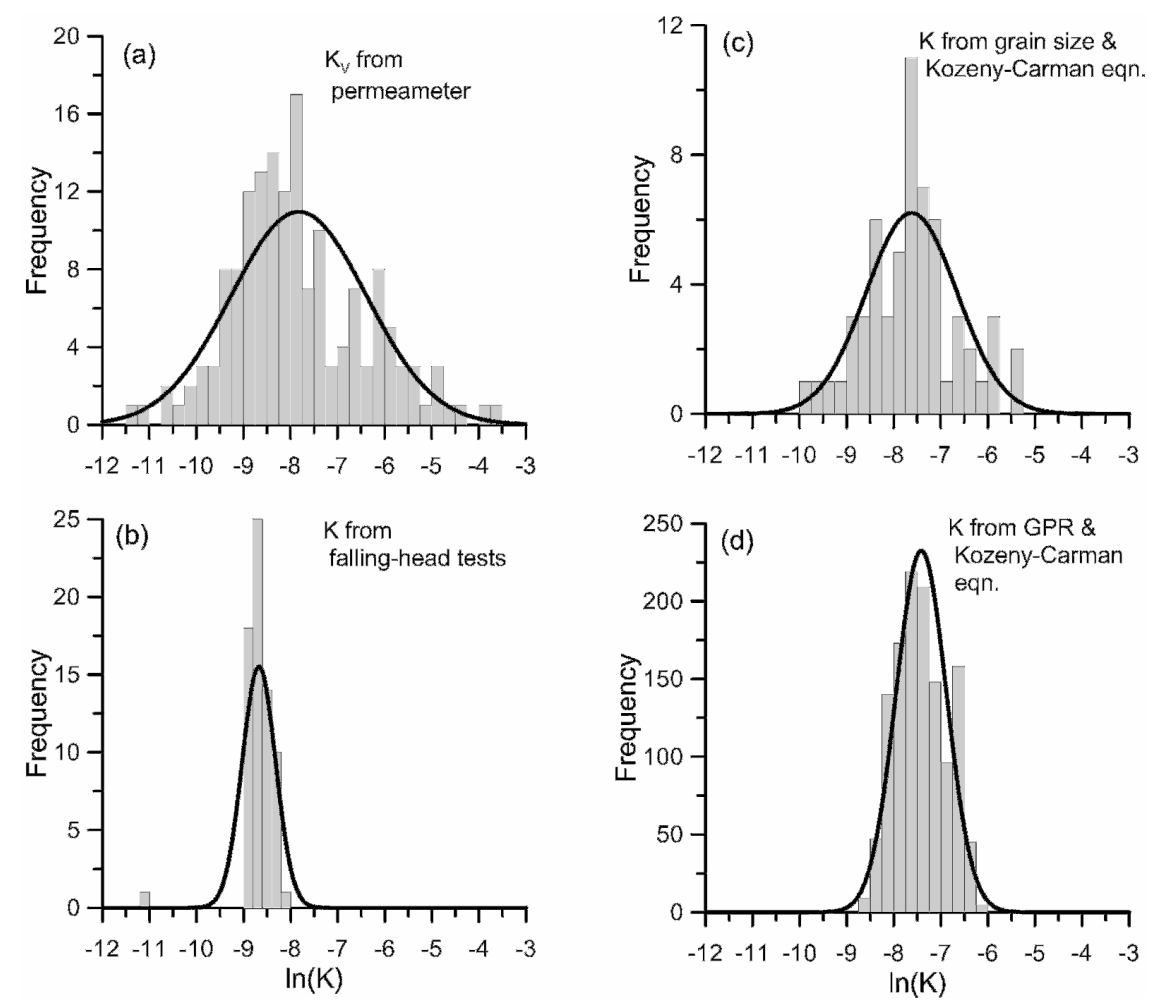

Figure 1-6 Distribution of $\ln (\mathrm{K})$ with the normal distribution curve shown by the thick line: (a) measured with the constant-head permeameter, (b) measured by in situ falling-head tests, and estimated from (c) grain-size analyses and (d) cross-hole groundpenetrating radar using the Kozeny-Carman equation. 


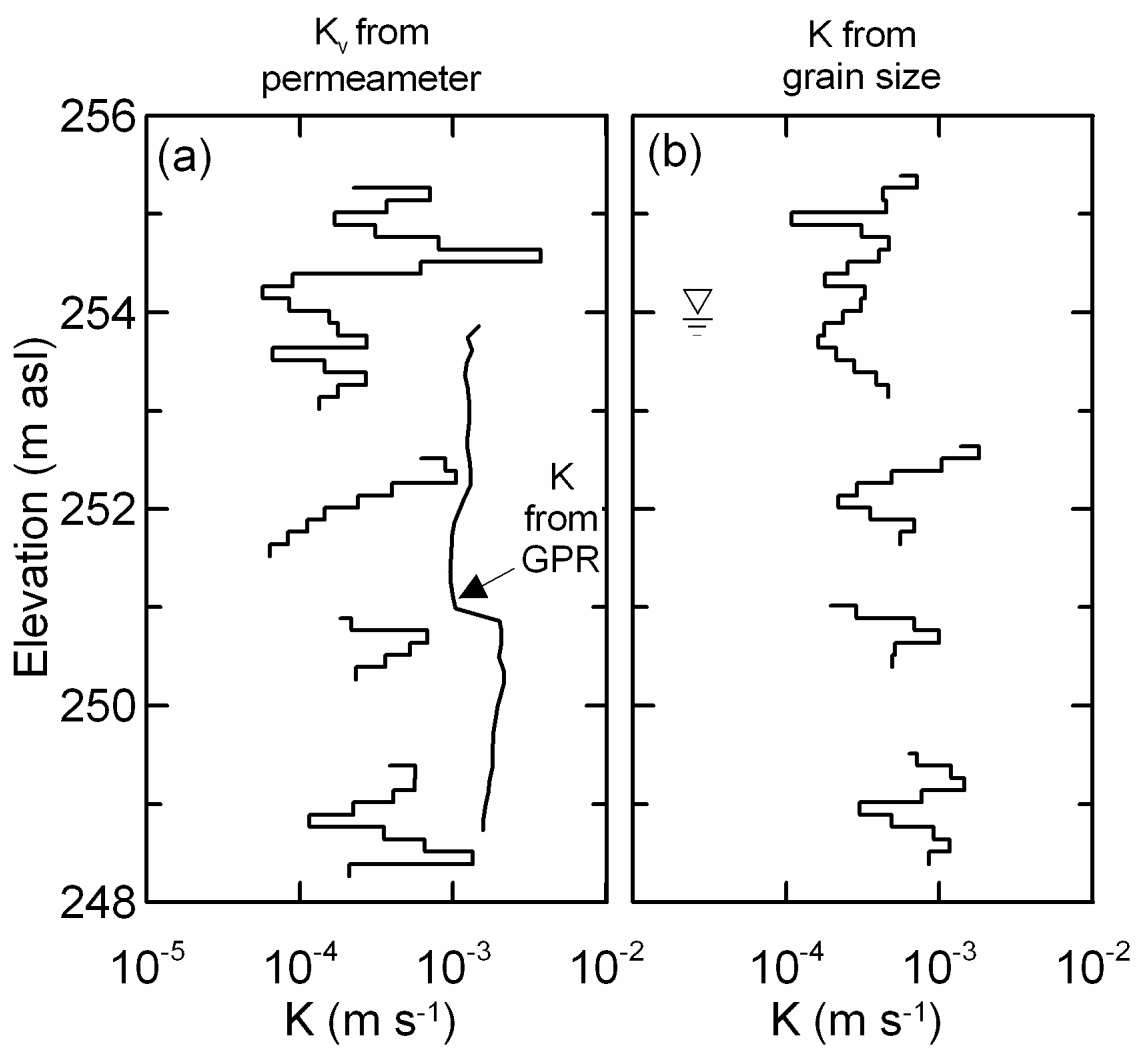

Figure 1-7 Vertical profiles of hydraulic conductivity $K$ at 10EC. (a) $K_{\mathrm{v}}$ measured by permeameter. Values of $K$, estimated from the ground-penetrating radar (GPR) using the Kozeny-Carman empirical equation, are from a zero-offset profile completed between M17 and M19. These are representative of an average of the porous medium between the two wells. (b) $K$ estimated from grain size using the Kozeny-Carman equation. These are depth-averaged values from the cores for wells M17 and M19.

These hydraulic conductivity values illustrate the effect of measurement scale. The mean hydraulic conductivity increases as the volumetric scale (support volume) of the test increases. The hydraulic conductivity from the 2-day pumping test is approximately two-orders of magnitude higher than values from most of the other tests. Similar differences between laboratory- and field-measured hydraulic conductivities have been reported for other glacial outwash aquifers. For example, Bradbury and Muldoon (1990) and Rehfeldt et al. (1992) reported differences of one- to two-orders of magnitude, and Wolf et al. (1991) and Rovey and Niemann (2001) reported differences of one-order of magnitude. These differences result, in part, from the volume of aquifer influenced 
during the test and how the heterogeneities encountered within this volume are spatially averaged by the test (e.g., Desbarats, 1994; Sánchez-Vila et al., 1996; Rovey and Niemann, 2001; Beckie and Harvey, 2002; Molz et al., 2005). To investigate the dependency of our measured hydraulic conductivities on the measurement scale, we chose the test volume as a measure of scale (Bradbury and Muldoon, 1990; SchulzeMakush et al., 1999). Permeameter and grain size measurements were made on sections of core with an average test volume of $1.24 \times 10^{-3} \mathrm{~m}^{3}$ ( 0.1-m-long by 0.125 -m-diameter).

We estimated the support volumes for the falling-head test and pumping test by calculating the volume of porous media required to accommodate or supply the volume of fluid injected or removed, during the test (Schulze-Makush et al., 1999). While we recognize that this method is an approximation of the volume of aquifer influenced by these two tests, it is simple to implement and provides a basis for comparing results. Methods that are more rigorous for estimating support volumes for pumping and slug tests have been developed for specific flow conditions (e.g., Desbarats, 1994; Beckie, 2001; Beckie and Harvey, 2002; Molz et al., 2005). However, applying these methods to the test conditions present at our site is outside of the scope of this research.

The GPR support volume can be approximated by the volume of the first Fresnel zone (Williamson, 1991; Červený and Soares, 1992; Reynolds, 2000). For a homogeneous medium, the Fresnel volume $V$ depends on the path lengths of the ray trace $L$ and the wavelength of the radar signal $\gamma$ and is given by (Huisman et al., 2003)

$$
V=\frac{4}{3} \pi a b c
$$

where $a, b$, and $c$ are the semi-axes of the ellipsoid defined as

$$
\begin{aligned}
& a=\frac{1}{2}\left(\frac{\gamma}{2}+L\right), \\
& b, c=\frac{1}{2}\left(\frac{\gamma^{2}}{4}+L \gamma\right)^{1 / 2} .
\end{aligned}
$$


The wavelength $\gamma$ can be calculated from the load frequency of the transmitter GPR pulse $f_{\mathrm{L}}$ and the velocity in the medium separating the transmitter and receiver as $v / f_{\mathrm{L}}$. The Fresnel zone is a circular region formed by the cross-section of the Fresnel volume in a plane perpendicular to the ray path. The maximum diameter of the Fresnel zone along the ray path is given by $2 b$ and is considered to be the spatial resolution in tomography. Given the measured load frequency (45-50 MHz for ZOPs and 90-110 MHz for MOPs) and the survey geometry, the average Fresnel volume for the $100 \mathrm{MHz}$ antennas was 17.8 $\mathrm{m}^{3}$ (Fresnel zone equal to $2.5 \mathrm{~m}$ ), and $1.2 \mathrm{~m}^{3}$ for the $200 \mathrm{MHz}$ antennas (Fresnel zone of $0.9 \mathrm{~m})$. The observed wave velocity, and hence hydraulic conductivity, is an average of the porous medium within the Fresnel volume.

Figure 1-8 illustrates the relationship between measured hydraulic conductivity and the test support volume. The hydraulic conductivity increases with the support volume up to an upper bound, after which the hydraulic conductivity remains approximately constant and the medium may be considered quasi-homogeneous. This upper bound occurs at a volume of approximately $10^{4} \mathrm{~m}^{3}$, but is highly dependent on the type of porous media (Schulze-Makuch et al. 1999). We have assumed the upper bound for this outwash is represented by the hydraulic conductivity obtained from the pumping test that has a support volume of $1.36 \times 10^{4} \mathrm{~m}^{3}$. Included in Figure $1-8$ is a value of hydraulic conductivity we determine, in a later section of this chapter, from the observed thermal plumes at this site. The data below the upper bound vary as some power of the support volume (Neuman, 1994) and may be described by the empirical relationship

$$
K=c V^{m}
$$

where $c$ is a coefficient characteristic of the porous medium, $V$ is the support volume, and $m$ is a scaling exponent (Schulze-Makuch et al., 1999). From a regression of the eight data points $c$ is $10^{-2.84}$ and $m$ is 0.22 . The correlation coefficient $r$ of the relationship is 0.92. From Eq. (1.9) the value of hydraulic conductivity may be estimated at any scale of 
interest. The value we determine for $m$ of 0.22 is less than 0.5 reported for glacial outwash sediments in Wisconsin (Schulz-Makush et al., 1999), but it is within the range reported by Schulz-Makush and Cherkauer (1998). This suggests one should exercise caution when applying this relationship at sites where the coefficients have not been determined with site-specific data.

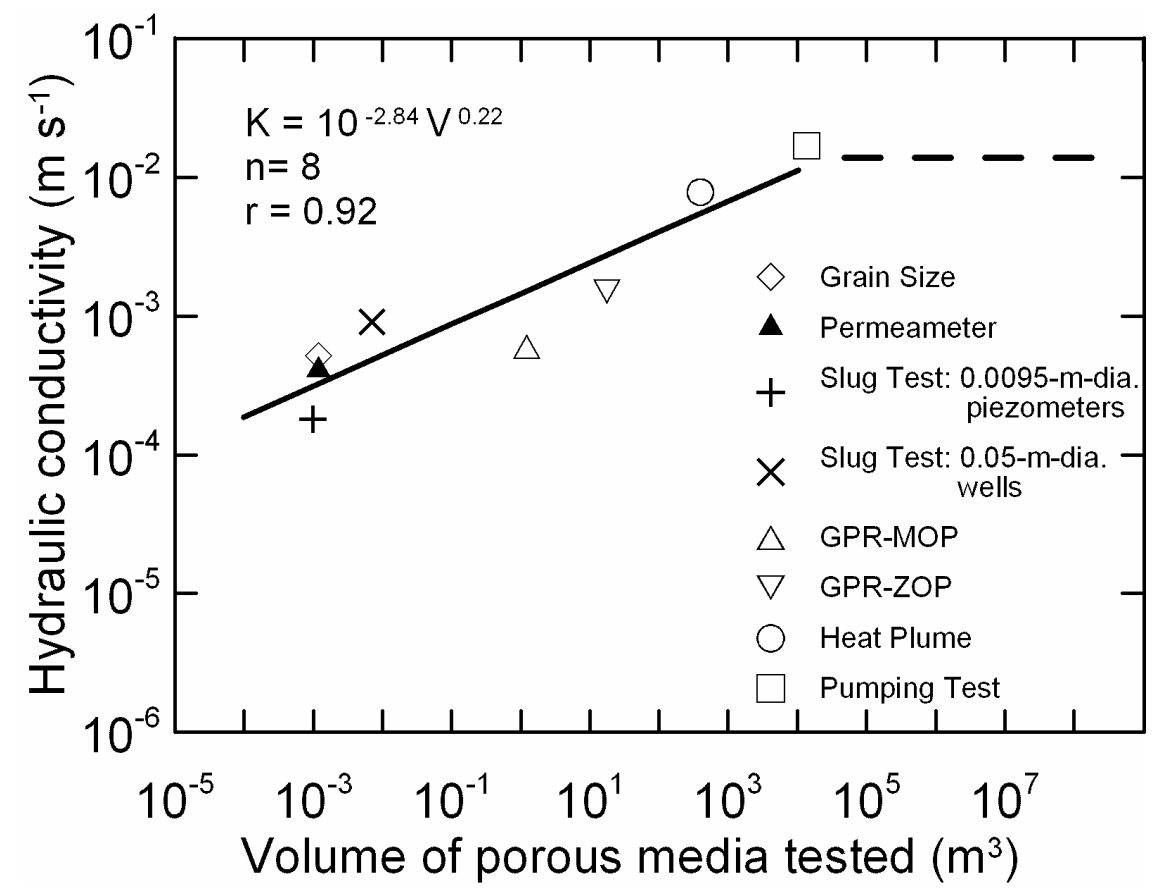

Figure 1-8 Relationship of hydraulic conductivity to scale of measurement in glacial outwash sand and gravel. The upper bound, shown by the dashed line, is assumed to be the hydraulic conductivity obtained from the constant-rate pumping test which has a support volume $>10^{4} \mathrm{~m}^{3}$ (Schulz-Makuch et al., 1999).

While hydraulic conductivity increases with measurement scale, the variance of $\ln (\mathrm{K})$ decreases as larger and larger heterogeneities are averaged by the test. Our data from the falling-head test is the exception having the smallest support volume and variance. Two factors may have contributed to this. The diameter of the piezometer tubes for these tests was $0.0095 \mathrm{~m}$ and the small diameter may have restricted the flow within the piezometer tubes, resulting in a lower estimate of hydraulic conductivity (Butler, 1997). The narrow distribution of the falling-head-determined conductivities probably 
results from the zone of disturbed aquifer surrounding the piezometer. Drilling and well installation mixes the aquifer material around the piezometer. As a result, the falling-head test yields hydraulic conductivity values that are less variable and biased by this disturbed zone toward the mean hydraulic conductivity of the porous medium. These factors must be considered when using the falling-head test data.

The observed increase in hydraulic conductivity with support volume illustrates the need for considering the scale of the field problem under investigation and carefully selecting methods for measuring hydraulic conductivity that will ensure they meet the needs of the investigation. While methods for up scaling have been proposed, they should be used judiciously and supported by the collection and analysis of site-specific data.

\section{Aquifer thermal properties}

We calculated the mean heat capacity of the aquifer solids $C_{\mathrm{s}}$ from the known mineral composition, and values for the specific heat and density of the mineral phases (Table 1-

4) using Eq. (1.3). The estimated heat capacities have a narrow range from $2.205 \times 10^{6} \mathrm{~J}$ $\mathrm{m}^{-3} \mathrm{~K}^{-1}$ for the average value for sand to $2.235 \times 10^{6} \mathrm{~J} \mathrm{~m}^{-3} \mathrm{~K}^{-1}$ for both gravel and till (Table 1-5), suggesting that a value of $2.22 \times 10^{6} \mathrm{~J} \mathrm{~m}^{-3} \mathrm{~K}^{-1}$ is representative of the heat capacity of the aquifer solids. Assuming values of $4174 \mathrm{~J} \mathrm{~kg}^{-1} \mathrm{~K}^{-1}$ and $1000 \mathrm{~kg} \mathrm{~m}^{-3}$ for the specific heat and density of water, respectively (de Vries, 1963), and a porosity of 0.29 , we estimated the heat capacity and $95 \%$ confidence interval of the saturated aquifer to be $(2.79 \pm 0.01) \times 10^{6} \mathrm{~J} \mathrm{~m}^{-3} \mathrm{~K}^{-1}$.

Table 1-4 Values of specific heat and density for minerals found in the outwash and till.

\begin{tabular}{|c|c|c|c|c|c|c|}
\hline \multirow[b]{2}{*}{ Mineral } & \multicolumn{4}{|c|}{ Specific heat $c_{\mathrm{s}}$} & \multicolumn{2}{|c|}{ Density $\rho$} \\
\hline & $\begin{array}{l}\text { Čermák and } \\
\text { Rybach (1982) }\end{array}$ & $\begin{array}{l}\text { Mercer et al. } \\
(1982)\end{array}$ & $\begin{array}{l}\text { Robie et al. } \\
(1978)\end{array}$ & $\begin{array}{l}\text { Helgeson et al. } \\
(1978)\end{array}$ & Horai (1971) & Clark (1966) \\
\hline & & & & & -1 & $\mathrm{~m}^{-3}$ \\
\hline Calcite & 793 & 786 & 834 & 818 & 2721 & 2712 \\
\hline Dolomite & & & 854 & 853 & 2857 & 2866 \\
\hline Anorthite & 700 & & 760 & 757 & 2769 & 2762 \\
\hline Hornblende & & 817 & & & 3254 & \\
\hline Quartz & 698 & 787 & 690 & 740 & 2647 & 2533 \\
\hline Clay minerals & 870 & & & & 2900 & 2834 \\
\hline
\end{tabular}

Table 1-5 Average thermal properties of the porous medium solids.

Thermal conductivity of porous medium solids, $8_{\mathrm{s}} \quad$ Heat capacity of porous medium solids, $C_{\mathrm{s}}$ 


\begin{tabular}{|c|c|c|c|c|c|c|}
\hline Source & Sand & Gravel & Till & Sand & Gravel & Till \\
\hline & 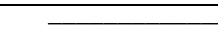 & $-\mathrm{W} \mathrm{m} \mathrm{m}^{-1} \mathrm{~K}^{-1}$ & 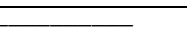 & 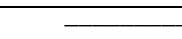 & $-\mathrm{kJ} \mathrm{m}^{-3} \mathrm{~K}^{-1}$ & 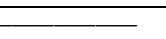 \\
\hline $\begin{array}{l}\text { Mineral } \\
\text { composition }\end{array}$ & $3.99 \pm 0.16(17) \neq$ & $3.83 \pm 0.13(6)$ & $4.05 \pm 0.14$ & $2205 \pm 25(17)$ & $2235 \pm 14(6)$ & $2235 \pm 36$ \\
\hline $\begin{array}{l}\text { Divided-bar } \\
\text { apparatus }\end{array}$ & $4.22 \pm 0.10(24)$ & $3.94 \pm 0.12$ & $3.72 \pm 0.59$ & & & \\
\hline
\end{tabular}

Thermal conductivity of the aquifer solids $\lambda_{\mathrm{s}}$, determined from the divided bar apparatus, ranged between 3.38 and $4.81 \mathrm{~W} \mathrm{~m}^{-1} \mathrm{~K}^{-1}$. The thermal conductivities are normally distributed (Shapiro-Wilk test, $W_{.05,41}^{*}=0.941<W=0.982$, with $p$ value $=0.7$ ) and the mean value and $95 \%$ confidence interval are $4.09 \forall 0.09 \mathrm{~W} \mathrm{~m}^{-1} \mathrm{~K}^{-1}$. The standard deviation of the measured values is $0.29 \mathrm{~W} \mathrm{~m}^{-1} \mathrm{~K}^{-1}$. We found $\lambda_{\mathrm{s}}$ was dependent on the grain size and mineral composition of the porous medium and could be assigned to three groups; till, gravel, and fine to coarse sand (Markle et al., 2006). The mean values of $\lambda_{s}$ for these groups are summarized in Table 1-5.

The apparent thermal conductivity $\lambda$, of a porous medium can be estimated by a variety of empirical equations and mixing formulas (e.g., Woodside and Messmer, 1961; Hashin and Shtrikman, 1962; de Vries, 1963; Johansen, 1975; Campbell et al., 1994). Using Akaike's information criterion (Akaike, 1973), we found the Campbell et al. (1994) model to be the best-approximating model for the porous medium at this site (Markle et al., 2006). Using the Campbell model we obtained estimates of apparent thermal conductivity in the saturated aquifer ranging from 2.14 to $2.69 \mathrm{~W} \mathrm{~m}^{-1} \mathrm{~K}^{-1}$ with a mean of $2.42 \mathrm{~W} \mathrm{~m}^{-1} \mathrm{~K}^{-1}$. We estimated $\lambda$ in the till to be $1.90 \mathrm{~W} \mathrm{~m}^{-1} \mathrm{~K}^{-1}$. In the unsaturated zone, $\lambda$ ranged from $2.6 \mathrm{~W} \mathrm{~m}^{-1} \mathrm{~K}^{-1}$ in the capillary fringe (estimated from grain size distributions to be $0.01-0.05 \mathrm{~m}$ above the water table) to $1.4 \mathrm{~W} \mathrm{~m}^{-1} \mathrm{~K}^{-1}$ at the ground surface. In the unsaturated zone, $\lambda$ varies directly with the moisture content.

We also estimated $\lambda$ using the background temperature profiles collected at the up gradient multilevel well, M0 (Fig. 1-9). As a periodic temperature variation propagates through the subsurface, the amplitude of the temperature variation decreases with depth while the time lag $t_{\mathrm{d}}$, between the peak temperature at depth $z$ and the ground surface, increases with depth. 


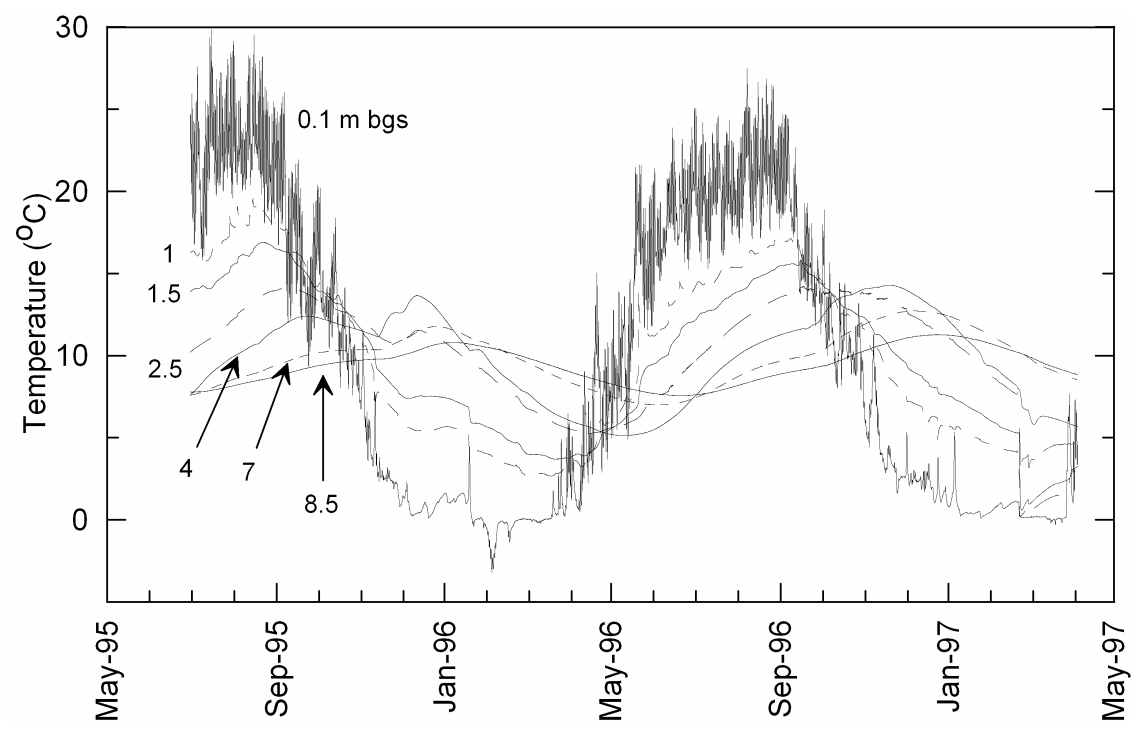

Figure 1-9 Temperature profile for the up-gradient multilevel well M0. The depths of the thermistors are reported as metres bgs (below ground surface). For clarity, the temperatures for only seven of the 12 thermistors are shown.

Assuming heat transport from the surface vertically through the aquifer is mainly by conduction, the time lag is

$$
t_{d}=\left(\frac{z}{2}\right)\left(\frac{P C_{0}}{\pi \lambda}\right)^{1 / 2},
$$

where $P$ is the period of the temperature variation (Ingersoll et al., 1954). The velocity at which the temperature variation propagates through the aquifer is

$$
\frac{z}{t_{d}}=2\left(\frac{\pi \lambda}{P C_{0}}\right)^{1 / 2}
$$

In a homogeneous medium, plotting $z$ versus $t_{\mathrm{d}}$ yields a straight line with a slope equal to the right-hand side of Eq. (1.11). A plot of $z$ versus $t_{\mathrm{d}}$ obtained for the nine thermistors within the saturated zone yields a fitted line with a slope of $0.0506 \mathrm{~m} \mathrm{~d}^{-1}$ and a coefficient 
of determination $r^{2}$ of 0.976 . Given a period of $365 \mathrm{~d}$ and a heat capacity of $2.79 \times 10^{6} \mathrm{~J}$ $\mathrm{m}^{-3} \mathrm{~K}^{-1}$, the apparent thermal conductivity of the saturated porous medium is estimated to be $2.40 \mathrm{~W} \mathrm{~m}^{-1} \mathrm{~K}^{-1}$, which is in excellent agreement with the average value of $2.42 \mathrm{~W} \mathrm{~m}^{-1}$ $\mathrm{K}^{-1}$ we estimated using the Campbell et al. (1994) model. Use of this method assumes that vertical heat transport is by conduction only with no convective heat transport. During large infiltration events, such as spring snowmelt and increased precipitation in the late fall, this assumption may not be valid. Under these conditions, the apparent thermal conductivity will be over-estimated. Lag times may be determined by comparing the times at which maximum or minimum temperatures occur at the depths of interest. If these peaks are influenced by significant recharge events, the lag times may be biased due to convective heat flux. To reduce the influence of individual recharge events on our estimates of time lag, we made use of the entire temperature record by cross-correlating the surface temperature signal with those measured at depth. As well, we investigated the influence that convective heat transport, due to vertical flow from recharge, may have on estimates of apparent thermal conductivity using the solution to the conductionconvection heat transport equation proposed by Stallman (1965). We completed simulations using a thermal conductivity and heat capacity of $2.4 \mathrm{~W} \mathrm{~m}^{-1} \mathrm{~K}^{-1}$ and $2.79 \times 10^{6} \mathrm{~J} \mathrm{~m}^{-3} \mathrm{~K}^{-1}$, respectively, for the saturated aquifer with vertical fluxes ranging from $0 \mathrm{~m} \mathrm{~s}^{-1}$ (pure conduction) to $1.27 \times 10^{-7} \mathrm{~m} \mathrm{~s}^{-1}$ (4000 $\mathrm{mm} \mathrm{yr}^{-1}$ ). Following the procedure outlined above and plotting $z$ versus $t_{\mathrm{d}}$, we obtained estimates of apparent thermal conductivity that were within $1 \%$ of the true conductivity provided the flux was $<6.34 \times 10^{-8} \mathrm{~m} \mathrm{~s}^{-1}\left(2000 \mathrm{~mm} \mathrm{yr}^{-1}\right)$. For flux rates of $1.27 \times 10^{-7} \mathrm{~m} \mathrm{~s}^{-1}$, the estimated apparent conductivity was $5 \%$ larger than true conductivity. The recharge rate at this site

is between 300 and $400 \mathrm{~mm} \mathrm{yr}^{-1}$. This suggests that the apparent thermal conductivity we estimated using this method, will not been influenced significantly by the convective transport of heat vertically through the subsurface.

\section{Water level monitoring}

The observed water levels in the pond (Fig. 1-10) and two down-gradient monitoring wells (M8 and M13) suggest the hydraulic head in the aquifer is controlled mainly by 
seasonal and short term variations in precipitation. During the 22-month monitoring period, the water table fluctuated by $0.7 \mathrm{~m}$ resulting in a $10 \%$ variation in the saturated thickness of the aquifer and yielding an average saturated thickness of $\sim 5 \mathrm{~m}$. The horizontal gradient between the pond and M13 ranged from $9 \times 10^{-4}$ to $1.5 \times 10^{-3} \mathrm{~m} \mathrm{~m}^{-1}$, and the mean annual hydraulic gradient is estimated to be $(1.2 \pm 0.1) \times 10^{-3} \mathrm{~m} \mathrm{~m}^{-1}$. While the calculated mean annual vertical gradients were generally $<5 \times 10^{-4} \mathrm{~m} \mathrm{~m}^{-1}$, in most cases the observed head differences were smaller than the uncertainty in measured water levels $( \pm 0.003 \mathrm{~m})$. Therefore, we do not consider the calculated vertical gradients to be significantly different from zero. During large recharge events, such as spring snowmelt, we observed vertical gradients as large as $0.3 \mathrm{~m} \mathrm{~m}^{-1}$, but these gradients quickly returned to near zero after recharge ceased. Thus, the hydraulic head distributions we observed suggest that flow through the outwash aquifer is predominantly horizontal. With an average porosity of 0.29 and using the hydraulic conductivity from the constant discharge test of $1.7 \times 10^{-2} \mathrm{~m} \mathrm{~s}^{-1}$, the estimated average linear groundwater velocity at $10^{\circ} \mathrm{C}$ is $7 \times 10^{-}$ ${ }^{5} \mathrm{~m} \mathrm{~s}^{-1}\left(6.1 \mathrm{~m} \mathrm{~d}^{-1}\right)$. For the streambed piezometers, the average vertical hydraulic gradient was $-0.1 \mathrm{~m} \mathrm{~m}^{-1}$ at each location (negative values indicating discharging conditions), and discharging conditions were predominant throughout the year. 


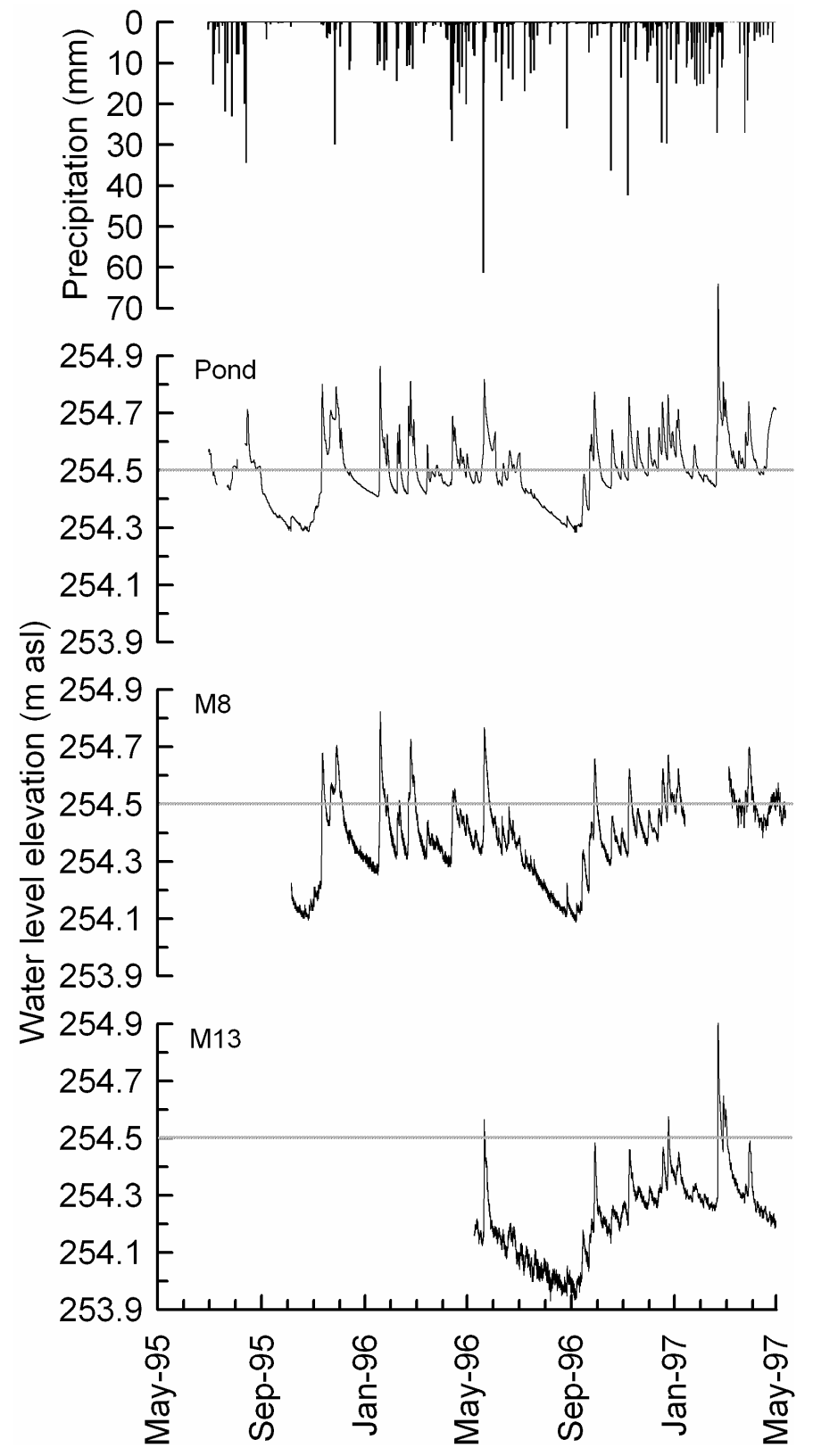

Figure 1-10 Water levels in the pond and two down-gradient monitoring wells (M8 and M13). Precipitation is shown at the top.

\section{Air - ground surface temperature and heat flux}

The air temperature, near-surface soil temperature, and soil heat flux measured at M14, are shown in Figure 1-11. The mean annual air temperature, measured $2 \mathrm{~m}$ ags, was $7.6^{\circ} \mathrm{C}$ with minimum and maximum daily average values of $-19.5^{\circ} \mathrm{C}$ and $27.6^{\circ} \mathrm{C}$, respectively. The mean annual soil temperature at $0.02 \mathrm{~m}$ bgs was $10.9^{\circ} \mathrm{C}$, with minimum and 
maximum daily average temperatures of $-7.9^{\circ} \mathrm{C}$ and $30.2^{\circ} \mathrm{C}$, respectively. The average annual near-surface soil temperature is $3.3^{\circ} \mathrm{C}$ higher than the air. This has been observed in a number of studies (e.g., Beltrami, 2001; Schmidt et al., 2001; Zhang et al., 2001) and several empirical relationships predict a $1-3^{\circ} \mathrm{C}$ difference between air and soil temperature (e.g., McDole and Fosberg, 1974; Kluender et al., 1993; Isard and Schaetzl, 1995). This difference is due to several factors including the heating of the soil by the solar radiation, the greater heat capacity of the soil compared to the air, and the insolating effect of the snow cover during the winter which decouples the soil temperature from the air temperature. Furthermore, the heat exchange across the ground surface is seasonally variable, and depends on the stage of vegetation growth and the amount of snow cover. The soil heat flux reaches maximum values in July and August ranging from up to $180 \mathrm{~W}$ $\mathrm{m}^{-2}$ during the day down to $-70 \mathrm{~W} \mathrm{~m}^{-2}$ at night (Fig. 1-11c). In the winter (December to March), the heat flux drops to near zero due to the insulating effect of the snow cover, the suppression of conductive heat transport through the release of latent energy during soil freezing, and the zero-curtain effect caused by water infiltration during spring snowmelt (e.g., Goodrich, 1982; Outcalt et al., 1990; Kane et al., 2001; Smerdon et al., 2003). During this period, there is little heat exchange between the air and near-surface soils. These data show that heat flux across the ground surface is seasonally variable and that the air temperature may not be representative of the near-surface soil temperature. Where possible, direct measurements of the near surface soil temperatures and heat flux should be collected. 

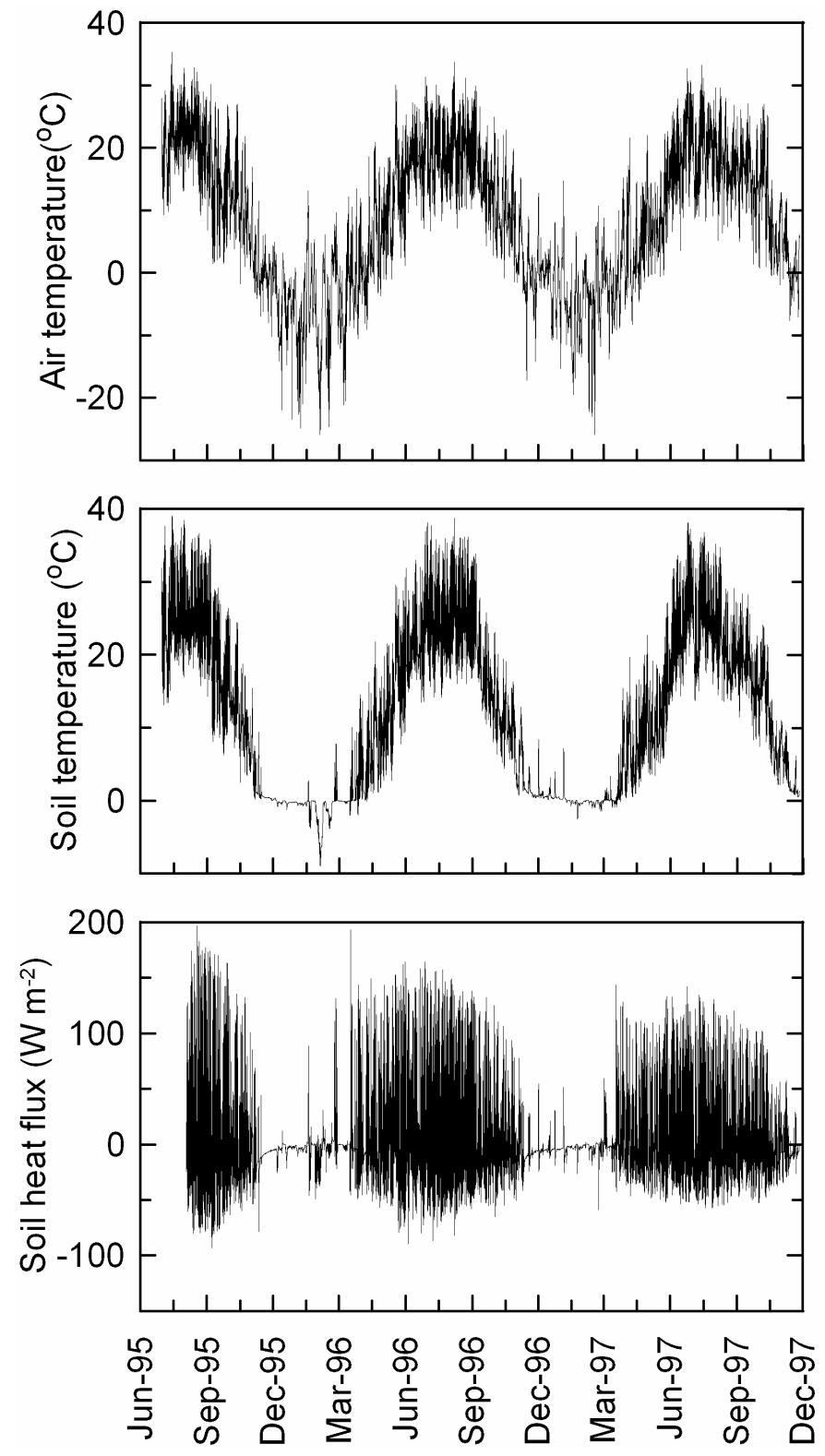

Figure 1-11 Shown are: (top) Air temperature at $2 \mathrm{~m}$ ags (above ground surface), (middle) near-surface soil temperature $0.02 \mathrm{~m}$ bgs (below ground surface), and (bottom) soil heat flux $0.05 \mathrm{~m}$ bgs at M14. 


\section{Pond water temperatures}

The pond temperatures show the magnitude of the thermal perturbation caused by the presence of the pit (Fig. 1-12). The water temperature near the surface of the pond ranges from near $0^{\circ} \mathrm{C}$ in February to over $30^{\circ} \mathrm{C}$ in August. The pond is stratified for about five months of the year between the beginning of May, when the surface of the pond begins to warm, and mid- to late September, when the pond begins to cool. For the remainder of the year the temperature is uniform throughout the depth. At the pond surface, the mean annual temperature is $11.7^{\circ} \mathrm{C}$ and the amplitude is $30^{\circ} \mathrm{C}\left(0.5-30.5^{\circ} \mathrm{C}\right)$. Near the bottom of the pond (5.65 $\mathrm{m}$ below the pond surface), the average temperature is $10.4^{\circ} \mathrm{C}$ and the amplitude is attenuated by $\sim 31 \%$ relative to the surface temperature and is $20.6^{\circ} \mathrm{C}(2.1-$ $22.7^{\circ} \mathrm{C}$ ). The pond temperature is uniform during the winter (December to March) and has an average temperature of $4^{\circ} \mathrm{C}$. In contrast, the annual temperature amplitude of the groundwater is much smaller at M0, located approximately $27 \mathrm{~m}$ up gradient of the pond (Fig. 1-9). At a depth of $0.1 \mathrm{~m}$ bgs, the mean annual soil temperature at $\mathrm{M} 0$ is $11^{\circ} \mathrm{C}$ and the amplitude is $20.9^{\circ} \mathrm{C}\left(2.1-23.0^{\circ} \mathrm{C}\right)$. At a depth of $6 \mathrm{~m} \mathrm{bgs}$ (equivalent to the bottom thermistor in the pond), the mean temperature at $\mathrm{M} 0$ is $9.3^{\circ} \mathrm{C}$ and the amplitude is $4.8^{\circ} \mathrm{C}$ $\left(7.0-11.8^{\circ} \mathrm{C}\right)$ and has been attenuated by $\sim 77 \%$ relative to the surface temperature. The lower attenuation of the surface temperature wave in the pond in comparison to the aquifer markedly alters the natural thermal regime. This temperature perturbation in the pond moves into the aquifer down gradient of the pond as shown in the following sequence of figures. 


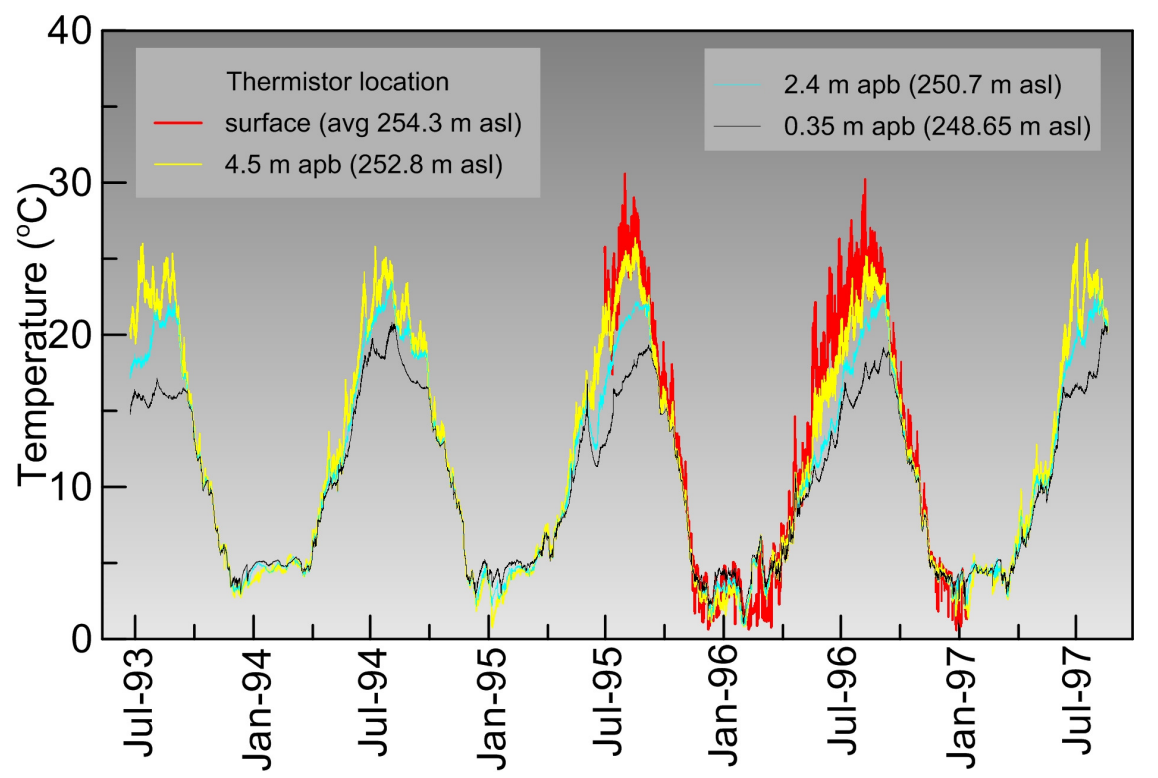

Figure 1-12 Pond temperature profile at the surface and 4.5, 2.4, and $0.35 \mathrm{~m}$ apb (above the pond bottom).

\section{Thermal plume monitoring}

Figure 1-13 shows the temperature distribution in the aquifer during a one-year period. These results, collected in the first 22-month monitoring period, show two distinct thermal pulses moving from the pond through the aquifer - a cool winter pulse followed by a warm summer pulse. The plumes persist above background groundwater temperature for up to $250 \mathrm{~m}$ down gradient of the pond, and for a period of 11-months after entering the aquifer. Between December and March the low rate of heat exchange across the ground surface is evident by the temperature of the near-surface soil which is relatively stable compared to the remainder of the year. During this period the plume temperature is not moderated by heat exchange across the ground surface. Other significant features are the annual surface temperature wave moving down into the aquifer in the summer and fall, and the overall cooling of the aquifer in April. This cooling is the result of the combined effect of the latent heat of melting of the snow and ice, which absorbs heat from the aquifer, and the convective flux of cold water infiltrating into the aquifer after the spring snowmelt. 

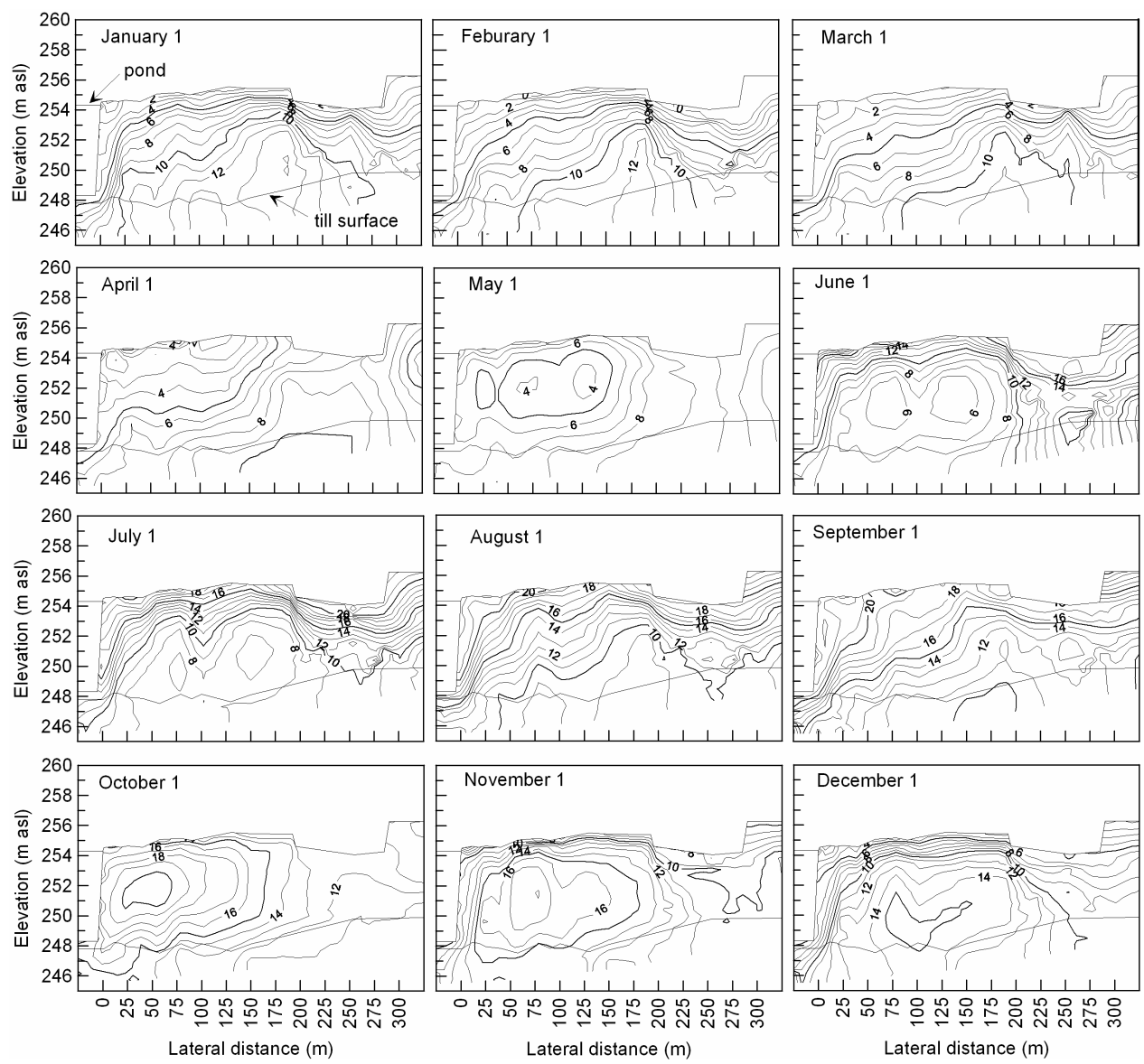

Figure 1-13 Thermal plume migration along section A-A' for period January to December 1996.

Much like chemical tracer tests, we can use the observed thermal plumes to estimate the plume transport rate and gain insight into the influence of thermal retardation on this rate. To estimate the plume transport rate, we cross-correlated the periodic temperature signals measured in the pond with the temperature signals measured at similar elevations in the aquifer. However, as the annual temperature variation in the pond moves horizontally through the aquifer mainly by convection with the groundwater flow, it combines with the annual surface temperature variation, moving vertically through the aquifer mainly by conduction. As these two temperature signals combine, the thermal plume from the pond is gradually attenuated (e.g., the warm thermal plume entering the aquifer from the pond in the summer is cooled by the cold temperature wave moving from the surface in the fall and winter), and identifying the pond signal within the 
observed temperature signal in the aquifer becomes increasingly difficult as the distance down gradient from the pond increases. As well, any convective heat flow vertically into the aquifer will tend to enhance the plume attenuation. Cross correlation of the temperature signals measured at a depth of approximately $4.5 \mathrm{~m} \mathrm{bgs}(250 \mathrm{~m}$ asl), provided good estimates of lag times for wells within the first $80 \mathrm{~m}$ of the aquifer (Table $1-6)$.

Table 1-6 Lag times determined from cross-correlation of the periodic annual temperature variation in the pond with the periodic temperature variation in the aquifer down gradient of the pond.

\begin{tabular}{lccccc}
\hline Well & Distance from pit face & Lag time $\dagger$ & Maximum lag value & $\begin{array}{c}\text { Standard error of cross } \\
\text { correlation }\end{array}$ & Plume velocity \\
\hline & $\mathrm{m}$ & $\mathrm{d}$ & & & $\mathrm{m} \mathrm{d}^{-1}$ \\
$\mathrm{M} 2$ & 11.2 & 7.8 & 0.972 & 0.0456 & 0.7 \\
$\mathrm{M} 3$ & 18.2 & 32.9 & 0.926 & 0.1321 & 0.1430 \\
$\mathrm{M} 4$ & 24.9 & 34.8 & 0.914 & 0.1784 & 1.4 \\
M5 & 40.2 & 44.9 & 0.902 & 0.2539 & 0.8 \\
M6 & 79.2 & 06 & 0.871 &
\end{tabular}

For our analysis, we considered the results of the cross-correlation to be good provided the maximum lag value was $>0.85$ (where a lag value of 1 is a perfect fit) and the standard error was $<0.3$. For the wells beyond $80 \mathrm{~m}$, we found cross-correlation became less reliable as the lag value decreased and the standard error increased. Using data from the first $80 \mathrm{~m}$, the lag times yield an average observed plume velocity of approximately $1.2 \mathrm{~m} \mathrm{~d}^{-1}$. This observed plume velocity is less than the groundwater velocity, due in part to attenuation of the thermal plume by vertical heat transport, but more importantly due to thermal retardation resulting from the contrast between the heat capacity of the porous medium solids and the pore water. The thermal retardation factor $R$ is given by

$$
R=\frac{C_{0}}{S \phi c_{w} \rho_{w}},
$$

where $S$ is the saturation, and the plume migration velocity is 


$$
v_{p}=\frac{v}{R},
$$

where $v$ is the average linear groundwater velocity. With an average porosity of 0.29 , an estimated heat capacity $C_{0}$ for the saturated aquifer of $2.79 \times 10^{6} \mathrm{~J} \mathrm{~m}^{-3} \mathrm{~K}^{-1}$, and assuming values of $4174 \mathrm{~J} \mathrm{~kg}^{-1} \mathrm{~K}^{-1}$ and $1000 \mathrm{~kg} \mathrm{~m}^{-3}$ for the specific heat and density of water, respectively, the estimated retardation factor is 2.3. This agrees well with values of $R$ obtained in similar aquifer materials: 2.0 for fine sands and 2.3 for sands and gravel (Andrews and Anderson, 1979); 1.9 for the Borden sand (Molson et al., 1992), and 2.0 for sands and gravels (Parr et al., 1983). These data suggest that the range of values for $R$ is narrow for saturated sand and gravel aquifers and, as a first approximation, it may be reasonable to assume $R$ is equal to 2. For $R$ equal to 2.3, we estimate the average groundwater velocity to be $2.8 \mathrm{~m} \mathrm{~d}^{-1}$, about half the value of $6.1 \mathrm{~m} \mathrm{~d}^{-1}$ estimated from the hydraulic conductivity measured by the constant discharge test. This difference may be related to the difference in support volumes (Table 1-3) and the differences in the dimensionality of the tests. As shown by stochastic theory (Gelhar, 1993) and theoretically (Neuman, 1994) the effective hydraulic conductivity depends on the dimensionality of the test. Rovey and Niemann (2001) suggest that in heterogeneous aquifers tracer tests measure a two-dimensional hydraulic conductivity that is the geometric mean of the hydraulic conductivity field, while pumping tests measure a threedimensional conductivity that is larger and lies between the geometric and arithmetic mean. Thus use of the hydraulic conductivity measured by the pumping test may overestimate the thermal plume velocity.

\section{Groundwater - surface water interaction}

Groundwater temperatures that we measured suggest that the thermal plumes at this site reach background temperatures after migrating $\sim 250 \mathrm{~m}$ through the gravel and sand aquifer. The nearest stream is $\sim 400 \mathrm{~m}$ down gradient of the pit and Tricks Creek is $\sim 750$ $\mathrm{m}$. Thus, the thermal effects from this pit are not impacting the surface water temperatures in either the tributary or Tricks Creek; however, as extraction proceeds south toward the tributary and Tricks Creek, impacts may occur. In addition there are 
several active pits within the watershed (Fig. 1-3) that could alter the stream temperature if adequate separation distances are not maintained. Thermal changes that Tricks Creek and associated tributaries can tolerate must be quantified before appropriate resource management decisions can be made. As a first step toward this objective, we measured the stream temperature and the groundwater temperature (1 m below the streambed) at four locations within the watershed; MP1, MP2, MP4, and MP5 (Fig. 1-14a to 1-14d). For MP1, MP2, and MP4, data for the period July 1995 to May 1997 are shown, and for MP5 data for the period December 2004 to June 2006 are shown. MP1, MP2, and MP5 are located in the upper reaches of the watershed where we typically expect temperature conditions to be suitable for spawning and incubation. The streambed at both MP1 and MP5 is, however, fine grained making these sites unsuitable for spawning. The streambed at MP2 and MP4 is predominantly gravel and thus suitable for spawning. We have presented the data for MP1 and MP5 to emphasize the variation in the groundwater surface water interactions across the watershed. 

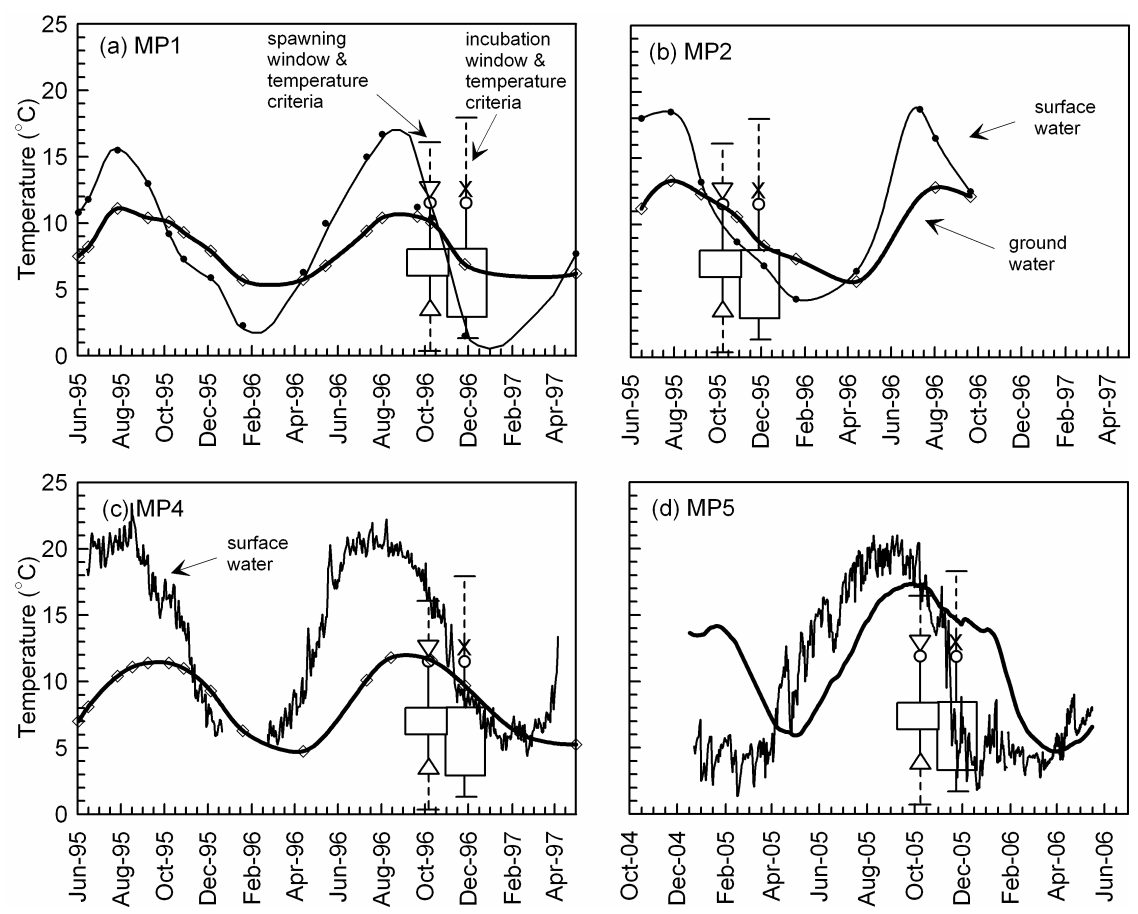

Figure 1-14 Surface water temperature and groundwater temperature (1 $\mathrm{m}$ below the base of the streambed) at (a) MP1, (b) MP2, (c) MP4, and (d) MP5. Also shown are the spawning and incubation time periods and temperature criteria for brook trout. The top and bottom of the boxes define the optimum temperature range for spawning and incubation (Hokanson et al., 1973; Witzel and MacCrimmon, 1983), and the width defines the time period during which spawning and incubation commonly occur. The open triangles define the upper and lower temperatures at which brook trout commonly spawn in Ontario (Witzel and MacCrimmon, 1983). The open circle shows the ET50 (Hokanson et al., 1973; Scott and Crossman, 1973), which is the upper mean effective temperature giving the median sublethal response, and the $\times$ shows the LT50 (Hokanson et al., 1973) which is the temperature at which 50\% normal hatch occurs. Finally, the horizontal bars indicate the temperatures beyond which spawning and incubation are unsuccessful (Hokanson et al., 1973; Curry et al., 2002.

For both benthic invertebrates and fish, the critical habitat requirements are concentrated largely in the early stages of development. For fish this includes the spawning and early rearing periods (Baxter and McPhail, 1999), and for macroinvertebrates this includes the egg and pupal development stages (Vannote and Sweeney, 1980). The thermal requirements for brook trout are given in Figure 1-14. Temperature data for macroinvertebrates are not as detailed as for brook trout. Of the macroinvertebrates found in Tricks Creek (Ephemeroptera, Plecoptera, Trichoptera, and 
Diptera), Plecoptera and Trichoptera are the most temperature sensitive. These two freshwater invertebrates tend to be restricted to cool-water habitats with a temperature range of $0-20^{\circ} \mathrm{C}$ (Quinn et al., 1994; Hogg and Williams, 1996). The upper lethal temperature for many cool-water insects is $<24^{\circ} \mathrm{C}$ (Nebeker and Lemke, 1968; Gaufin and Hern, 1971, deKozlowski and Bunting, 1981; Quinn et al., 1994). While these data provide upper limits on the range, several studies have shown that small temperature changes alter the structure of the macroinvertebrate community. For example, a large scale field experiment conducted in a first order cool-water stream in southern Ontario, showed that mean annual temperature changes as small as $2.1-2.4^{\circ} \mathrm{C}$ result in measurable changes that adversely affected the invertebrate community (Hogg and Williams, 1996). Other studies have reported measurable differences in growth rates for Ephemeroptera and Tricoptera for temperature changes of only $2^{\circ} \mathrm{C}$ (Vannote and Sweeney, 1980), while a temperature difference of $4^{\circ} \mathrm{C}$ (above the mean of $8.4^{\circ} \mathrm{C}$ ) virtually eliminated a mayfly in the family leptophlebiidae (Rempel and Carter, 1986). These data suggest that temperatures below $20^{\circ} \mathrm{C}$ are desirable and that small changes $\left(2-4^{\circ} \mathrm{C}\right)$ may adversely affect macroinvertebrates found in cool, headwater streams.

Figure 1-14a to 1-14c show that the groundwater moderates the stream temperature by discharging water that is cooler in the summer and warmer in the winter than the stream temperature at sites MP1, MP2, and MP4. The annual temperature amplitude of the discharging groundwater at MP5 is larger than at the three other sites and is nearly the same as the amplitude for the stream. We believe this is due to different discharge conditions at MP5. Here the floodplain is 90-m wide and the stream lies along the southern edge. At this location, the stream is not incised as much as it is at the other sites. In the floodplain, a $0.5-1 \mathrm{~m}$ thick silt layer, blanketed by approximately $0.5 \mathrm{~m}$ of peat and muck, overlies the sand and gravel aquifer. The silt layer restricts the groundwater discharge in to the stream. As a result, groundwater discharge is diffuse within the riparian zone where it mixes with infiltrating precipitation and enters the stream as near-surface flow through the soil and peat in the floodplain. Thus in the summer, the groundwater warms as it flows laterally through the peat and mixes with infiltration, prior to entering the stream. The warm discharging groundwater and the fine 
streambed substrate at MP5 result in conditions that are poor for spawning.

Brook trout begin spawning in mid-September and continue until mid- to late November. At all these sites except MP5, the temperature in the hyporheic zone ranges between $8.5^{\circ} \mathrm{C}$ and $13^{\circ} \mathrm{C}$ during October and November. By mid-November, the groundwater is approximately $2^{\circ} \mathrm{C}$ warmer than the surface water. The measured stream and groundwater temperatures show that the temperatures at MP1 are within the optimum ranges for spawning and incubation, and at MP2 they are within the range brook trout commonly spawn. At MP4 the stream temperature is near the maximum range. At MP5, both groundwater and surface water exceed the maximum temperature. In the winter (December to March), the average surface water temperature is $4^{\circ} \mathrm{C}$ but drops to about $1^{\circ} \mathrm{C}$ on occasion. While temperatures $<1^{\circ} \mathrm{C}$ can be tolerated by incubating brook trout (Curry et al., 2002), in areas of low groundwater discharge egg mortality increases dramatically (Curry et al., 1995; Power et al., 1999). During the winter, the mean groundwater temperature remains between $6^{\circ} \mathrm{C}$ and $8^{\circ} \mathrm{C}$ at MP1, MP2, and MP4. This warm groundwater maintains the water temperature in the hyporheic zone within or near optimum incubation temperatures, prevents the formation of anchor ice, and provides thermal conditions suitable for alevin growth prior to emergence from the substrate in late March to early April. Under these temperature conditions the incubation period is approximately $60 \mathrm{~d}$ (Garside, 1966). In the summer (June to September) the groundwater is between $5^{\circ} \mathrm{C}$ and $10^{\circ} \mathrm{C}$ cooler than the surface water. This provides thermal moderation of the high surface water temperatures, particularly during July and August.

These data highlight the importance of the temperature of the discharging groundwater on the spawning and incubation conditions. While not all the locations we monitored are used for spawning, groundwater temperature increases of only $2-3^{\circ} \mathrm{C}$ would shift water temperatures outside of the acceptable ranges. In particular, at MP5 even a small increase in groundwater temperature in the fall would push temperatures well above the maximum temperatures for spawning. At MP4, an increase of $1-2^{\circ} \mathrm{C}$ in groundwater temperature would result in temperatures that exceed the LT50 for incubation. These small temperature changes could alter the structure of the biotic communities by reducing the benthic invertebrate diversity, adult size and fecundity, and 
adversely affecting the productivity of the stream by decreasing the survivability of the fish eggs and alevins. Thus, even small temperature changes may cause unacceptable impacts to the biotic community within this creek. This suggests that the setback between aggregate pits and sections of the creek used for spawning should exceed $250 \mathrm{~m}$, recognizing that larger setbacks will be required where groundwater velocities are higher.

\section{Summary and Conclusions}

We observed thermal plumes migrating through a glacial-outwash sand and gravel aquifer in which the groundwater velocity is approximately $2.8 \mathrm{~m} \mathrm{~d}^{-1}$. The average apparent thermal conductivity of the outwash sand and gravel is $2.42 \mathrm{~W} \mathrm{~m}^{-1} \mathrm{~K}^{-1}$ and the thermal retardation factor was estimated to be 2.3. In this aquifer, the thermal plume velocity $\left(\sim 1.2 \mathrm{~m} \mathrm{~d}^{-1}\right)$ is less than half the groundwater velocity due to thermal retardation. Under these conditions the cool and warm thermal plumes persist for up to 11 months after entering the aquifer and migrate up to $250 \mathrm{~m}$ down gradient. At this site, the groundwater discharges to streams that are well beyond this distance, and thus are not affected by these thermal plumes. If, however, within this zone a stream was present and aquatic animals such as brook trout and cool-water macroinvertebrates were relying on the cool ground-water discharge, then thermal alterations may adversely affect these animals.

Our results indicate that laboratory-measured hydraulic conductivities are up to two-orders of magnitude smaller than field-measured hydraulic conductivities for this heterogeneous glacial outwash aquifer. The laboratory-measured hydraulic conductivities should be considered lower bounds on the aquifer hydraulic conductivity. Our data show that large-scale field-measured hydraulic conductivities will provide better estimates of hydraulic conductivity for predicting thermal plume velocities; however, velocities estimated with hydraulic conductivity from pumping tests may overestimate plume velocities and should be considered an upper bound on the hydraulic conductivity. While methods of scaling between laboratory-measured and field-measured hydraulic conductivities may be useful, one should verify the validity of the relationship for a 
particular site with multi-scale, site-specific data.

In this watershed, the temperature of the discharging groundwater ranges between $5^{\circ} \mathrm{C}$ and $17^{\circ} \mathrm{C}$ and moderates the stream temperatures. Comparison of the measured temperatures within the hyporheic zone and the stream shows that temperature changes of $2-3^{\circ} \mathrm{C}$ could shift temperatures beyond the maximum temperatures for brook trout spawning and adversely alter the structure of the macroinvertebrate community. These data emphasize that even small temperature changes may adversely impact the stream productivity especially if the stream temperatures are already near the upper or lower tolerable temperatures. As shown here, temperature conditions vary across the watershed. Establishing the thermal regime within the stream, the interaction of the groundwater and surface water, the spatial distribution of thermally sensitive aquatic animals, and the linkages to the stream environment is necessary to assess potential impacts on stream productivity from thermal disturbances to the discharging groundwater. Given the potential for small changes in groundwater temperature to negatively impact the benthic and fish community in this creek, quantification of the transport distance of the thermal disturbance from the aggregates pits is very important so that informed conservation and resource management decisions can be made.

This study demonstrated that aggregate extraction can impact stream temperatures if sufficient separation distances are not provided, and that these temperature changes may adversely affect the macroinvertebrate community and incumbent brook trout populations. The cumulative effects of several operations within the watershed are still unknown. Quantifying these effects will require a highly integrated study so that we may understand the groundwater - surface water interaction within the watershed and the links to the ecology in the context of the stream environment. 


\section{References}

Acornley, R.M. 1999. Water temperatures within spawning beds in two chalk streams and implications for salmonid egg development. Hydrological Processes 13:439446.

Akaike, H. 1973. Information theory as an extension of the maximum likelihood principle. p. 267-281. In B.N. Petrov and F. Csaki (eds.) Second symposium in Information Theory. Akadémiai Kiadó, Budapest.

Aldridge, D.F., D.W. Oldenburg. 1993. Two-dimensional tomographic inversion with finite-difference traveltimes. Journal of Seismic Exploration 2:257-274.

Anderson, M.P., J.S. Aiken, E.K. Webb, D.M. Mickelson. 1999. Sedimentology and hydrogeology of two braided stream deposits. Sedimentary Geology 129:187199.

Andrews C.B., M.P. Anderson. 1979. Thermal alteration of groundwater caused by seepage from a cooling lake. Water Resources Research 15:595-602.

Barnett, P.J. 1992. Quaternary geology of Ontario. p. 1011-1088. In P.C. Thurston, H. R. Williams, R.H. Sutcliffe, and G.M. Stott (eds.) Geology of Ontario. Ontario Geological Survey, Special Volume 4, Part 2. Publications Ontario, Toronto, Ontario.

Baxter, J.S., J.D. McPhail. 1999. The influence of redd site selection, ground water upwelling, and over-winter incubation temperature on survival of bull trout (Salvelinus confluentus) from egg to alevin. Canadian Journal Zoology 77:12331239.

Beckie, R. 2001. A comparison of methods to determine measurement support volumes. Water Resources Research 37:925-936.

Beckie, R., C.F. Harvey. 2002. What does a slug test measure: An investigation of instrument response and the effects of heterogeneity. Water Resources Research 38 (12), 1290, doi:10.1029/2001WR001072.

Beltrami, H. 2001. On the relationship between ground temperature histories and meteorlogical records: A report on the Pomquet station. Global Planetary Change 29:327-349.

Boggs, J.M., S.C. Young, D.J. Benton, Y.C. Chung. 1990. Hydrogeologic characterization of the MADE site. Report EN-6915, Electric Power Resource Institute, Palo Alto, California. 
Bradbury, K.R., M.A. Muldoon. 1990. Hydraulic conductivity determinations in unlithified glacial and fluvial materials. p. 138-151. In D.M. Nielsen and A.I. Johnson (eds.) Ground Water and Vadose Zone Monitoring, ASTM STP 1053. American Society for Testing and Materials, Philidelphia.

Brunke, M., T. Gonser. 1997. The ecological significance of exchange processes between rivers and groundwater. Freshwater Biology 37:1-33.

Butler, J.J., Jr. 1997. The Design, Performance, and Analysis of Slug Tests. CRC Press LLC, Baca Raton, Florida.

Campbell, G.S., J.D. Jr. Jungbauer, W.R. Bidlake, R.D. Hungerford. 1994. Predicting the effect of temperature on soil thermal conductivity. Soil Science 158:307-313.

Carman, P.C. 1937. Fluid flow through granular beds. Transactions, Institution of Chemical Engineers, London 15:150-166.

Carman, P.C. 1956. Flow of gas through porous media, Butterworths, London.

Carrier, W.D. III., 2003. Goodbye, Hazen; Hello, Kozeny-Carman. Journal of Geotechnical and Geoenvironmental Engineering ASCE 129 (11), 1054-1056, doi:10.1061/(ASCE) 1090-0241(2003)129:11(1054).

Čermák, V., L. Rybach. 1982. Thermal properties. p. 305-343. In Angenheister (ed.) Landolt-Börnstein Numerical data and functional relationships in science and technology, Physical properties of rocks, Volume 1a. Springer, New York.

Červený, V., J.E.P. Soares. 1992. Fresnel volume ray tracing. Geophysics 57:902-915.

Chadwick, M.A., J.W. Feminella. 2001. Influence of salinity and temperature on the growth and production of a freshwater mayfly the Lower Mobile River, Alabama. Limnology and Oceanography 46:532-542.

Clark, S.P., Jr. 1966. Thermal conductivity. In S.P. Clark, Jr. (ed.) Handbook of physical constants, revised edition, Geological Society of America Memoir 97. GSA, New York.

Cooper, A.J., W.D. Fitzgerald. 1977. Quaternary geology of the Goderich area, Southern Ontario. Ontario Geological Survey Preliminary Map P. 1232.

Cunjak, R.A., G. Power. 1986. Winter habitat utilization by stream resident brook trout (Salvelinus fontinalis) and brown trout (Salmo trutta). Canadian Journal of Fisheries and Aquatic Science 43:1970-1981. 
Curry, R.A., L.G. Noakes, G.E. Morgan. 1995. Ground water and the incubation and emergence of brook trout (Salvelinus fontinalis). Canadian Journal of Fisheries and Aquatic Sciences 52:1741-1749.

Curry R.A., D.A. Scruton, K.D. Clarke. 2002. The thermal regimes of brook trout incubation habitats and evidence of changes during forestry operations. Canadian Journal of Forest Research 32:1200-1207, doi:10.1139/X02-046.

de Vries, D.A. 1963. Thermal properties of soils. p. 210-235. In W.R. Van Wijk (ed.) Physics of Plant Environment, North-Holland, Amsterdam.

deKozlowski, S.J., D.L. Bunting II. 1981. Laboratory study on the thermal tolerance of four southeastern stream insect species (trichoptera, ephemeroptera). Hydrobiologia 70:141-145.

Desbarats, A.J. 1994. Spatial averaging of hydraulic conductivity under radial flow conditions. Mathematical Geology 26:1-21.

Elliott, J.M., 1994. Quantative Ecology and Brown trout. Oxford University Press: Oxford.

Environment Canada, 2005. Canadian climate normals 1971-2000. website: $<$ http://climate.weatheroffice.ec.gc.ca/climate_normals $>$.

Feng, S., P.N. Sen. 1985. Geometrical model of conductive and dielectric properties of partially saturated rocks. Journal of Applied Physics 58:3236-3243.

Garside, E.T. 1966. Effects of oxygen in relation to temperature on the development of embryos of brook trout and rainbow trout. Journal of Fisheries Resources Board of Canada 23:1121-1134.

Gaufin, A.R., S. Hern. 1971. Laboratory studies on tolerance of aquatic insects to heated waters. Journal of the Kansas Entomological Society 44:240-245.

Gelhar, L.W. 1993. Stochastic Subsurface Hydrogeology. Englewood Cliffs, New Jersey. Prentice Hall.

Goodrich, L.E. 1982. The influence of snow cover on the ground thermal regime. Canadian Geotechnical Journal 19:421-432.

Hashin, Z., S. Shtrikman. 1962. A variational approach to the theory of the effective magnetic permeability of multiphase materials. Journal of Applied Physics 33: 3125-3131. 
Helgeson, H.C., J.M. Delany, H.W. Nesbitt, D.K. Bord. 1978. Summary and critique of the thermodynamic properties of rock-forming minerals. American Journal of Science 278A:1-229.

Hess, K.M., S.H. Wolf, M.A. Celia, S.P. Garabedian. 1991. Macrodispersion and spatial variability of hydraulic conductivity in a sand and gravel aquifer, Cape Cod, Massachusetts. Environmental Research Brief EPA/600//M-91/005, U.S. Environmental Protection Agency, Washington, D.C.

Hess, K.M., S.H. Wolf, M.A. Celia. 1992. Large-scale natural gradient tracer test in sand and gravel, Cape Cod, Massachusetts 3 . Hydraulic conductivity variability and calculated macrodispersivities. Water Resources Research 28:2011-2027.

Hogg, I.D., D.D. Williams. 1996. Response of stream invertebrates to a global-warming thermal regime: An ecosystem-level manipulation. Ecology 77:395-407.

Hokanson, K.E.F., J.H. McCormick, B.R. Jones, J.H. Tucker. 1973. Thermal requirements for maturation, spawning, and embryo survival of the brook trout, Salvelinus fontinalis. Journal of Fisheries Resources Board of Canada 30:975984.

Holmes, R.M. 2000. The importance of ground water to stream ecosystem function. p.137-145. In: J.B. Jones and P.J. Mulholland (eds.) Groundwater and Streams. Academic Press, San Diego, CA.

Horai, K. 1971. Thermal conductivity of rock-forming minerals. Journal of Geophysical Research 76:1278-1308.

Huisman, J.A., S.S. Hubbard, J.D. Redman, A.P. Annan. 2003. Measuring soil water content with ground-penetrating radar: A review. Vadose Zone Journal 2:476491.

Hunt, R.J., D.A. Wilcox. 2003. Ecohydrology - why hydrologists should care. Ground Water 41:289.

Hunt, R.J., M. Strand, J.F. Walker. 2006. Measuring groundwater-surface water interaction and its effect on wetland stream benthic productivity, Trout Lake watershed, northern Wisconsin, USA. Journal of Hydrology, 320, doi:10.1016/j.jhydrol.2005.07.029.

Hynes, H.B.N. 1983. Groundwater and stream ecology. Hydrobiologia 100:93-99.

Ingersoll, 1.R., O.J. Zobel, A.C. Ingersoll. 1954. Heat conduction with engineering, 
geological, and other applications. The University of Wisconsin Press, Madison, Wisconsin, USA, $325 \mathrm{p}$.

Isard, S.A., R.J. Schaetzl. 1995. Estimating soil temperatures and frost in the lake effect snowbelt region, Michigan, USA. Cold Regions Science and Technology 23:317332.

Johansen, O. 1975. Thermal conductivity of soils, Ph.D. diss. Trondheim, Norway, (CRREL Draft Translation 637, 1977), ADA 044002.

Kane, D.L., K.M. Hinkel, D.J. Goering, L.D. Hinzman, S.I. Outcalt. 2001. Nonconductive heat transfer associated with frozen soils. Global Planetary Change 29:275-292.

Kluender, R.A., L.C. Thompson, D.M. Steigerwald. 1993. A conceptual model for predicting soil temperatures. Soil Science 156:10-19.

Kozeny, J. 1927. Überkapillare Leitung des Wassers im Boden: Sitzungsberichte Wiener Akademie, 136:217-306.

Lakly, M.B., J.V. McArthur. 2000. Macroinvertebrate recovery of a post-thermal stream: habitat structure and biotic function. Ecological Engineering 15:S87-S100.

Liberty, B.A., T.E. Bolton. 1971. Paleozoic geology of the Bruce peninsula area, Ontario. Geological Survey of Canada, Memoir 360, 163 p.

Luckner, L., W.M. Schestakow. 1991. Migration processes in the soil and groundwater zone. Lewis Publishers Inc., Chelsea, Michigan, USA., 485 p.

Lynch, J.A., G.B. Rishel. 1984. Thermal alteration of streams draining clearcut watersheds: quantification and biological implications. Hydrobiologia 111:161169.

Markle, J.M., R.A. Schincariol, J.H. Sass, J.W. Molson. 2006. Characterizing the twodimensional thermal conductivity distribution in a sand and gravel aquifer. Soil Science Society of America 70:1281-1294.

Marten, P.S. 1992. Effect of temperature variation on the incubation and development of Brook Trout eggs. The Progressive Fish-Culturist 54:1-6.

McDole, R.E., M.A. Fosberg. 1974. Soil temperatures in selected southeastern Idaho soils: I. Evaluation of sampling techniques and classification of soils. Soil Science Society of America Proceedings 38:480-491. 
Mercer, J.W., S.D. Thomas, B. Ross. 1982. Parameters and variables appearing in repository siting models, Report NUREG/CR-3066, U.S. Nuclear Regulatory Commission, Rockville, Maryland, 244 p.

Moench, A.F. 1997. Flow to a well of finite diameter in a homogeneous, anisotropic water table aquifer. Water Resources Research 33:1397-1407.

Moench, A.F. 2004. Importance of the vadose zone in analyses of unconfined aquifer tests. Ground Water 42:223-233.

Molson, J.W., E.O. Frind, C.D. Palmer. 1992. Thermal energy storage in an unconfined aquifer 2. Model development, validation, and application. Water Resources Research 28:2857-2867.

Molz, F.J., J. Guan, J. Wang. 2005. Spatial weighting functions: Transient hydraulic tests and heterogeneous media. Ground Water 43:215-221.

Nebeker, A.V., A. Lemke. 1968. Preliminary studies on the tolerance of aquatic insects to heated waters. Journal of the Kansas Entomological Society 41:413-418.

Neuman, S.P. 1994. Generalized scaling of permeabilities: Validation and effect of support scale. Geophysical Research Letters 21:349-352.

Nislow, K.H., W.H. Lowe. 2006. Influences of logging history and riparian forest characteristics on macroinvertebrates and brook trout (Salvelinus fontinalis) in headwater streams (New Hapmshire, U.S.A.), Freshwater Biology 51, 388-397, doi:10.1111/j.1365-2427.2005.01492.x.

Noakes, D.L.G. 1989. Early life history and behaviour of charrs. Physiology and Ecology Japan (Spec. Vol.) 1:173-186.

Oldenborger, G.A., R.A. Schincariol, L. Mansinha. 2003. Radar determination of the spatial structure of hydraulic conductivity. Ground Water 41:24-32.

Outcalt, S.I., F.E. Nelson, K.H. Hinkel. 1990. The zero-curtain effect: Heat and mass transfer across an isothermal region in freezing soil. Water Resources Research 26 :1509-1516.

Parr, A.D., F.J. Molz, J.G. Melville. 1983. Field determination of aquifer thermal energy storage parameters. Ground Water 21:22-35.

Power, G. 1980. The brook charr, Salvelinus fontinalis. p. 141-203. In E.K. Balon (ed.) Charrs. Salmonid fishes of the genus Salvelinus, Dr. W. Junk Publishers, The Hague, the Netherlands. 
Power, G., R.S. Brown, J.G. Imhof. 1999. Groundwater and fish - insights from northern North America. Hydrological Processes 13:401-422.

Pugsley, C.W., H.B.N. Hynes. 1986. Three-dimensional distribution of winter stonefly nymphs, allocapnia pygmaea, within the substrate of a southern Ontario river. Canadian Journal of Fisheries and Aquatic Sciences 43:1812-1817.

Quinn, J.M., G.L. Steele, C.W. Hickey, M.L. Vickers. 1994. Upper thermal tolerances of twelve New Zealand stream invertebrates species. New Zealand Journal of Marine and Freshwater Research 28:391-397.

Rehfeldt, K.R., J.M. Boggs, L.W. Gelhar. 1992. Field study of dispersion in a heterogeneous aquifer 3 . Geostatistical analysis of hydraulic conductivity. Water Resources Research 28:3309-3324.

Rempel, R.S., J.C.H. Carter. 1986. An experimental study on the effect of elevated temperature on the heterotrophic and autotrophic food resources of aquatic insects in a forested stream. Canadian Journal of Zoology 64:2457-2466.

Reynolds, J.M. 2000. An Introduction to Applied and Environmental Geophysics. Chichester, United Kingdom. John Wiley and Sons. pp. 806.

Robie, R.A., B.S. Hemingway, J.R. Fisher. 1978. Thermodynamic properties of minerals and related substances at $298.15 \mathrm{~K}$ and $1 \mathrm{bar}\left(10^{5}\right.$ pascals $)$ pressure and at higher temperatures. U.S. Geological Survey Bulletin 1452, pp.456.

Rovey, C.W., W.L. Niemann. 2001. Wellskins and slug tests: where's the bias? Journal of Hydrology 243:120-132.

Sánchez-Vila, X., J. Carrera, J.P. Girardi. 1996. Scale effects in transmissivity. Journal of Hydrology 183:1-22.

Sass, J.H., A.H. Lachenbruch, R.J. Munroe. 1971. Thermal conductivity of rocks from measurements on fragments and its application to heat-flow determinations. Journal of Geophysical Research 76:3391-3401.

Schmidt, W.L., W.D. Gosnold, J. Enz. 2001. A decade of air-ground temperature exchange from Fargo, North Dakota. Global Planetary Change 29:311-325.

Schulze-Makuch, D., D.A. Carlson, D.S. Cherkauer, P. Malik. 1999. Scale dependency of hydraulic conductivity in heterogeneous media. Ground Water 37:904-919.

Schulze-Makuch, D., D.S. Cherkauer. 1998. Variations in hydraulic conductivity with 
scale of measurement during aquifer tests in heterogeneous, porous carbonate rocks. Hydrogeology Journal 6:204-215.

Scott, W.B., E.J. Crossman. 1973. Freshwater Fishes of Canada, Fisheries Research Board of Canada Bulletin 184, Ottawa.

Sen, P.N., C. Scala, M.H. Cohen. 1981. A self-similar model for sedimentary rocks with application to the dielectric constant of fused glass beads. Geophysics 46:781795.

Shiozawa, S., G.S. Campbell. 1991. On the calculation of mean particle diameter and standard deviation from sand, silt, and clay fractions. Soil Science 152:427-431.

Smerdon, J.E., H.N. Pollack, J.W. Enz, M.J. Lewis. 2003. Conduction-dominated heat transport of the annual temperature signal in soil. Journal of Geophysical Research 108 (B9),2431, doi:10.1029/2002JB002351.

Stallman, R.W. 1965. Steady one-dimensional fluid flow in a semi-infinite porous medium with sinusoidal surface temperature. Journal of Geophysical Research 70: 2821-2827.

Taylor, B.R., A.N. Dykstra. 2005. Effects of hot ground water on a small swamp-stream in Nova Scotia, Canada. Hydrobiologia 545:129-144.

Vannote, R.L., B.W. Sweeney. 1980. Geographic analysis of thermal equilibria: A conceptual model for evaluating the effect of natural and modified thermal regimes on aquatic insect communities, The American Naturalist 115:667-695.

Williamson, P.R. 1991. A guide to the limits of resolution imposed by scattering in ray tomography. Geophysics 56:202-207.

Witzel, L.D., H.R. MacCrimmon. 1983. Redd-site selection by brook trout and brown trout in southwestern Ontario streams. Transactions of the American Fisheries Society 112:760-771.

Wolf, S.H., M.A. Celia, K.M. Hess. 1991. Evaluation of hydraulic conductivities calculated from multiport-permeameter measurements, Ground Water 29:516525.

Woodside, W., J.H. Messmer. 1961. Thermal conductivity of porous media. I. Unconsolidated sands. Journal of Applied Physics 32:1688-1706.

Yang, Zen-Fen. 1995. Application of the Heatflow model in a sand and gravel aquifer. MSc. thesis. Department of Earth Sciences, University of Waterloo, Waterloo, 
ON.

Zhang, T., R.G. Barry, D. Gilichinskt, S.S. Bykhovets, V.A. Sorokovikov, Jingping Ye. 2001. An amplified signal of climatic change in soil temperatures during the last century at Irkutsk, Russia. Climate Change 49:41-76. 


\section{Prologue to Chapter 2}

Chapter 2 was published in the Soil Science Society of America Journal in 2006 (Markle, Schincariol, Sass, and Molson, 2006) and provides a detailed description of the methods used to measure the thermal conductivity of the porous media solids $\lambda_{\mathrm{s}}$. These values are used to predict the apparent thermal conductivity $\lambda$ of variably-saturated soils using four mixing models and the best model is chosen using the information-theoretic approach. The selected model is integrated into a finite-element groundwater flow and heat transport model, and the influence of a heterogeneous thermal conductivity field is investigated. The thermal conductivities presented in this chapter are used as input to the numerical groundwater flow and transport modeling, presented in Chapter 3.

\section{References}

Markle, J.M., R.A. Schincariol, J.H. Sass, J.W. Molson. 2006. Characterizing the twodimensional thermal conductivity distribution in a sand and gravel aquifer. Soil Science Society of America 70:1281-1294. 


\section{Chapter 2}

\section{Introduction}

The temperature of discharging groundwater is an important factor in determining if temperature-sensitive aquatic animals can be supported in groundwater discharge areas such as river, streams, and wetlands (e.g., Garside, 1966; Acornley, 1999; Power et al., 1999). Many temperature-sensitive species have narrow ranges of thermal tolerance and even small increases in discharging groundwater temperature can degrade the habitat. For example, the optimum spawning and incubation temperature for brook trout (Salvelinus fontinalis) lies between 6 and $9^{\circ} \mathrm{C}$, with $50 \%$ mortality above $11.7^{\circ} \mathrm{C}$ (Hokanson et al., 1973). The temperature of the soil and groundwater within the upper 10 to $20 \mathrm{~m}$ of the subsurface is controlled by the annual variation in the amount of heat transferred at the ground surface. Any disturbance that alters energy transfer at the ground surface may alter groundwater temperature and adversely affect temperature-sensitive aquatic animals present in discharge areas. This work is part of an investigation into the potential thermal disturbance to groundwater that may result from aggregate extraction operations in a glaciofluvial outwash sand and gravel aquifer in southwestern Ontario, Canada. The groundwater from this aquifer discharges to a wetland and stream, supporting a coolwater fishery. The excavation of aggregate material below the water table involves removal of the forest cover and soil, followed by excavation of the unsaturated and saturated porous medium. Removal of the forest cover and the unsaturated porous medium increases the amount of solar radiation reaching the water table (e.g., Deardorff, 1978; Kaufmann et al., 2003; Nitoiu and Beltrami, 2005) and eliminates the insulating effect of the unsaturated zone. This results in an energy transfer across the air-water interface of the pond that is many times larger than the energy transfer across the water table under forested conditions. As a result, the temperature of the water in the pond is different from the groundwater under forested conditions. In the summer months, the water in the pond is much warmer and in the winter it is colder. Under the influence of the hydraulic gradient in the surrounding aquifer, the water in these ponds moves back into the groundwater system. It then moves through the aquifer as a series of alternating 
warm and cool thermal plumes. If this thermally altered groundwater discharges before reaching background temperature, it may adversely affect the aquatic biota in the discharge area.

The movement of thermal plumes through the subsurface is controlled by the groundwater velocity and by the aquifer thermal properties. Key thermal properties are the volumetric heat capacity and thermal conductivity. Since an aquifer is a granular medium consisting of solid, liquid, and gaseous phases, the volumetric heat capacity and thermal conductivity will depend on the volumetric proportions of these components. The volumetric heat capacity of an aquifer can be calculated accurately from the heat capacities and volume fractions of these three phases (Smith, 1939, 1942; Woodside and Messmer, 1961; de Vries, 1963). The apparent thermal conductivity, $\lambda$, is more complicated to calculate. It depends mainly on the mineral composition of the aquifer solids, and the porosity and degree of saturation. To a lesser extent, it depends on the bulk density of the aquifer solids, the shapes, sizes and arrangement of the solid particles, the contact area between the particles, the interfacial contact between the solid and liquid phases, the vapor diffusion in the unsaturated pores, and the temperature and pressure conditions (Smith 1939, 1942; de Vries, 1963; Hopmans and Dane, 1986). There are several methods available for estimating the apparent thermal conductivity of unconsolidated porous media. The most common methods include the direct measurement of conductivity using probes, and the estimation of conductivity using either empirical or mixing models. In situ transient line source probes have been used successfully in fine-textured porous media to measure thermal conductivity (Lubimova et al., 1961; Sass et al., 1981; Bristow et al., 1994); however, none of the currently available probes are durable enough for in situ measurements in coarse-textured media with cobbles and boulders. Measurement of thermal conductivity in these materials requires the use of alternate methods.

Predicting thermal transport through the subsurface is often accomplished with numerical finite-difference or finite-element models (e.g., Andrews and Anderson, 1979; Molson et al., 1992). As input to numerical simulations, these models require values of 
the apparent thermal conductivity for a variety of porous media across saturation conditions that range from nearly dry to fully saturated. If the thermal conductivity for the individual components is known, values of the apparent conductivity can be calculated using mixing models (e.g., de Vries, 1963; Gori, 1983; Campbell et al., 1994), a number of which were evaluated in this study.

Our goal was to simulate the migration of a thermal plume (emanating from a nearby aggregate pit) through the shallow aquifer using a finite-element numerical model. As input to the model, we required values of thermal conductivity for the glaciofluvial outwash sand and gravel aquifer. The main objectives of this study were to characterize the two-dimensional distribution of the apparent thermal conductivity in the aquifer, to evaluate the suitability of four candidate models for calculating the thermal conductivity, and to assess the influence of heterogeneous thermal conductivity on heat transport using numerical simulations.

\section{Site Description}

The study area is located in the Tricks Creek watershed of southwestern Ontario, 180 $\mathrm{km}$ west of Toronto (Fig. 2-1). The watershed is characterized by undulating topography. Before being cleared for agriculture, the area was covered by mixed deciduous forest. Presently, $12 \%$ of the watershed is forested. Tricks Creek lies within a wetland complex that encompasses an area of $\sim 105$ ha (4\% of the watershed). Tricks Creek is characterized by cool water and supports resident brook and rainbow trout (Oncorhynchus mykiss) populations. The creek and wetland are situated in a former glacial outwash channel in which the upper $6 \mathrm{~m}$ of the subsurface consist of glaciofluvial outwash deposits of sands and gravels. The outwash material overlies $30 \mathrm{~m}$ of silty clay till. The outwash sands and gravels were deposited in a meltwater channel at the ice margin in the last retreat of the ice sheet during the Wisconsinan glaciation, which occurred in this area approximately $13000 \mathrm{yr}$ ago (Barnett, 1992). The sands and gravels are mixtures of predominantly carbonate and quartz minerals, and form an unconfined aquifer. Within the study area the groundwater flows to the southwest and discharges to 
the wetland and creek (Fig. 2-1). Several aggregate operations are active along the western edge of the wetland and are upgradient of the creek.

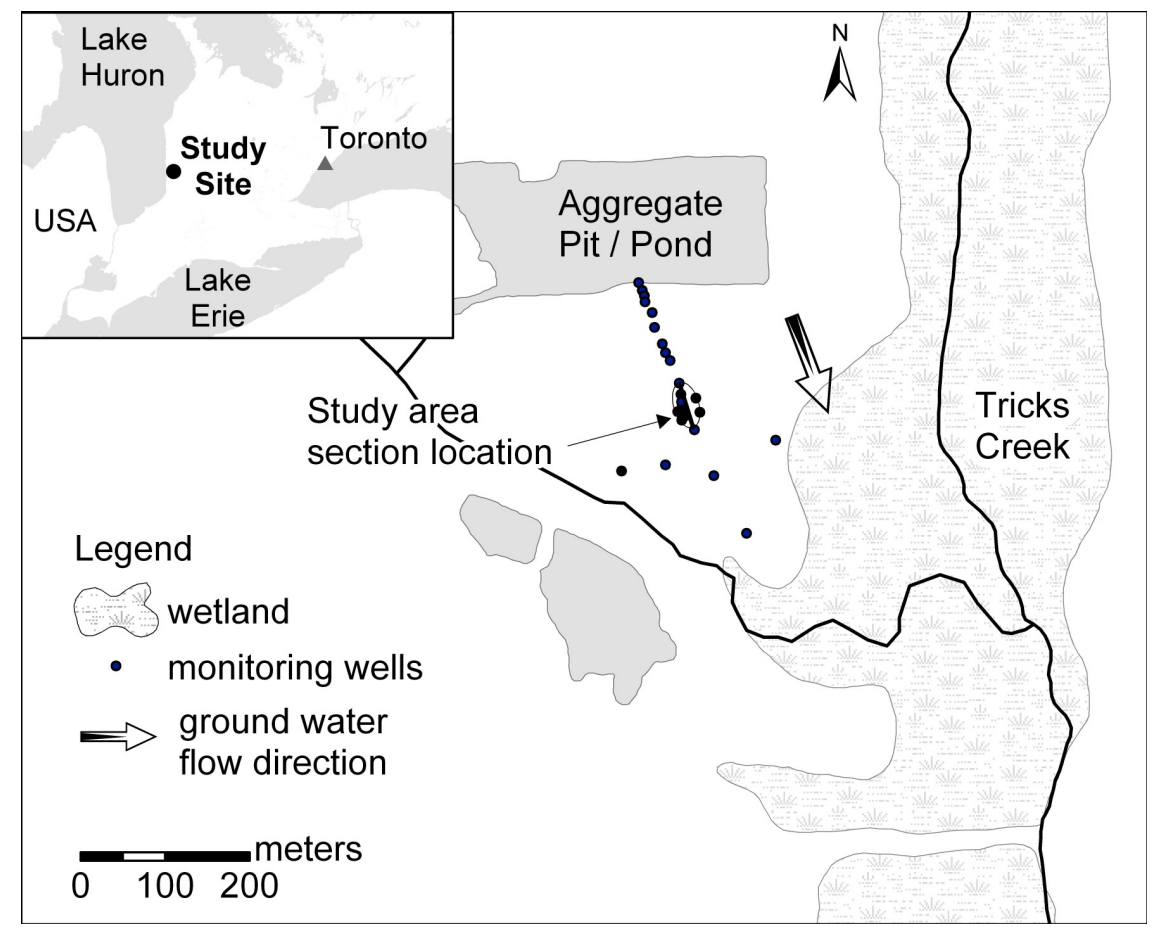

Figure 2-1 Site location map.

\section{Materials and Methods}

\section{Field and laboratory methods}

\section{Thermal conductivity of soil solids}

The presence of gravel, cobbles, and boulders in the outwash aquifer made the use of in situ probes impractical. For this investigation, we determined that it was more practical to recover aquifer material during drilling and complete measurements of thermal conductivity in the laboratory. Aquifer samples were collected using a truck-mounted drill rig equipped with hollow stem augers and a split-barrel sampler. The tip of the sampler preceded the augers during drilling and a PVC (polyvinyl chloride) sleeve inside the core barrel provided for the retrieval of aquifer cores, $0.126 \mathrm{~m}$ in diameter and $1.52 \mathrm{~m}$ long, with minimal disturbance. 
Within the study area, the soils are of the Humo-Ferric Podzol great group (Agriculture and Agri-Food Canada, 1998). These have developed beneath mixed deciduous forest cover with undulating terrain under well-drained conditions. In the area of the boreholes, the A horizon and much of the B horizon have been excavated in preparation for aggregate extraction. Based on observations during drilling, all that remains is $\sim 0.05 \mathrm{~m}$ of the $\mathrm{B}$ horizon at the surface. Below the soil lies $6 \mathrm{~m}$ of parent geologic material composed of carbonate-rich, glaciofluvial outwash sand and gravel. The outwash can be subdivided into four stratigraphic units, based on particle size distribution. These include poorly sorted gravel with sand, and well-sorted coarse, medium, and fine sand. The outwash is underlain by glacial till, which was the lowest stratigraphic unit encountered during drilling. From each unit, we selected representative samples and measured the thermal conductivity of the aquifer solids, $\lambda_{\mathrm{s}}$, on 41 of these samples. The mass of each sample was between 2 and $4 \mathrm{~kg}$. We ground the samples to a particle size of $<1 \mathrm{~mm}$ (Sass et al., 1971) and measured the thermal conductivity (at $20^{\circ} \mathrm{C}$ ) using the steady-state divided-bar apparatus. This method involves filling a cylindrical cell with crushed material, saturating the sample with water under vacuum, and measuring the conductivity of the cell in the same manner as a cylinder of solid rock. The conductivity of the solid component is then backed out from the geometric mean of the water and solid mixture. Additional details of the apparatus and method verification can be found in Sass et al. (1971). Values measured with this apparatus are generally accurate to within $\pm 5 \%$. From a subset, 27 samples were ground to a particle size of 20 $\mu \mathrm{m}$, and the mineralogy was obtained using the XRD (X-ray diffraction) technique. The semiquantitative estimation of relative mineral abundance was based on the integrated peak areas after the removal of the background response. This method yields estimates that are within 15\% for the clay minerals and 5\% for the other minerals (Mitchell, 1976). Both the divided-bar and XRD methods involve crushing and mixing the aquifer solids. As a result, the derived value of thermal conductivity represents a bulk value for the solid fraction of the aquifer. These methods do not provide information on the thermal 
conductivity or the mineralogy of individual particles. Furthermore, they do not account for the influence of particle size and shape.

We selected the divided-bar apparatus to measure $\lambda_{\mathrm{s}}$ and the mineral composition to estimate $\lambda_{\mathrm{s}}$, since these two methods are commonly used for consolidated rocks, and the porous medium mineral composition is often known. Furthermore, most predictive models use a bulk thermal conductivity for the porous media solids. While the model proposed by de Vries (1963) can account for a porous medium having particles of different thermal conductivity, mineral composition, and shape, the majority of the results reported in the literature use a bulk thermal conductivity, and a common mineral composition and shape for the particles. The exception to this is the work by Tarnawski et al. (2000), in which the porous medium is modeled as a mixture of 20 unique types of particles having unique thermal conductivities, mineral compositions, and shapes. For most studies, however, particle shape and mineralogy are not known.

\section{Volumetric water content and porosity}

In our study, the volumetric water content, $\theta$, of the aquifer was estimated from a series of cross-hole GPR (ground-penetrating radar) surveys. The surveys were completed across six boreholes, which span a 12.3 - by 7.6-m portion of the aquifer, using a Sensors and Software (Mississauga, ON, Canada) pulseEKKO 100 GPR system equipped with borehole antennas (Fig. 2-1). We conducted three ZOP (zero-offset profile) surveys using antennas with a center frequency of $100 \mathrm{MHz}$, and five MOP (multiple-offset profile) surveys using $200 \mathrm{MHz}$ antennas. We began all the ZOP surveys at the ground surface and proceeded to the bottom of the borehole with a step size of $0.125 \mathrm{~m}$. For all the MOP surveys, we began just below the water table and proceeded to the bottom of the borehole with a step size of $0.25 \mathrm{~m}$. For each GPR trace, we picked the arrival time of the direct wave from which the electromagnetic wave velocity was extracted. The value of $\theta$ was calculated from the velocities using the BHS (Bruggeman-Hanai-Sen) mixing formula (Sen et al., 1981; Feng and Sen, 1985).

The interwell velocity structure, measured with ZOPs and MOPs, is different as a 
result of the different geometrical configuration used for each of these surveys. The ZOPs have only horizontal ray paths collected between each station in the boreholes. Since the travel time measured for each station reflects the average of the electromagnetic wave velocity between the boreholes, only vertical variations in these average values are measured by ZOPs. In contrast, MOPs have ray paths at many different angles between the boreholes. Inversion of travel times for these ray paths yields both vertical and horizontal variations in interwell velocity. Since only ZOPs were completed in the unsaturated zone, only the vertical variation in the velocity and water content could be measured. In the saturated zone, we completed ZOPs and MOPs. The travel times from these were inverted to reconstruct the horizontal and vertical interwell velocity structure using the tomographic inversion code Pronto (Aldridge and Oldenburg, 1993). The inversions were performed with the domain divided into $0.25-\mathrm{m}$ square cells. In the saturated zone, the water content is equal to the porosity, $\mathrm{N}$, and the BHS equation provides a direct estimate of the soil porosity. In the unsaturated zone, the moisture content is given by $\theta=\mathrm{N} S_{\mathrm{r}}$, where $S_{\mathrm{r}}$ is the degree of saturation, which ranges between 0 and 1. In this zone, the BHS equation provides an estimate of the water content only; therefore, we measured porosity directly on cores recovered from the unsaturated zone. The porosity was estimated from the difference in mass between saturated and oven-dried samples.

\section{Predictive models}

We evaluated four predictive models: one empirical model (Johansen, 1975) and three mixing models (de Vries, 1963; Gori, 1983; Campbell et al., 1994). Each of these models may be used to predict the apparent thermal conductivity in saturated and unsaturated porous media under variable temperature conditions. The empirical model by Johansen (1975) uses a form of interpolation between the apparent thermal conductivity of dry and saturated sediments. The mixing models by de Vries (1963) and Campbell et al. (1994) are based on the analog to the Maxwell model for the electrical conductivity of a mixture of spheres dispersed in a continuous fluid. The mixing model by Gori (1983) models the 
porous medium as a cubic space with a cubic centered solid grain surrounded by a mixture of air and water. Only the basic equations for the four models are presented below.

The apparent thermal conductivity of an unsaturated porous medium is given by Johansen (1975) as

$$
\lambda=K_{\mathrm{e}}\left(\lambda_{\text {sat }}-\lambda_{\text {dry }}\right)+\lambda_{\text {dry }},
$$

where $\lambda_{\text {sat }}$ is the thermal conductivity of the saturated porous medium, $\lambda_{\text {dry }}$ is the thermal conductivity of the dry porous medium, and $K_{\mathrm{e}}$ is the Kersten number. The thermal conductivity of the saturated porous medium is

$$
\lambda_{\text {sat }}=\lambda_{\mathrm{s}}^{1-\phi} \lambda_{\mathrm{w}}^{\phi},
$$

where $\lambda_{\mathrm{s}}$ is the thermal conductivity of the solids and $\lambda_{\mathrm{w}}$ is the thermal conductivity of water. The thermal conductivity of a dry, coarse porous medium is given by

$$
\lambda_{\text {dry }}=\frac{0.137 \rho_{\mathrm{d}}+64.7}{\rho_{\mathrm{s}}-0.947 \rho_{\mathrm{d}}} \pm 20 \%,
$$

where $\rho_{\mathrm{d}}$ is the dry bulk density $\left(\mathrm{kg} \mathrm{m}^{-3}\right)$ and $\rho_{\mathrm{s}}$ is the density of the solids $\left(\mathrm{kg} \mathrm{m}^{-3}\right)$. The form of $K_{\mathrm{e}}$ given by Johansen (1975) applies only when $S_{\mathrm{r}}>0.05$. Below this level, it underestimates the value of the thermal conductivity and alternate models must be used (Farouki, 1981, 1982); however, the use of different models produces discontinuities at 
the transition points and is cumbersome to implement. To overcome these problems, we implemented the following form of the Kersten number (Ewen and Thomas, 1987):

$$
K_{\mathrm{e}}=1-\exp \left(\beta S_{\mathrm{r}}\right)
$$

where $\beta$ is a fitting parameter. While this form of $K_{\mathrm{e}}$ provides a continuous equation that applies across the full range of saturation, Eq. (2.1) and (2.2) do not yield the same $\lambda$ at full saturation when $\beta>-4.5$. We eliminated this discrepancy by modifying Eq. (2.1) as follows

$$
\lambda=K_{\mathrm{e}}\left(\lambda_{\text {sat }}-\lambda_{\text {dry }}\right)+\lambda_{\text {dry }}+S_{\mathrm{r}}\left(\lambda_{\text {sat }}-\lambda_{S_{\mathrm{r}}=1}\right)
$$

Here $\lambda_{S_{\mathrm{r}}=1}$ is evaluated using the unmodified form of Eq. (2.1).

Of much greater complexity than the Johansen empirical model are the mixing models. In the mixing model by de Vries (1963), the apparent thermal conductivity is calculated using

$$
\lambda=\frac{\sum_{i=0}^{n} k_{i} x_{i} \lambda_{i}}{\sum_{i=0}^{n} k_{i} x_{i}},
$$

where $x_{i}$ is the volume fraction of each constituent (air, water, or soil particle or mineral fraction), $\lambda_{i}$ is the thermal conductivity of each constituent, and $n$ is the number of soil constituents. The weighting factor $k_{i}$ is the ratio of the average temperature gradient in the 
$i$ th component in the soil to the temperature gradient in the continuous medium and is related to the shape and conductivity of the component. All components with the same shape and conductivity are considered as one type and have common $\lambda_{i} \mathrm{~s}$ and $k_{i} \mathrm{~s}$. The subscript zero applies to the continuous medium surrounding the soil particles, which for dry soils is air and for moist to saturated soils is water. For the continuous medium $k_{0}=1$, and the remaining $k_{i} \mathrm{~s}$ are given by

$$
k_{i}=\frac{1}{3} \sum_{j=1}^{3}\left[1+\left(\frac{\lambda_{i}}{\lambda_{0}}-1\right) g_{j}\right]^{-1}
$$

where $g_{j}$ are the shape factors for the $i$ th component and $\lambda_{0}$ is the thermal conductivity of the continuous phase. The quantities $g_{j}$ depend on the ratio of the major axes of the ellipsoid for the soil component, and $g_{1}+g_{2}+g_{3}=1$. Most soil particles are spheroids having $g_{1}=g_{2}=m g_{3}$, where $m$ varies from 0.1 to 100 (de Vries, 1963). Thus only one shape factor must be estimated for each component.

In unsaturated porous media, the temperature gradients cause moisture movement across the air-filled pores, which redistribute the heat across the pores. This can be described by an apparent thermal conductivity of the air-filled pores due to heat transport by conduction through dry air $\lambda_{\mathrm{a}}$, and by the movement of vapor $\lambda_{\mathrm{vs}}$ in the pores containing moist air at a relative humidity $h$. Thus the apparent thermal conductivity is

$$
\lambda_{\text {app }}=\lambda_{\mathrm{a}}+h \lambda_{\mathrm{vs}}
$$

There are several different expressions for $h$ and $\lambda_{\mathrm{vs}}$ (e.g., de Vries, 1963; Hopmans and Dane, 1986; Campbell et al., 1994; Tarnawski et al., 2000). 
The most complicated aspect of implementing the de Vries model is the evaluation of $g_{j}$ used in Eq. (2.7) for the air pore-shape factors in unsaturated porous media. These shape factors are dependent on the water content and a transition occurs at the field capacity of the soil. De Vries (1963) and Hopmans and Dane (1986) provide detailed descriptions of the procedure required to evaluate $g_{j}$, and we followed these procedures in our implementation of the de Vries model. Less complex implementations are available (de Vries, 1963; Farouki, 1982), but these are derived for the quartz sand considered in the work by de Vries (1963) and they may not be applicable to other soils.

To reduce the complexity of the de Vries model, Campbell et al. (1994) introduced a continuous function for the $k_{i}$ s, which applies across the full range of water contents, and then used $g_{j}$ as an empirical fitting factor. While Campbell's modified form of the de Vries model is easier to implement, it introduces two new parameters, $q_{\mathrm{o}}$ and $x_{\mathrm{wo}}$. The parameter $x_{\mathrm{wo}}$ is the cutoff water content for liquid recirculation and gives the water content at which water starts to affect thermal conductivity. It can be calculated using the relationship for $x_{\text {wo }}\left(\mathrm{m}^{3} \mathrm{~m}^{-3}\right)$ given by Campbell et al. (1994) as

$$
x_{\mathrm{wo}}=0.267 d_{\mathrm{g}}^{-0.2},
$$

where $d_{\mathrm{g}}$ is the geometric mean particle diameter ( $\left.\mu \mathrm{m}\right)$ (Shiozawa and Campbell, 1991). The parameter $q_{\mathrm{o}}$ relates to the rapidity of the transition from air- to water-dominated conductivity and is treated as a fitting parameter (Campbell et al., 1994). Additional details of the Campbell model and the continuous function for the $k_{i}$ s are given in Campbell et al. (1994).

Gori (1983) developed a model based on a cubic grain inside a cubic space for unsaturated frozen porous media. This model has been adapted to consider latent heat transfer in unfrozen soils (Tarnawski et al., 2000). The Gori model was shown to provide good agreement with measured values of thermal conductivity for unsaturated soils at 
temperatures of 30 and $50^{\circ} \mathrm{C}$ (Tarnawski et al., 2000). The equations for this model are quite complex and are not presented here but they can be found in Tarnawski et al. (2000).

\section{Model evaluation and selection}

Our objectives for model evaluation and selection were to compare the apparent thermal conductivities predicted with the models to existing data, and to select the model that best represents a balance between bias (underfitting data with models having few parameters) and variance (overfitting data with models having many parameters). This was achieved by compiling applicable datasets of measured thermal conductivity from the literature, and using AIC (Akaike's information criterion; Akaike, 1973). The AIC is an information-theoretic procedure, based on Kullback-Leibler information theory, and it provides a method for objective model selection. When $n / K<40$, where $n$ is the sample size and $K$ is the number of estimated parameters in the model, the AIC for small sample size, $\mathrm{AIC}_{\mathrm{C}}$ (Table 2-1), is used (Burnham and Anderson, 2002). For all datasets in the literature, $n / K$ was $<40$. The first term in the equation for $\mathrm{AIC}_{\mathrm{C}}$ is a measure of the lack of fit. This term gets smaller as more parameters are added to the model to improve the fit to the data. As more parameters are introduced into the model, the remaining terms in the $\mathrm{AIC}_{\mathrm{C}}$ equation get larger (a penalty for adding more parameters) and parsimony is enforced. As the sample size $n$ increases these terms get smaller. Thus AIC provides a rigorous way to achieve a model of appropriate complexity for a dataset with a given sample size. Burnham and Anderson (2002) described the theoretical foundations of AIC and its application for model ranking, selection, and inference.

Two statistics were used to measure the goodness-of-fit of predicted thermal conductivity against measured conductivity. These included the correlation coefficient $r$ and the $\mathrm{AIC}_{\mathrm{C}}$ (Table 2-1). In practice, one computes the criterion $\mathrm{AIC}_{\mathrm{C}}$ for each model and selects the model with the smallest value. Two additional parameters, $\Delta_{i}$ and $w_{i}$, may be calculated from $\mathrm{AIC}_{\mathrm{C}}$ values. The $\Delta_{i}$ allow an easy ranking of the models from best to worst ( $\Delta=0$ for the best model). In general, models having $\Delta_{i} \leq 2$ are very good models, 
those for which $4 \leq \Delta_{i} \leq 7$ have less support, and models having $\Delta_{i} \geq 10$ can be eliminated as candidates (Burnham and Anderson, 2002; Anderson, 2003). The $w_{i}$, called Akaike weights, are considered as the likelihood or weight of evidence in favor of model $i$ being the best model for the situation being considered. These likelihoods are normalized and can be treated as probabilities. In addition, the ratio $w_{i} / w_{j}$ gives the relative likelihood of model $i$ vs. model $j$ and is termed the evidence ratio. The evidence ratio allows us to state that there is $w_{i} / w_{j}$ times more support for model $i$ than model $j$ (Poeter and Anderson, 2005). 
Table 2-1 Criteria for evaluating the fit of the apparent thermal conductivity, estimated with the candidate models, to the measured thermal conductivity.

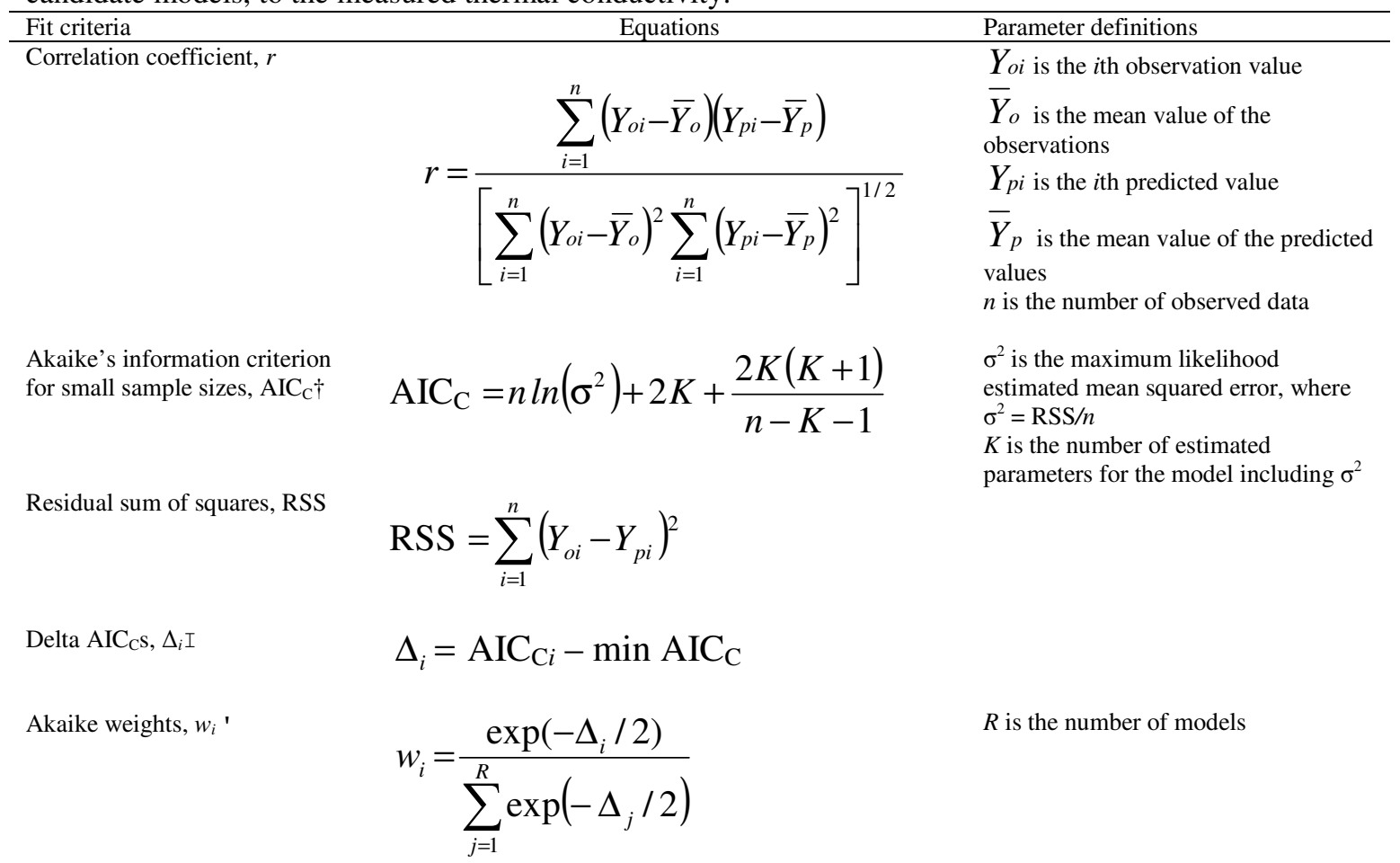

\footnotetext{
$\dagger$ This form of the expression applies to analyses using least squares estimation with normally distributed errors. In general, the model with the lowest $\mathrm{AIC}_{\mathrm{C}}$ value is the best model for the data set being considered.

I $\Delta_{i}$ represents the information lost by using model $i$ rather than the "best" model. As a rule of thumb, a $\Delta_{i}<2$ suggests the two models have similar support, values between 4 and 7 indicate the model with the larger $\mathrm{AIC}_{\mathrm{C}}$ has less support, and values $>10$ indicate the model has no support and can be neglected in the selection process (Burnham and Anderson, 2002, 2004).

' The Akaike weights range from 0 to 1 and indicate the probability that the model is the best among the models being considered.
} 


\section{Results and Discussion}

\section{Thermal conductivity of aquifer solids}

\section{Divided-bar method}

The thermal conductivity values measured using the divided-bar method are normally distributed and range between 3.38 and $4.81 \mathrm{~W} \mathrm{~m}^{-1} \mathrm{~K}^{-1}$. The mean value and $95 \%$ confidence interval are $4.08 \pm 0.09 \mathrm{~W} \mathrm{~m}^{-1} \mathrm{~K}^{-1}$ and the standard deviation is $0.29 \mathrm{~W} \mathrm{~m}^{-1}$ $\mathrm{K}^{-1}$ (Table 2-2). A box-whisker plot of measured thermal conductivity values suggests that $\lambda_{\mathrm{s}}$ may be correlated with the stratigraphic units (Fig. 2-2). To test this hypothesis, we conducted one-way unbalanced ANOVA. We excluded the data for the soil (S) from the analysis since there are only two samples. The Brown-Forsythe modification of Levene's test for the homogeneity of variance (Brown and Forsythe, 1974) indicated that the variances could be assumed equal $\left[F_{(4,34)}=2.035, p \leq 0.112\right]$.

Table 2-2 Thermal conductivity of the porous media solids $\left(\lambda_{\mathrm{s}}\right)$ measured by the divided-bar apparatus and estimated from the mineral composition measured by X-ray diffraction.

\begin{tabular}{|c|c|c|c|}
\hline \multirow{2}{*}{$\begin{array}{l}\text { Sample } \\
\text { identification }\end{array}$} & \multirow[b]{2}{*}{ Sample description $\dagger$} & \multicolumn{2}{|c|}{ Thermal conductivity of the porous media solids $\lambda_{s}$, } \\
\hline & & \multirow[t]{2}{*}{ Divided-bar apparatus } & Mineral composition \\
\hline & & & \\
\hline H1-R6-26 & clay silt till at till-aquifer contact $(\mathrm{T})$ & 4.08 & \\
\hline H1-R6 & clay silt till $(\mathrm{T})$ & 3.38 & 4.06 \\
\hline H9-R4 & clay silt till at till-aquifer contact $(\mathrm{T})$ & 3.99 & 3.92 \\
\hline H11-R5 & clay silt till at till-aquifer contact $(\mathrm{T})$ & 3.42 & 4.16 \\
\hline H3-R1b & dark brown humus rich fine sandy soil (S) & 3.81 & 5.01 \\
\hline H5-R1 & dark brown humus rich fine sandy soil $(\mathrm{S})$ & 3.87 & \\
\hline H1-R1 & fine sand $(\mathrm{FS})$ & 4.23 & 4.45 \\
\hline $\mathrm{H} 1-\mathrm{R} 2 \mathrm{~b}$ & fine sand (FS) & 4.39 & 4.64 \\
\hline H1-R5 & fine sand (FS) & 3.85 & 4.31 \\
\hline H3-R3a & fine sand (FS) & 4.42 & 4.09 \\
\hline H5-R4 & fine sand (FS) & 4.56 & \\
\hline H9-R4 & fine sand (FS) & 4.04 & 4.23 \\
\hline H1-R5 & medium sand (MS) & 4.56 & \\
\hline H1-R6 & medium sand (MS) & 3.98 & 3.59 \\
\hline H2-R2 & medium sand (MS) & 4.34 & 4.05 \\
\hline H5-R4 & medium sand (MS) & 4.27 & 3.88 \\
\hline H6-R5 & medium sand (MS) & 4.02 & 3.82 \\
\hline H8-R2 & medium sand (MS) & 4.15 & \\
\hline H8-R3 & medium sand (MS) & 4.81 & 4.37 \\
\hline H8-R4 & medium sand (MS) & 4.33 & \\
\hline H8-R4 & medium sand (MS) & 4.28 & 3.62 \\
\hline H9-R4 & medium sand (MS) & 3.98 & \\
\hline
\end{tabular}




\begin{tabular}{|c|c|c|c|}
\hline \multirow{2}{*}{$\begin{array}{l}\text { Sample } \\
\text { identification }\end{array}$} & \multirow[b]{2}{*}{ Sample description $\dagger$} & \multicolumn{2}{|c|}{ Thermal conductivity of the porous media solids $\lambda_{s}$, } \\
\hline & & Divided-bar apparatus & Mineral composition \\
\hline H1-R2a & coarse sand with minor gravel (CS) & 4.04 & 3.94 \\
\hline H1-R5 & coarse sand $(\mathrm{CS})$ & 3.95 & 3.83 \\
\hline H2-R5a & coarse sand $(\mathrm{CS})$ & 4.23 & 3.93 \\
\hline H2-R5a & coarse sand with minor gravel (CS) & 4.29 & \\
\hline H5-R3 & coarse sand $(\mathrm{CS})$ & 4.33 & \\
\hline H9-R2 & coarse sand (CS) & 4.28 & 3.91 \\
\hline H11-R5 & coarse sand (CS) & 4.06 & 3.84 \\
\hline H12-R5 & coarse sand (CS) & 3.93 & 3.34 \\
\hline H1-R3 & poorly sorted gravel with sand $(\mathrm{G})$ & 4.04 & 3.70 \\
\hline H1-R5 & poorly sorted gravel with sand $(\mathrm{G})$ & 3.80 & \\
\hline H3-R2a & poorly sorted gravel with sand $(\mathrm{G})$ & 4.06 & 3.85 \\
\hline H5-R2-tip & poorly sorted gravel with sand $(\mathrm{G})$ & 3.84 & \\
\hline H5-R2 & poorly sorted gravel with sand $(\mathrm{G})$ & 4.03 & 3.88 \\
\hline H5-R3 & poorly sorted gravel with sand $(\mathrm{G})$ & 3.74 & \\
\hline H6-R5 & poorly sorted gravel with sand (G) & 3.83 & 3.58 \\
\hline H11-R1 & poorly sorted gravel with sand and silt (G) & 4.22 & \\
\hline H11-R5 & $\begin{array}{l}\text { poorly sorted gravel with sand at till-aquifer } \\
\text { contact }(G)\end{array}$ & 3.68 & 3.92 \\
\hline H12-R3t & poorly sorted gravel with sand $(\mathrm{G})$ & 3.96 & \\
\hline H12-R3 & $\begin{array}{l}\text { poorly sorted gravel with coarse sand and cobbles } \\
\text { (G) }\end{array}$ & 4.14 & 4.05 \\
\hline
\end{tabular}

$\dagger$ Type of porous media: (T) till; (S) soil; (FS) fine sand; (MS) medium sand; (CS) coarse sand; and (G) gravel with sand. 


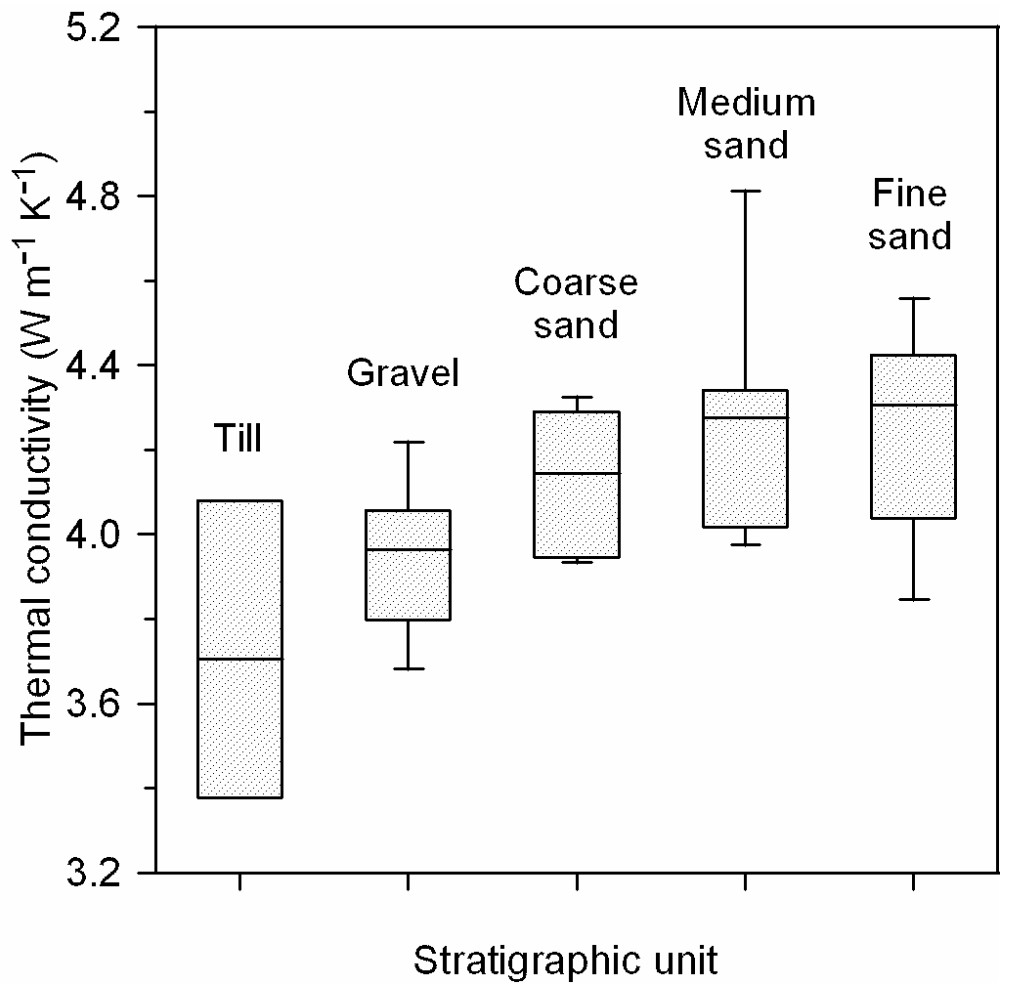

Figure 2-2 Box-whisker plot of the measured thermal conductivity for the solid fraction of porous media grouped by stratigraphic unit. The caps at the end of each box indicate the minimum and maximum values, the box is defined by the lower and upper quartiles (25th and 75th percentiles), and the line in the centre of the box is the median. No outliers were present in the data.

A significant difference between the mean thermal conductivity of the units was observed (Table 2-3). Post hoc MCT (multiple comparison tests) using the methods of Tukey, Scheffé, and Bonferroni (StatSoft, 2001), with an overall error rate of $\alpha=0.05$, suggested that the mean thermal conductivities of till (T), gravel and sand (G), and coarse sand (CS) were less than those of medium sand (MS) and fine sand (FS), and that $\mathrm{T} \sim \mathrm{G}$ $\sim \mathrm{CS}<\mathrm{MS} \sim \mathrm{FS}$. A nonparametric MCT (Conover, 1999) suggested T $\sim \mathrm{G}<$ $\mathrm{CS} \sim \mathrm{MS} \sim \mathrm{FS}$, based on the median thermal conductivities. Given the difficulty of distinguishing between coarse sand and medium sand in the field, it is more practical to group all sand units together. For till, only four measurements were made and the mean has a large standard deviation. We placed till in a separate group since additional measurements would probably decrease the standard deviation, and the MCTs would 
indeed identify till as a separate group. The basic statistical parameters for each of the grouped units are summarized in Table 2-4.

Table 2-3 The ANOVA table (one-way unbalanced analysis) shows the between-groups and within-groups sources of variance for the thermal conductivity data. The null hypothesis, $H_{0}$, was rejected at $p<0.05$.

\begin{tabular}{lcccc}
\hline Source of variance & Degrees of freedom & Sum of squares & Mean square & $F$ \\
\hline Between groups & 4 & 1.300 & 0.325 & 5.88 \\
Error (within groups) & 34 & 1.879 & 0.055 & 0.001 \\
Total & 38 & 3.179 & & \\
\hline
\end{tabular}

Table 2-4 Mean value $\pm 95 \%$ confidence interval, standard deviation, and upper and lower quartiles of the measured thermal conductivity of the porous media solids for individual stratigraphic units and for the grouped sand units.

\begin{tabular}{|c|c|c|c|c|c|}
\hline Stratigraphic unit $\dagger$ & $\begin{array}{l}\text { Measured thermal } \\
\text { conductivity } \ddagger\end{array}$ & $\begin{array}{l}\text { Number of } \\
\text { samples, } n\end{array}$ & $\begin{array}{l}\text { Standard } \\
\text { deviation }\end{array}$ & $\begin{array}{l}\text { 25th percentile or } \\
\text { lower quartile }\end{array}$ & $\begin{array}{l}\text { 75th percentile or } \\
\text { upper quartile }\end{array}$ \\
\hline & 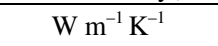 & & 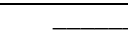 & 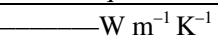 & 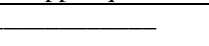 \\
\hline Till $(\mathrm{T})$ & $3.72 \forall 0.59$ & 4 & 0.37 & 3.40 & 4.04 \\
\hline Gravel and sand $(\mathrm{G})$ & $3.94 \forall 0.12$ & 11 & 0.17 & 3.81 & 4.05 \\
\hline Coarse sand (CS) & $4.14 \forall 0.14$ & 8 & 0.16 & 3.99 & 4.29 \\
\hline Medium sand (MS) & $4.27 \forall 0.19$ & 10 & 0.27 & 4.02 & 4.34 \\
\hline Fine sand $(\mathrm{FS})$ & $4.25 \forall 0.28$ & 6 & 0.27 & 4.04 & 4.42 \\
\hline $\begin{array}{l}\text { Fine, medium, and } \\
\text { coarse sand }\end{array}$ & $4.22 \forall 0.10$ & 24 & 0.23 & 4.03 & 4.33 \\
\hline
\end{tabular}

$\uparrow$ Type of porous medium: (T) till; (S) soil; (FS) fine sand; (MS) medium sand; (CS) coarse sand; and (G) gravel with sand. $\$$ Mean value $\pm 95 \%$ confidence interval. 


\section{Mineral composition method}

The mineral compositions for 27 outwash samples were primarily calcite, dolomite, quartz, and plagioclase feldspar (Table 2-5). Some samples contained hornblende, illite, montmorillonite, and chlorite or possibly kaolinite in minor quantities ( $<5 \%$ total). From the known mineral compositions, we estimated the bulk thermal conductivity for the aquifer solids $\lambda_{\mathrm{s}}$ (Table 2-2), having $n$ mineral components with a volume fraction $x_{i}$ and conductivity $\lambda_{i}$, using the geometric mean equation given by

$$
\lambda_{\mathrm{s}}=\prod_{i=1}^{n}\left(\lambda_{i}^{x_{i}}\right) .
$$

A wide range of values are cited in the literature for several rock-forming minerals in their monomineralic and rock form (Table 2-6); we used the underlined values in our calculations. In general, the thermal conductivities given for minerals in their monomineralic form are higher than those reported for rocks. This difference may be due to the presence of other minerals in the rocks tested, intragranular porosity in the rocks that reduces the thermal conductivity, or other factors. For calcite and dolomite, we selected the values of thermal conductivity reported for rocks, as these will account for the intragranular porosity common in these rocks.

Comparison of these calculated conductivities to those obtained using the dividedbar apparatus shows reasonable agreement, with the exception of the values for till. For the gravels and sands, the predicted and measured values are generally within $\pm 10 \%$, and have a correlation coefficient $r$ of 0.473 that is significant at $p<0.05$. For the till samples, the thermal conductivities estimated from the mineral composition are $\sim 20 \%$ larger than the measured values. This difference may be due to incomplete saturation of the till samples when measured using the divided-bar method. Air entrapped in the pore space will decrease the apparent thermal conductivity of the sample. Therefore, with the exception of the till, our results suggest that $\lambda_{\mathrm{s}}$ for the glacial outwash can be measured using the divided-bar apparatus or estimated using the geometric mean equation and the mineral composition of the aquifer solids. 
Table 2-5 Mineral composition of the porous media measured by X-ray diffraction and calculated using the integrated peak area method.

\begin{tabular}{|c|c|c|c|c|c|c|c|c|}
\hline \multirow[b]{3}{*}{$\begin{array}{l}\text { Sample } \\
\text { Identification } \dagger\end{array}$} & \multicolumn{8}{|c|}{ Mineralogical composition (volumetric fraction $\ddagger$ ) } \\
\hline & \multicolumn{2}{|c|}{ Carbonates } & \multirow{2}{*}{$\begin{array}{l}\text { Feldspars } \\
\text { Anorthite }\end{array}$} & \multirow{2}{*}{$\frac{\text { Amphiboles }}{\text { Hornblende }}$} & \multirow{2}{*}{$\frac{\text { Silicates }}{\text { Quartz }}$} & \multicolumn{3}{|c|}{ Micas-clays } \\
\hline & Calcite & Dolomite & & & & Illite & Montmorillonite & $\begin{array}{l}\text { Chlorite/ } \\
\text { kaolinite }\end{array}$ \\
\hline H1-R6 (T) & 0.32 & 0.39 & 0.06 & & 0.21 & 0.006 & & 0.005 \\
\hline H9-R4 (T) & 0.29 & 0.48 & 0.04 & 0.005 & 0.17 & 0.006 & & \\
\hline H11-R5 (T) & 0.35 & 0.32 & 0.01 & 0.002 & 0.29 & 0.015 & & 0.005 \\
\hline H3-R1b (S) & 0.05 & 0.05 & 0.15 & 0.007 & 0.71 & 0.01 & 0.008 & 0.023 \\
\hline H1-R1 (FS) & 0.20 & 0.48 & 0.01 & & 0.31 & & & \\
\hline H1-R2b (FS) & 0.18 & 0.27 & 0.06 & & 0.48 & & & \\
\hline H1-R5 (FS) & 0.24 & 0.28 & 0.09 & 0.008 & 0.37 & & 0.005 & \\
\hline H3-R3a (FS) & 0.16 & 0.31 & 0.10 & & 0.42 & & & \\
\hline H9-R4 (FS) & 0.26 & 0.39 & 0.07 & & 0.27 & & 0.005 & 0.005 \\
\hline H1-R6 (MS) & 0.28 & 0.34 & 0.12 & 0.007 & 0.25 & 0.003 & & \\
\hline H2-R2 (MS) & 0.28 & 0.37 & 0.05 & & 0.30 & & & \\
\hline H5-R4 (MS) & 0.40 & 0.54 & 0.01 & & 0.05 & & & \\
\hline H6-R5 (MS) & 0.33 & 0.38 & 0.06 & & 0.23 & & & \\
\hline H8-R3 (MS) & 0.22 & 0.25 & 0.05 & & 0.48 & & & \\
\hline H8-R4 (MS) & 0.35 & 0.38 & 0.19 & 0.003 & 0.07 & & 0.002 & \\
\hline H1-R2a (CS) & 0.34 & 0.52 & 0.05 & & 0.09 & & & 0.006 \\
\hline H1-R5 (CS) & 0.49 & 0.33 & 0.02 & & 0.16 & & & \\
\hline H2-R5a (CS) & 0.35 & 0.42 & 0.07 & & 0.15 & & & 0.003 \\
\hline H9-R2 (CS) & 0.26 & 0.37 & 0.17 & 0.005 & 0.19 & & & 0.004 \\
\hline H11-R5 (CS) & 0.37 & 0.33 & 0.05 & & 0.25 & & & \\
\hline H12-R5 (CS) & 0.35 & 0.30 & 0.14 & 0.006 & 0.20 & & & \\
\hline H1-R3 (G) & 0.43 & 0.35 & 0.04 & 0.006 & 0.17 & 0.005 & & \\
\hline H3-R2a (G) & 0.38 & 0.42 & 0.03 & & 0.16 & & & \\
\hline H5-R2 (G) & 0.42 & 0.31 & 0.03 & & 0.24 & & & \\
\hline H6-R5 (G) & 0.60 & 0.29 & 0.01 & & 0.10 & & & \\
\hline H11-R5 (G) & 0.45 & 0.35 & 0.005 & 0.011 & 0.18 & & & 0.005 \\
\hline H12-R3 (G) & 0.34 & 0.43 & 0.04 & 0.001 & 0.18 & & & 0.003 \\
\hline
\end{tabular}


Table 2-6 Thermal conductivity of selected minerals and rocks. The underlined values are considered to be representative of the minerals in the glaciofluvial outwash sands and gravels.

\begin{tabular}{|c|c|c|c|c|c|c|c|}
\hline \multirow[b]{2}{*}{ Group } & \multirow[b]{2}{*}{ Mineral } & \multirow[b]{2}{*}{ Form $\dagger$} & \multirow[b]{2}{*}{ Reference } & \multicolumn{3}{|c|}{ Thermal conductivity } & \multirow[b]{2}{*}{ Temperature } \\
\hline & & & & range & mean & $n$ & \\
\hline & & & & $\longrightarrow$ & ${ }^{1} \mathrm{~K}^{-1}$ & & EC \\
\hline \multirow[t]{5}{*}{ Silica } & quartz & & & & $\underline{6.00}$ & & \\
\hline & & mineral $(a) \dagger$ & Horai (1971) & & $\overline{7.69}$ & & 20 \\
\hline & & mineral $(\perp) \ddagger$ & Clauser and Huenges (1995) & & 6.15 & & 30 \\
\hline & & mineral $(\|)$ & Clauser and Huenges (1995) & & 10.17 & & 30 \\
\hline & & mineral & Tarnawski et al. (2000) & $5.65-6.25$ & 5.95 & & 20 \\
\hline \multirow[t]{15}{*}{ Carbonates } & calcite & & & & $\underline{3.00}$ & & \\
\hline & & mineral (a) & Horai (1971) & & 3.59 & 1 & 20 \\
\hline & & rock (limestone) & Hellwege and Angenheister (1982) & $0.6-4.4$ & 2.29 & 487 & \\
\hline & & rock (limestone) & Misener et al. (1951) & $1.96-2.97$ & 2.56 & 12 & 20 \\
\hline & & rock (limestone) & Judge (1971) & $2.68-3.93$ & 3.05 & & \\
\hline & & rock (limestone) & Sass et al. (1971) & & $3.43 \pm 0.62 \S$ & 4 & 25 \\
\hline & & rock (limestone) & Conaway and Beck (1977) & & 2.99 & 34 & 20 \\
\hline & dolomite & & & & 4.50 & & \\
\hline & & mineral (a) & Horai (1971) & & $\overline{5.51}$ & 1 & 20 \\
\hline & & mineral (a) & Clauser and Huenges (1995) & & $4.78 \pm 0.54$ & 70 & $25-35$ \\
\hline & & rock (dolostone) & Clark (1966) & $4.02-5.02$ & 4.60 & 5 & 20 \\
\hline & & rock (dolostone) & Judge (1971) & $3.39-5.56$ & 4.56 & & \\
\hline & & $\begin{array}{l}\text { rock (dolomitic } \\
\text { limestone) }\end{array}$ & Sass et al. (1971) & & $5.20 \pm 0.70$ & 11 & 25 \\
\hline & & rock (dolostone) & Conaway and Beck (1977) & & 4.55 & 34 & 20 \\
\hline & & rock (dolostone) & Hellwege and Angenheister (1982) & $1.6-6.6$ & 3.62 & 129 & \\
\hline \multirow[t]{5}{*}{ Alkali Feldspar } & orthoclase & & & & & & \\
\hline & & mineral (100) & Sass (1965) & & $2.34 \pm 0.11$ & 2 & 30 \\
\hline & & mineral (010) & Sass (1965) & & 2.68 & & 30 \\
\hline & & mineral (001) & Sass (1965) & & $2.30 \pm 0.30$ & 2 & 30 \\
\hline & & mineral (a) & Horai (1971) & & 2.31 & 1 & 20 \\
\hline \multirow[t]{4}{*}{ Plagioclase Feldspar } & albite & mineral (a) & Sass (1965) & & $\underline{2.34}$ & & 25 \\
\hline & & mineral (a) & Horai (1971) & $1.94-2.35$ & $\overline{2.14} \pm 0.22$ & 4 & 20 \\
\hline & anorthite & mineral (a) & Sass (1965) & & 2.72 & & 25 \\
\hline & & mineral (a) & Horai (1971) & & $\overline{1.68}$ & 1 & 20 \\
\hline \multirow[t]{2}{*}{ Amphibole } & hornblende & mineral (a) & Clauser and Huenges (1995) & & $\underline{2.91} \pm 0.09$ & 2 & 20 \\
\hline & & mineral (a) & Horai (1971) & $2.54-3.08$ & $2.81 \pm 0.38$ & 2 & 20 \\
\hline \multirow[t]{3}{*}{ Clay minerals } & biotite & mineral (a) & Horai (1971) & $1.70-2.34$ & $\underline{2.02} \pm 0.45$ & 2 & \\
\hline & chlorite & mineral (a) & Clauser and Huenges (1995) & & $\overline{5.25} \pm 0.15$ & 2 & \\
\hline & & mineral (a) & Horai (1971) & $4.34-6.18$ & $\overline{5.14} \pm 0.94$ & 3 & 20 \\
\hline
\end{tabular}




\begin{tabular}{|c|c|c|c|c|c|c|c|}
\hline \multirow[b]{2}{*}{ Group } & \multirow[b]{2}{*}{ Mineral } & \multirow[b]{2}{*}{ Form $\dagger$} & \multirow[b]{2}{*}{ Reference } & \multicolumn{3}{|c|}{ Thermal conductivity } & \multirow[b]{2}{*}{ Temperature } \\
\hline & & & & range & mean & $n$ & \\
\hline & & mineral & Clark (1966) & & 5.23 & & 20 \\
\hline & illiteđI & mineral (a) & Horai (1971) & $2.21-2.49$ & $\underline{2.32} \pm 0.15$ & 3 & 20 \\
\hline & kaolinite & & & & $\overline{5.25} \pm 0.15$ & & \\
\hline & montmo & & & & $5.25 \pm 0.15$ & & \\
\hline
\end{tabular}

$\perp$ Cited values are for randomly oriented crystals of the mineral unless it is denoted by (a) which indicates monomineralic aggregates.

$\ddagger$ Directions of anisotropy are specified by the minerals' optical a-, b-, or c-axes $(100,010,001)$ or by the thermal conductivity component normal or parallel to the direction of the maximum thermal conductivity $(\|, \perp)$.

$\S$ Where available, the mean and standard deviation are reported.

I Illite is structurally similar to muscovite and the value for muscovite (Horai, 1971) was used.

\# The thermal conductivity was assumed to be equal to that of chlorite. 


\section{Volumetric water content and porosity}

The two-dimensional distribution of volumetric water content in the saturated zone, measured with the GPR MOPs, shows three distinct layers with different water contents (Fig. 2-3a). Between $\sim 1.5$ and $4 \mathrm{~m}$ bgs (below ground surface), $\theta$ varies from 0.23 to 0.30 $\mathrm{m}^{3} \mathrm{~m}^{-3}$. Between 4 and $5.6 \mathrm{~m}$ bgs, $\theta$ increases to $0.35 \mathrm{~m}^{3} \mathrm{~m}^{-3}$, and below $5.6 \mathrm{~m} \mathrm{bgs}, \theta$ is $0.32 \mathrm{~m}^{3} \mathrm{~m}^{-3}$. Comparison of the variation in the water content (Fig. 2-3a) to the geological cross-section (Fig. 2-4a) suggests that $\theta$ is not directly correlated to the stratigraphic units. We speculate that these differences in $\theta$ may be related to variations in the depositional environment during deposition of the outwash sediments. Thus the GPR provides an important direct measurement of the aquifer water content.

(a)

(b)
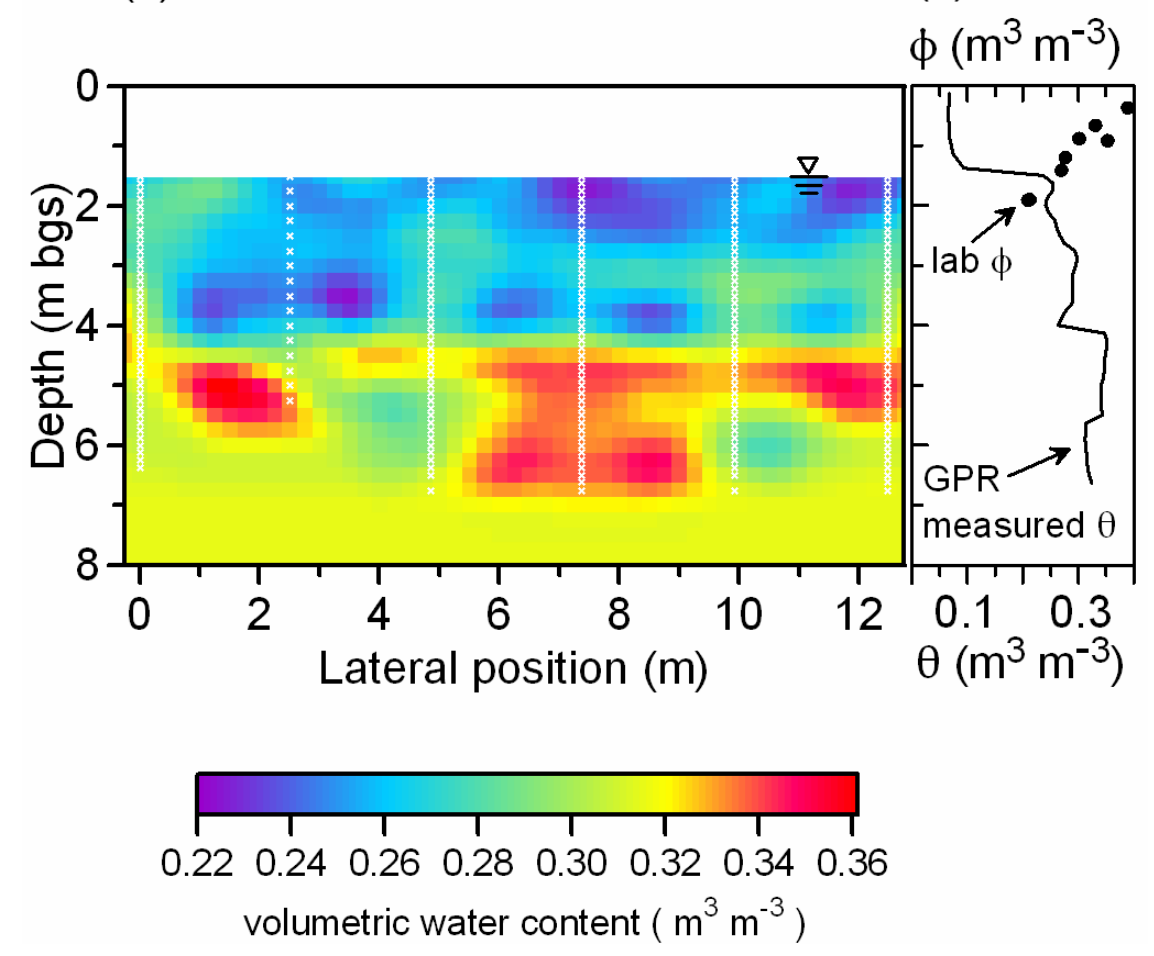

Figure 2-3 (a) Two-dimensional volumetric water content tomogram for the saturated zone, calculated from the interwell velocity tomogram and the Burggeman-Hanai-Sen mixing formula (Sen et al., 1981; Feng and Sen, 1985). The white $X$ s indicate the transmitter and receiver station locations. (b) Measured water content (2) variation vs. depth between the two boreholes on the right side of the section. In the saturated zone, the water content is equal to the porosity $(\mathrm{N})$. Above the water table, the porosity was 
measured in the laboratory on core collected during drilling. The locations and measured values of the porosity are indicated by filled circles.

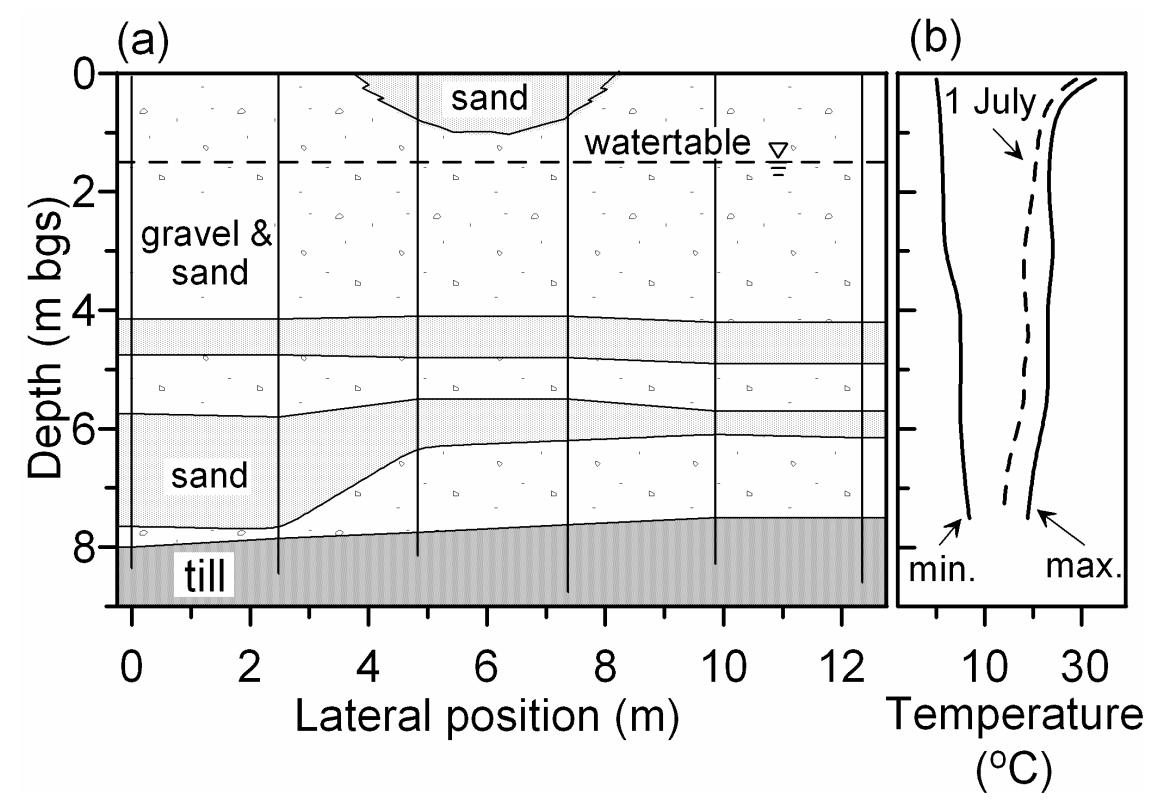

Figure 2-4 (a) Geological cross-section of the area over which the GPR (groundpenetrating radar) survey was completed. The three major stratigraphic units shown are gravel and sand, sand, and till. The vertical lines are the locations of the boreholes where core samples were collected during drilling and where the cross-hole GPR survey was conducted. The unsaturated zone is $\sim 1.5 \mathrm{~m}$ thick. (b) Envelope of the annual temperature variation (minimum observed temperature on the left and maximum on the right) and the temperature profile on 1 July for this section of the aquifer.

The volumetric water content in the saturated and unsaturated zones, measured with the ZOPs, shows the large contrast between these zones (Fig. 2-3b). In the unsaturated zone, the water content decreases rapidly from $0.25 \mathrm{~m}^{3} \mathrm{~m}^{-3}$ at the water table to $\sim 0.07 \mathrm{~m}^{3} \mathrm{~m}^{-3}$ above the capillary fringe. In the saturated zone, the variations in the water content that are evident in the ZOP, such as the higher water content layer between 4 and $5.6 \mathrm{~m} \mathrm{bgs}$, span the width of the tomogram (Fig. 2-3a). Porosity values in the unsaturated zone are sparse laterally and provide information on only the vertical variation. Porosity increases from $0.25 \mathrm{~m}^{3} \mathrm{~m}^{-3}$ at the water table to $0.4 \mathrm{~m}^{3} \mathrm{~m}^{-3}$ near the ground surface. 


\section{Evaluation of candidate models}

We chose six datasets that were representative of the types of sediments found at our site, and that had all the input data required by each of the candidate models (Table 2-7). We did not evaluate the models for gravel, as a dataset with sufficient information was not available. For most datasets, the thermal conductivity measurements are reported at a single temperature that is generally between 20 and $26^{\circ} \mathrm{C}$. For three soils we considered, measurements at more than one temperature are available: the quartz sand (de Vries, 1963); the loamy sand (Sepaskhah and Boersma, 1979); and the L-Soil (Campbell et al., 1994). While Campbell et al. (1994) completed measurement on 10 soils, all but the LSoil were finer grained than those at our site and were not considered. Hopmans and Dane (1986) measured the thermal conductivity of a Norfolk sandy loam at four different temperatures; however, there were too few measurements in this dataset for use in our analysis.

To evaluate the models, we compared the predicted apparent thermal conductivity with the measured conductivity for each soil across moisture conditions ranging from dry to saturated soil. In our analysis, we treated $\beta$ (Johansen's model), $g_{1}, q_{\mathrm{o}}$, and $x_{\text {wo }}$ (Campbell's model), $g_{1}, f_{\mathrm{c}}$ and $x_{\mathrm{c}}$ (de Vries' model), $\theta_{\mathrm{aw}}$ and $x_{\mathrm{c}}$ (Gori's model), and $\lambda_{\mathrm{s}}$ (all models) as fitting parameters. The best fit for each model to the soil data (Table 2-7) was obtained using the parameter estimation techniques in PEST, Version 9.01 (Doherty, 2005).

The correlation coefficients were generally $>0.95$ (Table $2-8$ ), indicating reasonable fits to the data with all the models. The $\mathrm{AIC}_{\mathrm{C}}$ values indicate that Campbell's model is the best approximating model for five of the datasets (quartz sand at $40^{\circ} \mathrm{C}$, loamy sand at 25 and $45^{\circ} \mathrm{C}$, Sandfly Creek sand, and Tottori dune sand), Johansen's model is the best model for two datasets (quartz sand at $20^{\circ} \mathrm{C}$ and Leighton Buzzard sand), and de Vries' model and Gori's model are each the best for one dataset (L-soil at $30^{\circ} \mathrm{C}$ and L-soil at $50^{\circ} \mathrm{C}$, respectively). For many of the datasets, there is no competitor to the top-ranked model $\left(\Delta_{i}>10\right)$. The exceptions, for which there is a competitor $\left(4<\Delta_{i}<\right.$ 7), are the quartz sand at $40^{\circ} \mathrm{C}$, the L-soil at $30^{\circ} \mathrm{C}$, and the Tottori dune sand. For the 
quartz sand at $40^{\circ} \mathrm{C}$, the weight for the top-ranked model (Campbell's model) is 0.99 , the weight for the second-ranked model (de Vries' model) is 0.072 , and the evidence ratio is 14 (there is 14 times more support for Campbell's model). For the Tottori dune sand, the weight for the top-ranked model (Campbell's model) is 0.84 , the weight for the secondranked model (Johansen's model) is 0.16 , and the evidence ratio is 5.1. In these two cases, there is strong support for Campbell's model. For the L-soil at $30^{\circ} \mathrm{C}$, the Campbell model has similar support to the de Vries model $\left(\Delta_{i}=0.8\right)$. Both models have similar Akaike weights and the evidence ratio for the de Vries model vs. the Campbell model is only 1.5. This is not strong evidence that the de Vries model is the best model. Since Campbell's model is the $\mathrm{AIC}_{\mathrm{C}}$-selected model for five of the nine datasets, and a strong competitor for another, we chose it as the "best-approximating model." 
Table 2-7 Parameter values input into the candidate models used to estimate the apparent thermal conductivity of the soil.

\begin{tabular}{|c|c|c|c|c|c|c|c|c|c|c|c|c|c|c|}
\hline \multirow[b]{2}{*}{ Soil } & \multirow[b]{2}{*}{ Reference } & \multirow[b]{2}{*}{$\begin{array}{l}\text { Porosity } \\
\mathrm{N}\end{array}$} & \multirow[b]{2}{*}{$\begin{array}{l}\text { Mineral } \\
\text { composition } \\
\text { of solids }\end{array}$} & \multirow[b]{2}{*}{$\begin{array}{c}\text { Dry } \\
\text { bulk } \\
\text { density } \\
\rho_{\mathrm{d}}\end{array}$} & \multirow[b]{2}{*}{$\begin{array}{c}\text { Density } \\
\text { of soil } \\
\text { particles } \\
\rho_{\mathrm{s}}\end{array}$} & \multirow[b]{2}{*}{$\begin{array}{c}\text { Thermal } \\
\text { conductivity of soil } \\
\text { solids } \dagger \\
\lambda_{\mathrm{s}}\end{array}$} & $\begin{array}{l}\text { Johansen } \\
\text { empirical } \\
\text { equation }\end{array}$ & \multicolumn{3}{|c|}{$\begin{array}{l}\text { Campbell et al. (1994) } \\
\text { modified de Vries model }\end{array}$} & \multicolumn{3}{|c|}{ de Vries (1963) } & \multirow{2}{*}{$\begin{array}{c}\text { Gori } \\
(1983) \ddagger \\
2_{\text {aw }}\end{array}$} \\
\hline & & & & & & & $\beta$ & $\begin{array}{l}\text { Shape } \\
\text { factor } \\
g_{j}\end{array}$ & $q_{\mathrm{o}}$ & $x_{\mathrm{wo}}$ & $\begin{array}{c}\text { Shape } \\
\text { factor } \\
g_{j}\end{array}$ & $f_{\mathrm{c}}$ & $x_{\mathrm{c}}$ & \\
\hline & & $\mathrm{m}^{3} \mathrm{~m}^{-3}$ & & $\mathrm{~kg} \mathrm{~m}^{-3}$ & $\mathrm{~kg} \mathrm{~m}^{-3}$ & $\mathrm{~W} \mathrm{~m}{ }^{-1} \mathrm{~K}^{-1}$ & & & & $\mathrm{~m}^{3} \mathrm{~m}^{-3}$ & & 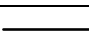 & $\mathrm{m}^{3} \mathrm{~m}^{-3}$ & $\bar{\square}$ \\
\hline Quartz sand & $\begin{array}{l}\text { de Vries } \\
(1963)\end{array}$ & 0.427 & $\begin{array}{l}0.89 \text { quartz } \\
0.11 \text { feldspar and } \\
\text { others }\end{array}$ & 1513 & 2640 & $\begin{array}{l}7.33 \text { at } 20^{\circ} \mathrm{C} \\
6.83 \text { at } 40^{\circ} \mathrm{C}\end{array}$ & -4.53 & 0.100 & 2.657 & 0.037 & 0.110 & 0.09 & 0.062 & 0.0001 \\
\hline Loamy sand & $\begin{array}{l}\text { Sepaskhah } \\
\text { and Boersma } \\
\text { (1979) }\end{array}$ & 0.475 & & 1690 & 2650 & $\begin{array}{l}3.35 \text { at } 25 \text { and } \\
45^{\circ} \mathrm{C}\end{array}$ & -1.19 & 0.074 & 7.388 & 0.211 & 0.183 & 0.50 & 0.25 & 0.0001 \\
\hline $\begin{array}{l}\text { Leighton } \\
\text { Buzzard sand }\end{array}$ & $\begin{array}{l}\text { Ewen and } \\
\text { Thomas } \\
\text { (1987) }\end{array}$ & 0.388 & 0.96 quartz & 1650 & 2700 & 6.60 at $20^{\circ} \mathrm{C}$ & -8.97 & 0.100 & 4.597 & 0.022 & 0.100 & 0.05 & 0.028 & 0.0295 \\
\hline L-Soil & $\begin{array}{l}\text { Campbell et } \\
\text { al. (1994) }\end{array}$ & 0.470 & & 1500 & 2650 & $\begin{array}{l}2.61 \text { at } 30 \text { and } \\
50^{\circ} \mathrm{C}\end{array}$ & -2.29 & 0.101 & 3.192 & 0.095 & 0.188 & 0.10 & 0.10 & 0.0274 \\
\hline $\begin{array}{l}\text { Sandfly } \\
\text { Creek sand }\end{array}$ & $\begin{array}{l}\text { Bristow } \\
(1998)\end{array}$ & 0.428 & $\begin{array}{l}0.49 \text { quartz, } \\
0.51 \text { albite, } \\
\text { K feldspar, } \\
\text { amphibole, mica- } \\
\text { illite, and smectite }\end{array}$ & 1520 & $\begin{array}{l}2660 \text { for } \\
\text { quartz } \\
\text { and } 2650 \\
\text { for other } \\
\text { minerals }\end{array}$ & $\begin{array}{l}5.90 \text { assumed to be } \\
\text { at } 25^{\circ} \mathrm{C}\end{array}$ & -2.79 & 0.125 & 7.086 & 0.059 & 0.125 & 0.179 & 0.085 & 0.03 \\
\hline $\begin{array}{l}\text { Tottori dune } \\
\text { sand }\end{array}$ & $\begin{array}{l}\text { Mori et al. } \\
\text { (2003) }\end{array}$ & 0.371 & & 1630 & 2655 & 3.65 at $20^{\circ} \mathrm{C}$ & -2.88 & 0.125 & 1.610 & 0.061 & 0.125 & 0.30 & 0.077 & 0.0001 \\
\hline
\end{tabular}

$\dagger$ Thermal conductivity estimated using PEST (Doherty, 2005).
$\neq$ The Gori model used the value of $x_{\mathrm{c}}$ determined for the de Vries model. 
Table 2-8 Summary of Akaike Information Criterion $\left(\mathrm{AIC}_{\mathrm{C}}\right)$ measures between the apparent thermal conductivity predicted by the candidate models and the measured values reported in the literature.

\begin{tabular}{|c|c|c|c|c|c|c|c|}
\hline Dataset soil type and reference & $n$ & Model & $\begin{array}{c}\text { Correlation } \\
\text { coefficient } \\
r \\
\end{array}$ & $\begin{array}{c}\text { Residual } \\
\text { Sum of } \\
\text { Squares } \\
\text { RSS } \\
\end{array}$ & $\begin{array}{c}\text { Akaike's } \\
\text { information } \\
\text { criterion } \\
\mathrm{AIC}_{\mathrm{C}}^{\dagger} \\
\end{array}$ & $\begin{array}{c}\text { Delta } \\
\text { AIC }_{C} \\
\Delta_{i}\end{array}$ & $\begin{array}{c}\text { Akaike } \\
\text { weights, } \\
w_{i}\end{array}$ \\
\hline $\begin{array}{l}\text { Quartz sand at } 20^{\circ} \mathrm{C} \\
\quad(\text { de Vries, 1963) }\end{array}$ & 14 & $\begin{array}{l}\text { Johansen (1975) } \\
\text { de Vries (1963) } \\
\text { Campbell et al. (1994) } \\
\text { Gori (1983) }\end{array}$ & $\begin{array}{l}0.996 \\
0.997 \\
0.993 \\
0.945\end{array}$ & $\begin{array}{l}9.602 \times 10^{-2} \\
1.002 \times 10^{-1} \\
1.457 \times 10^{-1} \\
5.338\end{array}$ & $\begin{array}{c}-61.4 \\
-51.7 \\
-46.4 \\
-1.1\end{array}$ & $\begin{array}{c}0 \\
9.7 \\
14.9 \\
60.3\end{array}$ & $\begin{array}{l}9.9 \times 10^{-1} \ddagger \\
7.8 \times 10^{-3} \\
5.7 \times 10^{-4} \\
8.0 \times 10^{-14}\end{array}$ \\
\hline $\begin{array}{l}\text { Quartz sand at } 40^{\circ} \mathrm{C} \\
\text { (de Vries, 1963) }\end{array}$ & 14 & $\begin{array}{l}\text { Johansen (1975) } \\
\text { de Vries (1963) } \\
\text { Campbell et al. (1994) } \\
\text { Gori (1983) }\end{array}$ & $\begin{array}{l}0.987 \\
0.997 \\
0.998 \\
0.946\end{array}$ & $\begin{array}{l}1.053 \\
7.684 \times 10^{-2} \\
5.287 \times 10^{-2} \\
5.593\end{array}$ & $\begin{array}{l}-27.8 \\
-55.4 \\
-60.6 \\
-0.4\end{array}$ & $\begin{array}{c}32.8 \\
5.2 \\
0 \\
60.2\end{array}$ & $\begin{array}{l}7.6 \times 10^{-8} \\
7.2 \times 10^{-2} \\
9.9 \times 10^{-1} \neq \\
8.4 \times 10^{-14}\end{array}$ \\
\hline $\begin{array}{l}\text { Loamy sand at } 25^{\circ} \mathrm{C} \\
\quad \text { (Sepaskhah and Boersma, 1979) }\end{array}$ & 13 & $\begin{array}{l}\text { Johansen (1975) } \\
\text { de Vries (1963) } \\
\text { Campbell et al. (1994) } \\
\text { Gori (1983) }\end{array}$ & $\begin{array}{l}0.944 \\
0.979 \\
0.996 \\
0.990\end{array}$ & $\begin{array}{l}5.546 \times 10^{-1} \\
1.152 \times 10^{-1} \\
2.100 \times 10^{-2} \\
3.370 \times 10^{-1}\end{array}$ & $\begin{array}{l}-32.3 \\
-42.9 \\
-65.0 \\
-34.5\end{array}$ & $\begin{array}{c}32.7 \\
22.1 \\
0 \\
30.5\end{array}$ & $\begin{array}{l}8.1 \times 10^{-8} \\
1.6 \times 10^{-5} \\
1.0 \ddagger \\
2.4 \times 10^{-7}\end{array}$ \\
\hline $\begin{array}{l}\text { Loamy sand at } 45^{\circ} \mathrm{C} \\
\quad \text { (Sepaskhah and Boersma, 1979) }\end{array}$ & 13 & $\begin{array}{l}\text { Johansen (1975) } \\
\text { de Vries (1963) } \\
\text { Campbell et al. (1994) } \\
\text { Gori (1983) }\end{array}$ & $\begin{array}{l}0.983 \\
0.973 \\
0.990 \\
0.982\end{array}$ & $\begin{array}{l}4.541 \times 10^{-1} \\
2.467 \times 10^{-1} \\
1.098 \times 10^{-1} \\
7.766 \times 10^{-1}\end{array}$ & $\begin{array}{l}-34.9 \\
-33.0 \\
-43.5 \\
-23.6\end{array}$ & $\begin{array}{c}8.6 \\
10.5 \\
0 \\
19.9\end{array}$ & $\begin{array}{l}1.4 \times 10^{-2} \\
5.1 \times 10^{-3} \\
9.8 \times 10^{-1} \ddagger \\
4.8 \times 10^{-5}\end{array}$ \\
\hline $\begin{array}{l}\text { Leighton Buzzard sand } \\
\text { (Ewen and Thomas, 1987) }\end{array}$ & 22 & $\begin{array}{l}\text { Johansen (1975) } \\
\text { de Vries (1963) } \\
\text { Campbell et al. (1994) } \\
\text { Gori (1983) }\end{array}$ & $\begin{array}{l}0.999 \\
0.991 \\
0.993 \\
0.889\end{array}$ & $\begin{array}{l}2.785 \times 10^{-1} \\
4.758 \times 10^{-1} \\
4.318 \times 10^{-1} \\
1.303 \times 10^{1}\end{array}$ & $\begin{array}{c}-88.8 \\
-70.6 \\
-72.7 \\
-1.2\end{array}$ & $\begin{array}{c}0 \\
18.2 \\
16.1 \\
87.6\end{array}$ & $\begin{array}{l}9.99 \times 10^{-1} \ddagger \\
1.1 \times 10^{-4} \\
3.3 \times 10^{-4} \\
9.4 \times 10^{-20}\end{array}$ \\
\hline $\begin{array}{l}\text { L-Soil at } 30^{\circ} \mathrm{C} \\
\quad \text { (Campbell et al. 1994) }\end{array}$ & 21 & $\begin{array}{l}\text { Johansen (1975) } \\
\text { de Vries (1963) } \\
\text { Campbell et al. (1994) } \\
\text { Gori (1983) }\end{array}$ & $\begin{array}{l}0.966 \\
0.973 \\
0.976 \\
0.974\end{array}$ & $\begin{array}{l}1.457 \times 10^{-1} \\
9.111 \times 10^{-2} \\
9.454 \times 10^{-2} \\
3.515 \times 10^{-1}\end{array}$ & $\begin{array}{l}-97.0 \\
-100.2 \\
-99.5 \\
-75.4\end{array}$ & $\begin{array}{c}3.3 \\
0 \\
0.8 \\
24.9\end{array}$ & $\begin{array}{l}1.0 \times 10^{-1} \\
5.3 \times 10^{-1} \ddagger \\
3.6 \times 10^{-1} \\
2.1 \times 10^{-6}\end{array}$ \\
\hline $\begin{array}{l}\mathrm{L}-\text { Soil at } 50^{\circ} \mathrm{C} \\
\quad(\text { Campbell et al. 1994) }\end{array}$ & 21 & $\begin{array}{l}\text { Johansen (1975) } \\
\text { de Vries (1963) } \\
\text { Campbell et al. (1994) } \\
\text { Gori (1983) }\end{array}$ & $\begin{array}{l}0.775 \\
0.910 \\
0.866 \\
0.952\end{array}$ & $\begin{array}{l}3.743 \\
1.168 \\
1.614 \\
5.349 \times 10^{-1}\end{array}$ & $\begin{array}{l}-28.8 \\
-46.7 \\
-39.9 \\
-66.6\end{array}$ & $\begin{array}{c}37.8 \\
19.9 \\
26.7 \\
0\end{array}$ & $\begin{array}{l}6.3 \times 10^{-9} \\
4.8 \times 10^{-5} \\
1.6 \times 10^{-6} \\
1.0 \ddagger\end{array}$ \\
\hline $\begin{array}{l}\text { Sandfly Creek sand } \\
\text { (Bristow, 1998) }\end{array}$ & 25 & $\begin{array}{l}\text { Johansen (1975) } \\
\text { de Vries (1963) } \\
\text { Campbell et al. (1994) } \\
\text { Gori (1983) }\end{array}$ & $\begin{array}{l}0.988 \\
0.997 \\
0.997 \\
0.953\end{array}$ & $\begin{array}{l}1.274 \times 10^{-1} \\
4.299 \times 10^{-2} \\
2.637 \times 10^{-2} \\
7.830\end{array}$ & $\begin{array}{l}-124.8 \\
-146.0 \\
-158.2 \\
-19.0\end{array}$ & $\begin{array}{c}33.4 \\
12.2 \\
0 \\
139.2\end{array}$ & $\begin{array}{l}5.7 \times 10^{-8} \\
2.2 \times 10^{-3} \\
9.98 \times 10^{-1} \ddagger \\
6.0 \times 10^{-31}\end{array}$ \\
\hline $\begin{array}{l}\text { Tottori dune sand } \\
\text { (Mori et al. 2003) }\end{array}$ & 21 & $\begin{array}{l}\text { Johansen (1975) } \\
\text { de Vries (1963) } \\
\text { Campbell et al. (1994) } \\
\text { Gori (1983) }\end{array}$ & $\begin{array}{l}0.991 \\
0.994 \\
0.995 \\
0.968\end{array}$ & $\begin{array}{l}9.628 \times 10^{-2} \\
1.413 \times 10^{-1} \\
6.022 \times 10^{-2} \\
1.452\end{array}$ & $\begin{array}{l}-105.7 \\
-91.0 \\
-108.9 \\
-45.6\end{array}$ & $\begin{array}{c}3.3 \\
17.9 \\
0 \\
63.3\end{array}$ & $\begin{array}{l}1.6 \times 10^{-1} \\
1.1 \times 10^{-4} \\
8.4 \times 10^{-1} \neq \\
1.5 \times 10^{-14}\end{array}$ \\
\hline
\end{tabular}

$\dagger$ The number of fitting parameters $K$ in each model used to evaluate $\mathrm{AIC}_{\mathrm{C}}$ was three for the Johansen model, five for the de Vries and Campbell models, and four for the Gori model. This includes $\sigma^{2}$, which is considered to be a fitting parameter (Burnham and Anderson, 2002).

$\ddagger$ Denotes the first ranked model. 


\section{Model predicted apparent thermal conductivities}

Using Campbell's model, we calculated the thermal conductivity for the glacial outwash aquifer. The three main units considered were sand, gravel and sand, and till (Fig. 2-4a). The unsaturated zone is approximately $1.5 \mathrm{~m}$ thick. For the simulations, $g_{1}$ and $q_{\mathrm{o}}$ were set equal to 0.100 and 4.0 for all soils. These are approximately the average values reported in Table 2-7. We investigated the implications of selecting these values through a sensitivity analysis with the sensitivity coefficient $S_{\mathrm{C}}$ given by

$$
S_{\mathrm{C}}=\frac{O-O_{\mathrm{b}}}{p-p_{\mathrm{b}}},
$$

where $O_{\mathrm{b}}$ is the model outcome for the parameter base value, $O$ is the model outcome for an alternate parameter value, $p_{\mathrm{b}}$ is the parameter base value, and $p$ is an alternate parameter value. Across the range of fitted values of $g_{1}$ for the Campbell model (Table 27 ), the sensitivity coefficient ranged from -0.5 to -4.7 (model-predicted $\lambda$ decreased as $g_{1}$ increased). If $g_{1}$ for the outwash actually lies near the extremes of this range, then our predicted values of $\lambda$ may vary by $\pm 20 \%$ for dry porous media and $\pm 3 \%$ for saturated porous media. The model is much less sensitive to $q_{\mathrm{o}}$, with $S_{\mathrm{C}}$ ranging from 0 to -0.18 . For $q_{\mathrm{o}}, \lambda$ varied by $\pm 5 \%$ in the unsaturated aquifer and less than $\pm 0.5 \%$ in the saturated aquifer across the range of fitted values.

Using Eq. (2.9) and grain size analyses from 60 sediment samples, we determined $x_{\text {wo }}$ to be equal to $0.04 \mathrm{~m}^{3} \mathrm{~m}^{-3}$ for gravel, $0.05 \mathrm{~m}^{3} \mathrm{~m}^{-3}$ for sand, and $0.25 \mathrm{~m}^{3} \mathrm{~m}^{-3}$ for till. The thermal conductivity values assigned to the aquifer solids were taken from Table 2-4. In the saturated zone, the measured values of $\phi$ and $\theta$ (Fig. 2-3a) were used with the exception of the bottom portion of the section occupied by till. Since the GPR did not extend to this depth, we set $\phi$ and $\theta$ equal to $0.4 \mathrm{~m}^{3} \mathrm{~m}^{-3}$, which is typical of tills in this area. In the unsaturated zone, we used the laboratory-measured values of the porosity and average values of $\theta$ from the ZOPs. Uniform values of $\phi$ and $\theta$ were assigned laterally across the unsaturated portion of the aquifer, and we used the temperature profile measured on 1 July. 
In the datasets we used for our model selection, conductivity values were all measured at temperatures between 20 and $50^{\circ} \mathrm{C}$. Throughout most of the subsurface profile at our site, the annual variation in temperature is below this range (Fig. 2-4b). Suitable datasets, with measurements $<20^{\circ} \mathrm{C}$, are not available and we were unable to evaluate the candidate models at these lower temperatures; however, until data are available with which to evaluate these models at temperatures $<20^{\circ} \mathrm{C}$, we have no reason to reject the Campbell model as the "best-approximating model" for our analysis.

The two-dimensional distribution of the apparent thermal conductivity of the glacial outwash sand and gravel for 1 July was calculated using the above approach (Fig. 2-5). The thermal conductivity of the saturated gravel and sand aquifer ranges from 2.14 to $2.69 \mathrm{~W} \mathrm{~m}^{-1} \mathrm{~K}^{-1}$, while $\lambda$ in the till is $1.90 \mathrm{~W} \mathrm{~m}^{-1} \mathrm{~K}^{-1}$. In the saturated zone, two distinct layers of different conductivity are evident. The upper layer extends from the water table down to $4 \mathrm{~m}$ bgs. In this layer, the mean and standard deviation of the apparent thermal conductivity, $\delta$ and $s$, are 2.53 and $0.08 \mathrm{~W} \mathrm{~m}^{-1} \mathrm{~K}^{-1}$ for the gravel and sand. The lower layer extends from $4 \mathrm{~m}$ bgs to the base of the section. In this layer, 8 and $s$ are 2.30 and $0.06 \mathrm{~W} \mathrm{~m}^{-1} \mathrm{~K}^{-1}$, for the gravel and sand, and 2.41 and $0.07 \mathrm{~W} \mathrm{~m}^{-1} \mathrm{~K}^{-1}$ for the sand. The $10 \%$ difference in 8 for the gravel and sand unit between the upper and lower layers is due to the difference in porosity. The influence of $\lambda_{\mathrm{s}}$ on the apparent thermal conductivity is visible in the bottom portion of the saturated zone, where 8 for the sand layers is $5 \%$ higher than for the gravel and sand layers. These values are in reasonable agreement with those obtained for aquifers of similar texture by Andrews and Anderson (1979) of $2.13 \mathrm{~W}$ $\mathrm{m}^{-1} \mathrm{~K}^{-1}$ for very fine to fine sand and $1.88 \mathrm{~W} \mathrm{~m}^{-1} \mathrm{~K}^{-1}$ for medium to coarse sand with gravel; by Palmer et al. (1992) of $2.1 \pm 0.3 \mathrm{~W} \mathrm{~m}^{-1} \mathrm{~K}^{-1}$ for Borden sand; and by Parr et al. (1983) of $2.29 \pm 0.19 \mathrm{~W} \mathrm{~m}^{-1} \mathrm{~K}^{-1}$ for sand and gravel. Given the relatively narrow range of $\lambda$ that we found, it may be sufficient to use literature-cited values of thermal conductivity and porosity for many investigations. As will be shown in the simulations below, however, for applications where small temperature differences are important, even the 5 
to $10 \%$ difference in $\lambda$ measured in this aquifer may influence heat transport significantly and require detailed measurements of the aquifer properties.

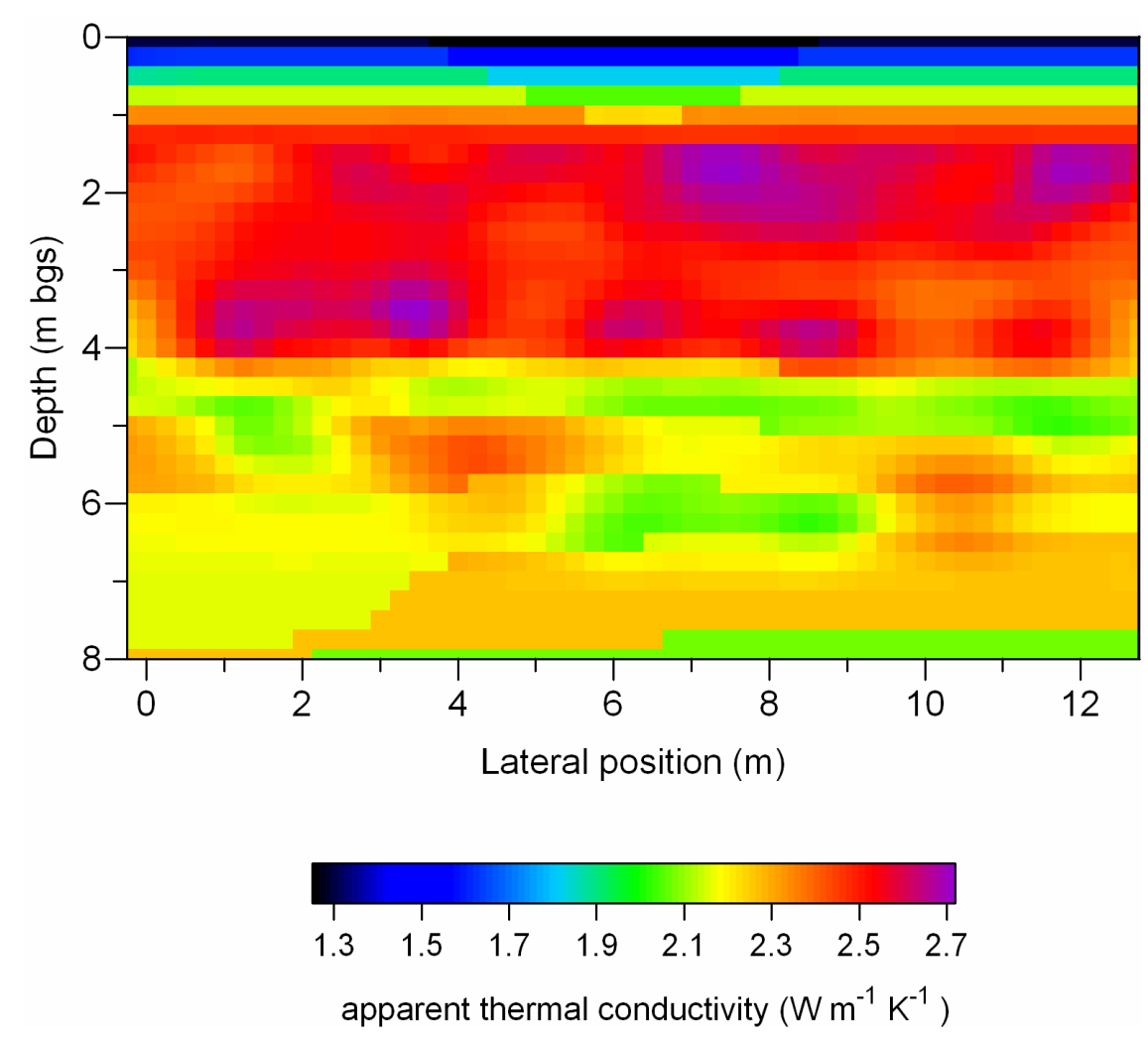

Figure 2-5 Two-dimensional distribution of the apparent thermal conductivity, $\lambda$ (W $\mathrm{m}^{-1} \mathrm{~K}^{-1}$ ) for the glaciofluvial outwash sand and gravel aquifer as calculated using the Campbell et al. (1994) model.

In the unsaturated zone, $\lambda$ drops from $2.6 \mathrm{~W} \mathrm{~m}^{-1} \mathrm{~K}^{-1}$ at the water table to $1.4 \mathrm{~W}$ $\mathrm{m}^{-1} \mathrm{~K}^{-1}$ at the ground surface. These values of $\lambda$ are 40 to $50 \%$ lower than in the saturated zone due to the lower water content above the capillary fringe. It must be recognized that, in areas where the subsurface temperatures are higher than those found at our site, the influence of heat transport by vapor diffusion will increase the apparent thermal conductivity in the unsaturated zone thereby decreasing the contrast between the saturated and unsaturated zones. 


\section{Numerical simulations of heat transport}

Using the measured thermal conductivity field, we investigated the influence of the heterogeneous thermal conductivity on heat transport within the two-dimensional study section. Simulations were completed using the finite-element numerical model Heatflow (Molson et al., 1992) after modifications were made to include the Campbell model for apparent thermal conductivity. The Heatflow model accounts for density-dependent groundwater flow, thermal advection, conduction through the porous medium, thermal buoyancy, and thermal retardation. For all simulations, we assumed a uniform hydraulic conductivity of $5.4 \times 10^{-4} \mathrm{~m} \mathrm{~s}^{-1}$ (at $10^{\circ} \mathrm{C}$ ) so that heat transport would be influenced by only heterogeneity of the aquifer thermal properties. We expect that heterogeneities in the aquifer hydraulic conductivity will increase dispersion. A hydraulic gradient of $0.01 \mathrm{~m}$ $\mathrm{m}^{-1}$ was applied across the section using constant heads at the lateral boundaries. The average measured porosity in the saturated zone (Fig. 2-3a) was 0.30, giving a mean groundwater velocity of $1.8 \times 10^{-5} \mathrm{~m} \mathrm{~s}^{-1}\left(1.55 \mathrm{~m} \mathrm{~d}^{-1}\right)$ at $10^{\circ} \mathrm{C}$. The top and bottom boundaries were assumed impermeable to flow. For the thermal transport simulations, a uniform fixed temperature of $10^{\circ} \mathrm{C}$ was assigned along the left boundary, except between 4.5 and $5.5 \mathrm{~m}$ below ground surface, where the temperature was set to $30^{\circ} \mathrm{C}$. All other boundaries were assigned a temperature gradient of zero. Throughout the domain, the initial temperature was set to $10^{\circ} \mathrm{C}$ and hydrodynamic dispersivities were set to zero. Simulations were completed using a grid of 221 by 133 nodes in the $x$ and $z$ directions, respectively, and using time steps of $0.02 \mathrm{~d}$. The temperature convergence criterion was $0.001^{\circ} \mathrm{C}$.

To investigate the influence of the heterogeneous thermal conductivity field on heat transport, we completed three sets of simulations. Each set comprised one simulation using a heterogeneous thermal conductivity field and one simulation using a homogeneous or uniform field. The differences in the temperatures between these two simulations were calculated at a time of $10 \mathrm{~d}$. For the homogeneous fields, thermal conductivities in the saturated and unsaturated zones were set equal to the geometric mean of the conductivities in the corresponding portions of the heterogeneous field. For 
the first set of simulations, the apparent thermal conductivities in the observed heterogeneous field (Fig. 2-5) were decreased by 10\%, and the thermal conductivities in the saturated and unsaturated portion of the homogeneous field were 2.16 and $1.57 \mathrm{~W}$ $\mathrm{m}^{-1} \mathrm{~K}^{-1}$, respectively. For the second set of simulations, the observed $\lambda$ field was used, and conductivities in the saturated and unsaturated portion of the homogeneous field were 2.40 and $1.73 \mathrm{~W} \mathrm{~m}^{-1} \mathrm{~K}^{-1}$, respectively. Finally, for the third set, thermal conductivities from the observed field were increased by $10 \%$, and conductivities in the saturated and unsaturated portion of the homogeneous field were 2.64 and $1.89 \mathrm{~W} \mathrm{~m}^{-1} \mathrm{~K}^{-1}$, respectively.

Temperatures predicted using the heterogeneous $\lambda$ fields (Fig. 2-6a, b, and c) were compared with the homogeneous $\lambda$ fields and differences were calculated (Fig. 2 $6 \mathrm{~d}$, e, and f). For the heterogeneous thermal conductivity field observed at our site, the plume front, defined by the $11^{\circ} \mathrm{C}$ contour, had migrated approximately $9.5 \mathrm{~m}$ across the model domain after $10 \mathrm{~d}$ (Fig. 2-6b). The maximum temperature difference between the plumes in the heterogeneous and homogeneous fields of $-0.36^{\circ} \mathrm{C}$ was centered at $8 \mathrm{~m}$, near the front of the plume (Fig. 2-6e). In this area, temperatures in the heterogeneous field plume were lower than in the uniform field plume due to increased thermal dispersion through the heterogeneous thermal conductivity field. While reducing the core temperatures, this increased thermal dispersion also increased the temperature, in the area directly above the plume, relative to the uniform field plume, thereby producing a positive temperature difference "halo." This halo was focused above the plume, probably due to thermal buoyancy, which causes the simulated plume to rise. 

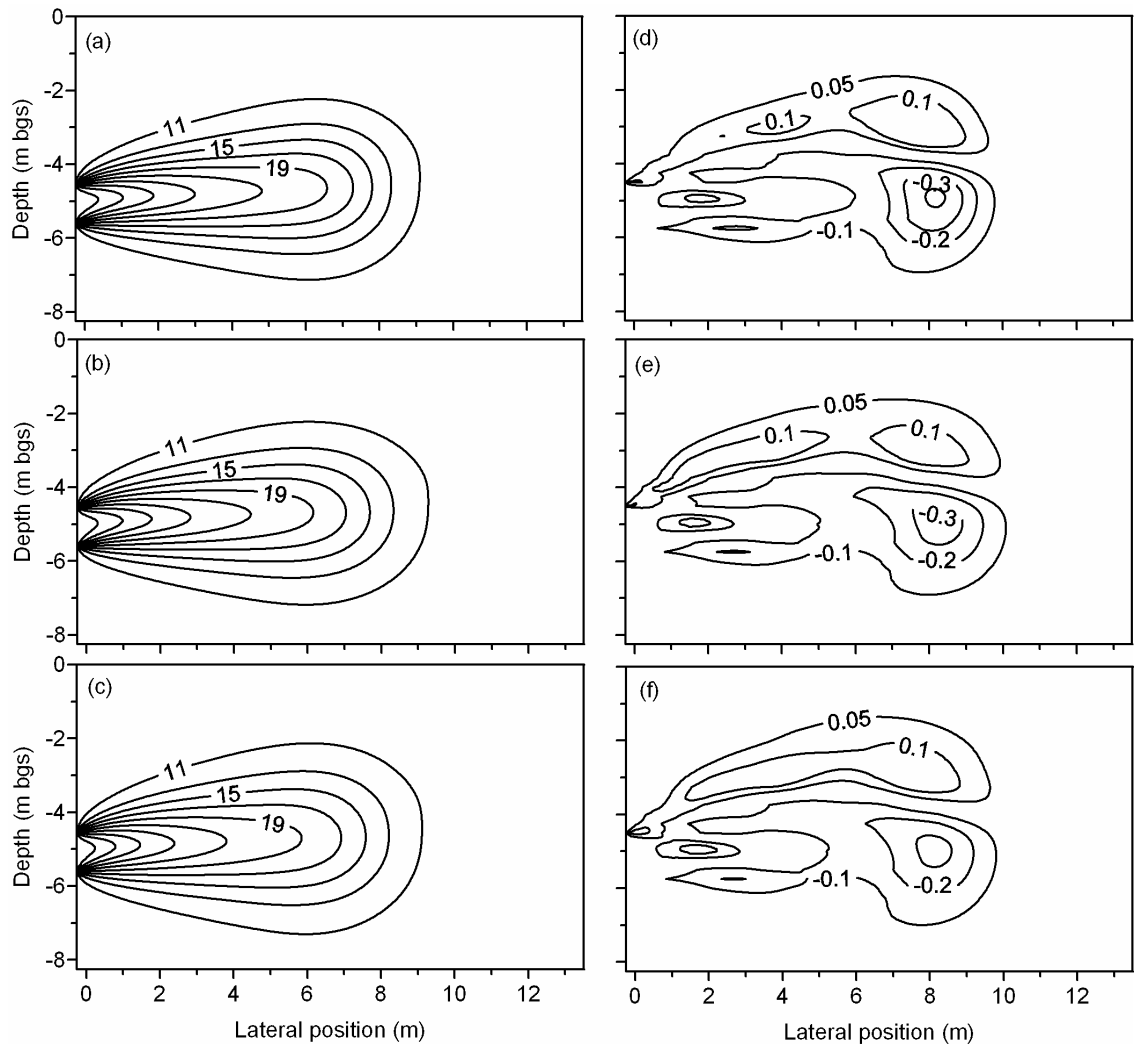

Figure 2-6 Simulated thermal plumes (left column) and corresponding temperature differences (right column) at $10 \mathrm{~d}$ using various thermal conductivity distributions. The thermal plumes on the left are shown using (a) a 10\% decrease in the observed field, (b) the observed field, and (c) a 10\% increase in the observed field. The corresponding temperature differences on the right were obtained by subtracting the plume temperatures simulated in the heterogeneous fields $(\mathrm{a}, \mathrm{b}$, and $\mathrm{c})$ from plume temperatures simulated using equivalent mean thermal conductivity fields in the saturated and unsaturated zones of (d) 2.16 and $1.57 \mathrm{~W} \mathrm{~m}^{-1} \mathrm{~K}^{-1}$, (e) 2.40 and $1.73 \mathrm{~W} \mathrm{~m}^{-1} \mathrm{~K}^{-1}$, and (f) 2.64 and $1.89 \mathrm{~W} \mathrm{~m}^{-}$ ${ }^{1} \mathrm{~K}^{-1}$, respectively.

When thermal conductivities in the heterogeneous field were decreased by $10 \%$ and the thermal plume (Fig. 2-6a) was compared to a uniform field case with a mean thermal conductivity in the saturated zone of $2.16 \mathrm{~W} \mathrm{~m}^{-1} \mathrm{~K}^{-1}$, the maximum temperature difference increased slightly to $-0.42^{\circ} \mathrm{C}$ (Fig. 2-6d). The positive temperature difference "halo" concurrently became smaller. The thermal plume in the uniform field dispersed less due to lower rates of heat conduction, thereby maintaining higher temperatures in the plume core. For lower thermal conductivities, the influence of the heterogeneities on 
thermal dispersion was therefore enhanced. In contrast, when thermal conductivity was increased by $10 \%$, dispersion of the thermal plume in the uniform field increased due to higher heat conduction rates. The influence of the heterogeneities on the thermal dispersion was decreased relative to the uniform case. The maximum temperature difference in this case was only $-0.33^{\circ} \mathrm{C}$, and the positive "halo" increased in area (Fig. 2-6f).

From other simulations, not shown here, we found that, as the plume transport distance increased, temperature differences increased in the frontal region of the plume. This is analogous to solute transport, where the size of the dispersion or mixing zone increases as the advective front moves farther from the source. While we considered only a two-dimensional system, we would expect increased thermal dispersion in a threedimensional thermal conductivity field. Furthermore, we did not investigate the influence of a heterogeneous hydraulic conductivity field, which would further increase the dispersion.

\section{Conclusions}

We developed a method for constructing the two-dimensional thermal conductivity field for a section of a glaciofluvial outwash deposit. The method involved a combination of field and laboratory measurements to determine the bulk thermal conductivity of the aquifer solids, the volumetric water content, and the porosity of the aquifer, as well as a model selection procedure using the information-theoretic approach. Using the $\mathrm{AIC}_{\mathrm{C}}$, the Campbell model was selected as the best-approximating model for predicting the apparent thermal conductivity of variably-saturated sands and gravels.

Thermal conductivities of aquifer solids were determined using two laboratory methods. Conductivity values measured directly with the divided-bar apparatus and estimated from the mineral composition were correlated, indicating that, where direct measurements are not available, estimating thermal conductivity from the mineral composition is a reasonable alternative. For this glacial outwash deposit, the thermal conductivities of the porous medium solids can be divided into three groups, which 
included fine to coarse sand having a mean thermal conductivity of $4.22 \pm 0.10 \mathrm{~W} \mathrm{~m}^{-1}$ $\mathrm{K}^{-1}$, gravel and sand having a mean conductivity of $3.94 \pm 0.12 \mathrm{~W} \mathrm{~m}^{-1} \mathrm{~K}^{-1}$, and till having a mean conductivity of $3.72 \pm 0.59 \mathrm{~W} \mathrm{~m}^{-1} \mathrm{~K}^{-1}$.

By combining measured thermal conductivities and site stratigraphy with the measured porosity, we were able to define a two-dimensional apparent thermal conductivity field (Fig. 2-5) for the glacial outwash deposit as input to a numerical model for simulating heat transport. In the saturated zone, the mean value and standard deviation of apparent thermal conductivity were 2.42 and $0.13 \mathrm{~W} \mathrm{~m}^{-1} \mathrm{~K}^{-1}$, respectively. For the moisture and temperature conditions present, the apparent thermal conductivities in the unsaturated zone were between 40 and 50\% lower than the apparent thermal conductivities in the saturated zone. Porosity strongly influenced the predicted twodimensional conductivity field, indicating that this parameter must be defined carefully.

The numerical simulations showed that, for short transport distances, using a mean thermal conductivity in place of a fully heterogeneous field would yield temperature differences of $<1^{\circ} \mathrm{C}$ relative to the fully heterogeneous field. For the homogeneous cases, predicted temperatures were higher in the plume core and lower along the plume fringes, indicative of reduced thermal dispersion; however, predicted temperature differences may increase with transport distance, plume scale, and in fully three-dimensional systems with heterogeneous aquifer thermal and hydraulic properties. Where small temperature differences are important, such as for temperature-sensitive aquatic environments, consideration of the heterogeneities in thermal conductivity may be necessary. These issues will be explored in future work. 


\section{References}

Acornley, R.M. 1999. Water temperature within spawning beds in two chalk streams and implications for salmoniod egg development. Hydrological Processes 13:439_ 446.

Agriculture and Agri-Food Canada. 1998. Canadian system of soil classification. Soil Classification Working Group. 3rd ed. NRC Research Press, Ottawa.

Aldridge, D.F., D.W. Oldenburg. 1993. Two-dimensional tomographic inversion with finite-difference traveltimes. Journal of Seismic Exploration 2:257-274.

Akaike, H. 1973. Information theory as an extension of the maximum likelihood principle. p. 267-281. In B.N. Petrov and F. Csaki (ed.) Second Symposium in Information Theory. Akadémiai Kiad $\therefore$, Budapest.

Anderson, D.R. 2003. Multimodel inference based on Kullback-Leibler information. pp. 366-370. In Proc. of MODFLOW and more 2003: Understanding Through Modeling, IGWMC, Colorado School of Mines, Golden, CO.

Andrews, C.B., M.P. Anderson. 1979. Thermal alteration of ground water caused by seepage from a cooling lake. Water Resources Research 15:595-602.

Barnett, P.J. 1992. Quaternary geology of Ontario. p. 1011-1088. In P.C. Thurston, H. R. Williams, R.H. Sutcliffe, and G. M. Stott (ed.) Geology of Ontario. Ontario Geological Survey, Special Volume 4, Part 2. Publications Ontario, Toronto, Ontario.

Bristow, K.L., G.J. Kluitenberg, R. Horton. 1994. Measurement of soil thermal properties with a dual-probe heat-pulse technique. Soil Science Society of America Journal 58:1288-1294.

Bristow, K.L. 1998. Measurement of thermal properties and water content of unsaturated soil using dual-probe heat-pulse probes. Agricultural and Forest Meteorology 89:75-84.

Brown, M.B., A.B. Forsythe. 1974. Robust tests for the equality of variances. Journal of America Statistical Association 69:264-267.

Burnham, K.P., D.R. Anderson. 2002. Model selection and multimodel inference: a practical information-theoretical approach. 2nd ed. Springer-Verlag, New York.

Burnham, K.P., D.R. Anderson. 2004. Multimodel inference: Understanding AIC and BIC in model selection. Sociological Methods and Research. 33:261-304. 
Campbell, G.S., J.D. Jungbauer, Jr., W.R. Bidlake, R.D. Hungerford. 1994. Predicting the effect of temperature on soil thermal conductivity. Soil Science 158:307-313.

Clark, S.P., Jr. 1966. Thermal conductivity. p. 459-482. In S.P. Clark, Jr. (ed.) Handbook of physical constants, Geological Society of America, Memoir 97. GSA, New York.

Clauser, C., E. Huenges. 1995. Thermal conductivity of rocks and minerals. p. 105-126. In T.J. Ahrens (ed.) Rock physics and phase relations: a handbook of physical constants. AGU Reference Shelf 3. American Geophysical Union, Washington, DC.

Conaway, J.G., A.E. Beck. 1977. Fine-scale correlation between temperature gradient logs and lithology. Geophysics 42:1401-1410.

Conover, W.J. 1999. Practical nonparametric statistics. 3rd ed. John Wiley \& Sons, Inc., New York.

Deardorff, J.W. 1978. Efficient prediction of ground surface temperature and moisture, with inclusion of a layer of vegetation. Journal of Geophysical Research 83:18891903.

de Vries, D.A. 1963. Thermal properties of soils. p. 210-235. In W.R. Van Wijk (ed.) Physics of Plant Environment. North-Holland, Amsterdam.

Doherty, J. 2005. PEST Model-independent parameter estimation, Users Manual: 5th ed. Watermark Numerical Computing, Brisbane, Australia.

Ewen, J., H.R. Thomas. 1987. The thermal probe - a new method and its use on a saturated sand. Géotechnique 37:91-105.

Farouki, O. 1981. Thermal properties of soils. CRREL Monograph 81-1. U.S. Cold Regions Research and Engineering Laboratory, Hanover, NH.

Farouki, O. 1982. Evaluation of methods for calculating soil thermal conductivity. CRREL Report 82-8. U.S. Cold Regions Research and Engineering Laboratory, Hanover, NH.

Feng, S., P.N. Sen. 1985. Geometrical model of conductive and dielectric properties of partially saturated rocks. Journal of Applied Physics 58:3236-3243.

Garside, E.T. 1966. Effects of oxygen in relation to temperature on the development of embryos of brook trout and rainbow trout. Journal of Fisheries Resources Board 
of Canada 23:1121-1134.

Gori, F. 1983. A theoretical model for predicting the effective thermal conductivity of unsaturated frozen soils. p. 363-368. In Proceedings of the Fourth International Conference on Permafrost. Fairbanks, Alaska, National Academy Press, Washington, DC.

Hellwege, K.H., G. Angenheister (ed.). 1982. Numerical Data and Functional Relationships in Science and Technology: Physical Properties of Rocks, Volume 1a. Springer-Verlag, New York.

Hokanson, K.E.F., J.H. McCormick, B.R. Jones, J.H. Tucker. 1973. Thermal requirements for maturation, spawning, and embryo survival of the brook trout, Salvelinus fontinalis. Journal of Fisheries Resources Board of Canada 30:975984.

Hopmans, J.W., J.H. Dane. 1986. Thermal conductivity of two porous media as a function of water content, temperature, and density. Soil Science 142:187-195.

Horai, K. 1971. Thermal conductivity of rock-forming minerals. Journal of Geophysical Research 76:1278-1308.

Johansen, O. 1975. Thermal conductivity of soils. Ph.D. diss. Trondheim, Norway, (CRREL Draft Translation 637, 1977), ADA 044002.

Judge, A.S. 1971. Geothermal measurements in a sedimentary basin. Ph.D. diss. University of Western Ontario, London, Ontario, Canada.

Kaufmann, R.K., L. Zhou, R.B. Mynemi, C.J. Tucker, D. Slayback, N.V. Shabanov. 2003. The effect of vegetation on surface temperature: A statistical analysis of NDVI and climate data. Journal of Geophysical Research Letters 30, 2147, doi:10.1029/2003GL018251, 2003.

Lubimova, H.A., L.M. Lusova, F.V. Firsov, G.N. Starkova, A.P. Shushpanov. 1961. Determination of surface heat flow in Mazesta (USSR). Annals of Geophysics XIV:157-167.

Misener, A.D., L.G.D. Thompson, R.J. Uffen. 1951. Terrestrial heat flow in Ontario and Quebec. Transactions of the American Geophysical Union 32:729-738.

Mitchell, J.K. 1976. Fundamentals of soil behavior. 2nd ed. John Wiley and Sons, Inc., New York.

Molson, J.W., E.O. Frind, C. Palmer. 1992. Thermal energy storage in an unconfined 
aquifer 2. Model development, validation and application. Water Resources Research 28:2857-2867.

Mori, Y., J.W. Hopmans, A.P. Mortensen, G.J. Kluitenberg. 2003. Multi-functional heat pulse probe for the simultaneous measurement of soil water content, solute concentration, and heat transport parameters. Vadose Zone Journal 2:561-571.

Nitoiu, D., H. Beltrami. 2005. Subsurface thermal effects of land use changes. Journal of Geophysical Research 110, FO1005, doi:10.1029/2004JF000151.

Palmer, C.D., D.W. Blowes, E.O. Frind, J.W. Molson. 1992. Thermal energy storage in an unconfined aquifer 1 . Field injection experiment. Water Resources Research 28:2845-2856.

Parr, A.D., F.J. Molz, J.G. Melville. 1983. Field determination of aquifer thermal energy storage parameters. Ground Water. 21:22-35.

Poeter, E., D. Anderson. 2005. Multimodel ranking and inference in ground water modeling. Ground Water. 43:597-605.

Power, G., R.S. Brown, J.G. Imhof. 1999. Groundwater and fish - insights from northern North America. Hydrological Processes 13:401-422.

Sass, J.H. 1965. The thermal conductivity of fifteen feldspar specimens. Journal of Geophysical Research 70:4064-4065.

Sass, J.H., A.H. Lachenbruch, R.J. Munroe. 1971. Thermal conductivity of rocks from measurements on fragments and its application to heat-flow determinations. Journal of Geophysical Research 76:3391-3401.

Sass, J.H., J.P. Kennelly, Jr., W.E. Wendt, T.H. Moses, Jr., J.P. Ziagos. 1981. In-situ determination of heat flow in unconsolidated sediments. Geophysics 46:76-83.

Sen, P.N., C. Scala, M.H. Cohen. 1981. A self-similar model for sedimentary rocks with application to the dielectric constant of fused glass beads. Geophysics 46:781795.

Sepaskhah, A.R., L. Boersma. 1979. Thermal conductivity of soils as a function of temperature and water content. Soil Science Society of America Journal 43:439444.

Shiozawa, S., G.S. Campbell. 1991. On the calculation of mean particle diameter and standard deviation from sand, silt and clay fractions. Soil Science 152:427-431. 
Smith, W.O. 1939. Thermal conductivity in moist soils. Soil Science Society of America Proceedings 4:32-40.

Smith, W.O. 1942. The thermal conductivity of dry soil. Soil Science 53:435-459.

StatSoft. 2001. Statistica electronic user's manual. Release 6.1. Tulsa, OK.

Tarnawski, V.R., F. Gori, B. Wagner, G.D. Buchan. 2000. Modelling approaches to predicting thermal conductivity of soils at high temperatures. International Journal of Energy Research 24:403-423.

Woodside, W., J.H. Messmer. 1961. Thermal conductivity of porous media. I. Unconsolidated sands. Journal of Applied Physics 32:1688-1706. 


\section{Prologue to Chapter 3}

Chapter 3 was prepared for submission to the Journal of Hydrology. In Chapter 3, the implementation of a three-dimensional finite-element numerical model is presented and used to study the movement of thermal plumes emanating from an aggregate. For any modelling study the development of a conceptual model, and the translation and implementation of the conceptual model into a three-dimensional numerical model are critical elements in obtaining a meaningful numerical representation of the physical system. These components are discussed in detail in this chapter. The numerical model used in this study is a three-dimensional finite-element model that accounts for coupled density-dependent groundwater flow and thermal energy transport by advection and conduction. Calibration and verification of the model is discussed and the calibrated model is used to simulate subsurface movement of thermal plumes from an aggregate pit though a shallow unconfined aquifer. 


\section{Chapter 3}

\section{Introduction}

The measurement of groundwater temperatures and the prediction of heat transport have found a variety of applications in hydrogeologic studies. These include identification of groundwater flow systems (e.g. Parson, 1970; Cartwright, 1974; Smith and Chapman, 1983; Woodbury and Smith, 1985), groundwater exploration studies (Cartwright, 1968), estimation of infiltration and vertical groundwater velocity (e.g. Bredehoeft and Papadopulos, 1965; Stallman, 1965; Sorey, 1971; Taniguchi, 1994; Lu and Ge, 1996; Reiter, 2001; Ferguson et al. 2003), evaluation of the feasibility of aquifer energy storage systems and geothermal energy supplies (e.g. Olmsted et al., 1975; Werner and Kley, 1977; Sass et al., 1981; Palmer et al., 1992; Molson et al., 1992; Ferguson and Woodbury, 2005), identification of zones of groundwater discharge and recharge to streams, lakes, and wetlands (e.g. Lapham, 1987; Silliman and Booth, 1993; Evans et al., 1995; Silliman et al., 1995; Constantz, 1998; Alexander and Caisse, 2003; Conant, 2004; Becker et al., 2004; Hatch et al. 2006; Keery et al., 2007), modeling of groundwatersurface water interactions (Brookfield et al., 2009) and assessment of impacts from anthroprogenic sources of heat such as power plant cooling lakes (Andrews and Anderson, 1979). The study by Andrews and Anderson, (1979) has several similarities to this study. They measured and modelled groundwater flow and heat transport from a cooling lake, through a sand aquifer, to a wetland. Groundwater velocities at their site ranged from 0 to $4.1 \times 10^{-2} \mathrm{~m} \mathrm{~d}^{-1}$. Changes in groundwater temperature persisted for about $100 \mathrm{~m}$ down gradient of the cooling lake and they observed changes to the wetland vegetation in response to changes in the temperature of the discharging groundwater. In this study, rather than having a cooling lake that is constructed on top of the aquifer, here the aquifer material has been removed, by the extraction of the aggregate, creating a water-filled pit such that the temperature perturbation, originating in the pit, penetrates the entire aquifer. As well the groundwater discharge is primarily to a cool-water stream. For cold or cool-water ecosystems, the temperature of the discharging groundwater may be critical (Hynes, 1983; Vannote and Sweeney, 1980; Power et al., 1999; Curry et al., 
2002) while the flora and fauna associated with a wetland are generally more tolerant of warm-water discharges. Thus, the potential impacts may be significantly different. Quantifying the potential effects these aggregate extraction operations may have on nearby cool-water aquatic systems is of fundamental importance to the citing and development of these operations. Modelling the movement of these plumes is a primary method used for evaluating the potential impacts these operations may have on the stream temperature.

The objectives of this study were to simulate the transport of thermal plumes emanating from aggregate pits through a shallow, unconfined glacial-outwash aquifer, to determine the distance over which these plumes persist in the subsurface, and to assess the important physical processes controlling heat transport in shallow unconfined aquifers. A multi-year field study that included monitoring groundwater temperatures and measuring aquifer physical parameters has been previously reported by Markle and Schincariol (2007), and Markle et al. (2006). Here field results are used to estimate parameter values, develop the conceptual model and implement the calibrated and validated numerical model towards a better understanding of the important physical processes controlling heat transport in shallow unconfined aquifers.

\section{Geological and Hydrogeological Setting of Tricks Creek}

A study of thermal plume migration through an unconfined aquifer was completed in the Tricks Creek watershed. The Tricks Creek watershed is a small headwater system (26 $\mathrm{km}^{2}$ ) located in southwestern Ontario, Canada, approximately $180 \mathrm{~km}$ west of Toronto (Fig. 3-1). Tricks Creek lies within a wetland complex that encompasses an area of approximately 105 ha (4\% of watershed). The headwaters originate in the northern portion of the wetland, flow to the south for approximately $4 \mathrm{~km}$, and discharge into the Bayfield River (43E34N55.5O N, 81E35N14O W). Two small tributaries enter the creek, one from the west and one from the east. The mean annual flow is $0.29 \mathrm{~m}^{3} \mathrm{~s}^{-1}$, with peak flows of $4.9 \mathrm{~m}^{3} \mathrm{~s}^{-1}$ (Appendix A1). Under summer flow conditions (June to September), the mean flow is $0.28 \mathrm{~m}^{3} \mathrm{~s}^{-1}$, the flow ranges between 0.10 and $2.9 \mathrm{~m}^{3} \mathrm{~s}^{-1}$, and is maintained mainly by groundwater discharge from the glacial outwash aquifer. An 
un-named creek lies along the south-west edge of the watershed and discharges to the Bayfield River at the same point as Tricks Creek. Tricks Creek is characterized by cool water with a mean annual temperature of $12.9^{\circ} \mathrm{C}$ and supports resident brook trout (Salvelinus fontinalis) and rainbow trout (Salmo Gairdneri) populations, and a diverse assemblage of macroinvertebrates, typical of a cool, headwater stream.

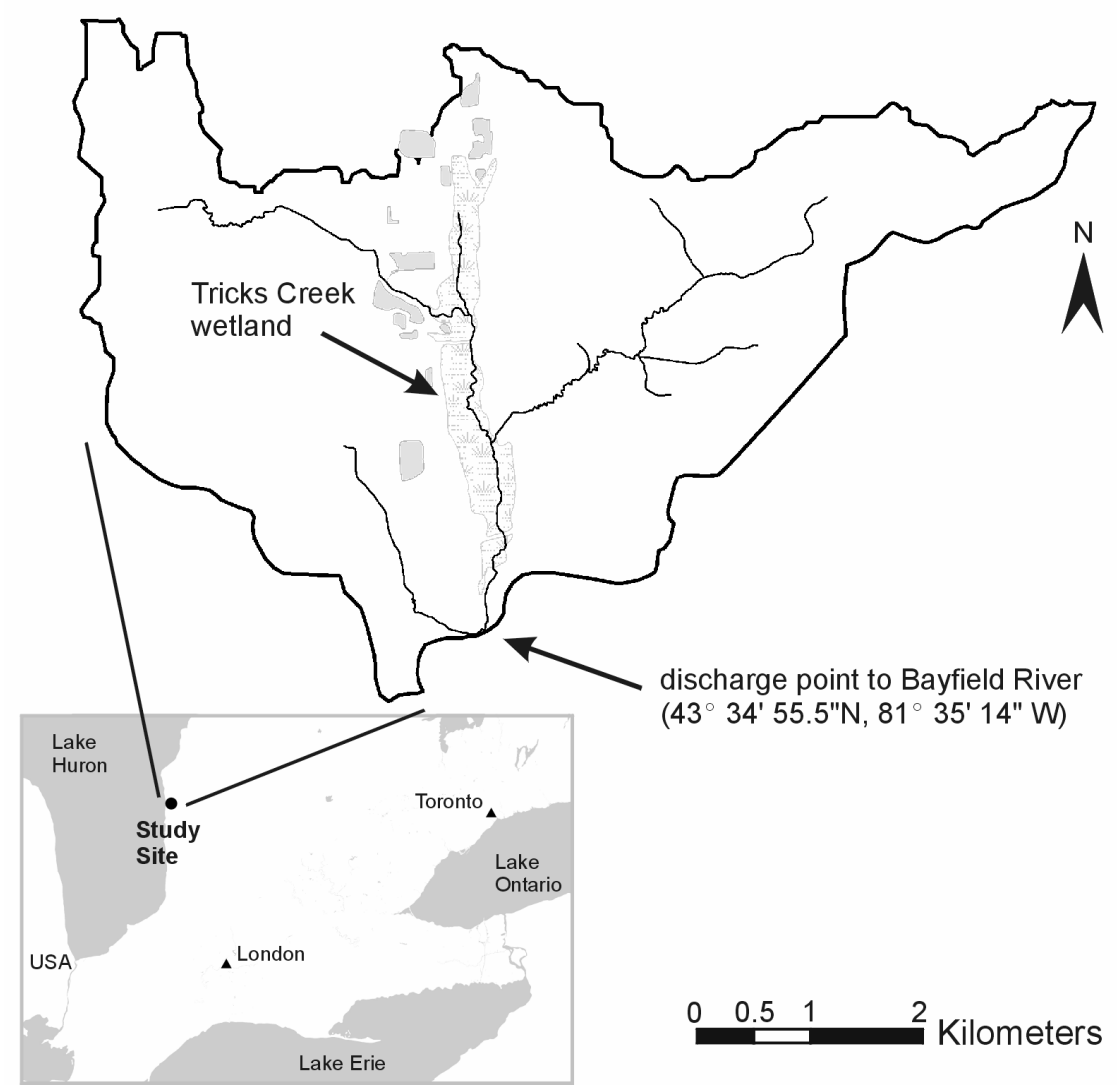

Figure 3-1 Study area location map and three-dimensional view of the Tricks Creek watershed and wetland.

The topography of the watershed is characterized by a broad north-south trending valley that slopes from an elevation of $\sim 260 \mathrm{~m}$ asl (above sea-level) at the northern boundary to $246 \mathrm{~m}$ asl along the southern boundary, and discharges into the Bayfield River at an elevation of $\sim 244 \mathrm{~m}$ asl (Fig. 3-1). The valley is bounded on the east and west by gently rising hills which reach maximum elevations of $300 \mathrm{~m}$ asl on the east and 285 $\mathrm{m}$ asl on the west. Climatic records for the Goderich, Ontario weather station (43E46N N, 
$81 \mathrm{E} 43 \mathrm{~N} \mathrm{~W}), 19 \mathrm{~km}$ to the northwest, and the Blyth, Ontario weather station (43E43N $\mathrm{N}, 81 \mathrm{E} 22 \mathrm{~N} \mathrm{~W}), 15 \mathrm{~km}$ to the northeast, indicate the mean monthly temperature from $1971-2000$ varied from a low of $-7.5^{\circ} \mathrm{C}$ in January to a high of $20.2^{\circ} \mathrm{C}$ in July. The average annual temperature is $6.8^{\circ} \mathrm{C}$ and the mean annual precipitation is $1184.3 \mathrm{~mm} \mathrm{yr}^{-1}$, with $350.4 \mathrm{~cm}$ as snow (Environment Canada, 2008; Appendix A2).

Tricks Creek and the surrounding wetland complex lie on the eastern edge of a former glacial-outwash channel that trends north-south. The glacial outwash, a mixture of predominantly carbonates and quartz rocks, forms a 9.8-km-long unconfined sand and gravel aquifer (Fig. 3-2). The glacial-outwash channel is incised into the till and spans two watersheds. To the north is the Bridgewater Creek watershed, with a catchment area of $\sim 54 \mathrm{~km}^{2}$. Groundwater and surface water, in this watershed, flow north and discharge to the Maitland River (approximately $5.5 \mathrm{~km}$ north of the study area). To the south is the Tricks Creek watershed. Groundwater and surface water, in this watershed, flow south and discharge to the Bayfield River. Separating the two watersheds are groundwater and surface-water divides situated approximately half way between the Maitland and Bayfield Rivers. The water table elevation at the groundwater divide is $\sim 255.5 \mathrm{~m}$ asl, dropping to $\sim 244 \mathrm{~m}$ asl at the southern end of the aquifer (Bayfield River) and $\sim 245 \mathrm{~m}$ asl at the northern end (Maitland River). The outwash deposits are laterally confined and unconformably overlie silty clay till moraines. These moraine deposits vary in thickness from approximately 25 to $45 \mathrm{~m}$. The underlying bedrock is middle Devonian age limestone, dolostone, and shale, and the bedrock surface slopes gently in a westerly direction $\left(0.006 \mathrm{~m} \mathrm{~m}^{-1}\right)$. The water table aquifer receives recharge only by precipitation that falls within the catchments, and all the groundwater eventually discharges to Tricks Creek and the Bayfield River, or to Bridgewater Creek and the Maitland River. 


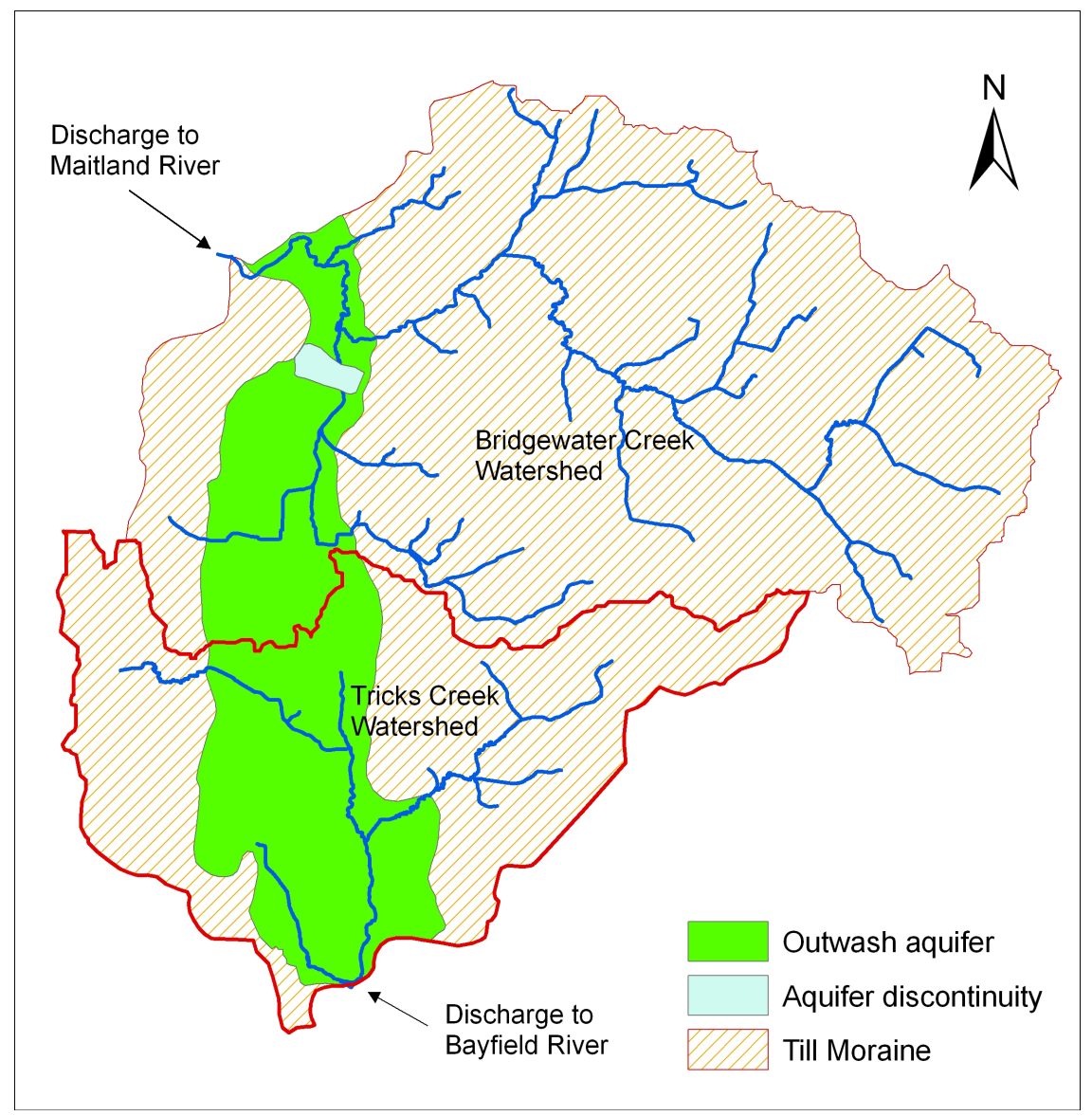

115

Figure 3-2 Glacial-outwash aquifer within the Tricks Creek and Bridgewater Creek watersheds.

Thermal plume migration down gradient of an existing aggregate operation within the Tricks Creek watershed was observed; details are reported in Markle and Schincariol, 2007. The site is located in the upper third of the Tricks Creek watershed (Fig. 3-3) and is one of several aggregate pits operating within the outwash aquifer. The site was ideal for the investigation as it was relatively isolated and secure, and the majority of the area down gradient of the pit was free of tree cover area so that monitoring well locations were not restricted. As well, the owners of the pit granted us unrestricted access. At this location the sand and gravel extraction proceeds by first clearing the forest cover and removing the soil and porous medium above the water table, and then excavating the porous medium below the water table creating a water-filled pit. The pond at our study site covers approximately 5 ha and varies between 4 and $6 \mathrm{~m}$ in depth. The pond is about 
$50 \mathrm{~m}$ west of the wetland and $100 \mathrm{~m}$ west of Tricks Creek. The regional potentiometric surface shows that the groundwater flow path from the pond to Tricks Creek is $\sim 750 \mathrm{~m}$ in length. The western tributary of Tricks Creek crosses this flow path about $400 \mathrm{~m}$ down gradient of the pit. The horizontal hydraulic gradient in the area of the pit is between 0.001 and $0.004 \mathrm{~m} \mathrm{~m}^{-1}$.

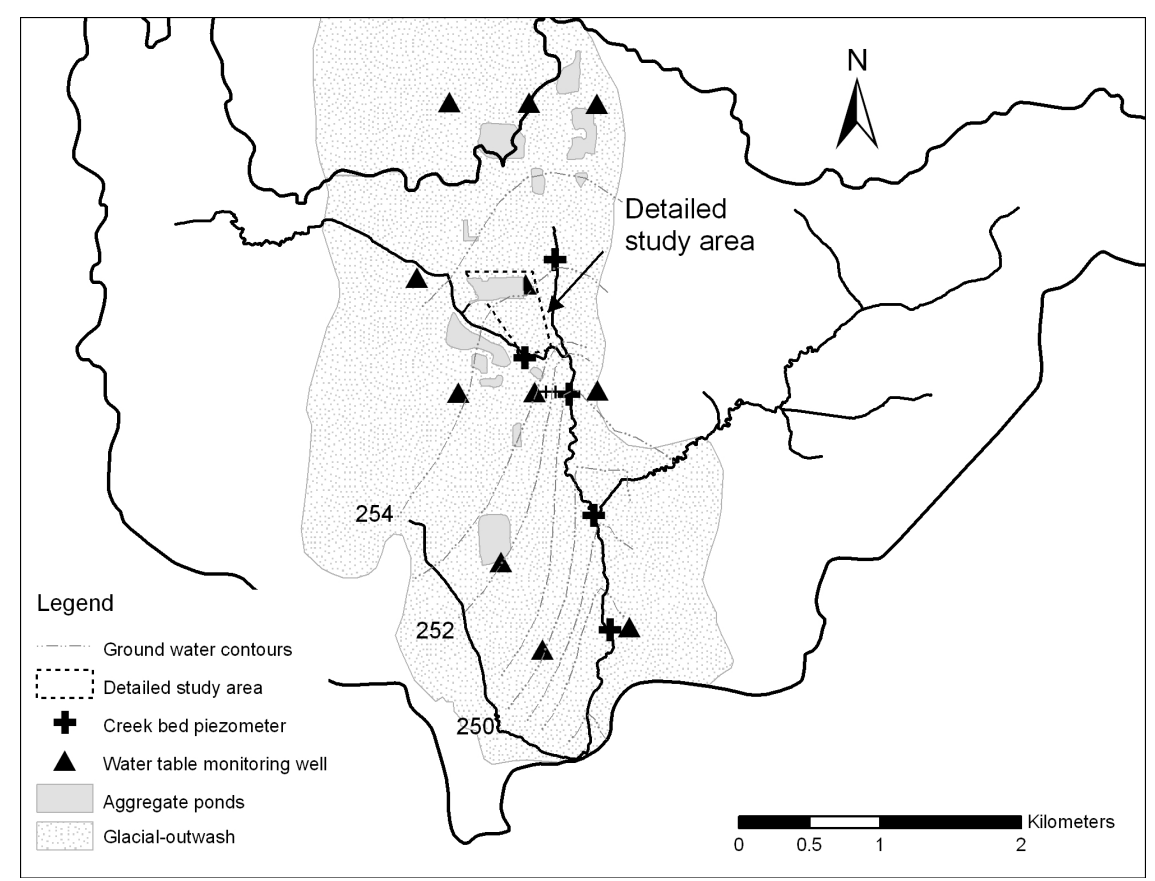

Figure 3-3 Study site location within the Tricks Creek watershed is shown by the dashed line. Also shown are the potentiometric surface and monitoring locations in the glacial-outwash aquifer.

\section{Conceptual Model}

Prior to building the numerical groundwater flow and transport model, a conceptual geologic model, based upon the field observations and available data, was developed.

The glacial-outwash channel is incised into the till and extends from the Bayfield River north beyond the watershed boundary to the Maitland River. The conceptual model was extended beyond the Tricks Creek watershed to include the northern portion of the outwash aquifer lying in the Bridgewater Creek watershed (Fig. 3-2). The conceptual model comprised three main surfaces: the ground surface (Fig. 3-4), constructed from a 10-m horizontal-resolution digital-elevation model supplemented with ground-elevation 
data collected during surveys of monitoring locations; the till surface (Fig. 3-5), constructed from the ground surface digital-elevation model on the exposed till moraines and domestic water well records and borehole data within the outwash channel; and the bedrock surface (Fig. 3-6), constructed from information contained in water wells records in the area. The outwash channel is in-filled with glacial outwash comprising coarse sand and gravel of varying thickness. The channel is incised into the till more deeply on the western side where the aquifer is up to 25-m-thick (Fig. 3-5). Approximately $1 \mathrm{~km}$ north of the Tricks Creek - Bridgewater Creek watershed boundary, the outwash aquifer thins and is absent for approximately $900 \mathrm{~m}$ before reappearing and continuing on to the Maitland River (Fig. 3-2). The aquifer is $\sim 1.8-\mathrm{km}$-wide within the Tricks Creek watershed and narrows to the north where it is 650-m-wide near the Maitland River. The till varies in thickness across these watersheds and, with the exception of the glacial outwash channel, it is at or near the ground surface (Fig. 3-4). The Bayfield River has eroded into the till, and at the confluence with Tricks Creek, the till is only 4-m-thick. Throughout most of the outwash channel, the till varies between 15 and 20-m-thick. At the Maitland River, the river has eroded the till and exposed the bedrock along portions of the river bottom. To the east of the outwash channel, the till varies between 20 and 40m-thick and to the west it varies between 50 and 75-m-thick. 


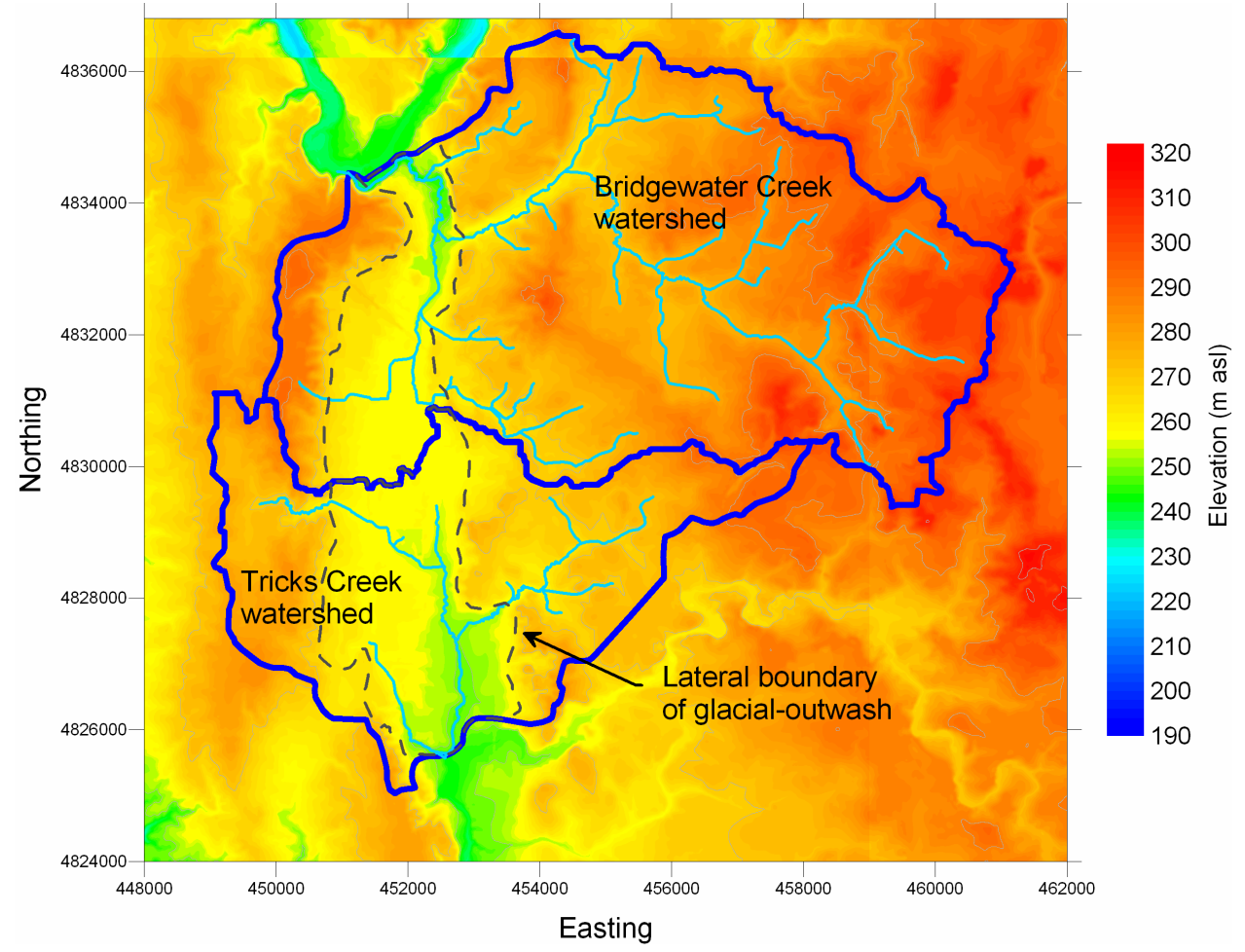

Figure 3-4 Ground surface elevation overlain by the stream network (light blue), Bridgewater Creek watershed in the north (dark blue), and Tricks Creek watershed in the south (dark blue). The boundary of the glacial-outwash is shown by the dashed line. 


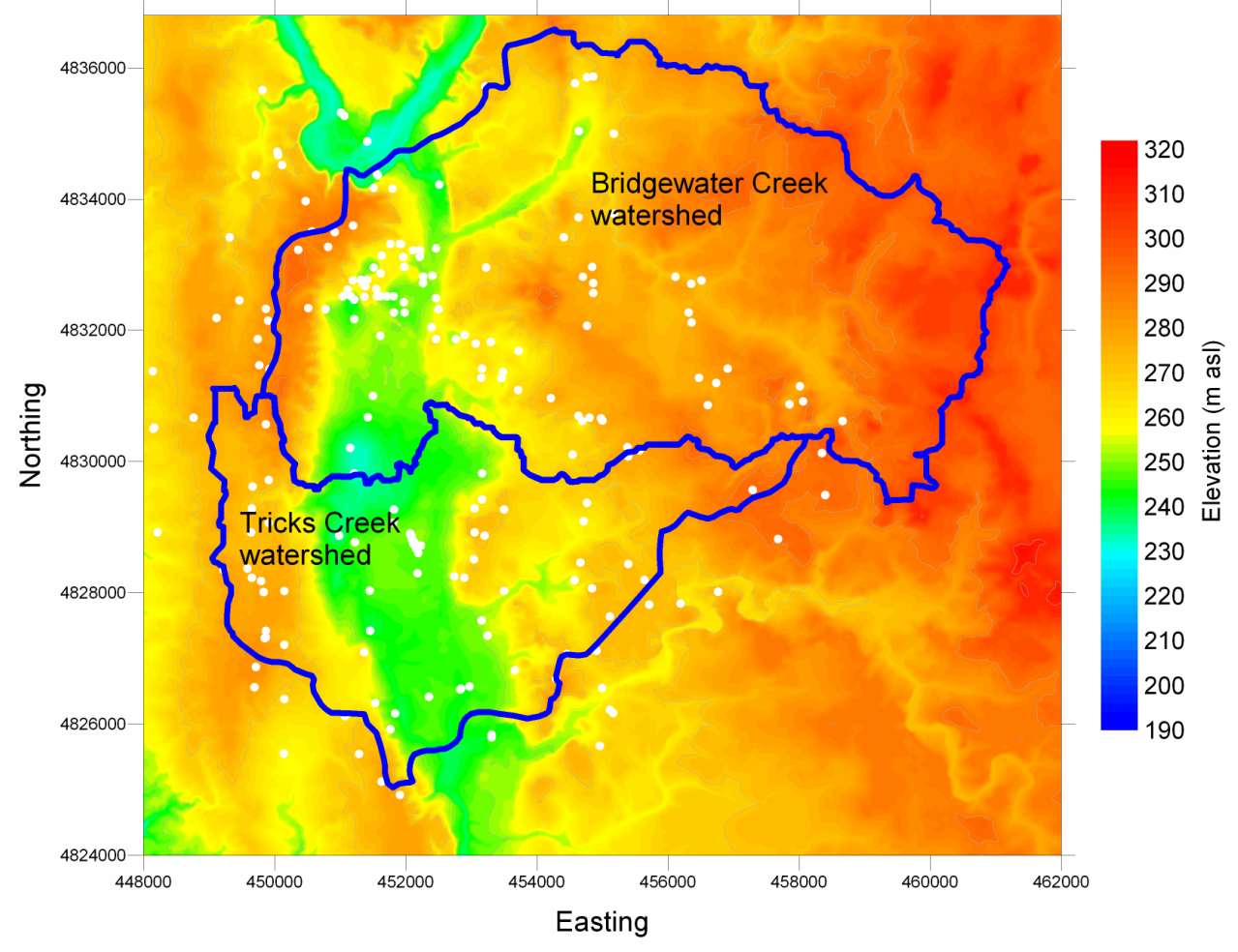

Figure 3-5 Till surface elevation overlain by Bridgewater Creek watershed in the north (dark blue), and Tricks Creek watershed in the south (dark blue). The glacialoutwash channel is the distinct north-south trending feature incised into the surface of the till moraine. The white dots indicate the location of water well record and borehole data used to construct the till surface. 


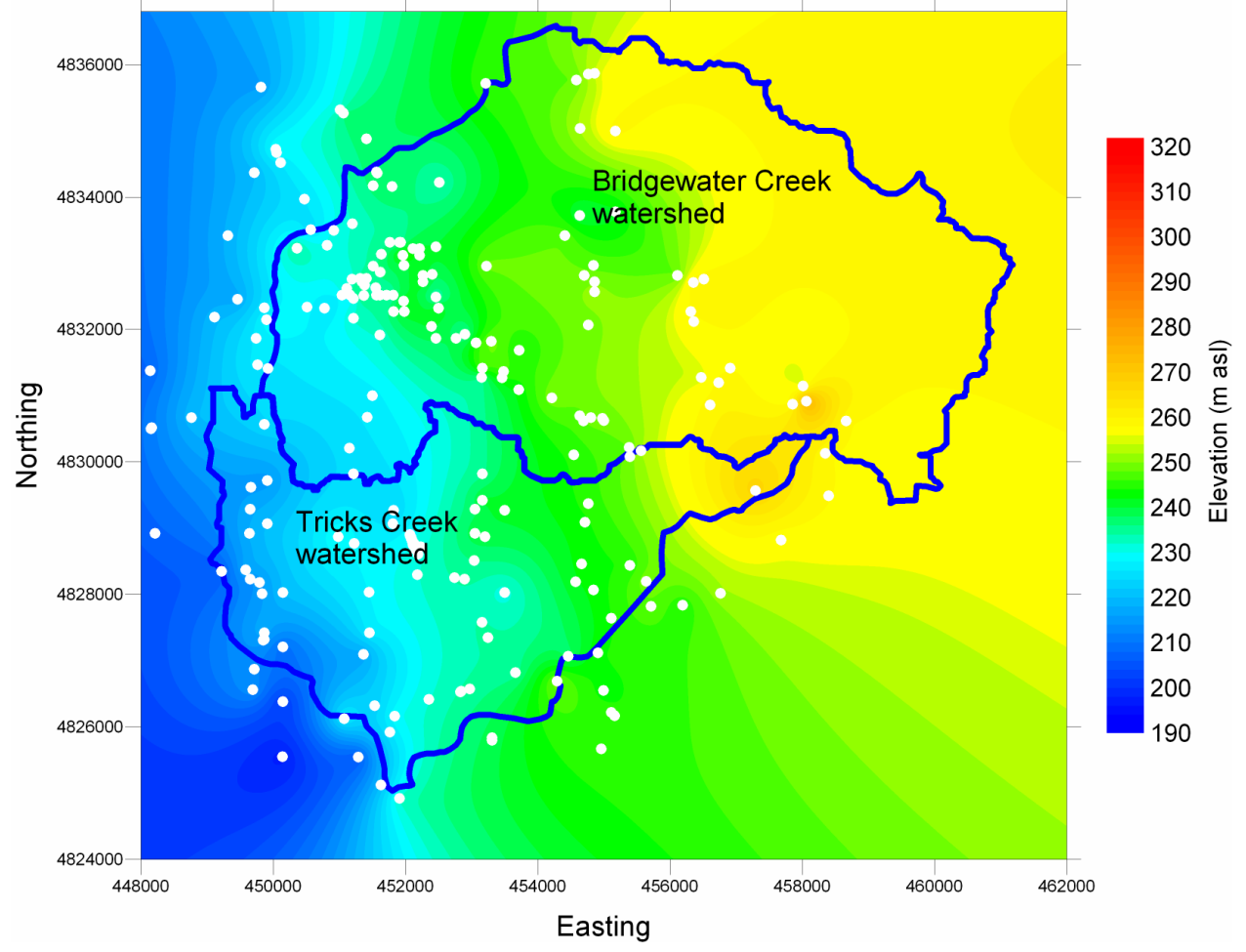

Figure 3-6 Bedrock surface elevation overlain by Bridgewater Creek watershed in the north (dark blue), and Tricks Creek watershed in the south (dark blue). The white dots indicate the location of water well record and borehole data used to construct the bedrock surface.

The base of the conceptual model corresponds to the till-bedrock interface (Fig. 36). The bedrock surface dips gently to the west and ranges in elevation from $210 \mathrm{~m}$ asl, along the western edge of the Tricks Creek watershed, to over $270 \mathrm{~m}$ asl at the eastern tip of the Bridgewater Creek watershed. Beneath the outwash aquifer, the bedrock surface ranges between $\sim 235 \mathrm{~m}$ asl along the eastern edge to $\sim 220 \mathrm{~m}$ asl along the western edge.

The conceptual model comprises two major hydrostratigraphic units. These are the unconfined glacial-outwash aquifer and the underlying clay-till aquitard. The tillbedrock interface is interpreted as the base of the conceptual model such that the bedrock is considered only a boundary. From the geologic descriptions in the water well records for the area, the till is relatively uniform, and thus homogeneous hydraulic and thermal properties were assigned to this layer. Based on observations during drilling and measurements of aquifer hydraulic and thermal properties, the glacial outwash is 
heterogeneous. An examination of exposed faces of outwash in aggregate pits, within the Tricks Creek watershed, indicates that there is a gradual transition from gravel and sand with cobbles, in the northern portion of the watershed, to fine and medium sand in the southern portion near the Bayfield River. Down-stream fining is a common transition in glacial outwash-channel deposits (Koteff, 1974; Barnett, 1992), and observations at this site are in agreement with the conclusion that this outwash channel formed approximately 13,000 years ago during a glacial advance and drained southward (Cooper and Fitzgerald, 1977).

The surface-water divide between the Tricks Creek and Bridgewater Creek watersheds (Fig. 3-2) is derived from the surface topography. Groundwater divides do not always coincide with watershed boundaries established from topography. As is shown later through the use of a 3-dimensional groundwater-flow model for the outwash aquifer, the groundwater divide in the outwash is approximately $1.1 \mathrm{~km}$ north of the surface-water divide. This groundwater divide is implemented as a model boundary in the implementation of the numerical model in subsequent sections.

Finally, the outwash aquifer is bounded laterally by till moraines. This creates a setting in which recharge may enter the glacial-outwash aquifer by (1) direct infiltration of precipitation falling on the ground surface within the highly permeable aquifer, (2) infiltration, at the lateral till-aquifer contact, of unchanneled overland flow and shallow subsurface flow from the upland till moraines, and (3) leakage from tributary streams that originate on the till moraines and flow on to the outwash aquifer as channeled flow (Morrissey et al., 1988). The conceptual model (Fig. 3-7) accounts for recharge entering the aquifer by each of these mechanisms. 


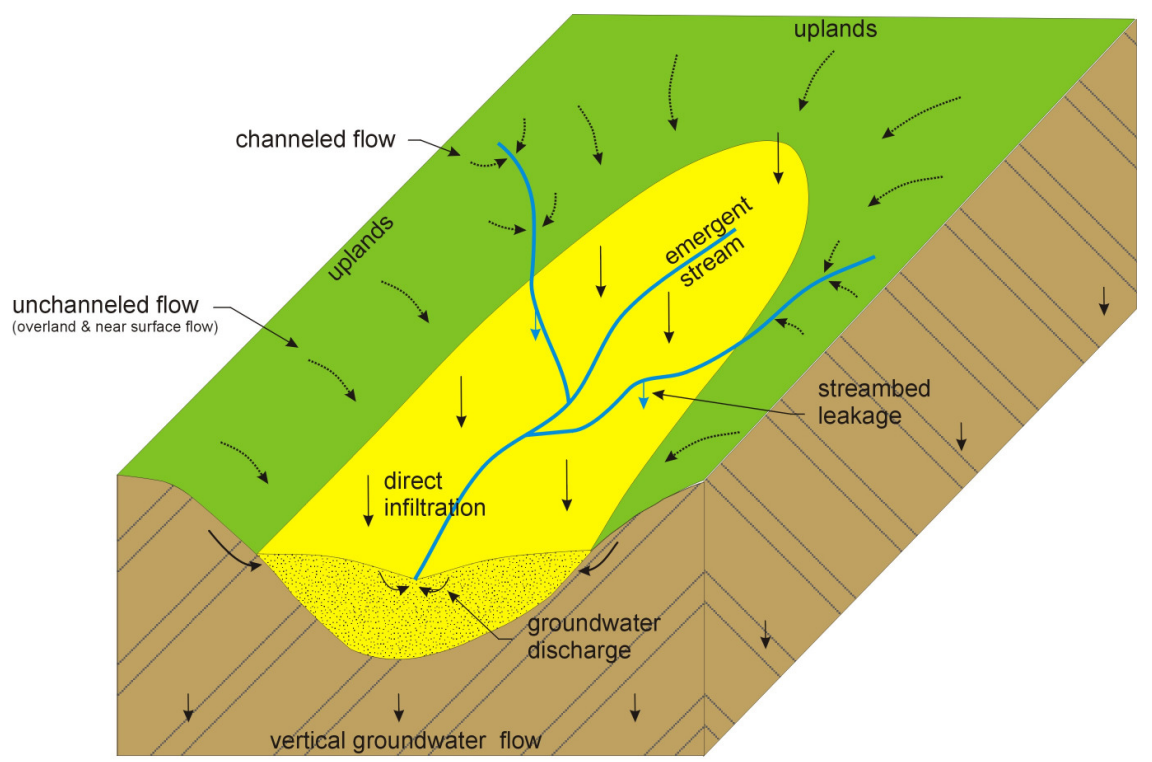

Valley-filled glacial-outwash aquifer

Glacial till moraine

Figure 3-7 Conceptual hydrogeological model of groundwater and surface water flow in a valley-filled glacial-outwash aquifer incised into a till moraine.

\section{Governing Equations and Numerical Model}

The numerical simulation of three-dimensional density-dependent groundwater flow and thermal energy transport requires the solution of the Darcy equation for densitydependent groundwater flow, and the continuity equation for the fluid and the heat. The three-dimensional density-dependent fluid flow equation is given as (Bear, 1972)

$$
\begin{aligned}
-\frac{\partial}{\partial x_{i}}\left[\frac{k_{i j} \rho(T)}{\mu(T)}\left(\frac{\partial p}{\partial x_{j}}+\rho_{w}(T) g \frac{\partial z}{\partial x_{j}}\right)\right]+Q \\
=-\rho_{w}(T)(\alpha+\theta \beta) \frac{\partial p}{\partial t}
\end{aligned}
$$

where $x_{\mathrm{j}}$ are the three-dimensional spatial coordinates (meters), $k_{\mathrm{ij}}$ is the permeability tensor $\left(\mathrm{m}^{2}\right), p$ is the pressure $\left(\mathrm{kg} \mathrm{m}^{-1} \mathrm{~s}^{-2}\right), g$ is the gravitational acceleration $\left(\mathrm{m} \mathrm{s}^{-2}\right), t$ is the time (s), $T$ is the temperature $\left({ }^{\circ} \mathrm{C}\right), \alpha$ is the aquifer compressibility $\left(\mathrm{m}^{2} \mathrm{~N}^{-1}\right), \beta$ the 
fluid compressibility $\left(\mathrm{m}^{2} \mathrm{~N}^{-1}\right)$, and $\theta$ the aquifer porosity (-). The temperature-

dependent variables are $\rho_{\mathrm{w}}(T)$ the fluid density $\left(\mathrm{kg} \mathrm{m}^{-3}\right)$, and $\mu(T)$ the dynamic viscosity of the fluid $\left(\mathrm{kg} \mathrm{m}^{-1} \mathrm{~s}^{-1}\right) . Q$ is a point source/sink mass flux term $\left(\mathrm{kg} \mathrm{m}^{-3} \mathrm{~s}^{-1}\right)$ which is written as

$$
Q=\sum_{k=1}^{N} Q_{k}(t) \rho_{w}(T) \delta\left(x_{k}^{\prime}, y_{k}^{\prime}, z_{k}^{\prime}\right)
$$

where $Q_{\mathrm{k}}(t)$ is the time-dependent injection or withdrawal rate $\left(\mathrm{s}^{-1}\right), \delta$ is the Dirac delta function $\left(\mathrm{m}^{-1}\right)$, and $x_{\mathrm{k}}^{\prime}, y_{\mathrm{k}}^{\prime}, z_{\mathrm{k}}^{\prime}$ are the coordinates of the point source/sink.

The continuity equation for heat gives the thermal transport equation as

$$
\begin{aligned}
\frac{\partial}{\partial x_{i}}\left[\left(\kappa+\frac{D_{i j}}{R}\right) \frac{\partial T}{\partial x_{j}}\right] & -\left(\frac{v_{i}}{R} \frac{\partial T}{\partial x}\right)+ \\
& \sum_{k=1}^{N} \frac{Q_{k}(t)\left[T_{k}(t)-T(t)\right]}{R \theta} \delta\left(x_{k}^{\prime}, y_{k}^{\prime}, z_{k}^{\prime}\right)=\frac{\partial T}{\partial t}
\end{aligned}
$$

where $\kappa$ is the aquifer thermal diffusivity $\left(\mathrm{m}^{2} \mathrm{~s}^{-1}\right), R$ is the thermal retardation term, and $T_{\mathrm{k}}(t)$ is the temperature of the injection or withdrawal fluid. The complete development of these equations is given by Molson et al. (1992) and implemented in the finite-element numerical code, Heatflow (Molson et al., 1992; Molson and Frind, 2004). The Heatflow model accounts for density-dependent groundwater flow, thermal advection, conduction through the porous medium, thermal buoyancy, and thermal retardation. The model supports three-dimensional grids constructed with deformable hexahedral brick elements, and accommodates completely saturated flow, and either saturated or partially saturated transport.

For this study, major modifications were made to Heatflow. These included incorporating the presence of streams and ponds within the domain, including recharge from upland channeled and unchanneled flow, modifying the fluid mass balance routine, adding a more complete consistent velocity formulation (Knabner and Frolkovic, 1996), 
adding the ability to export and import subgrid boundary files, implementing a variety of utilities such as output of grid Peclet and Courant numbers, output of data at user specified observation points, enhanced grid deformation subroutine, implementation of the Campbell algorithm (Campbell et al., 1994) to account for temperature dependent thermal conductivity, adding dynamic memory allocation, and updating the code to meet Fortran90 standards. Appendix B provides a complete description of the constitutive equations, the implementation of the boundary conditions and initial conditions considered in this study, along with the resulting finite-element matrix equations.

Heatflow accommodates fully saturated flow, and either completely saturated or partially saturated transport, by defining unique flow and transport grids. In the case of fully saturated flow and transport, the flow and transport grids are identical. In the case of partially saturated transport, the top of the flow grid corresponds to the water table and the top of the transport grid extends up into the unsaturated zone, generally to the ground surface (Molson and Frind, 2004). In this case, the flow grid will have fewer elements in the vertical direction and the top boundary of the flow and transport grid will not coincide. As will be seen later in the Numerical Simulations section the boundary conditions for flow and for transport must reflect these different boundary surfaces.

\section{Verification of Modified Model}

The revised code was verified against several standard benchmark problems and tests used for density-dependent flow and transport codes. These included the one-dimensional analytical solution of heat transport by convection and conduction in a semi-infinite porous medium with a time varying boundary condition (Stallman, 1965); a onedimensional analytical solution to heat conduction in a finite domain with a non-uniform initial condition; and two tests of consistency suggested by Voss and Souza, (1987). As well, two new analytical solutions to the Elder short heater problem (Elder, 1967) are presented and used as benchmark tests. Appendix C provides a complete description of these benchmark problems and tests, and shows the agreement between Heatflow and these tests. 


\section{Modelling Strategy}

This study focused on the simulation of the thermal plume migration from one pit studied in detail as part of this investigation (Fig. 3-3). Maintaining reasonable run times and memory requirements ( $<12$ hours of run time and $<2$ GB memory) while satisfying grid Courant and Peclet criteria requirements precluded the completion of transient flow and transport simulations for the entire glacial-outwash aquifer. For heat transport the grid Courant and Peclet criteria are given by

$$
C_{i}=\frac{v_{i} \Delta t}{R \Delta x_{i}} \leq 1 \quad P_{i}=\frac{v_{i} \Delta x_{i}}{R \kappa_{i}+d_{i i}} \leq 2,
$$

where $C_{\mathrm{i}} \quad$ is the grid Courant number in the $i$-direction;

$P_{\mathrm{i}} \quad$ is the grid Peclet number in the $i$-direction;

$v_{\mathrm{i}} \quad$ is the velocity in the $i$-direction $\left[\mathrm{L} \mathrm{T}^{-1}\right]$;

$\Delta t \quad$ is the simulation time step [T];

$\Delta x_{\mathrm{i}} \quad$ is the grid spacing in the $i$-direction [L];

$R \quad$ is the thermal retardation term;

$\kappa_{i} \quad$ is the thermal diffusivity $\left[\mathrm{L}^{2} \mathrm{~T}^{-1}\right]$, and

$d_{\mathrm{ii}} \quad$ is the hydrodynamic dispersion $\left[\mathrm{L}^{2} \mathrm{~T}^{-1}\right]$.

For the large model domains, satisfying the grid Peclet number results in grids and run times that are impractical. To overcome this issue a telescopic mesh refinement, or subdomain, approach was adopted whereby a larger model is used to establish the boundary conditions for a model covering a smaller area or domain (Ward et al., 1987). For the large models, the Peclet criterion is not satisfied and the resulting heat transport simulations are not reliable. For the sub-domain grids, the grid can be refined so the Peclet criterion is satisfied and the time step reduced to satisfy the Courant criterion.

In this study, four separate three-dimensional model domains were constructed. The first model domain incorporated the outwash aquifer from the Bayfield River north to the point along Bridgewater Creek where the aquifer thins and becomes discontinuous 
prior to reappearing and continuing north to the Maitland River (Fig. 3-8a). This model domain was used to complete steady-state groundwater flow simulations. Heat transport was not considered at this stage since the purpose was to simulate threedimensional groundwater flow within the entire outwash aquifer from which the location of the groundwater divide between the Tricks Creek and Bridgewater Creek watersheds could be established. Once the location of the groundwater divide was established, a watershed scale model was created covering the Tricks Creek watershed (Fig. 3-8b). Using this watershed scale model, both steady-state groundwater flow and transient groundwater flow and heat transport simulations, were completed. A smaller area or subdomain (Fig. 3-8c) was extracted from the transient flow and transport simulation by saving the head and temperature at the nodes defined by the lateral boundaries of the subdomain at each time step. These values are then used as the time varying boundary conditions along the lateral boundaries of the sub-domain grid. From this initial subdomain a smaller sub-domain was established (Fig. 3-8d) containing the detailed study area. This final sub-domain was used to model transient groundwater flow and heat transport in the area down gradient of the pit. 


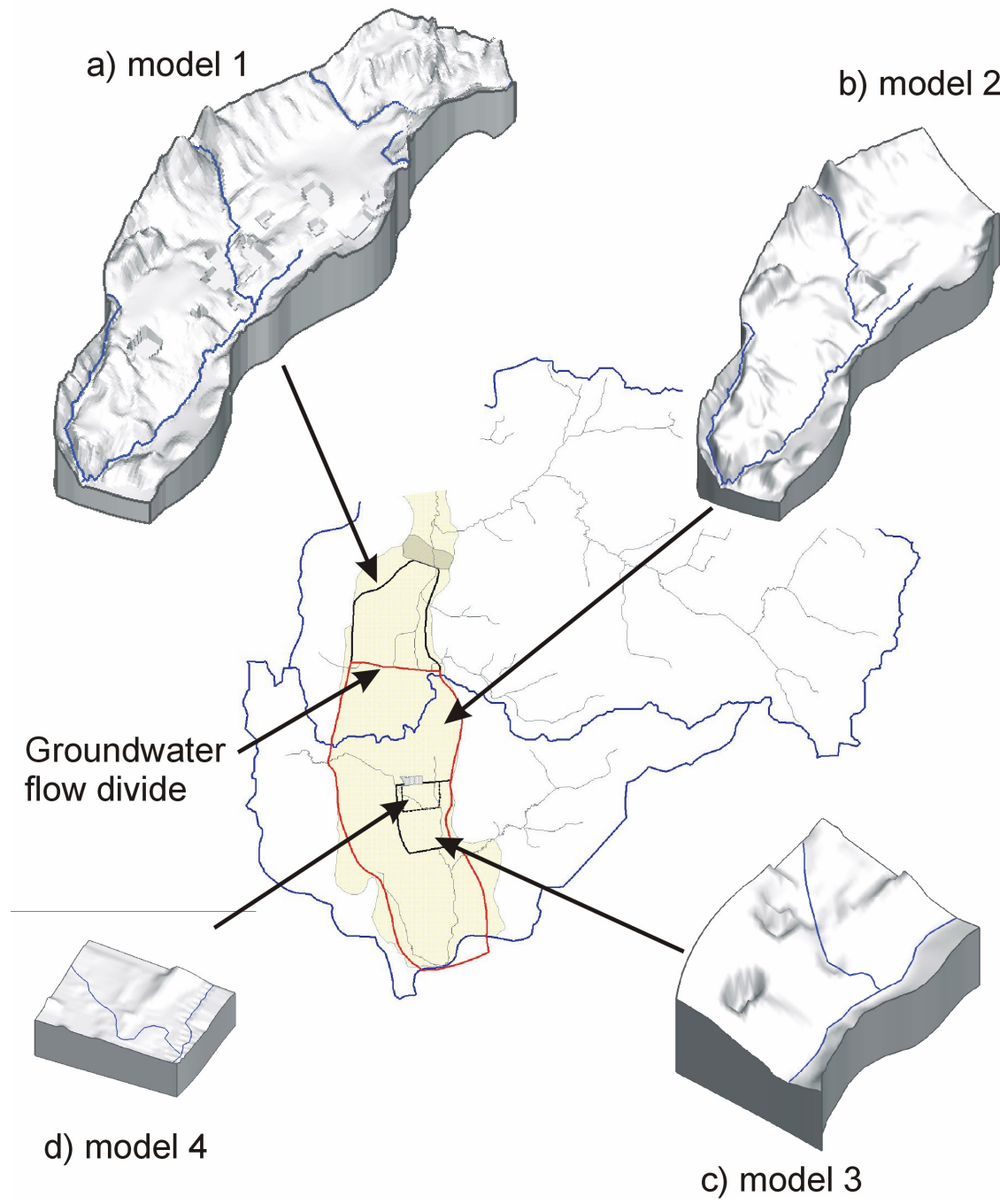

Figure 3-8 Three-dimensional model domains showing telescopic mesh refinement: a) model 1 - outwash aquifer spanning Tricks Creek and Bridgewater Creek watersheds; b) model 2 - Tricks Creek groundwater catchment; c) model 3 - sub-domain 1; and d) model 4 - final sub-domain focusing on detailed study area.

\section{Model Grid Design}

This section presents the design of the four 3-dimensional model grids starting with the large-scale steady-state groundwater flow model grid (Fig. 3-8a) and progressing to the final transient groundwater flow and heat transport sub-domain grid (Fig. 3-8d). The steady-state groundwater flow model incorporates the outwash aquifer from the Bayfield River north to the area where the outwash aquifer pinches out in the Bridgewater Creek watershed. It is bounded laterally by the edge of the saturated outwash aquifer and does 
not include the adjacent till moraines. The model domain $\left(12.58 \mathrm{~km}^{2}\right)$ was divided horizontally into 250 elements in the north-south direction, 60 elements in the east-west direction and vertically into 25 separate layers (10 layers within the till and 15 layers within the outwash sand and gravel aquifer). The mesh comprises a total of 375,000 hexahedral brick elements with 398,086 nodes (Table 3-1). The brick element mesh was built using GridBuilder (McLaren, 2007) and Grok (Therrien et al. 2007). The base of the domain coincides with the bedrock surface and ranges in altitude between 220 to $240 \mathrm{~m}$ asl along the western edge of the domain and 230 to $240 \mathrm{~m}$ asl along the eastern edge.

From the results of steady-state groundwater flow simulations, the location of the groundwater divide was established (Fig. 3-8a). The groundwater flow divide was used as the northern boundary for model 2, a watershed scale model covering 10.33 ha (Fig. 3$8 \mathrm{~b})$. The grid spacing for model 1 and model 2 was the same yielding a grid with 180 elements in the north-south direction, 60 elements in the east-west direction. For the watershed grid transient groundwater flow and heat transport simulations were completed. This required the addition of 5 new grid layers within the unsaturated zone such that the grid comprised 30 layers (10 layers within the till, 15 layers within the outwash sand and gravel aquifer, and 5 layers in the unsaturated zone). The resulting mesh comprises a total of 324,000 hexahedral brick elements with 342,271 nodes.

Model 2 was used to establish the hydraulic and thermal boundaries for the first groundwater flow and transport sub-domain model, model 3 (Fig. 3-8c). The northern boundary was set near the down gradient (southern) edge of the pond and the eastern boundary of the sub-domain was set coincident with the eastern edge of the saturated outwash aquifer. Preliminary transport simulations using a 3-dimensional generic rectangular domain demonstrated that within $1000 \mathrm{~m}$ down gradient of a pit, the lateral dispersion and conduction of a thermal plume from a constant temperature source was limited to $200 \mathrm{~m}$. Based on this finding and given the groundwater flow in a southsoutheast direction in this sub-domain, the western boundary was located $\sim 100 \mathrm{~m}$ west of the pond edge and extended $\sim 900$ m east to the outwash moraine contact (the eastern edge of model 2). The up gradient edge of the sub-domain (north end) was set at the down gradient edge of the pond and extends $\sim 1200 \mathrm{~m}$ south along a north-south grid line 
to the southern edge of the sub-domain. Time varying specified head and temperature boundary conditions were set along all four boundaries of the sub-domain. These specified values were obtained in the previous watershed scale model (model 2). During the transient simulation for model 2 , the head and temperatures at the nodes corresponding to the boundaries for the subgrid were saved to output files at each time step. These saved heads and temperatures were then read for each time step during the transient simulation for the sub-grid model (model 3). The hydraulic and thermal boundary conditions on the upper and lower boundaries of the sub-domain were the same as those used in the watershed scale model. The sub-domain mesh was refined and comprises a total of 148,500 elements with 158,286 nodes, and incorporates an area of 1.11 ha. Within the model domain, the ground surface ranges in altitude from $258 \mathrm{~m}$ asl at the northern east and southwest corners of the domain. Tricks Creek cuts across the eastern portion of the domain entering the northern end at $\sim 255 \mathrm{~m}$ asl and exiting the southern end at $\sim 251.4 \mathrm{~m}$ asl. The western tributary enters the sub-domain in the northwest corner at $254.5 \mathrm{~m}$ asl and merges with Tricks Creek at $253.9 \mathrm{~m}$ asl. The aquifer thickness ranges from $\sim 8 \mathrm{~m}$ along the western edge of the domain to near 0 along the eastern boundary. The till thickness varies from $21.4 \mathrm{~m}$ along the western boundary to $\sim 19 \mathrm{~m}$ along the eastern boundary.

From the transient flow and transport simulations of model 3, the boundary conditions for the final sub-grid model, model 4, were created. The sub-domain mesh for model 4 was refined and comprises a total of 678,240 elements with 712,845 nodes, and incorporates an area of 0.32 ha. 
Table 3-1 Model domains considered in study.

\begin{tabular}{|c|c|c|c|c|c|c|c|}
\hline \multirow{2}{*}{$\begin{array}{l}\text { Model } \\
\text { domain }\end{array}$} & \multirow[t]{2}{*}{ Scale } & \multirow{2}{*}{$\begin{array}{c}\text { Area } \\
\mathrm{km}^{2}\end{array}$} & Number of elements & \multirow{2}{*}{$\begin{array}{l}\text { Number } \\
\text { of layers }\end{array}$} & \multirow{2}{*}{$\begin{array}{l}\text { Number } \\
\text { of } \\
\text { elements }\end{array}$} & \multirow{2}{*}{$\begin{array}{l}\text { Number } \\
\text { of nodes }\end{array}$} & \multirow[t]{2}{*}{ Simulation type } \\
\hline & & & E-W & & & & \\
\hline model 1 & Tricks Creek and Bridgewater Creek watersheds $\dagger$ & 12.58 & 250 & $25 \S$ & 375,000 & 398,086 & 1. steady-state flow \\
\hline model 2 & Tricks Creek watershed $\dagger$ & 10.33 & 180 & $\begin{array}{l}25 \S \\
30 \pi\end{array}$ & $\begin{array}{l}270,000 \\
324,000\end{array}$ & $\begin{array}{l}287,066 \\
342,271\end{array}$ & $\begin{array}{l}\text { 1. steady-state flow } \\
\text { 2. transient flow and transport }\end{array}$ \\
\hline model 3 & Sub-domain $1 \ddagger$ & 1.11 & 110 & $\begin{array}{l}25 \S \\
30 \pi\end{array}$ & $\begin{array}{l}123,750 \\
148,500\end{array}$ & $\begin{array}{l}132,756 \\
158,286\end{array}$ & 1. transient flow and transport \\
\hline model 4 & Sub-domain $2 \ddagger$ & 0.32 & 314 & $\begin{array}{l}25 \S \\
30 \mathbb{I}\end{array}$ & $\begin{array}{l}565,200 \\
678,240\end{array}$ & $\begin{array}{l}597,870 \\
712,845\end{array}$ & 1. transient flow and transport \\
\hline
\end{tabular}




\section{Surface Water Features}

Surface water features within the model domains include streams and ponds. Streams were incorporated using a head-dependent boundary condition, and leakage is added to, or extracted from, stream nodes that lie along the top surface of the flow grid. The leakage at each node is given by

$$
Q_{i}=-K_{S} A_{i}\left(\frac{h_{\text {stream }}-h_{i}}{b_{S}}\right)
$$

where $K_{S}$ is the vertical hydraulic conductivity of the leaky layer or streambed conductance, $b_{\mathrm{S}}$ is the thickness, $h_{\text {stream }}$ the head specified in the stream, $h_{\mathrm{i}}$ is the head in the aquifer at node $i$, which is calculated by the model, and $A_{\mathrm{i}}$ is the area of the stream attributed to node $i$. If the head in the aquifer is greater than the head in the stream, the model removes water at these nodes; otherwise, it adds water. For the models developed here, where the head in the aquifer is less than the head in the stream, streams are implemented using two distinct approaches. For streams that emerge within the model domain (i.e., Tricks Creek emerges in the northern portion of the watershed), if the head in the aquifer drops below the bottom of the stream, then the stream is assumed to be disconnected from the aquifer and leakage is set to zero. For streams that emerge within upland areas outside the model domain, such as on till moraines, overland-channeled runoff from the moraine may flow on to and across the aquifer. This runoff is available for infiltration even where the water table is below the base of the stream. Under these conditions, the flux at the stream nodes is specified and a water balance of the channeled upland flow is used to evaluate if fluid is available for infiltration.

In this study, ponds are treated as high hydraulic conductivity elements (high $K$ approach). This method yields good results for seepage lakes for both steady-state and transient simulations provided the contrast between the aquifer and the lake elements is greater than three orders of magnitude (Anderson et al. 2002). The method was verified through a series of simulations completed using Heatflow but not shown here. The explicit incorporation of ponds into the numerical model would require use of the Navier- 
Stokes open-water flow equations which would result in nonlinear finite-element flow equations. Incorporating the Navier-Stokes equations into Heatflow would be a significant effort and would require the use of newer solvers capable of handling nonsymmetric coefficient matrices. This was beyond the scope of this study and is a potential avenue for future research. Thus, while it is not possible to model these ponds explicitly with the current version of Heatflow, the high $K$ approach yields good results for the setting considered in this work.

\section{Numerical Simulations}

Three-dimensional steady-state groundwater flow was simulated for model 1 and model 2. As well transient flow and thermal transport was simulated for model 2 (Fig. 3-8a and 3-8b, Table 3-1). Transient flow and heat transport were simulated for model 3 and model 4 (Fig. 3-8c and 3-8d). For model calibration, results of simulated heads, groundwater gradients, stream discharge, and aquifer temperatures were compared to field data collected for the period October 1995 to October 1996. Model validation was completed using data from a second time period June 2001 to December 2003. For the validation simulation, the size of the pond was enlarged to account for pit expansion that occurred as aggregate was extracted and the recharge boundary condition was alter to reflect recharge conditions during this period without additional calibration.

\section{Hydraulic properties of the glacial outwash and till}

The mean $K$ 's from multi-scale tests in the outwash aquifer range from $1.8 \times 10^{-4}$ to $1.7 \times 10^{-2} \mathrm{~m} \mathrm{~s}^{-1}$ in the horizontal directions $\left(K_{\mathrm{x}}\right.$ and $\left.K_{\mathrm{y}}\right)$ and the vertical anisotropy ratio $\left(K_{\mathrm{X}} / K_{\mathrm{z}}\right)$, determined from a 52-hour pumping test, is 5:1 (Markle and Schincariol, 2007). An initial horizontal hydraulic conductivity of $1.0 \times 10^{-3} \mathrm{~m} \mathrm{~s}^{-1}$ was selected and assigned as a uniform property to the outwash aquifer. During calibration, this value was adjusted and zones of specific hydraulic conductivity were created where necessary.

The lateral and vertical hydraulic conductivities of the till were estimated from grain size analysis, falling-head tests, and calibration to temperature profiles obtained in three bedrock wells (Appendix F). Estimates for $K_{\mathrm{x}}$ and $K_{\mathrm{y}}$ range from $10^{-11}$ to $\sim 10^{-7} \mathrm{~m} \mathrm{~s}^{-}$ 
${ }^{1}$ and $K_{\mathrm{z}}$ is $\sim 3 \times 10^{-8} \mathrm{~m} \mathrm{~s}^{-1}$. For the flow and transport simulations, $K_{\mathrm{x}}$ and $K_{\mathrm{y}}$ were

assigned a value of $10^{-7} \mathrm{~m} \mathrm{~s}^{-1}$ and were not adjusted during calibration. $K_{\mathrm{z}}$ was set equal to $1 \times 10^{-8} \mathrm{~m} \mathrm{~s}^{-1}$ and was allowed to vary between $1 \times 10^{-8}$ and $3 \times 10^{-8} \mathrm{~m} \mathrm{~s}^{-1}$. Similarly, the porosity for the aquifer and till were set to 0.29 and 0.4, respectively (Markle and Schincariol, 2007), and were not adjusted during the calibration.

For the transient flow simulations, the top layer of elements was assigned a specific yield of 0.09 . This value was based on an analysis of observed rises in the water table in response to individual recharge events and analysis of the 48-hour pumping test. The remaining elements within the aquifer were assigned a specific storage of $10^{-3} \mathrm{~m}^{-1}$, estimated from a two-day constant-discharge pumping test, and the specific storage of the till was $10^{-4} \mathrm{~m}^{-1}$.

\section{Hydraulic properties of the streams and ponds}

The streambed conductance $K_{S}$ was estimated from seepage-meter measurements and streambed temperature data. Water levels measured in streambed piezometers at five locations (MP1 to MP5), show groundwater discharge conditions persist throughout the year. From seepage-meter measurements at sites MP2, MP3 and MP4, estimates of vertical hydraulic conductivities range from $10^{-10}$ to $\sim 10^{-8} \mathrm{~m} \mathrm{~s}^{-1}$. These values are unrealistically low based on observations of the streambed material during the installation of streambed piezometers. Difficulties in obtaining reliable estimates from seepage meters are well documented (Landon et al., 2001; Schincariol and McNeil, 2006). Estimates from streambed temperature measurements yield values of $K_{S}$ ranging from $5 \times 10^{-6}$ to $2 \times 10^{-5} \mathrm{~m} \mathrm{~s}^{-1}$ which match field observations. Therefore, an initial value of $10^{-5}$ $\mathrm{m} \mathrm{s}^{-1}$ was selected and adjusted during calibration. Field observations indicate that the streambed material in Tricks Creek and the tributaries is finer in the northern portion of the creek and coarsens to the south in response to an increase in the gradient of the streambed. Thus, streambed conductance was adjusted to reflect this observation.

Streambed elevations were calculated from surveyed values at the streambed piezometers, gauging stations, and other surface observation points. Stream width was also measured at these locations. Between these data points, values were interpolated. 
Surface-water elevations were measured at the gauging stations at 30-minute intervals and at the streambed piezometers and surface-water observation points every few weeks.

The ponds were treated as high hydraulic conductivity zones, and the ratio of the hydraulic gradient across the ponds to the regional gradient aquifer was kept below 0.001 (Anderson et al., 2002) by assigning a $K$ of $10 \mathrm{~m} \mathrm{~s}^{-1}$ to the elements within the ponds. The top layer of elements was assigned a specific yield of 1 and the underlying elements were assigned a specific storage coefficient, $S_{\mathrm{S}}$, of $4.6 \times 10^{-6} \mathrm{~m}^{-1}$ at $0^{\circ} \mathrm{C}$, given by

$$
S_{S}=\rho g \beta_{w}\left(T=0^{\circ} C\right),
$$

where $\rho$ is the density of water, $g$ is the gravitational acceleration, and $\beta_{\mathrm{w}}$ is the compressibility of water.

\section{Hydraulic boundary conditions}

Heatflow supports a three-dimensional domain discretized using deformable hexahedral brick elements. As a result, each model grid has six model boundaries for which boundary conditions must be specified; four lateral boundaries, a bottom boundary and a top boundary. Where possible, model boundaries were set coincident with natural hydraulic boundaries. Table 3-2 summarizes the boundary conditions for each model and these boundary conditions are discussed in the following sections. 
Table 3-2 Boundary conditions for the groundwater flow and transport models.

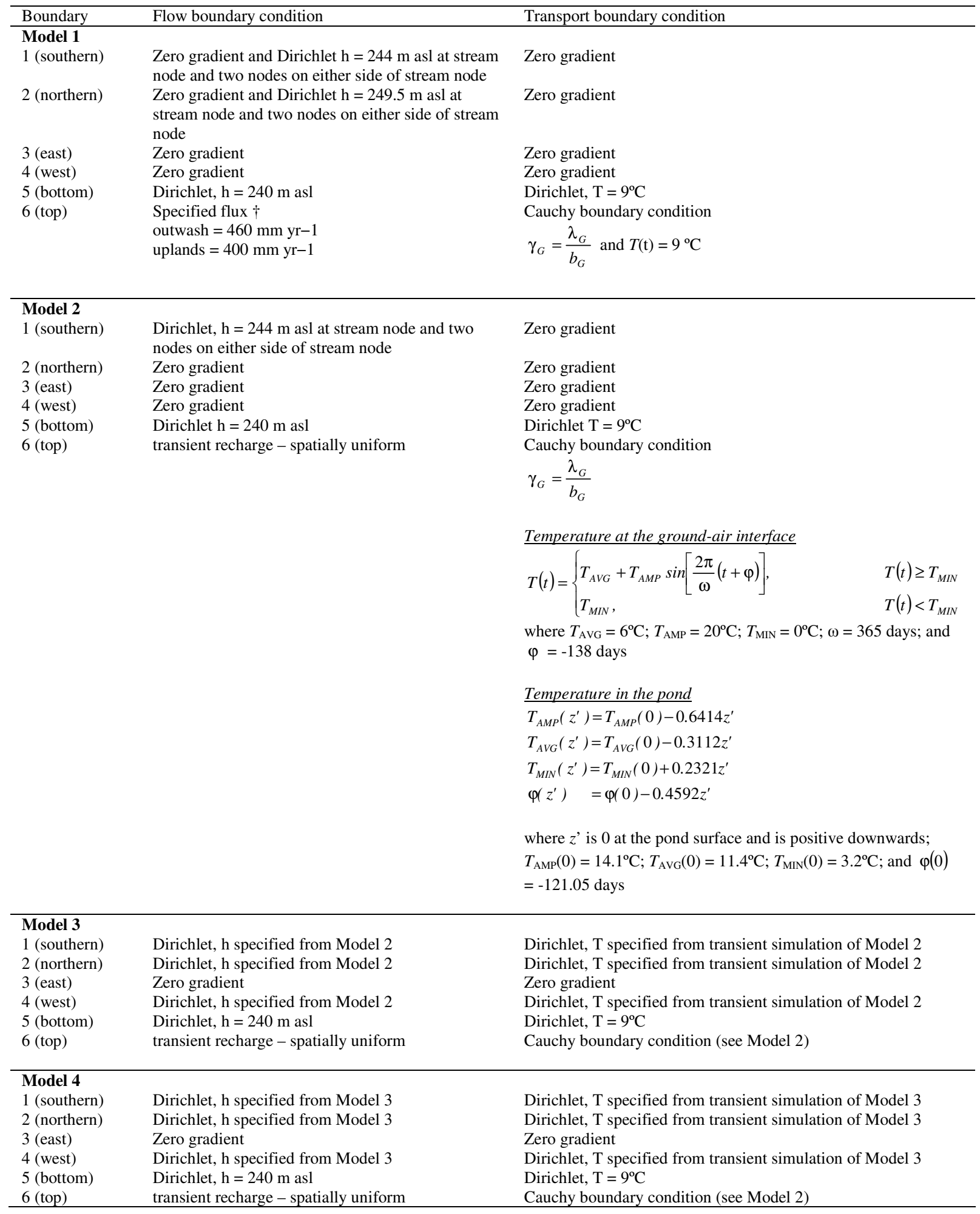


Model 1 and Model 2

With the exception of the northern portion of the model domains, model 1 and 2 share common boundaries and boundary conditions. To the south, where the aquifer terminates at the Bayfield River, the till surface rises and the water table drops below the base of the outwash channel into the till. The till restricts the discharge of groundwater from the outwash directly into the Bayfield River and groundwater discharge is focused along Tricks Creek and the un-named stream to the west. To reflect these conditions, the majority of the southern boundary was set as a no flow boundary with a fixed head of 244 $\mathrm{m}$ asl set at the stream node corresponding to the outlet of Tricks Creek. As well, the two nodes on either side of this stream node were assigned a specified head of $244 \mathrm{~m}$ asl to account for seepage through the aquifer material immediately adjacent to the stream channel. This conceptual model of groundwater-surface water interaction involving groundwater flow parallel to the stream channel and within the hyporheic zone was discussed by Woessner (2000).

To the north, the aquifer thins and in some areas pinches out (Fig. 3-2), and then reappears approximately $300 \mathrm{~m}$ further north and continues on to the Maitland River. Where the aquifer pinches out, the water table drops to the till surface and the discharge of groundwater from the outwash is restricted to Bridgewater Creek which flows north across the till surface. Model 1 extends to this point in the aquifer and to reflect these conditions, the majority of the northern boundary in model 1 was set as a no flow boundary with a fixed head of $249.5 \mathrm{~m}$ asl set at the stream node where Bridgewater Creek flows beyond the outwash and across the till. As well, two nodes on either side of the discharge point were assigned Dirichlet conditions of $249.5 \mathrm{~m}$ asl. Steady-state flow simulations for model 1 were used to establish the groundwater divide between the two watersheds to model domain spans. This groundwater divide defines a no flux boundary at which the northern boundary for model 2.

The east and west lateral boundaries of models 1 and 2 correspond to the sides of the outwash aquifer. These lateral boundaries extend vertically to the base of the till and are assumed to be no-flow boundaries. The bottom boundary corresponds to the tillbedrock interface. The potentiometric head in the bedrock slopes to the west, varying 
from $\sim 250 \mathrm{~m}$ asl along the eastern edge of the domain to $230 \mathrm{~m}$ asl along the western edge of the domain. For the model simulations a uniform head of $240 \mathrm{~m}$ asl, the average of the potentiometric surface measured in the bedrock across the domain, was specified across the bottom boundary. The measured head in the outwash aquifer is greater than that in the bedrock resulting in a downward vertical flux from the outwash aquifer, through the till and into the bedrock.

The top boundary of the flow domain corresponds to the water table. It and receives recharge from precipitation and is set as a specified flux boundary. The recharge applied across this boundary is equal to the recharge due to precipitation falling directly on the porous glacial outwash plus the recharge from unchanneled and channeled flow from the adjacent till uplands. For the steady-state model, a constant recharge (equal to the annual average recharge) was applied, while a transient recharge was applied for the transient simulations. Recharge is discussed in more detail in a subsequent section.

\section{Model 3 and Model 4}

Model 3 and model 4 are extracted as sub-domains from larger model domains (model 3 from simulations of model 2 and model 4 from simulations of model 3). As a result, for the sub-domain models these lateral boundaries are determined during the larger scale simulations and read in as specified head boundaries during the sub-domain simulations. The lateral boundaries for the sub-domain models were placed such that their presence would not interfere with the migration of the thermal plume in the detailed study area. As with models 1 and 2, the bottom boundary corresponds to the till-bedrock interface with a specified head corresponding to the potentiometric surface measured in the bedrock across the base of the model domain. Similarly the top boundary is a recharge boundary and corresponds to the transient water table.

\section{Recharge}

Reliable estimates of recharge are critical if a calibrated model with appropriate values of $K$ is to be obtained. At this site, the glacial-outwash aquifer is recharged by (1) direct infiltration of precipitation falling on the ground surface within the highly permeable 
aquifer, (2) infiltration, at the lateral till-aquifer contact, of unchanneled overland flow and shallow subsurface flow from the upland till moraines, and (3) leakage from tributary streams that originate on the till moraines and flow on to the outwash aquifer as channeled flow (Morrissey et al., 1988).

Recharge from the upland till moraines was estimated by assuming that all runoff from the adjacent moraines is available as recharge to the sand and gravel aquifer either at the lateral aquifer-moraine contact or through recharge along the stream channels originating on the till and flowing on to the aquifer. From the topography of the uplands, 18 sub-basins were established along the entire outwash aquifer (Fig. D-1, Appendix D). Sub-basins for channeled flow were established by defining the upland catchment above the point where the stream crosses from the uplands on to the aquifer. The remaining areas on the uplands form the sub-basins for unchanneled flow. Ten sub-basins contribute channeled flow to the aquifer and eight contribute unchanneled flow. The recharge for each sub-basin was apportioned to the aquifer. It was assumed that any surface runoff and shallow recharge occurring within these sub-basins moves either toward upland tributaries (sub-basins SB1 to SB8) and flows onto the aquifer, or to gullies and low topographic areas (sub-basins SB9 to SB18) and infiltrates at the aquifer-moraine contact.

The entire glacial-outwash aquifer covers $16.7 \mathrm{~km}^{2}$ and receives recharge from $53.7 \mathrm{~km}^{2}$ of channeled uplands and $10.5 \mathrm{~km}^{2}$ of unchanneled uplands. For models 1 and 2 , recharge from the uplands was adjusted to reflect the portion of the channeled and unchanneled upland flow captured by each particular model domain.

\section{Upland Runoff Available for Infiltration}

Runoff rates from the eastern and western uplands were estimated from streamflow measurements at the gauging station located on the eastern tributary. The gauging station was located at the aquifer-moraine contact, located $\sim 450 \mathrm{~m}$ up stream of the confluence with Tricks Creek, and the catchment above this station drains an area of $\sim 7.8 \mathrm{~km}^{2}$. Within this catchment the tributary cuts into the till and receives overland flow and shallow subsurface flow from the till moraine. The average upland surface runoff measured at this station between 1993 and 1997 was $9.9 \times 10^{-2} \mathrm{~m}^{3} \mathrm{~s}^{-1}\left(1.27 \times 10^{-2} \mathrm{~m}^{3} \mathrm{~s}^{-1}\right.$ 
$\mathrm{km}^{-2}$ or $400 \mathrm{~mm} \mathrm{yr}^{-1} \mathrm{~m}^{-2}$ ) and is equal to the sum of the rejected recharge minus evapotranspiration. This represents the amount of runoff available for infiltration to the outwash through either channeled or unchanneled flow from the upland till. While this is slightly less than estimates of runoff from uplands in the glaciated Northeastern United States (Randall and Johnson, 1988) which range from $1.64 \times 10^{-2}$ to $2.73 \times 10^{-2} \mathrm{~m}^{3} \mathrm{~s}^{-1} \mathrm{~km}^{-2}$ (517 to $862 \mathrm{~mm} \mathrm{yr}^{-1}$ ), the slopes of the uplands at this site are much smaller and may not promote as much runoff.

\section{Channeled upland flow}

Recharge of channeled flow originating on the uplands was simulated using two approaches. Where the tributary is intermittent across the aquifer, water level measurements show that the water table is generally $1.5 \mathrm{~m}$ below the streambed. Upland runoff from storm flows will infiltrate through the streambed as it moves across the aquifer. Williams and Morrissey (1996) report that upland tributaries loose between $2.79 \times 10^{-5}$ and $1.39 \times 10^{-4} \mathrm{~m}^{3} \mathrm{~s}^{-1}$ per $\mathrm{m}$ of stream reach on outwash aquifers in Northeastern United States. Therefore, for tributaries that originate on the uplands and flow onto the outwash aquifer, and provided the water table is below the base of the stream, the model applied a specified recharge of $10^{-4} \mathrm{~m}^{3} \mathrm{~s}^{-1}$ per $\mathrm{m}$ of stream reach at the stream nodes. For this portion of the stream, a water balance was maintained as water flows along the stream reach. When all the overland flow has infiltrated, the flux was set to zero. For the remainder of the tributary, the stream is simulated as a head-dependent flux boundary. Where the eastern tributary to Tricks Creek flows on to the outwash, the water table is near the ground surface and at the confluence with Tricks Creek the channel is a groundwater discharge zone. Channeled flow in this tributary has little opportunity to infiltrate and was not included as a potential source of recharge in the models.

\section{Unchanneled upland flow}

Unchanneled flow from the upland till moraines occurs as (1) overland flow during spring snowmelt and large precipitation events, and (2) shallow flow through the 
weathered till and, where present, through tile drains. Saturated lateral flow in the unweathered till is negligible given the downward vertical hydraulic gradients in the till are much larger than the lateral gradients. The estimate of channeled runoff from the uplands (400 $\mathrm{mm} \mathrm{yr}^{-1} \mathrm{~m}^{-2}$ ) was assumed to be similar to the maximum potential unchanneled runoff. Recharge from unchanneled runoff in similar hydrogeologic settings in the Northeastern United States ranges from 4 to $40 \%$ of the total recharge to the aquifer (Williams and Morrissey, 1996). The recharge from each sub-basin was apportioned to the first two rows of nodes on the top of the flow grid and along the lateral boundary of the model domain corresponding to the aquifer-moraine contact.

\section{Direct Infiltration}

During precipitation events little or no overland flow was observed across the outwash aquifer. Therefore, it was assumed that almost all precipitation that is not lost to evapotranspiration, recharges the aquifer. Recharge from direct precipitation on the aquifer was applied as a specified flux to the top layer of nodes of the flow grid. From a water balance, the direct recharge to the aquifer was estimated to be $460 \mathrm{~mm} \mathrm{yr}^{-1}$, or $\sim 40 \%$ of the average-annual precipitation (Appendix E). For transient flow simulations, recharge was estimated by adjusting the daily precipitation (Fig. A1-2a, Appendix A) by the daily evapotranspiration estimated using the FAO56-PM combination method (Allen et al., 1998) described in Appendix E. For both the steady-state and transient flow simulations the applied recharge was spatially uniform across the model domain.

The discharge measured at TR-Q provides a constraint on the total recharge to the aquifer. The total recharge to the catchment above this gauging station $\left(23.96 \mathrm{~km}^{2}\right)$ from direct infiltration, plus channeled and unchanneled runoff, cannot exceed the baseflow discharge plus the vertical flux to the underlying till. At TR-Q, the average and median baseflow between 1993 and 1997 are 0.28 and $0.23 \mathrm{~m}^{3} \mathrm{~s}^{-1}$, respectively, and annually baseflow ranges between 0.10 and $2.9 \mathrm{~m}^{3} \mathrm{~s}^{-1}$ (Appendix E2).

\section{Thermal boundary conditions}

The model requires specification of the thermal boundaries on the six external faces of 
the model domain. For models 1 and 2, the lateral boundaries were treated as thermally insulated boundaries (i.e., no heat flux across these boundaries). For subdomain models 3 and 4, the lateral boundaries were specified temperatures determined during the transient simulations of model 2 and model 3, respectively. The bottom boundary was assigned a specified temperature of $9^{\circ} \mathrm{C}$ for all four models. This temperature was based on measurements in bedrock wells in the study area (Appendix F).

The transfer of energy across the air-ground interface and the resulting heat flux into the ground is combination of surface energy flux components (Brutsaert, 1982) given by

$$
G=L_{e} E+H+R_{n}-A_{h}
$$

where $G \quad$ is the specific energy flux across the air-ground interface $\left[\mathrm{M} \mathrm{T}^{-3}\right]$;

$L_{\mathrm{e}} E \quad$ is latent heat flux $\left[\mathrm{M} \mathrm{T}^{-3}\right]$;

$H \quad$ is sensible and latent heat flux $\left[\mathrm{M} \mathrm{T}^{-3}\right]$;

$R_{\mathrm{n}} \quad$ is the shortwave and longwave radiation $\left[\mathrm{M} \mathrm{T}^{-3}\right]$, and

$A_{\mathrm{h}} \quad$ is the energy advection into the subsurface $\left[\mathrm{M} \mathrm{T}^{-3}\right]$.

For many practical purposes several of these terms can be ignored yielding a simplified energy balance at the ground surface (i.e., Brutsaert, 1982; Chung and Horton, 1987). In Heatflow a common approach is implemented whereby the top boundary was treated as a third-type Cauchy boundary condition (Appendix B). The convective heat flux is assumed to be linearly proportional to the temperature gradient across the ground surface and thermal energy transfer into the domain from recharge is considered (Carslaw and Jaeger, 1959; Andrews, 1978). In Heatflow this boundary condition takes the form

$$
\lambda_{z z}^{u}\left(\frac{\partial T}{\partial z}\right)=\left(\gamma_{G}+q_{R} c_{w} \rho_{w}\right)\left(T_{A}-T\right) \quad \text { on top surface }
$$

where $\lambda_{z z}^{u} \quad$ is the thermal conductivity of the unsaturated zone $\left[\mathrm{M} \mathrm{L} \mathrm{T}^{-3} \Theta^{-1}\right]$;

$\gamma_{\mathrm{G}}$ is the linear heat transfer coefficient at the ground surface $\left[\mathrm{M} \mathrm{T}^{-3} \Theta^{-1}\right]$; 
$q_{\mathrm{R}} \quad$ is the surface recharge flux rate $\left[\mathrm{L} \mathrm{T}^{-1}\right]$;

$c_{\mathrm{w}} \quad$ is the specific heat capacity of water $\left[\mathrm{L}^{2} \mathrm{~T}^{-2} \Theta^{-1}\right]$;

$\rho_{\mathrm{w}} \quad$ is the density of water $\left[\mathrm{M} \mathrm{L}^{-3}\right]$;

$T_{\mathrm{A}} \quad$ is the known atmospheric temperature $[\Theta]$, and

$T \quad$ is the unknown surface temperature within the subsurface $[\Theta]$.

The linear heat transfer coefficient is defined as

$$
\gamma_{G}=\frac{\lambda_{G}}{b_{G}},
$$

where $\lambda_{\mathrm{G}} \quad$ is the thermal conductivity of the transition layer $\left[\mathrm{M} \mathrm{L} \mathrm{T}^{-3} \Theta^{-1}\right.$, and

$b_{\mathrm{G}} \quad$ is the thickness of the transition layer [L].

From Eq. (3.8), the surface heat flux depends on the relative temperature difference at the air-ground surface interface and requires transient air temperature as an input. The air temperature is not a good predictor of the near-surface soil temperature especially during the winter when the insulating effect from the snow cover decouples the soil temperature from the air temperature (Markle and Schincariol, 2007). Therefore, the transient temperature measured $0.02 \mathrm{~m}$ bgs was used in this study (Fig. 3-9). The linear heat transfer coefficient was treated as a fitting parameter and was varied during calibration. The time varying temperature at the top boundary is given by

$$
T(t)=f(t), \quad f(t)= \begin{cases}T_{A V G}+T_{A M P} \sin \left[\frac{2 \pi}{\omega}(t+\varphi)\right], & T(t) \geq T_{M I N} \\ T_{M I N}, & T(t)<T_{M I N}\end{cases}
$$

where $t \quad$ is time [T];

$T_{\mathrm{AVG}}$ is average annual temperature at the top boundary $[\Theta]$;

$T_{\mathrm{AMP}}$ is the amplitude of the annual temperature $[\Theta]$;

given by $T_{M A X}-T_{A V G}$;

$T_{\mathrm{MIN}}$ is the minimum temperature $[\Theta]$; 
$\omega \quad$ is the frequency $\left[\mathrm{T}^{-1}\right]$, and

$\varphi \quad$ is the phase shift [T].

Fitting Eq. (3.9) to the measured average daily temperature at the ground surface (Fig. 39) yields values of $6^{\circ} \mathrm{C}, 20^{\circ} \mathrm{C}, 0^{\circ} \mathrm{C}, 365$ days and -138 days for $T_{\mathrm{AVG}}, T_{\mathrm{AMP}}, T_{\mathrm{MIN}}, \omega$ and $\varphi$, respectively. It is important to noted that $T_{\mathrm{AVG}}$ is not the average surface temperature, which is $10.9^{\circ} \mathrm{C}$ (Markle and Schincariol, 2007), but is the average value of the fitted sinusoidal function including that portion of the function below $T_{\mathrm{MIN}}$.

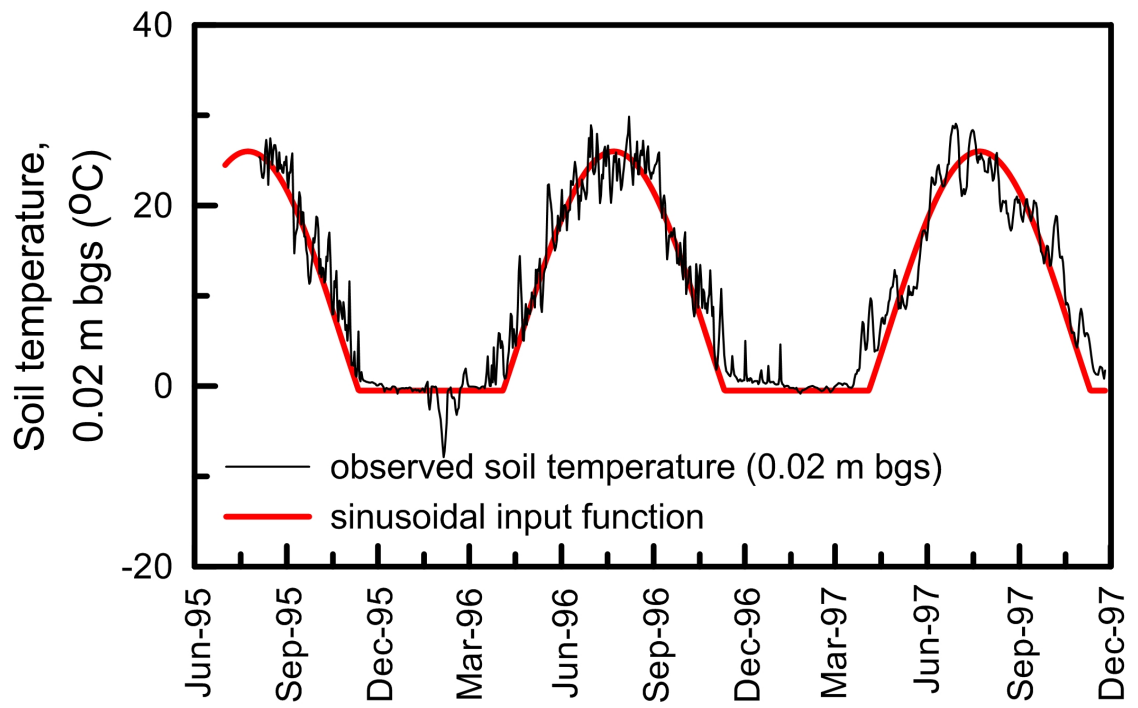

Figure 3-9 Comparison of observed mean daily surface temperature at $0.02 \mathrm{~m}$ bgs with simplified model input sinusoidal temperature function.

Heatflow does not account for open-water flow and thermal energy transport in surface water bodies such as the ponds considered in this research. As previously discussed, flow through these features was approximated using the high $K$ approach. Similarly, thermal energy transport must be approximated. Following the high $K$ approach, we explored a high thermal conductivity method which involved increasing the thermal conductivity of the pond elements and altering $\gamma_{G}$ above the pond surface. This approach proved to be unsuccessful as an acceptable match to the observed pond temperatures could not be obtained. Therefore, we specified the temperatures at the pond 
nodes using Eq. (3.9) after $T_{\mathrm{AVG}}, T_{\mathrm{AMP}}, T_{\mathrm{MIN}}$, and $\varphi$ were modified to account for the vertical variation in temperature (thermal stratification) observed within the pond. From the observed pond temperature (Fig. 3-10), the following functions were developed to account for the vertical variation in the pond temperature

$$
\begin{aligned}
& T_{A M P}\left(z^{\prime}\right)=T_{A M P}(0)-0.6414 z^{\prime} \\
& T_{A V G}\left(z^{\prime}\right)=T_{A V G}(0)-0.3112 z^{\prime} \\
& T_{M I N}\left(z^{\prime}\right)=T_{M I N}(0)+0.2321 z^{\prime} \\
& \varphi\left(z^{\prime}\right)=\varphi(0)-0.4592 z^{\prime}
\end{aligned}
$$

where $z$

is 0 at the pond surface and is positive downwards;

$$
\begin{array}{ll}
T_{\mathrm{AMP}}(0) & \text { is } 14.1^{\circ} \mathrm{C} ; \\
T_{\mathrm{AVG}}(0) & \text { is } 11.4^{\circ} \mathrm{C} ; \\
T_{\mathrm{MIN}}(0) & \text { is } 3.2^{\circ} \mathrm{C} \text {; and } \\
\varphi(0) & \text { is }-121.05 \text { days. }
\end{array}
$$

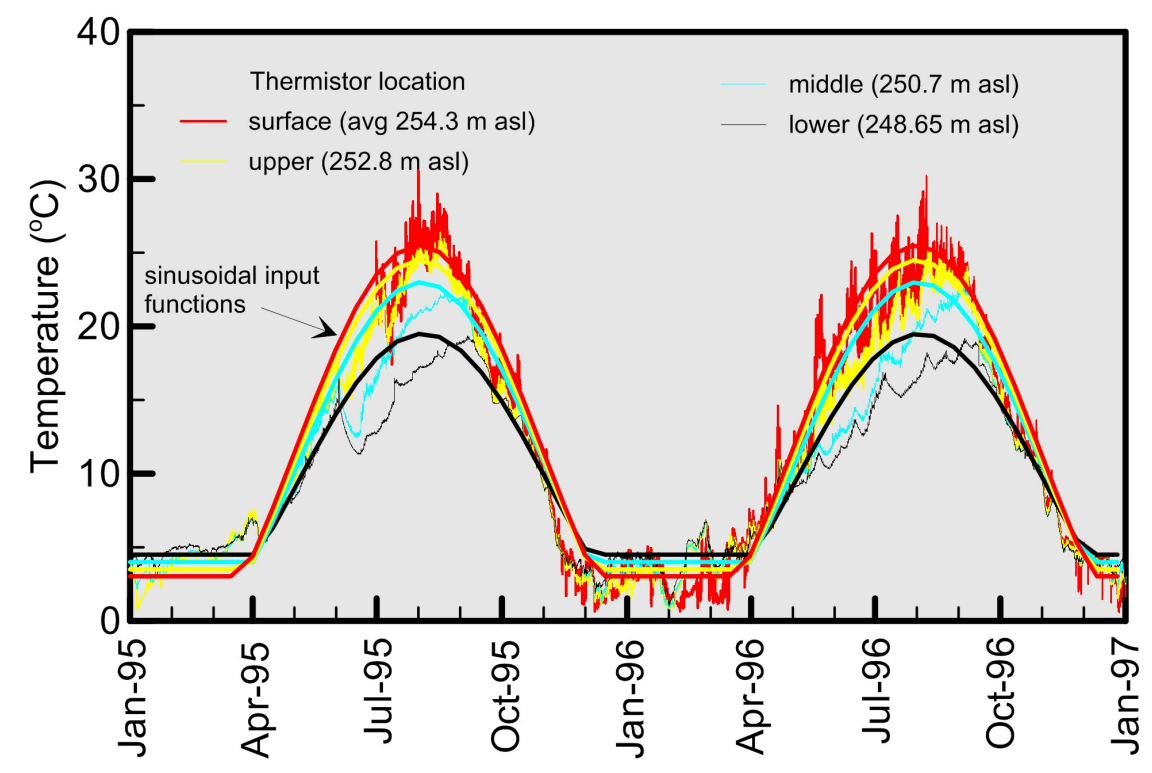

Figure 3-10 Comparison of observed pond temperatures with simplified model input sinusoidal temperature functions (smooth lines). 


\section{Thermal properties of the glacial outwash and till}

The thermal properties of the outwash sand and gravel, and the till were reported previously (Markle et al., 2006). The apparent thermal conductivity, $\lambda$, for the saturated sand and gravel outwash was set at $2.42 \mathrm{~W} \mathrm{~m}^{-1} \mathrm{~K}^{-1}$. In the unsaturated zone the $\lambda$ varied from $2.42 \mathrm{~W} \mathrm{~m}^{-1} \mathrm{~K}^{-1}$ at the water table to $1.7 \mathrm{~W} \mathrm{~m}^{-1} \mathrm{~K}^{-1}$ at the ground surface. $\lambda$ in the till was $1.9 \mathrm{~W} \mathrm{~m}^{-1} \mathrm{~K}^{-1}$. The porosity in the outwash was $0.29 \mathrm{~m}^{3} \mathrm{~m}^{-3}$ and the porosity of the till was set to $0.4 \mathrm{~m}^{3} \mathrm{~m}^{-3}$, typical of tills in this area. The solid matrix specific heat and matrix density were estimated from the mineral composition (Markle et al., 2006). The solid matrix specific heat for both the outwash and the till was $805 \mathrm{~J} \mathrm{~kg}^{-1}{ }^{\circ} \mathrm{C}^{-1}$, and the solid matrix density was 2760 and $2765 \mathrm{~kg} \mathrm{~m}^{-3}$, respectively.

\section{Initial Conditions}

\section{Steady-State and Transient Groundwater Flow Models}

For the steady-state flow simulations with model 1, the initial hydraulic head was set to $252 \mathrm{~m}$ asl, which is the average head measured across the model domain. A spatiallyuniform steady-state recharge rate of $1.46 \times 10^{-8} \mathrm{~m} \mathrm{~s}^{-1} \mathrm{~m}^{-2}\left(460 \mathrm{~mm} \mathrm{yr}^{-1}\right)$, equal to the average annual recharge, was applied to the ground surface and the water table was allowed to deform until the solution converged to the steady-state flow solution. As these were steady-state simulations, a uniform temperature of $9^{\circ} \mathrm{C}$ was assign throughout the model domain and the temperature at the top and bottom boundaries was fixed at $9^{\circ} \mathrm{C}$. These results were then used as the initial conditions for the watershed scale (model 2) steady-state groundwater flow simulations, and subsequent transient groundwater flow and transport simulations.

For the transient groundwater flow and transport simulations with model 2, a uniform temperature of $9^{\circ} \mathrm{C}$ was assign throughout the model domain as an initial condition. A sinusoidal time-varying temperature (Eq. 3.6) was specified uniformly across the ground surface. For the sub-grid scale flow and transport model the initial conditions were taken directly from the watershed scale simulation. 


\section{Transient Transport}

The steady-state flow simulation with model 2 had an initial uniform $9^{\circ} \mathrm{C}$ temperature distribution. This uniform temperature distribution does not reflect the true subsurface temperature profile established in response to the daily and annual temperature fluctuations at the ground surface, and is a poor initial condition for the temperature distribution in transient flow and transport simulations. To establish a realistic subsurface temperature profile for use as an initial condition, the transient version of model 2 was run for several years until a quasi steady-state temperature profile was reached. A quasi steady-state temperature was judged to be reached when the temperature and head differences on a specified day between subsequent years was less than $0.01^{\circ} \mathrm{C}$ and 0.01 $\mathrm{m}$, respectively. The results of these simulations were then used as the initial condition in calibration runs for model 2.

\section{Model Calibration and Validation}

\section{Calibration procedure}

Water levels measured between October 1995 and October 1996 in 20 monitoring wells and 3 surface water monitoring points, and stream discharge measured at TR-Q, were used during calibration (Fig. 3-11). Averages of these water levels were used in the calibration of the steady-state flow model (Table G-1). The transient flow model was calibrated to transient water levels during the same period. These data included water levels measured at 30-minute intervals with pressure transducers and data loggers at three locations. Since the focus of this study was on the Tricks Creek watershed, water level data within the Bridgewater Creek watershed were not collected. However, water level data are available for several monitoring wells and surface water monitoring points at the landfill site in the area where the aquifer pinches out (CRA, 2006). Data from one monitoring well and one surface water monitoring station at the landfill were used to constrain the steady-state groundwater flow model in this area. 


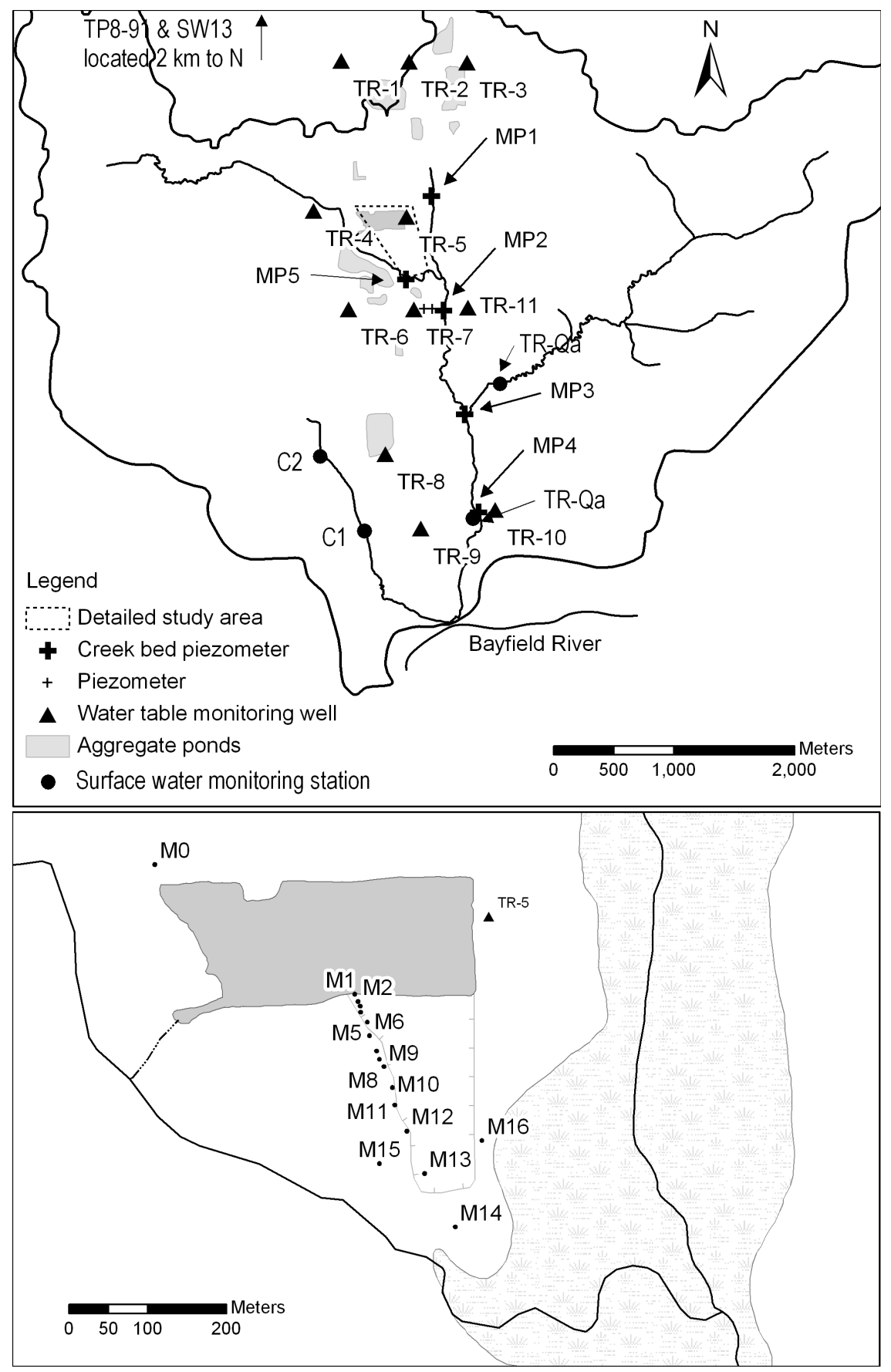

Figure 3-11 Monitoring locations used for model calibration. Monitoring locations across the watershed are shown at the top and monitoring wells in detailed study area are shown at the bottom.

Each of the steady-state flow models was calibrated with the assistance of PEST (Doherty, 2005) as well the trial and study approach. The aquifer hydraulic conductivity 
and streambed hydraulic conductivity were varied until an acceptable match was obtained to measured hydraulic heads, hydraulic gradients and stream discharge rates. One option when calibrating a groundwater flow model is to apply a high degree of spatial variability to the hydraulic conductivity. While providing an excellent fit to the field observations, this most often results in a highly parameterized model for which the resulting parameterization is non-unique, and the predictive capabilities of the model are poor (Burham and Anderson, 2002; Oreskes, 2003; Hill, 2006). Therefore, during calibration, the general philosophy of constructing as simple a model as possible was used (Burham and Anderson, 2002) and the guidance provided by Hill and Tiedeman (2007) and ASTM Guide D 5981-96 (ASTM, 1996), for obtaining a calibrated model, was followed. Complexity to the hydraulic conductivity field was added only where it was required to match the observed head data and could be supported by knowledge of the geology and depositional environment.

As previously noted the glacial outwash sand and gravel was deposited in an outwash channel that flowed from the north to the south. Field observations confirm the downstream fining of the outwash material and support this depositional environment. This information was incorporated into the model, while trying to maintain both model simplicity and model agreement with the known variation in the distribution of hydraulic conductivity along the length of the channel, by using the regularization feature of PEST. Using this method, a regional smoothing constraint was imposed on the hydraulic conductivity to limit the spatial variability of the optimized hydraulic conductivity field. This was achieved by minimizing the difference between neighbouring pairs of hydraulic conductivity values. This imposed a preferred state on the parameter distribution while sacrificing, to some degree, the goodness of the fit to the field hydraulic heads.

For the steady-state simulations, the goodness-of-fit between observed and simulated hydraulic heads was evaluated using the root mean squared error (RMS), or the standard deviation, given by

$$
R M S=\left[\frac{1}{N} \sum_{i=1}^{N}\left(c a l_{i}-o b s_{i}\right)^{2}\right]^{1 / 2}
$$


where $N \quad$ is the number of observation points used in the calibration;

$\mathrm{cal}_{\mathrm{i}} \quad$ is the simulated value; and

$o b s_{\mathrm{i}} \quad$ is the measured or observed value.

For the steady-state flow models a RMS of $0.25 \mathrm{~m}$ (the average standard deviation of the water level measurements) was considered to be acceptable. The magnitude of the errors relative to the overall model response was determined by computing the ratio of the RMS to the total hydraulic head drop over the aquifer. A small ratio indicates the errors in the hydraulic head represent only a small fraction of the overall model response (Anderson and Woessner, 1992). Qualitative measures of the calibration included plotting the observed hydraulic head verses the simulated head.

The transient groundwater flow model and transport model was calibrated to transient water levels measured at 21 monitoring points during this period as well as the measured transient temperatures. For these comparisons, the overall shape of the simulated water levels and temperatures was compared to the measured water levels and subsurface temperatures to assess the calibration. Thus the calibration (goodness-of-fit) was qualitatively evaluated rather than the more formalized quantitative method used for calibration of the steady-state flow models. During the calibration of the transport models some changes in the hydraulic properties were required (i.e., the hydraulic conductivity of the outwash, the stream leakance values). Where change were made in parameters during the calibration of the transport models, these changes were reflected back to the steady-state groundwater flow simulations to ensure calibration was consistent across all simulations.

\section{Final calibrated model parameters}

Steady-state groundwater flow models - models 1 and 2

Table 3-3 summarizes the final parameters for the calibrated models, and the goodness of fit of the observed to simulated hydraulic head data for the steady-state groundwater flow model is shown in Figure 3-12 (see Appendix G for calibration point statistics and locations). The two points identified with the open circle fall into that portion of the 
model domain in the Bridgewater Creek watershed north of the groundwater divide.

The overall agreement between the observed and simulated values is very good (RMS = 0.18). As well the agreement spans the entire range in heads and no regions with poor fit are present that would suggest poor calibration in particular regions of the model domain.

Table 3-3 Parameter values for the calibrated models.

\begin{tabular}{|c|c|c|c|}
\hline \multirow[b]{2}{*}{ Symbol } & \multirow[b]{2}{*}{ Parameter } & \multicolumn{2}{|c|}{ Value } \\
\hline & & Outwash aquifer & Till aquitard \\
\hline & Hydraulic parameters & & \\
\hline$K_{\mathrm{x}}, K_{\mathrm{y}}$ & Hydraulic conductivity $\dagger$ & $\begin{array}{l}8.59 \times 10^{-5} \text { at the Bayfield River to } \\
6.85 \times 10^{-3} \mathrm{~m} \mathrm{~s}^{-1} \text { at the groundwater } \\
\text { divide }\end{array}$ & $1.0 \times 10^{-7} \mathrm{~m} \mathrm{~s}^{-1}$ \\
\hline$K_{\mathrm{z}}$ & Vertical hydraulic conductivity $\dagger$ & $K_{\mathrm{z}} / 5$ & $\begin{array}{l}1.0 \times 10^{-8} \mathrm{~m} \mathrm{~s}^{-1} \text { in the southern } 1 / 2 \text { of } \\
\text { the domain and } 2 \times 10^{-8} \mathrm{~m} \mathrm{~s}^{-1} \text { in the } \\
\text { northern } 1 / 2 \text { of the domain }\end{array}$ \\
\hline$\phi$ & Porosity & 0.29 & 0.40 \\
\hline$S_{\mathrm{Y}}$ & Specific Yield $\dagger$ & $0.09 \ddagger$ & \\
\hline$S_{\mathrm{S}}$ & Specific Storage $\dagger$ & $1.0 \times 10^{-3} \mathrm{~m}^{-1} \ddagger$ & $1.0 \times 10^{-4} \mathrm{~m}^{-1} \ddagger$ \\
\hline \multirow[t]{2}{*}{$\Phi_{S B}=\frac{K_{S B}}{b_{S B}}$} & $\begin{array}{l}\text { Linear leakage coefficient of streambed } \\
\text { layer } \dagger\end{array}$ & $1.0 \times 10^{-7}$ to $5.0 \times 10^{-5} \mathrm{~s}^{-1}$ & \\
\hline & Hydrodynamic dispersion parameters & & \\
\hline$\alpha_{\mathrm{L}}$ & Longitudinal dispersivity & $0.1 \mathrm{~m}$ & $0.1 \mathrm{~m}$ \\
\hline$\alpha_{\mathrm{TH}}$ & Transverse horizontal dispersivity & $0.01 \mathrm{~m}$ & $0.01 \mathrm{~m}$ \\
\hline \multirow[t]{2}{*}{$\alpha_{\mathrm{TV}}$} & Transverse vertical dispersivity & $0.005 \mathrm{~m}$ & $0.005 \mathrm{~m}$ \\
\hline & Thermal Parameters & & \\
\hline$\lambda$ & $\begin{array}{l}\text { Apparent thermal conductivity (saturated } \\
\text { zone) }\end{array}$ & $2.42 \mathrm{~W} \mathrm{~m}^{-1} \mathrm{~K}^{-1}$ & $1.9 \mathrm{~W} \mathrm{~m}^{-1} \mathrm{~K}^{-1}$ \\
\hline$\lambda$ & $\begin{array}{l}\text { Apparent thermal conductivity } \\
\text { (unsaturated zone) } \dagger\end{array}$ & $\begin{array}{l}2.4 \mathrm{~W} \mathrm{~m}^{-1} \mathrm{~K}^{-1} \text { at water table } \\
\text { decreasing to } 1.5 \mathrm{~W} \mathrm{~m}^{-1} \mathrm{~K}^{-1} \text { at the } \\
\text { ground surface }\end{array}$ & \\
\hline$\gamma_{G}=\frac{\lambda_{G}}{b_{G}}$ & $\begin{array}{l}\text { Linear heat transfer coefficient for } \\
\text { ground surface } \dagger\end{array}$ & $\begin{aligned} \lambda_{\mathrm{G}} & =1.5 \mathrm{~W} \mathrm{~m} \mathrm{~W}^{-1} \mathrm{~K}^{-1} \\
b_{\mathrm{G}} & =0.9 \mathrm{~m}\end{aligned}$ & \\
\hline \multirow[t]{2}{*}{$\gamma_{S B}=\frac{\lambda_{S B}}{b_{S B}}$} & $\begin{array}{l}\text { Linear heat transfer coefficient for } \\
\text { streambed }\end{array}$ & $\begin{array}{l}\lambda_{\mathrm{SB}}=2.4 \mathrm{~W} \mathrm{~m}^{-1} \mathrm{~K}^{-1} \\
b_{\mathrm{SB}}=0.1 \mathrm{~m}\end{array}$ & \\
\hline & Matrix and water properties & & \\
\hline$\rho_{\mathrm{S}}$ & Matrix density & $2760 \mathrm{~kg} \mathrm{~m}^{-3}$ & $2765 \mathrm{~kg} \mathrm{~m}^{-3}$ \\
\hline $\mathrm{c}_{\mathrm{S}}$ & Specific heat of solid matrix & $805 \mathrm{~J} \mathrm{~kg}^{-1} \mathrm{~K}^{-1}$ & $805 \mathrm{~J} \mathrm{~kg}^{-1} \mathrm{~K}^{-1}$ \\
\hline$c_{W}$ & Specific heat of water & $4174 \mathrm{~J} \mathrm{~kg}^{-1} \mathrm{~K}^{-1}$ & \\
\hline
\end{tabular}




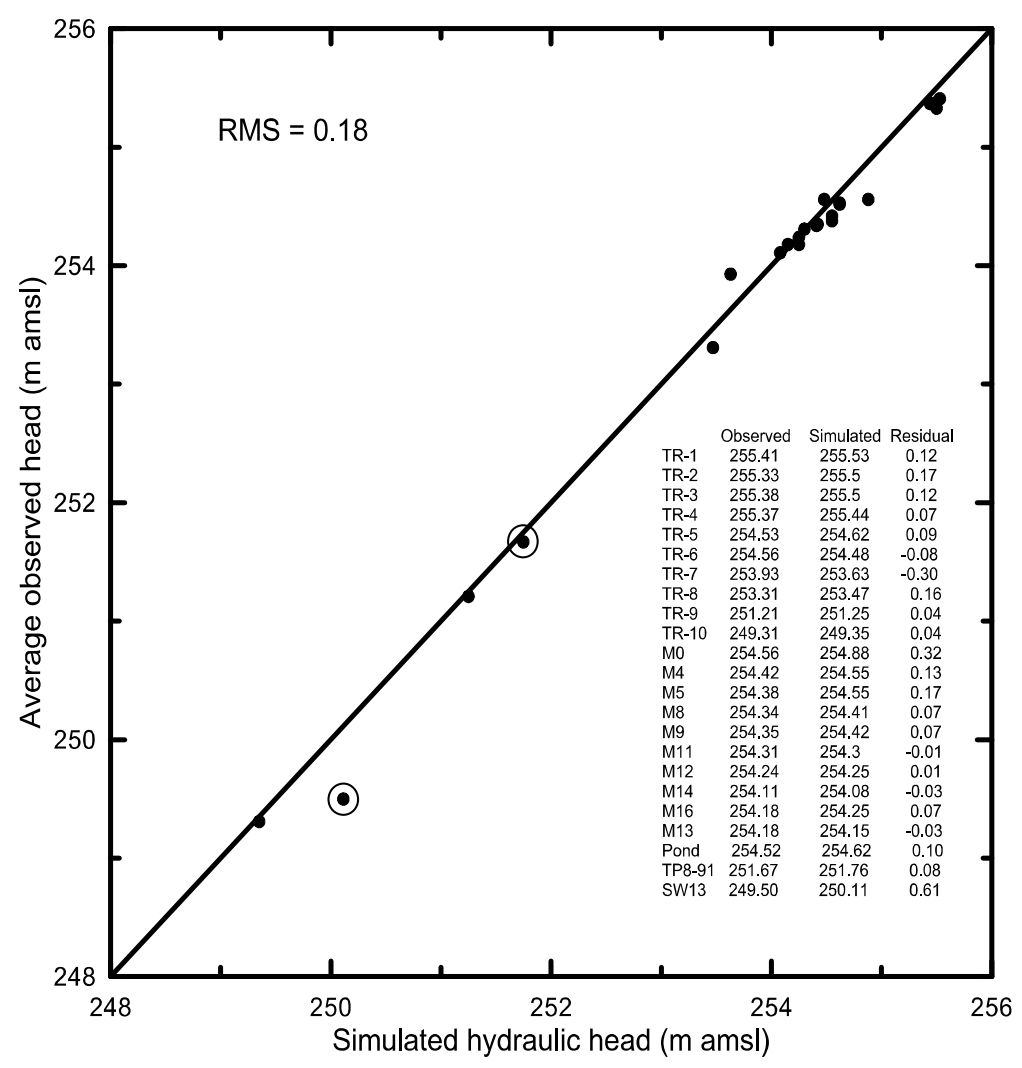

Figure 3-12 Residual plot of the calibrated steady-state groundwater flow model.

Figure 3-13 shows the steady-state water table for the final calibrated model 1 . There is a significant difference between the surface water and groundwater divides. The groundwater divide is located approximately $1200 \mathrm{~m}$ north of the surface water divide. This demonstrates the danger of assuming surface and ground water flow divides always coincide. Independent checks, either through conducting simulations or collecting sufficient water level data, should be completed. 


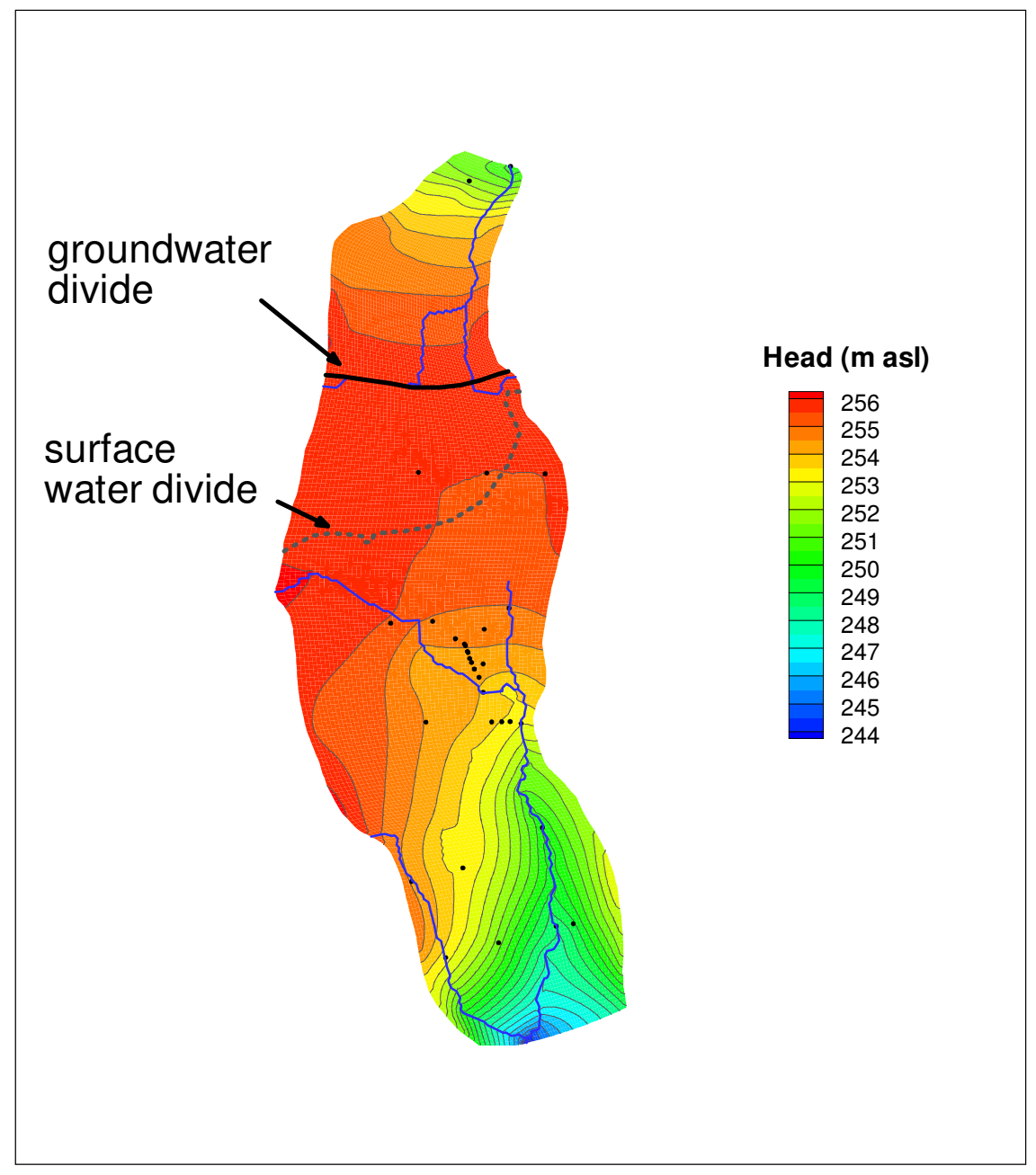

Figure 3-13 3-d domain for steady-state groundwater flow model. RMS $=0.18 \mathrm{~m}$ and RMS/total head drop $=0.02$. Black dots denote the location of calibration points.

The shape and trends in the steady-state water table compare well with the observed water table (Markle and Schincariol, 2007). The influence of Tricks Creek as a discharge zone in the lower portion of the watershed is evident by the groundwater contours. The stream discharge predicted at TR-Q is $0.11 \mathrm{~m}^{3} \mathrm{~s}^{-1}$ which compares well with the range in baseflow of 0.15 to 0.28 estimated from the measured discharge at TR-Q.

For the calibrated model, the hydraulic conductivity $\left(K_{\mathrm{x}}\right.$ and $\left.K_{\mathrm{y}}\right)$ in the glacial outwash aquifer (Fig. 3-14) ranged from $8.59 \times 10^{-5} \mathrm{~m} \mathrm{~s}^{-1}$ at the Bayfield River in the southern end of the domain, to $6.85 \times 10^{-3} \mathrm{~m} \mathrm{~s}^{-1}$ near the groundwater flow divide. From this point to the northern end of the model domain the hydraulic conductivity was 
constant. The vertical hydraulic conductivity, $K_{\mathrm{z}}$, in the outwash aquifer was $K_{\mathrm{x}} / 5$.

Through out the model domain $K_{\mathrm{x}}$ and $K_{\mathrm{y}}$ for the till were $1 \times 10^{-7} \mathrm{~m} \mathrm{~s}^{-1}$ and $K_{\mathrm{z}}$ ranged from $1 \times 10^{-8} \mathrm{~m} \mathrm{~s}^{-1}$, in the southern half of the model domain to $2 \times 10^{-8} \mathrm{~m} \mathrm{~s}^{-1}$ in the northern half.

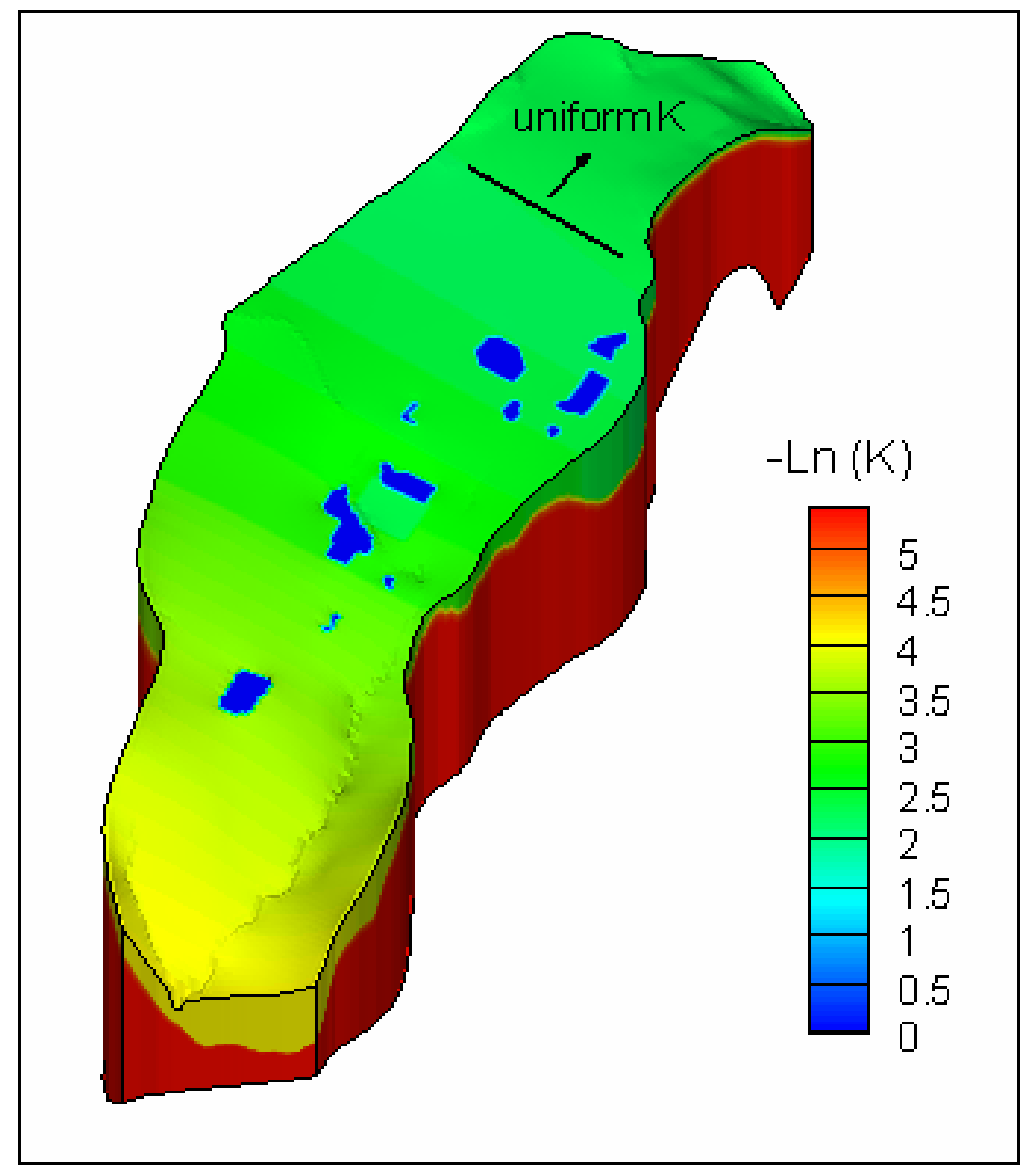

Figure 3-14 Hydraulic conductivity distribution, $-\operatorname{Ln}\left(K_{\mathrm{x}}\right) \mathrm{m} \mathrm{s}^{-1}$, for the final calibrated steady-state groundwater flow model.

The streambed leakance factor $\Phi_{S}$ was adjusted from the initial value of $10^{-4} \mathrm{~s}^{-1}$ during calibration. Final values ranged from $10^{-7}$ to $1.8 \times 10^{-5} \mathrm{~s}^{-1}$ but no clear pattern is evident in the distribution of $\Phi_{S}$. The hydraulic conductivity and $\Phi_{S}$ distribution from model 1 were translated directly into model 2 and yield essentially the same calibration results and they are not repeated here. 


\section{Transient Groundwater Flow Model - Model 2}

The hydraulic properties from the calibrated steady-state flow version of model 2 were used as the basis for the calibration of the transient flow version of model 2. A variable recharge was applied at the ground surface and the specific storage values of $10^{-3} \mathrm{~m}^{-1}$ and $10^{-5} \mathrm{~m}^{-1}$ were assigned to the outwash aquifer and the till, respectively. For the top layer of elements in the flow grid a specific yield of 0.09 was assigned. While a transient surface temperature and transient pond temperature yield a transient flow and transport model, only the hydraulic head from these simulations was considered in the calibration for this model since the Peclet criterion could not be satisfied. The Peclet criterion was satisfied only for model 4 . The calibration results for a select number of monitoring points is shown below (see Appendix G for remaining monitoring points). 

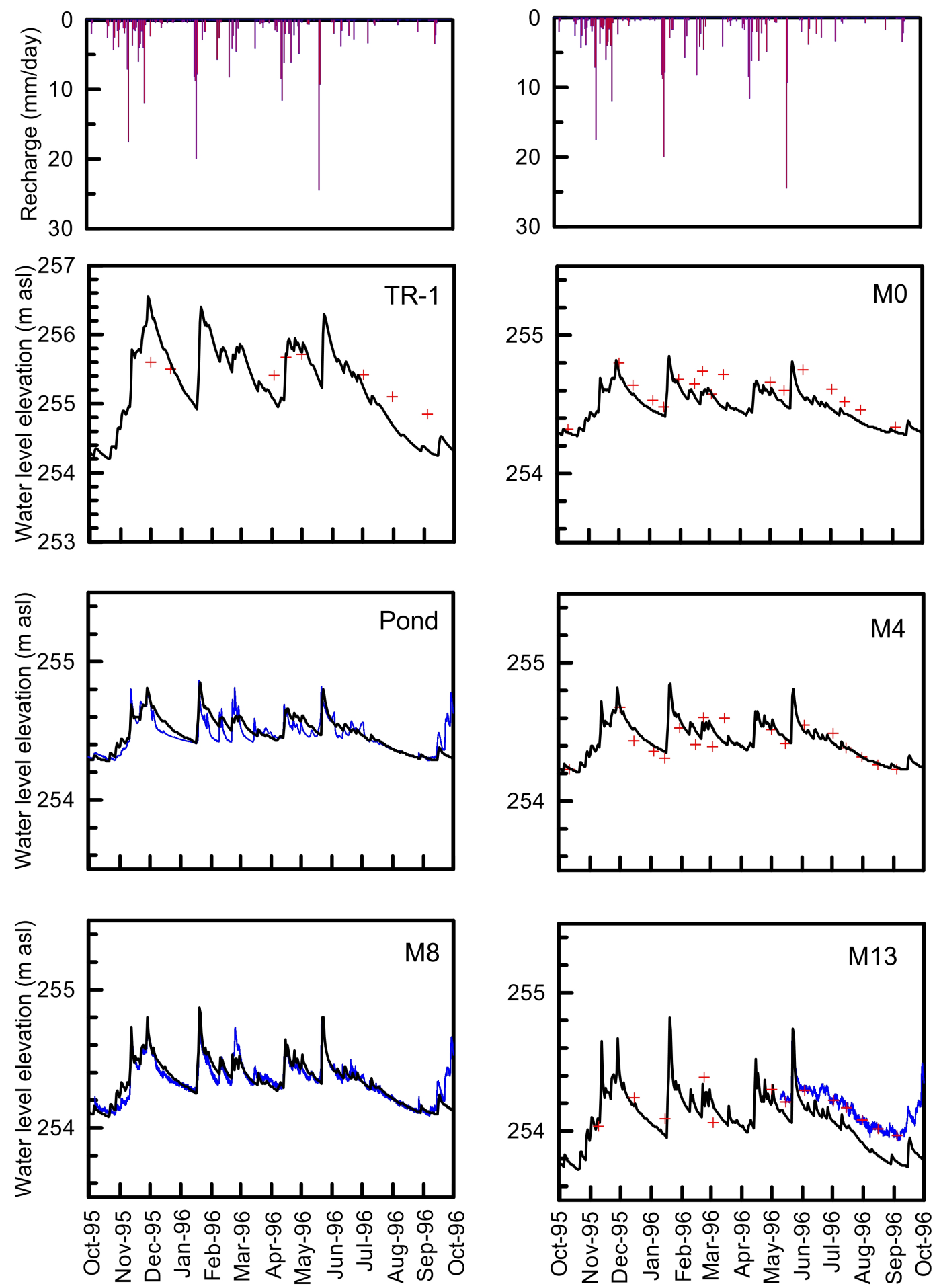

Figure 3-15 Model 2 calibrated transient water levels for selected monitoring wells: TR-1 (northern portion of watershed); M0 to M8 (monitoring points in detailed study area); and TR-9 (southern portion of watershed). The black line is the simulation result, the blue is the observed water level recorded with a pressure transducer, and the red symbols are manual measurements. Daily recharge is shown at the top. 
Within the detailed study area the match to the hydraulic heads is quite good and generally within $0.20 \mathrm{~m}$ of the observed head. The shape of the simulated transient response (water table rise in response to recharge events and subsequent recession) compares well to the observed water levels in the pond and at M0, M4 and M8. In the northern and southern portions of the watershed the matches are not quite as good with difference of up to $0.8 \mathrm{~m}$, but the simulated transient response captures the behaviour of the observed water table response to recharge events. Given the good agreement in the calibration points near the detailed study area, the area of particular interest, these results provide an acceptable match for the watershed scale model.

\section{Transient Groundwater Flow and Transport - Model 3 and Model 4}

Model 3 was extracted as sub-domain from the calibrated transient flow and transport model 2. The boundaries of the sub-domain were set along the north at the southern (down gradient) edge of the Township pit, along the east at the edge of the glacial outwash, along the south approximately $1500 \mathrm{~m}$ down gradient of the pit, and along the west approximately $100 \mathrm{~m}$ west of the pit. Model 4 was, in turn, extracted as a subdomain from Model 3. The northern boundary coincided with that use for Model 3, the eastern boundary was set approximately $25 \mathrm{~m}$ east of Tricks Creek, the southern boundary was set approximately $500 \mathrm{~m}$ down gradient of the pit face and the western boundary coincided with the western edge of the pit. For the sub-domain models these lateral boundaries are determined during the larger scale simulations and read in as specified head boundaries during the sub-domain simulations. The lateral boundaries for the sub-domain models were placed such that their presence would not interfere with the migration of the thermal plume in the detailed study area. As with models 1 and 2, the bottom boundary corresponds to the till-bedrock interface with a specified head corresponding to the potentiometric surface measured in the bedrock across the base of the model domain. Similarly the top boundary is a recharge boundary and corresponds to the transient water table.

The final calibrated groundwater temperatures from Model 4 at the calibration 
points (Fig. 3-16) are compared to the observed temperatures below (Fig. 3-17).

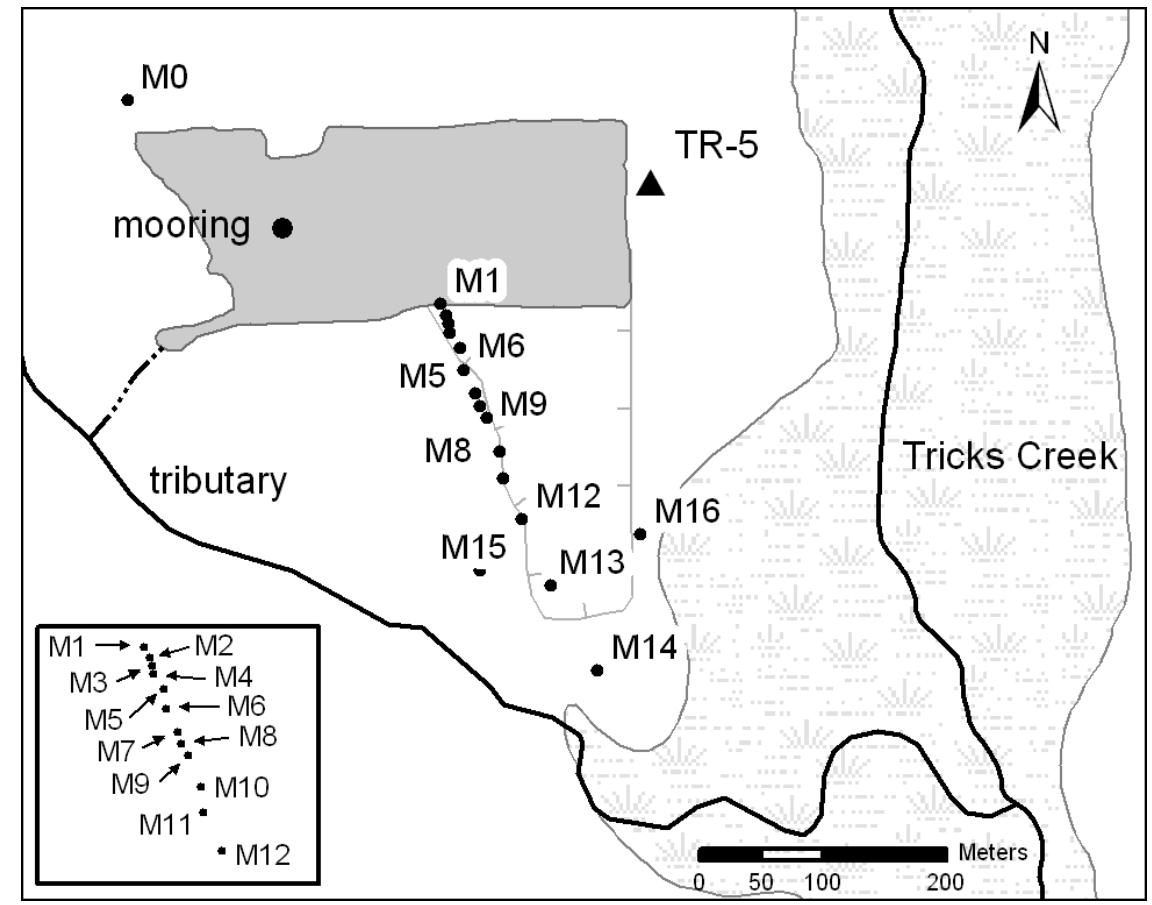

Figure 3-16 Model 4 calibrated locations. 

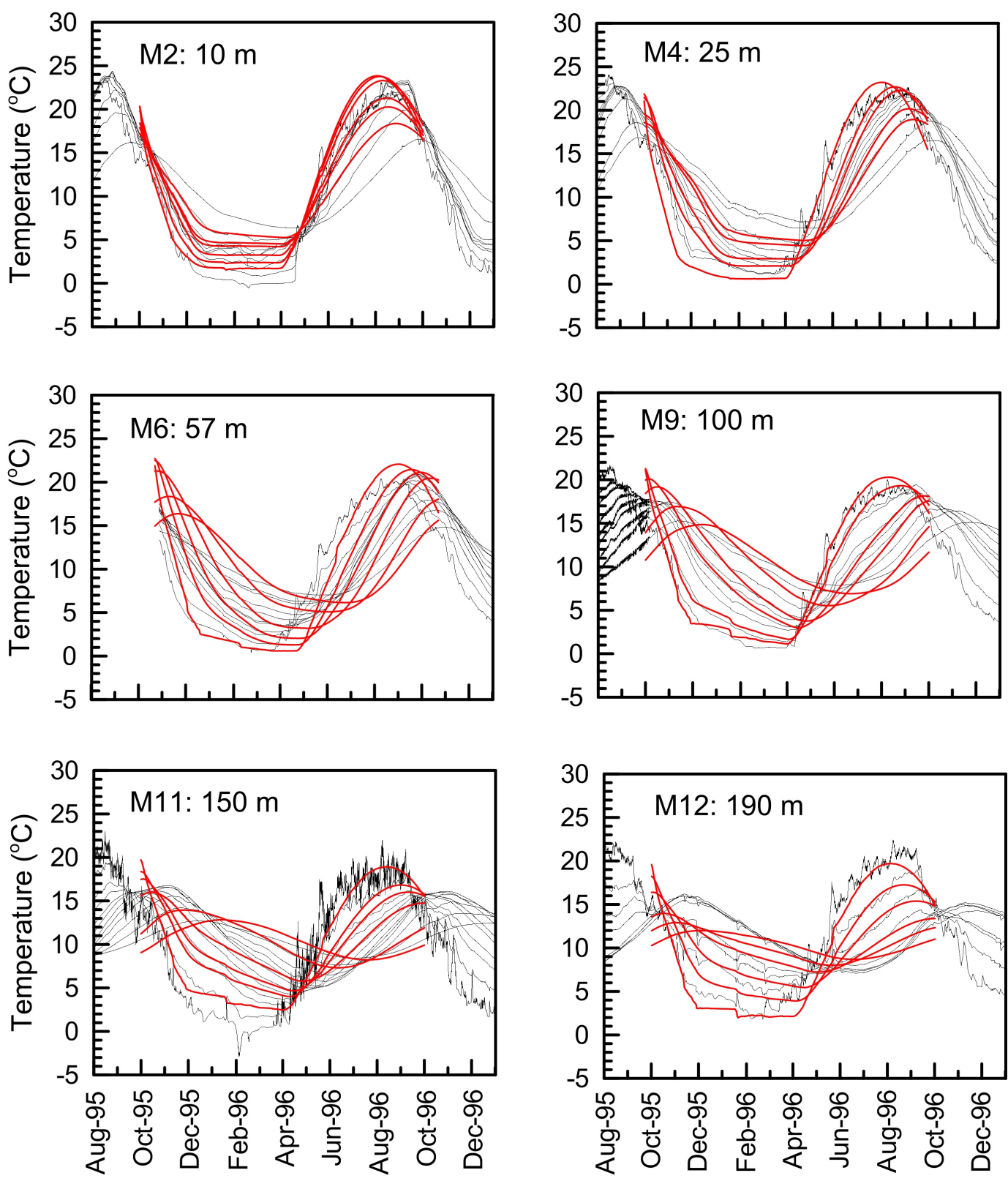

Figure 3-17 Model 4 calibrated transient ground water temperatures for selected monitoring wells down gradient of the pond. The black lines show the observed temperatures and the red show the simulated temperatures. The top left shows the calibration plot for the monitoring well M2 located $10 \mathrm{~m}$ down gradient of the pond and the bottom right shows the calibration plot for a well M12 located $190 \mathrm{~m}$ down gradient.

Reasonable agreement between the observed and the simulated temperatures was obtained. Differences do exist, in particular in the period October to December 1995 where simulated temperatures tend to be higher than observed temperatures. These 
differences may be attributed to the simplified temperature input functions used for the ground surface and the pond which do not capture the observed temperature variations. As well, the simplified temperature functions do not account for the natural variation in temperatures between each year. Rather these simplified functions impose a consistent and uniform temperature variation that is identical from year to year. Overall the nature of the groundwater temperature variation is captured and the arrival times of the peak temperatures are reasonably well predicted. Furthermore, the simulated groundwater velocity in the area down gradient of the pond is $\sim 2.5 \mathrm{~m} \mathrm{~d}^{-1}$ which compares well with the estimated groundwater velocity based on measured hydraulic conductivity and observed plume velocity of $2.8 \mathrm{~m} \mathrm{~d}^{-1}$ (Markle and Schincariol, 2007). Based on these factors we consider the calibration of Model 4 for transient groundwater flow and heat transport to be acceptable.

\section{Model Validation}

Between 1998 and 2000, a significant volume of aggregate was extracted from the southern (down gradient) face of the pit (Fig. 3-18).

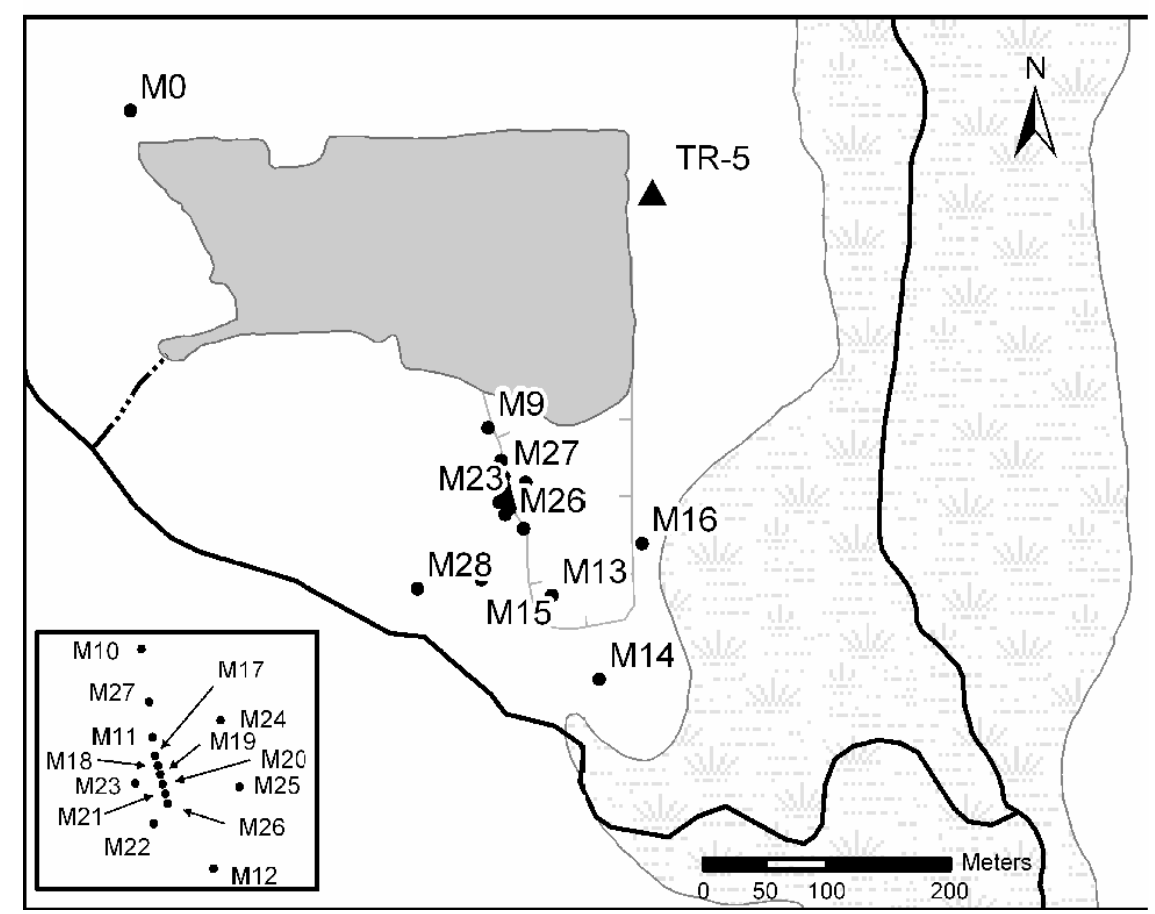

Figure 3-18 Model validation points and the final pond configuration. 
This resulted in the removal of the first $35 \mathrm{~m}$ of the monitoring well network (M1-M8). Subsequently, extraction ceased, and 12 additional wells (M17-M28) were installed and a second detailed monitoring event was completed between 6 June 2001 and 7 January 2003. The data collected during this period was used as a model validation dataset. Model 4 was altered to account for the southerly expansion of the pit and the recharge for the period 1 October 2001 to 1 October 2002 was used to complete a transient simulation. Figure 3-19 shows that the simulated groundwater temperatures also matched the observed temperature for this period well. 

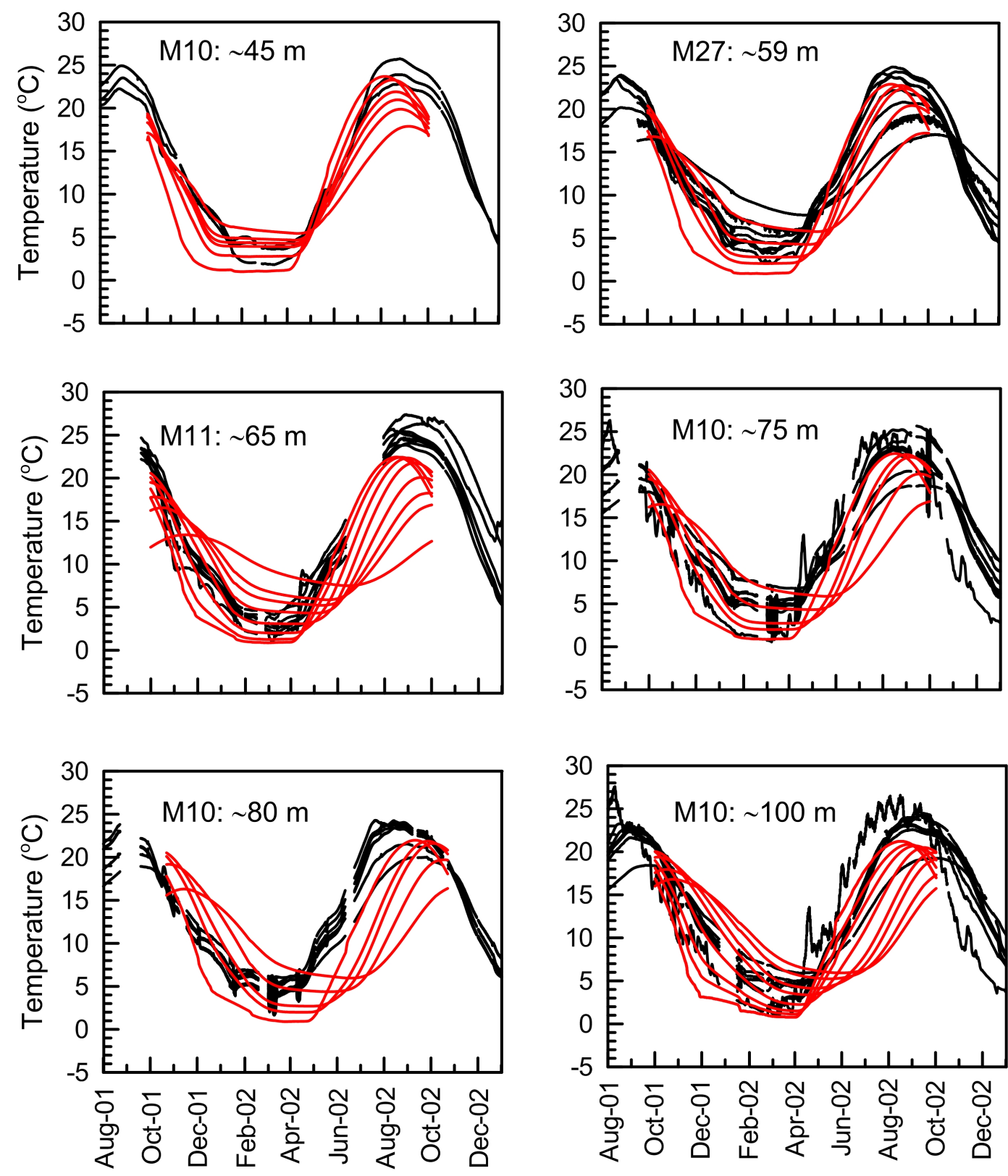

Figure 3-19 Model 4 transient ground water temperatures for the validation simulation at selected monitoring wells down gradient of the expanded pond. The black lines show the observed temperatures and the red show the simulated temperatures. The top left shows the temperature plot for the monitoring well M10 located $\sim 45 \mathrm{~m}$ down gradient of the pond and the bottom right shows the temperature plot for a well M12 located $\sim 100 \mathrm{~m}$ down gradient. 


\section{Results and Discussion}

\section{Influence of Hydraulic Conductivity}

To investigate the influence of hydraulic conductivity (hence groundwater velocity) on the distance of plume movement, simulations were completed for which the calibrated $K$ field was increased by $50 \%$ and decreased by $50 \%$ (Fig. 3-20). Changes of this magnitude are well within the variation seen for hydraulic conductivity and are not so large that the model will go too far out of calibration. It is recognized that hydraulic conductivity changes by a couple orders of magnitude from fine sands to coarse sand and gravel, but changes of this magnitude would result in a model well outside of the calibration range. All other parameters remained the same. For these simulations, the groundwater velocity in the area down gradient for the pond was $0.35 \mathrm{~m} \mathrm{~d}^{-1}(K$ decreased by $50 \%)$ and $3.9 \mathrm{~m}$ $\mathrm{d}^{-1}$ ( $K$ increased by $50 \%$ ) in comparison to $\sim 2.5 \mathrm{~m} \mathrm{~d}^{-1}$ for the calibrated run (base case). At each observation point in Fig. 3-20 responses are shown at points in the upper, middle and lower portions of the aquifer. Comparison of the simulated groundwater temperatures for these two cases shows that, in the upper portion of the aquifer, there are only minor temperature differences. Deeper in the aquifer differences are obvious particularly within the region spanning 25 to $100 \mathrm{~m}$ down gradient of the pond (observation points $\mathrm{M} 2$ to M9). The temperature perturbation from the pond arrives earlier and the peak is larger for the higher $K$ case (increased by $50 \%$ ) within this region due to less thermal dispersion. For the lower K case, the longer travel times provide for more heat transport by conduction and increased thermal dispersion which attenuates the peak height within the plume. In the down gradient direction the influence of the pond decreases (observation points M11 and M12) and has largely disappeared by $190 \mathrm{~m}$. This is in reasonable agreement with the observations that the temperature perturbation of the pond is evident up to $250 \mathrm{~m}$ down gradient of the pond (Markle and Schincariol, 2007). 

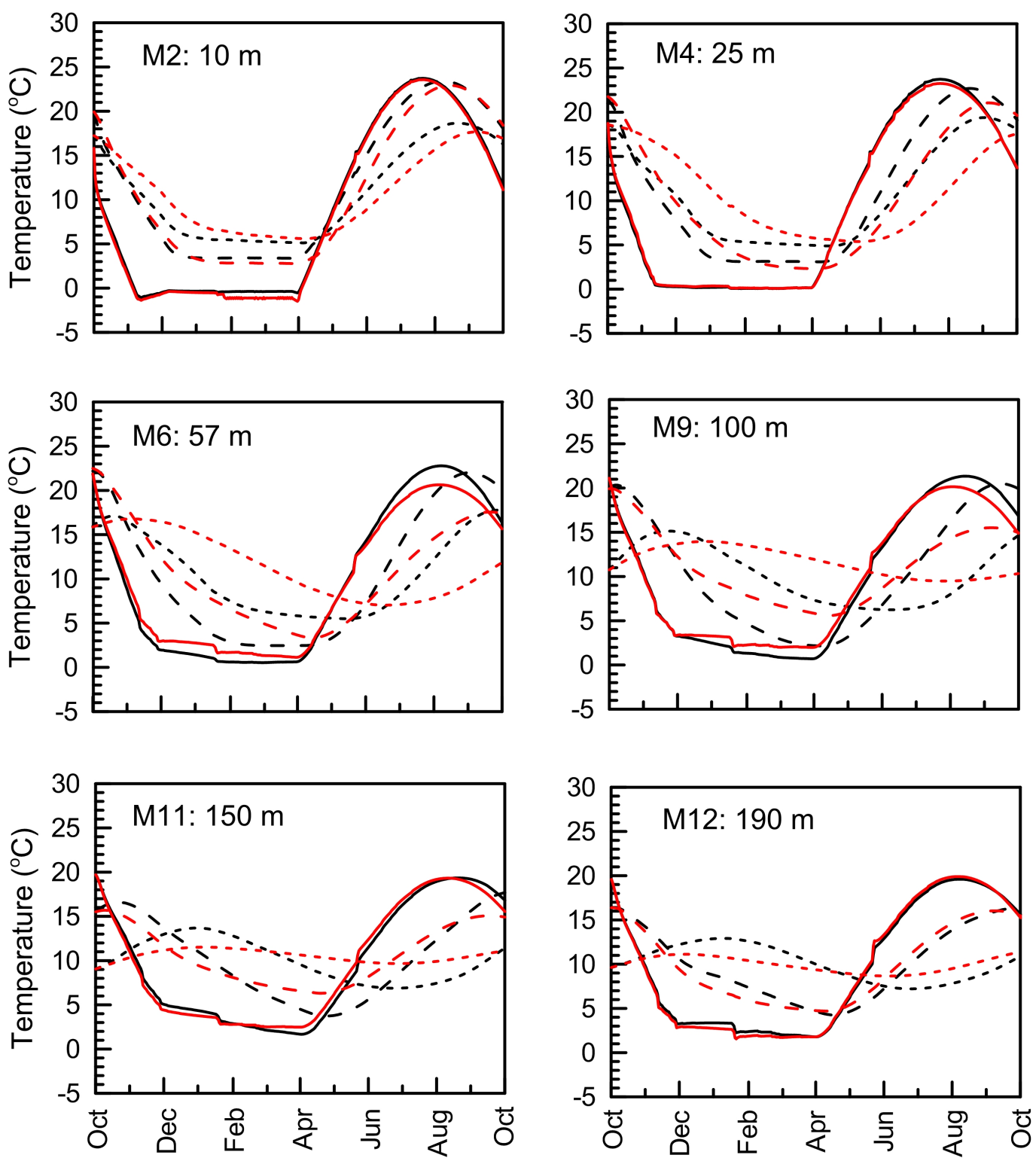

Figure 3-20 Comparison of simulated temperatures for $K$ decreased by $50 \%$ (red lines) and $K$ increased by $50 \%$ (blue lines) at points in the upper (solid line), middle (long dash) and lower (short dash) portions of the aquifer.

\section{Influence of Thermal Conductivity}

To investigate the influence of the apparent thermal conductivity $\lambda$, (hence thermal conduction in the porous medium) on the distance of plume movement, simulations were completed by varying $\lambda\left(2.42 \mathrm{~W} \mathrm{~m}^{-1} \mathrm{~K}^{-1}\right)$ used in the calibrated model. Based on the measured values of $\lambda$ for this aquifer (Markle et al., 2006), and consideration of the range 
of values reported in the literature for porous media ranging from sand to sand and gravel, $\lambda$ was varied by $\pm 10 \%$. Comparison of the simulated groundwater temperatures for these two cases shows no observable temperature differences within the first $60 \mathrm{~m}$ down gradient of the pond (observation points M2 to M6). Only minor differences are present further down gradient at M9 to M12 (Fig. 3-21). For these simulations the groundwater velocity was $\sim 2.5 \mathrm{~m} \mathrm{~d}^{-1}$ and the temperature response in the aquifer is dominated by the convective heat transport. These results suggest that in convection dominated systems, such as those typically present in areas of aggregate extraction, the thermal conductivity of the aquifer material has a minor influence on the subsurface temperature which is controlled mainly by the groundwater velocity and convective heat transport. 

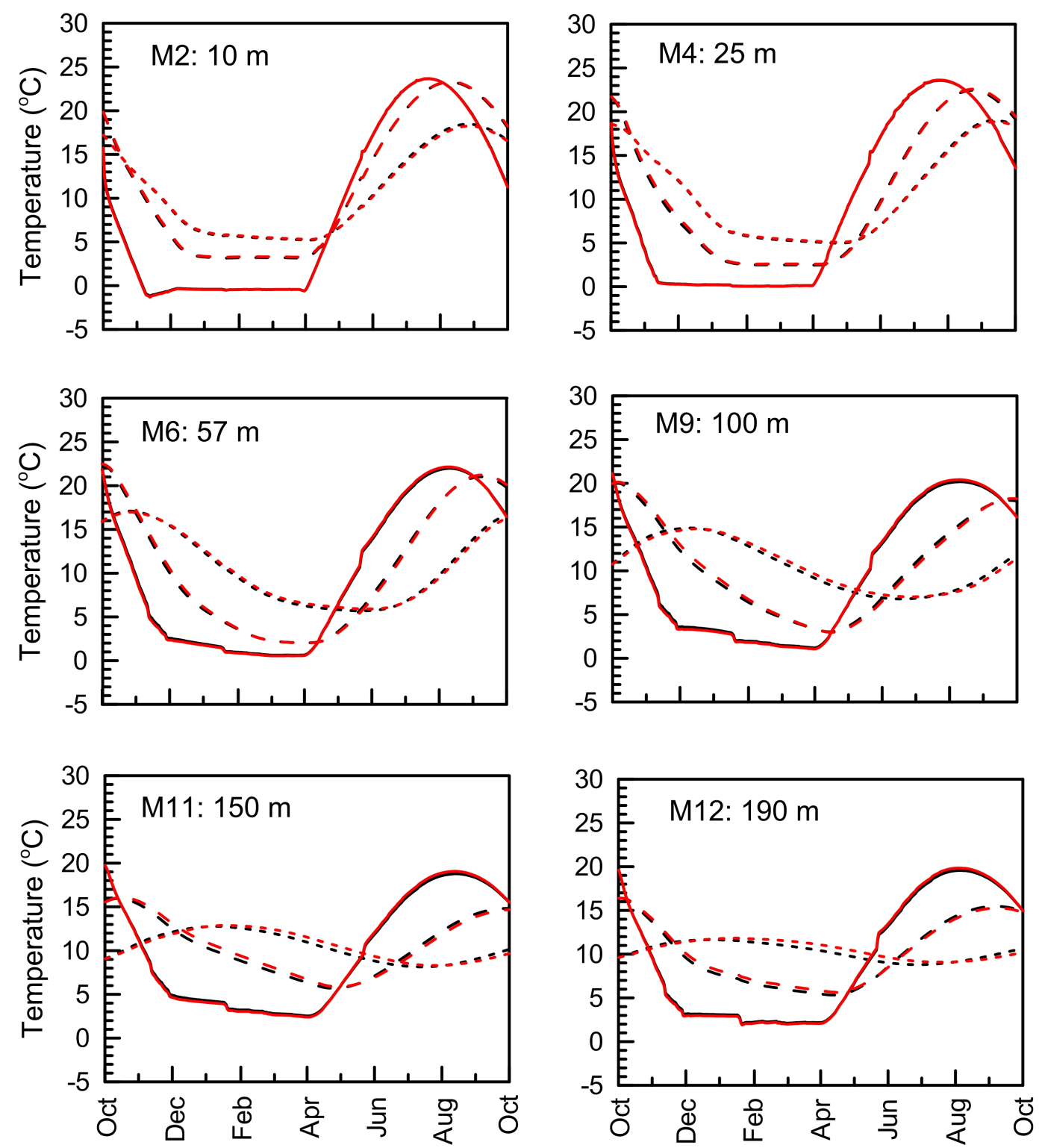

Figure 3-21 Comparison of simulated temperatures for $\lambda=2.18 \mathrm{~W} \mathrm{~m}^{-1} \mathrm{~K}^{-1}$ (red lines) and $\lambda=2.66 \mathrm{~W} \mathrm{~m}^{-1} \mathrm{~K}^{-1}$ (blue lines) at points in the upper (solid line), middle (long dash) and lower (short dash) portions of the aquifer.

\section{Summary and Conclusions}

Three-dimensional groundwater flow and heat transport of thermal plume migration through an unconfined glacial-outwash sand and gravel aquifer was simulated. The groundwater velocities in this outwash aquifer are relatively high $\left(\sim 2.8 \mathrm{~m} \mathrm{~d}^{-1}\right.$ in the study area). Satisfying the grid Peclet criterion thus required a finely discretized grid which 
precluded the completion of heat transport simulations that incorporated the entire outwash aquifer. To maintain reasonable run times and memory requirements it was necessary to adopt a telescopic mesh refinement process starting with a large scale steady-state groundwater flow model and moving to a small scale transient groundwater flow and heat transport model. While approximations were made to incorporate the overland flow to the aquifer from adjacent till uplands, and the temperature in the pond and at the ground surface were approximated by fitting modified sinusoidal functions to measured data, a calibrated groundwater flow and heat transport model was obtained.

Our results indicated that in this outwash aquifer where the groundwater velocity is relatively high, the temperatures in the aquifer within the first $100 \mathrm{~m}$ of the pond are dominated by the convective transport of the temperature perturbation in the pond. Within this zone the annual temperature variation at the ground surface (largely transported by conduction) is masked by that of the pond. Beyond $100 \mathrm{~m}$ the temperature perturbation from the pond is attenuated through thermal retardation and thermal dispersion to the point where the influence of the temperature variation at the ground surface becomes evident in the measured subsurface temperatures. The simulations are in good agreement with the observed subsurface temperatures and support the conclusion that convective groundwater transport from a pond may influence subsurface temperatures well beyond $100 \mathrm{~m}$ down gradient.

These results suggest that in aquifers with larger groundwater velocities (typical of settings with aggregate extraction) significant effort should be focused on obtaining representative estimates of the groundwater velocity (i.e., large scale hydraulic conductivity, porosity and hydraulic gradient) as the groundwater velocity plays a dominant role in the distance of thermal plume transport. While hydraulic conductivity (and hence groundwater velocity) may be obtained by several methods, the groundwater velocities in the calibrated flow and transport model $\left(\sim 2.5 \mathrm{~m} \mathrm{~d}^{-1}\right)$ best match our estimates of groundwater velocity obtained from the observed thermal plume movement (i.e., $\sim 2.5 \mathrm{~m} \mathrm{~d}^{-1}$ ) which is essentially an aquifer tracer test where heat is the tracer. Hydraulic conductivity obtained from a pumping test was a factor of two too large, suggesting care must be taken in selecting the appropriate methods of estimating 
hydraulic conductivity. Thermal conductivity appears to be less important in these settings where convection dominants plume transport and estimates of thermal conductivity based on the measured values presented here and in other studies may be acceptable provided the porous media are similar.

The results from the numerical simulations are in good agreement with the groundwater temperatures measured at this site down gradient of the pit and presented in Chapter 1. The observed temperatures indicate that the thermal plume migrates between 150 and $250 \mathrm{~m}$ down gradient of the pond. The numerical simulations indicate the thermal plume persists for up to $150 \mathrm{~m}$. At this site, the groundwater discharges to streams that are well beyond this distance, and thus are not affected by these thermal plumes. Where pits are within $250 \mathrm{~m}$ of the stream, these results suggest there is the potential for the thermal plumes to alter the temperature of the groundwater discharging to the stream. 


\section{References}

Alexander, M.D., D. Caissie. 2003. Variability and comparison of hyporheic water temperatures and seepage fluxes in a small Atlantic salmon stream. Ground Water 41:72-83.

Andrews, C.B. 1978. The impact of the use of heat pumps on ground water temperatures. Ground Water 16:437-443.

Andrews, C.B., M.P. Anderson. 1979. Impact of a power plant on the groundwater system of a wetland. Ground Water 16:105-111.

Andrews, C.B., M.P. Anderson. 1979. Thermal alteration of groundwater caused by seepage from a cooling lake. Water Resources Research 15:595-602.

Anderson, M.P., R.J. Hunt, J.T. Krohelski, K. Chung. 2002. Using high hydraulic conductivity nodes to simulate seepage lakes. Gound Water 40:119-124.

ASTM International. 1996. Standard guide for calibrating a ground-water flow model application, Designation: D 5981-96 (Reapproved 2002). ASTM Subcommittee D18.21 on Ground Water and Vadose Zone Investigations. ASTM International, West Conshohocken, Pennsylvania.

Barnett, P.J. 1992. Quaternary geology of Ontario. p. 1011-1088. In P.C. Thurston, H. R. Williams, R.H. Sutcliffe, and G.M. Stott (eds.) Geology of Ontario. Ontario Geological Survey, Special Volume 4, Part 2. Publications Ontario, Toronto, Ontario.

Becker, M.W., T. Georgian, H. Ambrose, J. Siniscalchi, K. Fredrick. 2004. Estimating flow and flux of ground water discharge using water temperature and velocity. Journal of Hydrology 296:221-233.

Bredehoeft, S.S. Papadopulos. 1965. Rates of vertical groundwater movement estimated from the earth's thermal profile. Water Resources Research 1:325-328.

Brookfield, A.E., E.A. Sudicky, Y.-J. Park, B. Conant Jr. 2009. Thermal transport modelling in a fully integrated surface/subsurface framework. Hydrological Processes 23:2150-2164.

Brutsaert, W. 1982. Evaporation into the atmosphere. Kluwer Academic Publishers. The Netherlands.

Burnham, K.P., D.R. Anderson. 2002. Model selection and multimodel inference: a practical information-theoretical approach. 2nd ed. Springer-Verlag, New York. 
Campbell, G.S., J.D. Jungbauer, Jr., W.R. Bidlake, R.D. Hungerford. 1994. Predicting the effect of temperature on soil thermal conductivity. Soil Science 158:307-313.

Carslaw, H.S., J.C. Jaeger. Conduction of heat in solids. 2nd ed. Oxford University Press, New York.

Cartwright, K. 1968. Thermal prospecting for ground water. Water Resources Research 4:395-401.

Cartwright, K. 1974. Tracing shallow groundwater systems by soil temperatures. Water Resources Research 10:847-855.

Chung, S.O., R. Horton. 1987. Soil heat and water flow with a partial surface mulch. Water Resources Research 23:2175-2186.

Conant, B. Jr. 2004. Delineating and quantifying ground water discharge zones using streambed temperatures. Ground Water 42:243-257.

Constantz, J. 1998. Interaction between stream temperature, streamflow, and groundwater exchanges in alpine streams. Water Resources Research 34:1609-1615.

Cooper, A.J., W.D. Fitzgerald. 1977. Quaternary geology of the Goderich area, Southern Ontario. Ontario Geological Survey Preliminary Map P. 1232.

Conestoga-Rovers and Associates. March 2006. 2005 Annual Monitoring Report - MidHuron Landfill Site.

Curry R.A., D.A. Scruton, K.D. Clarke. 2002. The thermal regimes of brook trout incubation habitats and evidence of changes during forestry operations. Canadian Journal of Forest Research 32:1200-1207, doi:10.1139/X02-046.

Doherty, J. 2005. PEST Model-independent parameter estimation, Users Manual: 5th ed. Watermark Numerical Computing, Brisbane, Australia.

Evans, E.C., M.T. Greenwood, G.E. Petts. 1995. Thermal profiles within river beds. Hydrological Processes 9:19-25.

Ferguson, G., A.D. Woodbury, G.L.D. Matile. 2003. Estimating deep recharge rates beneath an interlobate moraine using temperature logs. Ground Water 41:640-646.

Ferguson, G., A.D. Woodbury. 2005. Thermal sustainability of groundwater-source cooling in Winnipeg, Manitoba. Canadian Geotechnical Journal 42:1290-1301. 
Hatch, C.E., A.T. Fisher, J.S. Revenaugh, J. Constantz, C. Ruehl. 2006. Quantifying surface water-groundwater interactions using time series analysis of streambed thermal records: Method development. Water Resources Research 42:W10410, doi:10.1029/2005WR004787.

Hill, M.C. 2006. The practical use of simplicity in developing ground water models. Ground Water 44:775-781.

Hill, M.C., C.R. Tiedeman. 2007. Effective groundwater model calibration: with analysis of data, sensitivities, predictions, and uncertainty. John Wiley and Sons, New Jersey. p. 455.

Hunt, R.J., D.P. Krabbenhoft, M.P. Anderson. 1996. Groundwater inflow measurement in wetland systems. Water Resources Research 32:495-507.

Hynes, H.B.N. 1983. Groundwater and stream ecology. Hydrobiologia 100:93-99.

Keery, J., A. Binley, N. Crook, J.W.N. Smith. 2006. Temporal and spatial variability of groundwater-surface water fluxes: Development and application of an analytical method using temperature time series. Journal of Hydrology 336:1-16.

doi:10.1016/j.jhydrol.2006.12.003.

Koteff, C. 1974. The morphologic sequence concept and deglaciation of southern New England, in Coates, D.R., ed., Glacial geomorphology: Binghamton, N.Y., State University of New York, Published in Geomorphology, p. 121-144.

Lapham, W.W. 1987. Use of temperature profiles beneath streams to determine rates of vertical groundwater flow and vertical hydraulic conductivity. U.S. Geological Survey Water-Supply Paper 2337. pp. 34.

Landon, M.K., D.L. Rus, F.E. Harvey. 2001. Comparison of instream methods for measureing hydraulic conductivity in sandy streambeds. Ground Water 39:870885.

Leismann, H.M., E.O. Frind. 1989. A symmetric-matrix time integration scheme for efficient solution of advection-dispersion problems. Water Resources Research 25:1133-1139.

Lu, N., S. Ge. 1996. Effect of horizontal heat and fluid flow on the vertical temperature distribution in a semiconfining layer. Water Resources Research 32:1449-1453.

Markle , J.M., R.A. Schincariol, J.H. Sass, J.W. Molson. 2006. Characterizing the twodimensional thermal conductivity distribution in a sand and gravel aquifer. Soil Science Society of America Journal 70:1281-1294. 
Markle, J.M., R.A. Schincariol. 2007. Thermal plume transport from sand and gravel pits - Potential thermal impacts on cool water streams. Journal of Hydrology 338:174195.

McLaren, R.G. 2007. GridBuilder, A pre-processor for 2-d triangular element, finiteelement programs, Groundwater Simulation Group, University of Waterloo. pp. 83.

Molson, J.W., E.O. Frind, C. Palmer. 1992. Thermal energy storage in an unconfined aquifer 2. Model development, validation and application. Water Resources Research 28:2857-2867.

Molson, J.W., E.O. Frind. 2004. HEATFLOW Version 2.0, Density-dependent flow and thermal energy transport model in three dimensions, User Guide. pp. 77.

Morrissey, D.J., A.D. Randall, J.H. Williams. 1988. Upland runoff as a major source of recharge to stratified drift in the glaciated Northeast, in Regional Aquifer Systems of the United States, Northeast Glacial Aquifers, edited by A.D. Randall and A.I. Johnson. American Water Resources Association, Monograph Series, no. 11:17-36.

Olmsted, F.H., P.A. Glancy, J.R. Harrill, F.E. Rush, A.S. VanDenburgh. 1975. Preliminary hydrogeology appraisal of selected hydrothermal systems in northern and central Nevada. U.S.G.S. Open-File Report 75-56:128-147.

Oreskes, N. 2003. The role of quantitative models in science, in Models in Ecosystem Science, edited by C.D. Canham, J.J. Cole, and W.K. Lauenroth. Princeton University Press. pp. 13-31.

Palmer, C.D., D.W. Blowes, E.O. Frind, J.W. Molson. 1992. Thermal energy storage in an unconfined aquifer 1. Field injection experiment. Water Resources Research 28:2845-2856.

Parsons, M.L. 1970. Groundwater thermal regime in a glacial complex. Water Resources Research 6:1701-1720.

Power, G., R.S. Brown, J.G. Imhof. 1999. Groundwater and fish - insights from northern North America. Hydrological Processes 13:401-422.

Randall, A.D., A.I. Johnson. 1988. The Northeast glacial aquifer RASA project - An overview of results through 1987, in Randall, A.D., and Johnson, A.I. editors, Regional Aquifer Systems of the United States, Northeast Glacial Aquifers. American Water Resources Association, Monograph Series, no. 11:1-15.

Reiter, M. 2001. Using precision temperature logs to estimate horizontal and vertical 
groundwater flow components. Water Resources Research 37:663-674.

Sass, J.H., J.P. Kennelly, Jr., W.E. Wendt, T.H. Moses, Jr., J.P. Ziagos. In-situ determination of heat flow in unconsolidated sediments. Geophysics 46:76-83.

Silliman, S.E., D.F. Booth. 1993. Analysis of time-series measurements of sediment temperature for identification of gaining vs. losing portions of juday Creek, Indiana. Journal of Hydrology 146:131-148.

Silliman, S.E., J. Ramirez, R.L. McCabe. 1995. Quantifying downflow through creek sediments using temperature time series: one-dimensional solution incorporating measured surface temperature. Journal of Hydrology 167:99-119.

Smith, L., D.S. Chapman. 1983. On the thermal effects of groundwater flow 1. Regional scale systems. Journal of Geophysical Research 88:593-608.

Sorey, M.L. 1971. Measurement of vertical groundwater velocity from temperature profiles in wells. Water Resources Research 7:963-970.

Stallman, R.W. 1965. Steady one-dimensional fluid flow in a semi-infinite porous medium with sinusoidal surface temperature. Journal of Geophysical Research 70:2821-2827.

Taniguchi, M. 1994. Estimated recharge rates from groundwater temperatures in the Nara basin, Japan. Applied Hydrogeology 4:7-13.

Therrien, R., R.G. McLaren, E.A. Sudicky, S.M. Panday. 2007. HydroGeoSphere, A three-dimensional numerical model describing fully-integrated subsurface and surface flow and solute transport. Groundwater Simulation Group, University of Waterloo. pp. 347.

Vannote, R.L., B.W. Sweeney. 1980. Geographic analysis of thermal equilibria: A conceptual model for evaluating the effect of natural and modified thermal regimes on aquatic insect communities, The American Naturalist 115:667-695.

Ward, D.S., D.R. Buss, J.W. Mercer, S.S. Hughes. 1987. Evaluation of a groundwater corrective action at the Chem-Dyne hazardous waste site using a telescopic mesh refinement modeling approach. Water Resources Research 23:603-617.

Werner, D., W. Kley. 1977. Problems of heat storage in aquifers. Journal of Hydrology 34:35-43.

Williams, J.H., D.J. Morrissey. 1996. Recharge of valley-fill aquifers in the glaciated Northeast from upland runoff. in Ritchey, J.D., Rumbaugh, J. editors, Subsurface 
fluid flow (Ground-water and vadose zone) modeling, ASTM STP 1288:97-113.

Woodbury, A.D., L. Smith. 1985. On the thermal effects of three-dimensional groundwater flow. Journal of Geophysical Research 90:759-767.

Woessner, W.W. 2000. Stream and fluvial plain ground water interactions: Rescaling hydrogeologic thought. Ground Water 38:423-429. 


\section{Chapter 4}

\section{Conclusions}

The research presented in this dissertation makes original contributions to understanding of the movement of thermal plumes from aggregate pits and to assessing their potential impacts on nearby cool- and cold-water streams. The contributions are:

1) Chapter 1 presents the results of a detailed field investigation. These field results provide evidence of the movement of thermal plumes and is the first comprehensive field investigation of thermal plume movement from aggregate pits. The data collected shows that the thermal plumes do persist for distances exceeding $100 \mathrm{~m}$ and may persist beyond $200 \mathrm{~m}$. The methods employed in this field investigation provide the basis upon which field studies at other locations may be designed. The linkage between the groundwater and stream habitat are discussed and provide a basis for considering what impacts thermal plumes may have on nearby cool- and cold-water streams. Cross-correlation of the pond temperature signal with the measured groundwater temperature signals down gradient of the pond is used to estimate the plume velocity. Furthermore, the thermal plume velocity is shown to lag the average linear groundwater velocity through the thermal retardation factor estimated to be 2.3 in this aquifer.

2) The aquifer hydraulic conductivity is measured at several scales and found the laboratory-measured hydraulic conductivities are up to two-orders of magnitude smaller than field-measured hydraulic conductivities. While field measured values provide better estimates for predicting thermal plume velocities care must be taken in selecting which field estimates are appropriate as estimates obtained from a pumping tests are over a factor of 2 larger than estimates obtained from observing the plume velocity and values used in the calibrated groundwater flow and transport model. 
3) Chapter 2 presents a method for constructing the two-dimensional thermal conductivity field for a glaciofluvial outwash deposit. The method couples field and laboratory methods to determine the bulk thermal conductivity of the aquifer solids, the volumetric water content, and the porosity of the aquifer with an approximating model for predicting the apparent thermal conductivity of variablysaturated soils. The Campbell model is shown to be the best-approximating model using the information-theoretic approach. The measured thermal conductivity values for the aquifer solids provide a dataset upon which to estimate the apparent thermal conductivity of similar porous media. Porosity was shown to strongly influence the thermal conductivity, indicating that in conduction dominated systems this parameter must be defined carefully.

4) The Campbell model is implemented into a finite-element density-dependent groundwater flow and thermal transport numerical model. Using the measured two-dimensional thermal conductivity field and the numerical model, we demonstrated that heterogeneous $\lambda$ fields increase the thermal dispersion analogous to solute transport.

5) A three-dimensional conceptual site model is developed in Chapter 3 and implemented in a modified version of Heatflow. Model calibration and verification demonstrate that thermal plumes from aggregate pits can be modelled successfully. Heatflow was compared to a number of benchmark tests to verify various physical processes were being considered correctly. A standards benchmark test for density-dependent thermal transport models is the Elder problem. New analytical solution to this problem are presented and Heatflow is shown to yield good agreement. This new analytical solution may be used as a benchmark test for other density-dependent thermal transport numerical models.

6) Simulation results indicated that in this outwash aquifer where the groundwater velocity is relatively high, the temperatures in the aquifer within the first $100 \mathrm{~m}$ of 
the pond are dominated by the convective transport of the temperature perturbation in the pond. Within this zone the annual temperature variation at the ground surface (largely transported by conduction) is masked by that of the pond. Beyond $100 \mathrm{~m}$ the temperature perturbation from the pond is attenuated by thermal retardation and thermal dispersion to the point where the influence of the temperature variation at the ground surface becomes evident in the measured subsurface temperatures. The simulations are in good agreement with the observed subsurface temperatures and support the conclusion that convective groundwater transport from a pond may influence subsurface temperatures well beyond $100 \mathrm{~m}$ down gradient.

7) The results from the numerical simulations are in good agreement with the groundwater temperatures measured at this site down gradient of the pit and presented in Chapter 1. The observed temperatures and simulated temperatures indicate that the thermal plume migrates up to $150 \mathrm{~m}$ down gradient of the pond. At this site, the groundwater discharges to streams that are well beyond this distance, and thus are not affected by these thermal plumes. Where pits are within $250 \mathrm{~m}$ of the stream, these results suggest there is the potential for the thermal plumes to alter the temperature of the groundwater discharging to the stream.

8) Simulation results demonstrate that where large groundwater velocities exist (typical of settings with aggregate extraction) heat transport is dominated by thermal convection and significant effort should be focused on obtaining representative estimates of the groundwater velocity (i.e., large scale hydraulic conductivity, porosity and hydraulic gradient). Groundwater velocity plays a dominant role in the distance of thermal plume transport. While hydraulic conductivity (and hence groundwater velocity) may be obtained by several methods, the groundwater velocities in the calibrated flow and transport model $\left(\sim 2.5 \mathrm{~m} \mathrm{~d}^{-1}\right)$ best match our estimates of groundwater velocity obtained from the observed thermal plume movement (i.e., $\sim 2.8 \mathrm{~m} \mathrm{~d}^{-1}$ ) which is essentially an 
aquifer tracer test where heat is the tracer. Hydraulic conductivity obtained from a pumping test was a factor of two too large, suggesting care must be taken in selecting the appropriate methods of estimating hydraulic conductivity. Thermal conductivity appears to be less important in these settings where convection dominants plume transport and estimates of thermal conductivity based on the measured values presented here and in other studies may be acceptable provided the porous media are similar.

\section{Future research recommendations}

The goal of this research was to establish a framework for assessing the potential impacts of below-water-table aggregate extraction on groundwater temperatures and nearby cool and cold-water streams. While many aspects of this work contributed to attaining this goal the following additional areas of research and investigation would further our understanding of the movement of thermal plumes.

1) Numerical simulations in Chapter 2 showed that, for short transport distances, using a mean thermal conductivity in place of a fully heterogeneous field yields small temperature differences of $<1^{\circ} \mathrm{C}$. It is possible that temperature differences may increase with transport distances, plume scale, and in fully three-dimensional systems with heterogeneous aquifer and thermal properties. Numerical investigations using fully three-dimensional systems could be completed by extending the methods developed here for the two-dimensional case. Results from this research would further our understanding of the need to consider the influence of heterogeneity in heat transport investigations.

2) While this research focused on the collection and analysis of field data and the implementation of a numerical model to a field situation further understanding of the various factors that influence heat transport in these settings is required. It is 
suggested that by using a simplified rectangular domain the influence of various parameters on the transport distance of the thermal plumes may be investigated sequentially so that the influence of each parameter/factor can be understood. Factors to be investigated include:

a) groundwater velocity beyond the range investigated here;

b) aquifer thermal conductivity;

c) aquifer thickness and depth of penetration of the pond into the aquifer; and

d) thickness of the unsaturated zone down gradient of the pond and the nature of the vegetation cover in this area. A thin unsaturated zone may provide for larger thermal exchange with the aquifer and thus attenuate the thermal plume more readily. Alternatively removal of much of the unsaturated zone may have a negative impact on the groundwater temperatures in this portion of the aquifer. Similarly the type of vegetation cover in the area will affect the thermal exchange at the air-ground interface and influence the attenuation of thermal plumes in the underlying aquifer.

3) The numerical model implemented here was successfully calibrated and verified; however, the current version of Heatflow has several limitations:

a) the flow and thermal transport are not modeled explicitly in the ponds. As a result flow through the ponds must be approximated using the high hydraulic conductivity approach and temperature in the ponds is not a direct outcome of the model but must be imposed as a boundary condition. Implementing these features may require the solution of the Navier-Stokes equations which is beyond the numerical methods capabilities of implemented in Heatflow (i.e., the conjugate gradient solver and the Leismann time-weighting scheme). Major modifications to Heatflow would be required or alternative codes may now be available with these capabilities or to which these capabilities can be added.

b) grid creation is a very labourious process with Heatflow and the prismatic block grid used in Heatflow results in an overly large number of elements and nodes. For prismatic block grids, refinement in the grid around one feature results in 
refinement that must expand to the lateral edges of the grid. Grids generated using prismatic triangles do not suffer from this limitation. Thus a numerical model which uses triangular prisms may be a more efficient numerical model. 


\section{Appendix A: Watershed Hydrology and Climate}

\section{Appendix A1: Basin characteristics and stream discharge}

The 30-minute average stream stage was measured at two stream gauging stations (Fig. A1-1) during the period April 1993 to January 1997. TR-Qa measures the flows in the eastern tributary $450 \mathrm{~m}$ above the point where it joins Tricks Creek. The catchment for this station includes the Town of Clinton and the soils are predominantly tills. At TR-Q the discharge in Tricks Creek is measured. The discharge at this station includes the discharge measured at TR-Qa as well as contributions from the remainder of the watershed which includes the glacial outwash sand and gravel and the upland till along the western portion of the watershed. The stage data were converted to stream discharges (Fig. A1-2) using rating curves developed at each station during the period 1993 to 1997.

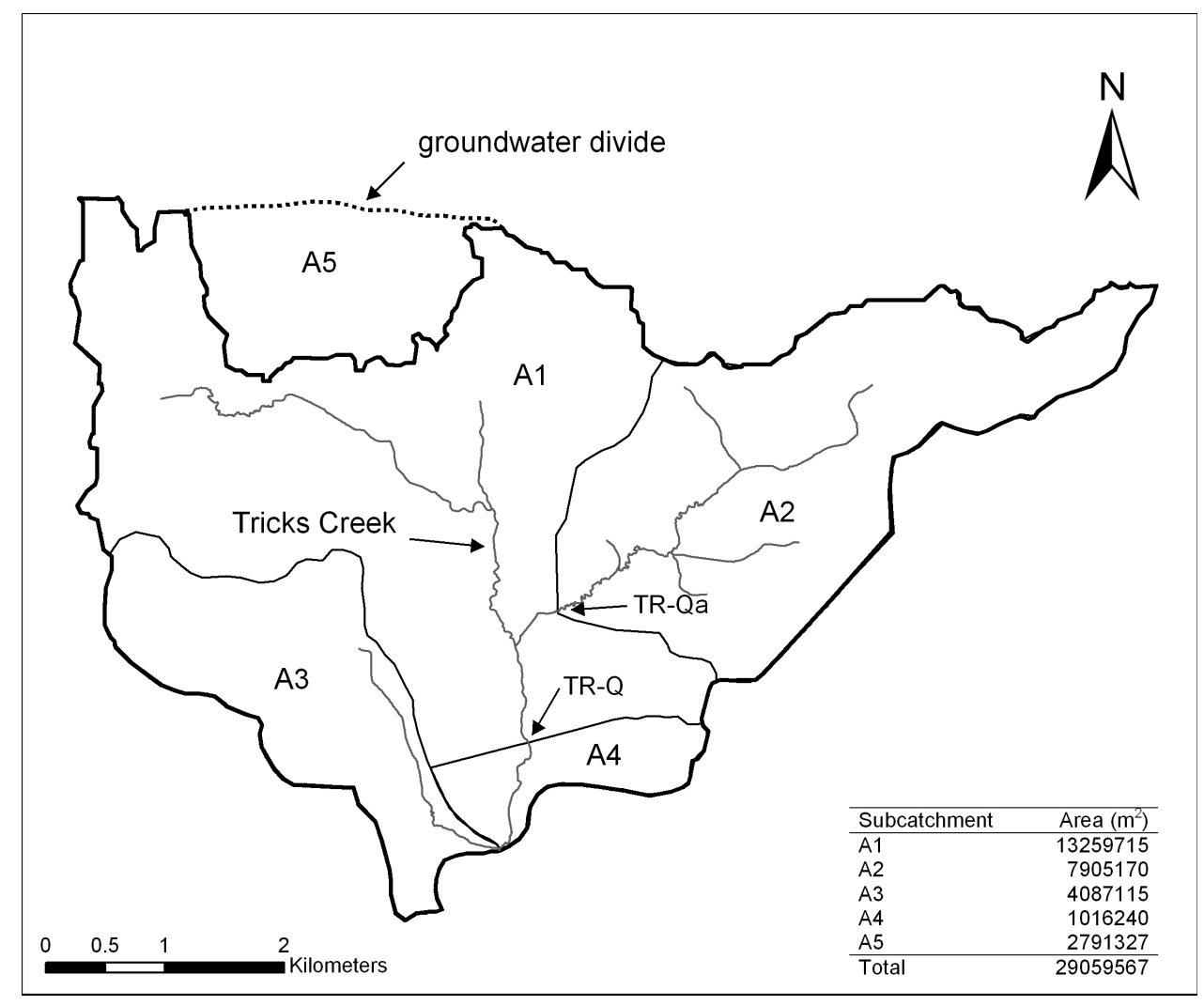

Figure A1-1 Tricks Creek watershed, subcatchments, and stream gauging stations TR$\mathrm{Q}$ and TR-Qa. 

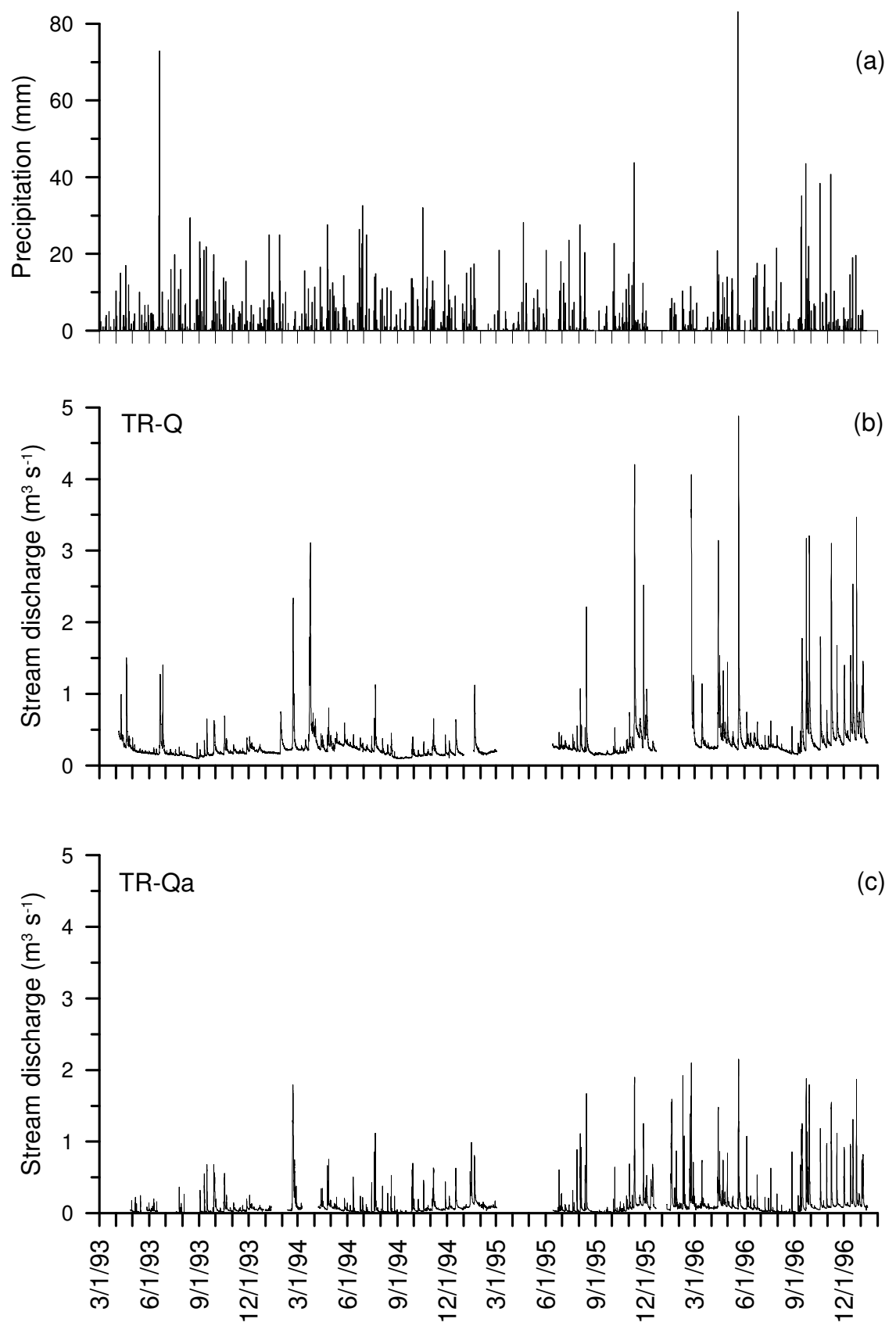

Figure A1-2 (a) Daily precipitation, and stream discharge at stations (b) TR-Q and (c) TR-Qa.

The catchment areas, average discharge, and annual discharge normalized for the catchment area are given below (Table A1-1). The average annual precipitation during this period was $1166 \mathrm{~mm}$. 
Table A1-1 Summary of catchment areas and discharges for the period 1993 to 1997.

\begin{tabular}{lccccc}
\hline Station & $\begin{array}{c}\text { Catchment } \\
\text { area } \dagger\end{array}$ & $\begin{array}{c}\text { Groundwater } \\
\text { catchment } \\
\text { area } \neq\end{array}$ & $\begin{array}{c}\text { Area of aquifer } \\
\text { within } \\
\text { groundwater } \\
\text { catchment }\end{array}$ & $\begin{array}{c}\text { Average } \\
\text { daily } \\
\text { discharge }\end{array}$ & $\begin{array}{c}\text { Annual } \\
\text { discharge per } \\
\text { unit area of } \\
\text { catchment }\end{array}$ \\
\hline TR-Q & 21164885 & 23956212 & 18824885 & 0.293 & $\mathrm{~m} \mathrm{yr}^{-1}$ \\
TR-Qa & 7905170 & 7905170 & 0 & 0.099 & 0.437 \\
TR-Q - TR-Qa & 13259715 & 16051042 & - & 0.194 & 0.461 \\
\hline
\end{tabular}

$\dagger$ catchment area derived from topography.

‡ groundwater catchment area derived from topography and steady state groundwater flow model.

Between 1993 and 1997, the average annual flow at TR-Qa accounted for approximately $31 \%$ of the flow measured at TR-Q, and under baseflow conditions the portion of the flow contributed by the catchment above TR-Qa is approximately $27 \%$. The catchment above TR-Qa $\left(\mathrm{A} 2=7905170 \mathrm{~m}^{2}\right)$ accounts for $37 \%$ of the catchment area above TR-Q $\left(\mathrm{A} 1+\mathrm{A} 2=21164885 \mathrm{~m}^{2}\right)$. This suggests that the catchment area drained by TR-Q (excluding the subcatchment drained by TR-Qa) contributes a larger portion of the flow to Tricks Creek. The discharges per unit area of catchment measured at TR-Q and TR-Qa are 0.437 and $0.393 \mathrm{~m} \mathrm{yr}^{-1}$, respectively. These values include both groundwater discharge to the stream $\left(Q^{\mathrm{bf}}\right)$ as well as overland flow and direct precipitation $\left(\mathrm{R}_{0}\right)$. The portion of the discharge measured at TR-Q that is contributed by subcatchment A1 may be isolated by subtracting the flow at TR-Qa. The annual discharge per unit area from subcatchment $\mathrm{A} 1$ is then $0.461 \mathrm{~m} \mathrm{yr}^{-1}$, which is $17 \%$ larger than that for the catchment above TR-Qa.

This difference in discharges may be related to the different soils in the two subcatchments. The soil in the catchment above TR-Qa (drained by the eastern tributary) is mainly till (low permeability). In some areas the till is overlain by a thin layer of ice contact stratified drift (sand and gravel). The low permeability of the till limits the contribution to baseflow from this area in comparison to the outwash above TR-Q. As well, the low permeability of the soils slow the infiltration rate such that a larger portion of the precipitation in this catchment is on or near the ground surface and is readily available for evapotranspiration.

In 2005, Environment Canada established a surface water discharge monitoring station on Tricks Creek where the gauging station established for this study (TR-Q) had 
been. Comparison of discharge data for the two periods shows similar flow characteristics.

\section{Appendix A2: Climate data (Temperature and Precipitation)}

Climate data from three nearby meteorlogical stations was obtained (Goderich, Blyth, and Exeter). These weather stations are maintained by Environment Canada. For each station daily and monthly values for temperature and precipitation are recorded and have been acquired for the period 1990 to 2005 at the Blyth and Exeter stations. At the Goderich station, daily values for temperature, precipitation, and wind speed and direction are available only between 1995 and present, while hourly values for temperature, dew point, relative humidity, pressure, precipitation, and wind speed and direction are available for the period 1990 to present. The 30-year climate normals for the Blyth and Exeter stations are presented in Table A2-1 and A2-2, and 15-year climate normals for Goderich are given in Table A2-3 (Environment Canada, 2006).

Table A2-1 Blyth station 6120819 climate normals for 1971-2000.

\begin{tabular}{llllllllllllll}
\hline & Jan & Feb & Mar & Apr & May & Jun & Jul & Aug & Sep & Oct & Nov & Dec & Year \\
\hline Daily average $\left({ }^{\circ} \mathrm{C}\right)$ & -7.5 & -6.7 & -1.7 & 5.5 & 12.3 & 17.3 & 20.2 & 19.1 & 15.1 & 8.8 & 2.7 & -3.6 & 6.8 \\
Daily maximum $\left({ }^{\circ} \mathrm{C}\right)$ & -4.1 & -2.9 & 2.5 & 10.1 & 17.9 & 22.9 & 25.9 & 24.6 & 20.1 & 13 & 5.8 & -0.6 & 11.3 \\
Daily minimum $\left({ }^{\circ} \mathrm{C}\right)$ & -10.8 & -10.5 & -5.9 & 0.8 & 6.7 & 11.7 & 14.5 & 13.6 & 10 & 4.5 & -0.5 & -6.5 & 2.3 \\
Precipitation $(\mathrm{mm})$ & 127.8 & 78.8 & 73 & 81.8 & 90.2 & 85.1 & 72.7 & 105.9 & 115.4 & 92.8 & 121.2 & 139.8 & 1184.3 \\
\hline
\end{tabular}

Latitude: $43^{\circ} 43^{\prime} \mathrm{N}$, Longitude: $81^{\circ} 22^{\prime} \mathrm{W}$, Elevation $350.50 \mathrm{~m}$

Table A2-2 Exeter station 6122370 climate normals for 1971-2000.

\begin{tabular}{|c|c|c|c|c|c|c|c|c|c|c|c|c|c|}
\hline Temperature & Jan & $\mathrm{Feb}$ & Mar & Apr & May & Jun & Jul & Aug & Sep & Oct & Nov & Dec & Year \\
\hline Daily average $\left({ }^{\circ} \mathrm{C}\right)$ & -6 & -5.7 & -0.5 & 6.2 & 12.9 & 18 & 20.4 & 19.5 & 15.3 & 9.1 & 3.1 & -2.9 & 7.5 \\
\hline Daily maximum $\left({ }^{\circ} \mathrm{C}\right)$ & -2.4 & -1.8 & 3.7 & 11 & 18.6 & 23.6 & 25.8 & 24.7 & 20.5 & 13.6 & 6.5 & 0.4 & 12 \\
\hline Daily minimum $\left({ }^{\circ} \mathrm{C}\right)$ & -9.6 & -9.7 & -4.7 & 1.3 & 7.2 & 12.3 & 14.9 & 14.1 & 10.1 & 4.6 & -0.3 & -6.2 & 2.8 \\
\hline Precipitation (mm) & 80.4 & 53 & 65.9 & 79.5 & 77.4 & 77.7 & 84.9 & 85.7 & 114.5 & 86.5 & 92.1 & 91 & 988.5 \\
\hline
\end{tabular}

Latitude: $43^{\circ} 21^{\prime} \mathrm{N}$, Longitude: 8130’ W, Elevation $262.10 \mathrm{~m}$

Table A2-3 Goderich station 6122847 climate normals for 1990-2000.

\begin{tabular}{|c|c|c|c|c|c|c|c|c|c|c|c|c|c|}
\hline Temperature $\dagger$ & Jan & Feb & Mar & Apr & May & Jun & Jul & Aug & Sep & Oct & Nov & Dec & Year \\
\hline Daily average $\left({ }^{\circ} \mathrm{C}\right)$ & -3.8 & -3.5 & 0.0 & 6.0 & 11.8 & 17.5 & 19.3 & 19.1 & 16.0 & 10.2 & 4.3 & -1.0 & 8.0 \\
\hline Daily maximum $\left({ }^{\circ} \mathrm{C}\right)$ & -1.0 & -0.4 & 3.4 & 10.0 & 16.2 & 21.8 & 23.4 & 23.0 & 20.2 & 13.8 & 7.1 & 1.4 & 11.6 \\
\hline Daily minimum $\left({ }^{\circ} \mathrm{C}\right)$ & -6.6 & -6.9 & -3.7 & 1.8 & 7.2 & 13.0 & 14.7 & 14.3 & 11.0 & 6.3 & 1.5 & -3.6 & 4.1 \\
\hline Precipitation $(\mathrm{mm}) \ddagger$ & 80.9 & 62.3 & 63.2 & 61.0 & 97.0 & 78.4 & 70.4 & 63.7 & 81.1 & 73.1 & 83.1 & 82.1 & 896.3 \\
\hline
\end{tabular}

Latitude: $43^{\circ} 46^{\prime} \mathrm{N}$, Longitude: $81^{\circ} 42^{\prime} \mathrm{W}$, Elevation $213.4 \mathrm{~m}$

$\dagger$ temperature normals were calculated from hourly data collected between 1990 and 2006 at Goderich station 6122847.

$\ddagger$ precipitation normals were calculated from data collected at five stations in Goderich. These stations operated at different times between 1866 and present. 
At Blyth $350.4 \mathrm{~mm}$ of the precipitation occurs as snow, at Exeter $182.7 \mathrm{~mm}$ occurs as snow, and at Goderich $213.0 \mathrm{~mm}$ occurs as snow.

\section{References}

Environment Canada. National Climate Data and Information Archive. website: http://climate.weatheroffice.gc.ca/climate_normals. 


\section{Appendix B: Numerical Method}

This appendix presents an overview of the design of the finite element numerical model Heatflow (Molson et al., 1992) as well as additions and modifications made as part of this study. The model incorporates 3-dimensional, non-isothermal, density-dependent, groundwater flow in the saturated domain, and thermal transport in the saturated and unsaturated domain. It is worth pointing out at this point that Heatflow solves flow in the saturated zone only. To obtain the thermal transport solution in the unsaturated zone, Heatflow prescribes the velocities in the elements above the water table internally. These velocities do not result from the solution of the unsaturated flow equation but rather are interpolated and are a simplification to flow in the unsaturated zone. The model also accounts for the interaction of 1-dimensional streams with the saturated domain.

\section{Appendix B1: Equations for Flow in Saturated Porous Media}

The governing equation for transient, three-dimensional, non-isothermal, densitydependent, groundwater flow through saturated porous media may be developed using Darcy's equation and the continuity equation for fluid mass flux, and is given by Molson et al. (1992) as

$$
\frac{\partial}{\partial x_{i}}\left[K_{i j}(T)\left(\frac{\partial h^{*}}{\partial x_{j}}+\rho_{r}(T) \tilde{n}_{j}\right)\right]-\sum_{k=1}^{N} Q_{k}(t) \delta\left(x_{k}^{\prime}, y_{k}^{\prime}, z_{k}^{\prime}\right)=S_{S} \frac{\partial h^{*}}{\partial t}
$$

where the following expressions are defined as

$$
\begin{array}{ll}
K_{i j}(T)=\frac{k_{i j} \rho_{0} g}{\mu(T)} & \text { is the hydraulic conductivity tensor }\left[\mathrm{L} \mathrm{T}^{-1}\right] ; \\
h^{*}=\frac{p}{\rho_{0} g}+z & \text { is the equivalent freshwater head [L], } \\
\text { as defined by (Frind, 1982); } \\
\rho_{r}(T)=\frac{\rho_{w}(T)}{\rho_{0}}-1 \quad \text { is a relative density [-]; }
\end{array}
$$


$S_{S}=\rho_{0} g(\alpha+\phi \beta) \quad$ is the specific storage coefficient of

where

$k_{\mathrm{ij}} \quad$ is the intrinsic permeability tensor for the porous medium $\left[\mathrm{L}^{2}\right]$;

$\rho_{0} \quad$ is the reference density $\left[\mathrm{M} \mathrm{L}^{-3}\right]$,

which is assumed to be $1000 \mathrm{~kg} \mathrm{~m}^{-3}$, the density of water at $0^{\circ} \mathrm{C}$;

$g \quad$ is the gravitational acceleration $\left[\mathrm{L} \mathrm{T}^{-2}\right]$;

$\mu(T)$ temperature-dependent dynamic viscosity of the fluid $\left[\mathrm{M} \mathrm{L}^{-1} \mathrm{~T}^{-1}\right]$;

$p \quad$ is the pressure head $\left[\mathrm{M} \mathrm{L}^{-1} \mathrm{~T}^{-2}\right]$;

$T \quad$ is the temperature $[\Theta]$;

$z \quad$ is the elevation above a datum [L];

$x_{\mathrm{j}} \quad$ are the principal directions $(\mathrm{x}, \mathrm{y}, \mathrm{z})[\mathrm{L}]$;

$\rho_{\mathrm{w}}(T)$ is the temperature-dependent density of the fluid $\left[\mathrm{M} \mathrm{L}^{-3}\right]$;

$t \quad$ is time [T];

$\alpha \quad$ is the porous medium compressibility $\left[\mathrm{M}^{-1} \mathrm{~L} \mathrm{~T}^{2}\right]$;

$\beta \quad$ is the compressibility of fluid $\left[\mathrm{M}^{-1} \mathrm{~L} \mathrm{~T}^{2}\right]$;

$\phi \quad$ is the porosity of the porous medium $\left[\mathrm{L}^{3}\right.$ voids $\mathrm{L}^{-3}$ porous media $]$;

$\tilde{n}_{j} \quad$ is the unit gravity vector often given as $\{0,0,-1\}$;

$N \quad$ is the number of well node point sources in the domain;

$Q_{\mathrm{k}} \quad$ is the volumetric fluid source at well node $k\left[\mathrm{~T}^{-1}\right]$,

which is the volume fluid injected per time / volume aquifer at point source $k . Q_{\mathrm{k}}$ is positive when fluid is injected;

$\delta \quad$ is the dirac delta function specifying a point source/sink, and $x_{k}^{\prime}, y_{k}^{\prime}, z_{k}^{\prime} \quad$ are the coordinates of the point injection or withdrawal.

In Heatflow, the three-dimensional coordinate system is assumed to be co-linear with the principal directions of anisotropy. Thus, the hydraulic conductivity tenor has no cross terms and is simply 
$K_{i j}=\left[\begin{array}{ccc}K_{x x} & 0 & 0 \\ 0 & K_{y y} & 0 \\ 0 & 0 & K_{z z}\end{array}\right]$

While not written as a function of temperature in the tensor, the hydraulic conductivity is temperature dependent.

To complete the transient flow equation, the following initial condition is required

$$
h^{*}=h(x, y, z) \text { at } t=0 \text { in } V
$$

where $V$ is the problem domain.

As well, boundary conditions along $\Gamma$, the boundary surface of the domain, are required to complete the problem statement. In this study, the following types of boundary conditions are considered.

\section{Dirichlet boundary condition ( $1^{\text {st }}$ type):}

This boundary condition is used when the hydraulic head can be prescribed on the boundary. It is expressed as

$$
h^{*}=h_{1}(x, y, z, t) \quad \text { on } \Gamma_{1}
$$

where $h_{1}(x, y, z, t)$ is the Dirichlet head on the Dirichlet boundary surface $\Gamma_{1}$.

\section{Neumann boundary condition ( $2^{\text {nd }}$ type):}

This boundary condition is employed when the flux at the boundary, resulting from the pressure-head gradient, is known as a function of time. It is written as

$$
-\bar{n} K_{i j}(T) \frac{\partial h^{*}}{\partial x_{j}}=q_{2}(x, y, z, t) \text { on } \Gamma_{2}
$$


where $q_{2}(x, y, z, t)\left[\mathrm{L} \mathrm{T}^{-1}\right]$ is the Neumann flux normal to the Neumann boundary surface $\Gamma_{2}$, and $\bar{n}$ is the unit vector normal to the boundary surface and is defined as positive inward.

\section{Cauchy boundary condition ( $3^{\text {rd }}$ type):}

This boundary condition is used when the flux resulting from the total hydraulic head gradient is known on a surface as a function of time. It is written as

$$
-\bar{n} K_{i j}(T)\left(\frac{\partial h^{*}}{\partial x_{j}}+\rho_{r}(T) \tilde{n}_{j}\right)=q_{3}(x, y, z, t) \text { on } \Gamma_{3}
$$

where $q_{3}(x, y, z, t)$ is the Cauchy flux $\left[\mathrm{L} \mathrm{T}^{-1}\right]$ across the Cauchy boundary surface $\Gamma_{3}$.

The Cauchy boundary condition may be used to represent several different boundaries. These include a leaky boundary where $q_{3}$ is proportional to the steady-state head drop across an aquitard (storage in the aquitard is zero), and a stream or river boundary where the stream bed is clogged (colmated) by a thin layer. These are described below.

\section{Leaky boundary condition:}

At the boundary with a leaky aquitard, the leakage flux is proportional to the total head drop across the aquitard for steady-state flow conditions. Assuming that the leakage flux is in the vertical direction and that flux into the domain is a positive quantity gives

$$
\begin{aligned}
-\bar{n} K_{i j}(T)\left(\frac{\partial h^{*}}{\partial x_{j}}+\rho_{r}(T) \tilde{n}_{j}\right) & =q_{3, L}(x, y, z, t) \quad \text { on } \Gamma_{3, L} \\
q_{3, L} & =K_{z z}^{L}\left(\frac{h_{L}-h^{*}}{b_{L}}+\rho_{r}(T)\right)
\end{aligned}
$$

where $q_{3, \mathrm{~L}} \quad$ is the fluid flux across the leaky boundary $\left[\mathrm{L} \mathrm{T}^{-1}\right]$; 
$K_{z z}^{L} \quad$ is the hydraulic conductivity of the leakage layer $\left[\mathrm{L}^{2} \mathrm{~T}^{-1}\right]$;

$b_{L} \quad$ is the thickness of the leaky layer [L];

$h^{*} \quad$ is the unknown head at the top of the aquifer (bottom of leaky layer) [L], and

$h_{L} \quad$ is the known head on the top of the leaky layer [L].

The flux $q_{3, \mathrm{~L}}(x, y, z, t)\left[\mathrm{L} \mathrm{T}^{-1}\right]$ into the domain is a positive quantity along the leaky boundary $\Gamma_{3, \mathrm{~L}}$.

\section{Stream/River boundary condition:}

This boundary is used where there is a thin layer of medium separating the streambed and the subsurface.

$$
\begin{aligned}
-\bar{n} K_{i j}(T)\left(\frac{\partial h^{*}}{\partial x_{j}}+\rho_{r}(T) \tilde{n}_{j}\right) & =q_{3, S}(x, y, z, t) \quad \text { on } \Gamma_{3, S} \\
q_{3, S} & =\Phi_{S}\left(h_{S}-h^{*}\right)
\end{aligned}
$$

where $q_{3, S} \quad$ is the fluid flux across the streambed [ $\mathrm{L} \mathrm{T}^{-1}$;

$\Phi_{\mathrm{S}} \quad$ is the linear fluid transfer coefficient through the thin layer on the streambed $\left[\mathrm{T}^{-1}\right]$, or streambed leakance factor given by

$$
\Phi_{S}=\frac{K_{S}}{b_{S}}, \text { and }
$$

$K_{\mathrm{S}} \quad$ is the hydraulic conductivity of the thin layer $\left[\mathrm{L} \mathrm{T}^{-1}\right]$;

$b_{\mathrm{S}} \quad$ is the thickness of the thin layer [L];

$h_{\mathrm{S}} \quad$ is the known hydraulic head in the stream [L];

$h^{*} \quad$ is the unknown hydraulic head at the interface with the subsurface [L], and

$\Gamma_{3, \mathrm{~S}} \quad$ is the boundary surface at the stream-subsurface interface.

In this case, it is assumed that the layer is thin and thus the gravity head term is 
negligible. The total flow across the interface of the stream bed and the subsurface is given by

$Q_{S}=q_{3, S} A_{S}$

where $Q_{\mathrm{S}} \quad$ is the total flow across the interface $\left[\mathrm{L}^{3} \mathrm{~T}^{-1}\right]$, and

$A_{\mathrm{S}} \quad$ is the area of the wetted stream-subsurface interface $\left[\mathrm{L}^{2}\right]$.

For all these boundary conditions $h^{*}, h_{\mathrm{L}}$, and $h_{\mathrm{S}}$ are time-dependent, and $\Gamma$ is the boundary surface of the domain given by $\Gamma=\Gamma_{1}+\Gamma_{2}+\Gamma_{3}+\Gamma_{3, L}+\Gamma_{3, S}$.

\section{Appendix B2: Equations for Thermal Transport in Variably-Saturated}

\section{Porous Media}

The governing equation for thermal transport through variably-saturated porous media is developed from the statement of thermal energy conservation. The convective form of the governing equation for thermal transport through variably-saturated porous media is given by Molson et al. (1992) as

$$
\frac{\partial}{\partial x_{i}}\left[\left(\kappa_{i j}+\frac{D_{i j}}{R}\right) \frac{\partial T}{\partial x_{j}}\right]-\left(\frac{v_{i}}{R} \frac{\partial T}{\partial x_{i}}\right)+\sum_{k=1}^{N} \frac{Q_{k}(t)\left[T_{k}(t)-T\right]}{R \phi} \delta\left(x_{k}^{\prime}, y_{k}^{\prime}, z_{k}^{\prime}\right)=\frac{\partial T}{\partial t}
$$

where the following expressions are defined as

$$
\begin{aligned}
\kappa_{i j}=\frac{\lambda_{i j}}{C_{0}+L \omega \rho_{w} \partial W_{u} / \partial T} \quad & \text { is the equivalent thermal diffusivity tensor } \\
& \text { of the porous medium }\left[\mathrm{L}^{2} \mathrm{~T}^{-1}\right], \\
& \text { which commonly has units of }\left[\mathrm{m}^{2} \mathrm{~s}^{-1}\right] ;
\end{aligned}
$$

$C_{0}=S \phi \rho_{w} c_{w}+(1-\phi) \rho_{s} c_{s} \quad$ is the heat capacity of the porous medium, 
considered on either a per-unit-mass or per-unit-volume basis; that is, mass-specific $\left[\mathrm{J} \mathrm{kg}^{-1}{ }^{\circ} \mathrm{C}^{-1}\right]$ or volume-specific heat capacity $\left[\mathrm{J} \mathrm{m}^{-3}{ }^{\circ} \mathrm{C}^{-1}\right]$;

$$
\begin{gathered}
R=\frac{C_{0}+L \omega \rho_{w} \partial W_{u} / \partial T}{S \phi \rho_{w} c_{w}} \quad \text { is the thermal retardation factor due to the aquifer } \\
\text { heat capacity [-], and }
\end{gathered}
$$

where

$D_{\mathrm{ij}} \quad$ is the hydrodynamic dispersion tensor $\left[\mathrm{L}^{2} \mathrm{~T}^{-1}\right]$;

$\phi \quad$ is the aquifer porosity $\left[\mathrm{L}^{3}\right.$ voids $\mathrm{L}^{-3}$ porous media];

$T \quad$ temperature $[\Theta]$;

$v_{\mathrm{i}} \quad$ is the average linear groundwater velocity $\left[\mathrm{L} \mathrm{T}^{-1}\right]$;

$\mathrm{S} \quad$ is the saturation level, $\theta_{w} / \phi[-]$;

$\theta_{\mathrm{w}}$ is the volumetric content of water $\left[\mathrm{L}^{3}\right.$ water $\mathrm{L}^{-3}$ porous media];

$c_{\mathrm{i}} \quad$ is the specific heat capacity of water and solids $\left[\mathrm{L}^{2} \mathrm{~T}^{-2} \Theta^{-1}\right]$, which commonly has units of $\left[\mathrm{J} \mathrm{kg}^{-1}{ }^{\circ} \mathrm{C}^{-1}\right]$;

$\rho_{\mathrm{i}} \quad$ is the density of water and solids $\left[\mathrm{M} \mathrm{L}^{-3}\right]$, which commonly has units of $\left[\mathrm{kg} \mathrm{m}^{-3}\right]$;

$L \quad$ is the latent heat of water $\left[\mathrm{L}^{2} \mathrm{~T}^{-2}\right]$;

$\omega \quad$ is the total aquifer moisture content $\left[\mathrm{L}^{3}\right.$ water $\mathrm{L}^{-3}$ porous media $]$;

$W_{u} \quad$ is the fraction of total moisture unfrozen and expressed as a function of temperature [-];

$\lambda_{\mathrm{ij}} \quad$ is the effective or apparent thermal conductivity tensor [ $\mathrm{M} \mathrm{L} \mathrm{T}^{-3} \Theta^{-1}$ ], which commonly has units of $\left[\mathrm{W} \mathrm{m}^{-1}{ }^{\circ} \mathrm{C}^{-1}\right]$;

$Q_{\mathrm{k}} \quad$ is the volumetric fluid source at well node point source $k\left[\mathrm{~T}^{-1}\right]$, which is the volume fluid injected per time / volume aquifer;

$T_{\mathrm{k}} \quad$ is the time-dependent temperature of the injection/withdrawal fluid $[\Theta]$;

$N \quad$ is the number of point sources or sinks, and 
$\delta \quad$ is the dirac delta function specifying a point source/sink $\left[\mathrm{L}^{-1}\right]$, and $x_{k}^{\prime}, y_{k}^{\prime}, z_{k}^{\prime} \quad$ are the coordinates of the point injection or withdrawal [L].

Where fluid is being withdrawn at the well nodes, $T=T_{\mathrm{k}}$, and the source/sink term vanishes. While $\rho_{\mathrm{w}}(T)$ is written simply as $\rho_{\mathrm{w}}$ in the above expressions, the fluid density is temperature-dependent in the transport model.

In Heatflow, the apparent thermal conductivity tensor is assumed to be given by

$$
\lambda_{i j}=\left[\begin{array}{ccc}
\lambda_{x x} & 0 & 0 \\
0 & \lambda_{y y} & 0 \\
0 & 0 & \lambda_{z z}
\end{array}\right]
$$

and the equivalent thermal diffusivity tensor is given by

$$
\kappa_{i j}=\left[\begin{array}{ccc}
\kappa_{x x} & 0 & 0 \\
0 & \kappa_{y y} & 0 \\
0 & 0 & \kappa_{z z}
\end{array}\right]
$$

For the dispersion model implemented in this study, the dispersion tensor is given by

$$
D_{i j}=\left[\begin{array}{ccc}
D_{x x} & D_{x y} & D_{x z} \\
D_{y x} & D_{y y} & D_{y z} \\
D_{z x} & D_{z y} & D_{z z}
\end{array}\right]
$$

where $D_{\mathrm{ij}}$ is symmetric. Individual entries are given by

$$
\begin{aligned}
& D_{x x}=\frac{1}{\bar{v}}\left(\alpha_{L} v_{x}^{2}+\alpha_{T H} v_{y}^{2}+\alpha_{T V} v_{z}^{2}\right) \\
& D_{y y}=\frac{1}{\bar{v}}\left(\alpha_{T H} v_{x}^{2}+\alpha_{L} v_{y}^{2}+\alpha_{T V} v_{z}^{2}\right)
\end{aligned}
$$


$D_{z z}=\frac{1}{\bar{v}}\left(\alpha_{T V} v_{x}^{2}+\alpha_{T H} v_{y}^{2}+\alpha_{L} v_{z}^{2}\right)$

and the off-diagonal entries are

$$
\begin{aligned}
& D_{x y}=D_{y x}=\left(\alpha_{L}-\alpha_{T H}\right) \frac{v_{x} v_{y}}{v} \\
& D_{x z}=D_{z x}=\left(\alpha_{L}-\alpha_{T V}\right) \frac{v_{x} v_{z}}{v} \\
& D_{y z}=D_{z y}=\left(\alpha_{L}-\alpha_{T V}\right) \frac{v_{y} v_{z}}{v}
\end{aligned}
$$

where $v=\left(v_{x}^{2}+v_{y}^{2}+v_{z}^{2}\right)^{1 / 2}$.

More complex models for dispersion may be implemented, but these are rarely justified with the data available for field scale investigations. Also, several references define an apparent thermal conductivity tensor that combines (B2.3b) and (B2.4a). In this study, I have kept them separate for clarity.

To complete the transient transport equation, the following initial condition is required,

$$
T=T(x, y, z) \text { at } t=0 \text { in } V
$$

where $V$ is the problem domain.

As well, boundary conditions are required along $\Gamma$, the boundary surface of the problem domain, to complete the problem statement. The following boundary conditions are considered.

\section{Dirichlet boundary condition:}

This boundary condition is used when the temperature can be prescribed on the boundary. It is expressed as 


$$
T=T_{1}(x, y, z, t) \quad \text { on } \Gamma_{1}
$$

where $T_{1}(x, y, z, t)$ is the specified temperature on the Dirichlet boundary surface $\Gamma_{1}$.

\section{Neumann boundary condition:}

This boundary condition is employed when the thermal gradient at a boundary is known as a function of time. It is written as

$$
-\bar{n} \lambda_{i j} \frac{\partial T}{\partial x_{j}}=q_{2}^{h}(x, y, z, t) \text { on } \Gamma_{2}
$$

where $q_{2}^{h}(x, y, z, t)$ is the heat flux $\left[\mathrm{M} \mathrm{T}^{-3}\right]$ normal to the Neumann boundary surface $\Gamma_{2}$, and $\bar{n}$ is the unit outward normal vector to the boundary surface.

\section{Leaky boundary condition:}

The heat flux across the interface between a leaky aquitard and the aquifer is proportional to the temperature gradient across the aquitard. Heat convection due to fluid flux across the interface is negligible since at the interface the temperature in the aquitard will be very close or equal to that in the aquifer. Thus, there will be no net gain or loss of heat due to convection. Assuming that the heat flux is at steady state and in the vertical direction across the interface yields a Neumann type boundary condition given by

$$
\begin{aligned}
-\bar{n} \lambda_{i j} \frac{\partial T}{\partial x_{j}} & =q_{2, L}^{h}(x, y, z, t) \quad \text { on } \Gamma_{2, L} \\
q_{2, L}^{h} & =\frac{\lambda_{L}}{b_{L}}\left(T_{L}-T\right)
\end{aligned}
$$

where $q_{2, L}^{h} \quad$ is the heat flux across the interface with the leaky aquitard $\left[\mathrm{M} \mathrm{T}^{-3}\right]$; 
$T_{\mathrm{L}} \quad$ is the known temperature on the far side of the leaky aquitard $\left[{ }^{\circ} \mathrm{C}\right]$;

$T \quad$ is the unknown temperature at the interface with the subsurface $\left[{ }^{\circ} \mathrm{C}\right]$;

$\lambda_{\mathrm{L}} \quad$ is the thermal conductivity of the leaky layer $\left[\mathrm{M} \mathrm{L} \mathrm{T}^{-3}{ }^{\circ} \mathrm{C}^{-1}\right]$, and

$b_{\mathrm{L}} \quad$ is the thickness of the leaky layer [L].

The flux $q_{2, L}^{h}(x, y, z, t)$ into the domain is a positive quantity along the leaky boundary $\Gamma_{2, \mathrm{~L}}$

\section{Cauchy boundary condition ( $3^{\text {rd }}$ type):}

This boundary condition is used to describe the total heat-flux rate along the boundary. It is written as

$$
\bar{n}\left(\rho_{w} c_{w} q_{i} T-\lambda_{i j} \frac{\partial T}{\partial x_{j}}\right)=q_{3}^{h}(x, y, z, t) \quad \text { on } \Gamma_{3}
$$

where $q_{3}^{h}(x, y, z, t)$ is the heat flux normal to the Cauchy boundary surface $\Gamma_{3}$, and $\bar{n}$ is the unit outward normal vector to the boundary surface.

\section{Air-ground surface interface boundary condition:}

At the ground surface the following Cauchy equation describes thermal exchange between the atmosphere and the ground surface. At this interface, heat exchange results from conduction due to the difference in temperatures at the air-subsurface interface, and convection of heat with infiltrating water. The complex physical processes that contribute to heat exchange at the ground surface are often simplified by assuming heat conduction and convection occur across a thin transition layer (much like a colmated layer on a streambed). Dispersion across this layer is ignored and it is assumed that the temperature of the fluid moving across this layer is unchanged. This type of boundary condition is given by 


$$
\begin{aligned}
\bar{n}\left(\rho_{w} c_{w} q_{i} T-\lambda_{i j} \frac{\partial T}{\partial x_{j}}\right) & =q_{3, G}^{h}(x, y, z, t) \text { on } \Gamma_{3, G} \\
q_{3, G}^{h} & =\rho_{w} c_{w} q_{R} T_{R}+\frac{\lambda_{G}}{b_{G}}\left(T_{A}-T\right)
\end{aligned}
$$

where $q_{3, G}^{h} \quad$ is the heat flux across the ground surface $\left[\mathrm{M} \mathrm{T}^{-3}\right]$;

$T_{\mathrm{A}} \quad$ is the known atmospheric temperature $[\Theta]$;

$T_{\mathrm{R}} \quad$ is the known temperature of infiltrating water $[\Theta]$;

$T \quad$ is the unknown surface temperature within the subsurface $[\Theta]$;

$\lambda_{\mathrm{G}} \quad$ is the thermal conductivity of the transition layer $\left[\mathrm{M} \mathrm{L} \mathrm{T}^{-3} \Theta^{-1}\right.$;

$b_{\mathrm{G}} \quad$ is the thickness of the transition layer [L], and

$q_{\mathrm{R}} \quad$ is the surface recharge flux rate $\left[\mathrm{L} \mathrm{T}^{-1}\right]$.

Defining a linear heat transfer coefficient as

$$
\gamma_{G}=\frac{\lambda_{G}}{b_{G}}
$$

rearranging, and considering transport at the ground surface in the vertical direction only, with $q_{\mathrm{i}}=q_{\mathrm{R}}$ gives

$$
\begin{aligned}
-\bar{n} \lambda_{i j} \frac{\partial T}{\partial x_{j}} & =\lambda_{z z}^{u}\left(\frac{\partial T}{\partial z}\right) \\
\lambda_{z z}^{u}\left(\frac{\partial T}{\partial z}\right) & =\gamma_{G}\left(T_{A}-T\right)+q_{R} c_{w} \rho_{w}\left(T_{R}-T\right)
\end{aligned}
$$

where $\lambda_{z z}^{u} \quad$ is the thermal conductivity of the unsaturated zone $\left[\mathrm{M} \mathrm{L} \mathrm{T}^{-3} \Theta^{-1}\right]$;

$\gamma_{\mathrm{G}}$ is the linear heat transfer coefficient at the ground surface $\left[\mathrm{M} \mathrm{T}^{-3} \Theta^{-1}\right]$;

$T_{\mathrm{A}} \quad$ is the known atmospheric temperature $[\Theta]$;

$T_{\mathrm{R}} \quad$ is the known temperature of infiltrating water $[\Theta]$; 
$T \quad$ is the unknown surface temperature within the subsurface $[\Theta]$, and

$q_{\mathrm{R}} \quad$ is the surface recharge flux rate $\left[\mathrm{L} \mathrm{T}^{-1}\right]$.

In some cases, it is reasonable to assume that the infiltrating water temperature is equal to the air temperature $T_{\mathrm{R}}=T_{\mathrm{A}}$. Then $(\mathrm{B} 2.6 \mathrm{e})$ is

$$
\lambda_{z z}^{u}\left(\frac{\partial T}{\partial z}\right)=\left(\gamma_{G}+q_{R} c_{w} \rho_{w}\right)\left(T_{A}-T\right) \quad \text { on } \Gamma_{3, G}
$$

\section{Subsurface-Stream/River interface boundary condition:}

This boundary is used where there is a thin layer of medium separating the stream and the saturated subsurface. The energy exchange across this Cauchy-type boundary is given by

$$
\lambda_{z z}^{u}\left(\frac{\partial T}{\partial z}\right)=\left(\gamma_{S B}+q_{S} c_{w} \rho_{w}\right)\left(T_{S}-T\right) \quad \text { on } \Gamma_{3, S}
$$

where $\gamma_{\mathrm{SB}}$ is the linear heat transfer coefficient at the stream bed - subsurface interface $\left[\mathrm{M} \mathrm{T}^{-3} \Theta^{-1}\right]$, given by

$$
\gamma_{S B}=\frac{\lambda_{S B}}{b_{S B}} \text {, and }
$$

$\lambda_{\mathrm{SB}} \quad$ is the thermal conductivity of the stream bed layer $\left[\mathrm{M} \mathrm{L} \mathrm{T}^{-3} \Theta^{-1}\right.$;

$b_{\mathrm{SB}} \quad$ is the thickness of the stream bed layer [L], and

$T_{\mathrm{S}} \quad$ is the known stream temperature $[\Theta]$;

$q_{\mathrm{S}} \quad$ is the fluid flux between the stream and subsurface $\left[\mathrm{L} \mathrm{T}^{-1}\right]$.

For these Cauchy-type boundary conditions given by (B2.6e to g), $T, T_{\mathrm{R}}, T_{\mathrm{S}}$ and $T_{\mathrm{A}}$ are time-dependent. Where the temperature between the stream and subsurface are continuous, the continuity of temperature should be imposed at the interface. The boundary surface of the domain $\Gamma$ is given as $\Gamma=\Gamma_{1}+\Gamma_{2}+\Gamma_{3}+\Gamma_{3, G}+\Gamma_{3, L}$.

The governing equation for the transport of heat through a variably-saturated 
porous medium (B2.1) along with the initial conditions (B2.5) and the boundary conditions (B2.6a to A2.6g) comprise a system of equations for describing heat transport through variably-saturated porous media with energy exchange between the air and the porous media at the ground surface, and/or streams and the saturated porous media. 


\section{Appendix B3: Basis of the Galerkin Finite Element Method}

In this study, the groundwater flow equation (B1.1) and thermal transport equation (B2.1) are solved using the Galerkin finite-element (FE) technique with the method of weighted residuals.

In the finite element method, the complex groundwater flow and transport equations may be approximated by means of a simple spatial interpolation function, defined in terms of nodal values. The errors introduced by this interpolation function are minimized on average over the problem domain. To generate the algebraic equations of the unknown nodal values, we apply the Weighted Residual Method to a partial differential equation of the form:

$L(u)-F=0$

where $L \quad$ is a Cartesian differential operator;

$u \quad$ is the field variable, and

$F \quad$ is a known function.

I now express the trial (approximate) solution as:

$u(x, y, z, t) \approx \hat{u}(x, y, z, t)=\sum_{j=1}^{n} u_{j}(t) N_{j}(x, y, z)$

where $u_{\mathrm{j}} \quad$ are the unknown values of the field variable at the nodes;

$N_{\mathrm{j}} \quad$ are the interpolation (or basis) functions, and

$n \quad$ is the total number of nodes in the problem domain.

When the approximate solution is substituted into the differential equation, the differential equation is no longer satisfied exactly

$L(\hat{u}(x, y, z, t))-F(x, y, z)=R(x, y, z) \neq 0$ 
where $R \quad$ is the error due to the approximating function.

In the method of weighted residuals we force the weighted average of the residuals at the nodes to be equal to zero

$$
\int_{V} R(x, y, z) W_{i}(x, y, z) d V=0, \quad i=1,2 \ldots, n
$$

where $V \quad$ is the problem domain, and

$W_{\mathrm{i}}(x, y, z) \quad$ are a set of $n$ weighting functions corresponding to the $n$ nodes.

To evaluate (B3.4) we must specify the form of the approximate solution $\hat{u}$ (which makes use of interpolation functions $N_{\mathrm{j}}$ ) and the weighting function $W_{\mathrm{i}}$. In the Galerkin Method, the weighting functions $W_{\mathrm{i}}$ and the interpolation functions $N_{\mathrm{j}}$ are chosen to be identical ( $W_{\mathrm{i}}$ is equal to $N_{\mathrm{i}}$ ). This choice results in a symmetrical coefficient matrix for the groundwater flow equation (Zienkiewicz et al., 2005). The Galerkin equation is then

$$
\iiint_{V}[L(\hat{u}(x, y, z))-F(x, y, z)] N_{i}(x, y, z) d V=0
$$

and substituting the approximating function into the Galerkin equation gives

$$
\iiint_{V}\left[L\left(\sum_{j=1}^{n} u_{j}(t) N_{j}(x, y, z)\right)-F(x, y, z)\right] N_{i}(x, y, z) d V=0
$$

The Galerkin equation gives $n$ equations in $n$ unknowns that may be solved for the unknown nodal values $u_{\mathrm{j}}$. 


\section{Appendix B4: Derivation of the Galerkin Finite Element Equation}

\section{for Groundwater Flow}

From the groundwater flow equation we can define the following

$$
L\left(h^{*}\right)=\frac{\partial}{\partial x_{i}}\left[K_{i j}(T)\left(\frac{\partial h^{*}}{\partial x_{j}}+\rho_{r}(T) \tilde{n}_{j}\right)\right]-\sum_{k=1}^{N} Q_{k}(t) \delta\left(x_{k}^{\prime}, y_{k}^{\prime}, z_{k}^{\prime}\right)-S_{S} \frac{\partial h^{*}}{\partial t}=0
$$

Using the finite element method we substitute the following approximate trial solution for the hydraulic head

$h^{*}(x, y, z, t) \approx \hat{u}(x, y, z, t)=\sum_{j=1}^{n} u_{j}(t) N_{j}(x, y, z)$

where $N_{j}(x, y, z)$ are linear basis functions and $n$ is the number of nodes in the finite element mesh. We define the residuals as

$\iiint_{V} L\left(h^{*}\right) W_{i} d V=R \quad \mathrm{i}=1,2, \ldots, n$

or

$\iiint_{V}\left\{\frac{\partial}{\partial x_{i}}\left[K_{i j}(T)\left(\frac{\partial \hat{u}}{\partial x_{j}}+\rho_{r}(T) \tilde{n}_{j}\right)\right]-\sum_{k=1}^{N} Q_{k}(t) \delta\left(x_{k}^{\prime}, y_{k}^{\prime}, z_{k}^{\prime}\right)-S_{S} \frac{\partial \hat{u}}{\partial t}\right\} W_{i}(x, y, z) d V=R$

where $W_{i}(x, y, z) \quad$ are weighting functions, and

$d V=d x d y d z$.

Applying the Galerkin method of weighted residuals, we choose weighting 
functions $W_{\mathrm{i}}$ with the same form as the linear basis functions $N_{\mathrm{j}}$ in (B4.2), and we force the weighted average of the residuals to be zero over the problem domain. The stepby-step details are not shown here but may be found in several references on finite element methods (see for example Istok, 1989; Voss and Provost, 2003; Zienkiewicz et al., 2005; Diersch, 2005). Approximating the time derivative by the standard finitedifference time-integration scheme gives the finite element equation for saturated groundwater flow written in matrix form as

$$
\begin{aligned}
\left(\varepsilon\left[S_{i j}\right]+\frac{1}{\Delta t}\left[P_{i j}\right]\right)\left\{u_{j}\right\}^{n+1} & =\left(-(1-\varepsilon)\left[S_{i j}\right]+\frac{1}{\Delta t}\left[P_{i j}\right]\right)\left\{u_{j}\right\}^{n} \\
& -(1-\varepsilon)\left\{G_{i}\right\}^{n}-\varepsilon\left\{G_{i}\right\}^{n+1} \\
& +(1-\varepsilon)\left\{F_{i}\right\}^{n}+\varepsilon\left\{F_{i}\right\}^{n+1} \\
& -(1-\varepsilon)\left\{F_{i}^{Q}\right\}^{n}-\varepsilon\left\{F_{i}^{Q}\right\}^{n+1}
\end{aligned}
$$

where $\left\lfloor S_{i j}\right\rfloor \quad$ is the global conductance or stiffness matrix;

$\left\lfloor P_{i j}\right\rfloor \quad$ is the global capacitance or mass matrix;

$\left\{G_{i}\right\}$ is the body force or density-gravity vector;

$\left\{F_{i}\right\}$ is the flux vector resulting from all the boundary conditions;

$\left\{F_{i}{ }^{Q}\right\}$ is the flux vector resulting from internal sources/sinks;

$n \quad$ is the time at the $n$th time level $[-]$;

$n+1 \quad$ is the time at the $n+1$ time level [-], and

$\varepsilon \quad$ is the time-weighting factor, ranging from 0 to 1.0 .

Expanding the indices gives

$$
\begin{aligned}
& {\left[S_{i j}\right]=\sum_{e} \iiint_{V^{e}}\left[K_{x x}(T) \frac{\partial N_{i}}{\partial x} \frac{\partial N_{j}}{\partial x}+K_{y y}(T) \frac{\partial N_{i}}{\partial y} \frac{\partial N_{j}}{\partial y}+K_{z z}(T) \frac{\partial N_{i}}{\partial z} \frac{\partial N_{j}}{\partial z}\right] d V} \\
& {\left[P_{i j}\right]=\sum_{e} \iiint_{V^{e}} N_{i} S_{S} N_{j} d V}
\end{aligned}
$$


$\left\{G_{i}\right\}=\sum_{e} \iiint_{V^{e}}\left[K_{x x}(T) \frac{\partial N_{i}}{\partial x}\left(\left\langle\rho_{r}(T) n_{x}\right\rangle\right)+K_{y y}(T) \frac{\partial N_{i}}{\partial y}\left(\left\langle\rho_{r}(T) n_{y}\right\rangle\right)+K_{z z}(T) \frac{\partial N_{i}}{\partial z}\left(\left\langle\rho_{r}(T) n_{z}\right\rangle\right)\right]^{203} d V$

$\left\{F_{i}\right\}=\sum_{e} \iint_{\Gamma^{e}} N_{i} q_{n} d \Gamma$

$\left\{F_{i}^{Q}\right\}=\sum_{e} \sum_{k=1}^{N} Q_{k}^{\prime}\left(x_{k}^{\prime}, y_{k}^{\prime}, z_{k}^{\prime}, t\right)$

Depending on the choice of $\varepsilon$ several different finite difference formulations are defined. Setting $\varepsilon=0.0$ yields the forward-difference (explicit) scheme which is only conditionally stable even for linear problems. It is generally not recommended for nonlinear problems and is not considered in this study. Setting $\varepsilon=0.5$, one obtains the Crank-Nicholson (centred-in-time) scheme. The Crank-Nicholson scheme has a truncation error of $0\left(\Delta \mathrm{t}^{2}\right)$, but its propagation-of-error characteristics frequently lead to oscillatory nonlinear instability. For $\varepsilon=1.0$, one obtains the backward-difference (fully implicit) scheme. The backward-difference approach has a truncation error of $0(\Delta t)$, and is quite resistant to oscillatory nonlinear instability.

\section{Appendix B5: Derivation of the Finite-Element Equations for Thermal Transport}

The standard procedure in solving the FE equations for transport is to approximate the temporal differential term by means of finite difference and to weight the spatial terms between two successive time levels. The most common weighting scheme is the CrankNicolson, where old and new time levels are weighted equally. If the advective term is centrally weighted, the matrix will be unsymmetric and cannot be solved using efficient matrix solvers that are available for symmetric matrices. To achieve a symmetric matrix the advective term may be placed entirely at the old time level. However, without compensation, such a scheme will not produce accurate results and may not be stable. Leismann and Frind (1989) proposed a time integration scheme along with compensating 
measures to over come these problems. The Leismann scheme is particularly efficient in that it generates a symmetric coefficient matrix for the transport problem, thereby reducing storage requirements and enabling the use of efficient symmetric matrix solvers. Matrix symmetry is achieved by placing the advective term at the old time level while numerical errors are compensated for with an artificial dispersion term. In Heatflow, Molson et al. (1992) solve the transport equation using the temporal integration scheme of Leismann and Frind (1989) along with the standard Galerkin finite element method with rectangular prism elements, and linear basis and weighting functions.

\section{Implementation of the Leismann and Frind (1989) time integration scheme}

In Heatflow, Molson et al. (1992) make use of the second-order scheme proposed by Leismann and Frind (1989) and has the general form

$$
\begin{aligned}
\frac{T^{n+1}}{\Delta t}+\varepsilon_{v} v_{i} \frac{\partial T^{n+1}}{\partial x_{i}} & -\varepsilon_{d} D_{i j} \frac{\partial^{2} T^{n+1}}{\partial x_{i} \partial x_{j}}-\varepsilon_{a} D_{i j}^{*} \frac{\partial^{2} T^{n+1}}{\partial x_{i} \partial x_{j}} \\
& -\frac{T^{n}}{\Delta t}+\left(1-\varepsilon_{v}\right) v_{i} \frac{\partial T^{n}}{\partial x_{i}}-\left(1-\varepsilon_{d}\right) D_{i j} \frac{\partial^{2} T^{n}}{\partial x_{i} \partial x_{j}}-\left(1-\varepsilon_{a}\right) D_{i j}^{*} \frac{\partial^{2} T^{n}}{\partial x_{i} \partial x_{j}}=0
\end{aligned}
$$

where $n$ and $n+1 \quad$ represent the old and new time levels, $t$ and $t+\Delta t$, respectively;

$$
\begin{array}{ll}
\Delta t & \text { is the time step [T]; } \\
D_{\mathrm{ij}}^{*} & \text { is the artificial diffusion tensor }\left[\mathrm{L}^{2} \mathrm{~T}^{-1}\right] ; \\
\varepsilon_{\mathrm{v}} & \text { time weighting factor related to advection [-]; } \\
\varepsilon_{\mathrm{d}} & \text { time weighting factor related to dispersion [-], and } \\
\varepsilon_{\mathrm{a}} & \text { time weighting factor related to artificial diffusion [-]. }
\end{array}
$$

To obtain a symmetric matrix $\varepsilon_{\mathrm{v}}$ must be set to 0 . For second-order accuracy $\varepsilon_{\mathrm{d}}$ and $\varepsilon_{\mathrm{a}}$ should be set to $1 / 2$ (Leismann and Frind, 1989).

The time weighted equation is spatially weighted using the standard Galerkin finite element method and the resulting matrix equation is solved for nodal values of 
$T^{\mathrm{n}+1}$.

Starting with thermal transport equation (B2.1)

$\frac{\partial}{\partial x_{i}}\left[\left(\kappa_{i j}+\frac{D_{i j}}{R}\right) \frac{\partial T}{\partial x_{j}}\right]-\left(\frac{v_{i}}{R} \frac{\partial T}{\partial x_{i}}\right)+\sum_{k=1}^{N} \frac{Q_{k}(t)\left[T_{k}(t)-T\right]}{R \phi} \delta\left(x_{k}^{\prime}, y_{k}^{\prime}, z_{k}^{\prime}\right)=\frac{\partial T}{\partial t}$

and expressing the time derivative using (B5.1) gives

$$
\begin{gathered}
\frac{\partial}{\partial x_{i}}\left[\varepsilon_{d}\left(\kappa_{i j}+\frac{D_{i j}}{R}\right)+\varepsilon_{a} D_{i j}^{*}\right] \frac{\partial T^{n+1}}{\partial x_{j}}-\varepsilon_{v} \frac{v_{i}}{R} \frac{\partial T^{n+1}}{\partial x_{i}} \\
+\varepsilon_{q} \sum_{k=1}^{N} \frac{Q_{k}^{n+1}\left(T_{k}^{n+1}-T^{n+1}\right)}{R \phi} \delta\left(x_{k}^{\prime}, y_{k}^{\prime}, z_{k}^{\prime}\right)-\frac{T^{n+1}}{\Delta t} \\
=-\frac{\partial}{\partial x_{i}}\left[\varepsilon_{d}^{\prime}\left(\kappa_{i j}+\frac{D_{i j}}{R}\right)+\varepsilon_{a}^{\prime} D_{i j}^{*}\right] \frac{\partial T^{n}}{\partial x_{j}}+\varepsilon_{v}^{\prime} \frac{v_{i}}{R} \frac{\partial T^{n}}{\partial x_{i}} \\
-\varepsilon_{q}^{\prime} \sum_{k=1}^{N} \frac{Q_{k}^{n}\left(T_{k}^{n}-T^{n}\right)}{R \phi} \delta\left(x_{k}^{\prime}, y_{k}^{\prime}, z_{k}^{\prime}\right)-\frac{T^{n}}{\Delta t}
\end{gathered}
$$

where $D_{i j}^{*}=v_{i} v_{j} \frac{\Delta t}{2} \quad$ is the artificial diffusion term $\left[\mathrm{L}^{2} \mathrm{~T}^{-1}\right]$;

$\Delta t$ is the time step [T];

$\varepsilon \quad$ is the time weighting factor at the $n+1$ time level [-], and

$\varepsilon^{\prime}=1-\varepsilon \quad$ is the time weighting factor at the $n$ time level [-].

Specifically, the optimum time weighting factors for second order accuracy are $\varepsilon_{d}=1$;

$\varepsilon_{a}=1 / 2$

$\varepsilon_{v}=0$, and

$\varepsilon_{q}=1 / 2$. 
From the time-discretized transport equation (B5.2) we can define the following

$$
L\left(T^{*}\right)=\beta\left(T^{n+1}\right)-\beta\left(T^{n}\right)=0
$$

where

$$
\begin{aligned}
& \beta\left(T^{n+1}\right)=\left[\varepsilon_{d}\left(\kappa_{i j}+\frac{D_{i j}}{R}\right)+\varepsilon_{a} D_{i j}^{*}\right] \frac{\partial^{2} T^{n+1}}{\partial x_{i} \partial x_{j}}-\varepsilon_{v} \frac{v_{i}}{R} \frac{\partial T^{n+1}}{\partial x_{i}}+\varepsilon_{q} \sum_{k=1}^{N} \frac{Q_{k}^{n+1}\left(T_{k}^{n+1}-T^{n+1}\right)}{R \phi} \delta\left(x_{k}^{\prime}, y_{k}^{\prime}, z_{k}^{\prime}\right)-\frac{T^{n+1}}{\Delta t} \\
& \beta\left(T^{n}\right)=-\left[\varepsilon_{d}^{\prime}\left(\kappa_{i j}+\frac{D_{i j}}{R}\right)+\varepsilon_{a}^{\prime} D_{i j}^{*}\right] \frac{\partial^{2} T^{n}}{\partial x_{i} \partial x_{j}}+\varepsilon_{v}^{\prime} \frac{v_{i}}{R} \frac{\partial T^{n}}{\partial x_{i}}-\varepsilon_{q}^{\prime} \sum_{k=1}^{N} \frac{Q_{k}^{n}\left(T_{k}^{n}-T^{n}\right)}{R \phi} \delta\left(x_{k}^{\prime}, y_{k}^{\prime}, z_{k}^{\prime}\right)-\frac{T^{n}}{\Delta t}
\end{aligned}
$$

Applying the Galerkin method of weighted residuals, we chose weighting functions $W_{\mathrm{i}}$ with the same form as the linear basis functions $N_{\mathrm{j}}$ and we force the weighted average of the residuals to be zero over the problem domain which gives the finite element thermal transport equation as

$$
\iiint_{V}\left\{\beta\left(\hat{T}^{n+1}\right)-\beta\left(\hat{T}^{n}\right)\right\}_{N_{i}}(x, y, z) d V=0
$$

Equation (B5.8) can be written in matrix form as

$$
\begin{gathered}
\left(\left[M_{i j}^{d}\right]+\left[M_{i j}^{a}\right]+\left[M_{i j}^{v}\right]+\left[M_{i j}^{Q}\right]+\frac{1}{\Delta t}\left[M_{i j}^{t}\right]\right)\{T\}^{n+1}=-\left(\left[M_{i j}^{d}\right]+\left[M_{i j}^{a}\right]+\left[M_{i j}^{v}\right]+\left[M_{i j}^{Q}\right]-\frac{1}{\Delta t}\left[M_{i j}^{t}\right]\right)\{T\}^{n} \\
+\left\{F_{i}\right\}+\left\{F_{i}^{Q}\right\}^{n}+\left\{F_{i}^{Q}\right\}^{n+1}
\end{gathered}
$$

In the Leismann time weighting scheme, the boundary conditions appearing in the heat flux vector $\{F\}$ are formulated on the basis of the physical conditions alone and are not weighted using the Leismann scheme. For second order accuracy these terms should 
be centrally weighted (Leismann and Frind, 1989), with $\varepsilon=1 / 2$. Applying timeweighting to the flux vector, and accounting for $\varepsilon_{d}=1$ and $\varepsilon_{v}=0$, gives

$$
\begin{aligned}
\left(\left[M_{i j}^{d}\right]+\left[M_{i j}^{a}\right]+\left[M_{i j}^{Q}\right]+\frac{1}{\Delta t}\left[M_{i j}^{t}\right]\right)\{T\}^{n+1}=-\left(\left[M_{i j}^{a}\right]+\left[M_{i j}^{v}\right]+\left[M_{i j}^{Q}\right]-\frac{1}{\Delta t}\left[M_{i j}^{t}\right]\right)\{T\}^{n} \\
+(1-\varepsilon)\left\{F_{i}\right\}^{n}+\varepsilon\left\{F_{i}\right\}^{n+1} \\
+\left\{F_{i}^{Q}\right\}^{n}+\left\{F_{i}^{Q}\right\}^{n+1}
\end{aligned}
$$

where $M$ and $F$ represent matrices and vectors, respectively, and specifically

$\left[M_{i j}^{d}\right]$ is the physical dispersion term;

$\left[M_{i j}^{a}\right]$ is the artificial dispersion term;

$\left[M_{i j}^{v}\right]$ is the advective term;

$\left[M_{i j}^{Q}\right]$ is the internal source/sink term;

$\left[M_{i j}^{t}\right]$ is the mass storage term;

$\left\{F_{i}\right\}$ is the heat flux vector resulting from all the boundary conditions;

$\left\{F_{i}^{Q}\right\}$ is the heat flux vector resulting from internal well point source/sinks, with $n+1$ and $n$ corresponding to the $\beta\left(\hat{T}^{n+1}\right)$ and $\beta\left(\hat{T}^{n}\right)$ terms, and

$\varepsilon \quad$ is the time weighting factor applied to the boundary flux terms.

After expanding the indices the terms in (B5.9) are

$$
\begin{aligned}
{\left[M_{i j}^{d}\right]=} & \sum_{e}\left\langle\iiint_{V}\left\{\left[\varepsilon_{d}\left(\kappa_{x x}+\frac{D_{x x}}{R}\right)\right] \frac{\partial N_{i}}{\partial x} \frac{\partial N_{j}}{\partial x}+\left[\varepsilon_{d}\left(\kappa_{x y}+\frac{D_{x y}}{R}\right)\right] \frac{\partial N_{i}}{\partial x} \frac{\partial N_{j}}{\partial y}+\left[\varepsilon_{d}\left(\kappa_{x z}+\frac{D_{x z}}{R}\right)\right] \frac{\partial N_{i}}{\partial x} \frac{\partial N_{j}}{\partial z}\right\} d V\right. \\
& +\iiint_{V}\left\{\left[\varepsilon_{d}\left(\kappa_{y x}+\frac{D_{y x}}{R}\right)\right] \frac{\partial N_{i}}{\partial y} \frac{\partial N_{j}}{\partial x}+\left[\varepsilon_{d}\left(\kappa_{y y}+\frac{D_{y y}}{R}\right)\right] \frac{\partial N_{i}}{\partial y} \frac{\partial N_{j}}{\partial y}+\left[\varepsilon_{d}\left(\kappa_{y z}+\frac{D_{y z}}{R}\right)\right] \frac{\partial N_{i}}{\partial y} \frac{\partial N_{j}}{\partial z}\right\} d V \\
& \left.+\iiint_{V}\left\{\left[\varepsilon_{d}\left(\kappa_{z x}+\frac{D_{z x}}{R}\right)\right] \frac{\partial N_{i}}{\partial z} \frac{\partial N_{j}}{\partial x}+\left[\varepsilon_{d}\left(\kappa_{z y}+\frac{D_{z y}}{R}\right)\right] \frac{\partial N_{i}}{\partial z} \frac{\partial N_{j}}{\partial y}+\left[\varepsilon_{d}\left(\kappa_{z z}+\frac{D_{z z}}{R}\right)\right] \frac{\partial N_{i}}{\partial z} \frac{\partial N_{j}}{\partial z}\right\} d V\right\}
\end{aligned}
$$




$$
\begin{aligned}
& {\left[M_{i j}^{a}\right]=} \sum_{e}\left\langle\iiint_{V}\left\{\varepsilon_{a} D_{x x}^{*} \frac{\partial N_{i}}{\partial x} \frac{\partial N_{j}}{\partial x}+\varepsilon_{a} D_{x y}^{*} \frac{\partial N_{i}}{\partial x} \frac{\partial N_{j}}{\partial y}+\varepsilon_{a} D_{x z}^{*} \frac{\partial N_{i}}{\partial x} \frac{\partial N_{j}}{\partial z}\right\} d V\right. \\
&+\iiint_{V}\left\{\varepsilon_{a} D_{y x}^{*} \frac{\partial N_{i}}{\partial y} \frac{\partial N_{j}}{\partial x}+\varepsilon_{a} D_{y y}^{*} \frac{\partial N_{i}}{\partial y} \frac{\partial N_{j}}{\partial y}+\varepsilon_{a} D_{y z}^{*} \frac{\partial N_{i}}{\partial y} \frac{\partial N_{j}}{\partial z}\right\} d V \\
&\left.+\iiint_{V}\left\{\varepsilon_{a} D_{z x}^{*} \frac{\partial N_{i}}{\partial z} \frac{\partial N_{j}}{\partial x}+\varepsilon_{a} D_{z y}^{*} \frac{\partial N_{i}}{\partial z} \frac{\partial N_{j}}{\partial y}+\varepsilon_{a} D_{z z}^{*} \frac{\partial N_{i}}{\partial z} \frac{\partial N_{j}}{\partial z}\right\} d V\right\rangle \\
& {\left[M_{i j}^{v}\right]=} \sum_{e} \iiint_{V}\left\{\varepsilon_{v} \frac{v_{x}}{R} \frac{\partial N_{j}}{\partial x}+\varepsilon_{v} \frac{v_{y}}{R} \frac{\partial N_{j}}{\partial y}+\varepsilon_{v} \frac{v_{z}}{R} \frac{\partial N_{j}}{\partial z}\right\} N_{i} d V \\
& {\left[M_{i j}^{Q}\right]=} \sum_{e}\left\{\varepsilon_{q} \sum_{k=1}^{N} \frac{Q_{k}^{\prime}\left(x_{k}^{\prime}, y_{k}^{\prime}, z_{k}^{\prime}, t^{n+1}\right)}{R^{e} \phi^{e}}\right\} \\
&\left\{F_{i}^{Q}\right\}^{n+1}= \sum_{e}\left\{\varepsilon_{q} \sum_{k=1}^{N} \frac{Q_{k}^{\prime}\left(x_{k}^{\prime}, y_{k}^{\prime}, z_{k}^{\prime}, t^{n+1}\right) T_{k}^{n+1}}{R^{e} \phi^{e}}\right\} \\
& {\left[F_{i j}^{t}\right]=} \sum_{e} \iiint_{V} N_{i} N_{j} d V \\
& {\left[F_{i}=\sum_{\Gamma^{e}} \iint_{i} N_{i} q_{n}^{h} d \Gamma\right.}
\end{aligned}
$$

\section{Appendix B6: Solution Strategy}

Equations (B4.4) and (B5.9) form a transient, nonlinear system that is coupled through Darcy's equation. Nonlinearities exist in the temperature-dependent hydraulic conductivity $K_{\mathrm{ij}}(T)$, and the relative and absolute densities $\rho_{\mathrm{r}}(T)$ and $\rho_{\mathrm{w}}(T)$ (Molson et al., 1992). As well, the unconfined position of the water table constitutes a further nonlinearity. In Heatflow, these nonlinearities in the coupled equations are accommodated by centering the nonlinear terms in time and iterating the solution to the specified convergence tolerance. 
In groundwater flow and transport models where the elements are relatively simple and undeformed, the integrations in (B4.5a to d) and (B5.10a to f) may be performed analytically which results in relatively quick execution times. For cases where the elements are deformed and there are large numbers of nodes, the integrations are performed numerically. The numerical integrations are made easier if the interpolation functions for the elements are expressed in a local coordinate system. This is most easily done by transforming the matrix equations from a global coordinate system to a local coordinate system, performing the numerical integration in local coordinates, and transforming the results back to the global coordinated system. Heatflow provides for both integration schemes.

In this study, the treatment of the expressions in the gravity-density term, Eq. (B4.6c) appearing in the finite-element groundwater equation, were modified in Heatflow. A brief discussion of the resulting expressions used in both the numerical and analytical integration schemes, within Heatflow, is presented below. A discussion on local and global coordinates, basis functions, weighting functions, and coordinate transforms may be found in several references on finite element methods (e.g., Istok, 1989; Voss and Provost, 2003; Zienkiewicz et al., 2005; Diersch, 2005). As well, the treatment of the remaining terms appearing in the groundwater flow equation and the heat transport equation can be found in these references and elsewhere, and are not presented here.

\section{Consistent evaluation of the head gradient and density-gravity terms}

The equation for groundwater flow (B1.1) contains the term

$$
\frac{\partial h^{*}}{\partial x_{j}}+\rho_{r}(T) \tilde{n}_{j}
$$

As previously noted, the calculation of this term requires some special attention, for density-dependent flow. This results from the lower-order spatial approximation attainable for head gradients which can conflict with the high-order spatial approximation 
attainable in the gravity or buoyancy term. A detailed explanation showing how this difference in the order of the spatial approximation arises is given by Diersch (2005). Several modified schemes termed consistent velocity approximation have been developed to address this issue (i.e., Voss and Souza, 1987; Herbert et al., 1998; Leijnse, 1992; Knabner and Frolkovič, 1996). In the original version of Heatflow implemented by Molson et al., (1992), the consistency in the spatial interpolation of the terms $\rho_{r}(T) \tilde{n}_{j}$ and $\partial h^{*} / \partial x_{j}$, Eq. (B1.1), or $\partial \hat{u} / \partial x_{j}$, Eq. (B4.4), was handled by averaging the nodal temperatures for each element, $\bar{T}^{e}=\sum_{i=1}^{8} T_{i}^{e}$, and using this average to calculate the relative density, effectively yielding an average relative density at the element centroid. This procedure was similar to that used by Frind (1982) where the relative density was calculated using an average concentration in each element. In this study, to maintain consistent spatial discretization of the density-gravity term $\rho_{r}(T) \tilde{n}_{j}$ and the head gradient term $\partial \hat{u} / \partial x_{j}$, I have adopted a more rigorous approach proposed by Knabner and Frolkovič (1996). They proposed integral functions of the relative density-gravity term to obtain the same spatial variability (consistency) as the head gradient term. These integral functions and the associated basis functions are provided below for the 3D linear hexahedral (brick) element.

In the finite element method, basis functions, weighting functions, and their derivatives may be described in local element geometry. In the 3D local coordinate system used in Heatflow, each local element is a two-by-two-by-two cube. The origin of the $3 \mathrm{D}$ local coordinates, $\varepsilon, \eta$, and $\zeta$, is at the centre of the element, and node numbers and coordinates are shown below. 


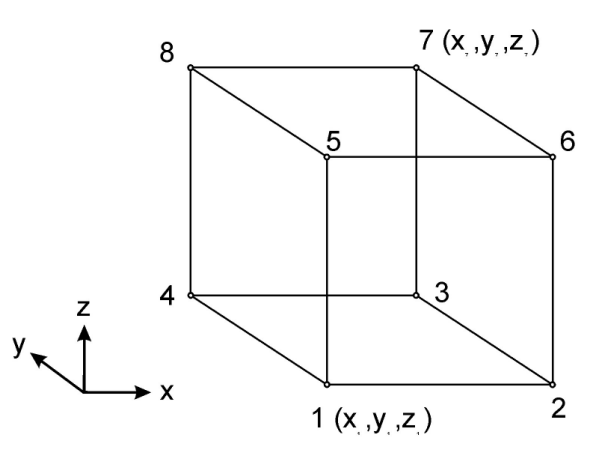

Global coordinates

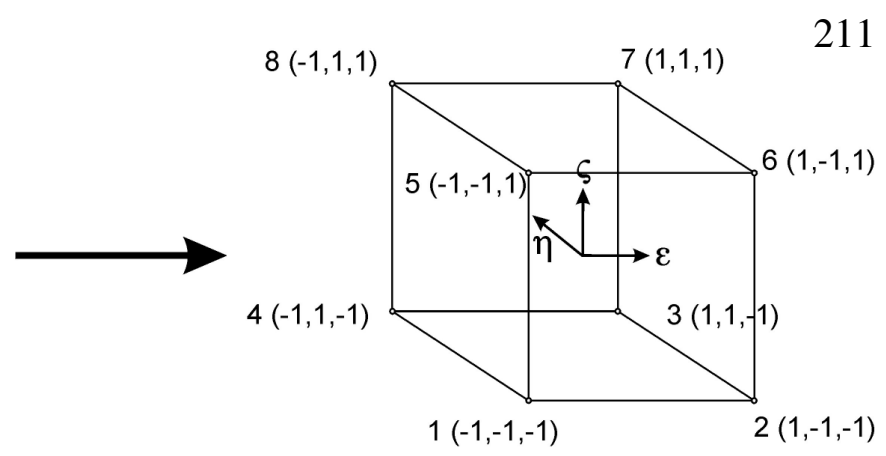

Local coordinates

Figure B6-1 Finite element in global and local coordinates.

In their development of the consistent velocity approximation, Knabner and

Frolkovič (1996) introduced the following integral functions for the density-gravity term

$$
\begin{aligned}
& H_{\varepsilon}=H_{\varepsilon}(\varepsilon, \eta, \zeta)=\int_{0}^{\varepsilon} \rho_{r}(\theta, \eta, \zeta) n_{\varepsilon}(\theta, \eta, \zeta) d \theta \\
& H_{\eta}=H_{\eta}(\varepsilon, \eta, \zeta)=\int_{0}^{\eta} \rho_{r}(\varepsilon, \theta, \zeta) n_{\eta}(\varepsilon, \theta, \zeta) d \theta \\
& H_{\zeta}=H_{\zeta}(\varepsilon, \eta, \zeta)=\int_{0}^{\zeta} \rho_{r}(\varepsilon, \eta, \theta) n_{\zeta}(\varepsilon, \eta, \theta) d \theta
\end{aligned}
$$

which leads to

$$
\left\{\begin{array}{l}
\frac{\partial H_{\varepsilon}}{\partial \varepsilon} \\
\frac{\partial H_{\eta}}{\partial \eta} \\
\frac{\partial H_{\zeta}}{\partial \zeta}
\end{array}\right\}=\rho_{r} \tilde{n}_{(\varepsilon, \eta, \zeta)}
$$

These integral functions provide the same spatial variability (consistent) for both the density-gravity term $\rho_{r}(T) \tilde{n}_{j}$ and the head term $\partial \hat{u} / \partial x_{j}$. 
Following the finite element method, the integral functions are interpolated by their nodal basis functions:

$$
\begin{aligned}
H_{\varepsilon} & =\sum_{j=1}^{8} H_{\varepsilon j} N_{j}(\varepsilon, \eta, \zeta) & \frac{\partial H_{\varepsilon}}{\partial \varepsilon} & =\sum_{j=1}^{8} H_{\varepsilon j} \frac{\partial N_{j}(\varepsilon, \eta, \zeta)}{\partial \varepsilon} \\
H_{\eta} & =\sum_{j=1}^{8} H_{\eta j} N_{j}(\varepsilon, \eta, \zeta) & \frac{\partial H_{\eta}}{\partial \eta} & =\sum_{j=1}^{8} H_{\eta j} \frac{\partial N_{j}(\varepsilon, \eta, \zeta)}{\partial \eta} \\
H_{\zeta} & =\sum_{j=1}^{8} H_{\zeta j} N_{j}(\varepsilon, \eta, \zeta) & \frac{\partial H_{\zeta}}{\partial \zeta} & =\sum_{j=1}^{8} H_{\zeta j} \frac{\partial N_{j}(\varepsilon, \eta, \zeta)}{\partial \zeta}
\end{aligned}
$$

and relative density term is evaluated by

$\rho_{r}(T)=\sum_{j=1}^{8} \rho_{r j} N_{j}(\varepsilon, \eta, \zeta)$

where $\rho_{r j} \quad$ is the relative density value at node $j$.

The integral functions at the corner nodes for the element in the local coordinate system are obtained by substituting (B6.3) into (B6.1a) and completing the integrations giving

$$
\begin{array}{rlrl}
H_{\varepsilon}(-1,-1,-1) & =H_{\varepsilon 1}=-\frac{1}{4} n_{\varepsilon}(-1,-1)\left(3 \rho_{r 1}+\rho_{r 2}\right) & H_{\eta}(-1,-1,-1)=H_{\eta 1}=-\frac{1}{4} n_{\eta}(-1,-1)\left(3 \rho_{r 1}+\rho_{r 4}\right) \\
H_{\varepsilon}(1,-1,-1)=H_{\varepsilon 2}=\frac{1}{4} n_{\varepsilon}(-1,-1)\left(\rho_{r 1}+3 \rho_{r 2}\right) & H_{\eta}(1,-1,-1)=H_{\eta 2}=-\frac{1}{4} n_{\eta}(1,-1)\left(3 \rho_{r 2}+\rho_{r 3}\right) \\
H_{\varepsilon}(1,1,-1)=H_{\varepsilon 3}=\frac{1}{4} n_{\varepsilon}(1,-1)\left(3 \rho_{r 3}+\rho_{r 4}\right) & H_{\eta}(1,1,-1)=H_{\eta 3}=\frac{1}{4} n_{\eta}(1,-1)\left(\rho_{r 2}+3 \rho_{r 3}\right) \\
H_{\varepsilon}(-1,1,-1)=H_{\varepsilon 4}=-\frac{1}{4} n_{\varepsilon}(1,-1)\left(\rho_{r 3}+3 \rho_{r 4}\right) & H_{\eta}(-1,1,-1)=H_{\eta 4}=\frac{1}{4} n_{\eta}(-1,-1)\left(\rho_{r 1}+3 \rho_{r 4}\right) \\
H_{\varepsilon}(-1,-1,1) & =H_{\varepsilon 5}=-\frac{1}{4} n_{\varepsilon}(-1,1)\left(3 \rho_{r 5}+\rho_{r 6}\right) & H_{\eta}(-1,-1,1)=H_{\eta 5}=-\frac{1}{4} n_{\eta}(-1,1)\left(3 \rho_{r 5}+\rho_{r 8}\right) \\
H_{\varepsilon}(1,-1,1)=H_{\varepsilon 6}=\frac{1}{4} n_{\varepsilon}(-1,1)\left(\rho_{r 5}+3 \rho_{r 6}\right) & H_{\eta}(1,-1,1)=H_{\eta 6}=-\frac{1}{4} n_{\eta}(1,1)\left(3 \rho_{r 6}+\rho_{r 7}\right) \\
H_{\varepsilon}(1,1,1)=H_{\varepsilon 7}=\frac{1}{4} n_{\varepsilon}(1,1)\left(3 \rho_{r 7}+\rho_{r 8}\right) \\
H_{\varepsilon}(-1,1,1)=H_{\varepsilon 8}=-\frac{1}{4} n_{\varepsilon}(1,1)\left(\rho_{r 7}+3 \rho_{r 8}\right) & H_{\eta}(1,1,1)=H_{\eta 7}=\frac{1}{4} n_{\eta}(1,1)\left(\rho_{r 6}+3 \rho_{r 7}\right) \\
H_{\eta}(-1,1,1)=H_{\eta 8}=\frac{1}{4} n_{\eta}(-1,1)\left(\rho_{r 5}+3 \rho_{r 8}\right)
\end{array}
$$




$$
\begin{aligned}
H_{\zeta}(-1,-1,-1) & =H_{\zeta 1}=-\frac{1}{4} n_{\zeta}(-1,-1)\left(3 \rho_{r 1}+\rho_{r 5}\right) \\
H_{\zeta}(1,-1,-1) & =H_{\zeta 2}=-\frac{1}{4} n_{\zeta}(1,-1)\left(3 \rho_{r 2}+\rho_{r 6}\right) \\
H_{\zeta}(1,1,-1) & =H_{\zeta 3}=-\frac{1}{4} n_{\zeta}(1,1)\left(3 \rho_{r 3}+\rho_{r 7}\right) \\
H_{\zeta}(-1,1,-1) & =H_{\zeta 4}=-\frac{1}{4} n_{\zeta}(-1,1)\left(3 \rho_{r 4}+\rho_{r 8}\right) \\
H_{\zeta}(-1,-1,1) & =H_{\zeta 5}=\frac{1}{4} n_{\zeta}(-1,-1)\left(\rho_{r 1}+3 \rho_{r 5}\right) \\
H_{\zeta}(1,-1,1) & =H_{\zeta 6}=\frac{1}{4} n_{\zeta}(1,-1)\left(\rho_{r 2}+3 \rho_{r 6}\right) \\
H_{\zeta}(1,1,1) & =H_{\zeta 7}=\frac{1}{4} n_{\zeta}(1,1)\left(\rho_{r 3}+3 \rho_{r 7}\right) \\
H_{\zeta}(-1,1,1) & =H_{\zeta 8}=\frac{1}{4} n_{\zeta}(-1,1)\left(\rho_{r 4}+3 \rho_{r 8}\right)
\end{aligned}
$$

In the version of Heatflow implemented by Molson et al. (1992), the spatial interpolation of the density-gravity term was kept consistent with the head gradient term by averaging the nodal temperatures within each element and calculating an average density at the element centroid. For this case, the density-gravity vector given by (B4.6c) can be expanded and written in local coordinates as

$$
\{G\}_{L}^{e}=\iiint\left[\begin{array}{ccc}
\frac{\partial N_{1}}{\partial \varepsilon} & \frac{\partial N_{1}}{\partial \eta} & \frac{\partial N_{1}}{\partial \zeta} \\
\vdots & \vdots & \vdots \\
\frac{\partial N_{8}}{\partial \varepsilon} & \frac{\partial N_{8}}{\partial \eta} & \frac{\partial N_{8}}{\partial \zeta}
\end{array}\right]\left[J^{-1}\right]^{T}\left[\begin{array}{ccc}
K_{x x}^{e} & 0 & 0 \\
0 & K_{y y}^{e} & 0 \\
0 & 0 & K_{z z}^{e}
\end{array}\right]\left[J^{-1}\right]\left\{\begin{array}{l}
\bar{\rho}_{r}^{e} n_{\varepsilon} \\
\bar{\rho}_{r}^{e} n_{\eta} \\
\bar{\rho}_{r}^{e} n_{\zeta}
\end{array}\right\} \operatorname{det} J d \varepsilon d \eta d \zeta
$$

where $\bar{\rho}_{r}^{e}$ is the average relative density in the element. The gravity vector $\left\{n_{\mathrm{x}}, n_{\mathrm{y}}, n_{\mathrm{z}}\right\}$ is given by $\{0,0,-1\}$ where the positive $\mathrm{z}$-axis is aligned with the vertical upward direction. In this study, I implemented the algorithm proposed by Knabner and Frolkovič (1996). Making use of Eq. (B6.1a) to (B6.3) with Eq. (B4.6c) yields the following density-gravity vector 


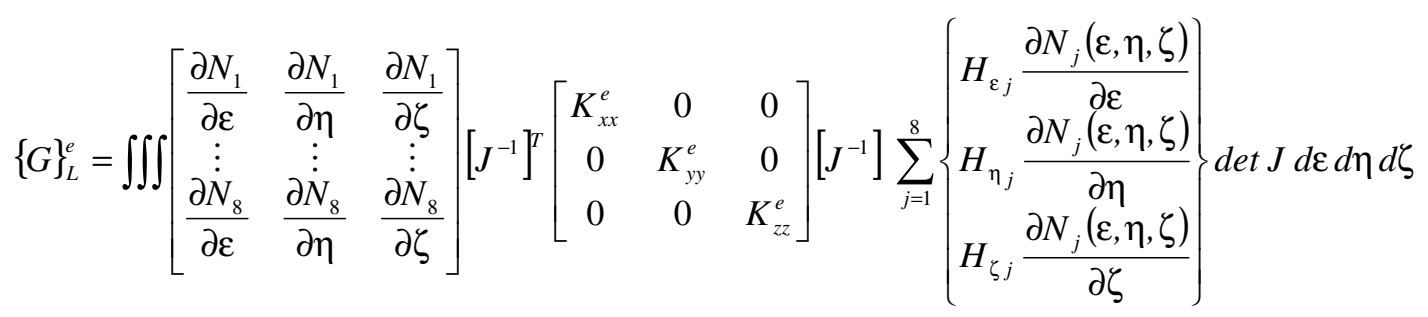


where

$$
\begin{aligned}
\sum_{j=1}^{8}\left\{\begin{array}{r}
H_{\varepsilon j} \frac{\partial N_{j}(\varepsilon, \eta, \zeta)}{\partial \varepsilon} H_{\eta_{j}} \frac{\partial N_{j}(\varepsilon, \eta, \zeta)}{\partial \eta} \\
\left.H_{\zeta j} \frac{\partial N_{j}(\varepsilon, \eta, \zeta)}{\partial \zeta}\right\}
\end{array}\right\} & \left\{\begin{array}{l}
\rho_{r} n_{\varepsilon} \\
\rho_{r} n_{\eta} \\
\rho_{r} n_{\zeta}
\end{array}\right\} \\
& =\left\{\begin{array}{r}
\frac{1}{8}\left[\begin{array}{r}
n_{\varepsilon}(-1,-1)\left(\rho_{r 1}+\rho_{r 2}\right)(1-\eta)(1-\zeta)+n_{\varepsilon}(1,-1)\left(\rho_{r 3}+\rho_{r 4}\right)(1+\eta)(1-\zeta) \\
+n_{\varepsilon}(-1,1)\left(\rho_{r 5}+\rho_{r 6}\right)(1-\eta)(1+\zeta)+n_{\varepsilon}(1,1)\left(\rho_{r 7}+\rho_{r 8}\right)(1+\eta)(1+\zeta)
\end{array}\right] \\
\frac{1}{8}\left[\begin{array}{r}
n_{\eta}(-1,-1)\left(\rho_{r 1}+\rho_{r 4}\right)(1-\varepsilon)(1-\zeta)+n_{\eta}(1,-1)\left(\rho_{r 2}+\rho_{r 3}\right)(1+\varepsilon)(1-\zeta) \\
+n_{\eta}(-1,1)\left(\rho_{r 5}+\rho_{r 8}\right)(1-\varepsilon)(1+\zeta)+n_{\eta}(1,1)\left(\rho_{r 6}+\rho_{r 7}\right)(1+\varepsilon)(1+\zeta)
\end{array}\right] \\
\frac{1}{8}\left[\begin{array}{r}
n_{\zeta}(-1,-1)\left(\rho_{r 1}+\rho_{r 5}\right)(1-\varepsilon)(1-\eta)+n_{\zeta}(1,-1)\left(\rho_{r 2}+\rho_{r 6}\right)(1+\varepsilon)(1-\eta) \\
+n_{\zeta}(1,1)\left(\rho_{r 3}+\rho_{r 7}\right)(1+\varepsilon)(1+\eta)+n_{\zeta}(-1,1)\left(\rho_{r 4}+\rho_{r 8}\right)(1-\varepsilon)(1+\eta)
\end{array}\right]
\end{array}\right\}
\end{aligned}
$$

Equation (B6.7) is solved for each element at the nodes, or for numerical integration at the Gauss points, and averaged for the element. The resulting gravity-density term is consistent with the head gradient term.

\section{Body force or density-gravity vector resulting from exact integration}

In the version of Heatflow implemented by Molson et al. (1992), where the positive zaxis is aligned vertically upward, the resulting elemental contribution of the densitygravity term resulting from the exact integration of (B6.5) is given as

$$
\{G\}^{e}=K_{z z}^{e} \bar{\rho}_{r} n_{z} \frac{L_{x} L_{y}}{4}\left\{\begin{array}{c}
-1 \\
-1 \\
-1 \\
-1 \\
1 \\
1 \\
1 \\
1
\end{array}\right\}
$$

To my knowledge the analytical expressions resulting from the exact integration 
of the density-gravity term (B6.7) for the algorithm of Knabner and Frolkovič (1996), are not available in the literature. Therefore, I completed the integrations in (B6.7) and present the density-gravity vector as follows

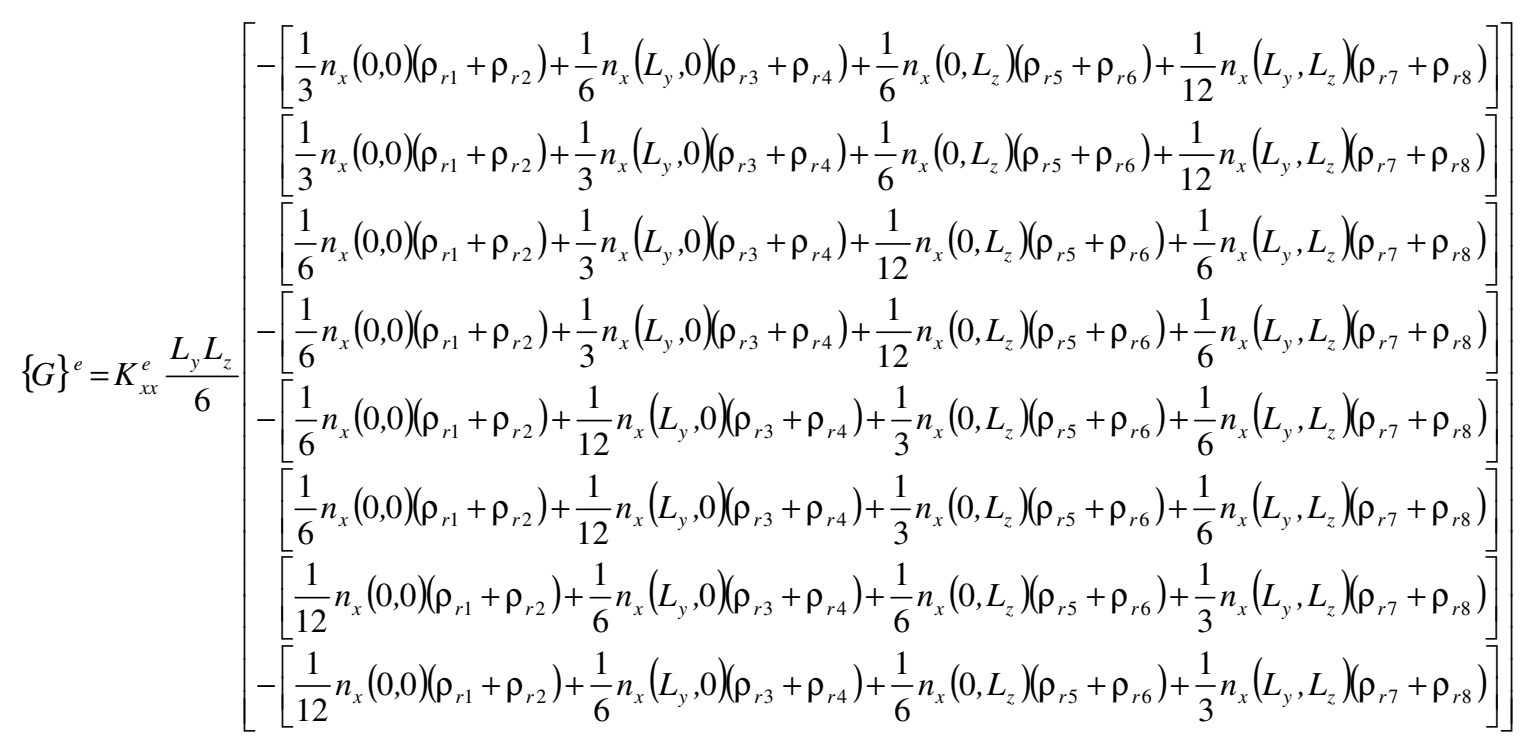

(B6.9a)

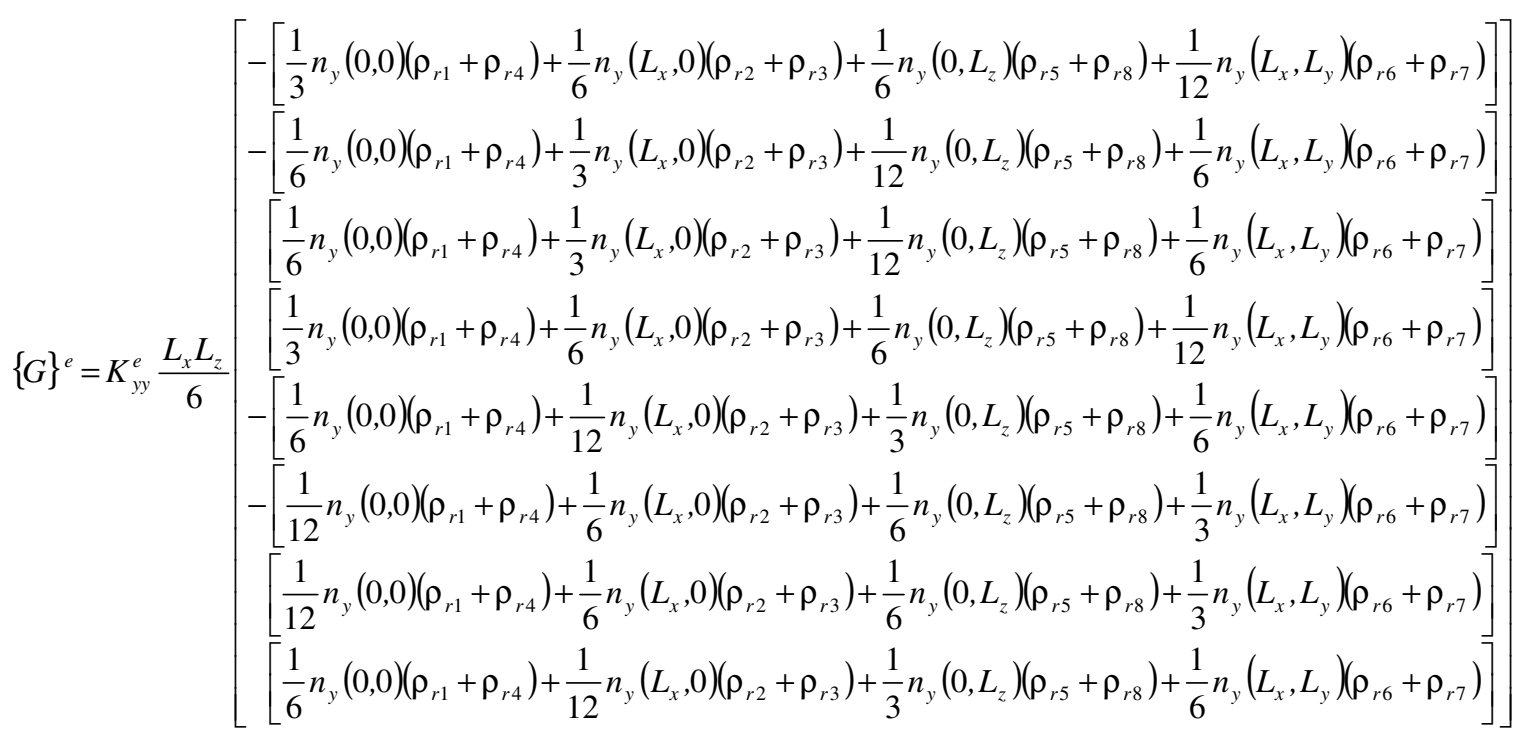

(B6.9b) 


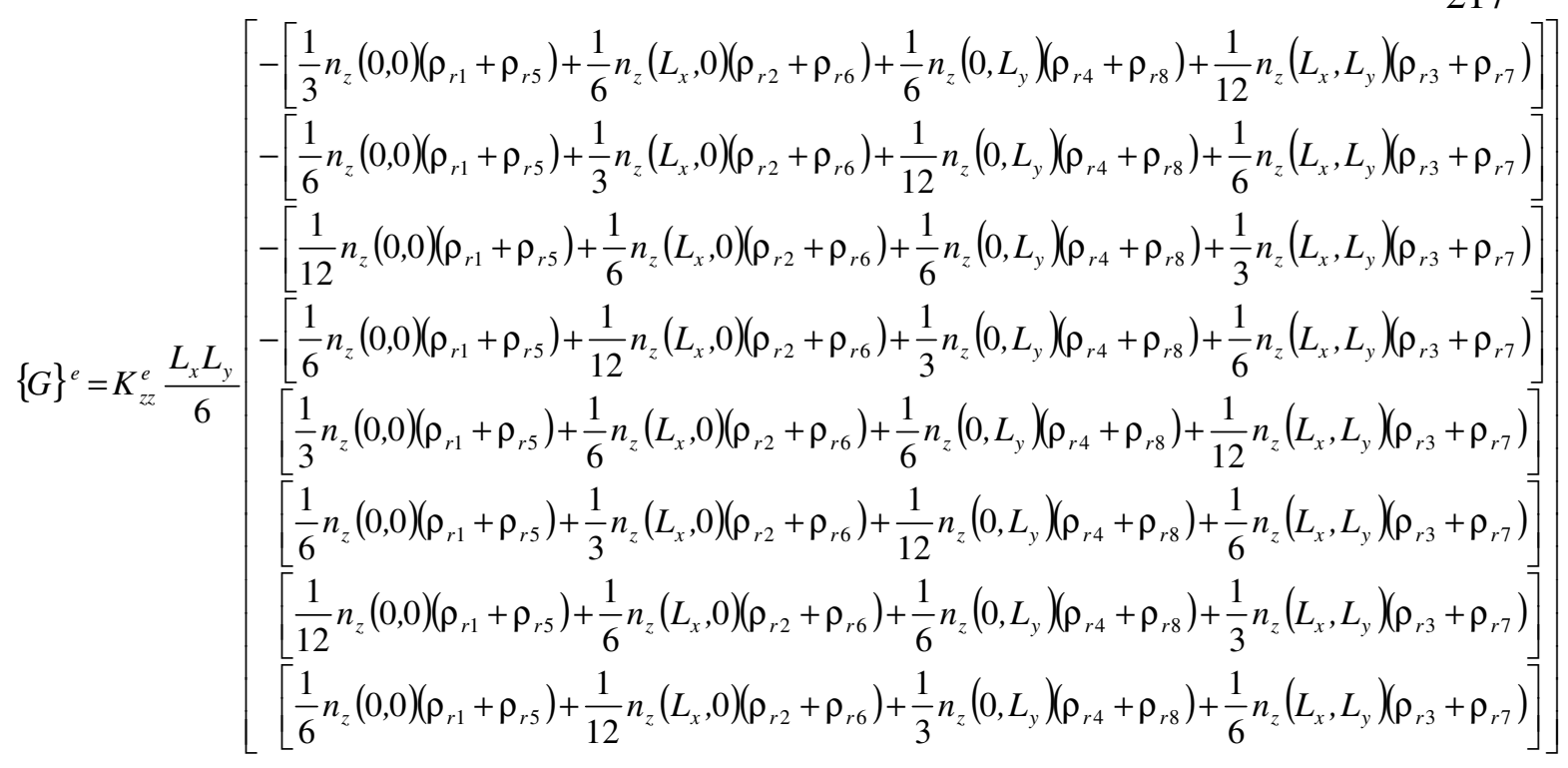

(B6.9a to c) are the new consistent formulations. For the orientation used in Heatflow, (B6.9a) and (B6.9b) are not used, and in (B6.9c) the gravity vector $n_{\mathrm{z}}(x, y)=-1$.

\section{References}

Istok, J., 1989. Groundwater Modeling by the Finite Element Method. Water Resources Monograph 13, American Geophysical Union.

Frind, E.O. 1982. Simulation of long-term transient density-dependent transport in groundwater. Advances in Water Resources 5:73-88.

Frolkovic, P., P. Knabner, C. Tapp, K. Thiele. 1997. Adaptive finite volume discretization of density driven flows in porous media.

Herbert, A.W., C.P. Jackson, D.A. Lever. 1988. Coupled groundwater flow and solute transport with fluid density strongly dependent on concentration. Water Resources Research 24:1781-1795.

Knabner, P., P. Frolkovic. 1996. Consistent velocity approximation for finite volume or element discretizations of density driven flow in porous media. In A.A. Aldama et. al., editor, Computational Methods in Water Resources XI, Volume 1 Computational Methods in Subsurface Flow and Transport Problems, Computational Mechanics Publications:93-100. 
Leijnse, A. 1992. Three-dimensional modeling of coupled flow and transport in porous media. PhD thesis. University of Notre Dame, Indiana.

Leismann, H.M., E.O. Frind. 1989. A symmetric-matrix time integration scheme for efficient solution of advection-dispersion problems. Water Resources Research 25:1133-1139.

Molson, J.W., E.O. Frind, C.D. Palmer. 1992. Thermal energy storage in an unconfined aquifer 2. Model development, validation, and application. Water Resources Research 28:2857-2867.

Diersch, H.-J.G. 2005. FEFLOW Finite Element Subsurface Flow and Transport Simulation System, White Papers Vol. 1:281 312.

Voss, C.I., A.M. Provost. 2003. SUTRA, A model for saturated-unsaturated, variabledensity ground-water flow with solute or energy transport. U.S. Geological Survey. Water-Resources Investigation Report 02-4231. 250 p.

Zienkiewicz. O.C., R.L. Taylor, J.Z. Zhu. 2005. The Finite Element Method: Its Basis and Fundamentals, Sixth Edition. Elsevier Publishing. 733 pp. 


\section{Appendix C: Benchmark Tests}

The benchmark problems presented here provide tests against which the performance of the finite element numerical code Heatflow may be compared. These comparisons help support the conclusion that the code is correctly solving the governing equations to variable-density groundwater flow and heat transport. As well, these benchmarks provided tests used to ensure changes to the Heatflow code, implemented in this study, did not inadvertently introduced errors into the code. It should be recognized that agreement between the simulations and these benchmark problems does not provide unequivocal confirmation that the simulation results for 3-dimensional flow and transport are correct, but it does help inspire confidence in the results.

\section{Appendix C1: One-dimensional heat conduction benchmark}

The ability of a numerical code to simulate pure conduction under hydrostatic conditions may be checked by comparison to the analytical solution of one-dimensional heat conduction in a finite domain (Fig. C1-1). Initially, the temperatures in the upper and lower halves of the domain are $20^{\circ} \mathrm{C}$ and $10^{\circ} \mathrm{C}$, respectively. The system is hydrostatic at all times and there is no flow. At the interface, heat conduction due to the temperature gradient will occur until the entire domain reaches an average steady state temperature of $15^{\circ} \mathrm{C}$. 


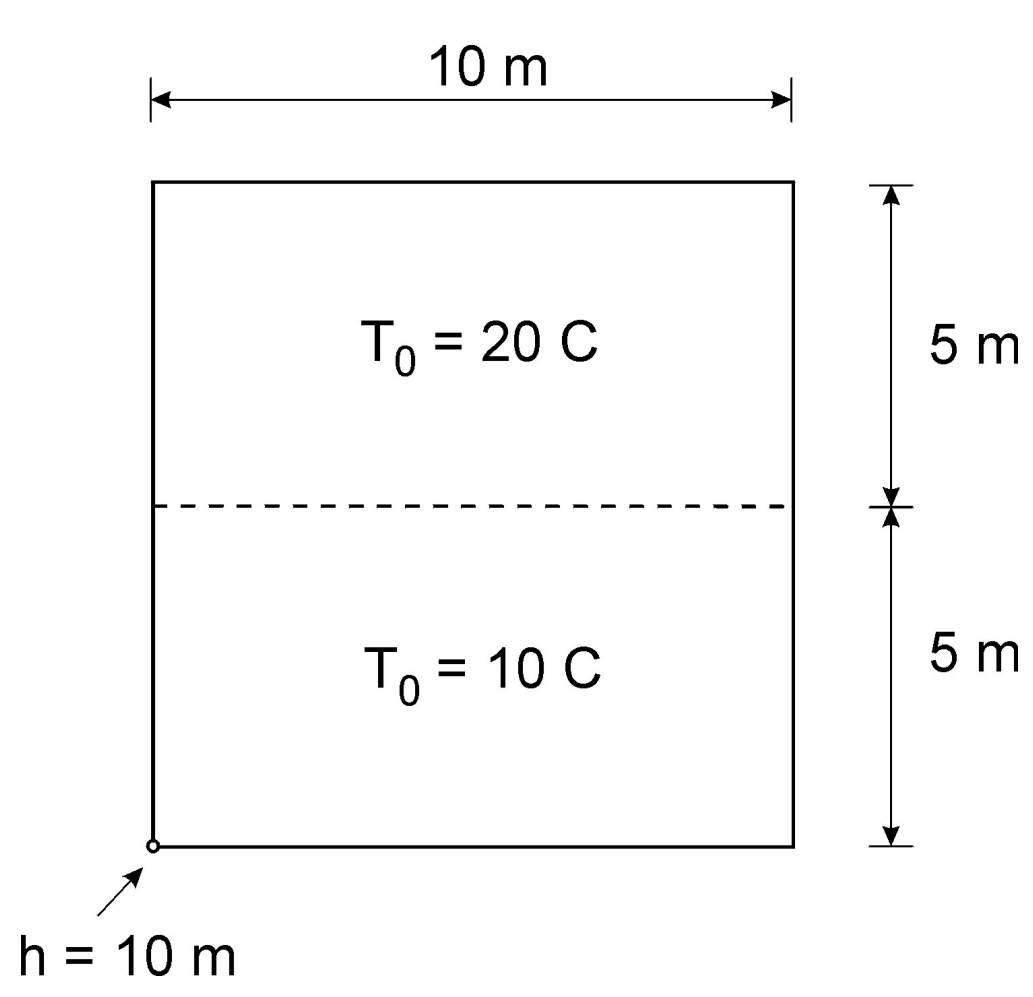

Figure C1-1 One-dimensional heat conduction in a finite domain benchmark.

For the purposes of this study, the domain modelled with Heatflow is a 2-dimensional domain with one element in the y-direction. The head is fixed at $10 \mathrm{~m}$ at the lower left corner node and all the boundaries are no flow and perfectly insulated. The choice of the hydraulic properties (i.e., permeability, porosity etc.) does not affect the solution.

The analytical solution of the heat conduction across the interface in a onedimensional finite domain may be adapted from Churchill (1972) and given by

$$
\begin{aligned}
T(z, t)= & T_{t}\left[\frac{1}{2}+\frac{2}{\pi} \sum_{n=1}^{\infty} \frac{(-1)^{n-1}}{2 n-1} \cos \frac{(2 n-1) \pi z}{l} \exp \left[-\frac{(2 n-1)^{2} \pi^{2} \kappa^{2} t}{4}\right]\right] \\
& +T_{b}\left[\frac{1}{2}-\frac{2}{\pi} \sum_{n=1}^{\infty} \frac{(-1)^{n-1}}{2 n-1} \cos \frac{(2 n-1) \pi z}{l} \exp \left[-\frac{(2 n-1)^{2} \pi^{2} \kappa^{2} t}{4}\right]\right]
\end{aligned}
$$

where $T_{\mathrm{t}} \quad$ is the temperature in the upper half of the domain;

$T_{\mathrm{b}} \quad$ is the temperature in the lower half of the domain;

$\kappa_{\mathrm{e}} \quad$ is the apparent thermal diffusivity given by $\lambda_{e} / C_{0}$, 
where $\lambda_{e}=\phi \lambda_{f}+(1-\phi) \lambda_{s} ; \quad C_{0}=\phi \rho_{f} c_{f}+(1-\phi) \rho_{s} c_{s}$

$t \quad$ is time, and

$z \quad$ is the vertical coordinate.

Table C1-1 lists the parameters used in the analytical and numerical models.

Table C1-1 Simulation parameters for heat conduction in a finite domain.

\begin{tabular}{|c|c|c|}
\hline Symbol & Parameter name & Value \\
\hline & domain length $^{\dagger}$ & $10 \mathrm{~m}$ \\
\hline & domain height & $10 \mathrm{~m}$ \\
\hline & element dimensions $^{\dagger}$ & $0.1 \times 0.1 \times 0.1 \mathrm{~m}$ \\
\hline & fluid & water \\
\hline$T_{\text {ref }}$ & reference temperature ${ }^{\dagger}$ & $4^{\circ} \mathrm{C}$ \\
\hline$\rho_{\text {ref }}$ & reference fluid density ${ }^{\dagger}$ & $1000 \mathrm{~kg} \mathrm{~m}^{-3}$ \\
\hline$n$ & porosity $^{\dagger}$ & 0.35 \\
\hline$\lambda_{\mathrm{e}}$ & 'apparent' or bulk thermal conductivity & $2.0 \mathrm{~J} \mathrm{~m}^{-1} \mathrm{~s}^{-1} \mathrm{~K}^{-1}$ \\
\hline$c_{\mathrm{f}}$ & specific heat capacity of fluid & $4174 \mathrm{~J} \mathrm{~kg}^{-1} \mathrm{~K}^{-1}$ at $4^{\circ} \mathrm{C}$ \\
\hline$c_{\mathrm{s}}$ & specific heat of solids & $800 \mathrm{~J} \mathrm{~kg}^{-1} \mathrm{C}^{-1}$ \\
\hline$\rho_{\mathrm{f}}$ & fluid density ${ }^{\dagger}$ & $1000 \mathrm{~kg} \mathrm{~m}^{-3}$ \\
\hline$\rho_{\mathrm{s}}$ & solid density ${ }^{\dagger}$ & $2630 \mathrm{~kg} \mathrm{~m}^{-3}$ \\
\hline$\mu$ & dynamic viscosity $^{\dagger}$ & $10^{-3} \mathrm{~kg} \mathrm{~m}^{-1} \mathrm{~s}^{-1}$ \\
\hline$K$ & hydraulic conductivity $^{\dagger}$ & $1.0 \times 10^{-5} \mathrm{~m} \mathrm{~s}^{-1}$ at $4^{\circ} \mathrm{C}$ \\
\hline$S_{\mathrm{s}}$ & specific storage $^{\dagger}$ & 0 \\
\hline$\alpha_{\mathrm{L}}$ & longitudinal dispersivity & $0.1 \mathrm{~m}$ \\
\hline$\alpha_{\mathrm{TH}}$ & transverse horizontal dispersivity & $0.1 \mathrm{~m}$ \\
\hline$\alpha_{\mathrm{TV}}$ & transverse vertical dispersivity & $0.1 \mathrm{~m}$ \\
\hline$T_{\mathrm{t}}$ & temperature in upper half of domain & $20^{\circ} \mathrm{C}$ \\
\hline$T_{\mathrm{b}}$ & temperature in bottom half of domain & $10^{\circ} \mathrm{C}$ \\
\hline
\end{tabular}

Note: $\dagger$ denotes parameters required for Heatflow but not for the analytical solution.

The results from the analytical model and Heatflow are shown in Fig. C1-2 at 20, 100, 200, and 400 days. As shown, the results compare very well. For both models, at early time the temperature near the interface changes rapidly due to the large temperature gradient. With increasing time, the gradient becomes smaller and the rate of temperature changes decreases. Both methods reach a steady state temperature profile at approximately 700 days (not shown here). 


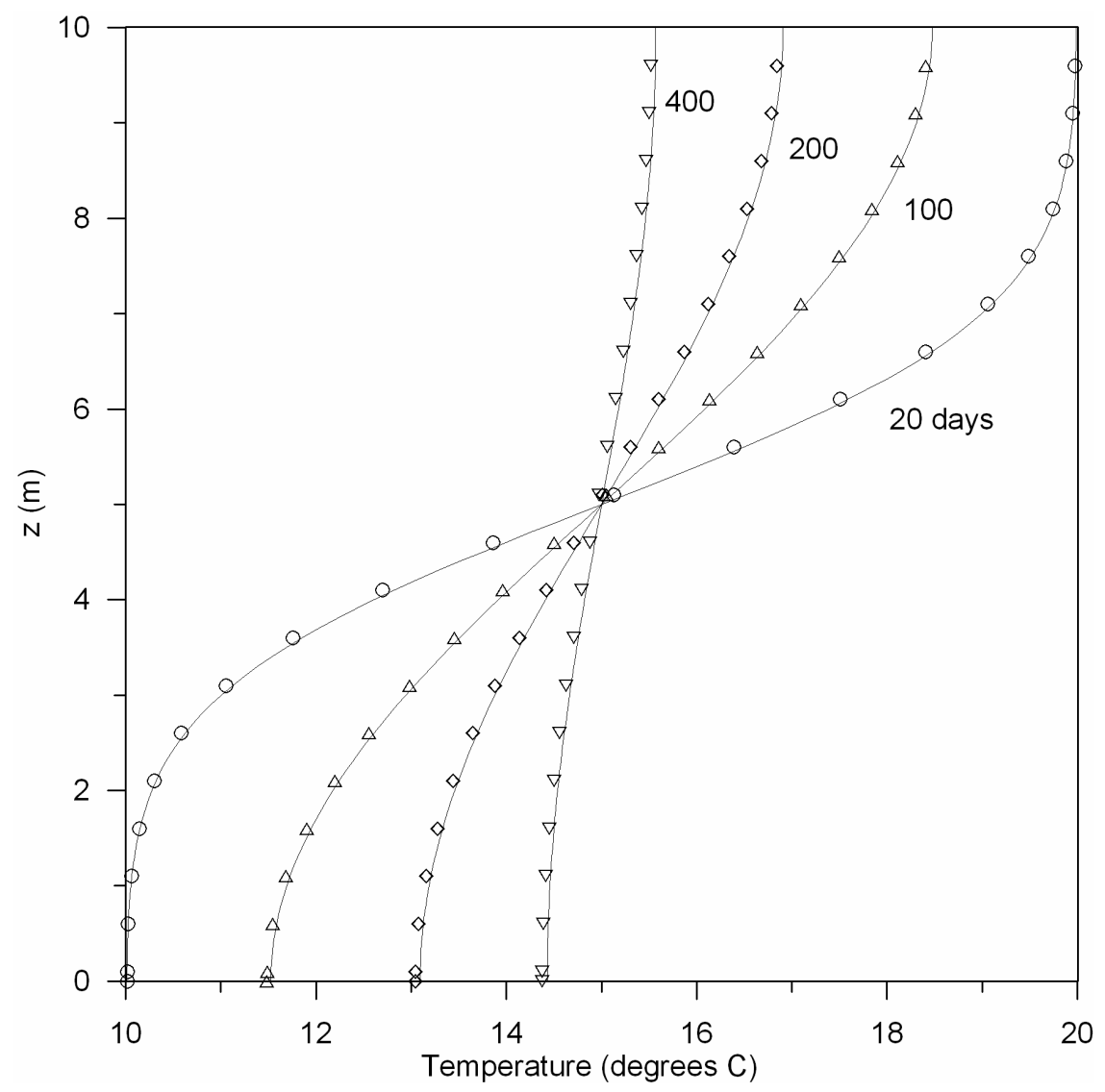

Figure C1-2 Temperature profile at 20, 100, 200 and 400 days for the analytical solution (lines) and numerical simulations (symbols). 


\section{Appendix C2: Checks of the consistency of the velocity approximation}

Voss and Souza (1987) suggested two steady-state tests to check the consistency of a models ability to handle density-driven flow. The first test is a simulation of a rectangular domain containing a less dense fluid layer above a denser fluid layer; and the second is the same system with open vertical sides and uniform horizontal flow. The first test checks the consistency under hydrostatic conditions with a stable density configuration and no flow across the boundaries. The correct solution is obtained only if the hydraulic gradient and the density-gravity terms are consistently approximated (Voss and Souza, 1987). The second test checks for consistency in a system where flow is parallel to the interface and mesh. In both cases, in the correct solution the interface will remain in a single row of elements. An inconsistent approximation of the density-gravity terms will result in spreading of the interface in both cases.

(a)

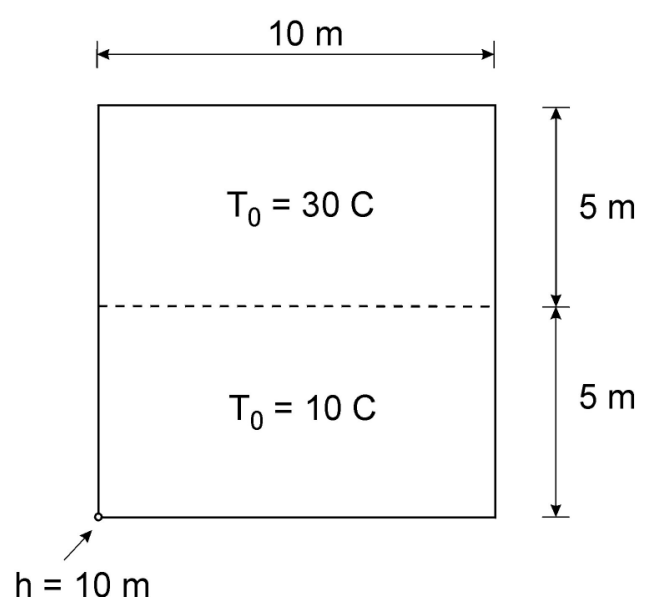

(b)

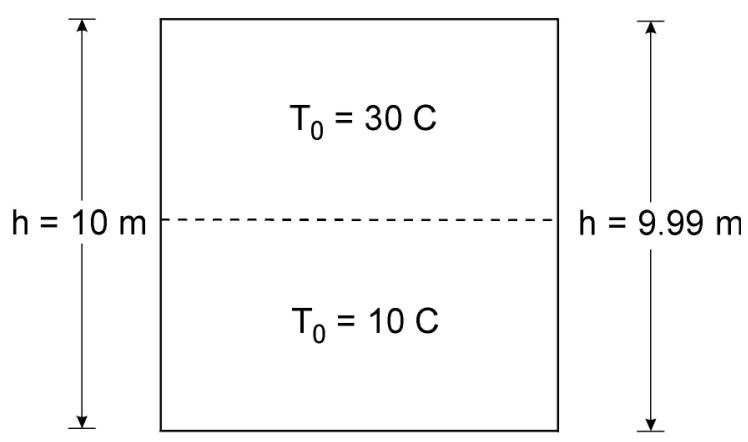

Figure C2-1 Configuration for two-dimensional steady state tests for consistent velocity approximation: (a) test 1: hydrostatic conditions; (b) test 2: parallel flow from the left with a head, $h$, of $10 \mathrm{~m}$ to the right with a head of $9.99 \mathrm{~m}$.

The simulation parameters are provided below (Table C2-1). For both tests, the apparent thermal conductivity is set to zero and the dispersivities are equal to the length of the largest element. 
Table C2-1 Simulation parameters for heat conduction in a finite domain.

\begin{tabular}{lll}
\hline Symbol & Parameter name & Value \\
\hline & domain length & $10 \mathrm{~m}$ \\
& domain height & $10 \mathrm{~m}$ \\
& element dimensions & $0.1 \times 0.1 \times 0.1 \mathrm{~m}$ \\
& fluid & water \\
$T_{\text {ref }}$ & reference temperature & $4{ }^{\circ} \mathrm{C}$ \\
$\rho_{\text {ref }}$ & reference fluid density & $1000 \mathrm{~kg} \mathrm{~m}^{-3}$ \\
& & \\
$n$ & porosity & 0.35 \\
$\lambda_{\mathrm{e}}$ & 'apparent' or bulk thermal conductivity & $0 \mathrm{~J} \mathrm{~m}^{-1} \mathrm{~s}^{-1} \mathrm{~K}^{-1}$ \\
$c_{\mathrm{f}}$ & specific heat capacity of fluid & $0 \mathrm{~J} \mathrm{~kg}^{-1} \mathrm{~K}^{-1}$ at $4^{\circ} \mathrm{C}$ \\
$c_{\mathrm{s}}$ & specific heat of solids & $0 \mathrm{~J} \mathrm{~kg}^{-1} \mathrm{C}^{-1}$ \\
$\rho_{\mathrm{f}}$ & fluid density & $1000 \mathrm{~kg} \mathrm{~m}^{-3}$ \\
$\rho_{\mathrm{s}}$ & solid density & $2630 \mathrm{~kg} \mathrm{~m}^{-3}$ \\
$\mu$ & dynamic viscosity & $10^{-3} \mathrm{~kg} \mathrm{~m}^{-1} \mathrm{~s}^{-1}$ \\
$K$ & hydraulic conductivity & $1.0 \times 10^{-5} \mathrm{~m} \mathrm{~s}^{-1}$ at $4^{\circ} \mathrm{C}$ \\
$S_{\mathrm{s}}$ & specific storage & 0 \\
$\alpha_{\mathrm{L}}$ & longitudinal dispersivity \\
$\alpha_{\mathrm{TH}}$ & transverse horizontal dispersivity & $0.1 \mathrm{~m}$ \\
$\alpha_{\mathrm{TV}}$ & transverse vertical dispersivity & $0.1 \mathrm{~m}$ \\
$T_{\mathrm{t}}$ & temperature in upper half of domain & $0.1 \mathrm{~m}$ \\
$T_{\mathrm{b}}$ & temperature in bottom half of domain & $30^{\circ} \mathrm{C}$ \\
\hline
\end{tabular}

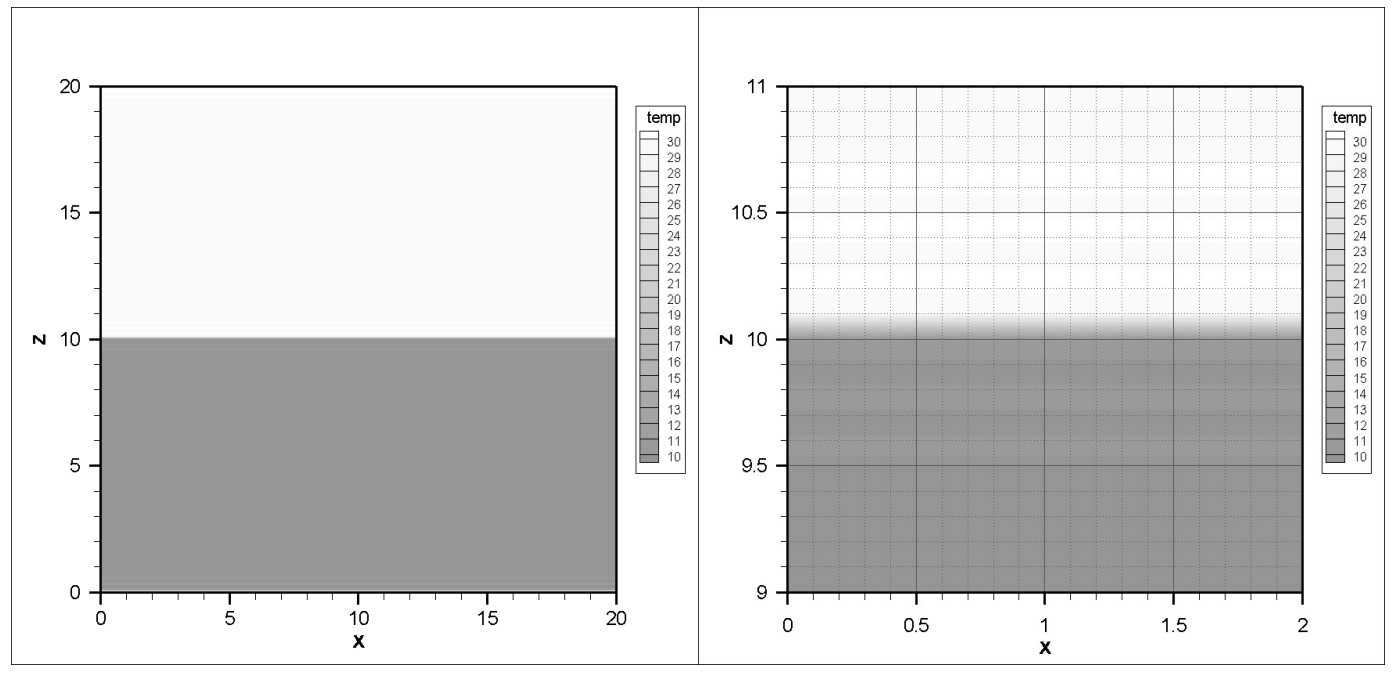

Figure C2-2 Temperature profile for steady state test 1 . The figure on the left shows the entire domain and the figure on the right shows the temperature gradient is constrained to a single row of cells. 
The correct solution to each test problem was obtained in both cases.

\section{Appendix C3: One-dimensional heat transport benchmark (Stallman,}

\section{5)}

Stallman developed an analytical solution to the subsurface temperature profile in a semiinfinite porous medium, in response to a sinusoidal surface temperature (Stallman, 1965). This solution provides a test of a numerical codes ability to simulate one dimensional heat convection and conduction in response to a time varying Dirichlet boundary (Fig. C3-1).

Ground surface boundary condition
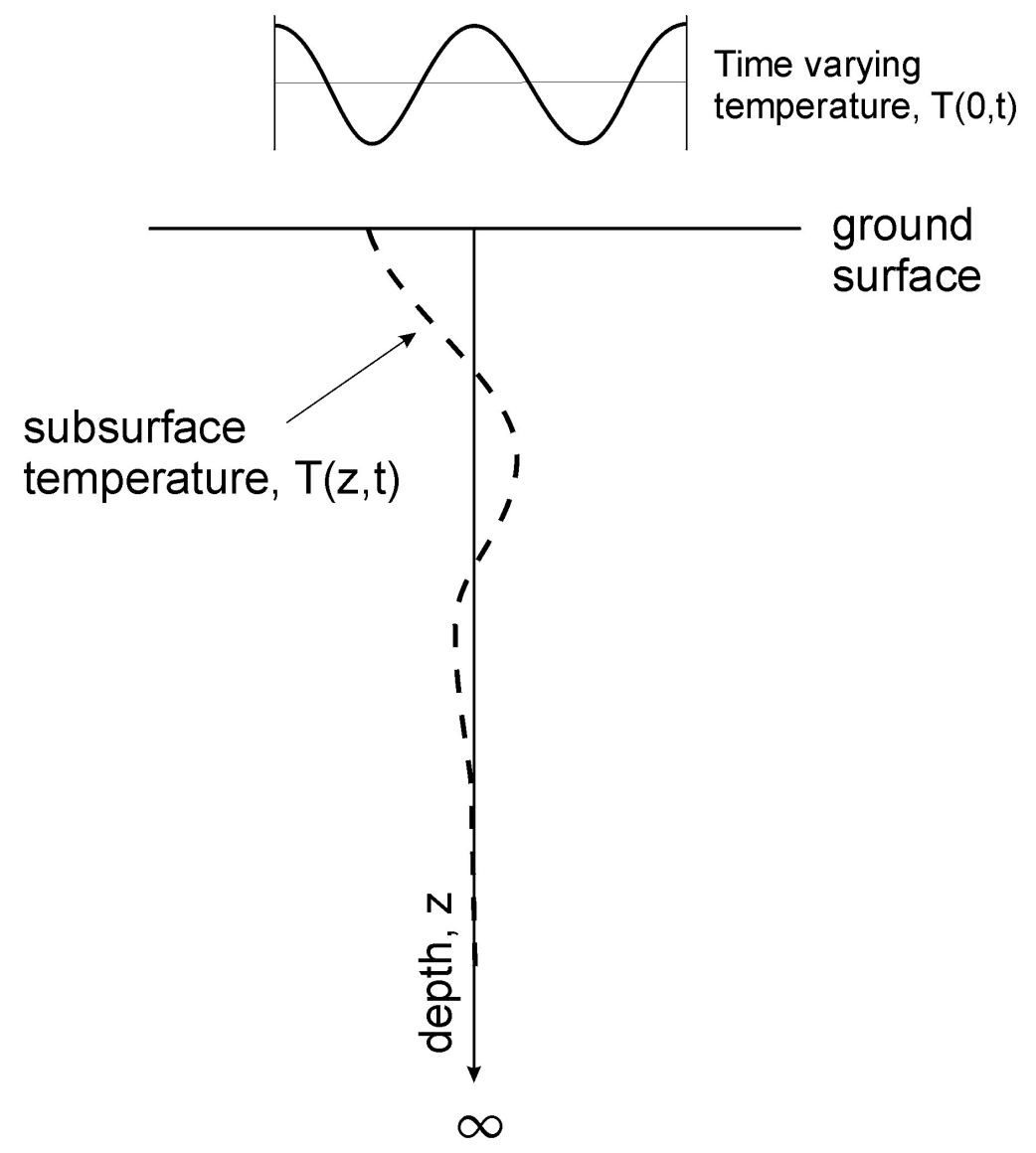

Figure C3-1 Subsurface temperature profile in a semi-infinite porous medium with a sinusoidal surface temperature (Stallman, 1965). 
Given a temperature variation at the ground surface described by

$T(0, t)=T_{s}+\Delta T \sin \left(\frac{2 \pi t}{\tau}\right)$

where $T_{\mathrm{s}} \quad$ is the average surface temperature;

$\Delta T \quad$ is the temperature amplitude;

$t \quad$ is time, and

$\tau \quad$ is the period.

The temperature variation with depth is given by Stallman (1965) as

$T(z, t)=\Delta T e^{-a z} \sin \left(\frac{2 \pi t}{\tau}-b z\right)+T_{\infty}$

where

$a=\left\{\left(\left(\frac{\pi c \rho}{\lambda_{e} \tau}\right)^{2}+\frac{1}{4}\left(\frac{q c_{f} \rho_{f}}{2 \lambda_{e}}\right)^{4}\right]^{1 / 2}+\frac{1}{2}\left(\frac{q c_{f} \rho_{f}}{2 \lambda_{e}}\right)^{2}\right\}^{1 / 2}-\left(\frac{q c_{f} \rho_{f}}{2 \lambda_{e}}\right)$

and

$b=\left\{\left[\left(\frac{\pi c \rho}{\lambda_{e} \tau}\right)^{2}+\frac{1}{4}\left(\frac{q c_{f} \rho_{f}}{2 \lambda_{e}}\right)^{4}\right]^{1 / 2}-\frac{1}{2}\left(\frac{q c_{f} \rho_{f}}{2 \lambda_{e}}\right)^{2}\right\}^{1 / 2}$

Table C3-1 Simulation parameters for the Stallman (1965) analytical solution.

\begin{tabular}{|c|c|c|}
\hline Symbol & Parameter name & Value \\
\hline & domain length & $70 \mathrm{~m}$ \\
\hline & fluid & water \\
\hline$T_{\text {ref }}$ & reference temperature ${ }^{\dagger}$ & $4^{\circ} \mathrm{C}$ \\
\hline$\rho_{\text {ref }}$ & reference fluid density ${ }^{\dagger}$ & $1000 \mathrm{~kg} \mathrm{~m}^{-3}$ \\
\hline$n$ & porosity ${ }^{\dagger}$ & 0.4 \\
\hline$\lambda_{\mathrm{e}}$ & 'apparent' or bulk thermal conductivity & $2.0 \mathrm{~J} \mathrm{~m}^{-1} \mathrm{~s}^{-1} \mathrm{~K}^{-1}$ \\
\hline$c_{\mathrm{f}}$ & specific heat capacity of fluid & $4174 \mathrm{~J} \mathrm{~kg}^{-1} \mathrm{~K}^{-1}$ at $4^{\circ} \mathrm{C}$ \\
\hline$c_{\mathrm{s}}$ & specific heat of solids & $800 \mathrm{~J} \mathrm{~kg}^{-1} \mathrm{C}^{-1}$ \\
\hline$\rho_{\mathrm{f}}$ & fluid density ${ }^{\dagger}$ & $1000 \mathrm{~kg} \mathrm{~m}^{-3}$ \\
\hline$\rho_{\mathrm{s}}$ & solid density ${ }^{\dagger}$ & $2630 \mathrm{~kg} \mathrm{~m}^{-3}$ \\
\hline$\mu$ & dynamic viscosity $^{\dagger}$ & $10^{-3} \mathrm{~kg} \mathrm{~m}^{-1} \mathrm{~s}^{-1}$ \\
\hline$K$ & hydraulic conductivity $^{\dagger}$ & $1.0 \times 10^{-5} \mathrm{~m} \mathrm{~s}^{-1}$ at $4^{\circ} \mathrm{C}$ \\
\hline$S_{\mathrm{s}}$ & specific storage $^{\dagger}$ & 0 \\
\hline$\alpha_{\mathrm{L}}$ & longitudinal dispersivity & $0.1 \mathrm{~m}$ \\
\hline$\alpha_{\mathrm{TH}}$ & transverse horizontal dispersivity & $0.1 \mathrm{~m}$ \\
\hline
\end{tabular}




\begin{tabular}{lll}
\hline Symbol & Parameter name & Value \\
\hline$\alpha_{\mathrm{TV}}$ & transverse vertical dispersivity & $0.1 \mathrm{~m}$ \\
$\mathrm{q}$ & specific flux & $4 \times 10^{-7} \mathrm{~m} \mathrm{~s}^{-1}$ downward \\
$\tau$ & period of oscillation of temperature at the ground surface & 365 days \\
$\Delta T$ & amplitude of the temperature variation at the ground & $10^{\circ} \mathrm{C}$ \\
& surface & $20^{\circ} \mathrm{C}$ \\
$T_{\mathrm{s}}$ & average ambient temperature at the ground surface & $15^{\circ} \mathrm{C}$ \\
$T_{\infty}$ & ambient temperature at depth & \\
\hline
\end{tabular}

Note: $\dagger$ denotes parameters required for Heatflow but not for analytical solution.

For this simulation, both the density and dynamic viscosity of the fluid were held constant to match the assumptions used in the analytical solution.

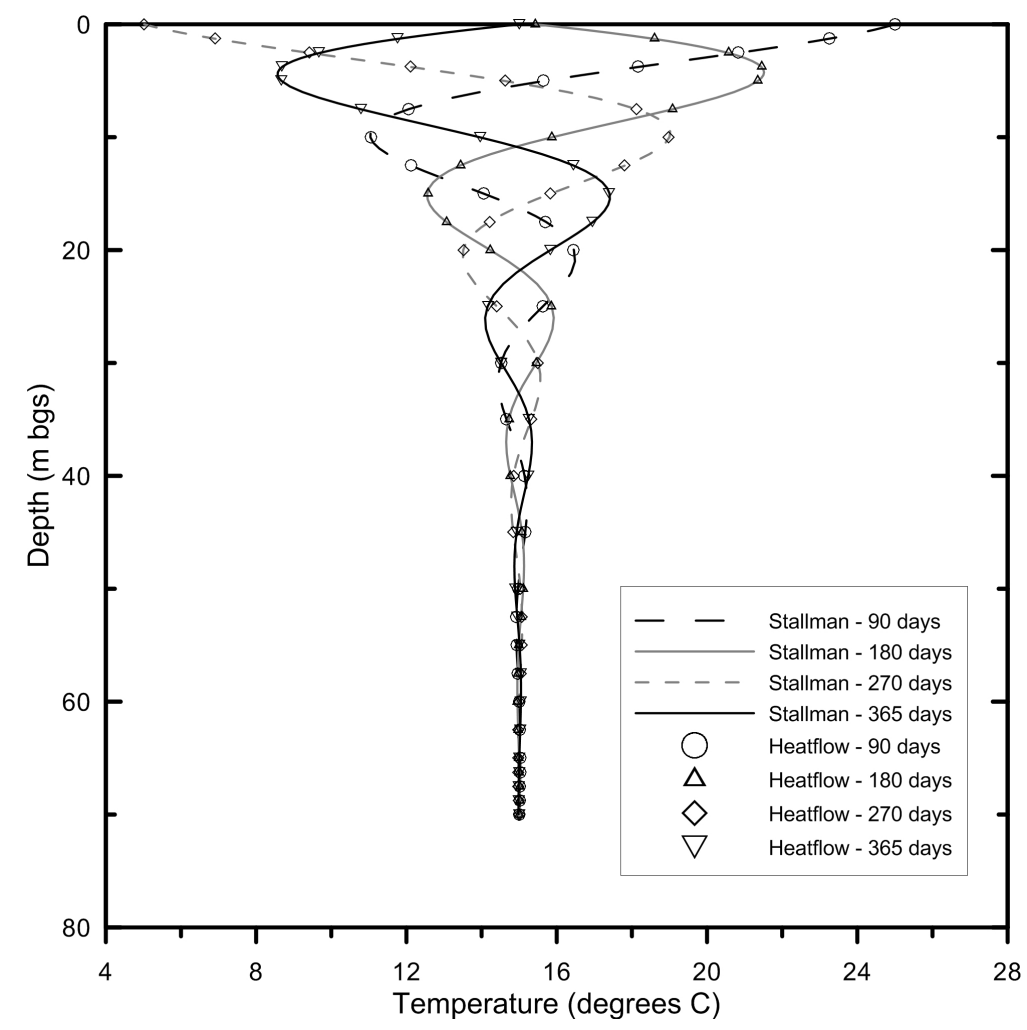

Figure C3-2 Comparison to Stallman's 1-D analytical solution.

Fig. C3-2 shows the analytical and numerical results are in excellent agreement indicating that the implementation of the time varying boundary condition in Heatflow is correct. As well, Heatflow accurately predicts the convective and conductive transport of heat in a one-dimensional system where the temperature dependency on density and viscosity is not considered. 


\section{Appendix C4: Elder Short Heater Problem (Elder, 1967)}

The Elder short heater problem (Elder, 1967) is based on experimental measurements and numerical simulations of free thermal convection in a Hele-Shaw cell. In this experiment, fluid flow is driven purely by fluid density differences arising from temperature differences within the domain. Elder's model was 20-cm-wide by 5-cm-high, and he used silicon oil as the fluid. Elder centred a 10-cm-long heater across the bottom boundary, at which he maintained a constant temperature, and the top boundary was cooled to maintain a constant temperature. He observed the resulting convection patterns. This apparatus was scaled by Voss and Souza (1987) to be representative of solutal transport in a large-scale aquifer problem (600-m-wide by 150 -m-high). Their scaled configuration has become the standard benchmark for verifying the ability of a numerical model to simulate free convection where bulk fluid flow is driven solely by fluid density differences. The original Elder problem has been adapted for solutal density-driven flow and transport (e.g., Diersch, 1981; Voss and Souza, 1987; Oldenburg and Pruess, 1994; Oldenburg and Pruess, 1995; Holzbecher, 1998; Kolditz et al., 1998; Frolkovič and De Schepper, 2001; Simpson and Clement, 2003; Al-Maktoumi et al., 2007; Woods and Carey, 2007; van Reeuwijk et al., 2009), thermohaline flow and transport (Diersch and Kolditz, 1998 and 2002), and free thermal convection (Oldenburg et al., 1995; Holzbecher, 1998; Graf, 2009).

Here, I consider free thermal convection. I employ the symmetry of the problem and consider only the right half of the rectangular domain (Fig. C4-1), such that the computational domain has dimensions $300 \mathrm{~m} \times 150 \mathrm{~m}$. The bottom left corner has coordinates $(\mathrm{x}, \mathrm{z})=(0,0)$ and the top right corner has coordinates $(300,150)$. The heater along the bottom extends from $(0,0)$ to $(150,0)$. 


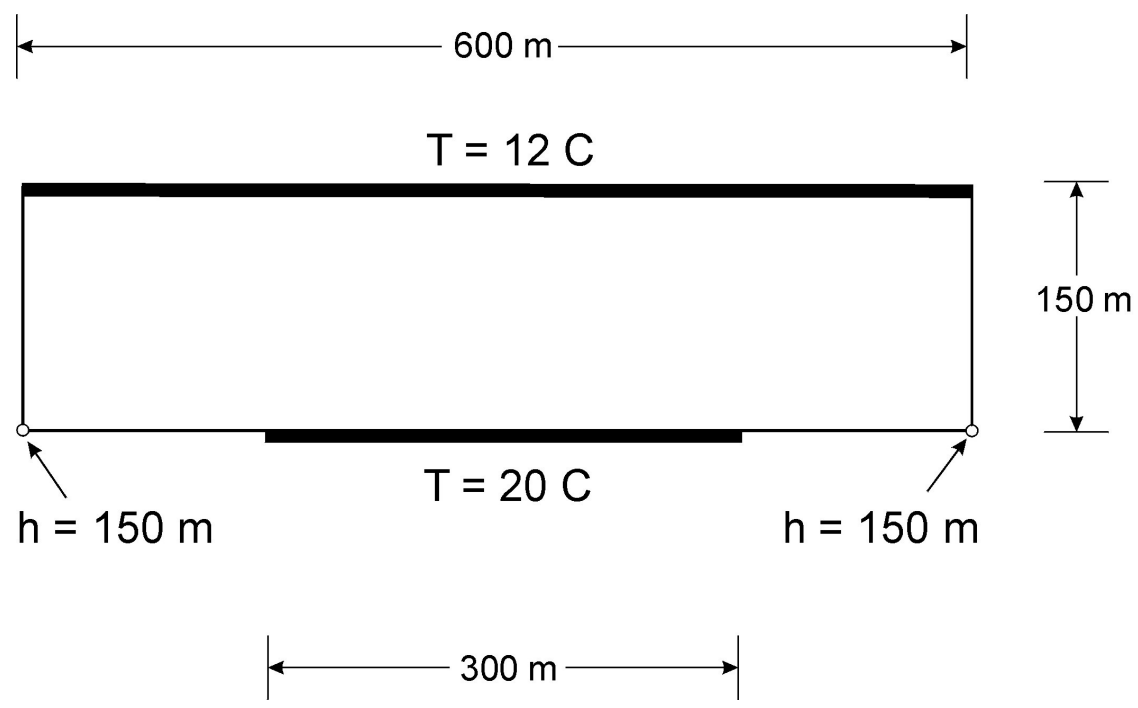

Figure C4-1 Configuration and boundary conditions for the Elder (1967) short heater thermal convection problem.

Initially, the temperature is $12^{\circ} \mathrm{C}$ inside the domain and the hydraulic head is 150 $\mathrm{m}$. The boundaries are all no flow with the exception of the bottom right corner $(300,0)$ at which a specified head value of $150 \mathrm{~m}$ is imposed. The temperature along the entire top boundary is fixed at $12^{\circ} \mathrm{C}$, and the temperature along the heated portion of the bottom boundary is fixed at $20^{\circ} \mathrm{C}$. The unheated portion of the bottom boundary may be considered as having a fixed temperature or as being perfectly insulating. These two cases will be discussed in more detail in subsequent sections. The two lateral boundaries are considered to be perfectly insulated. The heat capacity of the matrix is set to zero, analogous to pure fluid used in the Hele-Shaw cell, and the permeability is set to yield the desired Rayleigh number.

The remaining simulation parameters and boundary conditions representative of the Elder (1967) study and used in Heatflow are summarized below.

Table C4-1 Simulation parameters for the Elder (1967) short-heater thermal convection problem.

\begin{tabular}{lll}
\hline Symbol & Parameter name & Value \\
\hline$L$ & domain length & $600 \mathrm{~m}$ \\
$H$ & domain height & $150 \mathrm{~m}$ \\
& fluid & water
\end{tabular}




\begin{tabular}{lll}
\hline Symbol & Parameter name & Value \\
\hline$T_{\text {ref }}$ & reference temperature & $4{ }^{\circ} \mathrm{C}$ \\
$\rho_{\text {ref }}$ & reference fluid density & $1000 \mathrm{~kg} \mathrm{~m}^{-3}$ \\
$\mu$ & dynamic viscosity & $\sim 10^{-3} \mathrm{~kg} \mathrm{~m}^{-1} \mathrm{~s}^{-1}$ at $20^{\circ} \mathrm{C}$ \\
$k(R a=60)$ & permeability & $1.22 \times 10^{-11} \mathrm{~m}^{2}$ \\
$k(R a=400)$ & permeability & $8.18 \times 10^{-11} \mathrm{~m}^{2}$ \\
$k(R a=600)$ & permeability & $1.21 \times 10^{-10} \mathrm{~m}^{2}$ \\
$g$ & gravity & $9.81 \mathrm{~m} \mathrm{~s}^{-2}$ \\
$S_{\mathrm{s}}$ & specific storage & 0 \\
$\phi$ & porosity & 0.1 \\
$\lambda_{\mathrm{e}}$ & 'apparent' or bulk thermal conductivity & $1.49 \mathrm{~J} \mathrm{~m}^{-1} \mathrm{~s}^{-1} \mathrm{~K}^{-1}$ \\
$c_{\mathrm{f}}$ & specific heat capacity of fluid & $4190 \mathrm{~J} \mathrm{~kg}^{-1} \mathrm{~K}^{-1}$ at $12^{\circ} \mathrm{C}$ \\
$c_{\mathrm{S}}$ & specific heat of solids & $0 \mathrm{~J} \mathrm{~kg}^{-1} \mathrm{C}^{-1}$ \\
$\rho_{\mathrm{s}}$ & solid density & $2760 \mathrm{~kg} \mathrm{~m}^{-3}$ \\
$C_{\mathrm{s}}$ & heat capacity of solids & $0 \mathrm{~m}^{2} \mathrm{~s}^{-2} \mathrm{~K}^{-1}$ \\
$\beta$ & thermal expansion coefficient of fluid & $1.1 \times 10^{-4} \mathrm{C}^{-1}$ at $12^{\circ} \mathrm{C}$ \\
$\alpha_{\mathrm{L}}$ & longitudinal dispersivity & $0 \mathrm{~m}$ \\
$\alpha_{\mathrm{TH}}$ & transverse horizontal dispersivity & $0 \mathrm{~m}$ \\
$\alpha_{\mathrm{TV}}$ & transverse vertical dispersivity & $0 \mathrm{~m}$ \\
$T_{\mathrm{t}}$ & temperature at top of domain & $12^{\circ} \mathrm{C}$ \\
$T_{1}$ & temperature along heater & $20^{\circ} \mathrm{C}$ \\
$T_{0}$ & initial temperature & $12^{\circ} \mathrm{C}$ \\
\hline${ }^{\dagger}$ the permeability & was adopted from Oldenburg et al. (1995a) in which $R a \sim 600$.
\end{tabular}

The ratio between the buoyancy forces, driving free convection, and conductive forces is expressed by the 'global' thermal Rayleigh number (Lapwood, 1948), also called the Peclet number, given by

$$
\begin{aligned}
R a & =\frac{\text { driving force of bouyancy }}{\text { dissipative mechanisms of viscous drag \& conduction }} \\
& =\frac{k g H\left(\rho_{b}-\rho_{t}\right)}{\mu \kappa_{e}}
\end{aligned}
$$

where $\kappa_{e}$ is the apparent thermal diffusivity, and $\rho_{\mathrm{t}}$ and $\rho_{\mathrm{b}}$ are the density of the fluid at the top and bottom specified temperature boundaries, respectively. The original Elder experiment used Hele-Shaw plates with silicon oil. In this case, the apparent thermal diffusivity comprises the thermal properties of only the silicon oil and is given by

$$
\kappa_{e}=\frac{\lambda_{f}}{\rho_{f} c_{f}}
$$


Where the system being considered is a saturated porous medium, the thermal diffusivity comprises the thermal properties of the solids and fluid and is given by

$$
\kappa_{e}=\frac{\phi \lambda_{f}+(1-\phi) \lambda_{s}}{\phi \rho_{f} c_{f}+(1-\phi) \rho_{s} c_{s}}
$$

In the literature, for the both the solute and thermal transport versions of the Elder problem, the dynamic viscosity $\mu$ in Eq. (C4.1) is taken at $20^{\circ} \mathrm{C}$. While this may be appropriate for the solute versions, it seems more appropriate to take $\mu$ at the initial temperature for the thermal problem. However, to remain consistent with the published results, and to provide for direct comparison to those results, I have used $\mu\left(20^{\circ} \mathrm{C}\right)$ here. In Eq. (C4.2a) and (C4.2b) all the parameters are taken at the initial temperature, and in Eq. $(\mathrm{C} 4.2 \mathrm{~b}) c_{\mathrm{s}}$ is generally assumed to be 0 for the Elder problem.

Several investigators have found the computational results for the solutal transport version of this problem to be highly sensitive to the spatial and temporal discretization (e.g., Oldenburg and Pruess, 1995b; Holzbecher, 1998; Kolditz et al., 1998; Frolkovič and De Schepper, 2001; Diersch and Kolditz, 2002; Woods and Carey, 2007; AlMaktoumi et al., 2007), initial conditions (e.g., Frolkovič and De Schepper, 2001; Woods and Carey, 2007; van Reeuwijk et al., 2009), and the choice of the numerical algorithm used to approximate the transport equation, as well as the solver (e.g., Oldenburg and Pruess, 1995a; Holzbecher, 1998; Kolditz et al., 1998; Frolkovič and De Schepper, 2001; Diersch and Kolditz, 2002; Al-Maktoumi et al., 2007; Park and Aral, 2007). This has resulted in there being several solutions to the Elder problem presented in the literature. Until recently, there was no consensus on the correct numerical solution, making a quantitative comparison difficult. Based on work by Johannsen (2003), van Reeuwijk et al. (2009) used pseudospectral methods that avoid numerical and spatial discretization errors, to show the influence of the Rayleigh number $(R a)$ on the stability of the solution. They show that where $R a>76$, there exist more than one stable solution and that the solution to which the numerical code convergences depends on the initial conditions. 
Based on this work, they recommend that an improved and reproducible benchmark test case should have $R a<76$. They constructed a test case for $R a=60$ and present the solution for two different grid levels ( 4.5 and $\sim 5.5)$ at real time values of 6, 20, 60 and 200 years.

In this verification study, I follow the recommendations of van Reeuwijk et al. (2009) and completed benchmark tests of the Elder problem for $R a=0,60$, and 400. For $R a=400$, they suggest that two of the three known steady state solutions be reproduced by the model by altering the initial conditions. I also completed a series of simulations with $\mathrm{Ra}=600$ which were compared to results presented by Oldenburg et al. (1995a) and Graf (2009). In combination with these tests, I completed a mesh convergence study where the number of elements $N E$ at a given mesh level $l$ for the half domain is

$$
N E=2^{2 l+1}
$$

for a range in grid levels from 4 to 10 , Table C4-2.

Table C4-2 Summary of grid levels $l$ used in grid convergence study.

\begin{tabular}{cccc}
\hline $\begin{array}{c}\text { Grid } \\
\text { level }\end{array}$ & NE & $\begin{array}{c}\text { Number of elements } \\
\text { in x-direction }\end{array}$ & $\begin{array}{c}\text { Number of elements } \\
\text { in z-direction }\end{array}$ \\
\hline 4 & 512 & 32 & 16 \\
5 & 2048 & 64 & 32 \\
6 & 8192 & 128 & 64 \\
7 & 32768 & 256 & 128 \\
8 & 131072 & 512 & 256 \\
9 & 524288 & 1024 & 512 \\
10 & 2097152 & 2048 & 1024 \\
\hline
\end{tabular}

For all simulations presented in this appendix, I discretized the domain using a uniform grid with prismatic block elements. While the grid is 3-dimensional, only one element in the y-direction was considered to approximate a 2-dimensional domain. In all cases, I used the consistent velocity formulation proposed by Knabner and Frolkovič (1996) with analytical integration of the gravity-density vector and element coefficient matrices.

\section{Pure Conduction Benchmark Test,} Consistent Boundary Conditions on Bottom, $R \boldsymbol{a}=\mathbf{0}$ 
The case of pure conduction, $R a=0$, is possible only if there are no density differences and thus buoyancy induced flow (Holzbecher, 1998). In this case, the Elder problem is described by the heat conduction equation given as

$$
\frac{\partial T(x, z, t)}{\partial t}=\kappa_{e}\left(\frac{\partial^{2} T(x, z, t)}{\partial x^{2}}+\frac{\partial^{2} T(x, z, t)}{\partial z^{2}}\right)
$$

In the original Elder experiment, a portion of the bottom boundary was heated and the remainder was insulated. This creates a mixed boundary condition along the bottom boundary that makes obtaining an analytical solution difficult. In this section I present the solution to the boundary value problem with the temperatures specified along the heated and unheated portions of the bottom boundary, similar to the solution for a saline system presented by van Reeuwijk et al. (2009). In a subsequent section, I present the solution to the more difficult problem with a mixed boundary condition along the bottom.

Where the temperature along the bottom boundary is specified, the initial and boundary conditions are

$$
\begin{aligned}
& T(x, z, t)=T_{0}, \quad 0 \leq x \leq L, \quad 0 \leq z \leq H, \quad t=0 \\
& T(x, z, t)=T_{0}, \quad 0 \leq x \leq L, \quad z=H, \quad t>0 \\
& T(x, z, t)=f(x), \quad 0 \leq x \leq L, \quad z=0, \quad t>0 \\
& f(x)=\left\{\begin{array}{cc}
T_{0}, & 0 \leq x<x_{1} \\
T_{1}, & x_{1} \leq x \leq x_{2} \\
T_{0}, & x_{2}<x \leq L
\end{array}\right. \\
& \frac{\partial T(x, z, t)}{\partial x}=0, \quad x=0, \quad 0 \leq z \leq H, \quad t>0 \\
& \frac{\partial T(x, z, t)}{\partial x}=0, \quad x=L, \quad 0 \leq z \leq H, \quad t>0
\end{aligned}
$$

A schematic diagram of the problem and boundary conditions is shown below (Fig. C4$2)$. 


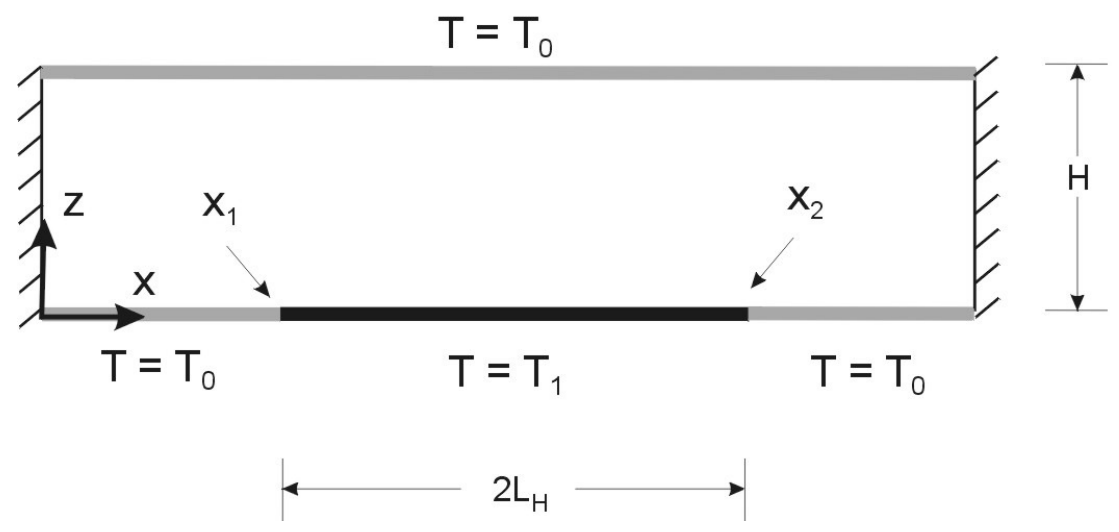

Figure C4-2 Schematic diagram of the problem domain and boundary conditions. In the original Elder problem, $L=2 H, L_{\mathrm{H}}=H$, and the heater is centred along the bottom. Furthermore, in the original problem the unheated portion of the bottom boundary was perfectly insulated, while in this case it is a specified temperature, $T_{0}$.

I define the following variables

$$
\begin{array}{llll}
x_{D}=\frac{x}{H}, & z_{D}=\frac{z}{H}, & t_{D}=\frac{\kappa_{e} t}{H^{2}}, & T_{D}=\frac{T-T_{0}}{T_{1}-T_{0}}, \\
x_{D 1}=\frac{x_{1}}{H}, & x_{D 2}=\frac{x_{2}}{H}, & L_{D}=\frac{L}{H}, & L_{D H}=\frac{L_{H}}{H} .
\end{array}
$$

and recasting (C4.4) and (C4.5) into dimensionless forms yields

$$
\frac{\partial T_{D}\left(x_{D}, z_{D}, t_{D}\right)}{\partial t_{D}}=\frac{\partial^{2} T_{D}\left(x_{D}, z_{D}, t_{D}\right)}{\partial x_{D}^{2}}+\frac{\partial^{2} T_{D}\left(x_{D}, z_{D}, t_{D}\right)}{\partial z_{D}^{2}}
$$


The initial and boundary conditions are

$$
\begin{aligned}
& T_{D}\left(x_{D}, z_{D}, t_{D}\right)=0, \quad 0 \leq x_{D} \leq L_{D}, \quad 0 \leq z_{D} \leq 1, \quad t_{D}=0 \\
& T_{D}\left(x_{D}, z_{D}, t_{D}\right)=0, \quad 0 \leq x_{D} \leq L_{D}, \quad z_{D}=1, \quad t_{D}>0 \\
& T_{D}\left(x_{D}, z_{D}, t_{D}\right)=f\left(x_{D}\right), \quad 0 \leq x_{D} \leq L_{D}, \quad z_{D}=0, \quad t_{D}>0 \\
& f\left(x_{D}\right)=\left\{\begin{array}{lc}
0, & 0 \leq x_{D}<x_{D 1} \\
1, & x_{D 1} \leq x_{D} \leq x_{D 2} \\
0, & x_{D 2}<x_{D} \leq L_{D}
\end{array}\right. \\
& \frac{\partial T_{D}\left(x_{D}, z_{D}, t_{D}\right)}{\partial x_{D}}=0, \quad x_{D}=0, \quad 0 \leq z_{D} \leq 1, \quad t_{D}>0 \\
& \frac{\partial T_{D}\left(x_{D}, z_{D}, t_{D}\right)}{\partial x_{D}}=0, \quad x_{D}=L_{D}, \quad 0 \leq z_{D} \leq 1, \quad t_{D}>0
\end{aligned}
$$

A solution to this system can be obtained through the use of the Laplace transform

$$
\hat{T}(s)=\int_{0}^{\infty} T(t) \exp (-s t) d t
$$

and the finite Fourier cosine transform

$$
\bar{T}(m)=\int_{0}^{L} T(x) \cos \left(g_{m} x\right) d x \quad g_{m}=\frac{m \pi}{L} \quad m=0,1,2 \ldots \quad 0 \leq x \leq L
$$

which has the inverse

$$
T(x)=\frac{\bar{T}(m=0)}{L}+\frac{2}{L} \sum_{m=1}^{\infty} \bar{T}(m) \cos \left(g_{m}\right)
$$

Applying the Laplace transform in time followed by the finite Fourier cosine transform in the $\mathrm{x}$-direction gives the following general solution

$$
\frac{d^{2} \overline{\hat{T}}_{D}\left(m, z_{D}, s\right)}{d z_{D}^{2}}=\xi_{m}^{2} \overline{\hat{T}_{D}}\left(m, z_{D}, s\right) \quad \xi_{m}^{2}=g_{m}^{2}+s
$$


with transformed boundary conditions

$$
\begin{array}{ll}
\overline{\hat{T}}\left(m, z_{D}, s\right)=0 & z_{D}=1 \\
\overline{\hat{T}}\left(m, z_{D}, s\right)=\frac{1}{s} \int_{0}^{L} f\left(x_{D}\right) \cos \left(g_{m} x_{D}\right) d x_{D} & z_{D}=0
\end{array}
$$

The general solution of Eq. (C4.12) has the form

$$
\overline{\hat{T}}_{D}\left(m, z_{D}, s\right)=A_{m} \cosh \left(\xi_{m} z_{D}\right)+B_{m} \sinh \left(\xi_{m} z_{D}\right)
$$

Applying the boundary conditions (C4.13) to (C4.14) gives the transformed solution

$$
\overline{\hat{T}}_{D}\left(m, z_{D}, s\right)=A_{m} \cosh \left(\xi_{m} z_{D}\right)-A_{m} \operatorname{coth}\left(\xi_{m}\right) \sinh \left(\xi_{m} z_{D}\right)
$$

where

$$
A_{m}=\frac{1}{s} \int_{0}^{L_{D}} f\left(x_{D}\right) \cos \left(g_{m} x_{D}\right) d x_{D}
$$

Applying the inverse Fourier transform (C4.11) gives

$$
\begin{aligned}
\hat{T}_{D}\left(x_{D}, z_{D}, s\right)= & \frac{L_{D H}}{s L_{D}}\left[\cosh \left(\xi_{m}^{\prime} z_{D}\right)-\operatorname{coth}\left(\xi_{m}^{\prime}\right) \sinh \left(\xi_{m}^{\prime} z_{D}\right)\right] \\
& +\frac{2}{s L_{D}} \sum_{m=1}^{\infty} \frac{1}{g_{m}}\left[\sin \left(g_{m} x_{D 2}\right)-\sin \left(g_{m} x_{D 1}\right)\right] \\
& \quad\left[\cosh \left(\xi_{m} z_{D}\right)-\operatorname{coth}\left(\xi_{m}\right) \sinh \left(\xi_{m} z_{D}\right)\right] \cos \left(g_{m} x\right)
\end{aligned}
$$

where $\xi_{m}^{\prime}=\sqrt{s}$.

This dimensionless solution is in Laplace transformed space. Rather than attempting to invert Eq. (C4.16) analytically, I use the Gaver-Stehfest algorithm to numerically invert Eq. (C4.16). Note that this solution is similar to that presented by van 
Reeuwijk et al. (2009) for the solute case with the following differences:

1. the equations and dimensionless variables account for heat conduction rather than solute diffusion;

2. the ratio between $L$ and $H$ is not fixed by the solution so that the size of the domain is variable;

3. the coordinates of the heater along the base are variable, and

4. the origin of the coordinate axis is along the heated side of the domain.

The solution given by $(\mathrm{C} 4.16)$ collapses to the Elder problem if $L_{\mathrm{D}}=2, x_{\mathrm{D} 1}=0$, and $x_{\mathrm{D} 2}=$ 1 (ie. $L=2 H, x_{1}=0$, and $x_{2}=H$ ).

Comparison of the numerical simulation results from Heatflow with those from the analytical solution for $R a=0$ shows excellent agreement (Fig. C4-3). The numerical results were obtained using a grid level of 6 and a time step $\Delta t$ of 30 days. 


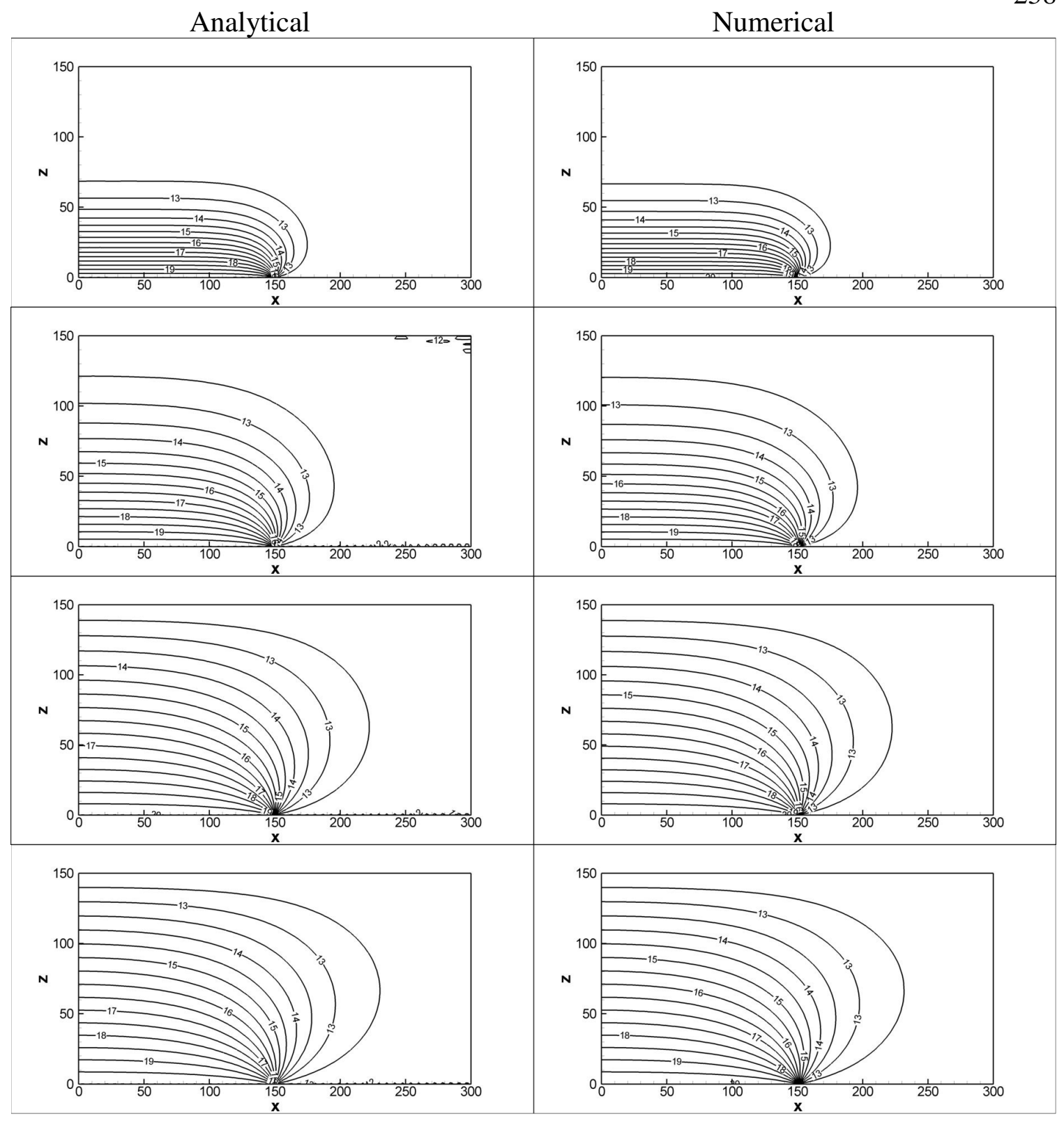

Figure C4-3 Elder solution for $R a=0$. On the left are results using the analytical solution (with $\mathrm{H}=150 \mathrm{~m} ; \mathrm{L}=300 \mathrm{~m} ; \mathrm{x}_{1}=0 ; \mathrm{x}_{2}=150 \mathrm{~m} ; \mathrm{T}_{0}=12^{\circ} \mathrm{C} ; \mathrm{T}_{1}=20^{\circ} \mathrm{C}$; and $\kappa_{e}=$ $\left.3.7674 \times 10^{-6} \mathrm{~m} \mathrm{~s}^{-2}\right)$, and on the right are results using Heatflow. The results are shown at time 6 years (top), 20, 60, and 200 years (bottom). 
Pure Conduction Benchmark Test,

\section{Mixed Boundary Conditions on Bottom, $R a=0$}

In the original Elder experiment, a portion of the bottom boundary was heated and the remainder was insulated. This creates a mixed boundary condition along the bottom boundary (Fig. C4-4).

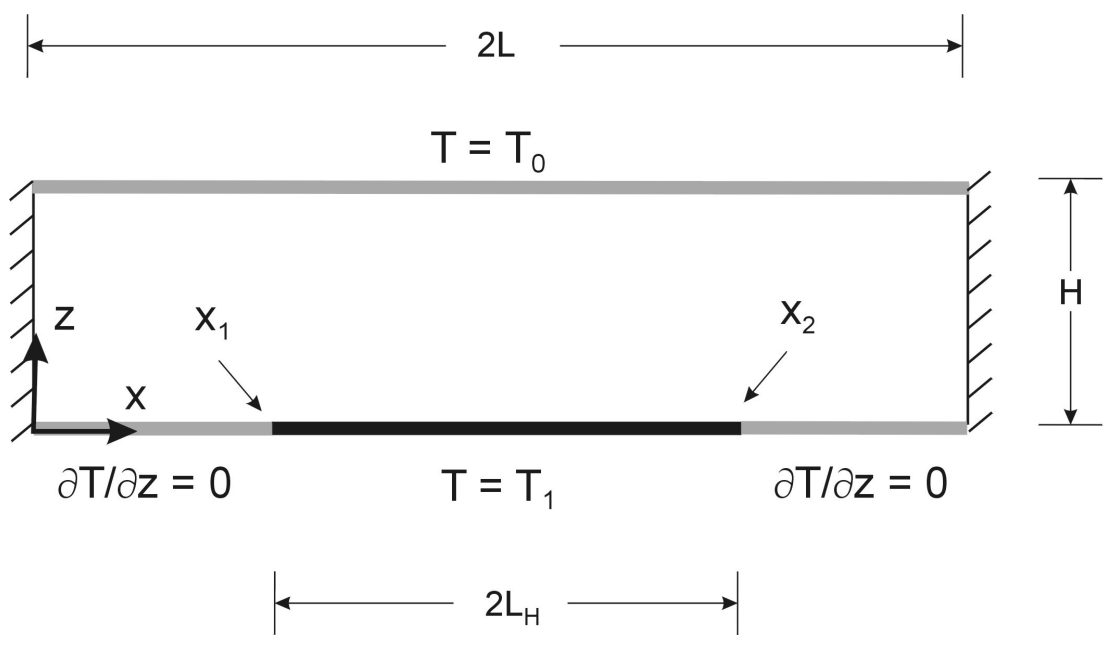

Figure C4-4 Schematic diagram of the problem domain and boundary conditions. In the original Elder problem, $L=2 H, L_{\mathrm{H}}=H$, and the heater is centred along the bottom. 
The governing equation and the initial and boundary conditions are

$$
\begin{aligned}
& \frac{\partial T(x, z, t)}{\partial t}=\kappa_{e}\left(\frac{\partial^{2} T(x, z, t)}{\partial x^{2}}+\frac{\partial^{2} T(x, z, t)}{\partial z^{2}}\right) \\
& T(x, z, t)=T_{0}, \quad 0 \leq x \leq L, \quad 0 \leq z \leq H, \quad t=0 \\
& T(x, z, t)=T_{0}, \quad 0 \leq x \leq L, \quad z=H, \quad t>0 \\
& T(x, z, t)=T_{1}, \quad x_{1} \leq x \leq x_{2}, \quad z=0, \quad t>0 \\
& \frac{\partial T(x, z, t)}{\partial z}=0, \quad 0<x<x_{1}, \quad x_{2}<x<L, \quad t>0 \\
& \frac{\partial T(x, z, t)}{\partial x}=0, \quad x=0, \quad 0 \leq z \leq H, \quad t>0 \\
& \frac{\partial T(x, z, t)}{\partial x}=0, \quad x=L, \quad 0 \leq z \leq H, \quad t>0
\end{aligned}
$$

The above mathematical model can not be solved directly using the integral transform techniques employed in the previous solution because of the mixed boundary conditions given by Eq. (C4.17c) along the heated portion of the bottom, and (C4.17d) along the insulated portion of the bottom. This can be overcome by recasting the boundary condition along the heater in terms of the heat flux, rather than as a specified temperature, as discussed below.

The total heat flow from the heater into the model domain is given by

$$
Q_{H}=\int_{x_{1}}^{x_{2}} q_{H}(x, t) d x, \quad x_{1} \leq x \leq x_{2}
$$

where the heat flux is equal to the heat flow rate per unit area of heater given by

$$
q_{H}(x, t)=-\left.\lambda e_{e} \frac{\partial T}{\partial z}\right|_{z=0}
$$

Using Eqs. (C4.18a and b), Eq. (C4.17c) can be transformed into a Neumann condition 
establishing a uniform description of the boundary conditions along the bottom boundary. Although the heat flux is unknown, it can be determined during the solution of the boundary value problem through the use of Eq. (C4.17c). Intuitively, it is recognized that $\frac{\partial T}{\partial z}$ changes along the heater and thus the heat flux $q_{H}(x, t)$ also changes, particularly near the ends of the heater where two-dimensional heat flow is more pronounced.

I define the following variables

$$
\begin{aligned}
& x_{D}=\frac{x}{H}, \quad z_{D}=\frac{z}{H}, \quad t_{D}=\frac{\kappa_{e} t}{H^{2}}, \quad T_{D}=\frac{T-T_{0}}{T_{1}-T_{0}}, \\
& x_{D 1}=\frac{x_{1}}{H}, \quad x_{D 2}=\frac{x_{2}}{H}, \quad L_{D}=\frac{L}{H}, \quad L_{D H}=\frac{L_{H}}{H}, \quad q_{D H}=\frac{q_{H} L}{\lambda_{e}\left(T_{1}-T_{0}\right)} \text {. }
\end{aligned}
$$

and recasting $(\mathrm{C} 4.4)(\mathrm{C} 4.17 \mathrm{a}$ to $\mathrm{e})$ and $(\mathrm{C} 4.18 \mathrm{a}$ and $\mathrm{b})$ into dimensionless form yields

$$
\frac{\partial T_{D}\left(x_{D}, z_{D}, t_{D}\right)}{\partial t_{D}}=\frac{\partial^{2} T_{D}\left(x_{D}, z_{D}, t_{D}\right)}{\partial x_{D}^{2}}+\frac{\partial^{2} T_{D}\left(x_{D}, z_{D}, t_{D}\right)}{\partial z_{D}^{2}},
$$

with initial and boundary conditions

$$
\begin{array}{llcl}
T_{D}\left(x_{D}, z_{D}, t_{D}\right)=0, & 0 \leq x_{D} \leq L_{D}, & 0 \leq z_{D} \leq 1, & t_{D}=0 \\
T_{D}\left(x_{D}, z_{D}, t_{D}\right)=0, & 0 \leq x_{D} \leq L_{D}, & z_{D}=1, & t_{D}>0 \\
T_{D}\left(x_{D}, z_{D}, t_{D}\right)=1, & x_{D 1} \leq x_{D} \leq x_{D 2}, & z_{D}=0, & t_{D}>0 \\
\frac{\partial T_{D}\left(x_{D}, z_{D}, t_{D}\right)}{\partial z_{D}}=0, & 0<x_{D}<x_{D 1}, & x_{D 2}<x_{D}<L_{D}, & t_{D}>0 \\
\frac{\partial T_{D}\left(x_{D}, z_{D}, t_{D}\right)}{\partial x_{D}}=0, & x_{D}=0, & 0 \leq z_{D} \leq 1, & t_{D}>0
\end{array}
$$


$\frac{\partial T_{D}\left(x_{D}, z_{D}, t_{D}\right)}{\partial x_{D}}=0, \quad x_{D}=L_{D}, \quad 0 \leq z_{D} \leq 1, \quad t_{D}>0$

$\left.\frac{\partial T_{D}}{\partial z_{D}}\right|_{z_{D}=0}=-q_{D H}\left(x_{D}, t_{D}\right)$

A solution to this system can now be obtained through the use of the integral transform method used previously and includes the Laplace transform

$\hat{T}(s)=\int_{0}^{\infty} T(t) \exp (-s t) d t$

and the finite Fourier cosine transform

$\bar{T}(m)=\int_{0}^{L} T(x) \cos \left(g_{m} x\right) d x \quad g_{m}=\frac{m \pi}{L} \quad m=0,1,2 \ldots \quad 0 \leq x \leq L$

which has the inverse

$T(x)=\frac{\bar{T}(m=0)}{L}+\frac{2}{L} \sum_{m=1}^{\infty} \bar{T}(m) \cos \left(g_{m}\right)$.

Applying the Laplace transform in time followed by the finite Fourier cosine transform in the $\mathrm{x}$-direction gives the following general solution

$\frac{d^{2} \overline{\hat{T}_{D}}\left(m, z_{D}, s\right)}{d z_{D}^{2}}=\xi_{m}^{2} \hat{\hat{T}}_{D}\left(m, z_{D}, s\right) \quad \xi_{m}^{2}=g_{m}^{2}+s$

with transformed boundary conditions

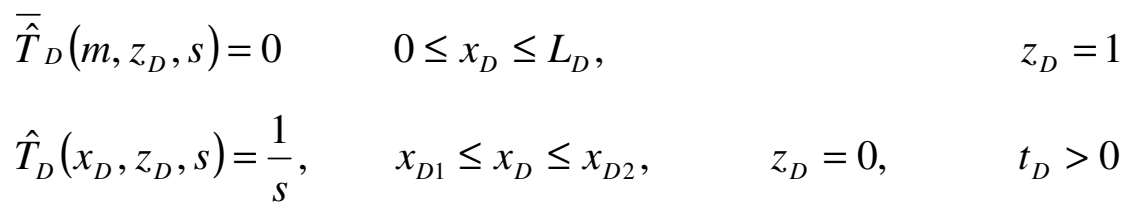


$\left.\frac{\partial \overline{\hat{T}}_{D}}{\partial z_{D}}\right|_{z_{D}=0}=-\int_{x_{D 1}}^{x_{D 2}} q_{D H}\left(x_{D}, s\right) \cos \left(g_{m} x_{D}\right) d x_{D}$

(C4.25)

The finite Fourier transform is not applied to Eq. (C4.21c) since it is not needed to obtain the general solution to Eq. (C4.23), but will be used later to solve for the heat flux $\hat{q}_{D H}\left(x_{D}, s\right)$.

The general solution of Eq. (C4.11) has the form

$\overline{\hat{T}}_{D}\left(m, z_{D}, s\right)=A_{m} \cosh \left(\xi_{m} z_{D}\right)+B_{m} \sinh \left(\xi_{m} z_{D}\right)$

and is subject to the remaining boundary conditions. Applying the boundary conditions (C4.24a) and (C4.25) gives the transformed solution

$\tilde{\hat{T}}_{D}\left(m, z_{D}, s\right)=\left[\frac{1}{\xi_{m}} \tanh \left(\xi_{m}\right) \cosh \left(\xi_{m} z_{D}\right)-\frac{1}{\xi_{m}} \sinh \left(\xi_{m} z_{D}\right)\right] \int_{x_{D 1}}^{x_{D 2}} \hat{q}_{D H}\left(x_{D}, s\right) \cos \left(g_{m} x_{D}\right) d x$

Applying the inverse Fourier transform (C4.10) gives

$$
\begin{aligned}
\hat{T}_{D}\left(x_{D}, z_{D}, s\right)=\frac{1}{L_{D} \xi_{m}^{\prime}} & {\left[\tanh \left(\xi_{m}^{\prime}\right) \cosh \left(\xi_{m}^{\prime} z_{D}\right)-\sinh \left(\xi_{m}^{\prime} z_{D}\right)\right] \int_{x_{D 1}}^{x_{D 2}} \hat{q}_{D H}\left(x_{D}, s\right) d x_{D} } \\
& +\frac{2}{L_{D}} \sum_{m=1}^{\infty} \frac{1}{\xi_{m}}\left\{\left[\tanh \left(\xi_{m}\right) \cosh \left(\xi_{m} z_{D}\right)-\sinh \left(\xi_{m} z_{D}\right)\right] \int_{x_{D 1}}^{x_{D 2}} \hat{q}_{D H}\left(x_{D}, s\right) \cos \left(g_{m} x_{D}\right) d x_{D}\right\} \cos \left(g_{m} x_{D}\right)
\end{aligned}
$$

where $\xi_{m}^{\prime}=\sqrt{s}$.

Now the heat flux $\hat{q}_{D H}\left(x_{D}, s\right)$ is unknown but can be obtained by dividing the heater into $M$ segments of length $\Delta x_{D i}$, where $i=1,2 \ldots . M$ (e.g., Gringarten and Ramey, 
1975; Dagan, 1978; Chang and Chen, 2003). The unknown function $\hat{q}_{D H}\left(x_{D}, s\right)$ is replaced by $\hat{q}_{i}(s), \quad i=1,2 \ldots . M$ such that

$\int_{x_{D 1}}^{x_{D 2}} \hat{q}_{D H}\left(x_{D}, s\right) d x_{D}=\sum_{i=1}^{M} \hat{q}_{i}(s) \Delta x_{D, i}$

The heat flux from each segment $\hat{q}_{i}(s)$ is now constant across each segment $\Delta x_{D i}$, but varies between segments along the length of the heater (Fig. C4-5).

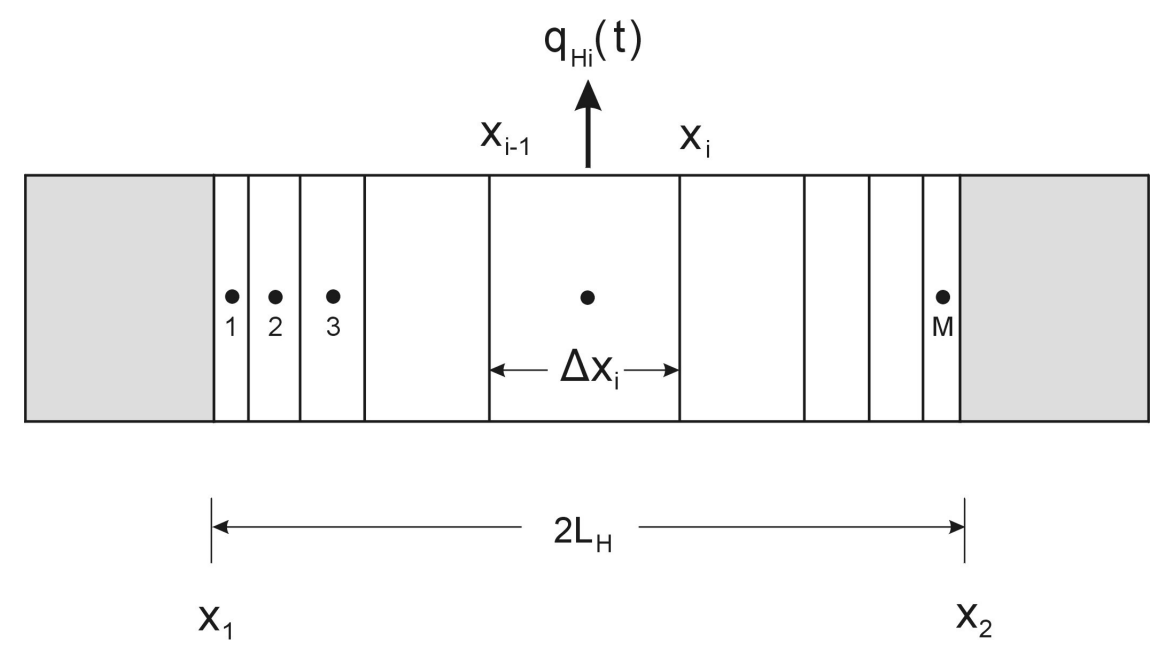

Figure C4-5 Schematic diagram of the discretization of the heater into $M$ segments. The heat flux $q_{H i}(t)$ along individual segments is constant, but may vary between segments. The dots denote the centre of the segments. A non-uniform discretization scheme is shown above.

Introducing Eq. (C4.29) into (C4.28) yields

$\hat{T}_{D}\left(x_{D}, z_{D}, s\right)=\hat{T}_{D 1}\left(x_{D}, z_{D}, s\right)+\hat{T}_{D 2}\left(x_{D}, z_{D}, s\right)$

where

$\hat{T}_{D 1}\left(x_{D}, z_{D}, s\right)=\frac{1}{L_{D} \xi_{m}^{\prime}}\left[\tanh \left(\xi_{m}^{\prime}\right) \cosh \left(\xi_{m}^{\prime} z_{D}\right)-\sinh \left(\xi_{m}^{\prime} z_{D}\right)\right] \sum_{i=1}^{M} \hat{q}_{D H, i}(s) \Delta x_{D, i}$

and 


$$
\begin{array}{r}
\hat{T}_{D 2}\left(x_{D}, z_{D}, s\right)=2 \sum_{i=1}^{M} \hat{q}_{D H, i}(s)\left\{\sum_{m=1}^{\infty} \frac{1}{m \pi \xi_{m}}\left[\tanh \left(\xi_{m}\right) \cosh \left(\xi_{m} z_{D}\right)-\sinh \left(\xi_{m} z_{D}\right)\right]\right. \\
\left.\left[\sin \left(g_{m} x_{D, i}\right)-\sin \left(g_{m} x_{D, i-1}\right)\right] \cos \left(g_{m} x_{D}\right)\right\}
\end{array}
$$

Recall Eq. (C4.24b) is

$$
\hat{T}_{D}\left(x_{D}, z_{D}, s\right)=\frac{1}{s}, \quad x_{D 1} \leq x_{D} \leq x_{D 2}, \quad z_{D}=0, \quad t_{D}>0
$$

The corresponding discretized heater equation is applied at the centre of each heater segment as

$\hat{T}_{D}\left(\bar{x}_{D, i}, z_{D}=0, s\right)=\frac{1}{s}, \quad x_{D 1} \leq \bar{x}_{D, i} \leq x_{D 2}, \quad i=1,2 \ldots . M$

where $\bar{x}_{D, i}=\left(x_{D, i}+x_{D, i-1}\right) / 2$ represents the centre of the $i$ th heater segment between $x_{D, i}$ and $x_{D, i-1}$.

For each heater segment there is one equation resulting from the application of (C4.32) to (C4.30). For $M$ segments there are $M$ equations, and the resulting system can be written as

$$
\left[a_{i, j}\right]\left\{\hat{q}_{i}\right\}=\left\{\frac{1}{s}\right\} \quad i, j=1,2 \ldots . M
$$

where

$$
\begin{aligned}
{\left[a_{i, j}\right]=} & \frac{\Delta x_{D, j}}{L_{D} \xi_{m}^{\prime}} \tanh \left(\xi_{m}^{\prime}\right) \\
& +2 \sum_{m=1}^{\infty} \frac{1}{m \pi \xi_{m}} \tanh \left(\xi_{m}\right)\left[\sin \left(g_{m} x_{D, j}\right)-\sin \left(g_{m} x_{D, j-1}\right)\right] \cos \left(g_{m} \bar{x}_{D, i}\right)
\end{aligned}
$$

From Eq. (C4.34), the heat flux for each segment is determined. These heat fluxes are then substituted into Eq. (C4.30) to allow for the calculation of the temperature at any 
point within the domain.

The solution given by $(\mathrm{C} 4.30)$ collapses to the Elder problem if $L_{\mathrm{D}}=2, x_{\mathrm{D} 1}=0$, and $x_{\mathrm{D} 2}=1$ (ie. $L=2 H, x_{1}=0$, and $x_{2}=H$ ). Comparison of the numerical simulation results from Heatflow with those from the analytical solution for $R a=0$ shows excellent agreement (Fig. C4-6). The numerical results were obtained using a grid level of 6 and a time step $\Delta t$ of 30 days. 
Analytical

Numerical

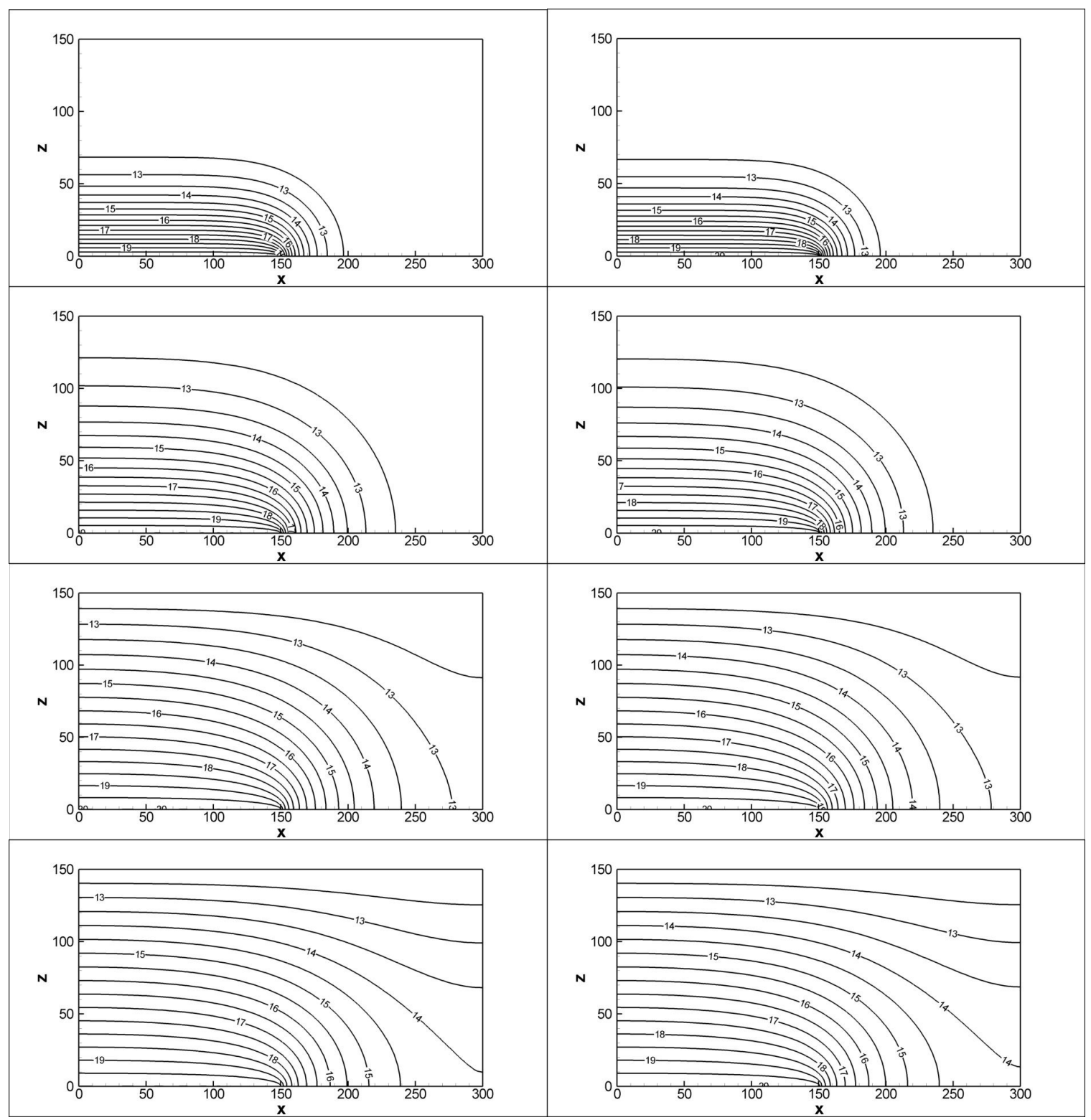

Figure C4-6 Elder solution for $R a=0$. On the left are results using the analytical solution and on the right are results using Heatflow. The results are shown at time 6 years (top), 20, 60, and 200 years (bottom).

For the above simulation, the analytical solution provided the heat flux for each heater segment. Fig. C4-7 shows the variation in the heat flux along the length of the heater. The significant increase in heat flux toward the end of the heater is due to the transition from predominantly one-dimensional flow along the left side of the heater to 
two-dimensional heat flow at the end of the heater.

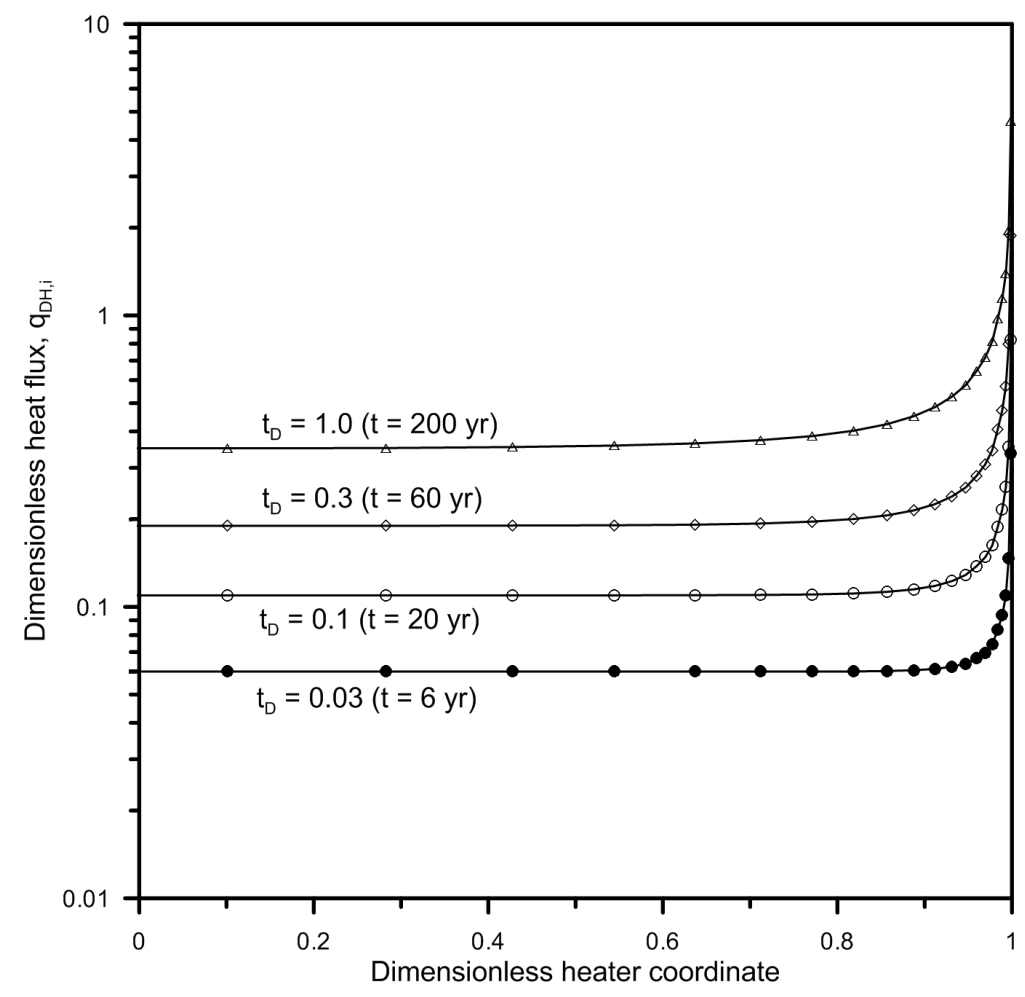

Figure C4-7 Dimensionless heat flux variation $q_{D H, i}\left(t_{D}\right)$ along the length of the heater and temporal variation of the dimensionless heat flux. The symbols denote the centre of the discretized heater segments (21 segments in this case), 0 represents the start of the heater, and 1 is the end of the heater. For this case, the start of the heater coincides with the left side of the domain (ie. $x_{\mathrm{D} 1}=0$ ). 
Low Rayleigh Number Benchmark Test, $R a=60$

Below, I present test results obtained for $R a=60$ using Heatflow. This Rayleigh number was obtained by setting the permeability $k=1.22 \times 10^{-11} \mathrm{~m}^{2}$. All the other parameters in Table C1-1 remained the same. These results are presented at 6, 20, 60, and 200 years for grid levels 4 to 9 , and at 6 and 20 years for grid level 10. The simulations presented here were completed using a time step, $\Delta t=30$ days. I completed additional simulations using $\Delta t<30$ days and found the results to be essentially the same.

Level 4

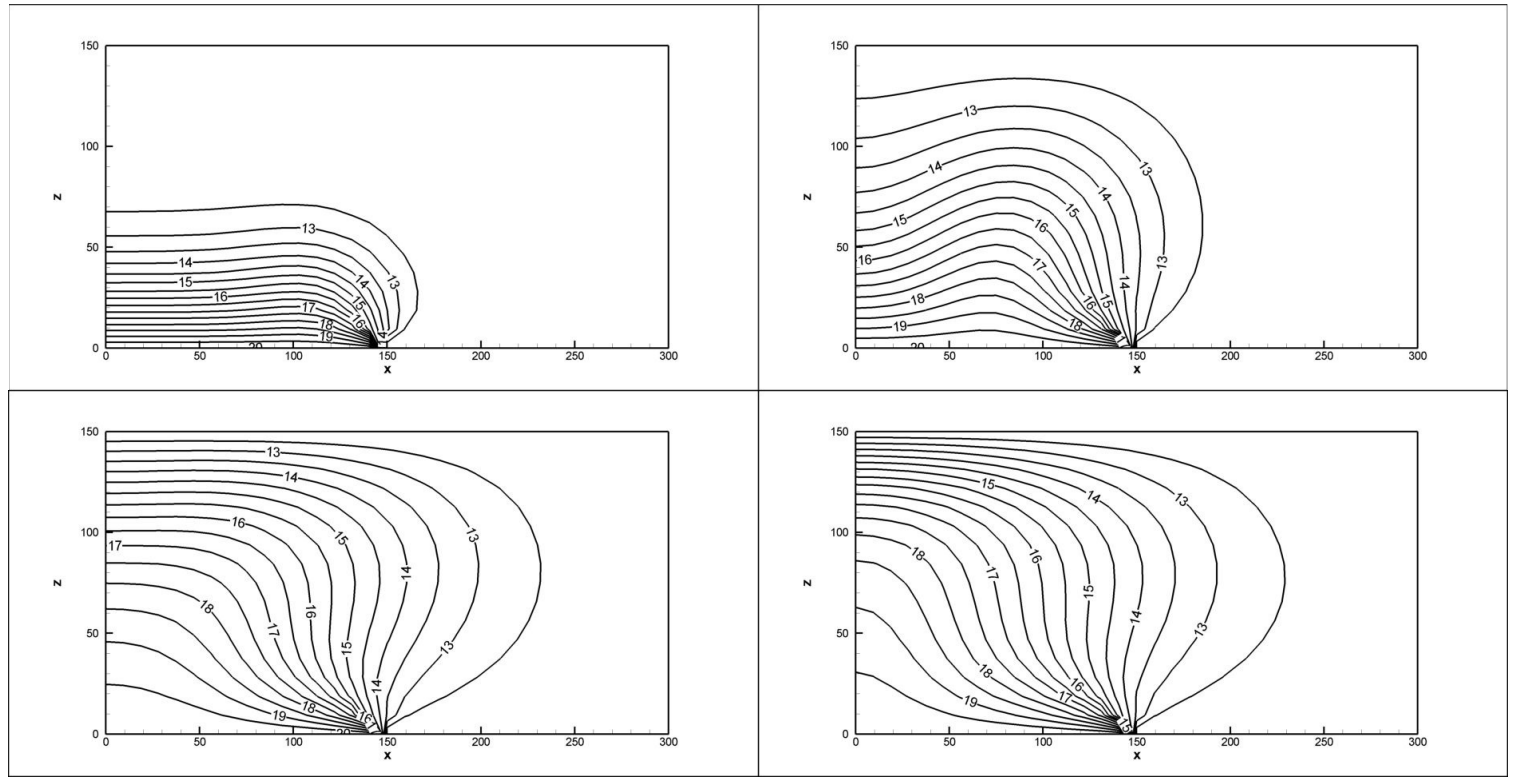


Level 5

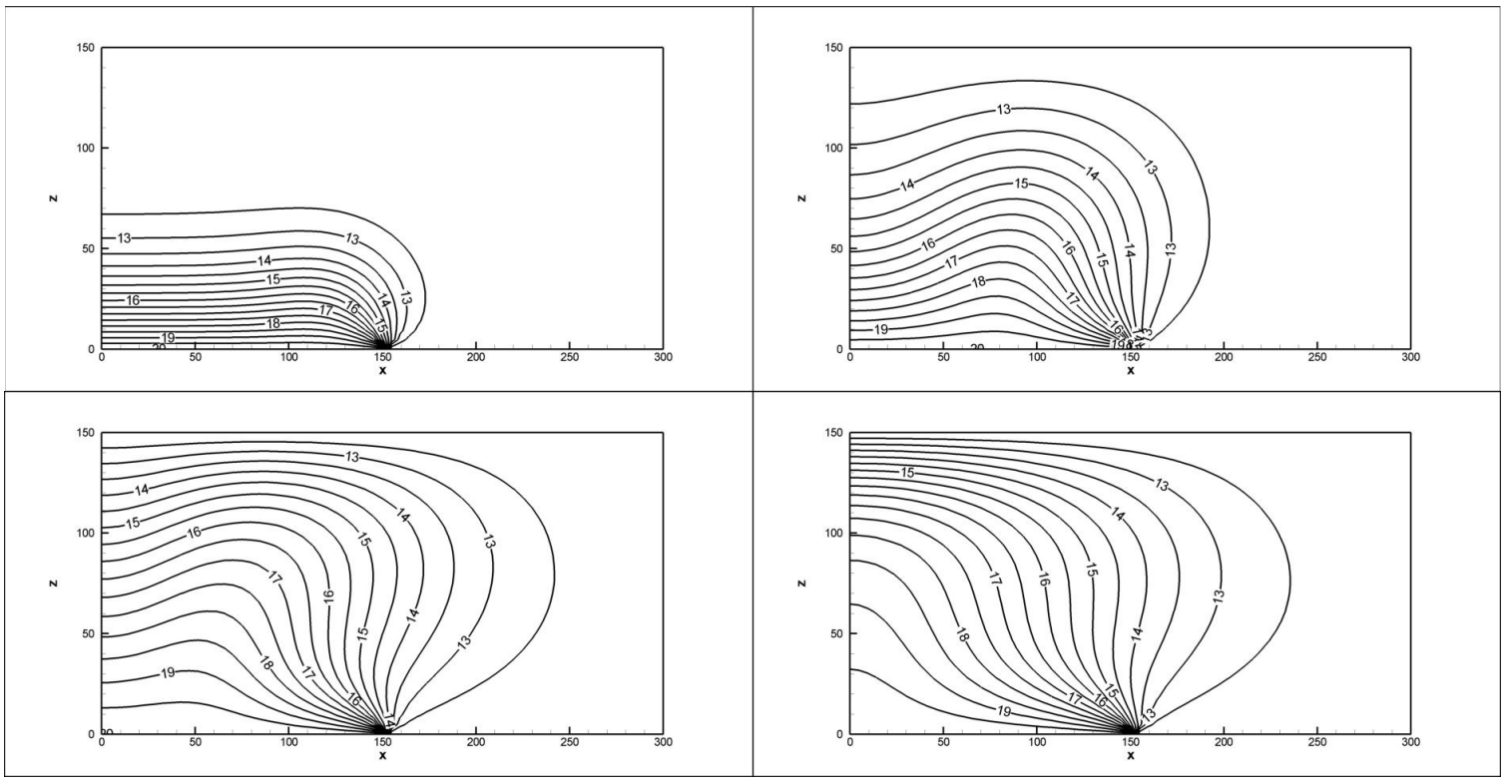

\section{Level 6}

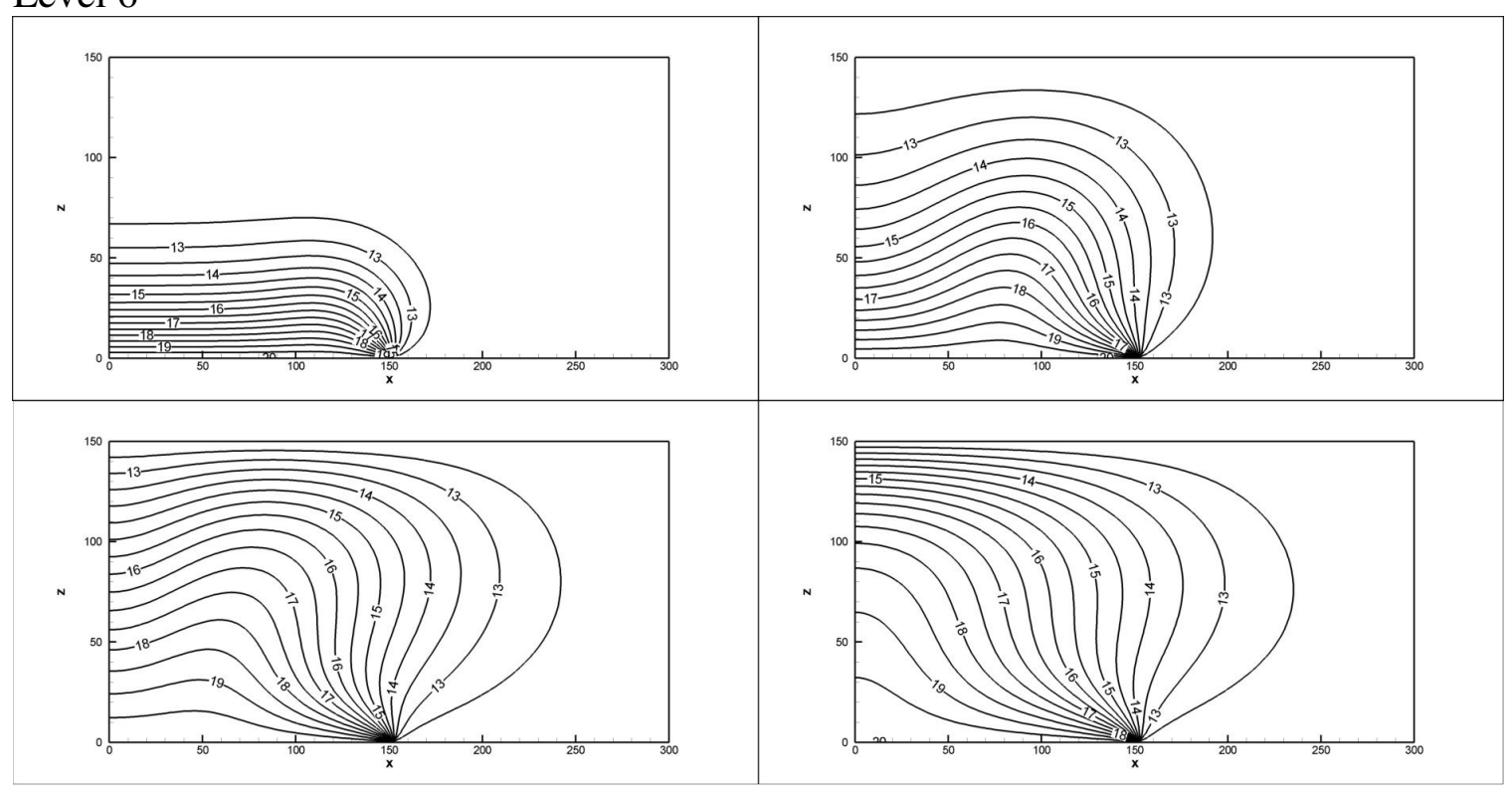


Level 7
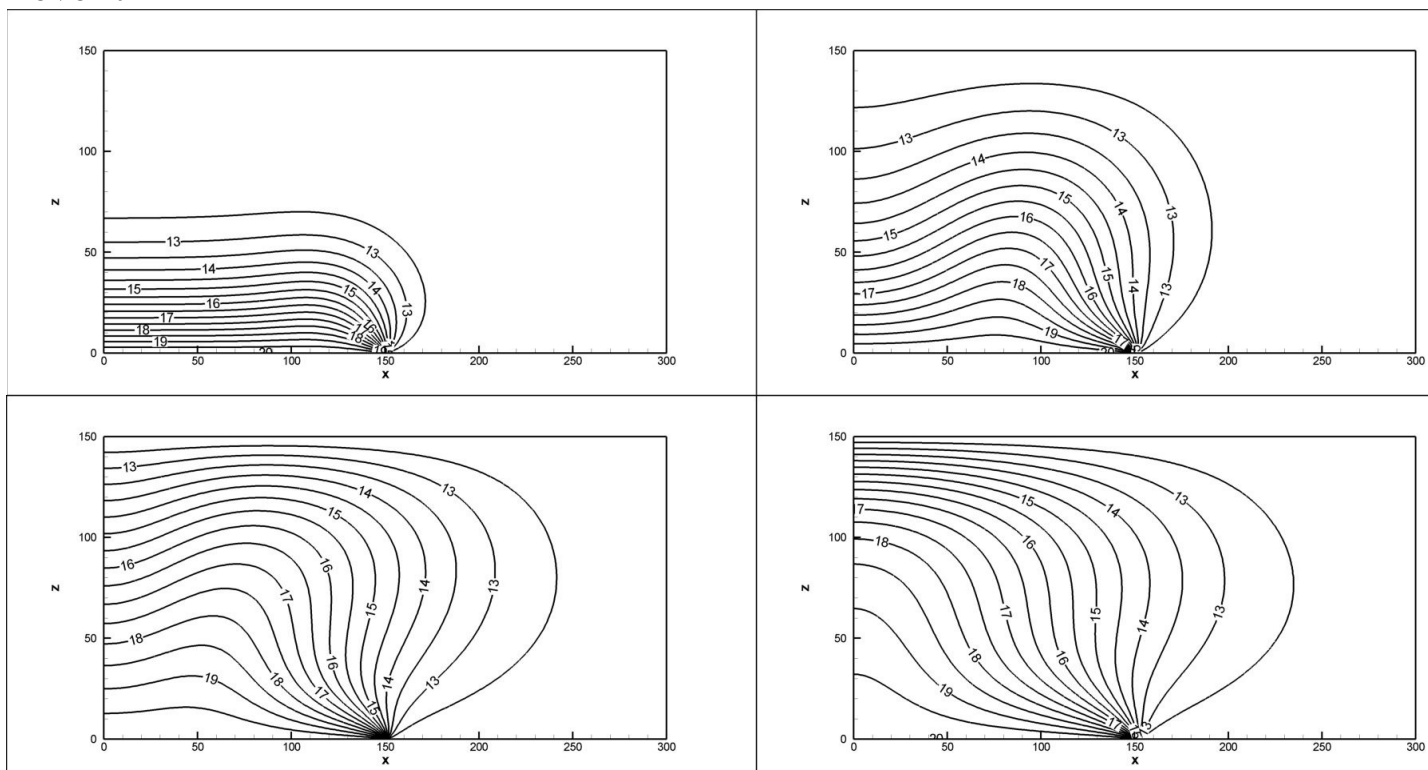

\section{Level 8}

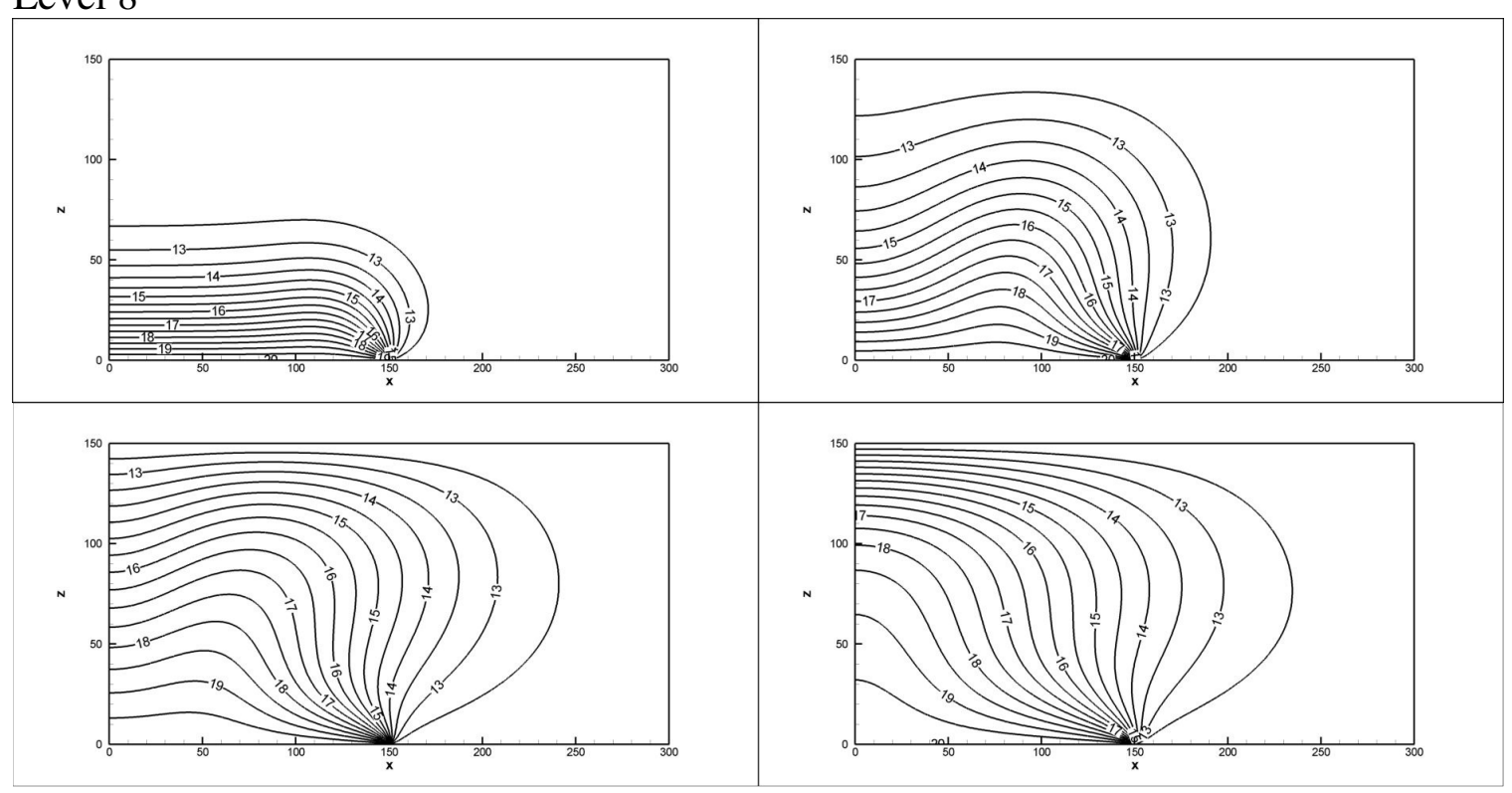


Level 9

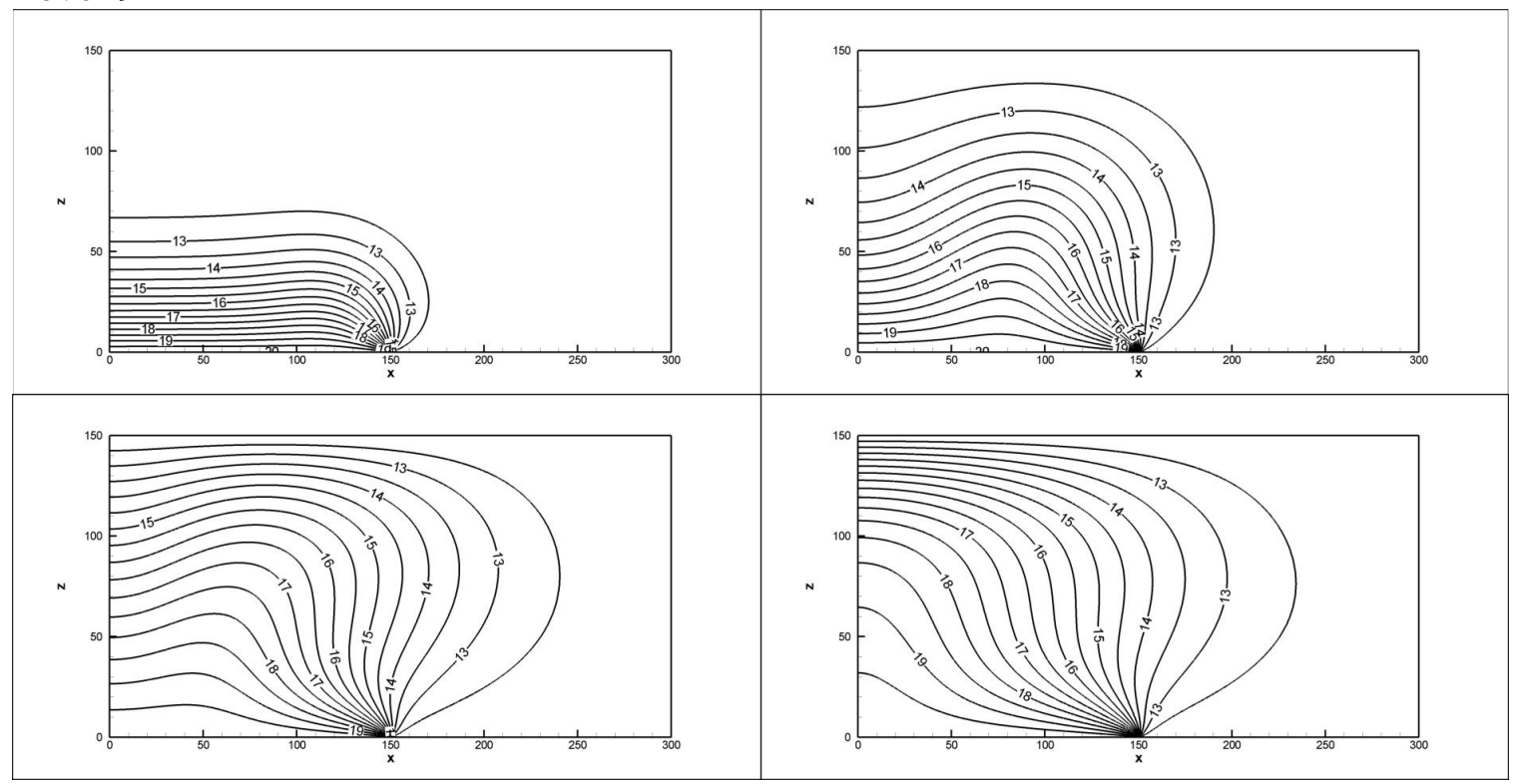

Level 10

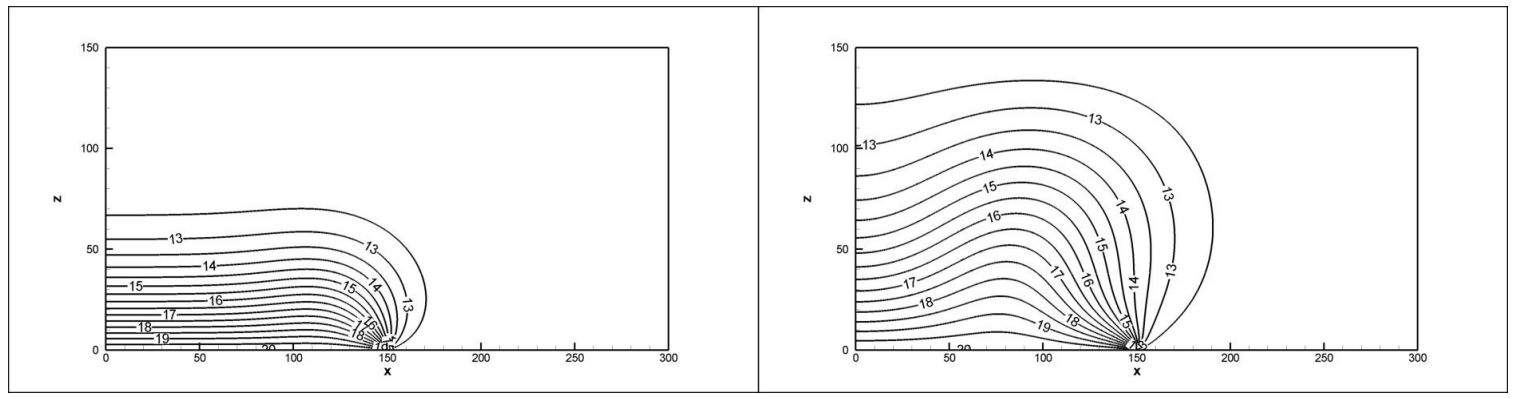

Figure C4-8 Temperature fields for Heatflow simulations with $R a=60$ and grid levels 4 to 9 for time $=6,20,60$, and 200 years (left to right).

The results presented above suggest that grid convergence is obtained by grid level 5 , with nearly identical results at all times for grid levels 5 to 10 . As will be shown in the next section $(R a>400)$, the influence of the grid discretization is more pronounced as the Rayleigh number increases. Also, these results compare well with the isoclines of concentration presented by van Reeuwijk et al. (2009). 
High Rayleigh Number Benchmark Test, $R a=400$

In this series of simulations the Rayleigh number was set equal to 400 to match that used in the majority of the solutal transport benchmark studies. This Rayleigh number was obtained by setting the permeability $k=8.18 \times 10^{-11} \mathrm{~m}^{2}$. All the other parameters in Table C4-1 remained the same. I studied the sensitivity of the results to the size of the time step ( $\Delta t=0.1,1,2,5,10$ and 30 days; results not shown here) and based on these results selected a time step of 2 days for these simulations. Simulations for grid levels 4 to 10 are presented below at times of 2, 4, 6, and 10 years.

Level 4

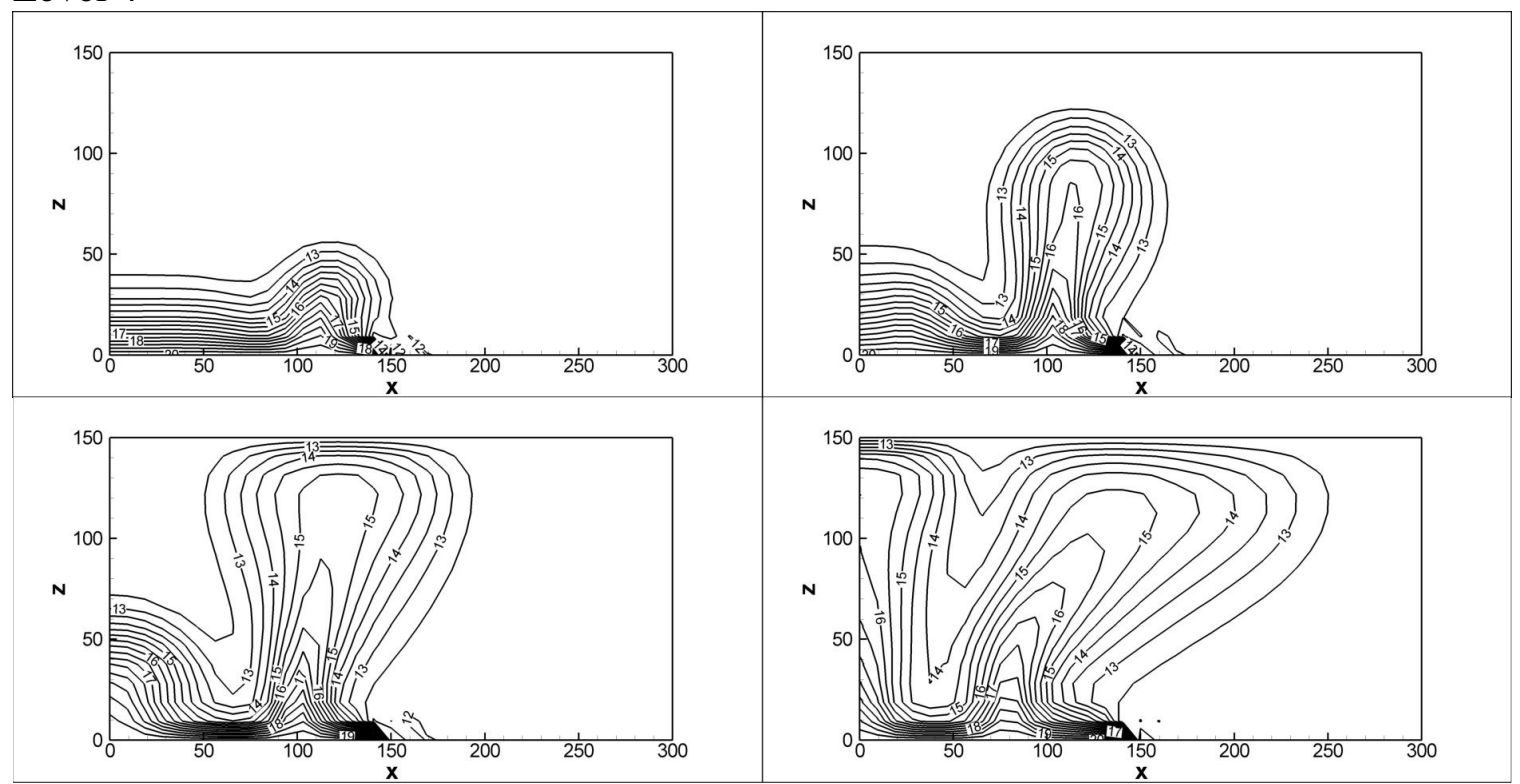


Level 5

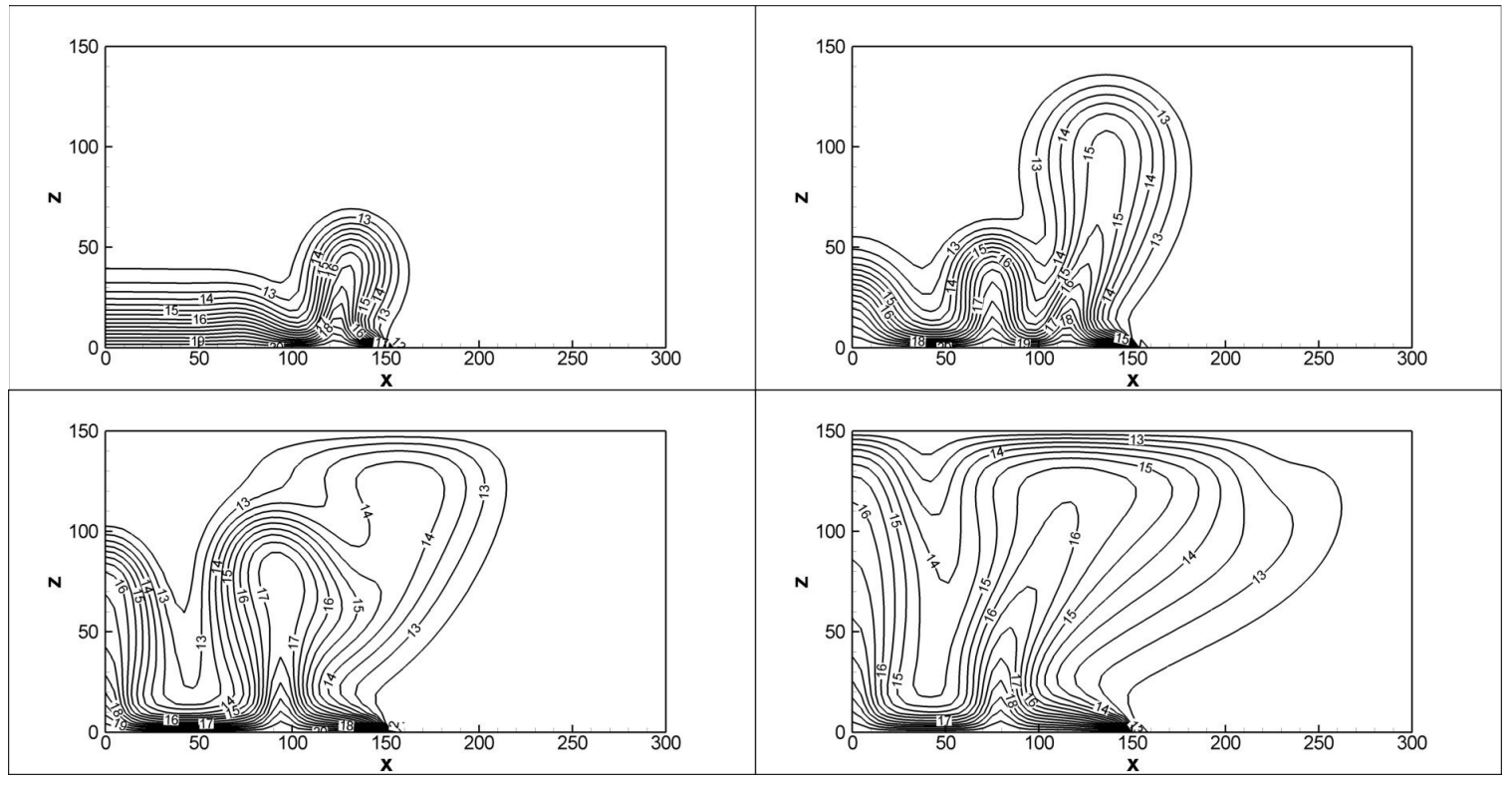

\section{Level 6}

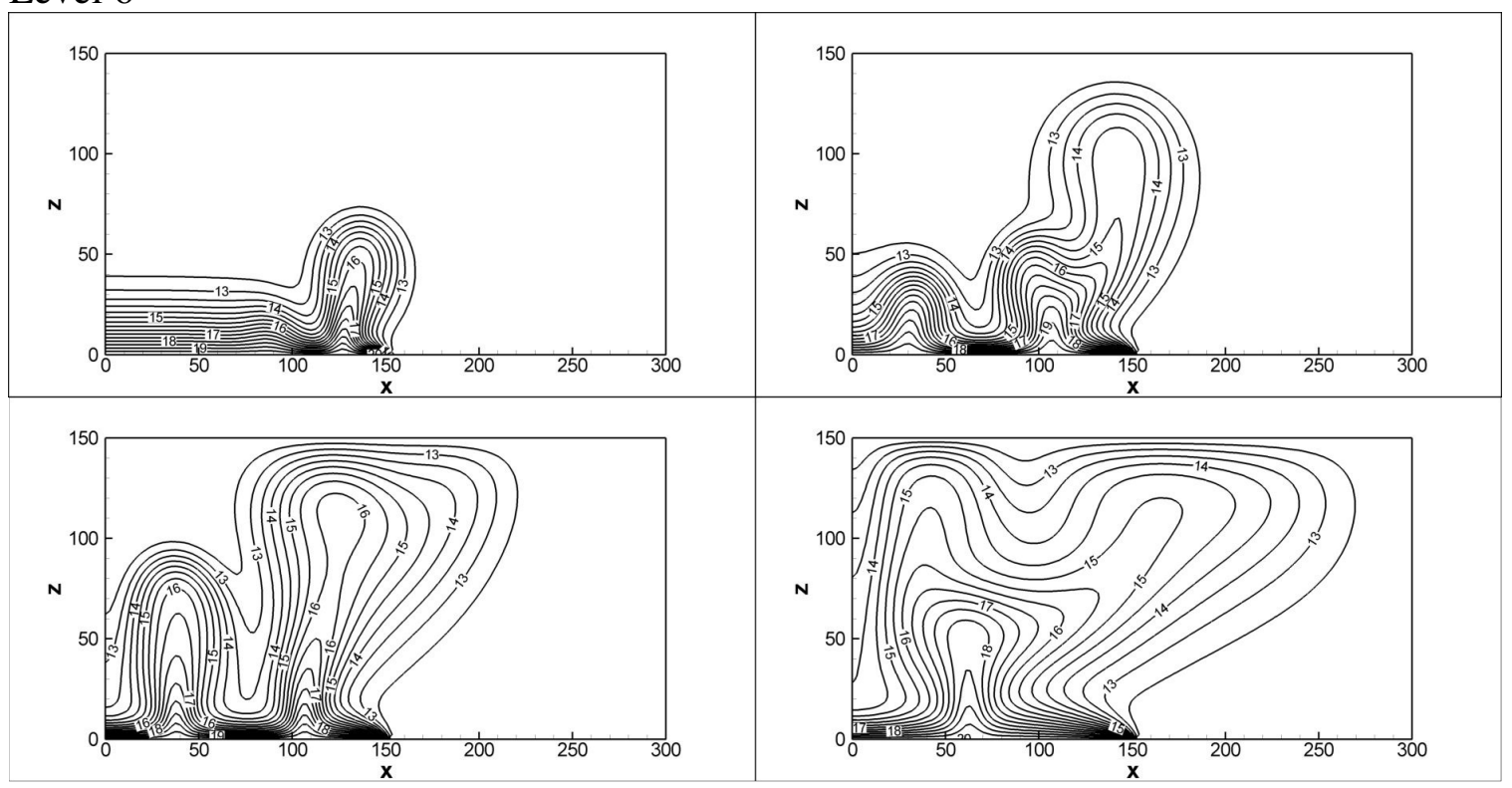


Level 7

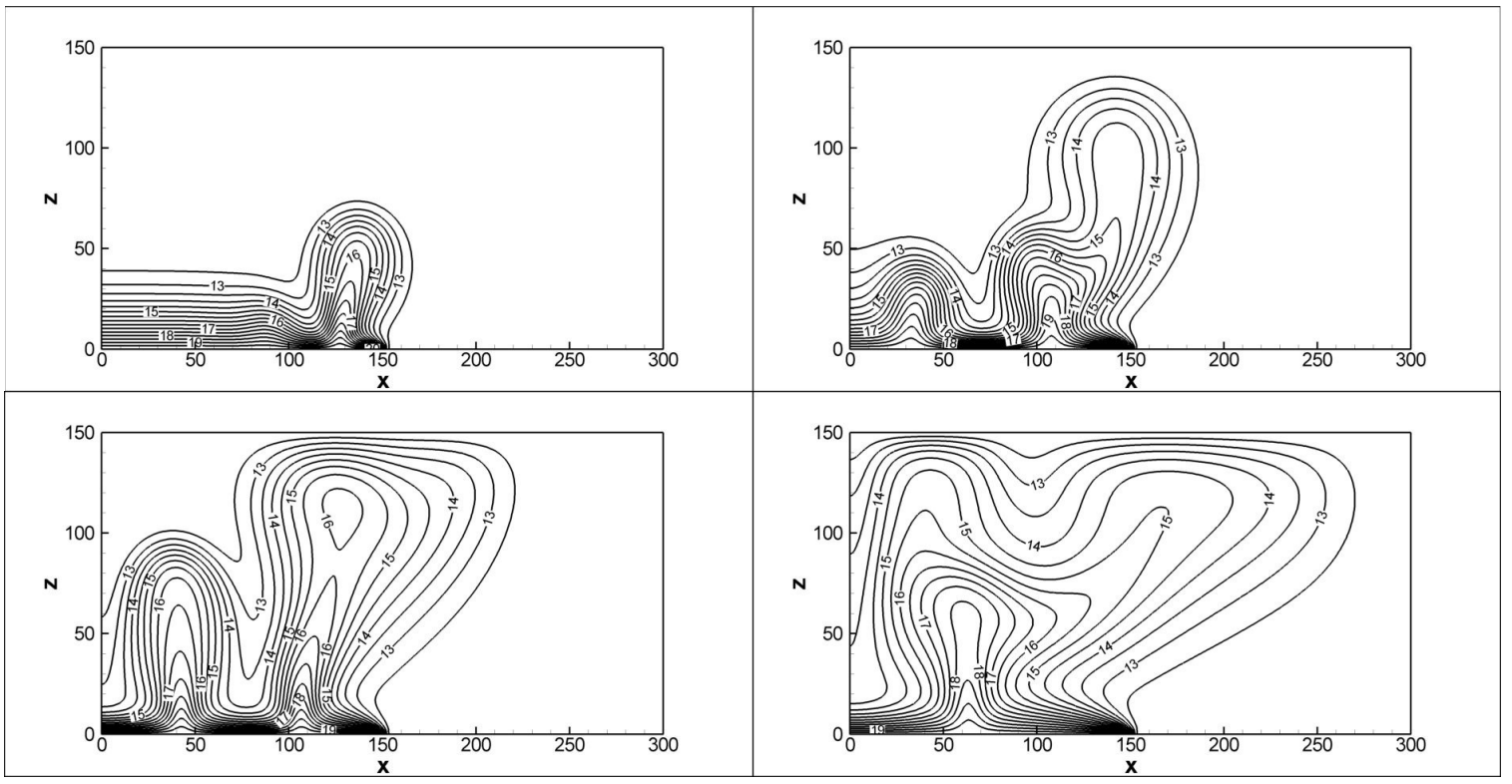

\section{Level 8}

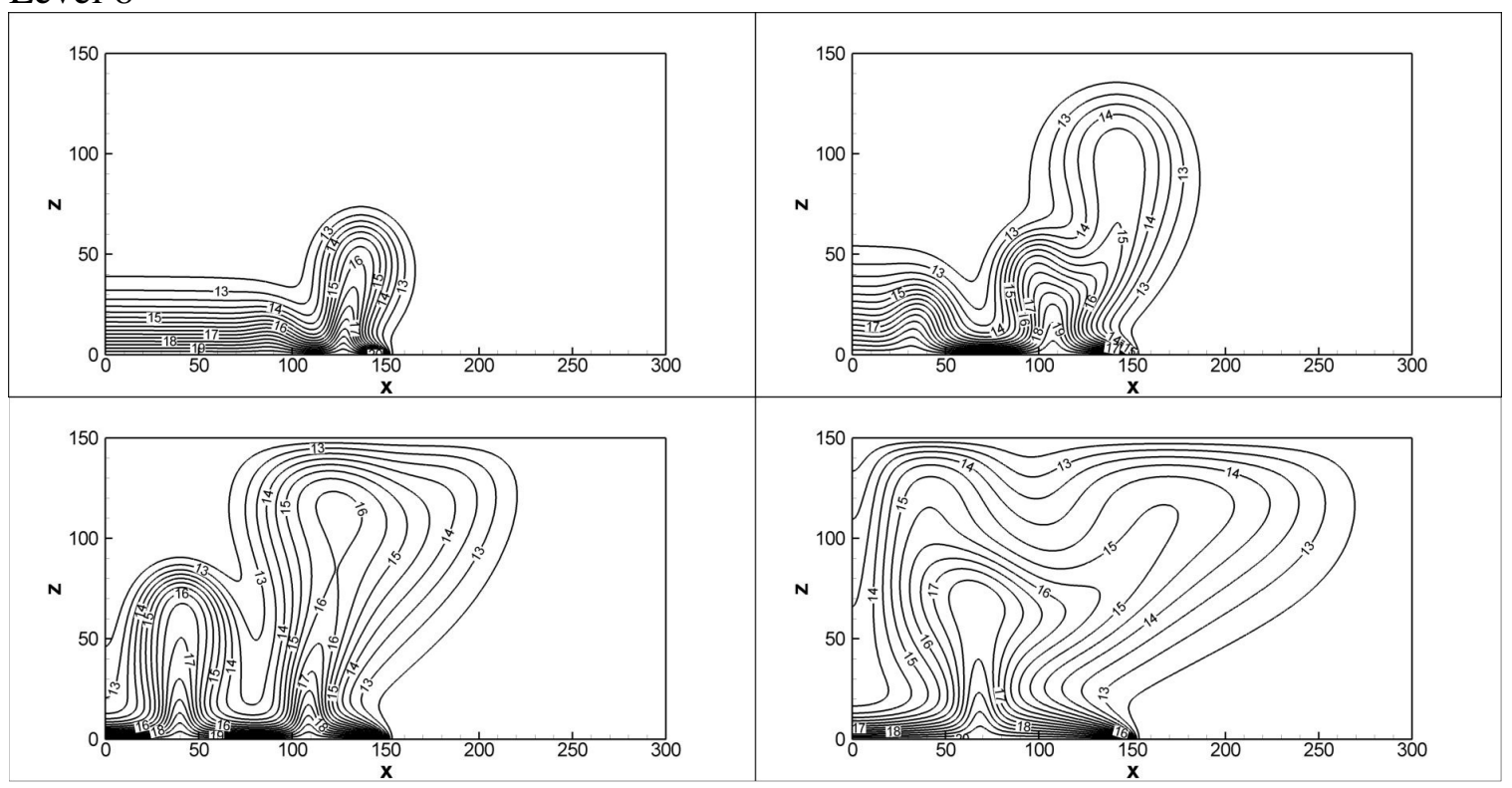


Level 9

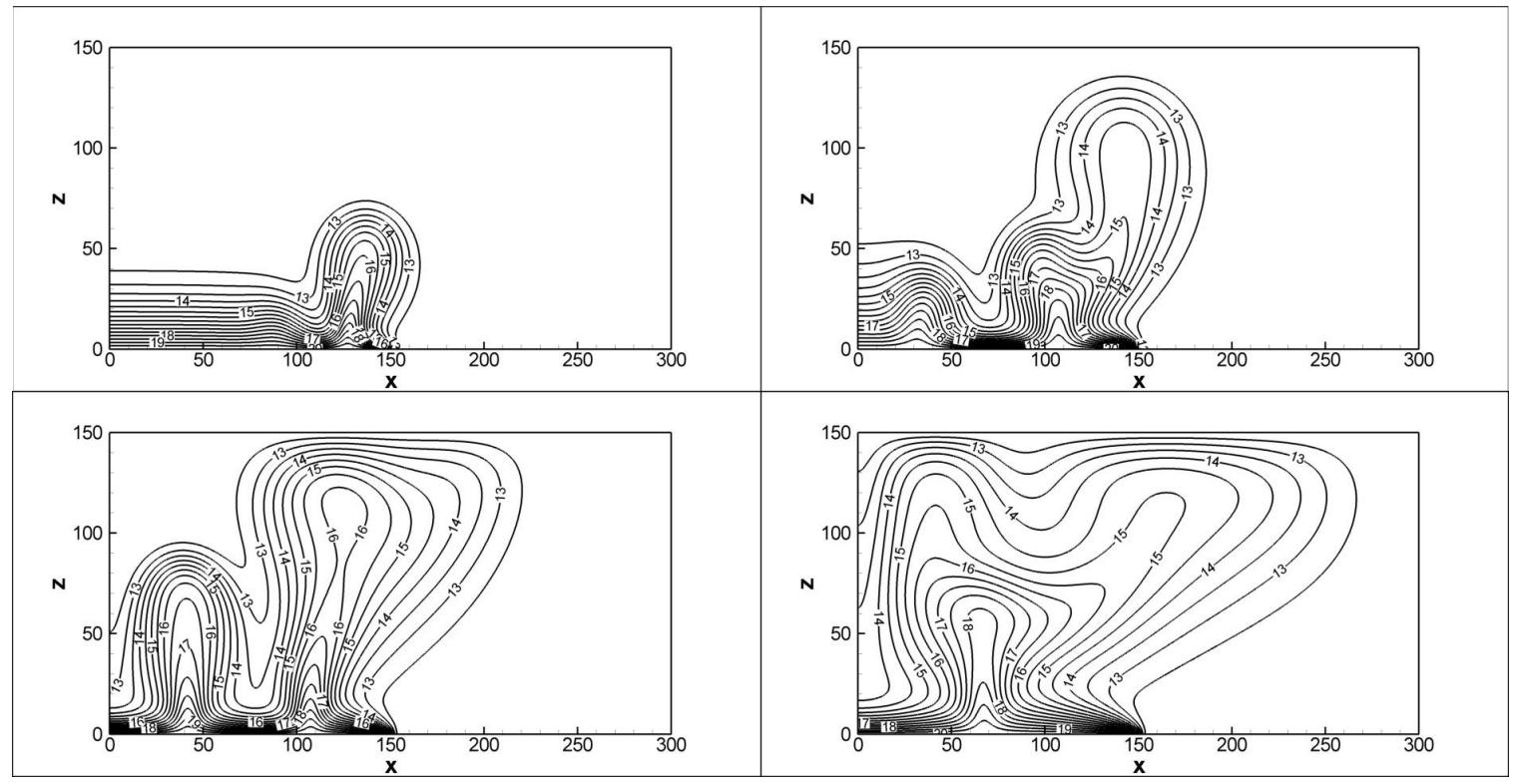

Level 10

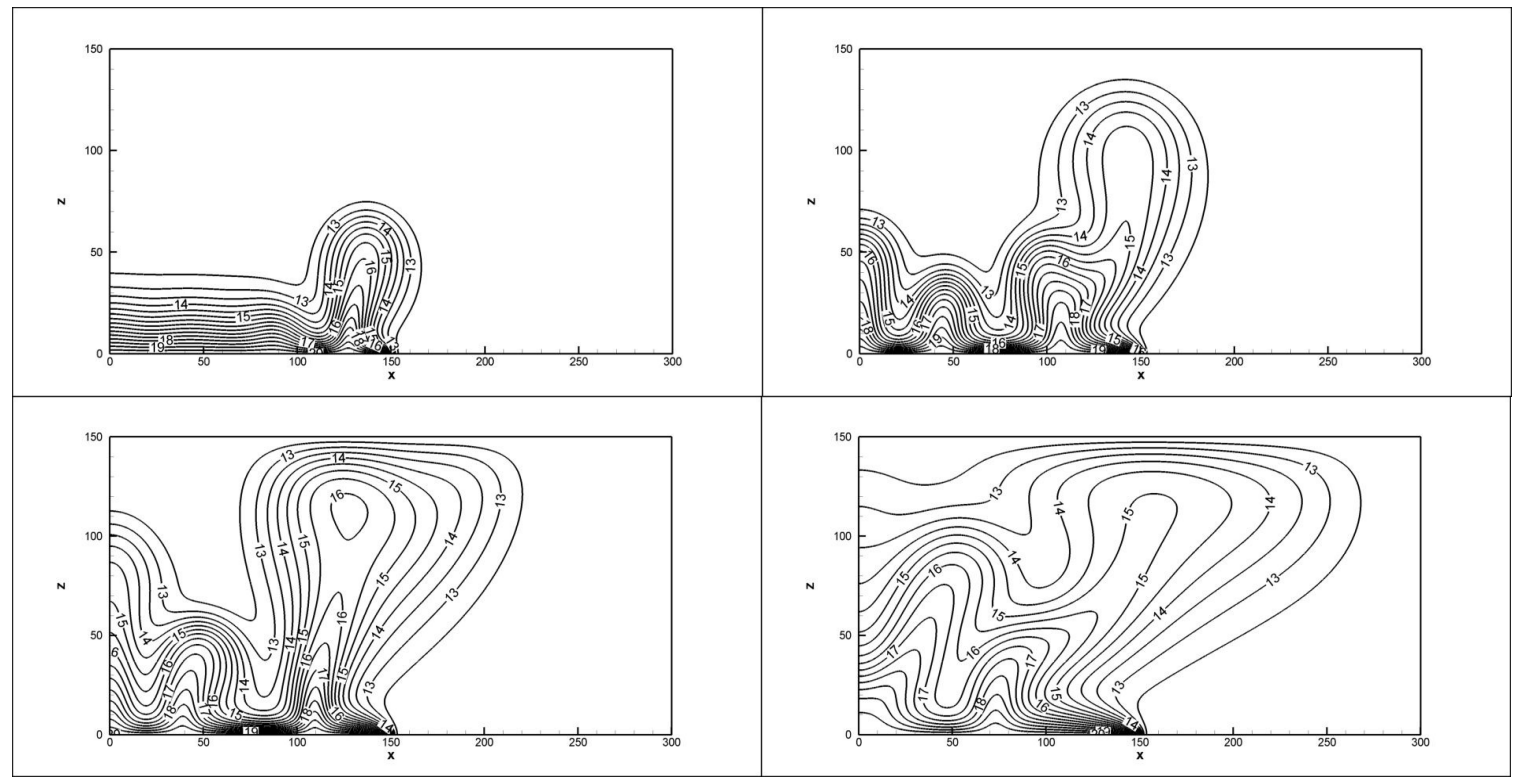

Figure C4-9 Temperature fields for Heatflow simulations with $R a=400$ and grid levels 4 to 10 for time $=2,4,6$, and 10 years (left to right).

Inspection of the above simulation results suggests that grid convergence may occur above level 7 and that the simulation results for grid level 7, 8 and 9 are similar.

However, significant differences appear at grid level 10 and these are believed to be related to the accumulation of numerical round-off errors. 
The above simulations present the solution commonly referred to as the $S_{2}$ solution (Johannsen, 2003) for up to 10 years. The steady state solution, attained at approximately 40 years is shown below along with the steady state solution for the $S_{1}$ solution. The subscript refers to the number of upward thermal plumes in the solution. To obtain the $S_{1}$ solution, I altered the initial conditions slightly by specifying a small area (2 elements by 2 elements) with an initial temperature of $13^{\circ} \mathrm{C}$.

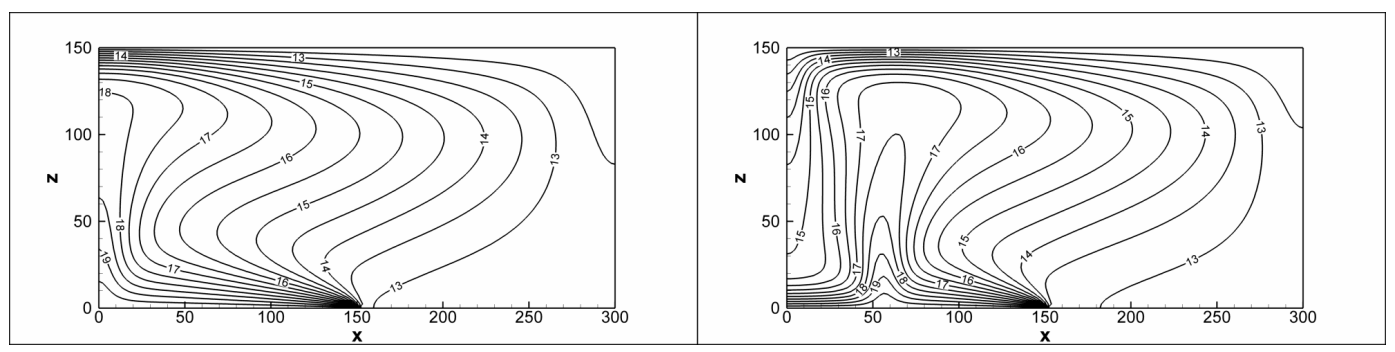

Figure C4-10 Steady state temperature fields for Heatflow simulations with $R a=400$ and grid level 6 at 40 years. On the left is the $S_{1}$ solution and on the right is the $S_{2}$ solution.

\section{High Rayleigh Number Benchmark Test, $R a=600$}

Simulations were completed using $R a=600$ (results not shown here). These results were compared directly with those of Oldenburg et al. (1995a) and Graf (2009) who report results at a similar Rayleigh number. Simulations were completed for grid levels ranging from 4 to 9 and time steps ranging from 0.1 to 30 days. The overall characteristics of the simulations are in good agreement with the numerical results reported by Oldenburg et al. (1995a) and Graf (2009). The most important observation from these simulations is that as $R a$ increases above 400, the solution becomes highly sensitive to the level of grid discretization and the time step $\Delta t$, making them less suitable benchmark tests.

In this appendix I have presented several analytical solutions to various heat transport scenarios (some new and some existing) and used these solutions as benchmark problems for verifying the numerical simulator Heatflow. In particular, I have demonstrated that Heatflow successfully reproduces the Elder problem for $R a=0,60$, and 400. As noted by others (e.g., Oldenburg and Pruess, 1995b; Holzbecher, 1998; Kolditz et al., 1998; Frolkovič and De Schepper, 2001; Diersch and Kolditz, 2002; 
Woods and Carey, 2007; Al-Maktoumi et al., 2007) as $R a$ increases above 60, the numerical solution becomes increasingly sensitive to the grid discretization and time step size.

\section{References}

Al-Maktoumi, A., D.A. Lockington, R.E. Volker. 2007. SEAWAT 2000: modelling unstable flow and sensitivity to discretization levels and numerical schemes. Hydrogeology Journal 15:1119-1129.

Chang, C.-C., C.-S. Chang. 2003. A flowing partially penetrating well in a finitethickness aquifer: a mixed-type initial boundary value problem. Journal of Hydrology 271:101-118.

Churchill, R.V. 1972. Operational Mathematics. McGraw-Hill, Inc.

Dagan, G. 1978. A note on packer, slug, and recovery tests in unconfined aquifers. Water Resources Research 14:929-934.

Diersch, H.-JG. 1981. Primitive variables finite element solutions of free convection flows in porous media. ASME Journal of Heat Transfer 61:325-337.

Diersch, H.-JG., O. Kolditz. 1998. Coupled groundwater flow and transport: 2. Thermohaline and 3D convection systems. Advances in Water Resources 21:401-425.

Diersch, H.-JG., O. Kolditz. 2002. Variable-density flow and transport in porous media: approaches and challenges. Advances in Water Resources 25:899-944.

Elder, J.W. 1967. Transient convection in a porous medium. Journal of Fluid Mechanics 27:609-623.

Frind , E.O. 1982. Simulation of long-term transient density-dependent transport in groundwater. Advances in Water Resources 5:73-88.

Frolkovič, P., H. De Schepper. 2001. Numerical modelling of convection dominated transport coupled with density driven flow in porous media. Advances in Water Resources 24:63-72.

Graf, T. 2009. Simulation of geothermal flow in deep sedimentary basins in Alberta. Energy Resources Conservation Board, ERCB/AGS Open File Report 2009-11.

Gringarten, A.C., H.J. Ramey. 1975. An approximate infinite conductivity solution for a 
partially penetrating line-source well. Society of Petroleum Engineers Journal 259:140-148.

Holzbecher, E.O.1998. Modeling density-driven flow in porous media. Springer Verlag, Berlin, Germany.

Johannsen, K. 2003. On the validity of the Boussinesq approximation for the Elder problem. Computational Geosciences 7:169-182.

Knabner, P., P. Frolkovič. 1996. Consistent velocity approximation for finite volume or element discretizations of density driven flow in porous media. In: Aldama AA et al., editors. Computational methods in water resources XI, vol. 1: Computational methods in subsurface flow and transport problems. Southhampton:

Computational Mechanics Publications:93-100.

Kolditz, O., R. Ratke, H.-JG. Diersch, W. Zielke. 1998. Coupled groundwater flow and transport: 1. Verification of variable-density flow and transport models. Advances in Water Resources 21:27-46.

Lapwood, E.R. 1948. Convection of a fluid in a porous medium. Proceedings of the Cambridge Philosophical Society. A 225:508-521.

Leijnse, A. 1992. Three-dimensional modeling of coupled flow and transport in porous media. PhD thesis. Indiana: University of Notre Dame.

Oldenburg, C.M., R.L. Hinkins, G.J. Moridis, K. Pruess. 1995a. On the development of MP-TOUGH2. Proceedings of TOUGH Workshop 1995, Lawrence Berkley Laboratory, Berkley, California. pp:252-258.

Oldenburg, C.M., K. Pruess. 1995b. Dispersive transport dynamics in a strongly coupled groundwater-brine flow system. Water Resources Research 31:289-302.

Park, C., M. Aral. 2007. Sensitivity of the solution of the Elder problem to density, velocity and numerical perturbations. Journal of Contaminant Hydrology 92:33-49.

Stallman, R.W. 1965. Steady one-dimensional fluid flow in a semi-infinite porous medium with sinusoidal surface temperature. Journal of Geophysical Research 70:2821-2827.

Simpson, M.J., T.P. Clement. 2003. Theoretical analysis of the worthiness of Henry and Elder problems as benchmarks of density-dependent groundwater flow models. Advances in Water Resources 26:17-31.

van Reeuwijk, M., S.A. Mathias, C.T. Simmons, J.D. Ward. 2009. Insights from a 
pseudospectral approach to the Elder problem. Water Resources Research 45:W04416, doi:10.1029/2008WR007421.

Voss, C.I., W.R. Souza. 1987. Variable density flow and solute transport simulation of regional aquifers containing a narrow freshwater-saltwater transition zone. Water Resources Research 23:1851-1866.

Woods, J.A., G.F. Carey. 2007. Upwelling and downwelling behavior in the Elder-VossSouza benchmark. Water Resources Research 43, W12403, doi:10.1029/2006WR004918. 


\section{Appendix D: Watershed Sub-basins}

\section{Estimating upland runoff}

In this section, I estimate the runoff from the uplands that is available for infiltration. The uplands (Fig. D-1) may be categorized as:

1) channeled flow sub-basins from which the runoff is transmitted to the aquifer via a creek or stream, and

2) unchanneled flow sub-basins from which the runoff moves to the aquifer by either overland sheet flow or shallow subsurface flow.

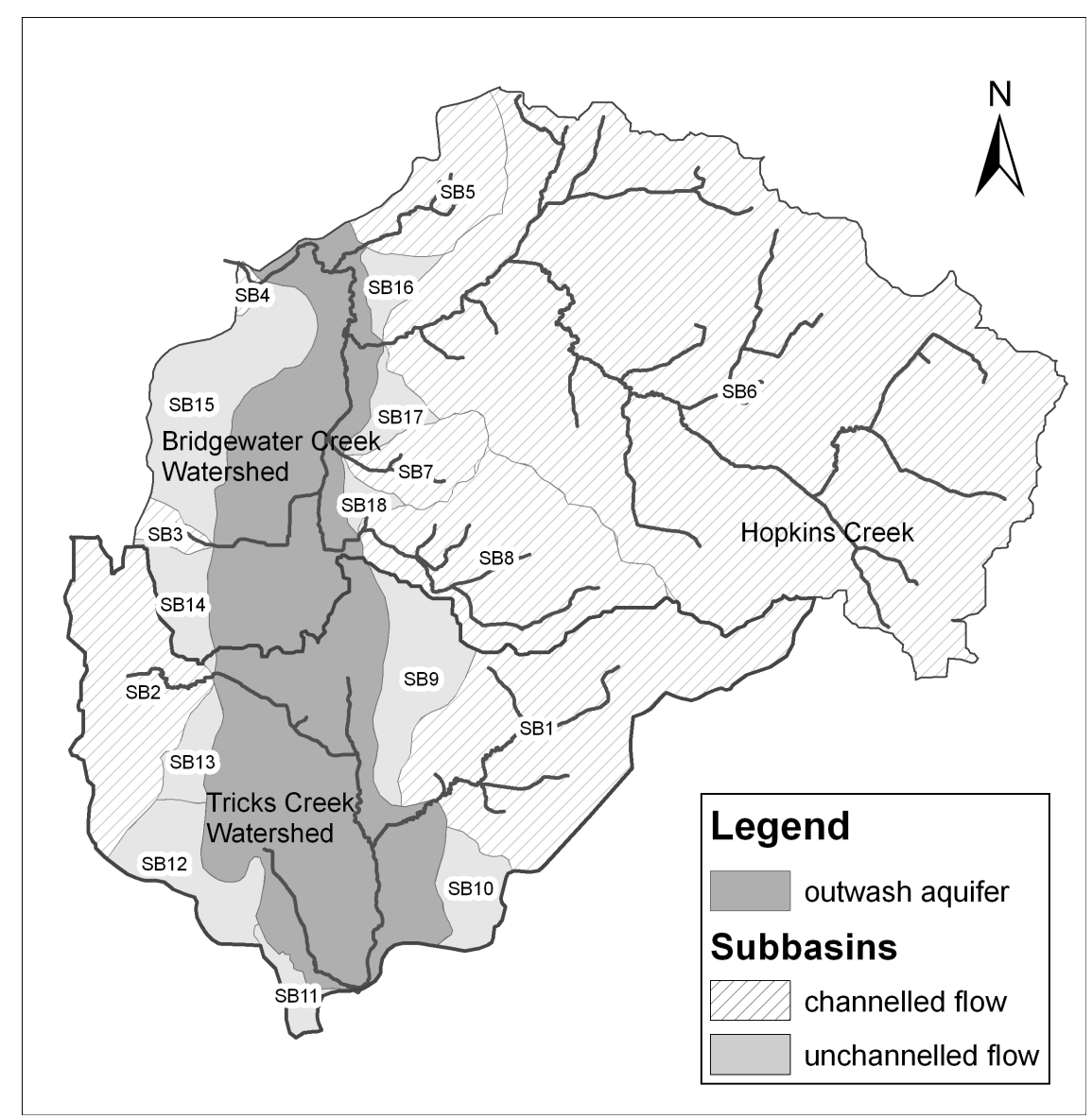

Figure D-1 Upland area contributing to recharge to the glacial-outwash aquifer via channeled and unchanneled flow from sub-basins bordering the aquifer within the Tricks Creek and Bridgewater Creek Watersheds. 
Table D-1 Summary of upland sub-basins, areas, and average annual runoff available for infiltration, for channeled and unchanneled flow.

\begin{tabular}{lllrl}
\hline Sub-basin & Basin type & Watershed & \multicolumn{1}{c}{ Area } & Total runoff \\
\hline & & & $\mathrm{m}^{2}-$ & $\mathrm{m}^{3} \mathrm{~s}^{-1} \bar{y}$ \\
\cline { 4 - 4 } SB1 & channeled & Tricks Creek & 7801244 & $9.91 \times 10^{-2}$ \\
SB2 & channeled & Tricks Creek & 3882091 & $4.93 \times 10^{-2}$ \\
SB3 & channeled & Bridgewater Creek & 346596 & $4.40 \times 10^{-3}$ \\
SB4 & channeled & Bridgewater Creek & 175495 & $2.23 \times 10^{-3}$ \\
SB5 & channeled & Bridgewater Creek & 2076471 & $2.64 \times 10^{-2}$ \\
SB6 & channeled & Bridgewater Creek & 33109238 & $4.20 \times 10^{-1}$ \\
SB7 & channeled & Bridgewater Creek & 1236631 & $1.57 \times 10^{-2}$ \\
SB8 & channeled & Bridgewater Creek & 5096051 & $6.47 \times 10^{-2}$ \\
SB9 & unchanneled & Tricks Creek & 1844874 & $2.34 \times 10^{-2}$ \\
SB10 & unchanneled & Tricks Creek & 933502 & $1.19 \times 10^{-2}$ \\
SB11 & unchanneled & Tricks Creek & 402996 & $5.12 \times 10^{-3}$ \\
SB12 & unchanneled & Tricks Creek & 1566947 & $1.99 \times 10^{-2}$ \\
SB13 & unchanneled & Tricks Creek & 592151 & $7.52 \times 10^{-3}$ \\
SB14 & unchanneled & Tricks Creek & 868236 & $1.10 \times 10^{-2}$ \\
SB15 & unchanneled & Bridgewater Creek & 2974287 & $3.78 \times 10^{-2}$ \\
SB16 & unchanneled & Bridgewater Creek & 506581 & $6.43 \times 10^{-3}$ \\
SB17 & unchanneled & Bridgewater Creek & 566435 & $7.19 \times 10^{-3}$ \\
SB18 & unchanneled & Bridgewater Creek & 255378 & $3.24 \times 10^{-3}$ \\
\hline
\end{tabular}

$\dagger$ The average annual runoff available for infiltration from both the channeled and unchanneled uplands is $1.27 \times 10^{-2} \mathrm{~m}^{3} \mathrm{~s}^{-1} \mathrm{~km}^{-2}\left(400 \mathrm{~mm} \mathrm{yr}^{-1}\right)$ which is equal to the discharge measured at TR-Qa during the period 1993-1997. 


\section{Appendix E: Recharge and Baseflow}

\section{Appendix E1: Groundwater recharge}

Recharge is a critical input to the groundwater flow models and is generally treated as a calibration parameter. To provide reasonable bounds on the recharge used for model calibration, I estimated the recharge using two techniques which include the:

1. water budget for the watershed, and

2. baseflow discharge with consideration of the flow from the upland till.

I chose these techniques based on the data that was available for the watershed and the relative success of these methods reported in the literature (e.g., Scanlon et al., 2002).

\section{Water budget}

The water budget method, based on a balance equation for the volume of water within a given region, can be stated as:

Volume water entering $=$ Volume water leaving + change in storage

The balance equation may be written more explicitly as:

$$
P+Q_{\text {in }}=E T+Q_{\text {out }}+\Delta S
$$

where $P$ is precipitation, $Q_{\text {in }}$ and $Q_{\text {out }}$ are water flow into and out of the region, respectively, $E T$ is evapotranspiration, and $\Delta S$ is the change in water storage within the region. Each component may be subdivided into several subcomponents given by

$$
\begin{aligned}
P+Q_{i n}^{s w}+Q_{i n}^{g w}= & E T^{s w}+E T^{u z}+E T^{g w} \\
& +R_{0}+Q^{b f}+Q_{\text {out }}^{g w} \\
& +\Delta S^{\text {snow }}+\Delta S^{s w}+\Delta S^{u z}+\Delta S^{g w}
\end{aligned}
$$


where $Q_{i n}^{s w}$ and $Q_{i n}^{g w}$ are surface water and groundwater flowing into the region, $E T^{\text {sw }}$ $E T^{\mathrm{uz}}$ and $E T^{\mathrm{gw}}$ are evapotranspiration from the surface water, unsaturated zone and groundwater respectively, $R_{0}$ is the runoff or overland surface-water flow, $Q_{\mathrm{bf}}$ is the groundwater discharge to the streams, $Q_{\text {out }}^{g w}$ is the groundwater flow out of the region, and $\Delta S^{\text {snow }}, \Delta S^{\mathrm{sw}}, \Delta S^{\mathrm{uz}}$, and $\Delta S^{\mathrm{gw}}$ are the changes in storage of water in the snow, surfacewater bodies, unsaturated zone, and saturated groundwater zones respectively. Groundwater recharge, $R$, includes any infiltrating water that reaches the saturated zone and can be written as

$$
R=Q_{o u t}^{g w}-Q_{i n}^{g w}+Q^{b f}+E T^{g w}+\Delta S^{g w}
$$

This equation states that any water that reaches the water table either flows out of the region as groundwater, is discharged to the surface water features as baseflow, is evapotranspired, or is retained in storage. Substitution of Eq. (E1.4) into Eq. (E1.3) gives

$$
R=P+Q_{i n}^{s w}-R_{0}-E T^{s w}-E T^{u z}-\Delta S^{s n o w}-\Delta S^{s w}-\Delta S^{u z}
$$

Values for each parameter may be measured directly or estimated indirectly from available data. It is often assumed that $\Delta S^{\text {snow }}, \Delta S^{\mathrm{sw}}$, and $\Delta S^{\mathrm{uz}}$ are all approximately zero on an annual basis. Thus, the following data are required to estimate the recharge:

- $\quad$ measured precipitation $(P)$;

- $\quad$ estimates of evapotranspiration $(E T)$;

- measured surface-water flow into and out of region $\left(Q_{i n}^{s w}\right.$ and $\left.Q_{\text {out }}^{s w}\right)$;

- drainage area above gauge stations, and

- change in groundwater storage over the period of the water balance (based on groundwater level data and assumed to be zero for a water year).

For the Tricks Creek watershed, with the exception of ET, I have measured these data during specific intervals in this study. In the following two sections, I present the method 
for estimating ET for the region followed by estimates of the recharge obtained using stream-flow partitioning and digital filtering.

\section{Evapotranspiration}

Estimating ET (evapotranspiration) generally involves two steps. First, an estimate of the potential evapotranspiration is made, where the potential evapotranspiration is either for a well-watered reference crop $\left(E T_{\mathrm{r}}\right.$ or $\left.E T_{\mathrm{o}}\right)$ or is a surface-dependent evapotranspiration $\left(E T_{\mathrm{s}}\right)$. Next an estimate of actual evapotranspiration $E T$ is obtained by multiplying the potential evapotranspiration by a crop coefficient. In general, the methods for estimating potential ET are classified into three groups: temperature, radiation, and combination methods. The temperature methods use air temperature as a surrogate for the amount of energy that is available for evapotranspiration (i.e., Thorthwaite, 1955a, b and 1957; Hamon, 1963; Hargreaves and Samani, 1985; Hargreaves, 1994). The radiation methods use solar radiation coupled with air temperature to predict ET (i.e., Hargreaves and Samani, 1985; Turc, 1961; Priestley and Taylor, 1972; Jensen and Haise, 1963), and finally the combination methods use radiation, air temperature, wind speed and relative humidity (Jensen et al., 1990; Allen et al,. 1998; Allen et al., 2000).

The accuracy of these methods is variable (i.e., Jensen et al., 1990; Feder et al., 1996; Vosmarty et al., 1998; Martin, 2000; Jacobs and Satti, 2001). In general, the combination methods give the best results provided the required data are available. In this study, I compared the above noted methods and selected the combination method FAO56-PM (Allen et al., 1998). This method provides an estimate of ET that is close to the average of all these methods, it allows for estimates of both daily and monthly ET, and it has well-documented methods for estimating data that have not been measured and are not directly available. The form of the FAO56-PM implemented is given below.

$$
E T_{S}=\frac{0.408 \Delta\left(R_{n}-G\right)+\gamma \frac{900}{T+273} u_{2}\left(e_{s}-e_{a}\right)}{\Delta+\gamma\left(1+0.34 u_{2}\right)}
$$


where $E T_{\mathrm{S}} \quad$ reference evapotranspiration $\left[\mathrm{mm} \mathrm{day}^{-1}\right]$;

$R_{\mathrm{n}} \quad$ net radiation at the crop surface $\left[\mathrm{MJ} \mathrm{m}^{-2}\right.$ day $\left.^{-1}\right]$;

$G \quad$ soil heat flux density $\left[\mathrm{MJ} \mathrm{m}^{-2}\right.$ day $\left.^{-1}\right]$;

$T$ mean daily air temperature at $2 \mathrm{~m}$ height $\left[{ }^{\circ} \mathrm{C}\right]$;

$u_{2} \quad$ wind speed at $2 \mathrm{~m}$ height $\left[\mathrm{m} \mathrm{s}^{-1}\right]$;

$e_{\mathrm{s}} \quad$ saturation vapour pressure $[\mathrm{kPa}]$;

$e_{\mathrm{a}} \quad$ actual vapour pressure $[\mathrm{kPa}]$;

$e_{\mathrm{s}}-e_{\mathrm{a}} \quad$ saturation vapour pressure deficit $[\mathrm{kPa}]$;

$\Delta \quad$ slope vapour pressure curve $\left[\mathrm{kPa}^{\circ} \mathrm{C}^{-1}\right]$, and

$\gamma \quad$ psychrometric constant $\left[\mathrm{kPa}^{\circ} \mathrm{C}^{-1}\right]$.

The potential evapotranspiration for specific crops under given weather conditions may be estimated by modifying the $E T_{\mathrm{r}}$ by a crop adjustment factor (Doorenbos and Pruitt, 1977; Allen et al., 2000)

$$
E T=K_{C} E T_{i},
$$

where $K_{\mathrm{C}}$ is the crop coefficient or adjustment factor which should correspond to the reference crop used to determine $E T_{\mathrm{i}}$, where $E T_{\mathrm{i}}$ is either $E T_{\mathrm{r}}$ or $E T_{\mathrm{s}}$ (Table E1-2).

Table E1-2 Crop adjustment factor.

\begin{tabular}{lc}
\hline Month & $K_{\mathrm{C}}$ \\
\hline January & 0.50 \\
February & 0.50 \\
March & 0.50 \\
April & 0.25 \\
May & 0.33 \\
June & 1.10 \\
July & 1.10 \\
August & 1.10 \\
September & 0.60 \\
October & 0.25 \\
November & 0.25 \\
December & 0.25 \\
\hline
\end{tabular}

From the climate normals for the Goderich weather station, the average annual crop 
adjusted evapotranspiration using the PM FAO56 method was $564 \mathrm{~mm} \mathrm{yr}^{-1}$ which is close to the average of all the methods tested. For 1994 to 1996, the estimated annual recharge was 405,387 and $484 \mathrm{~mm}$, respectively. Note that this is considered to be an estimate of the minimum potential recharge since the method assumes that water is always available to meet the potential evapotranspiration. 


\section{Appendix E2: Baseflow}

\section{Stream-flow partitioning and digital filter methods}

These methods use stream discharge data as input to provide estimates of stream baseflow. Assuming that stream baseflow, and hence recharge, is equal to groundwater recharge, one can use streamflow partitioning to estimated the baseflow from daily streamflow records. I completed streamflow partitioning using the program PART (Rutledge, 1998; 2005) to obtain estimates of monthly baseflow. Values for monthly stream discharge and baseflow, normalized for catchment area, are given in Table E2-1.

Table E2-1 Monthly stream discharge and baseflow normalized by catchment area at TR-Q and TR-Qa estimated by streamflow partitioning using PART.

\begin{tabular}{|c|c|c|c|}
\hline Time period and subcatchment & Monthly stream discharge & Monthly baseflow & Baseflow index \\
\hline Subcatchment A1+A2 & $\mathrm{mm}$ & & \\
\hline May 1993 & 24.4 & 23.6 & 96.9 \\
\hline June 1993 & 31.5 & 23.4 & 74.2 \\
\hline July 1993 & 20.1 & 19.6 & 97.5 \\
\hline August 1993 & 16.0 & 15.5 & 96.8 \\
\hline September 1993 & 23.1 & 18.0 & 78.0 \\
\hline October 1993 & 26.7 & 24.1 & 90.5 \\
\hline November 1993 & 23.9 & 22.6 & 94.7 \\
\hline December 1993 & 28.4 & 26.9 & 94.6 \\
\hline January 1994 & 27.2 & 24.4 & 89.7 \\
\hline February 1994 & 40.6 & 28.7 & 70.6 \\
\hline March 1994 & 58.2 & 38.6 & 66.4 \\
\hline April 1994 & 37.6 & 33.3 & 88.5 \\
\hline May 1994 & 39.4 & 36.1 & 91.6 \\
\hline June 1994 & 31.8 & 31.0 & 97.6 \\
\hline July 1994 & 32.0 & 28.7 & 89.7 \\
\hline August 1994 & 21.1 & 19.1 & 90.4 \\
\hline September 1994 & 14.5 & 13.2 & 91.2 \\
\hline October 1994 & 19.1 & 18.3 & 96.0 \\
\hline November 1994 & 23.4 & 19.8 & 84.8 \\
\hline December 1994 & 24.6 & 22.4 & 90.7 \\
\hline March 1996 & 38.9 & 36.1 & 92.8 \\
\hline April 1996 & 57.9 & 44.2 & 76.3 \\
\hline May 1996 & 58.4 & 41.7 & 71.3 \\
\hline June 1996 & 38.4 & 35.1 & 91.4 \\
\hline July 1996 & 33.3 & 31.8 & 95.4 \\
\hline August 1996 & 26.9 & 26.2 & 97.2 \\
\hline September 1996 & 61.2 & 38.4 & 62.7 \\
\hline October 1996 & 45.5 & 38.4 & 84.4 \\
\hline November 1996 & 60.7 & 45.5 & 74.9 \\
\hline December 1996 & 81.8 & 55.9 & 68.3 \\
\hline
\end{tabular}

Subcatchment A2 
March 1996

April 1996

May 1996

June 1996

July 1996

August 1996

September 1996

October 1996

November 1996

December 1996

Subcatchment A1

March 1996

April 1996

May 1996

June 1996

July 1996

August 1996

September 1996

October 1996

November 1996

December 1996
33.5

53.6

50.3

26.9

12.7

7.4

76.5

38.1

54.1

77.7

\section{8}

34.0

32.3

23.6

95.5

65.5

79.0

106.4

117.9

120.4
30.5

38.6

29.0

22.1

9.9

5.3

26.2

24.9

32.5

38.9

39.1

25.7

27.4

17.0

47.5

55.4

54.4

53.1

80.3

81.8
90.9

72.0

57.6

82.1

78.0

72.4

34.2

65.3

60.1

50.0

58.6

75.4

85.0

72.0

49.7

84.5

68.8

49.9

68.1

67.9

The baseflow for the water year October 1993 to 1994 is $326.6 \mathrm{~mm}$ which is in good agreement with estimates of recharge obtained by recession curve displacement (not shown here). Estimates of baseflow, normalized for catchment area, at TR-Qa (subcatchment A2) were on average 63\% of those estimated for TR-Q (subcatchments A1 \& A2). This demonstrates that the low permeability till in subcatchment A2 yields less baseflow compared to the high permeability sand and gravel in subcatchment A1. Furthermore, when the discharge from only subcatchment A1 (primarily outwash) is considered, the normalized baseflow is $25 \%$ greater than that estimated for $\mathrm{A} 1+\mathrm{A} 2$.

\section{Hydrograph separation using digital recursive filters}

Baseflow can be separated automatically by applying a digital recursive filter to the stream discharge time series (Nathan and McMahon, 1990; Chapman, 1991, 1999; Szilagyi et al., 2003). The objective of a digital filter is to filter out (remove) the highfrequency signal (assumed to be event flow) from the low-frequency signal (assumed to be baseflow) in the streamflow hydrograph. A number of recursive filters have been proposed (Lyne and Hollick, 1979; Chapman, 1991, 1999; Boughton, 1993; Wittenberg, 1999; Jakeman and Hornberger, 1993; Furey and Gupta, 2003). With the exception of the 
filter developed by Furey and Gupta (2003), the main weakness of the digital filters is that they are not physically based, but rather they are based on the assumption that streamflow is made up of high frequency components (the event flows) and a low frequency component (the baseflow). While this assumed model of baseflow may not strictly be correct, the main advantage of digital filters is the technique provides an objective and repeatable estimate of baseflow that is easily automated.

I implemented four recursive digital filters (Lyne and Hollick, 1979; Chapman, 1991, 1999; and Boughton, 1993) and applied them to the discharge measured at TR-Q during the period 1993 to 1997 . The streamflow in Tricks Creek is maintained almost entirely by baseflow for the majority of the year due to the high permeability of the outwash sand and gravel. The filters proposed by Chapman $(1991,1999)$ and Boughton (1993) have a maximum allowable limit of baseflow contribution of $61 \%$ of the total streamflow, which far under estimates that expected in this watershed. Testing confirmed this outcome and the filter proposed by Lyne and Hollick (1979) was the preferred filter for this watershed. A similar conclusion was reached by Szilagyi et al. (2003) for a watershed dominated by sandy soils. The Lyne and Hollick (1979) filter may be implemented in terms of baseflow and total stream discharge as follows,

$$
Q_{b f}(i)=\alpha Q_{b f}(i-1)+\frac{1-\alpha}{2}\left[Q_{\text {out }}^{s w}(i)+Q_{\text {out }}^{s w}(i-1)\right]
$$

where $\alpha$ is the filter constant and Eq. (E2.1) is subject to the following constraints

$$
\begin{aligned}
& Q_{b f}(i) \leq Q_{\text {out }}^{\text {sw }}(i) \\
& Q_{b f} \geq 0
\end{aligned}
$$

In general, the recursive filter may be applied several times to obtain an acceptable baseflow separation. With each pass of the filter, the direction in which the filter is applied alternates. For example, for the first pass it is applied to the stream discharge record in the forward direction, and for the second pass it is applied in the 
reverse direction. The reason for reversing the direction of the filter with each pass is to reduce the phase shift introduced into the data as the filter is not a zero-phase filter. There is debate about the number of passes that are appropriate for the filter (Spongberg, 2000); however, there appears to be no consensus.

To gain an understanding of the filter performance, several tests were completed varying the filter constant and the number of filter passes. In the first series of tests the filter was applied to the hydrograph comprising the 30-minute average stream discharge data with a filter constant of 0.990 and three filter passes (Fig. E2-1a). With each pass of the filter the discharge is increasingly attenuated. Smaller filter constants produced baseflow hydrographs that retained too much of the event flow peaks.

Comparison of the statistics for the measured discharge with the filtered baseflow discharge shows that the filter has the greatest affect on the maximum discharge (Table E2-2). At both TR-Q and TR-Qa, the filter reduces the maximum discharge by over $60 \%$. The reduction in the average and median values is much less and ranges between 5 and 30 percent.

Table E2-2 Comparison of measured and filtered discharge statistics for the hydraulic year 1993-1994†.

\begin{tabular}{|c|c|c|c|c|c|c|c|c|}
\hline \multirow[t]{2}{*}{ Station } & \multicolumn{4}{|c|}{ Measured discharge } & \multicolumn{4}{|c|}{ Filtered baseflow } \\
\hline & average & median & minimum & maximum & average & median & minimum & maximum \\
\hline TR-Q & 0.256 & 0.221 & 0.096 & 2.397 & 0.218 & 0.211 & 0.096 & 0.920 \\
\hline TR-Qa & 0.066 & 0.043 & 0.005 & 1.497 & 0.044 & 0.035 & 0.005 & 0.441 \\
\hline
\end{tabular}

$\dagger$ the hydraulic year includes 1 October 1993 to 30 September 1994.

$\ddagger$ baseflow values were obtained using 2 passes of the Lyne and Hollick recursive filter.

For the majority of the applications of digital filters appearing in the literature, the stream discharge data comprises average daily discharges rather than the 30-minute averages used above. Therefore average daily discharge values were calculated and the digital filters applied. I found that using daily discharge data a filter constant of 0.6 produced hydrograph separations similar to those shown above, indicating that the magnitude of the filter constant is dependent on the sample spacing (Fig. E2.1 a and b). 


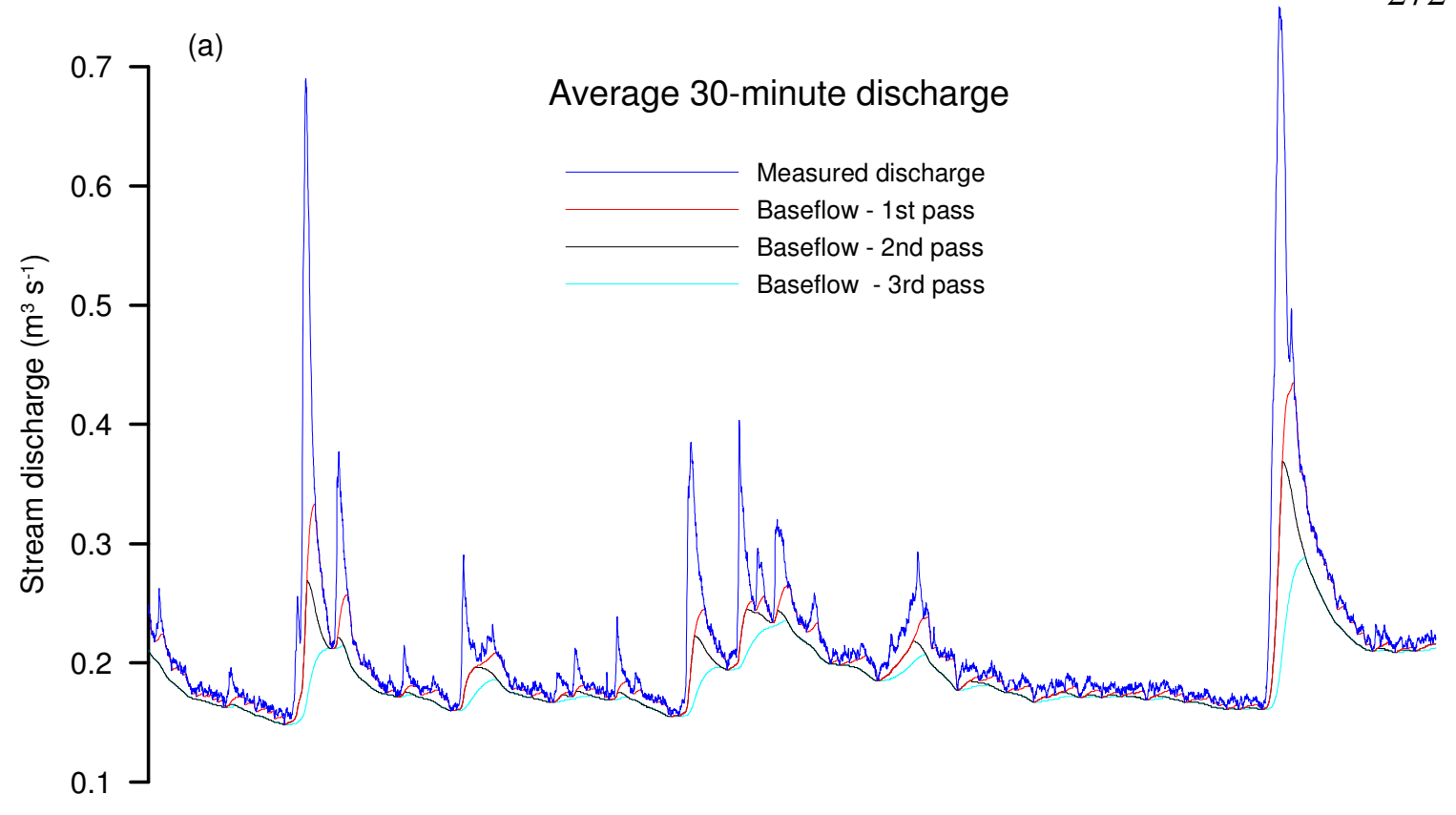

(b)

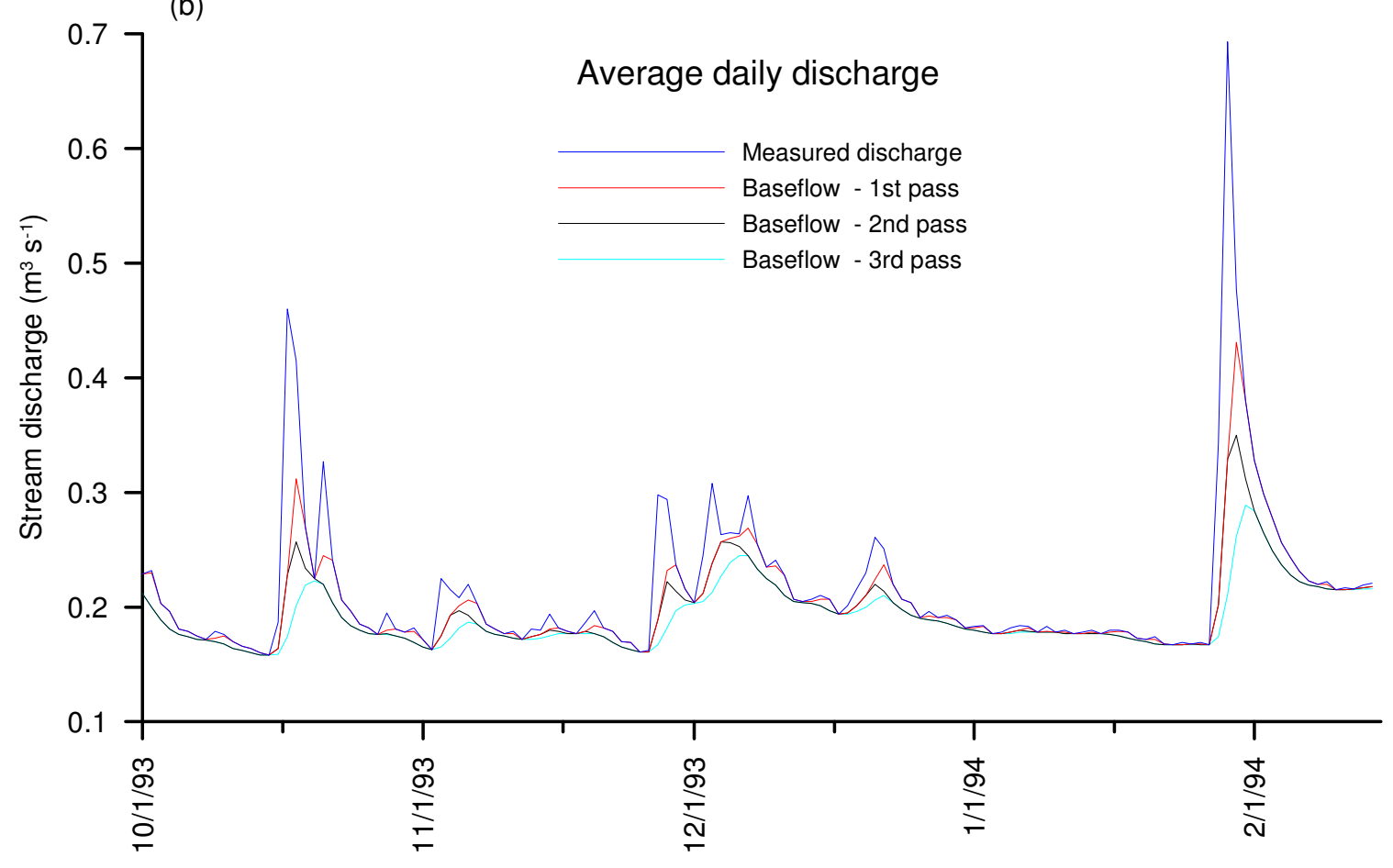

Figure E2-1 Baseflow hydrograph separation of the discharge at station TR-Q with Lyne and Hollick (1979) digital filter for (a) 30-minute average discharge measurements using a filter constant of 0.99 and (b) average daily discharge measurements using a filter constant of 0.60 . The blue line is the measured discharge and the red, black, and light blue are the baseflow after the $1^{\text {st }}, 2^{\text {nd }}$, and $3^{\text {rd }}$ pass of the digital filter. 
Table E2-3 Summary of monthly and annual stream discharge and baseflow, normalized by catchment area, and obtained by applying the Lyne and Hollick (1979) digital filter to the average daily discharge.

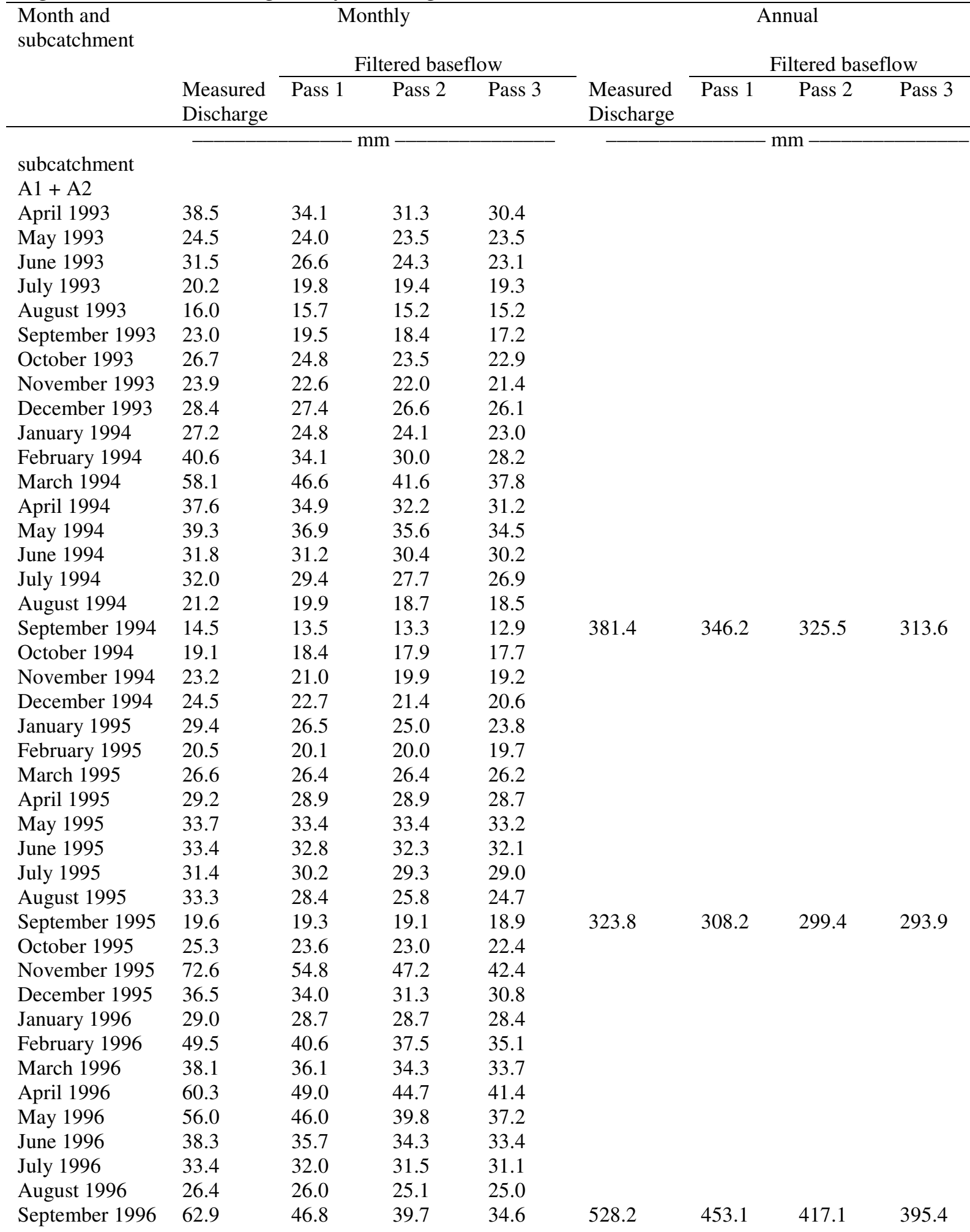




\begin{tabular}{|c|c|c|c|c|c|c|c|c|}
\hline \multirow{3}{*}{$\begin{array}{l}\text { Month and } \\
\text { subcatchment }\end{array}$} & \multicolumn{4}{|c|}{ Monthly } & \multicolumn{4}{|c|}{ Annual } \\
\hline & \multirow[b]{2}{*}{$\begin{array}{l}\text { Measured } \\
\text { Discharge }\end{array}$} & \multicolumn{3}{|c|}{ Filtered baseflow } & \multirow[b]{2}{*}{$\begin{array}{l}\text { Measured } \\
\text { Discharge }\end{array}$} & \multicolumn{3}{|c|}{ Filtered baseflow } \\
\hline & & Pass 1 & Pass 2 & Pass 3 & & Pass 1 & Pass 2 & Pass 3 \\
\hline October 1996 & 44.9 & 40.1 & $\overline{36.8}$ & 35.4 & & & $n-$ & 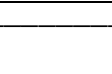 \\
\hline November 1996 & 60.3 & 50.2 & 45.2 & 42.7 & & & & \\
\hline December 1996 & 80.3 & 63.2 & 56.4 & 52.1 & & & & \\
\hline $\begin{array}{l}\text { Subcatchment } \\
\text { A2 }\end{array}$ & & & & & & & & \\
\hline May 1993 & 11.4 & 9.7 & 8.8 & 8.1 & & & & \\
\hline June 1993 & 6.9 & 5.0 & 3.8 & 3.5 & & & & \\
\hline September 1993 & 28.3 & 17.3 & 12.2 & 9.5 & & & & \\
\hline October 1993 & 19.8 & 15.2 & 13.1 & 11.7 & 217.1 & 170.9 & 146.2 & 131.5 \\
\hline November 1993 & 19.1 & 16.7 & 15.6 & 14.3 & & & & \\
\hline December 1993 & 17.3 & 16.4 & 15.6 & 15.3 & & & & \\
\hline February 1994 & 66.9 & 46.7 & 36.4 & 29.8 & & & & \\
\hline April 1994 & 34.5 & 30.0 & 26.9 & 25.5 & & & & \\
\hline May 1994 & 15.5 & 14.2 & 12.8 & 12.4 & & & & \\
\hline June 1994 & 7.4 & 6.3 & 5.4 & 5.0 & & & & \\
\hline July 1994 & 16.1 & 10.8 & 8.4 & 7.1 & & & & \\
\hline August 1994 & 6.6 & 5.0 & 4.1 & 3.8 & & & & \\
\hline September 1994 & 14.0 & 9.6 & 7.9 & 6.6 & & & & \\
\hline October 1994 & 25.6 & 19.5 & 16.8 & 14.9 & 136.6 & 112.1 & 98.8 & 91.4 \\
\hline November 1994 & 23.3 & 20.9 & 19.5 & 18.6 & & & & \\
\hline December 1994 & 45.6 & 37.1 & 32.8 & 29.6 & & & & \\
\hline January 1995 & 29.5 & 28.6 & 26.5 & 26.1 & & & & \\
\hline June 1995 & 14.5 & 12.6 & 11.6 & 11.0 & & & & \\
\hline July 1995 & 21.1 & 14.8 & 12.3 & 10.3 & & & & \\
\hline August 1995 & 16.8 & 12.0 & 9.4 & 8.5 & & & & \\
\hline September 1995 & 9.1 & 6.9 & 6.3 & 5.8 & & & & \\
\hline October 1995 & 36.7 & 20.4 & 18.1 & 14.5 & 416.9 & 311.1 & 262.1 & 234.4 \\
\hline November 1995 & 65.5 & 52.1 & 44.2 & 40.4 & & & & \\
\hline December 1995 & 57.3 & 44.5 & 37.1 & 33.3 & & & & \\
\hline March 1996 & 45.2 & 36.6 & 33.2 & 30.2 & & & & \\
\hline April 1996 & 44.3 & 38.2 & 33.7 & 32.6 & & & & \\
\hline May 1996 & 46.0 & 33.7 & 28.0 & 25.3 & & & & \\
\hline June 1996 & 17.8 & 15.5 & 13.8 & 13.3 & & & & \\
\hline July 1996 & 9.5 & 8.0 & 7.1 & 6.9 & & & & \\
\hline August 1996 & 30.4 & 17.1 & 12.0 & 8.3 & & & & \\
\hline September 1996 & 64.3 & 45.1 & 34.9 & 29.6 & & & & \\
\hline October 1996 & 58.3 & 40.3 & 33.6 & 30.1 & & & & \\
\hline November 1996 & 66.5 & 45.6 & 40.1 & 36.6 & & & & \\
\hline
\end{tabular}

Comparison of the monthly and annual baseflows estimated with the digital filter (Table E2-3) to those estimated with by streamflow partitioning (Table E2-1) using PART show good agreement. The annual trend in monthly baseflow values is similar for both methods with the lowest monthly baseflows occurring in September and the largest 
in March and April. The annual baseflow estimated by streamflow partitioning, for the water year October 1993 to September 1994, was $326.6 \mathrm{~mm}$, while the digital filter yields values of $346.2 \mathrm{~mm}$ after 1 pass of the filter, $325.5 \mathrm{~mm}$ after 2 filter passes, and $313.6 \mathrm{~mm}$ after 3 filter passes. This suggests that applying the filter twice will provide good estimates of baseflow at this site.

\section{References}

Allen, R.G., L.S. Periera, D. Raes, M. Smith. 1998. Crop evapotranspiration: Guidelines for computing crop requirements. Irrigation and Drainage Paper No. 56. FAO. Rome, Italy. 300 pp.

Allen, R.G., I.A. Walter, R. Elliot, B. Mecham, M.E. Jensen, D. Itenfisu, T.A. Howell, R. Snyder, P. Brown, S. Echings, T. Spofford, M. Hattendorf, R.H. Cuenca, J.L. Wright. 2000. Issues, requirements and challenges in selecting and specifying a standardized ET equation. in Evans, R.G., B.L. Benham, T.P. Trooien (ed.). Proceedings of the National Irrigation Symposium, ASCE, November 14-16, 2000. Phoenix, AZ:201-208.

Boughton, W.C. 1993. A hydrograph-based model for estimating the water yield of ungauged catchments. in Hydrology and Water Resources Symposium. Institution of Engineers Australia, Newcastle, NSW:317-324.

Chapman, T.G. 1991. Comment on "Evaluation of automated techniques for base flow and recession analyses" by R.J. Nathan and T.A. McMahon. Water Resources Research 27:1783-1784.

Chapman, T.G. 1999. a comparison of algorithms for stream flow recession and baseflow separation. Hydrological Processes 13:701-714.

Doorenbos, J., W.O. Pruitt. 1977. Crop water requirements. FAO Irrigation and Drainage Paper 24. Rome. 144 pp.

Feder, C.A., C.J. Vosmarty, B. Fekete. 1996. Intercomparison of methods for calculating potential evaporation in regional and global water balance models. Water Resources Research 32:2315-2321.

Furey, P.R., V.K. Gupta. 2003. Tests of two physically based filters for base flow separation. Water Resources Research 39(10). 1297. doi:10.1029/2002WR001621.

Hamon, W.R. 1963. Computation of direct runoff amounts from storm rainfall. 
International Association Sciientific Hydrology Publication 63:52-62.

Hargreaves, G.H., Z.A. Samani. 1985. Reference crop evapotranspiration from temperature. Applied Engineering in Agriculture 1:96-99.

Hargreaves, G.H. 1994. Defining and using reference evapotranspiration. Journal of Irrigation and Drainage Engineering, ASCE 120:1132-1139.

Jacobs, J.M., S.R. Satti. 2001. Final Technical Report. Department of Civil and Coastal Engineering, University of Florida. 114 pp.

Jakeman, A.J., G.M. Hornberger. 1993. How much complexity is warranted in a rainfallrunoff model?. Water Resources Research 29:2637-2649.

Jensen, M.E., H.R. Haise. 1963. Estimating evapotranspiration from solar radiation. Journal of Irrigation and Drainage Division of American Society of Civil Engineers 89 (IR4):15-41.

Jensen, M.E., R.D. Burman, R.G. Allen. (Editors). 1990. Evapotranspiration and irrigation water requirements. In: ASCE Manuals and Reports on Engineering Practices No. 70. ASCE, New York, NY. 360 pp.

Lyne, V., M. Hollick. 1979. Stochastic time-variable rainfall-runoff modeling. in Hydrology and Water Resources Symposium. Institution of Engineers Australia, Perth. 89-92.

Allen, R.G., I.A. Walter, R. Elliott, B. Mecham, M.E. Jensen, D. Itenfisu, T.A. Howelll, R. Snyder, P. Brown, S. Echings, T. Spofford, M. Hattendorf, R.H. Cuenca, J.L. Wright, D. Martin. 2000. Issues, Requirements and Challenges in Selecting and Specifying a Standardized ET Equation. pp. 201-208 in Evans, R.G., B.L. Benham, T.P. Trooien (ed.) Proceedings of the National Irrigation Symposium, ASCE, Nov. 14-16, 2000, Phoenix, AZ.

Nathan, R.J., T.A. McMahon. 1990. Evaluation of automated techniques for base flow and recession analysis. Water Resources Research 26:1465-1473.

Priestley, C.H.B., R.J. Taylor. 1972. On the assessment of surface heat flux and evaporation using large-scale parameters. Monthly Weather Review 100:81-92.

Rutledge, A.T. 1998. Computer programs for describing the recession of groundwater discharge and for estimating mean groundwater recharge and discharge from streamflow data-update: U.S. Geological Survey Water-Resources Investigations Report 98-4148. 43 pp.

Rutledge, A.T. 2005. Program user guide for Part. USGS. March 2005. 8 pp. 
Scanlon, B.R., R.W. Healy, P.G. Cook. 2002. Choosing appropriate techniques for quantifying groundwater recharge. Hydrogeology Journal. doi:10.1007/s100400010176-2:18-39.

Spongberg, M.E. 2000. Spectral analysis of base flow separation with digital filters. Water Resources Research 36:745-752.

Szilagyi, J., F.E. Harvey, J.F. Ayers. 2003. Regional estimation of base recharge to ground water using water balance and a base-flow index. Ground Water 41:504513.

Thornthwaite, C.W., J.R. Mather. 1955a. The water balance. Publications in Climatology. VIII(1). Drexel Institute of Climatology, Centeron NJ. 1-104.

Thornthwaite, C.W., J.R. Mather. 1955b. The water budget and its use in irrigation. In Water, The Yearbook of Agriculture. US Department of Agroculture. Washington DC. 346-358.

Thornthwaite, C.W., J.R. Mather. 1957. Instructions and tables for computing potential evapotranspiration and the water balance. Publications in Climatology. X(3). Drexel Institute of Climatology, Centeron NJ. 1-311.

Turc, L. 1961. Evaluation de besions en eau d'irrigation, evapotranspiration potentielle, formule climatique simplifice et mise a jour. Annales Agronomiques 12:13-49.

Vosmarty, C.J., C.A. Federer, A.L. Schloss. 1998. Potential evaporation functions compared on US watersheds: Possible implications for global-scale water balance and terrestrial ecosystem modeling. Journal of Hydrology 207:147-169.

Wittenberg, H. 1999. Baseflow recession as recharge as nonlinear storage processes. Hydrological Processes 13:715-726. 


\section{Appendix F: Bedrock Well Temperature Profiles}

\section{Appendix F1: Deep well temperature profiles}

I measured temperature profiles in three deeper wells (BR1, BR2, and BR3) that extend through the till and into the bedrock (Fig. F-1). These temperature profiles were obtained using a temperature logging system developed by Dr. John Sass. The system incorporates a highly accurate temperature sensor $\left(0.01^{\circ} \mathrm{C}\right)$ with a rapid response time $(<1 \mathrm{~s})$. In each well, the logging system recorded temperatures at $0.3 \mathrm{~m}$ intervals from just below the static water level to the bottom of the well. Temperatures were then recorded as the sensor was raised back up the well. Also shown are the minimum and maximum temperatures observed in the monitoring wells in the outwash aquifer (blue solid lines). These lines defined the temperature envelope within which the subsurface temperatures in the aquifer vary over an annual basis. Only temperatures from wells not influenced by the presence of a pit were considered in defining this envelope. 


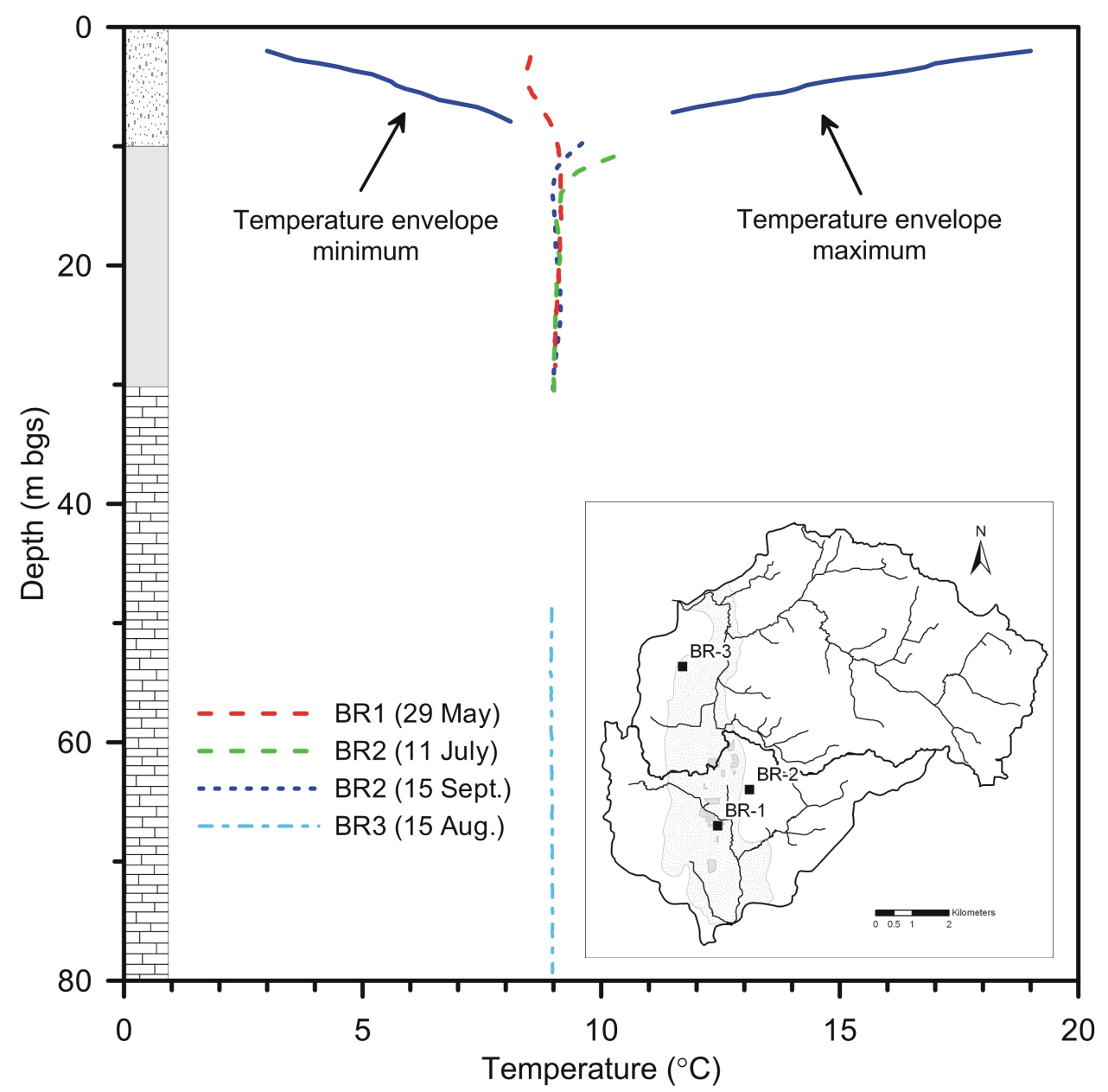

Figure F-1 Temperature profiles for bedrock wells (dashed lines) and the temperature envelope observed in the outwash aquifer in shallow monitoring wells. Outwash, till and bedrock are shown on the right-hand side.

The construction details for each well and water levels at the time the temperatures were measured are provided below.

Table F-1 Summary of deep well construction and borehole temperature log details.

\begin{tabular}{|c|c|c|c|c|c|}
\hline Well & interval & Geology formation & Casing depth & Water level & Logging date \\
\hline & $-\mathrm{m}$ bgs -- & & $-\mathrm{m}$ & gs & \\
\hline \multirow[t]{3}{*}{ BR1 } & 0 to 4.6 & outwash sand & 30.8 & 4.5 & 29 May 2001 \\
\hline & 4.6 to 29.3 & clay till & & & \\
\hline & 29.3 to 32.0 & limestone (Dundee Fm.) - top $1 \mathrm{~m}$ is fractured & & & \\
\hline \multirow[t]{2}{*}{ BR2 } & 0 to 18.2 & outwash sand & unknown & 46.2 & 11 July 2003 \\
\hline & 18.2 to 38.4 & clay till & & & 15 Sept. 2003 \\
\hline
\end{tabular}




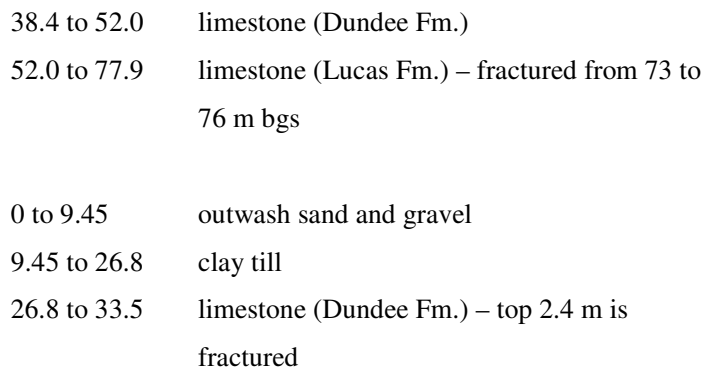

Within the study area, bedrock is typically found at depths between 25 to $35 \mathrm{~m}$ bgs (below ground surface). The upper portion of the bedrock is comprised of the Dundee formation which is approximately $25 \mathrm{~m}$ thick and overlies the Lucas formation found at depths between 52 and $55 \mathrm{~m}$ bgs. In general, the upper $1.5 \mathrm{~m}$ of the Dundee formation is fractured and the static water level in this zone ranges between 9 and $15 \mathrm{~m}$ bgs. Beneath the fractured zone, the Dundee formation is competent rock. The upper portion of the Lucas formation is also competent but it contains a fractured zone between 78 and $88 \mathrm{~m}$ bgs which is a productive aquifer in this area. The head within the fractured portion of the Lucas formation ranges between 40 and $60 \mathrm{~m}$ bgs. This 25 to $50 \mathrm{~m}$ head difference between the fractured zones in the Dundee and Lucas formations suggest the two fractured zones are hydraulically isolated by the intervening competent bedrock.

The temperature profiles at BR1 and BR3 span the till and the upper portion of the Dundee formation. In these profiles, the annual temperature signal from the ground surface was evident to depths of $20 \mathrm{~m}$ bgs. Below this depth, the temperature profiles merge together and at the base of the till the temperature is $\sim 9^{\circ} \mathrm{C}$ ranging between 8.98 and $9.04^{\circ} \mathrm{C}$. The deepest profile was obtained in $\mathrm{BR} 2$ where temperatures were measured from 49 to $79 \mathrm{~m}$ bgs. In this well, the static water level was $46.2 \mathrm{~m}$ bgs, which is below the till. Thus, no temperature data in the till was collected at BR2; however, this temperature profile provides information on the temperature in the deeper Lucas formation. In $\mathrm{BR} 2$, the temperature ranged from a low of $8.93^{\circ} \mathrm{C}$ at $54 \mathrm{~m} \mathrm{bgs}$ to $8.98^{\circ} \mathrm{C}$ at $79.3 \mathrm{~m}$ bgs, the bottom of the well. 


\section{Calculation of vertical hydraulic conductivity}

The temperature profiles may be used to estimate the vertical fluid and heat flux through the till and thereby an estimate of the vertical hydraulic conductivity $K_{\mathrm{z}}$. Two approaches were used to estimate the vertical fluid flux through the till. These include: fitting the temperature data to the analytical solutions developed by Bredehoeft and Papadopulos (1965) and Lu and Ge (1996), as adapted by Reiter (2001), and matching the measured temperature profiles with simulations conducted with Heatflow.

Curve fitting procedures after Reiter (2001)

Reiter (2001) detailed curve fitting procedures to expressions adapted from Bredehoeft and Papadopulos (1965)

$$
T=a_{1}+b_{1} e^{-z / c_{1}}
$$

where

$a_{1}=T_{1}-\frac{T_{2}-T_{1}}{e^{\beta}-1}, \quad b_{1}=\frac{T_{2}-T_{1}}{e^{\beta}-1}, \quad c_{1}=\frac{\lambda}{c_{w} \rho_{w} q_{z}}, \quad \beta=\frac{c_{w} \rho_{w} q_{z} L}{\lambda}$

and of Lu and Ge (1996)

$$
T=a_{2}+b_{2} e^{c_{2} z}-g_{3} z
$$

where

$$
\begin{array}{ll}
a_{2}=T_{1}-\frac{T_{2}-T_{1}}{e^{\beta}-1}-\frac{\varepsilon\left(T_{2}-T_{1}\right)}{e^{\beta}-1}, & b_{1}=\frac{T_{2}-T_{1}}{e^{\beta}-1}+\frac{\varepsilon\left(T_{2}-T\right)_{1}}{e^{\beta}-1}, \quad c_{2}=\frac{c_{w} \rho_{w} q_{z}}{\lambda}, \\
g_{3}=\frac{\left(T_{2}-T_{1}\right)}{L} \frac{q_{x} \Gamma_{x}}{q_{z}}, & \varepsilon=\frac{q_{x} \Gamma_{x}}{q_{z}\left(T_{2}-T_{1}\right) / L}
\end{array}
$$

and where

$T_{1}$ and $T_{2} \quad$ are the temperatures at the top and bottom of the interval $[\Theta]$;

$L \quad$ is the thickness of the interval [L];

$\lambda \quad$ is the apparent thermal conductivity of the saturated formation $\left[\mathrm{M} \mathrm{L} \mathrm{T}^{-3} \mathrm{~K}^{-1}\right.$ ], which is $1.9 \mathrm{~W} \mathrm{~m}^{-1} \mathrm{~K}^{-1}$ for the till; 


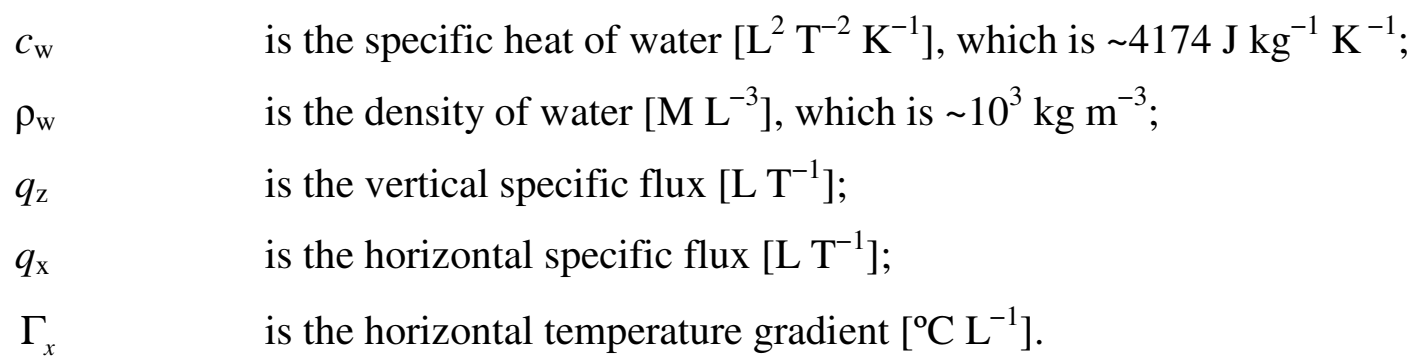

To limit the influence of the annual temperature fluctuation at the ground surface on the analysis, I used the temperature data between 20 and $25 \mathrm{~m}$ bgs from BR1 (Reiter, 2003). Best fits of Eq. (F1.1) and (F1.3) to these data yield estimates of the vertical specific flux, $q_{\mathrm{z}}$, of $1.72 \times 10^{-8} \mathrm{~m} \mathrm{~s}^{-1}$ (after Bredehoeft and Papadopulos, 1965), and $1.66 \times 10^{-8} \mathrm{~m} \mathrm{~s}^{-1}$ (after Lu and Ge, 1996). Assuming a vertical gradient of 0.53 downwards through the till, $K_{\mathrm{z}}$ is between $3.16 \times 10^{-8} \mathrm{~m} \mathrm{~s}^{-1}$ and $3.29 \times 10^{-8} \mathrm{~m} \mathrm{~s}^{-1}$ at $9^{\circ} \mathrm{C}\left(2.42 \times 10^{-8} \mathrm{~m} \mathrm{~s}^{-1}\right.$ and $2.52 \times 10^{-8} \mathrm{~m} \mathrm{~s}^{-1}$ at $\left.0^{\circ} \mathrm{C}\right)$.

\section{Temperature matching with Heatflow}

Using Heatflow, I attempted to match the observed temperature profiles. My objective with these simulations was to match the temperature profile through the till and bedrock as well as matching the range in temperatures observed in the outwash aquifer. I used a quasi 2-dimensional model domain (Fig. F-2) which was $20 \times 20 \times 80 \mathrm{~m}(\mathrm{x} \times \mathrm{y} \times \mathrm{z})$, and comprised $20 \times 1 \times 84$ elements. 
specified flux

\&

linear thermal exchange

(3rd type)

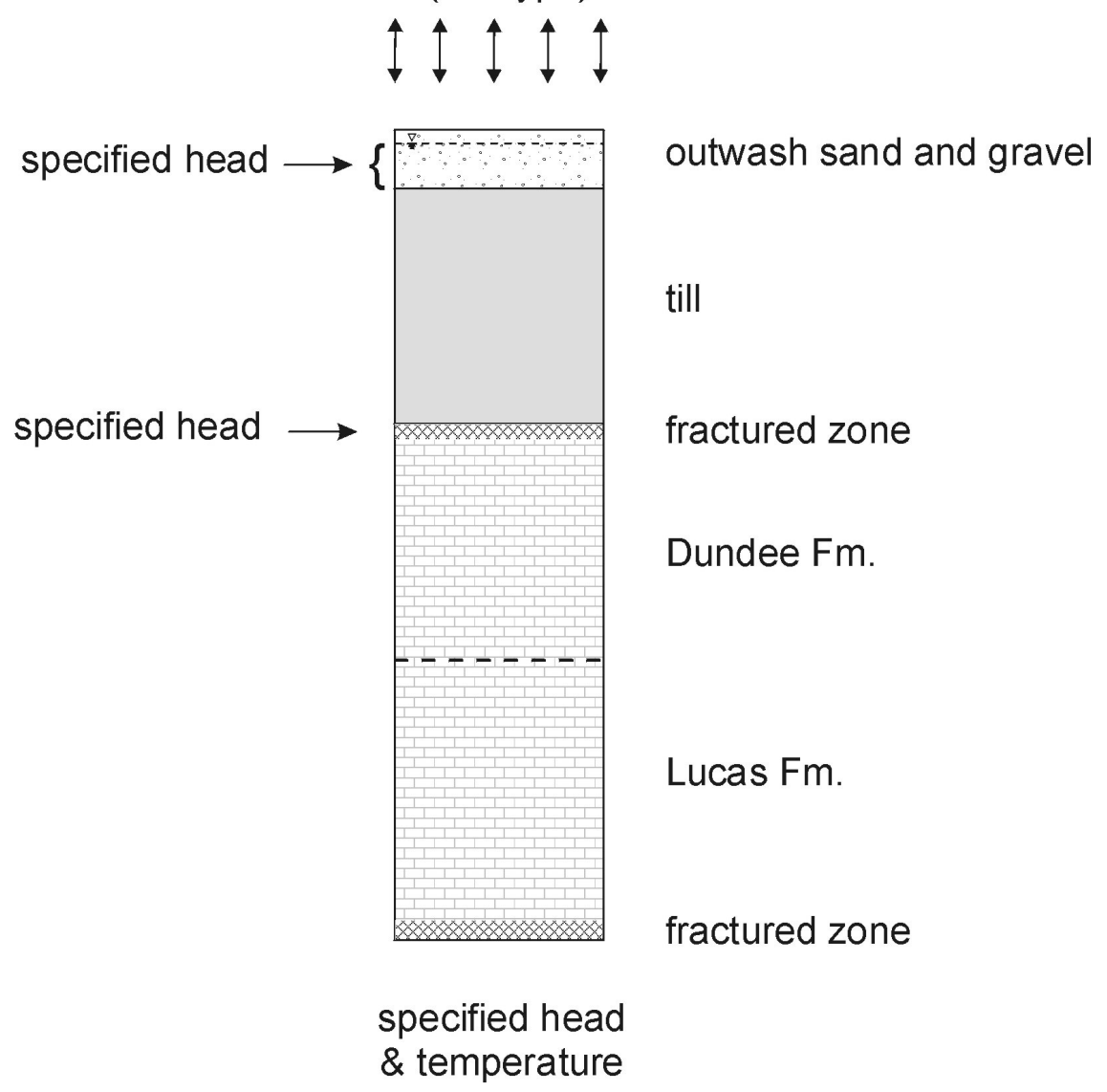

Figure F-2 Schematic diagram and boundary conditions for temperature profile matching.

The specification of the boundary conditions was guided by the measured field data. For the hydraulic boundaries I set a specified flux of $400 \mathrm{~mm} \mathrm{yr}^{-1}$ along the top. Along the saturated portion of the outwash sand and gravel the head was set at $78.5 \mathrm{~m}$ asl along both the left and right sides. To match the regional gradient in the upper fractured portion of the Dundee formation, the head at the left and right sides was fixed $66.575 \mathrm{~m}$ asl and $66.407 \mathrm{~m}$ asl, respectively. This also conformed to the vertical hydraulic gradient observed across the till between the outwash aquifer and the upper bedrock. The bottom of the model domain was set at the fractured zone in the Lucas formation and the head fixed at $27.625 \mathrm{~m}$ asl to match the vertical hydraulic gradient measured between the 
fractured zones in the Dundee and Lucas formations. All other boundaries were specified as zero flux boundaries. For the transport boundary conditions, a third type, linear thermal exchange boundary was set along the top (see Appendix B2 for complete development) and is given by

$$
\lambda_{z z}^{u}\left(\frac{\partial T}{\partial z}\right)=\left(\gamma_{G}+q_{R} c_{w} \rho_{w}\right)\left(T_{A}-T\right) \quad \text { on } \Gamma_{3, G}
$$

where $\lambda_{z z}^{u} \quad$ is the thermal conductivity of the unsaturated zone $\left[\mathrm{M} \mathrm{L} \mathrm{T}^{-3} \Theta^{-1}\right]$;

$T_{\mathrm{A}} \quad$ is the known atmospheric temperature $[\Theta]$;

$T \quad$ is the unknown surface temperature within the subsurface $[\Theta]$;

$\gamma_{\mathrm{G}}$ is the linear heat transfer coefficient at the ground surface $\left[\mathrm{M} \mathrm{T}^{-3} \Theta^{-1}\right]$;

$q_{\mathrm{R}} \quad$ is the surface recharge flux rate $\left[\mathrm{L} \mathrm{T}^{-1}\right]$;

$c_{\mathrm{w}} \quad$ is the specific heat capacity of water $\left[\mathrm{L}^{2} \mathrm{~T}^{-2} \Theta^{-1}\right]$, and

$\rho_{\mathrm{w}} \quad$ is the density of water $\left[\mathrm{M} \mathrm{L}^{-3}\right]$.

Defining a linear heat transfer coefficient as

$$
\gamma_{G}=\frac{\lambda_{G}}{b_{G}}
$$

where $\lambda_{\mathrm{G}} \quad$ is the thermal conductivity of the transition layer $\left[\mathrm{M} \mathrm{L} \mathrm{T}^{-3} \Theta^{-1}\right]$, and $b_{\mathrm{G}} \quad$ is the thickness of the transition layer [L].

The time varying temperature at the top boundary is given by

$$
T(t)=f(t), \quad f(t)= \begin{cases}T_{A V G}+T_{A M P} \sin \left[\frac{2 \pi}{\omega}(t+\varphi)\right], & T(t) \geq T_{M I N} \\ T_{M I N}, & T(t)<T_{M I N}\end{cases}
$$


where $t \quad$ is time [T];

$T_{\mathrm{AVG}}$ is average annual temperature at the top bpoundary $[\Theta]$;

$T_{\text {AMP }}$ is the amplitude of the annual temperature $[\Theta]$;

given by $T_{M A X}-T_{A V G}$;

$T_{\mathrm{MIN}} \quad$ is the minimum temperature $[\Theta]$;

$\omega \quad$ is the frequency $\left[\mathrm{T}^{-1}\right]$, and

$\varphi \quad$ is the phase shift [T].

Fitting the above curve to the measured average daily temperature at the ground surface (Fig. F-3) yields values of $6^{\circ} \mathrm{C}, 20^{\circ} \mathrm{C}, 0^{\circ} \mathrm{C}, 365$ days and -138 days for $T_{\mathrm{AVG}}, T_{\mathrm{AMP}}, T_{\mathrm{MIN}}$, $\omega$ and $\phi$, respectively.

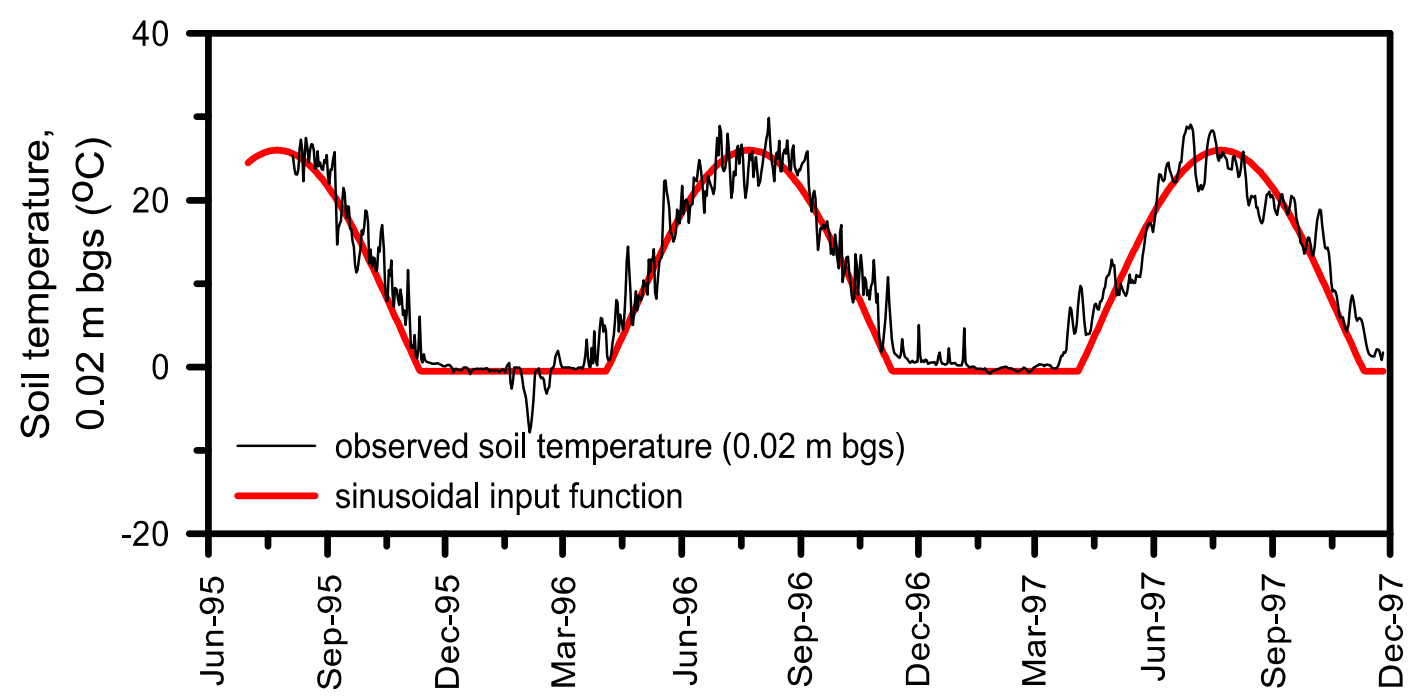

Figure F-3 Comparison of observed mean daily surface temperature at $0.02 \mathrm{~m} \mathrm{bgs}$ with simplified model input sinusoidal temperature function. 
The temperature along the left hand side of the upper fractured zone was fixed at $8.96^{\circ} \mathrm{C}$, and along the bottom boundary the temperature was fixed at $8.98^{\circ} \mathrm{C}$. All the remaining boundaries were specified as zero thermal flux boundaries. The hydraulic and thermal properties assigned to the formations are given in Table F-2.

Table F-2 Summary of formation parameters used for the temperature profile matching.

\begin{tabular}{|c|c|c|c|c|}
\hline Geology & Depth interval & Static water level & Hydraulic properties & Thermal properties $\dagger$ \\
\hline $\begin{array}{l}\text { unsaturated outwash } \\
\text { sand and gravel }\end{array}$ & $-\mathrm{n}$ & gs - & $\phi=0.29$ & $\begin{array}{l}\lambda=1.7 \text { to } 2.42 \mathrm{~W} \mathrm{~m}^{-1} \mathrm{~K}^{-1} \\
\mathrm{C}_{0}=1.66 \text { to } 2.79 \times 10^{6} \mathrm{~J} \mathrm{~m}^{-3} \mathrm{~K}^{-1}\end{array}$ \\
\hline $\begin{array}{l}\text { saturated outwash sand } \\
\text { and gravel }\end{array}$ & 1.5 to 7.5 & 1.5 & $\begin{aligned} \mathrm{K}_{\mathrm{x}} & =\mathrm{K}_{\mathrm{y}}=1.5 \times 10^{-4} \mathrm{~m} \mathrm{~s}^{-1} \\
\mathrm{~K}_{\mathrm{z}} & =3 \times 10^{-5} \mathrm{~m} \mathrm{~s}^{-1} \\
\mathrm{~S}_{\mathrm{S}} & =1 \times 10^{-3} \\
\phi & =0.29\end{aligned}$ & $\begin{array}{l}\lambda=2.42 \mathrm{~W} \mathrm{~m}^{-1} \mathrm{~K}^{-1} \\
\mathrm{C}_{0}=2.79 \times 10^{6} \mathrm{~J} \mathrm{~m}^{-3} \mathrm{~K}^{-1}\end{array}$ \\
\hline till & 7.5 to 30 & & $\begin{array}{l}\mathrm{K}_{\mathrm{x}}=\mathrm{K}_{\mathrm{y}}=1 \times 10^{-7} \mathrm{~m} \mathrm{~s}^{-1} \\
\mathrm{~K}_{\mathrm{z}}=\text { fitted parameter } \\
\mathrm{S}_{\mathrm{S}}=1 \times 10^{-5} \\
\phi=0.40\end{array}$ & $\begin{array}{l}\lambda=1.90 \mathrm{~W} \mathrm{~m}^{-1} \mathrm{~K}^{-1} \\
\mathrm{C}_{0}=3.01 \times 10^{6} \mathrm{~J} \mathrm{~m}^{-3} \mathrm{~K}^{-1}\end{array}$ \\
\hline $\begin{array}{l}\text { Dundee Fm. (top } 1.5 \mathrm{~m} \\
\text { fractured) }\end{array}$ & 30 to 31.5 & 11.0 & $\begin{aligned} \mathrm{K}_{\mathrm{x}} & =\mathrm{K}_{\mathrm{y}}=1 \times 10^{-5} \mathrm{~m} \mathrm{~s}^{-1} \\
\mathrm{~K}_{\mathrm{z}} & =1 \times 10^{-5} \mathrm{~m} \mathrm{~s}^{-1} \\
\mathrm{~S}_{\mathrm{S}} & =1 \times 10^{-4} \\
\phi & =0.2\end{aligned}$ & $\begin{array}{l}\lambda=3.1 \mathrm{~W} \mathrm{~m}^{-1} \mathrm{~K}^{-1} \ddagger \\
\mathrm{C}_{0}=3.01 \times 10^{6} \mathrm{~J} \mathrm{~m}^{-3} \mathrm{~K}^{-1} \ddagger\end{array}$ \\
\hline $\begin{array}{l}\text { competent sections of } \\
\text { the Dundee and Lucas } \\
\text { Fm. }\end{array}$ & 31.5 to 78 & & $\begin{array}{l}\mathrm{K}_{\mathrm{x}}=\mathrm{K}_{\mathrm{y}}=1 \times 10^{-7} \mathrm{~m} \mathrm{~s}^{-1} \\
\mathrm{~K}_{\mathrm{z}}=1 \times 10^{-7} \mathrm{~m} \mathrm{~s}^{-1} \\
\mathrm{~S}_{\mathrm{S}}=1 \times 10^{-5} \\
\phi=0.065 \ddagger\end{array}$ & $\begin{array}{l}\lambda=3.1 \mathrm{~W} \mathrm{~m}^{-1} \mathrm{~K}^{-1} \ddagger \\
\mathrm{C}_{0}=2.81 \times 10^{6} \mathrm{~J} \mathrm{~m}^{-3} \mathrm{~K}^{-1} \ddagger\end{array}$ \\
\hline $\begin{array}{l}\text { Lucas Fm. (fractured at } \\
78 \text { to } 87 \mathrm{~m} \mathrm{bgs} \text { ) }\end{array}$ & 78 to 80 & 52.4 & $\begin{array}{l}\mathrm{K}_{\mathrm{x}}=\mathrm{K}_{\mathrm{y}}=1 \times 10^{-5} \mathrm{~m} \mathrm{~s}^{-1} \\
\mathrm{~K}_{\mathrm{z}}=1 \times 10^{-5} \mathrm{~m} \mathrm{~s}^{-1} \\
\mathrm{~S}_{\mathrm{S}}=1 \times 10^{-4} \\
\phi=0.2\end{array}$ & $\begin{array}{l}\lambda=3.1 \mathrm{~W} \mathrm{~m}^{-1} \mathrm{~K}^{-1} \ddagger \\
\mathrm{C}_{0}=3.01 \times 10^{6} \mathrm{~J} \mathrm{~m}^{-3} \mathrm{~K}^{-1} \ddagger\end{array}$ \\
\hline
\end{tabular}

$\dagger$ thermal conductivity and heat capacity are apparent values (i.e., include fluid and matrix solids)

$\$$ bedrock formation parameters adapted from Judge (1972).

Fig. F-4 show the results obtained by varying the conditions imposed along the 
top boundary (i.e., heat transfer coefficients and annual temperature signal at the ground surface) and the vertical hydraulic conductivity of the till until an acceptable fit was obtained.

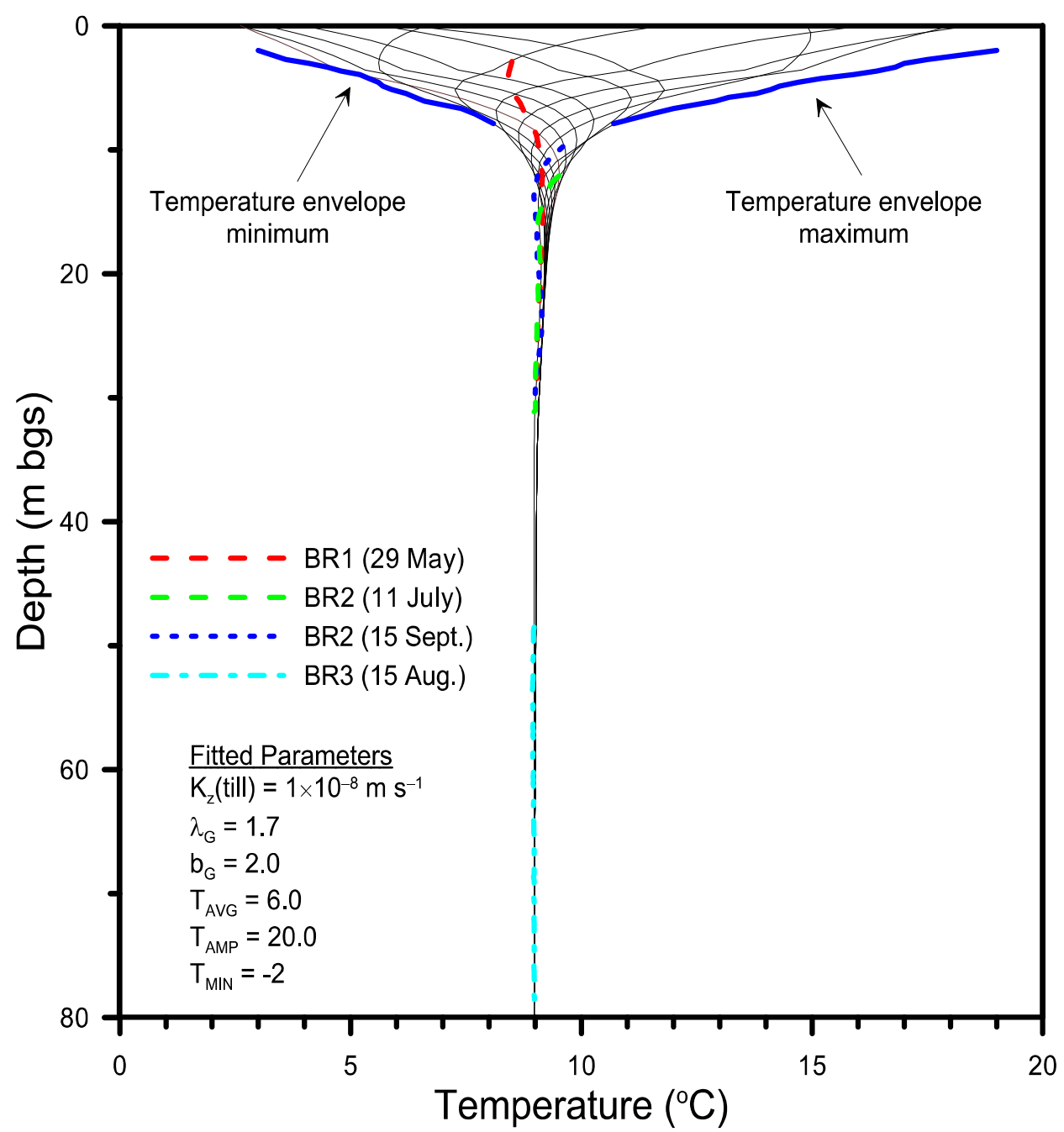

Figure F-4 Temperature profile match obtained with Heatflow to temperature profiles from deep wells BR1, BR2 and BR3, and to the maximum and minimum of the temperature envelope observed in the shallow wells that were not influenced by the presence of a pond.

The simulations indicate that once $K_{\mathrm{z}}$ is less than $3 \times 10^{-8} \mathrm{~m} \mathrm{~s}^{-1}$ at $9^{\circ} \mathrm{C}\left(\sim 2.3 \times 10^{-8} \mathrm{~m} \mathrm{~s}^{-1}\right.$ at $0^{\circ} \mathrm{C}$ ) the temperature profiles are relatively consistent. Thus, the temperature profiles provide an upper bound on $K_{\mathrm{z}}$ of $\sim 3 \times 10^{-8} \mathrm{~m} \mathrm{~s}^{-1}$ at $9^{\circ} \mathrm{C}$. This estimate is in good agreement with the values obtained using the curve fitting procedures (Reiter, 2001), and 
with the range in the value of bulk hydraulic conductivity for the till obtained from

other methods. For example, estimates of hydraulic conductivity range from $1.6 \times 10^{-7}$ and $2.5 \times 10^{-9} \mathrm{~m} \mathrm{~s}^{-1}$ at $9^{\circ} \mathrm{C}$ (Conestoga-Rover and Associates, 1989), based on grain-size analyses of till samples from the landfill north of the study area, and from $10^{-9}$ to $10^{-11} \mathrm{~m}$ $\mathrm{s}^{-1}$ at $9^{\circ} \mathrm{C}$ from field-based falling-head tests (Markle and Schincariol, 2007).

\section{References}

Bredehoeft, J.D., I.S. Papadopulos. 1965. Rates of vertical groundwater movement estimated from the Earth's thermal profile. Water Resources Research. 1:325-328.

Conestoga-Rovers and Associates. 1989. Hydrogeologic investigation, Holmesville landfill site.

Judge, A.S. 1972. Geothermal measurements in a sedimentary basin. Ph.D. thesis, University of Western Ontario, London, Ontario, Canada.

Lu, N., S. Ge. 1996. Effects of horizontal heat and fluid flow on the vertical temperature distribution of a semi-confining layer. Water Resources Research. 32:1449-1453.

Markle, J.M., R.A. Schincariol. 2007. Thermal plume transport from sand and gravel pits - Potential thermal impacts on cool water streams. Journal of Hydrology 338:174195.

Reiter, M. 2001. Using precision temperature logs to estimate horizontal and vertical groundwater flow components. Water Resources Research. 37:663-674. 


\section{Appendix G: Calibration Targets}

\section{Steady-state groundwater flow calibration targets}

Table G-1 Steady-state groundwater flow calibration targets

\begin{tabular}{|c|c|c|c|c|c|c|}
\hline $\begin{array}{l}\text { Calibration } \\
\text { point }\end{array}$ & Time period & $\begin{array}{l}\text { Number } \\
\text { of values }\end{array}$ & Minimum & Maximum & Mean \pm C.I $\dagger$ & $\mathrm{SD} \neq$ \\
\hline & & & & $-\mathrm{m}$ asl - & - & $-\mathrm{m}-$ \\
\hline \multirow[t]{2}{*}{ TR-1 } & Oct 1995 to Oct 1996 & 9 & 254.85 & 255.72 & $255.41 \pm 0.18$ & 0.28 \\
\hline & Jan 1991 to Sept 2003 & 84 & 254.46 & 255.88 & $255.20 \pm 0.08$ & 0.36 \\
\hline \multirow[t]{2}{*}{ TR-2 } & Oct 1995 to Oct 1996 & 9 & 254.78 & 255.60 & $255.33 \pm 0.18$ & 0.27 \\
\hline & Jan 1991 to Sept 2003 & 78 & 254.40 & 255.77 & $255.13 \pm 0.08$ & 0.36 \\
\hline \multirow[t]{2}{*}{ TR-3 } & Oct 1995 to Oct 1996 & 9 & 254.82 & 255.68 & $255.38 \pm 0.17$ & 0.26 \\
\hline & Jan 1991 to Sept 2003 & 83 & 254.47 & 255.90 & $255.22 \pm 0.08$ & 0.36 \\
\hline \multirow[t]{2}{*}{ TR-4 } & Oct 1995 to Oct 1996 & 11 & 254.64 & 256.02 & $255.37 \pm 0.27$ & 0.46 \\
\hline & Jan 1991 to Sept 2003 & 88 & 254.23 & 256.30 & $255.17 \pm 0.11$ & 0.50 \\
\hline \multirow[t]{2}{*}{ TR-5 } & Oct 1995 to Oct 1996 & 11 & 254.33 & 254.75 & $254.53 \pm 0.08$ & 0.13 \\
\hline & Jan 1991 to Sept 2003 & 86 & 253.99 & 254.90 & $254.46 \pm 0.04$ & 0.17 \\
\hline \multirow[t]{2}{*}{ TR-6 } & Oct 1995 to Oct 1996 & 9 & 254.20 & 254.76 & $254.56 \pm 0.11$ & 0.16 \\
\hline & Jan 1991 to Sept 2003 & 81 & 253.82 & 254.95 & $254.36 \pm 0.06$ & 0.27 \\
\hline \multirow[t]{2}{*}{ TR-7 } & Oct 1995 to Oct 1996 & 9 & 253.71 & 254.08 & $253.93 \pm 0.07$ & 0.11 \\
\hline & Jan 1991 to Sept 2003 & 76 & 253.31 & 254.36 & $253.83 \pm 0.04$ & 0.17 \\
\hline \multirow{2}{*}{ TR-8 } & Oct 1995 to Oct 1996 & 5 & 253.20 & 253.41 & $253.31 \pm 0.08$ & 0.09 \\
\hline & Jan 1991 to Sept 2003 & 60 & 252.81 & 253.69 & $253.14 \pm 0.06$ & 0.22 \\
\hline \multirow[t]{2}{*}{ TR-9 } & Oct 1995 to Oct 1996 & 9 & 250.90 & 251.38 & $251.21 \pm 0.10$ & 0.15 \\
\hline & Jan 1991 to Sept 2003 & 82 & 250.73 & 251.74 & $251.08 \pm 0.05$ & 0.21 \\
\hline \multirow[t]{2}{*}{ TR-10 } & Oct 1995 to Oct 1996 & 9 & 248.75 & 249.67 & $249.31 \pm 0.20$ & 0.30 \\
\hline & Jan 1991 to Sept 2003 & 82 & 248.31 & 249.74 & $249.17 \pm 0.07$ & 0.33 \\
\hline \multirow[t]{2}{*}{ Pond } & Oct 1995 to Oct 1996 & 17568 & 254.28 & 254.86 & $254.46 \pm 0.002$ & 0.11 \\
\hline & & 16 & 254.30 & 254.68 & $254.50 \pm 0.06$ & 0.11 \\
\hline M0 & Oct 1995 to Oct 1996 & 17 & 254.32 & 254.77 & $254.56 \pm 0.06$ & 0.13 \\
\hline M4 & Oct 1995 to Oct 1996 & 19 & 254.23 & 254.66 & $254.42 \pm 0.06$ & 0.12 \\
\hline M5 & Oct 1995 to Oct 1996 & 17 & 254.18 & 254.67 & $254.38 \pm 0.06$ & 0.13 \\
\hline \multirow[t]{2}{*}{ M8 } & Oct 1995 to Oct 1996 & 17298 & 254.09 & 254.82 & $254.34 \pm 0.002$ & 0.14 \\
\hline & & 19 & 254.13 & 254.60 & $254.35 \pm 0.06$ & 0.13 \\
\hline M9 & Oct 1995 to Oct 1996 & 19 & 254.13 & 254.64 & $254.35 \pm 0.07$ & 0.15 \\
\hline M11 & Oct 1995 to Oct 1996 & 19 & 254.09 & 254.57 & $254.31 \pm 0.06$ & 0.13 \\
\hline M12 & Oct 1995 to Oct 1996 & 19 & 254.00 & 254.54 & $254.24 \pm 0.06$ & 0.14 \\
\hline M13 & Dec 1995 to Dec 1996 & 15 & 253.97 & 254.39 & $254.18 \pm 0.06$ & 0.12 \\
\hline M14 & Oct 1995 to Oct 1996 & 19 & 253.89 & 254.35 & $254.11 \pm 0.06$ & 0.13 \\
\hline M16 & Oct 1995 to Oct 1996 & 18 & 253.94 & 254.46 & $254.18 \pm 0.06$ & 0.14 \\
\hline \multirow[t]{2}{*}{ MP-1 } & Oct 1995 to Oct 1996 & 4 & 254.51 & 254.77 & $254.65 \pm 0.12$ & 0.09 \\
\hline & Aug 1993 to July 1996 & 27 & 254.45 & 254.77 & $254.57 \pm 0.03$ & 0.12 \\
\hline \multirow[t]{2}{*}{ MP-2§ } & Oct 1995 to Oct 1996 & 0 & & & & \\
\hline & Aug 1993 to July 1996 & 19 & 251.40 & 251.70 & $251.57 \pm 0.04$ & 0.09 \\
\hline \multirow[t]{2}{*}{ MP-3§ } & Oct 1995 to Oct 1996 & 0 & & & & \\
\hline & Aug 1993 to Dec 1993 & 8 & 249.65 & 250.15 & $249.93 \pm 0.14$ & 0.20 \\
\hline MP-4§ & Oct 1995 to Oct 1996 & 0 & & & & \\
\hline
\end{tabular}




\begin{tabular}{|c|c|c|c|c|c|c|}
\hline $\begin{array}{l}\text { Calibration } \\
\text { point }\end{array}$ & Time period & $\begin{array}{l}\text { Number } \\
\text { of values }\end{array}$ & Minimum & Maximum & Mean \pm C.I $\dagger$ & $\mathrm{SD} \ddagger$ \\
\hline \multirow{3}{*}{ MP-5§ } & Aug 1993 to Aug 1995 & 22 & 248.02 & 248.25 & $248.14 \pm 0.01$ & 0.03 \\
\hline & Oct 1995 to Oct 1996 & 0 & & & & \\
\hline & Aug 2005 to Jan 2006 & 5 & 253.97 & 254.15 & $254.07 \pm 0.07$ & 0.08 \\
\hline \multirow[t]{2}{*}{ MP-6 } & Oct 1995 to Oct 1996 & 6 & 253.45 & 253.55 & $253.50 \pm 0.03$ & 0.04 \\
\hline & Aug 1993 to July 1996 & 32 & 253.37 & 253.96 & $253.48 \pm 0.06$ & 0.16 \\
\hline \multirow[t]{2}{*}{ MP-7§ } & Oct 1995 to Oct 1996 & 5 & 251.88 & 252.00 & $251.95 \pm 0.04$ & 0.05 \\
\hline & Aug 1993 to July 1996 & 33 & 251.55 & 252.00 & $251.93 \pm 0.05$ & 0.14 \\
\hline \multirow[t]{2}{*}{$\mathrm{C} 1 \S$} & Oct 1995 to Oct 1996 & 6 & 251.60 & 251.78 & $251.67 \pm 0.07$ & 0.07 \\
\hline & Aug 1993 to July 1996 & 34 & 251.42 & 252.63 & $251.71 \pm 0.02$ & 0.06 \\
\hline \multirow[t]{2}{*}{$\mathrm{C} 2 \S$} & Oct 1995 to Oct 1996 & 5 & 254.68 & 254.49 & $254.58 \pm 0.07$ & 0.08 \\
\hline & Aug 1993 to July 1996 & 24 & 254.20 & 254.90 & $254.47 \pm 0.05$ & 0.12 \\
\hline TP8-91 & Mar 1992 to Sept 2004 & 34 & 251.27 & 252.17 & $251.67 \pm 0.08$ & 0.23 \\
\hline SW13 & Apr 2002 to Sept 2004 & 5 & 249.2 & 249.53 & $249.50 \pm 0.11$ & 0.12 \\
\hline
\end{tabular}

Note: calibration points TP8-91 and SW13 are in Bridgewater Creek watershed.

$\dagger 95 \%$ confidence interval

\$ Standard deviation

$\S$ not used in estimating RMSE of calibration runs but used for qualitative comparison.

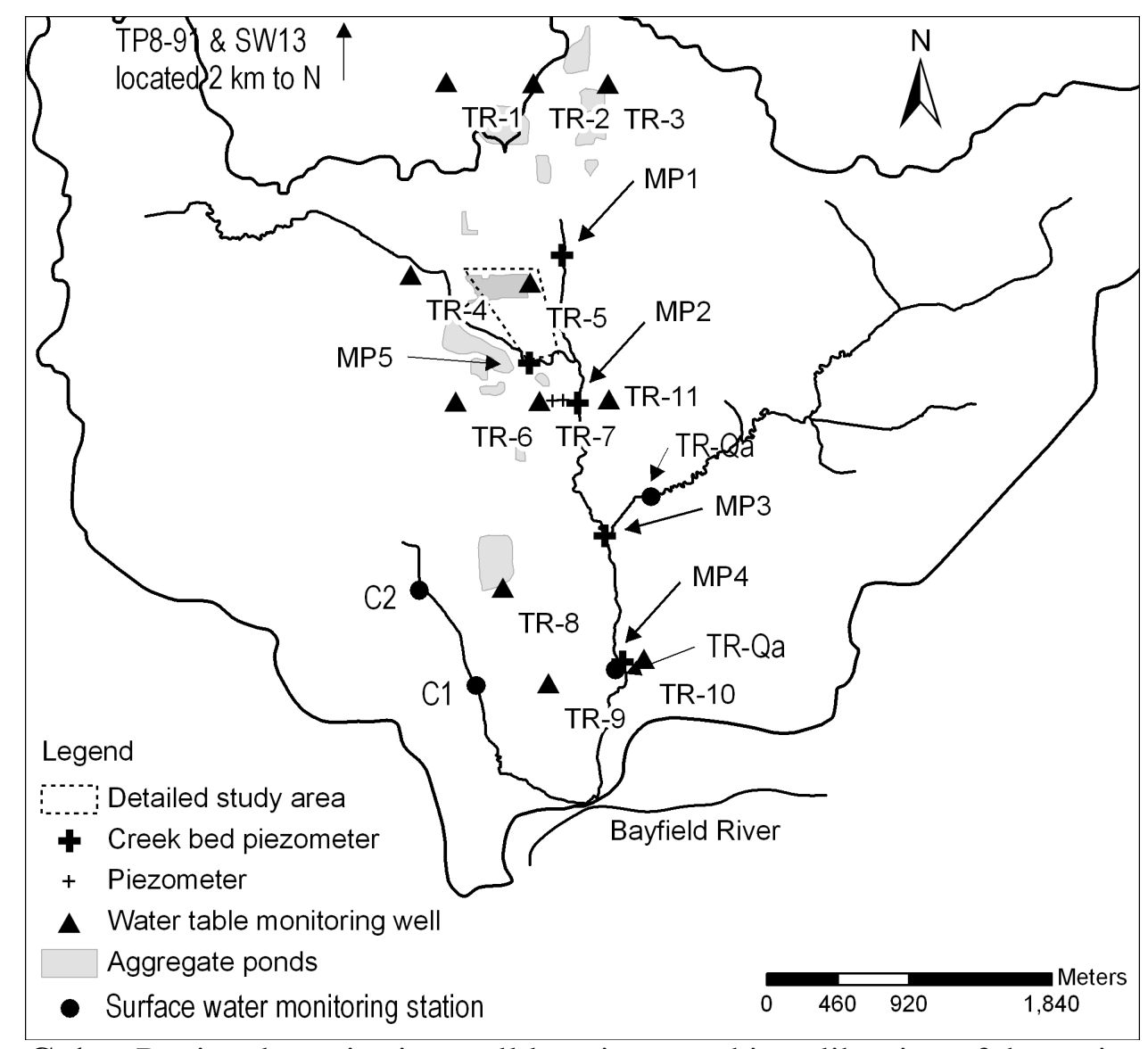

Figure G-1 Regional monitoring well locations used in calibration of the regional and watershed scale groundwater flow and transport models. 


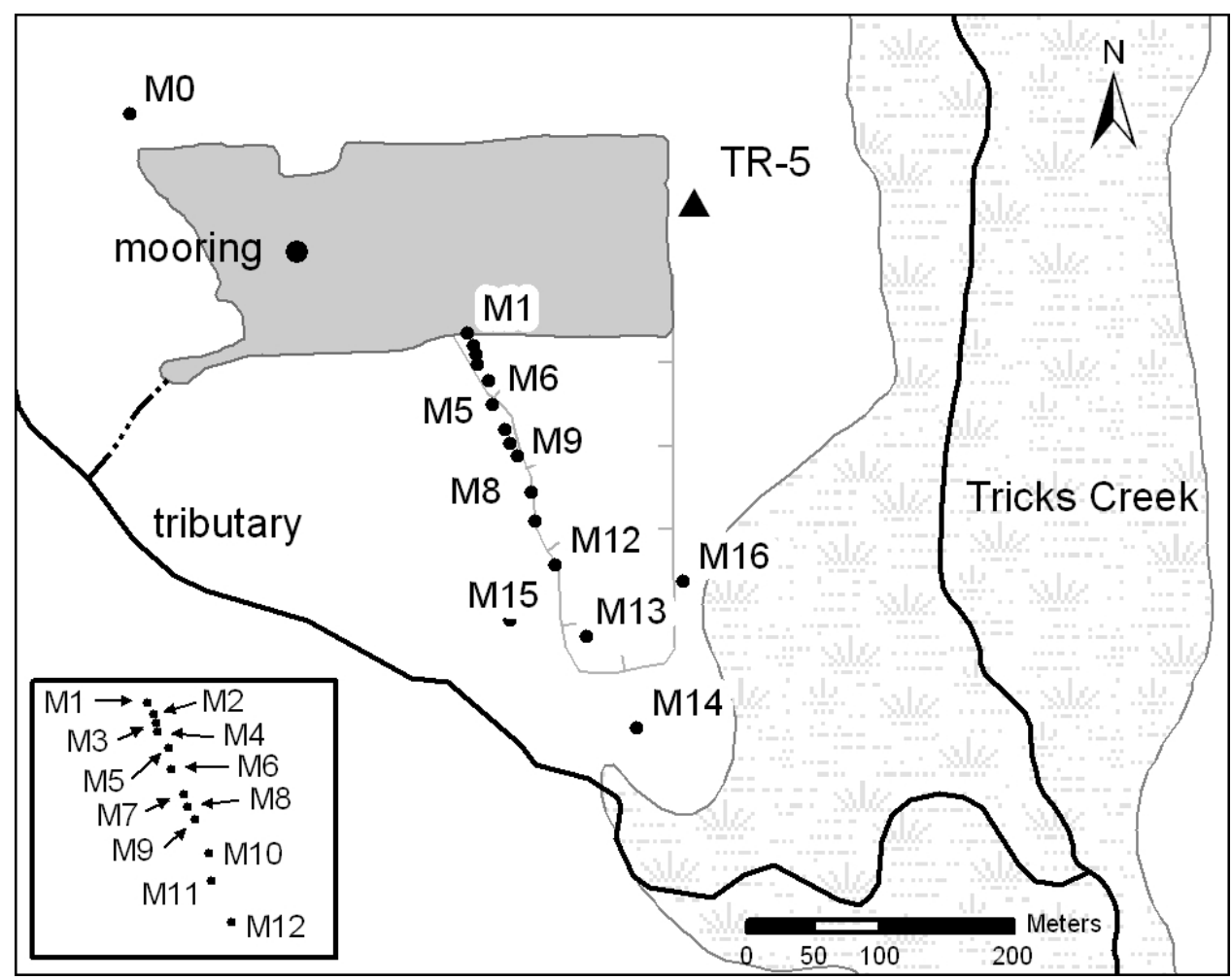

291

Figure G-2 Monitoring well locations in the detailed study area used in calibration of sub-grid groundwater flow and transport models. 
Steady-state groundwater flow simulations
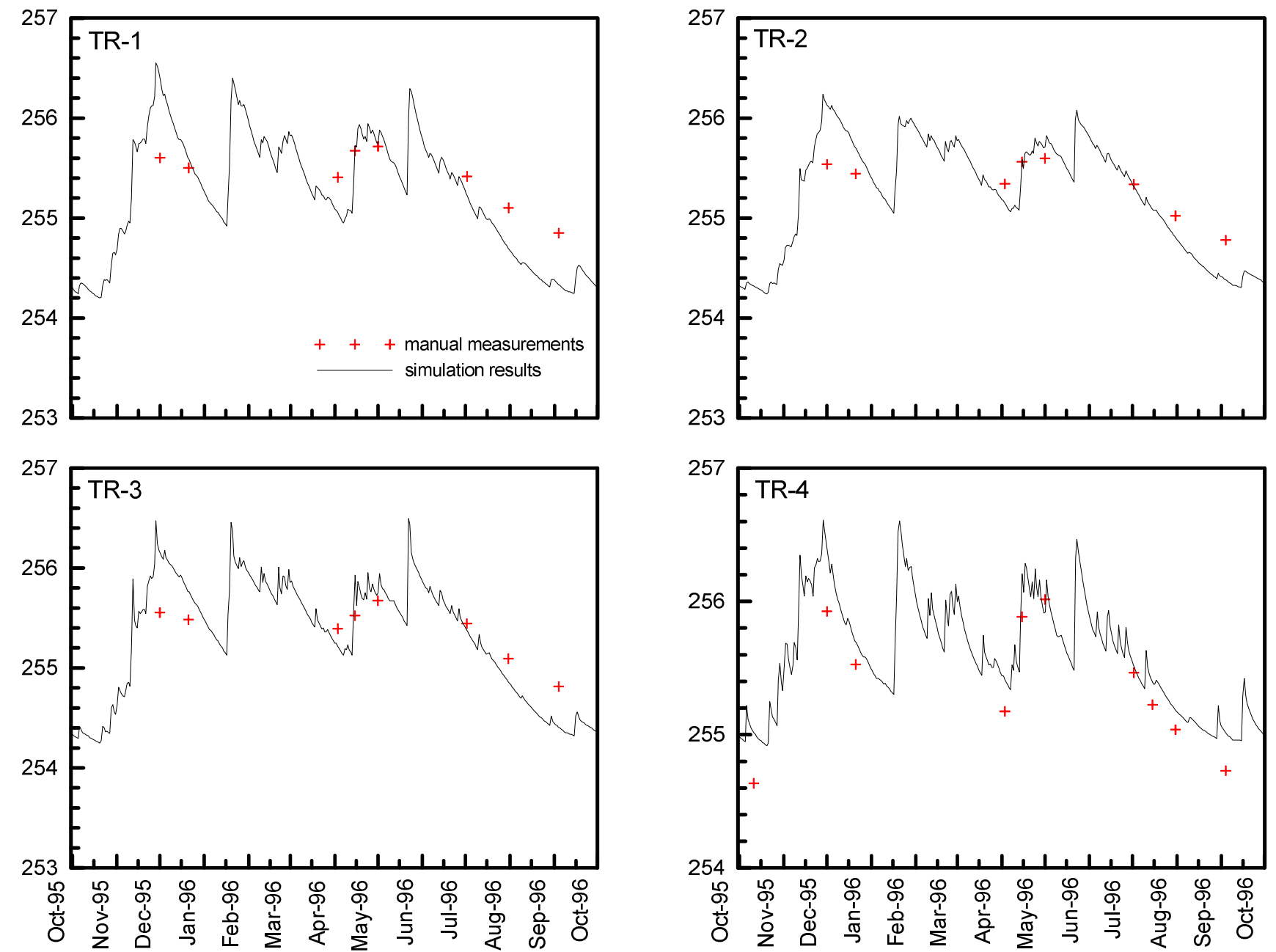

Figure G-3 Calibration plots of Model 2 for transient groundwater flow. 

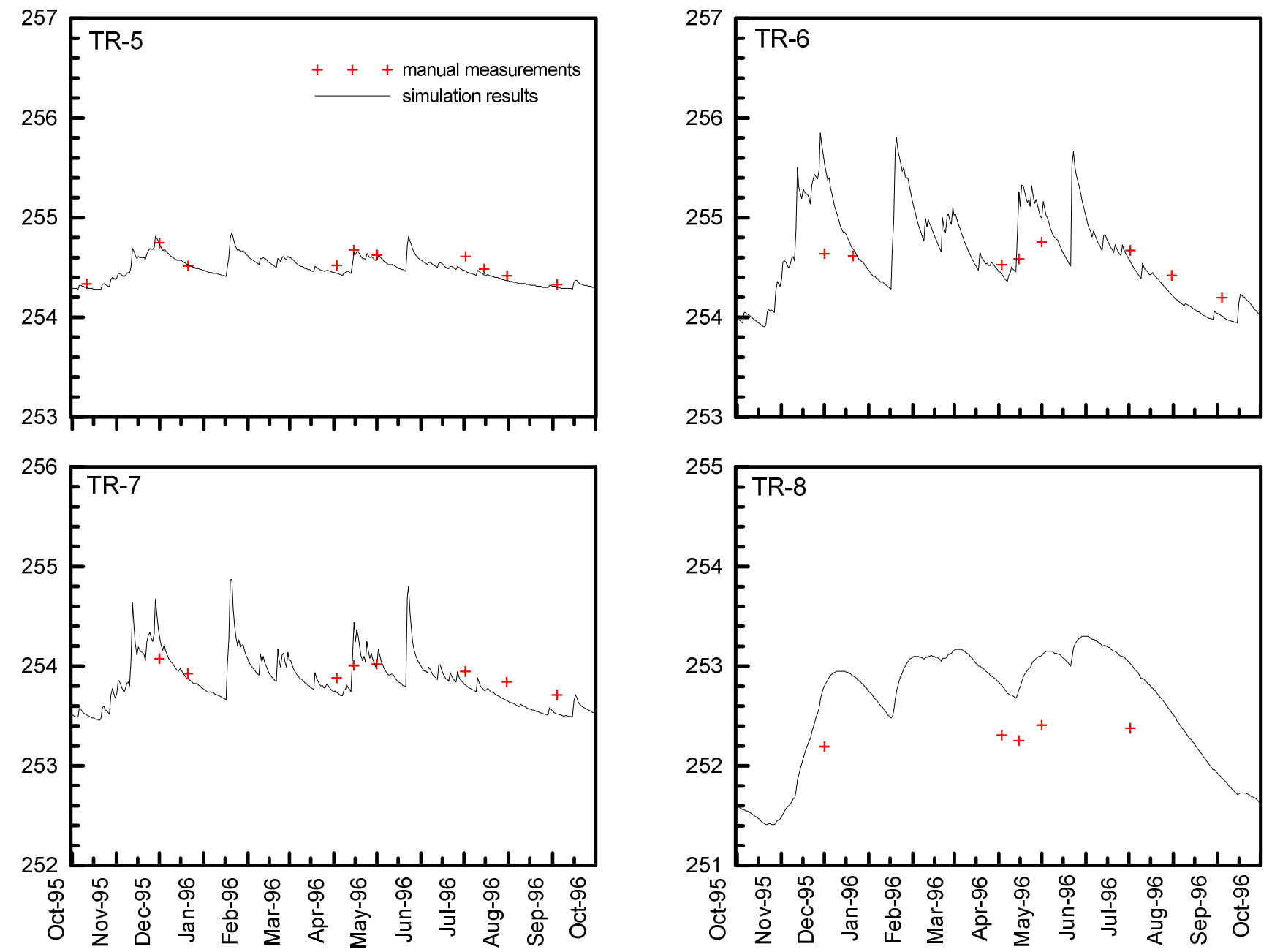

Figure G-4 Calibration plots of Model 2 for transient groundwater flow. 

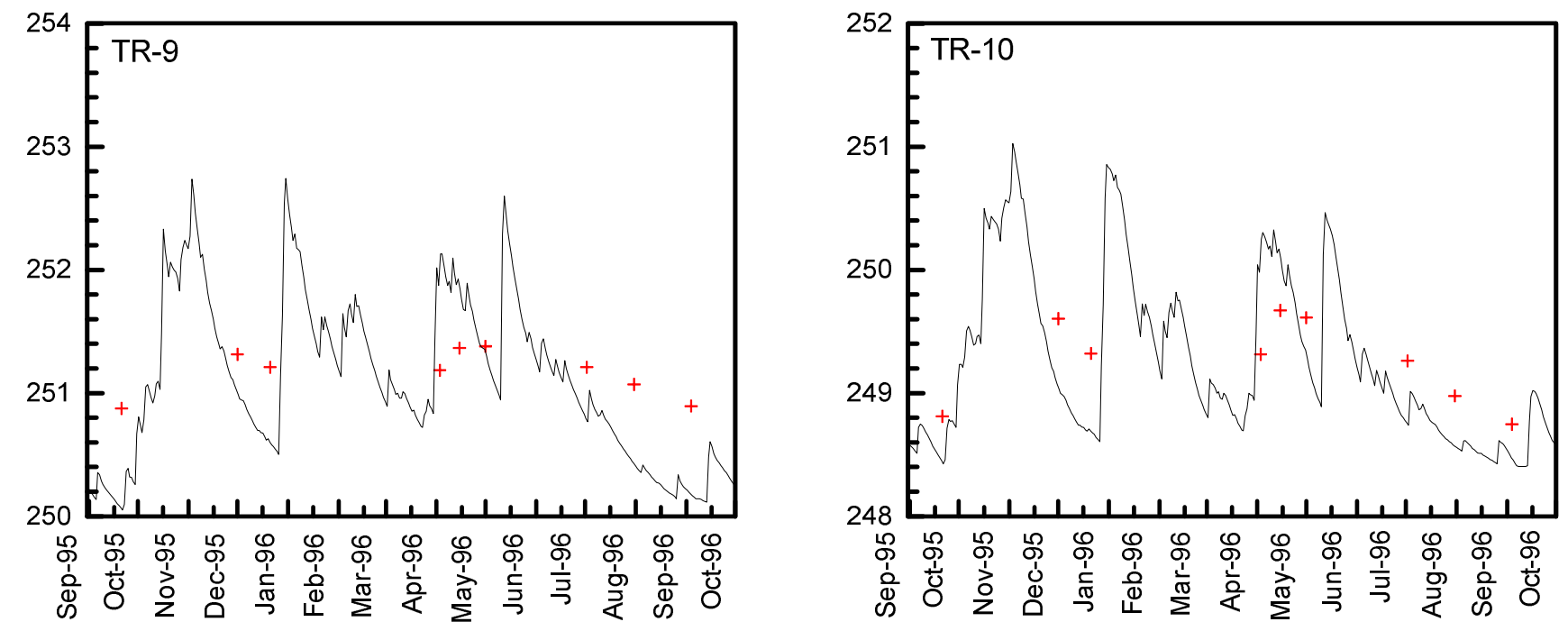

Figure G-5 Calibration plots of Model 2 for transient groundwater flow. 

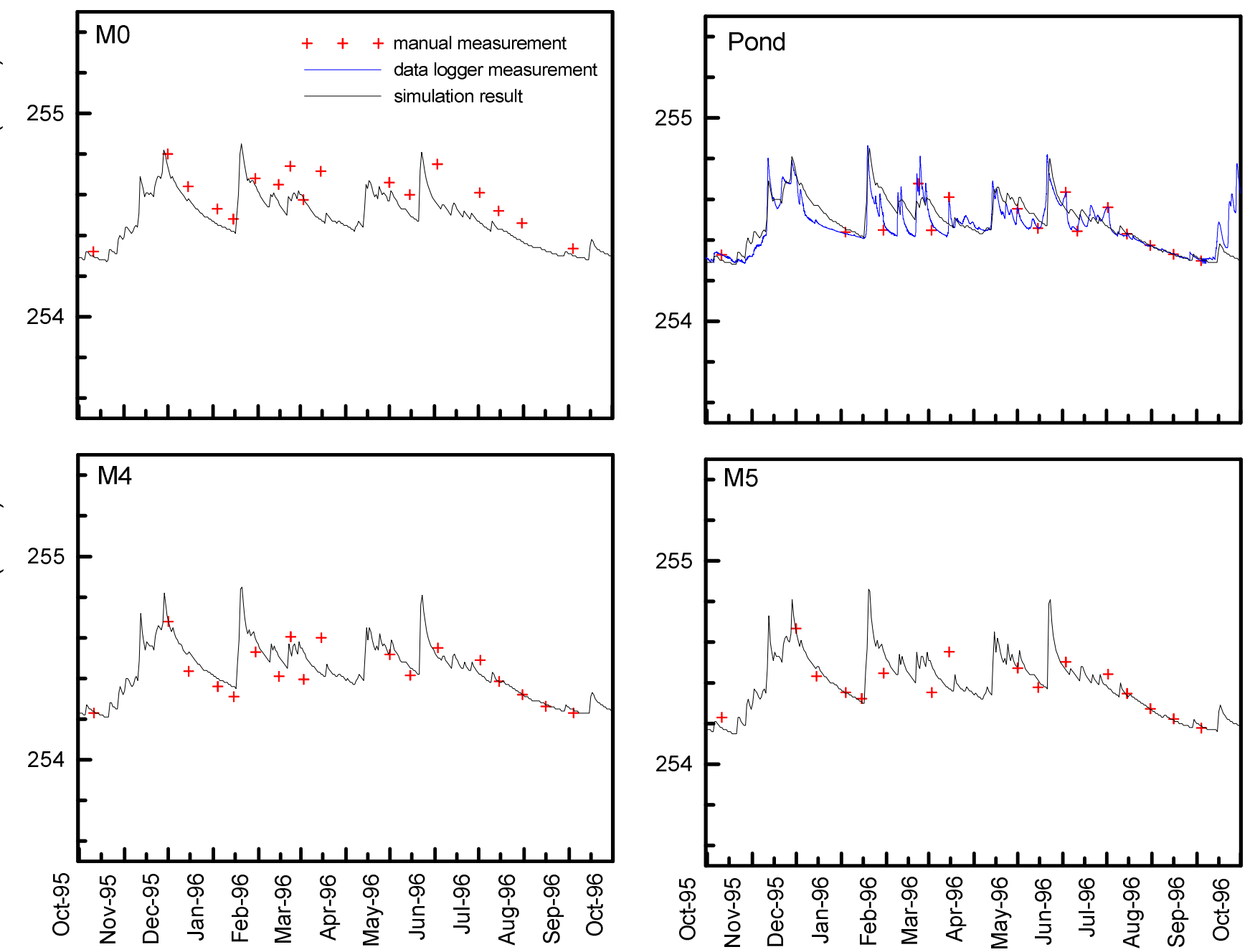

Figure G-6 Calibration plots of Model 2 for transient groundwater flow. 

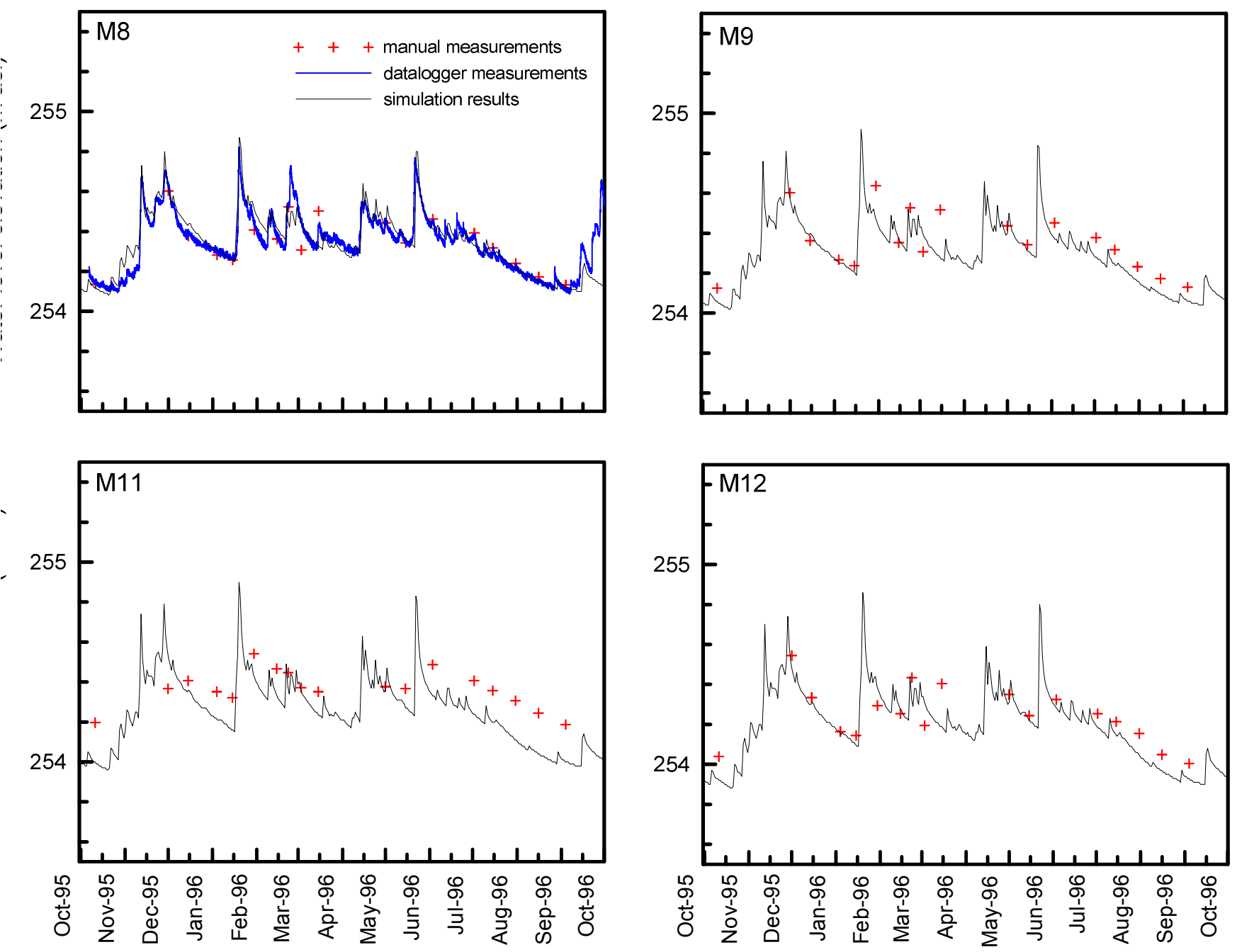

Figure G-7 Calibration plots of Model 2 for transient groundwater flow. 

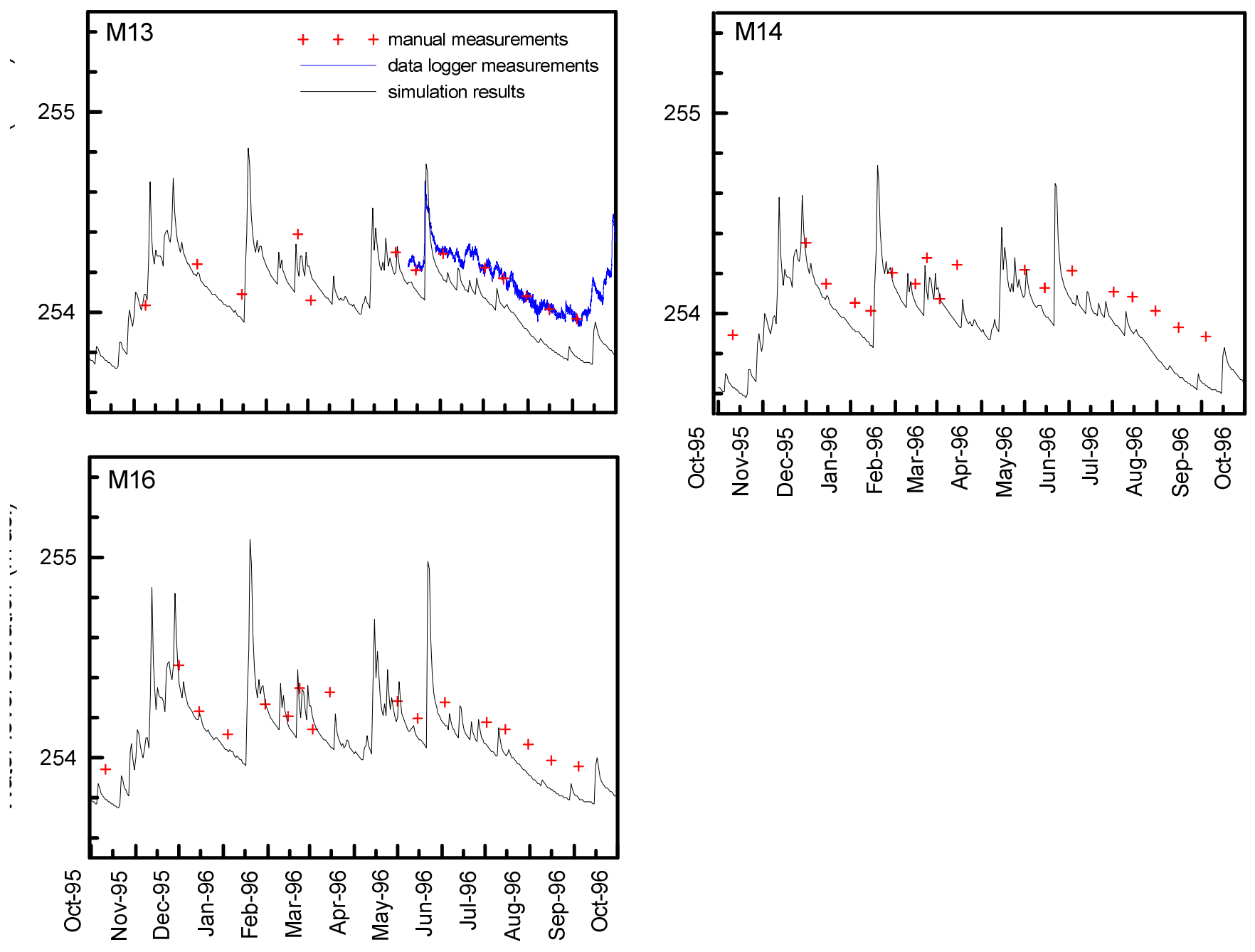

Figure G-8 Calibration plots of Model 2 for transient groundwater flow. 
Transient groundwater flow and thermal transport simulations
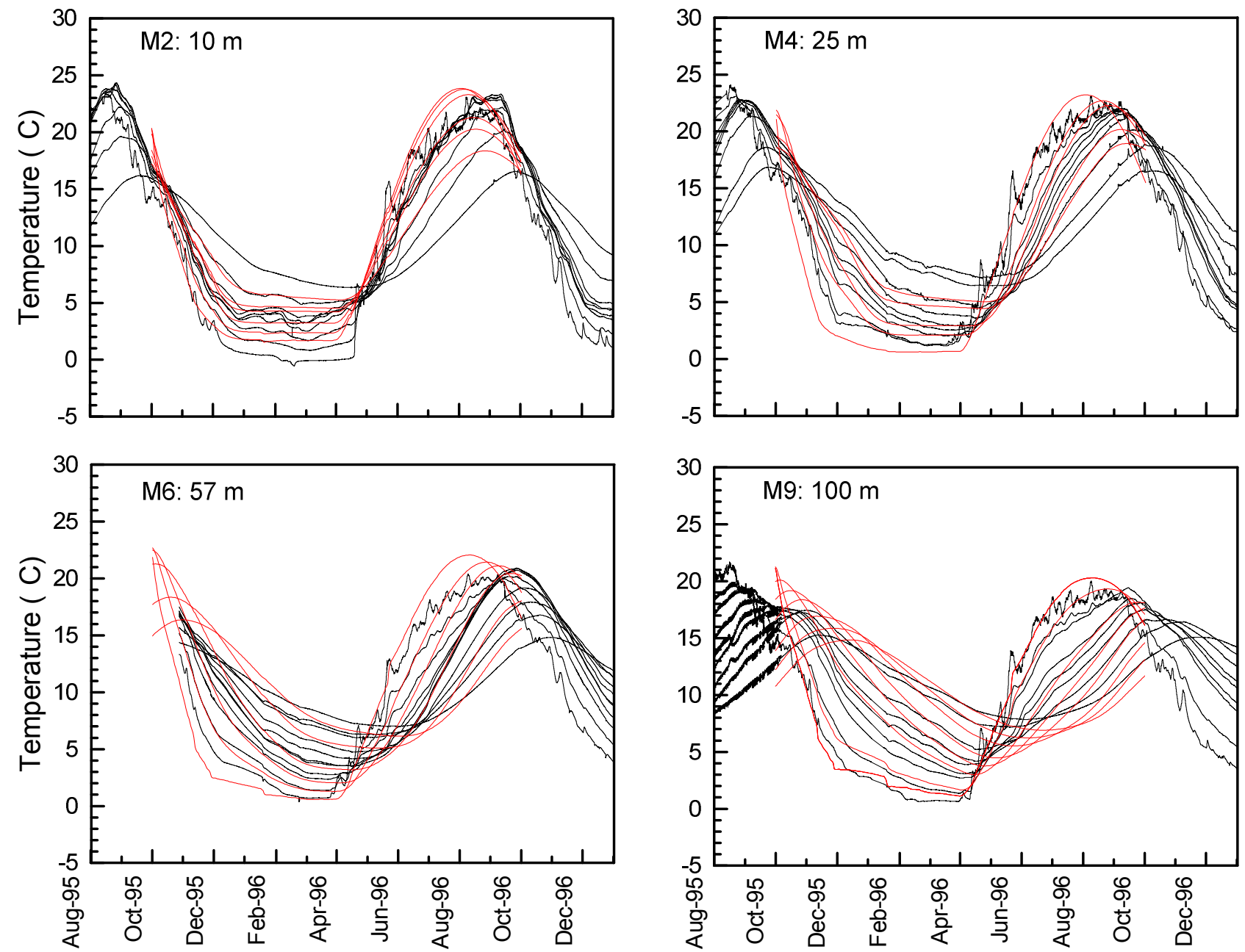

Figure G-9 Calibration plots of Model 4 for transient groundwater temperatures. 

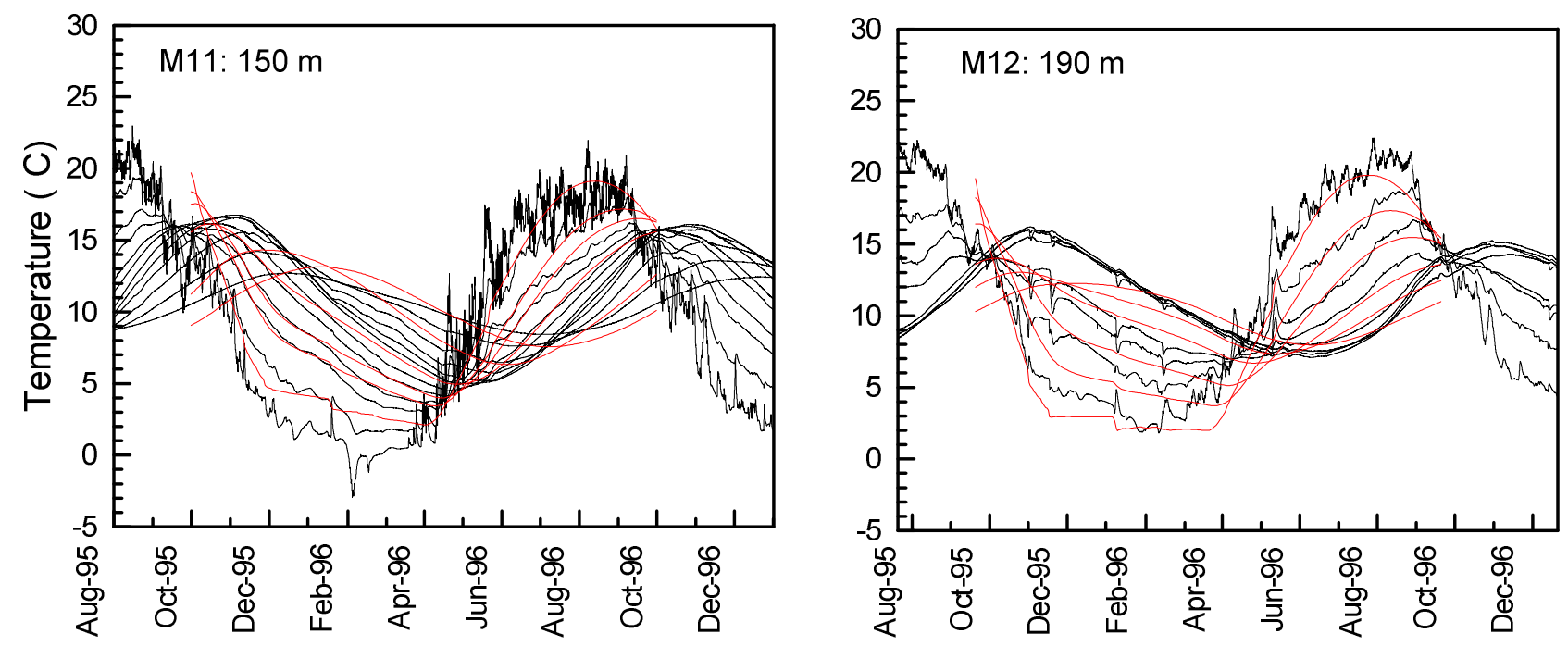

Figure G-10 Calibration plots of Model 4 for transient groundwater temperatures. 


\section{Curriculum Vitae}

Name:

Post-secondary

Education and Degrees:

Honours and

Awards:

Related Work Experience:
Jeffrey Markle

University of Waterloo

Waterloo, Ontario, Canada

1986 B.ASc. (Geological Engineering)

University of Western Ontario

London, Ontario, Canada

1993 M.Eng. (Geological Engineering)

2000-2002 OGSST Scholarship

2000 Student Research Grant

The Geological Society of America

2001 Student Research Grant

American Association of Petroleum Geologists

2001 Special University Scholarship (SUS)

University of Western Ontario

2001 Meloche Monnex / CCPE Scholarship

Ottawa, Ontario, Canada

2010 Amethyst Award - Ontario Public Service

Toronto, Ontario, Canada

1986-1988

Intera Technologies Ltd.

Environmental Engineer

Ottawa, Ontario, Canada

1988-2000

Ministry of the Environment

Hydrogeologist

London, Ontario, Canada

2000-2002

University of Western Ontario

$\mathrm{PhD}$. Candidate 
London, Ontario, Canada

Fall 2001

University of Western Ontario

Lecturer in Department of Earth Sciences

London, Ontario, Canada

2002-2010

Ministry of the Environment

Hydrogeologist

London, Ontario, Canada

2010-Present

Ministry of the Environment

District Engineer

London, Ontario, Canada

\section{Publications:}

\section{$\underline{\text { Peer Reviewed Journals }}$}

Markle, J.M., and R.A. Schincariol. 2007. Thermal plume transport from sand and gravel pits - Potential thermal impacts on cool water streams. Journal of Hydrology, doi:10.1016/j.jhydrol.2007.02.031, 338, $174-195$.

Markle, J.M., R.A. Schincariol, J.H. Sass and J.W. Molson. 2006. Characterization of the two-dimensional thermal conductivity distribution in a sand and gravel aquifer. Soil Science Society of America Journal, 70, 1281-1294.

Markle, J.M., R.K. Rowe and K.S. Novakowski. 1995. A Model for the Constant-Head Pumping Test Conducted in Vertically Fractured Media, International Journal for Numerical and Analytical Methods in Geomechanics, 19, 457-473.

\section{Conference Presentations}

Jones, D., R. McWatters, R.K. Rowe, and J.M. Markle. 2011. Migration of PCBs in a 24 year old PCB storage facility. paper submitted to Geo-Frontiers 2011 conference. Dallas, Texas, March 2011.

Jones, D., R. McWatters, R.K. Rowe, and J.M. Markle. 2010. PCB migration in a 25 year old PCB storage facility. paper accepted at $63^{\text {rd }}$ Canadian Geotechnical conference. Calgary, Alberta, 12-16 September 2010. 
Safari, E. R.K. Ro2

Safari, E., R.K. Rowe, and J.M. Markle. 2010. Antioxidants in a HDPE geomembrane used in a bottom liner and cover in a PCB containment landfill after 25 years. paper accepted at $63^{\text {rd }}$ Canadian Geotechnical conference. Calgary, Alberta, 12-16 September 2010.

Markle, J.M, R.A. Schincariol, J.H. Sass and J.W. Molson. 2004. Assessing the role of aquifer heterogeneity on heat transport in an unconfined aquifer, paper presented at the Geological Society of America annual meeting, Denver, Colorado, 10 November 2004.

Schincariol, R.A., G.A. Oldenborger, J. McNeil, and J.M. Markle. 2003. Assessing the spatial variability of hydraulic conductivity and its role in solute heat transport. paper presented at the Geological Society of America annual meeting, Seattle, Washington, 5 November 2003.

Schincariol, R.A., and J.M. Markle. 2002. Thermal ground water plume impacts on cold water streams from aggregate operations, paper presented at the Geological Society of America annual meeting, Denver, Colorado, 30 October 2002.

Markle, J.M., and R.A. Schincariol. 2001. A field investigation of temperature impacts from aggregate operations, 2001 A.D. Latornell Conservation Symposium - Water and Health: The Ripple Effect, 15 November 2001.

Neville, C.J., and J.M. Markle. 2000. Interpretation of constant-head tests: Rigorous and approximate analyses, First Joint IAH-CNC and CGS Groundwater Specialty Conference, Montréal, Québec, 15-18 October 2000.

Markle, J.M., R.K. Rowe and K.S. Novakowski. 1992. Analysis of Hydraulic Tests Conducted in Vertically Fractured Media, paper presented at the American Geophysical Union 1992 Fall Meeting, San Francisco, California, U.S.A., December 1992.

Essop, S. and J.M. Markle. 1989. Defining the Risks at Sites Affected by Ground Water Contamination, paper presented at the 3rd Annual Hazardous Materials Management Conference/Canada, Toronto, Ontario, Canada, September 1989. 